Universidade de São Paulo

Instituto de Astronomia, Geofísica e Ciências Atmosféricas

Departamento de Astronomia

Phillip Andreas Brenner Galli

\title{
Determinação de Distâncias Cinemáticas de Estrelas Pré-Sequência Principal em Regiões de Formação Estelar
}

São Paulo 

Phillip Andreas Brenner Galli

\section{Determinação de Distâncias Cinemáticas de} Estrelas Pré-Sequência Principal em Regiões de Formação Estelar

Tese apresentada ao Departamento de Astronomia do Instituto de Astronomia, Geofísica e Ciências Atmosféricas da Universidade de São Paulo como requisito parcial para a obtenção do título de Doutor em Ciências.

Área de Concentração: Astronomia

Orientador: Prof. Dr. Ramachrisna Teixeira

São Paulo 

À minha família. 



\section{Agradecimentos}

Uma tese de doutorado é resultado de muito tempo dedicado ao estudo e da colaboração de diversas pessoas. Quiseram as circunstâncias que tudo conspirasse a favor para que este trabalho se desenvolvesse bem desde o início. Eu sou imensamente grato a tudo que aconteceu ao longo dos últimos anos e da forma como ocorreu. Aproveito este momento para prestar os meus mais sinceros agradecimentos a todos aqueles que contribuíram de forma direta ou indireta para que esta tese se desenvolvesse.

Aos meus pais, Vladimir e Susanne, pelo amor, educação, apoio, incentivo e por garantir todas as condições necessárias para que eu escolhesse o meu caminho e lutasse até o fim. Obrigado pelo exemplo de pessoa, esforço, dedicação e competência nos quais me espelhei em muitos momentos da minha vida. À minha irmã, Catherine, e ao Bruno pelas conversas e o apoio incondicional em todos momentos que sempre precisei.

À Kelly, por estar sempre ao meu lado compartilhando os bons e os maus momentos da vida e por me apoiar nas minhas decisões. Obrigado pelo amor e paciência sobretudo no final desta tese e por aceitar que em muitos momentos eu tivesse que dedicar mais tempo ao trabalho.

Aos meus avós e tios, pelas conversas e o incentivo que sempre me proporcionaram. Obrigado por entenderem a minha ausência em muitos momentos importantes.

Ao meu orientador, Dr. Ramachrisna Teixeira, pela orientação no trabalho, amizade, estímulo e apoio constante. Obrigado por me introduzir na vida acadêmica e na astronomia profissional desde os meus primeiros anos da faculdade. Se não fosse por isso, eu estaria em outro lugar menos feliz e também menos realizado com o meu trabalho. 
À Dra. Christine Ducourant e ao Dr. Claude Bertout pela colaboração e amizade ao longo desta tese de doutorado. Obrigado por me acolherem muito bem em Bordeaux e Paris, pelo tempo e atenção dedicados a este projeto, pelos conselhos e conversas sempre muito produtivas e pelo exemplo de pesquisa que desenvolvem em astronomia.

Ao Dr. Johny Setiawan e Maren Mohler do Instituto Max-Planck (MPIA), por terem me recebido gentilmente em seu instituto e ajudado com a redução de dados e técnicas para o cálculo da velocidade radial.

Ao Dr. Andreas Quirrenbach e Dra. Sabine Reffert do Observatório de Heidelberg pelo tempo dedicado em discutir os resultados deste trabalho comigo e por ampliarem os meus horizontes de pesquisa.

Aos meus professores da graduação e pós-graduação, em particular do IAG/USP, que sempre se dedicaram ao máximo nos cursos ministrados. Obrigado Augusto, Beatriz, Boczko, Cesar, Enos, Gastão, Jacques, Jane, Janot, Nelson, Rama, Roberto, Ronaldo, Ruth, Sandra, Silvia, Thais e Zulema, pois o que sei de astronomia aprendi com vocês. Aos pesquisadores Jorge, Sylvio, Tatiana e Vera, com os quais infelizmente não tive a oportunidade de fazer um curso. Obrigado pela amizade e exemplo de pesquisador que sempre me motivaram ao longo doutorado. Ao Paulo e Michel, pelas conversas sobre ponto de convergência que muito me ajudaram para desenvolver uma parte importante deste trabalho. Ao Enos, por acompanhar o meu trabalho como relator do projeto e garantir que tudo caminhasse bem.

Aos colegas da pós-graduação, pela amizade, ajuda e conversas que contribuíram para que este momento fosse único e especial. Obrigado Alberto, Ana, Andressa F. Andressa J., Beatriz, Bruno, Cristiano, Daniel M., Daniel R., Eduardo, Felipe, Fernanda, Graziella, Luciene, Mairan, Oscar, Paulo, Pedro, Rafael, Rodolfo, Rodrigo, Vinicius, Tiago e Thais. Ao Bruno por dividir a organização do Journal Club comigo e ao Rodrigo por me substituir em alguns momentos no final desta tese. 
Ao Departamento de Astronomia do IAG/USP por garantir a estrutura necessária para o desenvolvimento desta tese através do pessoal de suporte técnico e secretariado. Obrigado Ana Carolina, Cida, Conceição, Luís, Marcel, Marcos, Marina, Patricia, Regina e Ulisses.

Aos funcionários do Observatório Abrahão de Moares, em especial ao Messias cujo trabalho observacional está certamente incluído nesta tese de doutorado.

À FAPESP pela bolsa de doutorado direto (2008/50074-2) concedida para este projeto e o apoio financeiro para a realização de estágios de pesquisa no exterior e a participação em reuniões científicas. Ao ESO pelo suporte financeiro e tempo de telescópio destinado às observações realizadas neste trabalho. 

"O período de maior ganho em conhecimento e experiência é o período mais difícil da vida de alguém."

Tenzin Gyatso

(Dalai Lama) 



\section{RESUMO}

Este trabalho tem como objetivo principal a determinação da distância de estrelas présequência principal em regiões de formação estelar próximas. A determinação precisa da distância individual das estrelas é necessária para obter os principais parâmetros físicos de cada estrela e para investigar a estrutura da Galáxia. Em particular, investigamos as regiões de formação estelar de Lupus e Ophiuchus que contém uma das associações mais ricas em estrelas $\mathrm{T}$ Tauri. A grande maioria das estrelas pré-sequência principal nessas regiões não foi observada pelo satélite Hipparcos devido à sua magnitude e também não têm paralaxe trigonométrica medida a partir do solo devido à distância em que se encontram.

O procedimento aqui empregado para a obter a distância individual das estrelas baseiase na estratégia de ponto de convergência e utiliza dados de movimento próprio e velocidade radial. Desenvolvemos uma nova versão do método de ponto de convergência que permite simultaneamente determinar a posição do ponto de convergência e selecionar os membros de um moving group. Partindo dos dados de movimento próprio e o novo método aqui desenvolvido investigamos as propriedades cinemáticas e realizamos uma análise de pertinência das estrelas em cada região estudada o que nos permitiu identificar um moving group com 114 estrelas em Lupus e 55 estrelas em Ophiuchus. Calculamos a distância para cada membro do grupo usando velocidades radiais publicadas, que foram complementadas com novas observações, e a velocidade espacial do moving grup para as estrelas com velocidade radial não conhecida. Calculamos as paralaxes com precisão de 1-2 mas o que implica em um erro relativo médio de $25 \%$ nas distâncias obtidas.

Finalmente, investigamos as propriedades dos diversos subgrupos e a estrutura tridimensional dos complexos de nuvens em Lupus e Ophiuchus, concluindo que existem efeitos de profundidade importantes. Utilizamos os novos resultados de distância para obter os parâmetros físicos (luminosidade, massa e idade) das estrelas e o diagrama-HR de cada região de formação estelar considerada, confirmando a distribuição de idade diferente das duas subclasses de estrelas $\mathrm{T}$ Tauri. Os resultados aqui obtidos representam um primeiro passo no sentido de melhor entender a estrutura das regiões de formação estelar e os estágios iniciais da formação de estrelas e planetas. 



\section{Abstract}

The main objective of this work is to determine the distance of pre-main sequence stars in nearby star-forming regions. A precise determination of the distance to individual stars is required to accurately determine the main physical parameters of each star and the structure of the Galaxy. Here we investigate the Lupus and Ophiuchus star-forming regions that contain one of the richest associations of T Tauri stars. Most pre-main sequence stars in these regions were neither observed by the HipPARCOs satellite due to their magnitude nor have any trigonometric parallax measured from the ground due to their distance.

The procedure that we use here to derive the distance to individual stars is based on the convergent point strategy and makes full use of proper motion and radial velocity data. We developed a new version of the convergent point search method that simultaneously determines the convergent point position and selects the most likely members of a moving group. Based on proper motion data and our new method we investigate the kinematic properties and perform a membership analysis of the stars in each star-forming region considered that allows us to identify a moving group with 114 stars in Lupus and 55 stars in Ophiuchus. We calculate the distance of each group member using published radial velocities, which we supplemented with new measurements, and the spatial velocity of the moving group for the remaining stars with unknown radial velocity. We derived parallaxes with accuracies of 1-2 mas yielding the average relative error of $25 \%$ on the distances.

Finally, we investigate the properties of the various subgroups and the three dimensional structure of the Lupus and Ophiuchus cloud complex and conclude that significant depth effects exist. We use the new distances to refine the physical parameters (luminosity, mass and age) of stars and the HR-diagram for each star-forming region considered confirming

the different age distribution of the two $\mathrm{T}$ Tauri subclasses. These results represent a first step towards better understanding the structure of star-forming regions and the early stages of star and planet formation. 



\section{SUMÁRIO}

1. Introdução . . . . . . . . . . . . . . . . . . . . . . . . . . . . . 19

2. Método do Ponto de Convergência . . . . . . . . . . . . . . . . . 25

2.1 Introdução . . . . . . . . . . . . . . . . . . . . . . 26

2.2 O Método Clássico (Jones 1971; de Bruijne 1999a) . . . . . . . . . . . . . . 29

2.2.1 Princípio de decomposição do movimento próprio . . . . . . . . . . 29

2.2.2 Algoritmo de Jones (1971) . . . . . . . . . . . . . . 30

2.2.3 Algoritmo de de Bruijne (1999a) . . . . . . . . . . . . . . . 31

2.3 Um Novo Método (Galli et al. 2012) . . . . . . . . . . . . . . . . . 33

2.3.1 Representação por grande círculo . . . . . . . . . . . . . . . . . 33

2.3.2 Algoritmo . . . . . . . . . . . . . . . . . 35

2.3.3 Correção devido à dispersão de velocidade . . . . . . . . . . . . . . 38

2.3.4 Otimização do método de ponto de convergência . . . . . . . . . . . 39

2.4 Simulações . . . . . . . . . . . . . . . . . . . . . . . . . . . . . . . 40

2.4.1 Construção de dados sintéticos . . . . . . . . . . . . . . . . . 40

2.4.2 Seleção dos membros e rejeição das estrelas de campo ...... . 42

2.4.3 Análise da posição do ponto de convergência . . . . . . . . . . . . . 45

2.4.3.1 Efeito da dispersão de velocidade . . . . . . . . . . 45

2.4.3.2 Precisão no ponto de convergência . . . . . . . . . . . . 47

2.4.4 Escolha de $\epsilon_{\min } \ldots \ldots \ldots$. . . . . . . . . . . . . . . . . . 49

2.4.5 Teste de normalidade da distribuição de $s \ldots . .52$

2.5 Aplicação ao Aglomerado Aberto das Híades . . . . . . . . . . . . . . . . . 53 
2.5.1 Matriz de covariância . . . . . . . . . . . . . 53

2.5.2 Análise do ponto de convergência . . . . . . . . . . . . . 54

2.5.3 Paralaxes cinemáticas . . . . . . . . . . . . . 56

2.6 Conclusão . . . . . . . . . . . . . . . . . . . . . . . . . . . . . . 58

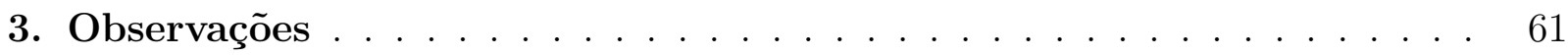

3.1 Programa Observacional . . . . . . . . . . . . . . . . . . . 62

3.2 Velocidade Radial . . . . . . . . . . . . . . . . . . . . . . . 63

3.3 Classificação do Perfil $\mathrm{H} \alpha$. . . . . . . . . . . . . . . . . . . . 66

3.4 Largura Equivalente do $\mathrm{Li} \mathrm{e} \mathrm{H} \alpha \ldots \ldots$. . . . . . . . . . . . . . 68

3.5 Binárias Espectroscópicas _ . . . . . . . . . . . . . . . . . . . . . . . 69

3.6 Sumário . . . . . . . . . . . . . . . . . . . . . . . . . . . . . 72

4. Um Estudo Cinemático da Região de Formação Estelar de Lupus . . . 73

4.1 Introdução . . . . . . . . . . . . . . . . . . . . . . . . . 74

4.2 Estrelas Pré-Sequência Principal em Lupus . . . . . . . . . . . . . . . . . . 76

4.2.1 Levantamento das estrelas candidatas . . . . . . . . . . . . . 76

4.2 .2 Movimentos próprios . . . . . . . . . . . . . . 76

4.2 .3 Divisão da amostra . . . . . . . . . . . . . . . . . . . . . . 77

4.2.4 Velocidades radiais . . . . . . . . . . . . . . . . . . 82

4.3 Análise do Ponto de Convergência . . . . . . . . . . . . . . . 83

4.3.1 Resultados preliminares . . . . . . . . . . . . . . . . . 84

4.3.2 Centaurus Superior-Lupus (UCL) . . . . . . . . . . . . . . 85

4.3.3 Lupus (dentro das nuvens) . . . . . . . . . . . . . 85

4.3.4 Lupus (fora das nuvens) . . . . . . . . . . . . . 86

4.3.5 Lupus (solução final) . . . . . . . . . . . . . . . . . . . 86

4.3.6 Simulações Monte Carlo . . . . . . . . . . . . . . . . 87

4.4 Distâncias . . . . . . . . . . . . . . . . . . . . . . 90

4.4.1 Comentário sobre as velocidades radiais . . . . . . . . . . . . . . . 90

4.4.2 Paralaxe calculada a partir da velocidade radial . . . . . . . . . . 91

4.4.3 Paralaxe calculada a partir da velocidade espacial . . . . . . . . 93

4.4.4 Comparação com o catálogo Hipparcos . . . . . . . . . . . . . . 95 
4.5 Discussão . . . . . . . . . . . . . . . . . . . . . . . . . . . . . . 101

4.5.1 Paralaxe das subclasses de estrelas pré-sequência principal . . . . . 101

4.5.2 Paralaxe dos subgrupos de Lupus . . . . . . . . . . . . . . . 103

4.5.3 Distribuição espacial das estrelas . . . . . . . . . . . . . . 107

4.5.4 Velocidade espacial das estrelas . . . . . . . . . . . . . . . 109

4.6 Conclusão . . . . . . . . . . . . . . . . . . . . . . . . . . . . . . . . . 110

5. Cinemática das Estrelas Pré-Sequência Principal em Ophiuchus . . 111

5.1 Introdução . . . . . . . . . . . . . . . . . . . . . . . . . . . . 112

5.2 Estrelas Pré-Sequência Principal em Ophiuchus . . . . . . . . . . . . . . . 114

5.2.1 Levantamento das estrelas candidatas . . . . . . . . . . . . . . . . 114

5.2 .2 Movimentos Próprios . . . . . . . . . . . . . . . . . . . . 114

5.2 .3 Velocidades Radiais . . . . . . . . . . . . . . . . . . . . . . . 118

5.3 Análise do Ponto de Convergência . . . . . . . . . . . . . . . . . . . . . . . 119

5.3 .1 Scorpius Superior (US) . . . . . . . . . . . . . . . 120

5.3 .2 Ophiuchus . . . . . . . . . . . . . . . . . 120

5.3 .3 Simulações Monte Carlo . . . . . . . . . . . . . . . . . . . . . . 121

5.4 Distâncias . . . . . . . . . . . . . . . . . . . . . . . . . . . . . . 123

5.4.1 Paralaxe calculada a partir da velocidade radial . . . . . . . . . 123

5.4.2 Paralaxe calculada a partir da velocidade espacial . . . . . . . . 125

5.4.3 Comparação com o catálogo Hipparcos . . . . . . . . . . . 126

5.5 Discussão . . . . . . . . . . . . . . . . . . . . . . . . . . . . . 130

5.5.1 Paralaxe das subclasses de estrelas pré-sequência principal $\ldots . .130$

5.5.2 Distribuição espacial das estrelas . . . . . . . . . . . . . . . . . . 132

5.5 .3 Lynds $1688(\mathrm{~L} 1688) \ldots \ldots \ldots \ldots \ldots$

5.5.4 Velocidade espacial das estrelas . . . . . . . . . . . . . 135

5.6 Conclusão . . . . . . . . . . . . . . . . . . . . . . . . . . . . . . . 136

6. Propriedades Físicas das Estrelas Jovens em Lupus e Ophiuchus . . . 137

6.1 Introdução . . . . . . . . . . . . . . . . . . . . . . . . . 138

6.2 Luminosidade . . . . . . . . . . . . . . . . . . . . . . . . 141

6.3 Diagrama-HR . . . . . . . . . . . . . . . . . . . . . . . . . . . 142 
6.4 Massa e Idade . . . . . . . . . . . . . . . . . . . . . . . 146

6.5 Comparação com Taurus-Auriga . . . . . . . . . . . . . . . . 153

6.6 Simulação do Diagrama-HR . . . . . . . . . . . . . . . . . 158

6.7 Distância Individual vs. Distância Média . . . . . . . . . . . . . . . . 161

6.8 Conclusão . . . . . . . . . . . . . . . . . . . . . 164

7. Considerações Finais . . . . . . . . . . . . . . . 165

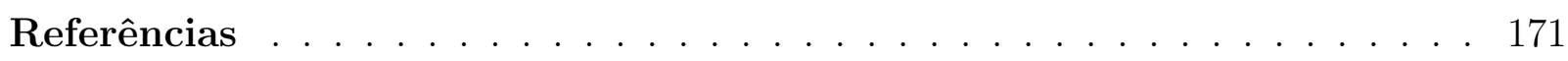

$\begin{array}{ll}\text { Apêndice } & 189\end{array}$

A. Lista de Acrônimos . . . . . . . . . . . . . . . . . . . . . . . 191

B. A new method for calculating the convergent point of a moving group 193

C. A kinematic study and membership analysis of the Lupus star-forming

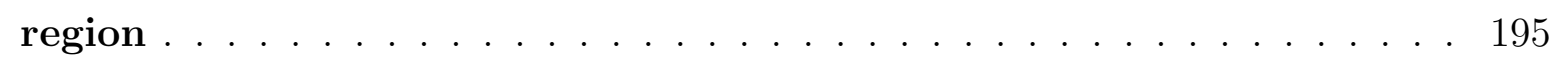

D. Proper motion and densification of the International Celestial Reference Frame in the direction of the Galactic bulge . . . . . . . . . . . . . 197 
Capítulo 1

\section{INTRODUÇÃO}

"A dúvida é o princípio da sabedoria."

Aristóteles

A astronomia tem como base as observações que fazemos dos astros desde aquelas mais rudimentares em um passado remoto até as mais sofisticadas que realizamos hoje em dia a partir do solo e do espaço. Quando medimos o brilho, a posição e o diâmetro dos astros obtemos grandezas aparentes cuja conversão em valores absolutos exige necessariamente o conhecimento da distância.

A distância é uma das grandezas mais fundamentais na astronomia, pois nos permite determinar parâmetros estelares de extrema importância e estimar as dimensões do Uni-

verso. É através da distância que podemos visualizar a distribuição espacial das estrelas, aglomerados de estrelas e consequentemente a estrutura da própria Galáxia. Conhecendo a distância de uma estrela calculamos a quantidade de energia que ela emite, ou seja, a sua luminosidade. Mais adiante, partindo da luminosidade obtemos os dois parâmetros físicos mais importantes na caracterização de uma estrela: massa e idade. Enquanto a massa da estrela determina a sua evolução, a idade nos permite identificar o estágio evolutivo em que se encontra. É também pelo conhecimento da distância que podemos calcular o movimento espacial das estrelas e investigar as propriedades cinemáticas e dinâmicas de grupos de estrelas e da Galáxia como um todo. 
A determinação da distância de uma estrela é uma tarefa difícil, mas pode ser alcançada por diversas estratégias. Ao observar uma estrela próxima ao longo do tempo, notamos que ela realiza um pequeno movimento de vaivém no céu com relação às estrelas "fixas" de fundo. A diferença de ângulo sob a qual a estrela é observada entre duas posições da Terra em sua órbita ao redor do Sol recebe o nome de paralaxe estelar, paralaxe trigonométrica ou simplesmente paralaxe. A maneira mais direta de obter a distância é a medida da paralaxe trigonométrica que usa o ângulo de paralaxe e a distância da Terra ao Sol. Essa medida quando realizada a partir do solo exige no mínimo um ano de observações distribuídas ao longo do tempo e fornece resultados com precisão em até 20\% (Ducourant et al. 2007). O satélite Hipparcos (ESA 1997) provocou uma revolução na determinação de paralaxes trigonométricas observando aproximadamente 120 mil estrelas. As expectativas são ainda muito mais audaciosas para a missão espacial GAIA que deverá observar 1 bilhão de estrelas na Galáxia e tem lançamento previsto para 2013. ${ }^{1}$ Hoje existem cerca de 30 mil estrelas com precisão em até 10\% nas paralaxes trigonométricas oriundas da missão HipPARCos. Estima-se que 150 milhões de estrelas terão a mesma precisão com os dados da missão GAIA (Turon et al. 2012). Uma variante do método da paralaxe trigonométrica tem sido recentemente empregada usando técnicas de radio-interferometria com grande linha de base (VLBA, do inglês Very Long Baseline Array) e fornece uma precisão em até $5 \%$ nas paralaxes obtidas (Loinard et al. 2008; Torres et al. 2009, 2012; Dzib et al. 2010, 2011).

A medida de distâncias têm privilegiado sobretudo as estrelas mais próximas e brilhantes. Isso é explicado por fatores observacionais, pois em geral é menos difícil observar as estrelas mais brilhantes e o alto brilho, por si só, já é um indicativo de proximidade. Pouco progresso recente tem sido feito com relação à determinação da distância de estrelas jovens (e fracas) que apresentam interesse particular no estudo dos estágios iniciais da formação de estrelas e planetas. Dentro desse contexto inclui-se, por exemplo, a população de estrelas T Tauri (TTS, do inglês T Tauri star) que foram descobertas por Joy (1945). As TTSs são estrelas pré-sequência principal (PMS, do inglês pre-main sequence) de baixa massa (até $2 M_{\odot}$ ) e tipo espectral tardio que se encontram em geral associadas às nuvens moleculares de regiões de formação estelar (Joy 1945; Bertout 1989; Hartmann 2009). Uma outra classe de estrelas PMS, são as estrelas Ae/Be de Herbig (HAeBe) que têm massa maior

\footnotetext{
${ }^{1}$ O catálogo final da missão GAIA estará disponível somente em 2021 (de Bruijne 2012).
} 
que as TTSs e tipo espectral mais recente (Herbig 1960; Hartmann 2009). O termo objeto estelar jovem (YSO, do inglês young stellar object) é utilizado para referir-se de um modo geral às TTSs, HAeBes e proto-estrelas (Strom 1972; Hartmann 2009). Uma característica marcante dessas estrelas é a existência de material (disco) circunestelar. Um parâmetro importante na evolução dos discos proto-planetários e a escala de tempo da formação de planetas é a idade da estrela que pode ser obtida conhecendo-se a sua distância (Preibisch 2012). A determinação da distância dessas estrelas é ainda mais crítica para aquelas que se encontram em regiões de formação estelar suficientemente afastadas ( $\sim 200$ pc) o que inviabiliza uma medida direta da paralaxe a partir do solo. É importante mencionar que a maior parte das estrelas PMS em regiões de formação estelar próximas não foi contemplada na missão Hipparcos, pois se encontra além do limite de detecção do satélite, e portanto não têm distância medida. Como devemos proceder para obter a distância dessas estrelas?

A distância de uma estrela também pode ser obtida de forma menos direta através do estudo cinemático de grupos de estrelas cujos membros compartilham o mesmo movimento espacial. Essa estratégia baseia-se no fato que os vetores de movimento próprio das estrelas convergem para um ponto específico na esfera celeste denominado ponto de convergência (Jones 1971; de Bruijne 1999a; Galli et al. 2012). Conhecendo-se as coordenadas do ponto de convergência, o movimento próprio e a velocidade radial das estrelas obtém-se a distância para cada membro do grupo. Essa estratégia nos permite calcular a distância individual de cada estrela e atingir limites maiores que aqueles obtidos via paralaxe trigonométrica.

Um passo importante nesta direção foi dado por Bertout \& Genova (2006) no estudo da região de formação estelar em Taurus-Auriga. Os autores identificaram um grupo com 94 estrelas PMS co-moventes e calcularam a distância para 67 estrelas usando dados de movimento próprio, velocidade radial e o ponto de convergência desses movimentos. Em um trabalho posterior, Bertout et al. (2007) utilizaram as distâncias calculadas para obter a distribuição de massa e idade das estrelas nessa região. Em particular, eles investigam uma relação entre as estrelas T Tauri clássicas (CTTS, do inglês classical T Tauri star) e as estrelas T Tauri fracas (WTTS, do inglês weak-emission line T Tauri star). A diferença entre os dois tipos deve-se à existência de um disco de acresção nas CTTSs, enquanto as WTTSs são magneticamente ativas e não apresentam indícios de acresção de matéria. Os resultados obtidos permitiram concluir que as CTTS evoluem para WTTS quando o 
disco é totalmente acretado pela estrela. Embora esse cenário evolutivo já fosse aceito há algum tempo (Bertout 1989), essa foi a primeira confirmação com dados observacionais. Além disso, Bertout et al. (2007) desenvolveram um modelo empírico para o tempo de vida do disco de acresção para as estrelas em Taurus-Auriga. Esse resultado ainda deverá ser confirmado com o estudo de outras regiões de formação estelar.

A região de Taurus-Auriga possui características peculiares como a formação de estrelas de baixa massa ou de massa intermediária de maneira isolada e sem interações dinâmicas com sistemas vizinhos. Um estudo semelhante de outras regiões de formação estelar com propriedades de densidade estelar e ambientes diferentes permitirá comparar e confrontar os resultados obtidos por Bertout et al. (2007) nessa região. Dentro desse contexto, este trabalho de tese tem como objetivo o cálculo da distância de estrelas PMS em duas regiões de formação estelar próximas: Lupus e Ophiuchus. Essas regiões pertencem a um complexo muito maior de nuvens moleculares que se estende no hemisfério sul celeste (vide Figura 1.1) e contém estrelas de alta e baixa massa que compõem a associação Scorpius-Centaurus. As distâncias aqui obtidas serão utilizadas em um segundo momento para a determinação das propriedades físicas das estrelas e a comparação com os resultados obtidos em TaurusAuriga. Embora a distância média ou aproximada para essas regiões seja conhecida na literatura (Comerón 2008; Wilking et al. 2008) o que realmente interessa para o estudo que será realizado é a distância individual de cada estrela. Para tanto, utilizamos a estratégia de ponto de convergência que nos permite obter a distância de cada estrela e investigar as propriedades cinemáticas dessas regiões de formação estelar. 


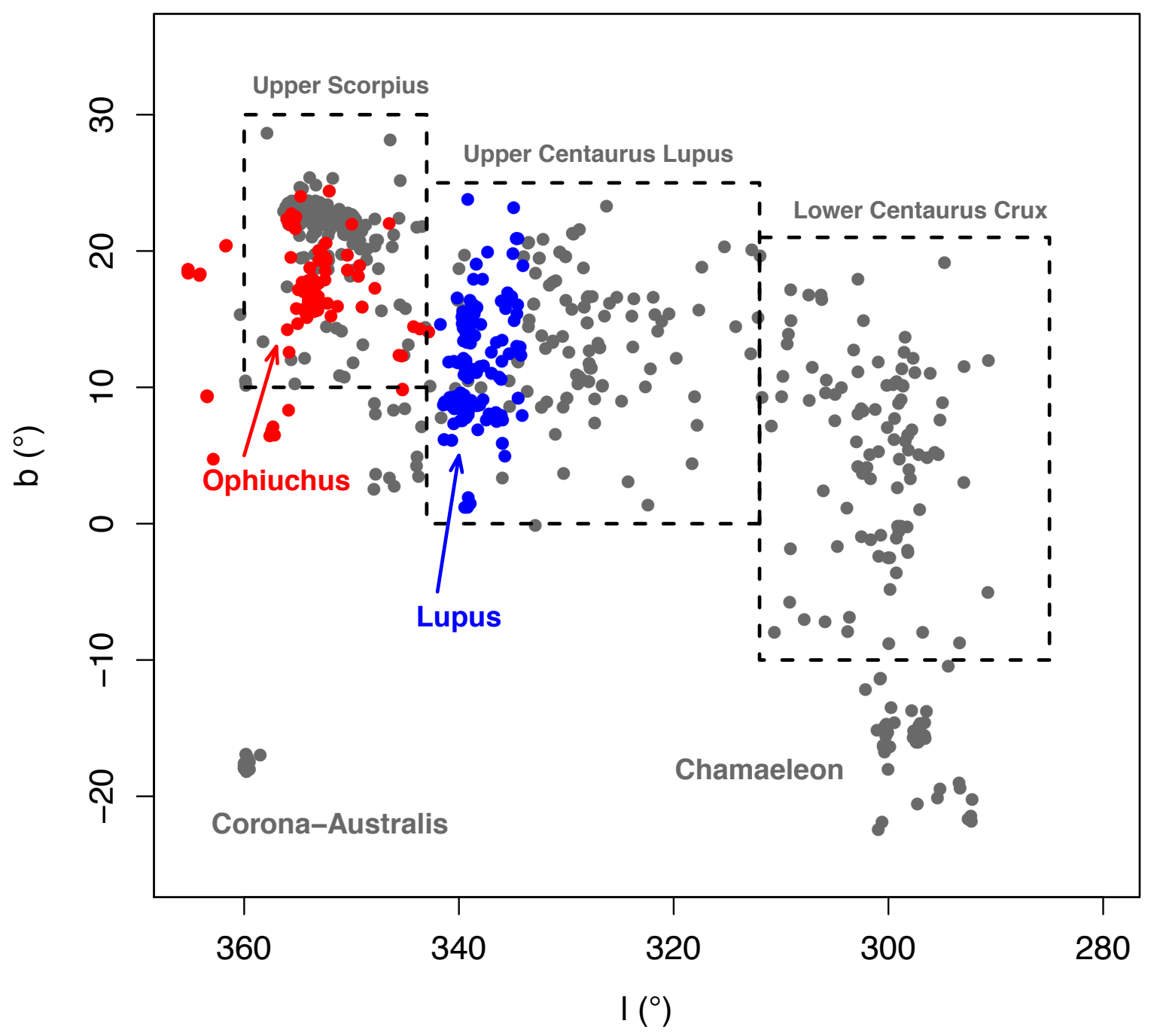

Figura 1.1: Localização em coordenadas galácticas das estrelas PMS nas regiões de formação estelar do hemisfério sul celeste (Lupus, Ophiuchus, Chamaeleon e Corona-Australis) e os subgrupos da associação Scorpius-Centaurus (Upper Scorpius, Upper Centaurus Lupus e Lower Centaurus Crux). As regiões que serão analisadas neste trabalho (Lupus e Ophiuchus) estão destacadas. 


\section{Organização da Tese}

Esta tese de doutorado está dividida em outros seis capítulos e quatro apêndices.

No capítulo 2 introduzimos a estratégia de ponto de convergência que será utilizada para calcular distâncias. Desenvolvemos uma nova versão do método de ponto de convergência que permite obter a posição do ponto de convergência e selecionar as estrelas que pertencem ao grupo. O novo método (Galli et al. 2012) é testado e comparado à versão clássica da literatura (Jones 1971; de Bruijne 1999a) com simulações e uma aplicação ao aglomerado aberto das Híades.

O capítulo 3 é dedicado às observações realizadas neste trabalho com o espectrógrafo FEROS no telescópio 2.2m (MPG/ESO) em La Silla (Chile). Apresentamos as velocidades radiais medidas para algumas estrelas PMS da amostra em estudo e os demais parâmetros resultantes das observações.

Nos capítulos 4 e $\mathbf{5}$ desenvolvemos a análise do ponto de convergência e o cálculo de distâncias para as estrelas PMS nas regiões de formação estelar de Lupus e Ophiuchus, respectivamente. Conforme ilustrado na Figura 1.1 existe uma sobreposição das estrelas nessas regiões com aquelas que pertencem à associação Scorpius-Centaurus. Utilizamos o novo método de ponto de convergência para realizar um estudo de pertinência das estrelas e separar as populações. Discutimos os resultados de distância obtidos e a identificação de estruturas em cada região.

No capítulo 6 apresentamos o cálculo da luminosidade, massa, idade e a construção do diagrama-HR das estrelas PMS em Lupus e Ophiuchus como uma primeira aplicação das distâncias aqui calculadas. Comparamos os resultados em Lupus e Ophiuchus com aqueles obtidos por Bertout et al. (2007) na região de Taurus-Auriga.

Finalmente, no capítulo 7 apresentamos as conclusões e perspectivas de continuação do trabalho com base nos resultados obtidos nesta tese de doutorado. Por último, apresentamos no Apêndice A uma lista dos acrônimos utilizados na redação deste documento e nos Apêndices B, C e D os artigos publicados (ou submetidos para publicação) que resultaram da tese de doutorado. 
Capítulo 2

\section{Método do Ponto de Convergência}

"A mente que se abre a uma nova idéia jamais volta ao seu tamanho original."

Albert Einstein

\section{Conteúdo}

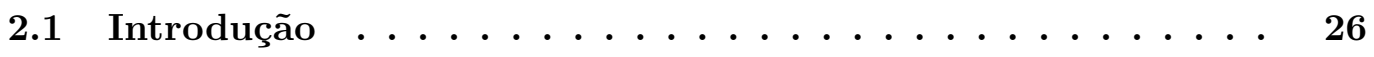

2.2 O Método Clássico (Jones 1971; de Bruijne 1999a) . . . . 29

2.3 Um Novo Método (Galli et al. 2012) . . . . . . . . . . 33

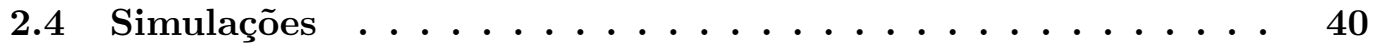

2.5 Aplicação ao Aglomerado Aberto das Híades . . . . . . . . 53

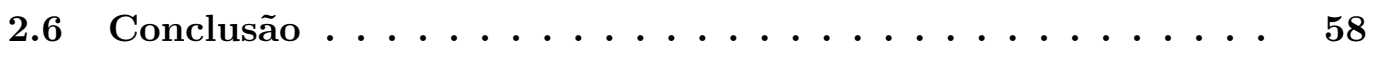

A determinação do ponto de convergência é um passo essencial para o cálculo de distâncias neste trabalho. Iniciamos este capítulo apresentando a estratégia que será utilizada para calcular distâncias cinemáticas a partir do ponto de convergência. Em seguida discutimos os métodos mais utilizados na literatura para obter o ponto de convergência de um grupo de estrelas e apresentamos um novo método que foi desenvolvido nesta tese de doutorado. A nova estratégia é testada e validada por simulações e uma aplicação ao aglomerado aberto das Híades. Um artigo com os resultados deste capítulo foi publicado (Galli et al. 2012) e se encontra em anexo (Apêndice B). 


\subsection{Introdução}

A vizinhança solar é formada por diversos grupos de estrelas cujos membros compartilham o mesmo movimento espacial ( $\mathrm{MG}$, do inglês moving group). A origem e evolução dessas estruturas é um assunto ainda em aberto e tem sido explicada por diferentes cenários que envolvem a dispersão de aglomerados, efeitos dinâmicos e eventos de acresção (Eggen 1996; Dehnen 2000; Fux 2001; Navarro et al. 2004). O interesse científico em estudá-las consiste em investigar a estrutura e a história de formação da Galáxia. Os MGs exibem dispersão de velocidade pequena, tipicamente alguns km/s (Mathieu 1986), e se destacam como regiões de alta densidade no espaço de velocidades (Antoja et al. 2008).

A velocidade de uma estrela que pertence ao MG é a soma de duas componentes: a velocidade $\mathbf{V}$ do grupo e a componente residual $\delta \mathbf{V}$, significativamente menor que a primeira. Assim, o movimento das estrelas que pertencem ao mesmo MG é essencialmente paralelo (vide Figura 2.1). Os movimentos paralelos no espaço quando projetados na esfera celeste convergem para um ponto denominado ponto de convergência ( $\mathrm{CP}$, do inglês convergent point). Enquanto os movimentos próprios são utilizados na determinação do CP, a velocidade radial nos permite investigar a expansão do MG (Blaauw 1964).

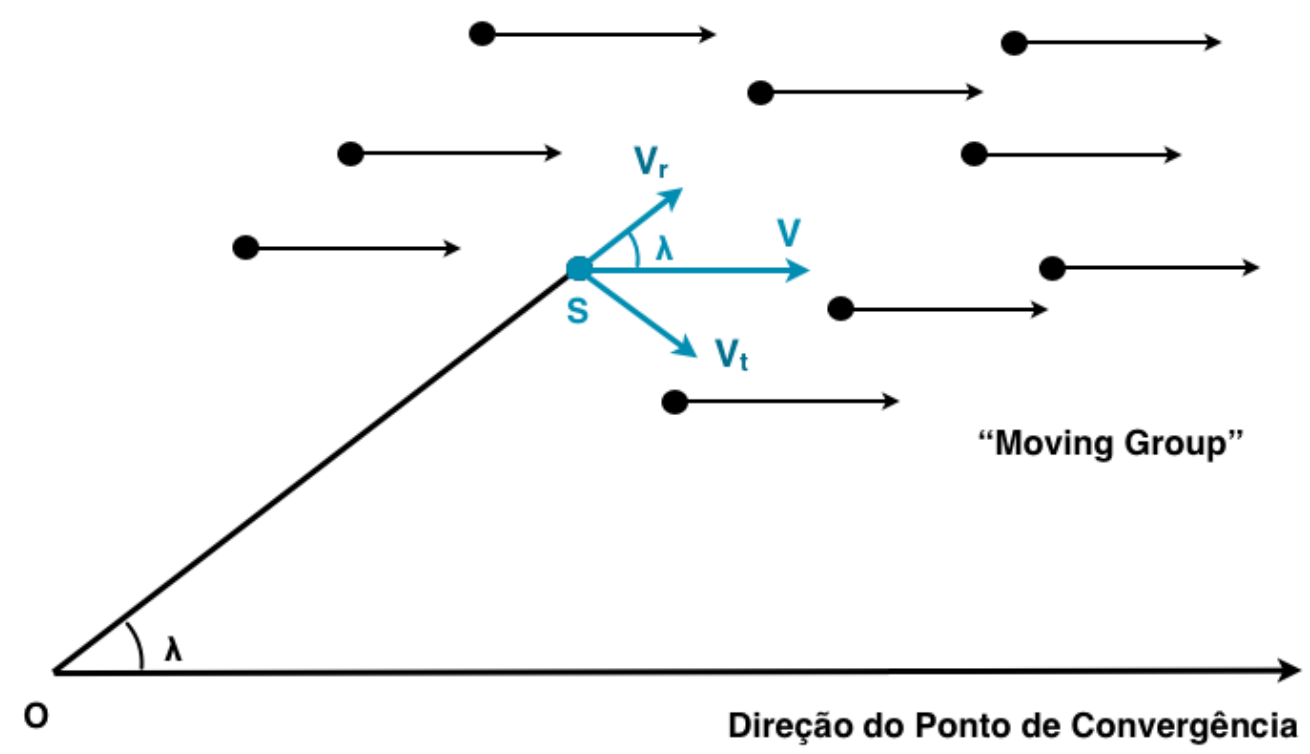

Figura 2.1: Representação esquemática de um MG. Colocamos em destaque uma estrela $S$, onde $\mathbf{V}_{\mathbf{r}}$, $\mathbf{V}_{\mathbf{t}}$ e $\mathbf{V}$ são, respectivamente, a velocidade radial, velocidade tangencial e velocidade espacial da estrela. 
Neste momento descrevemos o procedimento utilizado para obter a distância de uma estrela que pertence ao MG (por exemplo, a estrela S na Figura 2.1). A distância angular $\lambda$ entre a estrela $\mathrm{S}$ com coordenadas $(\alpha, \delta)$ e o $\mathrm{CP}\left(\alpha_{c p}, \delta_{c p}\right)$ é obtida pela lei dos cossenos aplicada ao triângulo esférico ilustrado na Figura 2.2:

$$
\cos \lambda=\sin \delta \sin \delta_{c p}+\cos \delta \cos \delta_{c p} \cos \left(\alpha_{c p}-\alpha\right)
$$

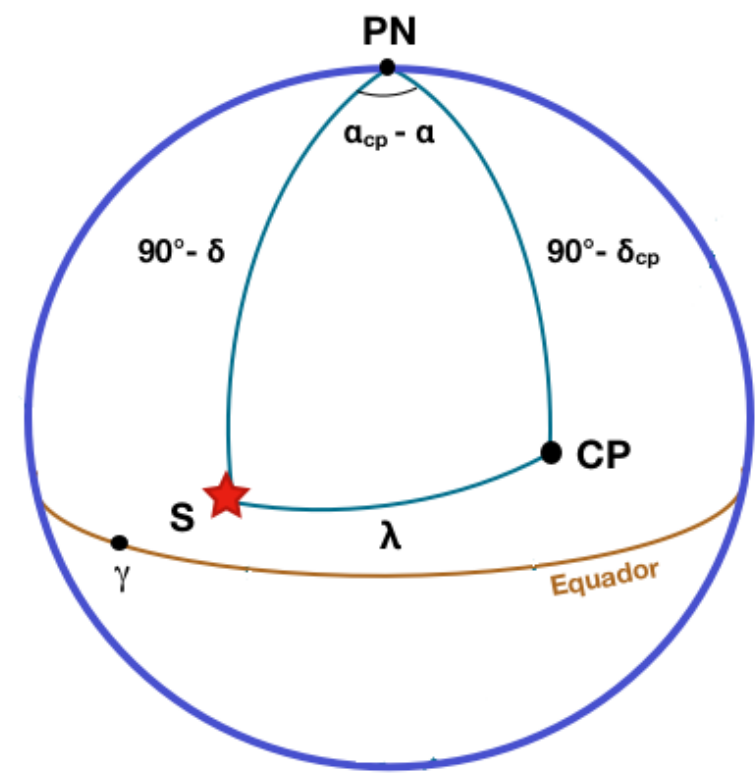

Figura 2.2: Representação esquemática do triângulo esférico formado pelo pólo norte (PN), o $\mathrm{CP}$ e a estrela S. Os lados do triângulo esférico e o ângulo interno estão indicados na figura.

A velocidade tangencial $\mathbf{V}_{\mathbf{t}}[\mathrm{km} / \mathrm{s}]$ de uma estrela é dada em função da sua paralaxe $\pi$ [mas] e do movimento próprio $\mu$ [mas/yr] por

$$
\left|\mathbf{V}_{\mathbf{t}}\right|=\frac{A|\mu|}{\pi}
$$

onde $A=4.74047 \mathrm{~km} \cdot \mathrm{yr} / \mathrm{s}$ é razão entre uma unidade astronômica em $\mathrm{km}$ e o número de segundos em um ano. No caso de movimentos paralelos $\lambda$ é também o ângulo entre a velocidade espacial $\mathbf{V}$ e a velocidade radial $\mathbf{V}_{\mathbf{r}}$ da estrela (vide Figura 2.1). Isso nos permite obter $\mathbf{V}_{\mathbf{t}}$ e $\mathbf{V}_{\mathbf{r}}$ em função de $\mathbf{V}$ e $\lambda$ pelas relações que seguem:

$$
\begin{aligned}
& \left|\mathbf{V}_{\mathbf{t}}\right|=|\mathbf{V}| \sin \lambda, \\
& \left|\mathbf{V}_{\mathbf{r}}\right|=|\mathbf{V}| \cos \lambda .
\end{aligned}
$$


Partindo das equações (2.2), (2.3) e (2.4) calculamos a paralaxe individual de cada estrela que pertence ao MG a partir da sua velocidade radial por

$$
\pi=\frac{A\left|\mu_{\|}\right|}{\left|\mathbf{V}_{\mathbf{r}}\right| \tan \lambda},
$$

onde $\mu_{\|}$é a componente do movimento próprio que aponta para o CP (vide seção 2.2.1).

Quando a velocidade radial da estrela não é conhecida ainda é possível estimar a sua paralaxe com base na velocidade espacial do MG, partindo da hipótese que as estrelas do MG compartilham o mesmo movimento espacial. A velocidade espacial do MG pode ser obtida a partir das estrelas com velocidade radial conhecida. A paralaxe calculada desse modo será representada neste trabalho por $\pi^{\prime}$ e ela é dada por

$$
\pi^{\prime}=\frac{A\left|\mu_{\|}\right|}{\left|\mathbf{V}_{M G}\right| \sin \lambda},
$$

onde $\mathbf{V}_{M G}$ é a velocidade espacial do MG. A paralaxe obtida pelas equações (2.5) e (2.6) recebe o nome de paralaxe cinemática. ${ }^{1}$

A distância de uma estrela (em unidades de parsec, $1 \mathrm{pc}=3.086 \times 10^{16} \mathrm{~m}$ ) é dada em função da sua paralaxe (em segundos de arco) por

$$
d[p c]=\frac{1}{\pi\left[{ }^{\prime \prime}\right]} .
$$

Quando a distância é obtida da paralaxe cinemática da estrela ela recebe o nome de distância cinemática. Portanto, conhecendo-se o movimento próprio, a velocidade radial e a distância angular da estrela ao CP pode-se obter a sua distância. Enquanto o movimento próprio e a velocidade radial da estrela são grandezas observacionais, o CP é um parâmetro que deve ser calculado.

A determinação do CP tem uma longa história na astronomia e diversas técnicas já foram propostas: Charlier (1916, vide Smart 1968), Seares (1945), Petrie (1949), Roman (1949), Brown (1950) e Bertiau (1958). A evolução dessas estratégias culminaram no método clássico de Jones (1971) que foi posteriormente revisado por de Bruijne (1999a). Tendo em vista a importância e a utilização desses métodos na literatura optamos por discutí-los em detalhe mais adiante (seção 2.2). Em seguida apresentamos uma nova estratégia para o cálculo do CP que foi desenvolvida neste trabalho (seção 2.3).

\footnotetext{
${ }^{1}$ Utilizamos simplesmente o termo paralaxe ao invés de paralaxe cinemática para se referir aos resultados aqui obtidos. Quando necessário fazemos a distinção entre paralaxe cinemática e paralaxe trigonométrica.
} 


\subsection{O Método Clássico (Jones 1971; de Bruijne 1999a)}

\subsubsection{Princípio de decomposição do movimento próprio}

Partimos de um conjunto com $N$ estrelas, onde a posição $(\alpha, \delta)$, o movimento próprio $\left(\mu_{\alpha} \cos \delta, \mu_{\delta}\right)$ e os respectivos erros são conhecidos. Os movimentos próprios das estrelas que pertencem ao MG definem o CP com coordenadas $\left(\alpha_{c p}, \delta_{c p}\right)$ na esfera celeste. O princípio básico para o cálculo do CP no método clássico é a decomposição do movimento próprio $\mu$ das estrelas em duas componentes: $\mu_{\|}$e $\mu_{\perp}$, respectivamente, paralela e perpendicular ao grande círculo que passa pelo CP e a estrela em questão (vide Figura 2.3). As componentes do movimento próprio são definidas pela transformação

$$
\left(\begin{array}{c}
\mu_{\|} \\
\mu_{\perp}
\end{array}\right)=\left(\begin{array}{cc}
\sin \theta & \cos \theta \\
-\cos \theta & \sin \theta
\end{array}\right)\left(\begin{array}{c}
\mu_{\alpha} \cos \delta \\
\mu_{\delta}
\end{array}\right)
$$

onde $\theta$ é o ângulo de posição do CP que é dado por

$$
\tan \theta=\frac{\sin \left(\alpha_{c p}-\alpha\right)}{\cos \delta \tan \delta_{c p}-\sin \delta \cos \left(\alpha_{c p}-\alpha\right)}
$$

No caso ideal, isto é, convergência exata dos movimentos próprios conclui-se que $\mu_{\perp}=0$ e $\mu_{\|}=\mu$. No entanto, verifica-se que em geral $\mu_{\perp} \neq 0$ devido à dispersão de velocidade do MG e os erros envolvidos na medida do movimento próprio. 


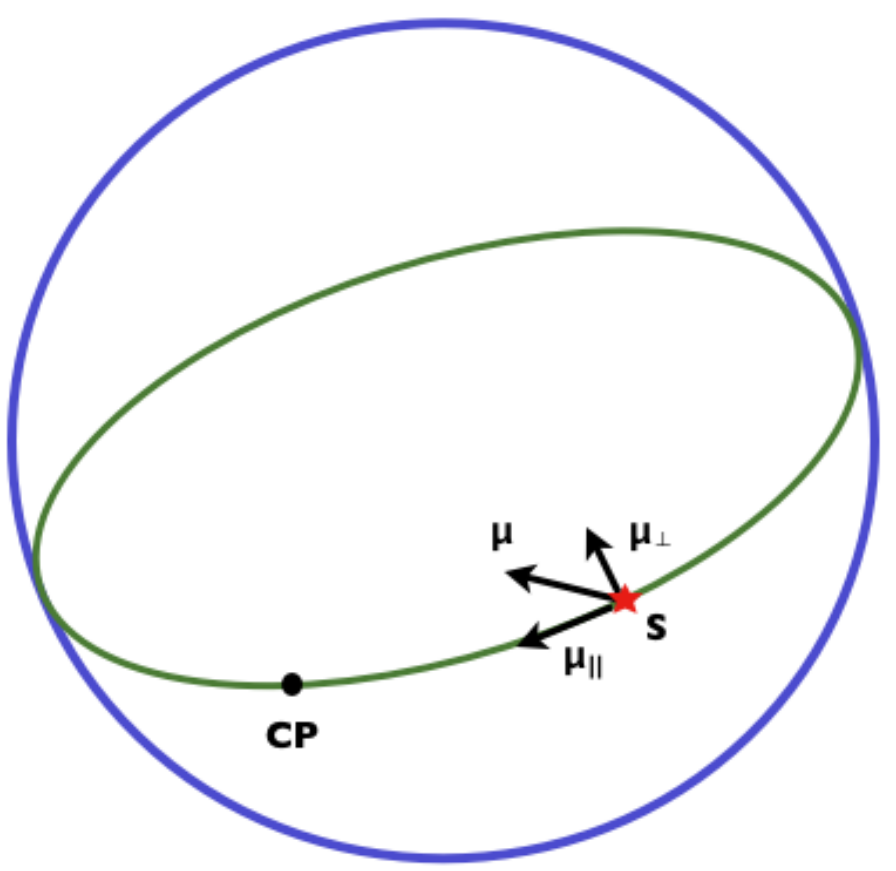

Figura 2.3: Representação esquemática do movimento próprio $\mu$ de uma estrela $\mathrm{S}$ decomposto nas componentes paralela $\mu_{\|}$e perpendicular $\mu_{\perp}$ ao grande círculo que passa pela estrela e o CP. Figura retirada de Galli et al. (2012).

\subsubsection{Algoritmo de Jones (1971)}

O algoritmo clássico para o cálculo do CP foi proposto por Jones (1971) com base em um método para a determinação simultânea do CP e seleção dos membros do MG. A estratégia consiste em determinar as coordenadas de máxima verossimilhança do CP baseado na comparação da componente perpendicular $\mu_{\perp}$ do movimento próprio das estrelas candidatas com o seu valor esperado igual a zero. O método consiste em sobrepor uma grade à esfera celeste onde cada ponto da grade é um ponto candidato a CP. Em cada ponto da grade calcula-se o valor de $X^{2}$ definido por

$$
X^{2}=\sum_{j=1}^{N} t_{\perp_{j}}^{2},
$$

onde $t_{\perp}=\mu_{\perp} / \sigma_{\perp}$ e $\sigma_{\perp}$ é a incerteza associada ao valor de $\mu_{\perp}$ para cada estrela $(j=1,2,3, . ., N)$. Jones (1971) assume que a distribuição de $t_{\perp}$ é normal e que $X^{2}$ segue uma distribuição de $\chi^{2}$ com $N-2$ graus de liberdade. Minimizar $X^{2}$ implica em maximizar a função de verossimilhança, portanto deve-se escolher o ponto da grade com o menor valor de $X^{2}$. No entanto, é possível que o menor valor de $X^{2}$ ocorra ao acaso devido à presença de 
estrelas na amostra que não pertencem ao MG. Para avaliar essa possibilidade calcula-se a probabilidade $\epsilon$ de $X^{2}$ exceder um dado valor observado. Essa probabilidade é dada por:

$$
\epsilon=\frac{1}{\Gamma\left[\frac{1}{2}(N-2)\right]} \int_{X^{2}}^{\infty} x^{\frac{1}{2}[N-2]-1} e^{-x} d x
$$

Se a probabilidade calculada for muito baixa, deve-se rejeitar a estrela com o maior valor de $\left|t_{\perp}\right|$, corrigir o tamanho da amostra e recomeçar o procedimento. Esse processo deve ser repetido até que $\epsilon$ atinja um valor mínimo $\epsilon_{\min }$ (a ser discutido na seção 2.4.4). Quando isso acontece o ponto da grade resultante do processo é o CP e as estrelas não rejeitadas são membros do MG. Portanto, a determinação do CP e a seleção dos membros do MG são dependentes entre si e realizadas simultaneamente.

O procedimento desenvolvido por Jones (1971) para a determinação do CP é um método robusto e de fácil implementação que evita os problemas de uma topologia mais complicada da função $X^{2}$ retornando sempre o ponto de mínimo global dentro da precisão utilizada. Entretanto, essa técnica é também reflexo do baixo poder computacional da época o que não permitia varrer a esfera celeste em passos muito pequenos. Hoje, com o avanço da tecnologia já é possível implementar o método de Jones (1971) de forma otimizada utilizando um passo tão pequeno quanto necessário. Uma desvantagem desse método é que ele não retorna a incerteza na solução de CP. Tipicamente essa grandeza seria dada pelo tamanho do passo usado para varrer a esfera celeste. No entanto, como o tamanho do passo escolhido pode ser infinitamente pequeno, esse valor deixa de representar um erro físico e/ou estatístico que deve estar associado à solução.

\subsubsection{Algoritmo de de Bruijne (1999a)}

A utilização de medidas precisas em astronomia permite concluir que nem todas estrelas que pertencem ao MG têm movimento próprio que aponta exatamente na direção do CP. Isso ocorre devido à dispersão de velocidade das estrelas no MG. Portanto, selecionar somente as estrelas com $\mu_{\perp}=0$ não permitirá determinar todos os membros. Tendo em vista esse problema, de Bruijne (1999a) adaptou o algoritmo de Jones (1971) para incluir a dispersão de velocidade do MG. Para tanto, é introduzida uma nova definição de $t_{\perp}$ : 


$$
t_{\perp}=\frac{\mu_{\perp}}{\sqrt{\sigma_{\perp}^{2}+\sigma_{i n t}^{2}}} .
$$

A dispersão de velocidade $\sigma_{i n t}$ em unidades de mas/yr é dada por

$$
\sigma_{i n t}=\frac{1000 \sigma_{v}}{A d}
$$

onde $\sigma_{v}$ é a dispersão de velocidade intrínseca unidimensional em unidades de $\mathrm{km} / \mathrm{s}$ e $d$ é uma estimativa da distância média das estrelas em unidades de parsec. Nesse contexto, o método atribui à cada estrela uma probabilidade de pertinência ao MG que é dada por

$$
p_{j}=\exp \left[-\frac{1}{2}\left(\frac{\mu_{\perp_{j}}^{2}}{\sigma_{\perp_{j}}^{2}+\sigma_{\text {int }}^{2}}\right)\right] .
$$

Uma outra modificação introduzida por de Bruijne (1999a) é a implementação de uma rotina de minimização da função $X^{2}$ que retorna a solução de $\mathrm{CP}$ e a sua incerteza na forma de uma matriz de covariância. Essa nova estratégia elimina o conceito de grade utilizado na versão anterior. O método foi ainda adaptado para melhor explorar os dados da missão Hipparcos visando a identificação de MGs nesse catálogo (vide seção 2.5.1).

As modificações introduzidas por de Bruijne (1999a) representam um grande avanço com relação ao método de Jones (1971), pois consideram a dispersão de velocidade do MG, retornam a solução com sua incerteza e substituem uma implementação numérica por uma rotina de minimização. No entanto, a inclusão do termo de dispersão da velocidade na definição de $t_{\perp}$ aumenta as probabilidades individuais de cada estrela. O fato de uma estrela ser aceita ou rejeitada depende não só da sua probabilidade individual de pertencer ao grupo, mas também da probabilidade total que é produto da probabilidade de todas estrelas do conjunto. Isso significa que se o MG em questão tem muitas estrelas com alta probabilidade de pertinência, mais estrelas que sejam membros falsos serão toleradas na solução. Em particular, o método tende a aceitar estrelas de campo mais distantes cujo movimento próprio e consequentemente a componente perpendicular $\mu_{\perp}$ são pequenos.

Uma outra crítica ao método é que ao introduzir o termo da dispersão de velocidade conforme sugerido por de Bruijne (1999a) admite-se que todas estrelas do grupo estão à mesma distância o que implica em aplicar a mesma correção a todas estrelas da amostra. Essa aproximação deixa de ser válida para MGs que ocupam um grande volume no espaço. 


\subsection{Um Novo Método (Galli et al. 2012)}

\subsubsection{Representação por grande círculo}

O movimento próprio é definido como sendo a projeção da componente tangencial do movimento de uma estrela na esfera celeste. O cálculo do movimento próprio decorre da medida da posição da estrela em diferentes épocas. Como a escala de tempo que separa as observações é muito pequena com relação àquela em que ocorrem variações no movimento da estrela assumimos que o movimento da estrela é uniforme. Consideramos que $\mathbf{r}=(x, y, z)=(\cos \alpha \cos \delta, \sin \alpha \cos \delta, \sin \delta)$ é posição de uma estrela com coordenadas $(\alpha, \delta)$ e movimento próprio $\left(\mu_{\alpha} \cos \delta, \mu_{\delta}\right)$ no sistema usual de coordenadas equatoriais onde os vetores unitários $\hat{\mathbf{x}}, \hat{\mathbf{y}}, \hat{\mathbf{z}}$ apontam respectivamente para o ponto vernal, o ponto no equador com $\alpha=90^{\circ}$ e o pólo norte do equador. A velocidade ângular da estrela é dada pela derivada temporal do vetor de posição

$$
\dot{\mathbf{r}}=\mu_{\alpha} \cos \delta\left(\begin{array}{c}
-\sin \alpha \\
\cos \alpha \\
0
\end{array}\right)+\mu_{\delta}\left(\begin{array}{c}
-\cos \alpha \sin \delta \\
-\sin \alpha \sin \delta \\
\cos \delta
\end{array}\right)
$$

O plano definido pelo vetores $\mathbf{r}$ e $\dot{\mathbf{r}}$ aparece na esfera celeste como um grande círculo ao longo do qual a estrela se movimenta. A idéia de representar o movimento de uma estrela na esfera celeste por um grande círculo foi proposta por Herschel em 1783 (Trumpler \& Weaver 1953) e tem sido utilizada mais recentemente por Abad et al. (2003); Abad \& Vieira (2005). No caso específico de um MG, onde os movimentos são paralelos, a intersecção dos grandes círculos das estrelas define dois pontos diametralmente opostos (vide Figura 2.4): o $\mathrm{CP}$, e o seu ponto espelho que neste trabalho recebe o nome de ponto de divergência (DP, do inglês divergent point). Assumindo que o CP tem coordenadas $\left(\alpha_{c p}, \delta_{c p}\right)$, então as coordenadas do DP são $\left(\alpha_{d p}, \delta_{d p}\right)=\left(\alpha_{c p}+180^{\circ},-\delta_{c p}\right)$. Essa simples visualização do movimento das estrelas na esfera celeste permite concluir que o problema em questão apresenta duas soluções geometricamente equivalentes. A distinção entre ambas depende da direção dos vetores de movimento próprio. 


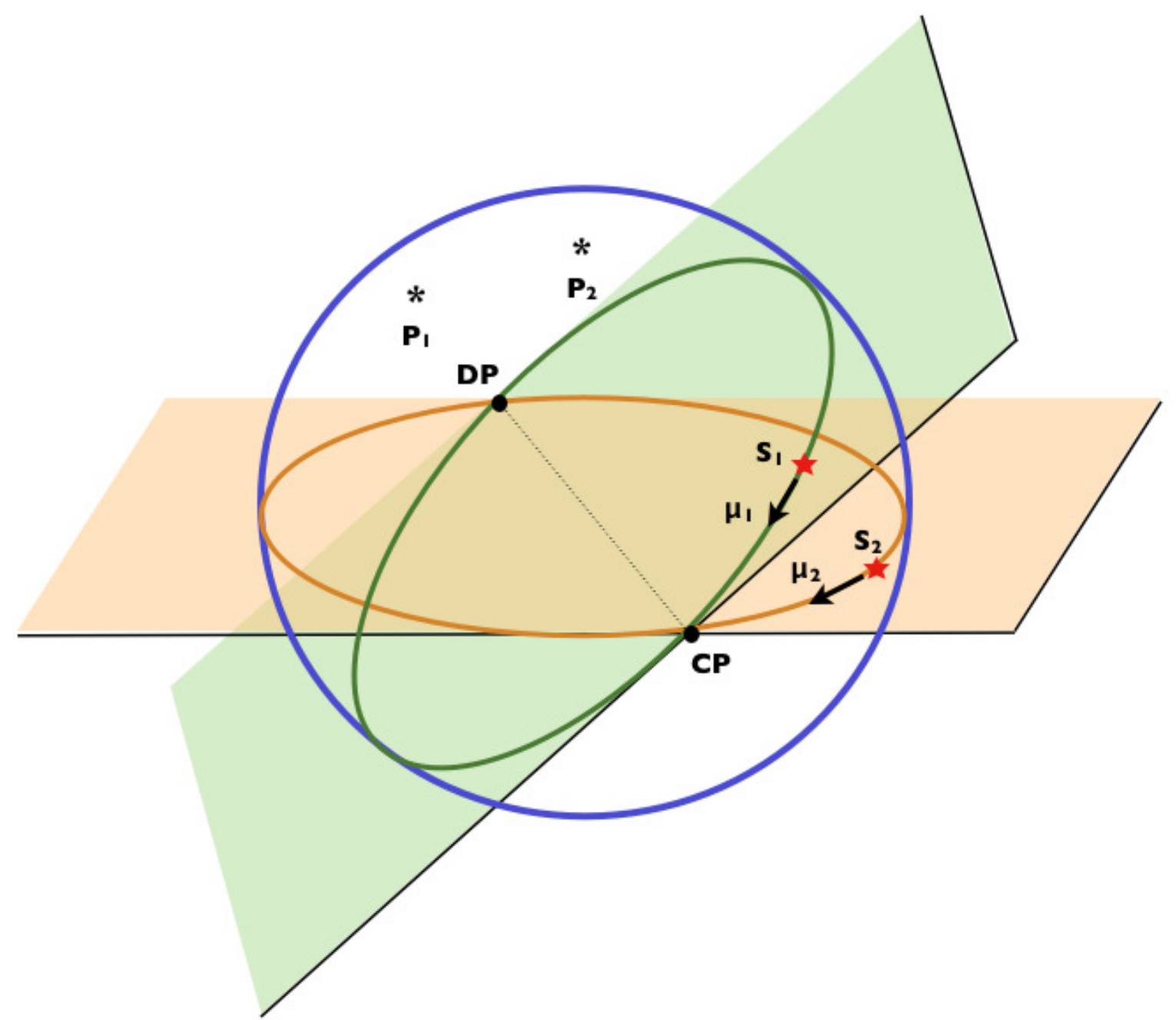

Figura 2.4: Representação esquemática do grande círculo de duas estrelas $S_{1}$ e $S_{2}$ com movimentos próprios $\mu_{1}$ e $\mu_{2}$. Os pólos dos grandes círculos são, respectivamente, $P_{1}$ e $P_{2}$. As soluções de CP e DP estão assinaladas na figura. Figura retirada de Galli et al. (2012).

O grande círculo de cada estrela é resultado da intersecção do plano definido por $\mathbf{r}$ e $\dot{\mathbf{r}}$ com a esfera celeste. No entanto, ele pode também ser representado pelo vetor normal ao plano que contém a estrela e as soluções do problema (CP e DP). O vetor normal ao plano definido por $\mathbf{r}$ e $\dot{\mathbf{r}}$ recebe o nome de vetor polar $\mathbf{p}$ e é dado por

$$
\mathbf{p}=\mathbf{r} \times \dot{\mathbf{r}}=\mu_{\alpha} \cos \delta\left(\begin{array}{c}
-\cos \alpha \sin \delta \\
-\sin \alpha \sin \delta \\
\cos \delta
\end{array}\right)-\mu_{\delta}\left(\begin{array}{c}
-\sin \alpha \\
\cos \alpha \\
0
\end{array}\right)
$$

O vetor polar define o pólo de cada grande círculo na esfera celeste. Os pólos dos grandes círculos das estrelas que pertencem ao mesmo MG estão localizados sobre um plano comum. 
A intersecção desse plano com a esfera resulta em um outro grande círculo: o grande círculo polar. Os pólos do grande círculo polar são as soluções de CP e DP. Esse é o princípio básico da nova estratégia de determinação do CP aqui desenvolvida.

\subsubsection{Algoritmo}

O algoritmo desenvolvido neste trabalho para o cálculo do CP tem como base o método da grade utilizado por Jones (1971) que é adaptado à nova estratégia. Trata-se de um método para a determinação simultânea do CP e seleção dos membros do MG. O método determina as coordenadas de máxima verossimilhança do CP baseado na representação do movimento de uma estrela na esfera celeste por um grande círculo. O procedimento consiste em interpolar o grande círculo polar cujos pólos são as soluções do problema. Para tanto, deve-se entender que o plano que contém as soluções do problema e aquele que define o grande círculo polar são ortogonais. Nesse contexto define-se $\boldsymbol{\kappa}=\kappa \hat{\mathbf{e}}_{\mathbf{c p}}$, onde $\kappa$ é o erro de ortogonalidade que representa a fração de $\mathbf{p}$, vetor polar normalizado, projetado na direção do CP definida pelo vetor unitário $\hat{\mathbf{e}}_{\mathbf{c p}}$. O erro de ortogonalidade é dado por

$$
\boldsymbol{\kappa}=\left(\mathbf{p} \cdot \hat{\mathbf{e}}_{\mathbf{c p}}\right) \hat{\mathbf{e}}_{\mathbf{c p}}=\underbrace{(\cos \theta)}_{\kappa} \hat{\mathbf{e}}_{\mathbf{c p}}
$$

onde $\theta$ é ângulo esférico entre os planos que contém $\mathbf{p}$ e $\hat{\mathbf{e}}_{\mathbf{c p}}$. O plano que contém as soluções do problema e aquele que define o grande círculo polar são ortogonais quando $\kappa=0$. Na prática, devemos minimizar o valor de $\kappa$ para encontrar o $\mathrm{CP}$.

Em seguida apresentamos as etapas necessárias para a determinação do $\mathrm{CP}\left(\alpha_{c p}, \delta_{c p}\right)$. O procedimento é descrito utilizando-se o sistema de coordenadas equatoriais e pode ser facilmente adaptado para os demais sistemas de coordenadas. 
1. Determine o pólo do grande círculo de cada estrela da amostra. As coordenadas do pólo $\left(\alpha^{p}, \delta^{p}\right)$ são dadas por

$$
\begin{gathered}
\alpha^{p}=\arctan \left(\frac{-\mu_{\alpha} \cos \delta \sin \delta \sin \alpha-\mu_{\delta} \cos \alpha}{-\mu_{\alpha} \cos \delta \sin \delta \cos \alpha+\mu_{\delta} \sin \alpha}\right), \\
\delta^{p}=\arctan \left(\frac{\mu_{\alpha} \cos ^{2} \delta}{\sqrt{\left(\mu_{\alpha} \cos \delta \sin \delta\right)^{2}+\mu_{\delta}^{2}}}\right) .
\end{gathered}
$$

A incerteza $\left(\sigma_{\alpha^{p}}, \sigma_{\delta^{p}}\right)$ associada às coordenadas do pólo é obtida em função dos erros de posição e movimento próprio por

$$
\begin{gathered}
\sigma_{\alpha^{p}}^{2}=\left(\frac{\partial \alpha^{p}}{\partial \alpha}\right)^{2} \sigma_{\alpha}^{2}+\left(\frac{\partial \alpha^{p}}{\partial \delta}\right)^{2} \sigma_{\delta}^{2}+\left(\frac{\partial \alpha^{p}}{\partial \mu_{\alpha^{*}}}\right)^{2} \sigma_{\mu_{\alpha^{*}}}^{2}+\left(\frac{\partial \alpha^{p}}{\partial \mu_{\delta}}\right)^{2} \sigma_{\mu_{\delta}}^{2}, \\
\sigma_{\delta^{p}}^{2}=\left(\frac{\partial \delta^{p}}{\partial \alpha}\right)^{2} \sigma_{\alpha}^{2}+\left(\frac{\partial \delta^{p}}{\partial \delta}\right)^{2} \sigma_{\delta}^{2}+\left(\frac{\partial \delta^{p}}{\partial \mu_{\alpha^{*}}}\right)^{2} \sigma_{\mu_{\alpha^{*}}}^{2}+\left(\frac{\partial \delta^{p}}{\partial \mu_{\delta}}\right)^{2} \sigma_{\mu_{\delta}}^{2},
\end{gathered}
$$

onde $\mu_{\alpha^{*}}=\mu_{\alpha} \cos \delta$.

2. Defina uma grade sobreposta à esfera celeste onde cada ponto $\left(i=1,2,3 \ldots, N_{\text {grade }}\right)$ é um candidato a CP. Inicie no ponto $i=1$ e assuma que esse é o CP.

3. Calcule para cada estrela da amostra $(j=1,2,3, \ldots, N)$ o valor de $s$, definido por

$$
s=\frac{\kappa}{\sigma_{\kappa}}
$$

onde $\kappa$ é o erro de ortogonalidade dado em (2.17). Aplicando a lei dos cossenos no triângulo esférico que contém o CP e o pólo do grande círculo da estrela em questão verifica-se que

$$
\kappa \equiv \cos \theta=\sin \delta_{c p} \sin \delta^{p}+\cos \delta_{c p} \cos \delta^{p} \cos \left(\alpha_{c p}-\alpha^{p}\right)
$$

A incerteza $\sigma_{\kappa}$ é obtida por propagação de erros levando em conta os erros nas coordenadas do pólo:

$$
\begin{aligned}
& \sigma_{\kappa}^{2}=\left[-\cos \delta_{c p} \cos \delta^{p} \sin \left(\alpha^{p}-\alpha_{c p}\right)\right]^{2} \sigma_{\alpha^{p}}^{2}+ \\
& +\left[\sin \delta_{c p} \cos \delta^{p}-\cos \delta_{c p} \sin \delta^{p} \cos \left(\alpha^{p}-\alpha_{c p}\right)\right]^{2} \sigma_{\delta^{p}}^{2} .
\end{aligned}
$$


4. Calcule o valor de $X^{2}$ neste ponto da grade $(i=1)$ que é dado por

$$
X^{2}=\sum_{j=1}^{N} s_{j}^{2}
$$

5. Calcule o valor de $X^{2}$ em cada ponto da grade repetindo as etapas anteriores.

6. Assume-se que $s$ segue uma distribuição normal (a validade dessa hipótese será discutida na seção 2.4.5) e que $X^{2}$ segue uma distribuição de $\chi^{2}$ com $N-2$ graus de liberdade. A distribuição de probabilidade para uma dada combinação de $\kappa_{j}$ e $\sigma_{\kappa_{j}}$ reduz-se a

$$
p_{j}=\frac{1}{\sqrt{2 \pi}} \exp \left(-\frac{1}{2} s_{j}^{2}\right) .
$$

A probabilidade total $P$ do conjunto de valores calculados de $s$ para um ponto da grade é dada por

$$
P=\prod_{j=1}^{N} p_{j}=\frac{1}{(2 \pi)^{N / 2}} \exp \left(-\frac{1}{2} X^{2}\right),
$$

e define a função de verossimilhança $\mathcal{L}$

$$
\mathcal{L} \equiv(2 \pi)^{N / 2} P=\exp \left(-\frac{1}{2} X^{2}\right)
$$

A maximização da função de verossimilhança corresponde à minimização de $X^{2}$. Defina o ponto na grade com o menor valor de $X^{2}$.

7. No entanto, é possível que o menor valor de $X^{2}$ ocorra ao acaso devido à presença de estrelas na amostra que não pertencem ao MG. Para avaliar essa possibilidade calcule a probabilidade $\epsilon$ de $X^{2}$ exceder um dado valor observado. Essa probabilidade é dada por

$$
\epsilon=\frac{1}{\Gamma\left[\frac{1}{2}(N-2)\right]} \int_{X^{2}}^{\infty} x^{\frac{1}{2}[N-2]-1} e^{-x} d x .
$$

8. Se $\epsilon<\epsilon_{\min }$ rejeite a estrela com o maior valor de $|s|$, corrija o tamanho da amostra $N \rightarrow N-1$ e recomece o procedimento do passo 3. Quando $\epsilon \geq \epsilon_{\min }$ o ponto da grade resultante do processo é o CP e as estrelas não rejeitadas são membros do MG. 


\subsubsection{Correção devido à dispersão de velocidade}

A estratégia de determinação do CP desenvolvida neste trabalho seleciona como membros do MG as estrelas cujo erro de ortogonalidade $\kappa$ se aproxima do valor esperado igual a zero. O erro de ortogonalidade é uma medida do ângulo entre o plano que contém os pólos de cada grande círculo e o plano definido pelo CP, DP e a estrela em questão. Quando $\kappa$ atinge o seu valor esperado esses planos são ortogonais. Para que os pólos dos grandes círculos das estrelas estejam todos sobre um mesmo plano é necessário assumir que as estrelas têm movimentos paralelos entre si. No entanto, nem todas estrelas que pertencem ao mesmo MG apresentam movimentos perfeitamente paralelos. Isso ocorre devido à dispersão de velocidade do grupo. Portanto, selecionar somente as estrelas com $\kappa=0$ não permitirá identificar todos os membros do MG. Consequentemente deve-se permitir que exista um pequeno desvio de $s$ com relação ao zero. Introduzimos uma nova definição dessa grandeza que é dada por

$$
s=\frac{\kappa}{\sqrt{\sigma_{\kappa}^{2}+\Delta \kappa^{2}}},
$$

onde $\Delta \kappa$ é uma estimativa da dispersão de velocidade unidimensional que se traduz na dispersão dos movimentos próprios $\left(\Delta \mu_{\alpha}, \Delta \mu_{\delta}\right)$ das estrelas. O termo de correção é dado por

$$
\Delta \kappa=\sqrt{\left(\left|\frac{\partial \kappa}{\partial \mu_{\alpha}^{*}}\right| \Delta \mu_{\alpha}^{*}\right)^{2}+\left(\left|\frac{\partial \kappa}{\partial \mu_{\delta}}\right| \Delta \mu_{\delta}\right)^{2}},
$$

onde $\mu_{\alpha}^{*}=\mu_{\alpha} \cos \delta$. A dispersão no movimento próprio das estrelas em cada componente devido à dispersão de velocidade do MG é quantificada pela equação (2.13) introduzida por de Bruijne (1999a, vide seção 2.2.3). Analogamente ao método clássico, atribuímos uma probabilidade de pertinência para cada estrela que pertence ao MG que é dada por

$$
p_{j}=\exp \left[-\frac{1}{2}\left(\frac{\kappa_{j}^{2}}{\sigma_{\kappa_{j}}^{2}+\Delta \kappa_{j}^{2}}\right)\right] .
$$

As estrelas com alta probabilidade de pertinência (membros em potencial) têm a sua probabilidade aumentada após a introdução da correção. Consequentemente a solução admite novos membros ainda que com menor probabilidade de pertinência. A correção devido à dispersão de velocidade do grupo proposta para esta nova estratégia é fisicamente semelhante àquela utilizada por de Bruijne (1999a) no método clássico e tem por origem os mesmos fatores. No entanto, ela difere no fato de ser uma correção individual aplicada 
a cada estrela, pois as derivadas parciais na equação (2.31) dependem da posição e do movimento próprio de cada estrela. Desse modo considera-se que as estrelas do grupo não estão todas à mesma distância visto que o movimento próprio é um indicativo de distância. Esse ponto é particularmente importante no caso de MGs que ocupam um grande volume no espaço.

\subsubsection{Otimização do método de ponto de convergência}

O algoritmo descrito na seção 2.3.2 retorna a posição aproximada do CP que é limitada pelo tamanho do passo usado para varrer a esfera celeste. Uma forma de resolver esse problema consiste em implementar uma rotina de minimização de $X^{2}$ resolvendo o problema de mínimos quadrados não-linear abaixo:

$$
\begin{aligned}
& \frac{\partial X^{2}}{\partial \alpha_{c p}}=0 \rightarrow \sum_{j=1}^{N} s_{j} \frac{\partial s_{j}}{\partial \alpha_{c p}}=0, \\
& \frac{\partial X^{2}}{\partial \delta_{c p}}=0 \rightarrow \sum_{j=1}^{N} s_{j} \frac{\partial s_{j}}{\partial \delta_{c p}}=0 .
\end{aligned}
$$

O procedimento que adotamos para resolver esse sistema de equações é baseado em uma implementação própria do método de Levenberg-Marquardt seguindo a descrição presente em Madsen et al. (2004) e Press et al. (1992). O modelo é aproximado para o caso linear por uma expansão em série de Taylor até primeira ordem e as coordenadas do CP são refinadas por sucessivas iterações. A existência de uma solução aproximada como ponto de partida é imprescindível nessa estratégia. Utilizamos a solução obtida com o algoritmo da grade (vide seção 2.3.2) que é ideal neste caso, pois já nos permite partir da vizinhança do ponto de mínimo global.

Uma vez encontrada a solução calculamos a matriz de covariância que resulta deste processo. Os elementos da diagonal principal fornecem a incerteza nas coordenadas do CP. O critério utilizado para interromper o processo iterativo de refinamento da solução depende da amplitude dos erros que resultam da matriz de covariância. A iteração até os limites de precisão da máquina não é necessária visto que o erro associado à posição do CP é muito maior. 
A estratégia desenvolvida neste trabalho combina (i) o método de máxima verossimilhança para a determinação simultânea da posição aproximada do CP e seleção dos membros do MG com (ii) uma rotina de minimização que retorna uma solução de CP mais refinada e a sua incerteza. Todas as derivadas utilizadas na rotina de minimização de $X^{2}$ foram implementadas de forma analítica.

\subsection{Simulações}

A determinação do CP e a seleção dos membros é influenciada por diversos fatores ligados às propriedades do MG e aos erros observacionais envolvidos no processo de medição. Nesta seção apresentamos os resultados de simulações com o objetivo de investigar a importância de cada parâmetro na solução e comparar os dois métodos de ponto de convergência (CPSM, do inglês convergent point search method): a estratégia clássica (decomposição do movimento próprio) e a nova estratégia (representação por grande círculo). A implementação da rotina de cálculo do CP e seleção dos membros do MG é feita conforme descrito na seção 2.3.4 para os dois CPSMs.

\subsubsection{Construção de dados sintéticos}

Para a construção dos dados sintéticos de MGs utilizamos o sistema de coordenadas Galácticas. Considera-se o conjunto de três vetores unitários $\hat{\mathbf{u}}, \hat{\mathbf{v}}, \hat{\mathbf{w}}$, onde $\hat{\mathbf{u}}$ aponta para o centro da Galáxia, $\hat{\mathbf{v}}$ é positivo na direção da rotação Galáctica e $\hat{\mathbf{w}}$ é normal ao plano Galáctico e aponta para o pólo norte Galáctico. A longitude Galáctica do centro do $M G$ varia de $0^{\circ}$ a $360^{\circ}$ em intervalos de $\Delta l=60^{\circ}$ com a latitude Galáctica constante em $b=0^{\circ}$. A escolha pelo plano Galáctico é justificada, pois as regiões de formação estelar da Galáxia se encontram mais próximas ao mesmo e é para as estrelas jovens que o CPSM será empregado neste trabalho. As distâncias individuais são obtidas de uma distribuição gaussiana onde o valor médio depende da configuração adotada. Consideramos três configurações de distância média nas simulações variando o tamanho angular do grupo projetado no céu conforme apresentado na Tabela 2.1. As estrelas estão distribuídas em uma região com extensão de 30 pc ao longo da linha de visada e a posição em coordenadas Galácticas $(l, b)$ é obtida de forma aleatória dentro dos limites do MG em cada configuração. 


\begin{tabular}{ccccc}
\hline$\#$ & Distância $(\mathrm{pc})$ & Tamanho Angular $l \times b\left(\mathrm{grau}^{2}\right)$ & $N_{M G}$ & $N_{\text {campo }}$ \\
\hline $\mathrm{A}$ & 100 & $20 \times 20$ & 200 & 1000 \\
$\mathrm{~B}$ & 200 & $15 \times 15$ & 100 & 500 \\
$\mathrm{C}$ & 400 & $10 \times 10$ & 50 & 250 \\
\hline
\end{tabular}

Tabela 2.1 - Distância média, tamanho angular do grupo projetado no céu, número de estrelas que pertencem ao MG e estrelas de campo para as três configurações (A,B,C) adotadas.

As componentes $(\mathrm{U}, \mathrm{V}, \mathrm{W})$ da velocidade espacial de cada estrela são obtidas independentemente por uma distribuição gaussiana com dispersão de velocidade isotrópica $\sigma_{v}=1 \mathrm{~km} / \mathrm{s}$ e a velocidade do MG (U,V,W $)_{M G}$ escolhida aleatoriamente. Uma vez definidas a velocidade, a posição e a distância das estrelas simuladas calculamos as componentes do movimento próprio $\left(\mu_{l} \cos b, \mu_{b}\right)$ e a velocidade radial $V_{r}$ de cada estrela. Os erros em posição e movimento próprio são obtidos analogamente de uma distribuição gaussiana com médias, respectivamente, de 1 mas e 1 mas/yr. As simulações também levam em conta o reflexo do movimento solar e a correção nos movimentos próprios devido ao efeito de rotação Galáctica. Adotamos para o movimento solar os valores $(U, V, W)_{\odot}=$ $(10.00,5.23,7.17) \mathrm{km} / \mathrm{s}$ de Dehnen \& Binney (1998) e para a rotação Galáctica as constantes de Oort $A=14.82 \mathrm{~km} \mathrm{~s}^{-1} \mathrm{kpc}^{-1}$ e $B=-12.37 \mathrm{~km} \mathrm{~s}^{-1} \mathrm{kpc}^{-1}$ dadas por Feast \& Whitelock (1997). A rotação Galáctica é introduzida usando as fórmulas de aproximação em primeira ordem de Green (1985).

Os conjuntos de dados sintéticos são construídos com estrelas que pertencem ao MG e estrelas de campo na proporção 1:5. O modelo de população de campo adotado é simples mas suficiente para os objetivos do trabalho. As estrelas de campo são colocadas na mesma região do céu em que se encontra o MG, têm movimentos aleatórios (em direção e amplitude) e os erros na posição e no movimento próprio são da mesma ordem de grandeza daqueles simulados para as estrelas do MG. A densidade total de estrelas em cada conjunto simulado é próxima daquela conhecida para o catálogo Hipparcos $\left(\sim 3\right.$ estrelas/grau $\left.{ }^{2}\right)$.

Ao todo foram realizadas 100 simulações Monte Carlo para cada uma das 18 configurações (vide Tabela 2.1 e início desta seção) investigadas neste trabalho, resultando em um total de 1800 simulações. O valor da dispersão de velocidade utilizado no cálculo do CP é o mesmo usado para a construção dos dados sintéticos. Adotamos o valor de $\epsilon_{\min }=0.954$ (conforme proposto por de Bruijne 1999a) para os dois CPSMs. O valor desse parâmetro 
será discutido mais adiante na seção 2.4.4. Iniciamos a análise dos dados simulados com um estudo de pertinência na seção 2.4.2 para comparar a eficiência de cada CPSM em identificar os membros do MG e eliminar as estrelas de campo nos conjuntos de dados sintéticos. Na seção 2.4.3 investigamos a posição do CP em função de diversos parâmetros (dispersão de velocidade, erros observacionais, posição no céu e tamanho da amostra).

\subsubsection{Seleção dos membros e rejeição das estrelas de campo}

As estrelas que pertencem ao mesmo MG exibem propriedades cinemáticas que nos permitem separá-las das estrelas de campo. É necessário distinguir entre dois tipos de população de campo: (i) estrelas de campo à mesma distância do MG e (ii) estrelas de fundo, mais distantes que o grupo em questão.

Consideramos primeiramente o caso em que a população de campo se encontra à mesma distância média do MG. A fração de estrelas de campo rejeitadas pelo CPSM para cada configuração da Tabela 2.1 é apresentada na Figura 2.5. A taxa de estrelas de campo que não são rejeitadas é de aproximadamente $20 \%$ para os dois CPSMs até 200 pc. À medida que a distância aumenta a eficiência em eliminar estrelas de campo diminui para os dois CPSMs. As simulações indicam que aproximadamente $80 \%$ dos membros são corretamente identificados pelos dois CPSMs. Esses resultados estão em perfeito acordo com aqueles obtidos por de Bruijne (1999a) para o CPSM clássico. Em alguns casos a quantidade de estrelas de campo que é eliminada pelo novo CPSM é maior do que aquela observada para o CPSM clássico.

Em seguida substituímos a população de campo à mesma distância média do MG por uma população de estrelas de fundo que se encontra duas vezes mais distante. Os resultados são apresentados na Figura 2.6. O novo CPSM elimina mais estrelas de campo do que o CPSM clássico e a diferença de desempenho entre os dois CPSMs aumenta com a distância. Esse resultado é explicado pelo processo de minimização de $X^{2}$ que no caso do CPSM clássico considera somente a amplitude de uma das componentes do movimento próprio (i.e., $\mu_{\perp}$ ). No entanto, nem todas estrelas com $\mu_{\perp}$ pequeno pertencem ao MG. Isso ocorre, porque as estrelas distantes têm movimentos próprios pequenos e consequentemente a componente $\mu_{\perp}$ é também pequena. Por outro lado, a representação por grandes círculos no novo CPSM considera a amplitude e a direção do vetor movimento próprio no processo 

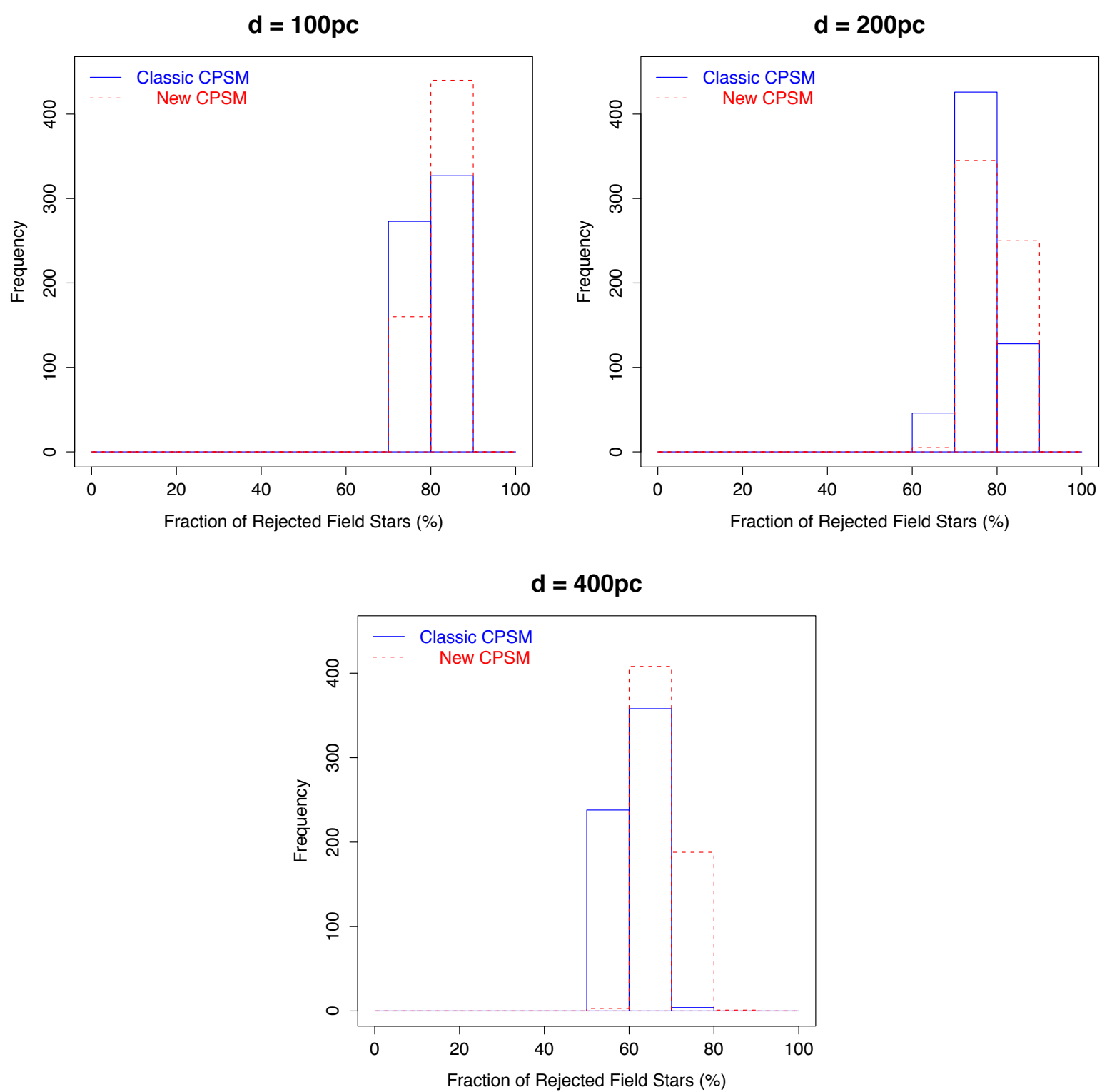

Figura 2.5: Fração de estrelas de campo rejeitadas pelos dois CPSMs. Os resultados são apresentados para MGs centrados em $l=60^{\circ}, 120^{\circ}, 180^{\circ}, 240^{\circ}, 300^{\circ}, 360^{\circ}$ e $b=0^{\circ}$ nas três configurações (A,B,C) de distância média consideradas. A população de campo se encontra à mesma distância média do MG.

Figura retirada de Galli et al. (2012). 

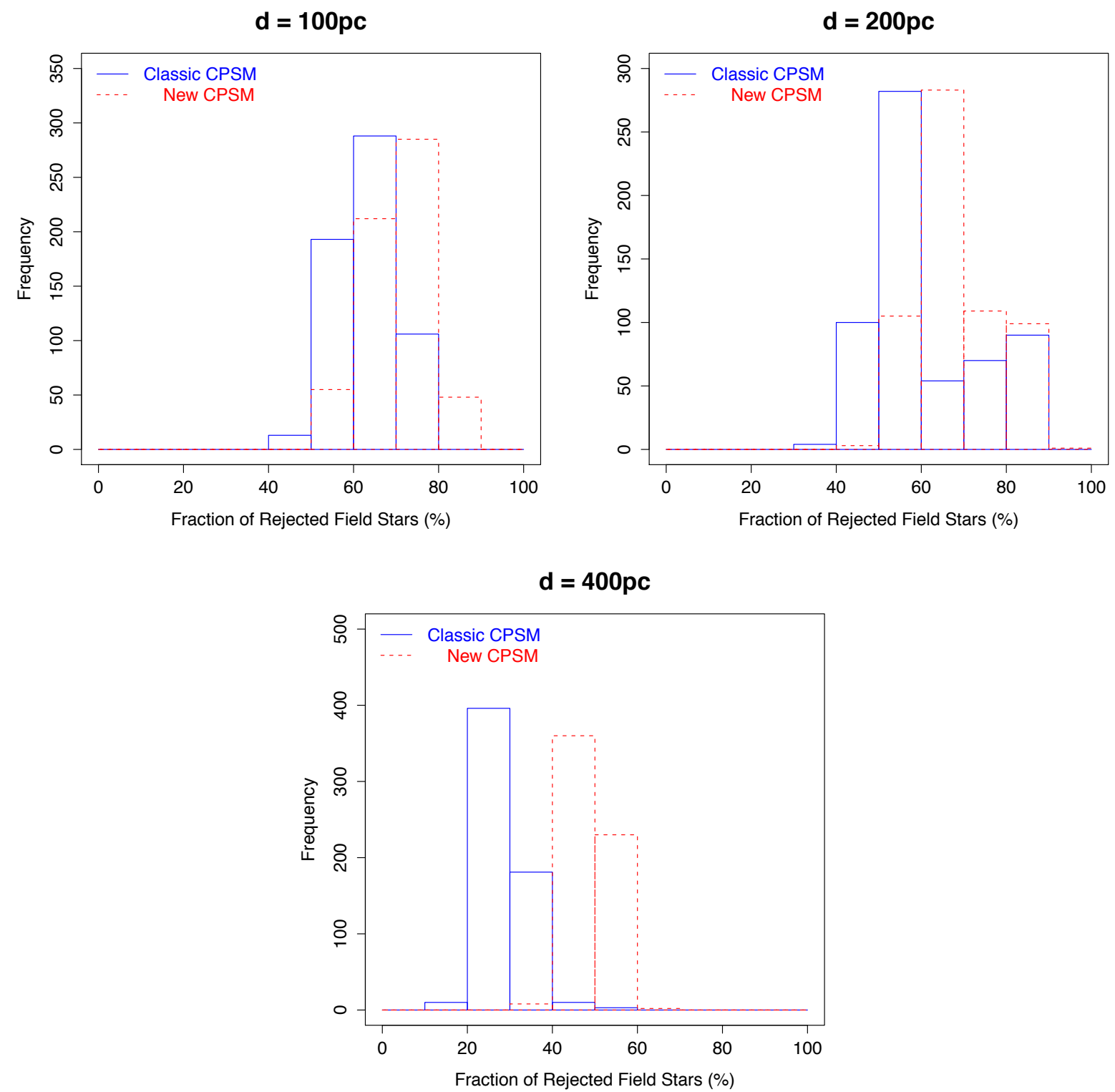

Figura 2.6: Fração de estrelas de campo rejeitadas pelos dois CPSMs. Os resultados são apresentados para MGs centrados em $l=60^{\circ}, 120^{\circ}, 180^{\circ}, 240^{\circ}, 300^{\circ}, 360^{\circ}$ e $b=0^{\circ}$ nas três configurações (A,B,C) de distância média consideradas. A população de campo se encontra duas vezes mais distante que o MG. Figura retirada de Galli et al. (2012). 
de minimização removendo o viés seletivo. Concluímos que o novo CPSM desenvolvido neste trabalho é mais eficiente na seleção dos membros do MG.

\subsubsection{Análise da posição do ponto de convergência}

Conforme observado na seção anterior, o CPSM permite identificar os membros do MG com uma contaminação estimada em $20 \%$ do número total de estrelas de campo. Apesar da alta taxa de sucesso na identificação dos membros verdadeiros, a posição calculada do CP fica deslocada da sua posição real devido às estrelas de campo que não foram eliminadas no processo. Isso ocorre em parte, porque a população de campo é dominante na amostra inicial. Para melhor investigar a solução de CP em função de outros fatores é necessário partir de um conjunto de estrelas onde o MG é dominante. Assim, construímos novos conjuntos de dados sintéticos (conforme descrito na seção 2.4.1) com 100 estrelas $\left(N_{M G}=80, N_{\text {campo }}=20\right)$ para prosseguir com a análise.

\subsubsection{Efeito da dispersão de velocidade}

A dispersão de velocidade das estrelas impede que os vetores de movimento próprio apontem exatamente na direção do CP. Investigamos esse efeito na solução obtida por cada CPSM em conjuntos de dados construídos com $\sigma_{v}=1 \mathrm{~km} / \mathrm{s}, \sigma_{v}=2 \mathrm{~km} / \mathrm{s}$ e $\sigma_{v}=3 \mathrm{~km} / \mathrm{s}$ (conforme descrito na seção 2.4.1). Os resultados obtidos são apresentados na Figura 2.7. As soluções de CP estão sobrepostas às curvas de contorno com $X^{2}$ constante calculadas para um MG sem dispersão de velocidade centrado na mesma posição e distância dos MGs simulados. Como exemplo consideramos o caso de um MG centrado em $(l, b)=\left(180^{\circ}, 0^{\circ}\right)$ a 100 pc de distância. O mesmo comportamento é observado para as demais configurações da Tabela 2.1. Como podemos observar pela Figura 2.7, as soluções obtidas pelos dois CPSMs se situam dentro da banda central com o menor valor de $X^{2}$, conforme esperado. Conclui-se que quanto maior a dispersão de velocidade (maior perturbação nos movimentos próprios), maior é a dispersão dos CPs calculados. O comportamento observado é semelhante para os dois CPSMs. 

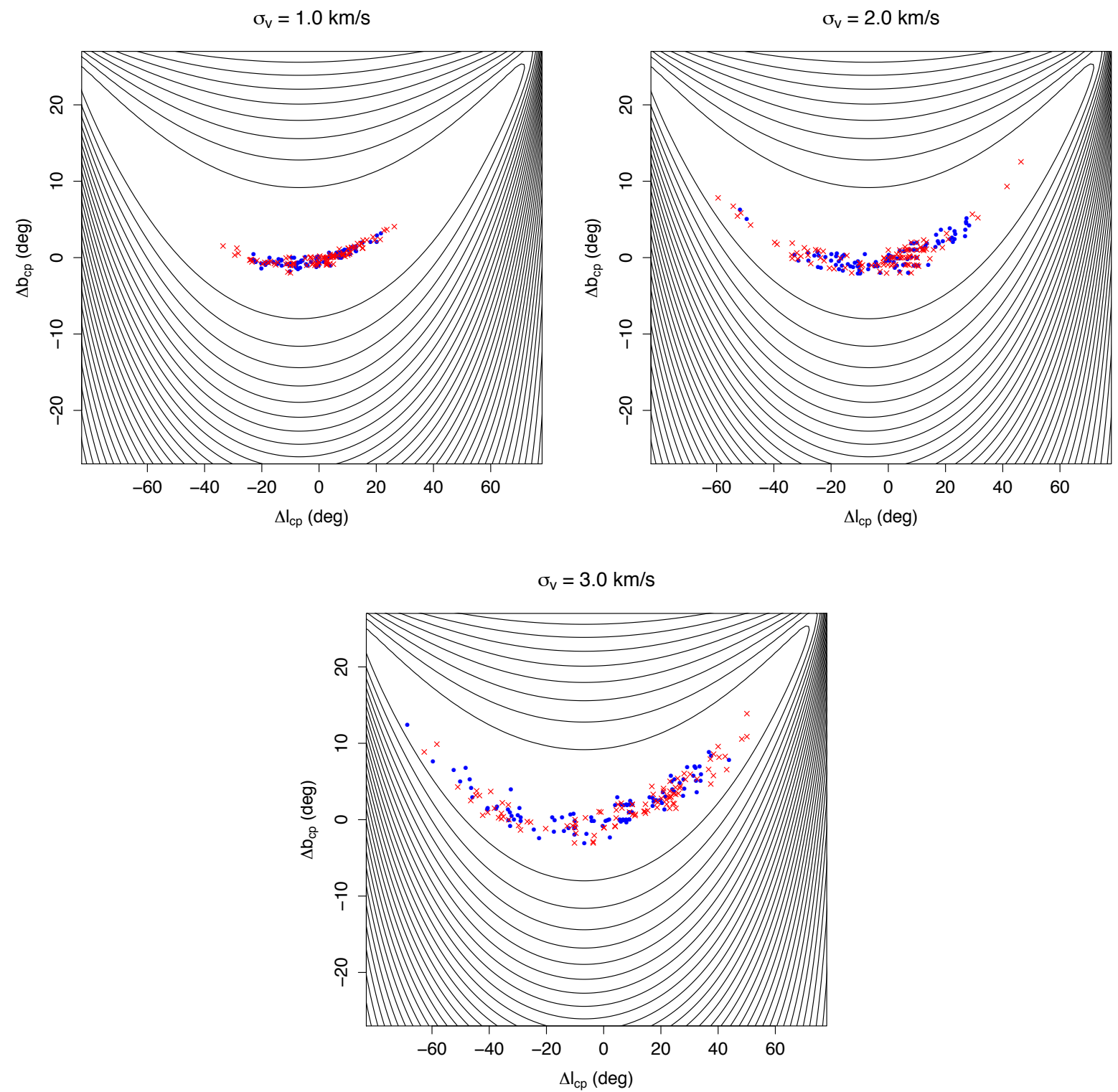

Figura 2.7: Posição relativa $\left(\Delta l_{c p}, \Delta b_{c p}\right)$ dos CPs para 100 simulações Monte Carlo com $\sigma_{v}=1 \mathrm{~km} / \mathrm{s}$, $\sigma_{v}=2 \mathrm{~km} / \mathrm{s}$ e $\sigma_{v}=3 \mathrm{~km} / \mathrm{s}$. Os símbolos azuis e vermelhos denotam, respectivamente, os resultados obtidos com o CPSM clássico e o novo CPSM. As linhas de contorno indicam valores constantes de $X^{2}$ linearmente espaçados em $\Delta X^{2}=2000$ para um MG com CP em $\left(\Delta l_{c p}, \Delta b_{c p}\right)=\left(0^{\circ}, 0^{\circ}\right)$. A posição relativa do CP $\left(l_{c p}^{s i m}, b_{c p}^{s i m}\right)$ para cada simulação é dada por $\Delta l_{c p}=l_{c p}^{s i m}-l_{c p}$ e $\Delta b_{c p}=b_{c p}^{s i m}-b_{c p}$. Figura retirada de Galli et al. (2012). 


\subsubsection{Precisão no ponto de convergência}

O erro na posição do CP é influenciado por diversos parâmetros que serão investigados neste momento. Inicialmente comparamos o erro obtido em cada CPSM definindo a grandeza $\Delta \sigma_{c p}=\sigma_{c p}^{\text {classico }}-\sigma_{c p}^{\text {novo }}$, onde

$$
\sigma_{c p}=\sqrt{\sigma_{l_{c p}}^{2}+\sigma_{b_{c p}}^{2}}
$$

Os resultados dessa análise são apresentados na Figura 2.8. O novo CPSM retorna uma solução mais precisa do que o CPSM clássico para $95 \%$ dos casos simulados $\left(\Delta \sigma_{c p}>0\right)$. Por outro lado, quando a distância aumenta o número de casos em que $\Delta \sigma_{c p}<0$ também aumenta. A nova estratégia proposta neste trabalho define o $\mathrm{CP}$ como a intersecção dos grandes círculos das estrelas. À medida que a distância aumenta, as estrelas ficam mais concentradas no céu e consequentemente o ponto de intersecção dos grandes círculos fica pior definido o que explica o resultado observado.

Existem outros parâmetros como por exemplo, os erros nos movimentos próprios, a quantidade de estrelas na amostra e a posição relativa entre as estrelas e o CP que também afetam a solução. A maneira mais eficiente de investigar a importância de cada parâmetro consiste em adotar um modelo simplificado que recebe o nome de MG ideal. Os efeitos da dispersão de velocidade, rotação Galáctica e contaminação por estrelas de campo são desconsiderados nesse modelo. Nesse caso os dois CPSMs retornam sempre a mesma solução de $\mathrm{CP}$ com $X^{2}=0$ e $\epsilon=1$. Em seguida investigamos o efeito de cada parâmetro mencionado acima na solução de CP. Enfatizamos que os resultados qualitativos desta investigação não dependem de nenhuma configuração de posição ou distância adotada. No entanto, a amplitude dos erros de CP apresentados na Figura 2.9 (eixo das ordenadas) é específica para a configuração A da Tabela 2.1 e um MG centrado em $(l, b)=\left(180^{\circ}, 0^{\circ}\right)$.

O primeiro parâmetro a ser investigado é o erro nos movimentos próprios. Construímos diferentes conjuntos de dados variando a qualidade dos movimentos próprios com erros relativos de $5 \%$ a 100\%. A Figura 2.9(a) mostra que os erros no CP aumentam linearmente com os erros nos movimentos próprios. 

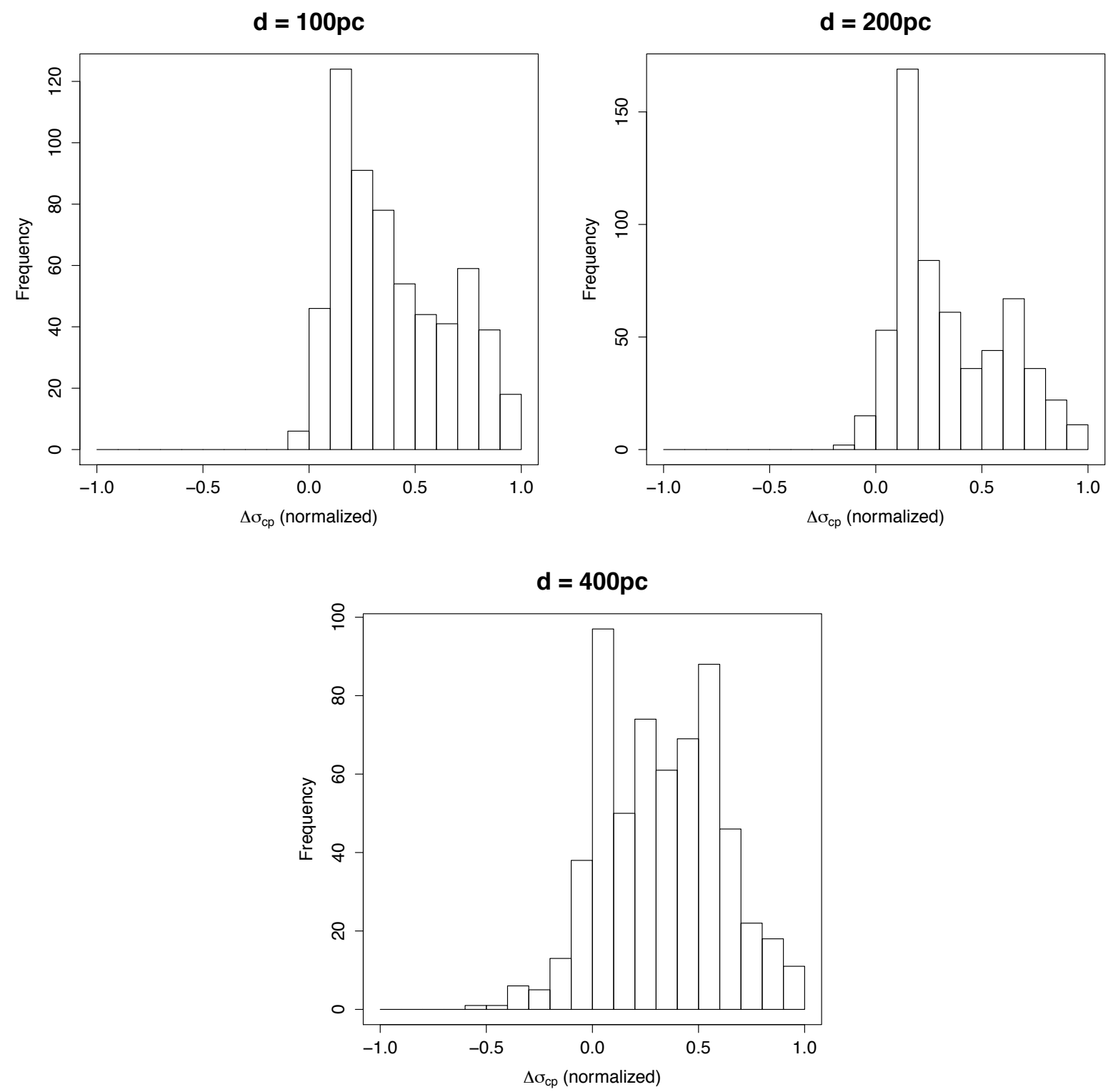

Figura 2.8: Comparação dos erros na solução de CP obtida com os dois CPSMs. Os resultados são apresentados para MGs centrados em $l=60^{\circ}, 120^{\circ}, 180^{\circ}, 240^{\circ}, 300^{\circ}, 360^{\circ} \operatorname{com} b=0^{\circ}$ nas três configurações $(\mathrm{A}, \mathrm{B}, \mathrm{C})$ de distância média consideradas. A grandeza $\Delta \sigma_{c p}$ está normalizada para indicar somente valores relativos entre os dois CPSMs. Figura retirada de Galli et al. (2012). 
Como mencionado anteriormente, a distância exerce um efeito na precisão do CP. As estrelas mais distantes têm movimentos próprios menores e o erro relativo é em geral pior. Por outro lado, a maior distância do MG tende também a concentrar as estrelas no céu. Qual dos efeitos é mais importante na determinação do CP ? Na prática não é possível separar esses dois efeitos, mas nas simulações conseguimos analisá-los individualmente. Construímos outros dois conjuntos de dados: no primeiro caso variamos a distância média ao MG com o tamanho angular fixo e no segundo caso, variamos o tamanho angular do MG com a distância fixa. A Figura 2.9(b) e (c) ilustra os resultados obtidos. Concluímos que a maior concentração das estrelas é o efeito dominante à medida que a distância aumenta.

Um outro parâmetro que contribui na solução é o número de estrelas utilizadas na determinação do CP. Foram construídos novos conjuntos de dados variando o tamanho da amostra de 10 a 300 estrelas. Os resultados dessa análise são apresentados na Figura 2.9(d). Observamos que para $N>200$ não existe uma melhora significativa na precisão do CP.

Por fim investigamos o efeito da posição relativa entre as estrelas do MG e o CP. Consideramos uma configuração específica dos parâmetros mencionados anteriormente e o caso particular em que as estrelas têm velocidade isotrópica o que define o CP com coordenadas $\left(l_{c p}, b_{c p}\right)=\left(45.00^{\circ}, 35.26^{\circ}\right)$. Foram construídos novos conjuntos de dados variando somente a longitude Galáctica do $M G$ de $0^{\circ}$ a $360^{\circ}$ em passos de $\Delta l=0.15^{\circ}$ e $b=0^{\circ}$. Os resultados são apresentados na Figura 2.9(e) e (f). Observamos que dois MGs defasados em $180^{\circ}$ apresentam os mesmos erros no CP. Isso ocorre porque a distância angular à solução mais próxima (CP ou DP) é a mesma. Portanto, a posição relativa entre as estrelas do MG e o CP influencia a precisão do resultado. A solução menos precisa ocorre para os MGs que se encontram a $90^{\circ}$ do $\mathrm{CP}$.

\subsubsection{Escolha de $\epsilon_{\min }$}

A seleção de estrelas pelo CPSM depende do valor de $\epsilon_{\min }$ considerado. Os resultados apresentados até o momento foram obtidos $\operatorname{com} \epsilon_{\min }=0.954$ para os dois CPSMs. Esse é o valor utilizado por de Bruijne (1999a) no CPSM clássico. Nesta seção será justificado o uso desse valor para o novo CPSM. 

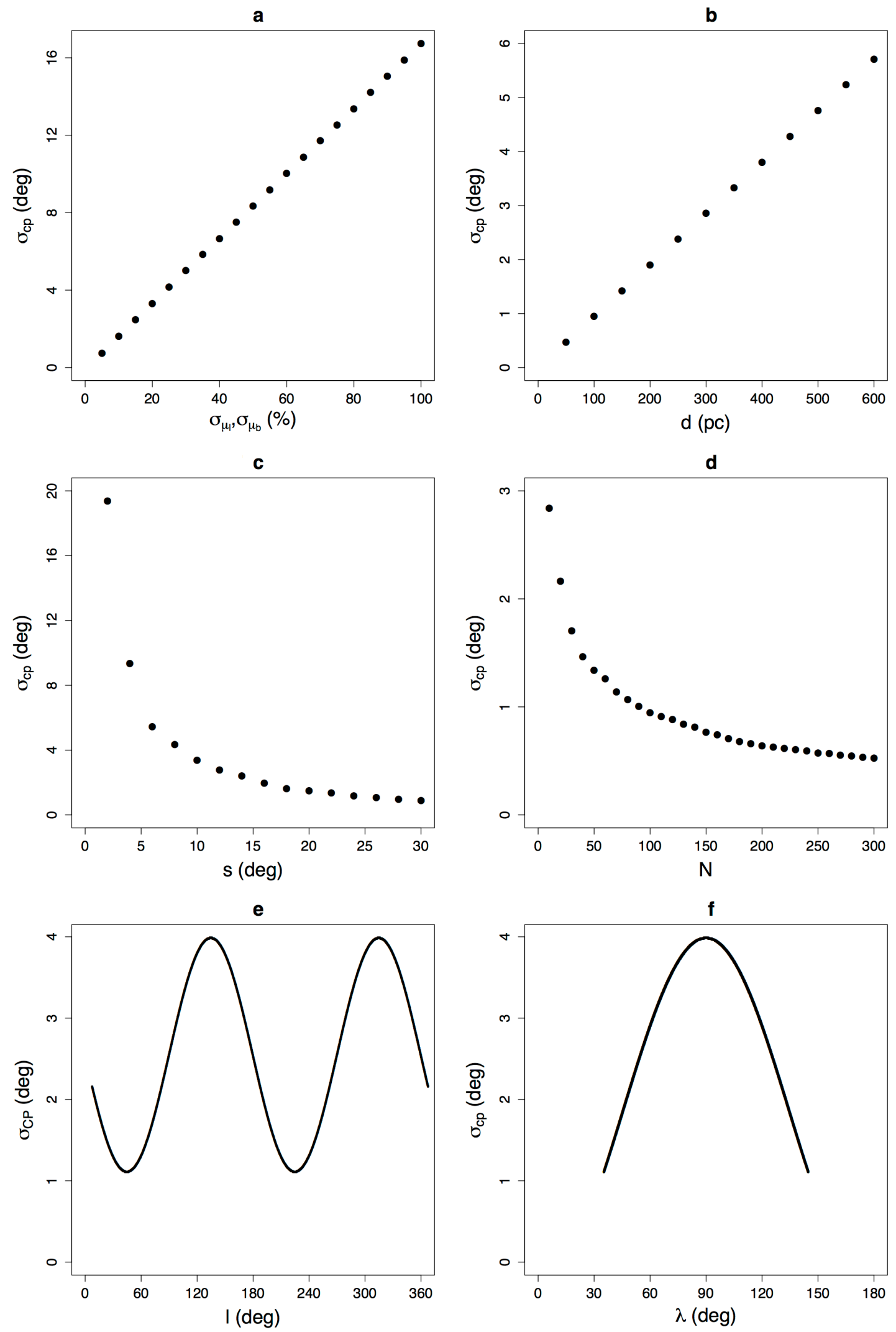

Figura 2.9: Precisão do ponto de convergência em função (a) dos erros no movimento próprio, (b) da distância média ao MG com tamanho angular fixo, (c) do tamanho angular do MG ( $\mathrm{s} \times \mathrm{s})$ com distância fixa, (d) do número de membros, (e) da longitude Galáctica do centro do MG (estrelas com velocidade isotrópica) e (f) da distância angular $\lambda$ do CP ao centro do MG. Figura retirada de Galli et al. (2012). 
Aplicamos os dois CPSMs nos conjuntos de dados sintéticos da seção 2.4.1 variando o valor de $\epsilon_{\min }$ entre 0 e 1 . Para cada valor considerado medimos a fração de membros do MG que foram identificados e as estrelas de campo rejeitadas. Essa análise é apresentada na Figura 2.10 para o caso de um MG centrado em $\left(180^{\circ}, 0^{\circ}\right)$ a 100 pc. O mesmo comportamento é observado para as demais configurações da Tabela 2.1. Como podemos notar, para $\epsilon_{\min }>0.954$ o número de membros do MG que são rejeitados continua aumentando enquanto a fração de estrelas de campo na amostra estabiliza para os dois CPSMs. Conclui-se que $\epsilon_{\min }=0.954$ é também adequado para o novo CPSM. Esse valor representa o melhor compromisso entre a quantidade de membros do MG que são identificados e as estrelas de campo que são rejeitadas.

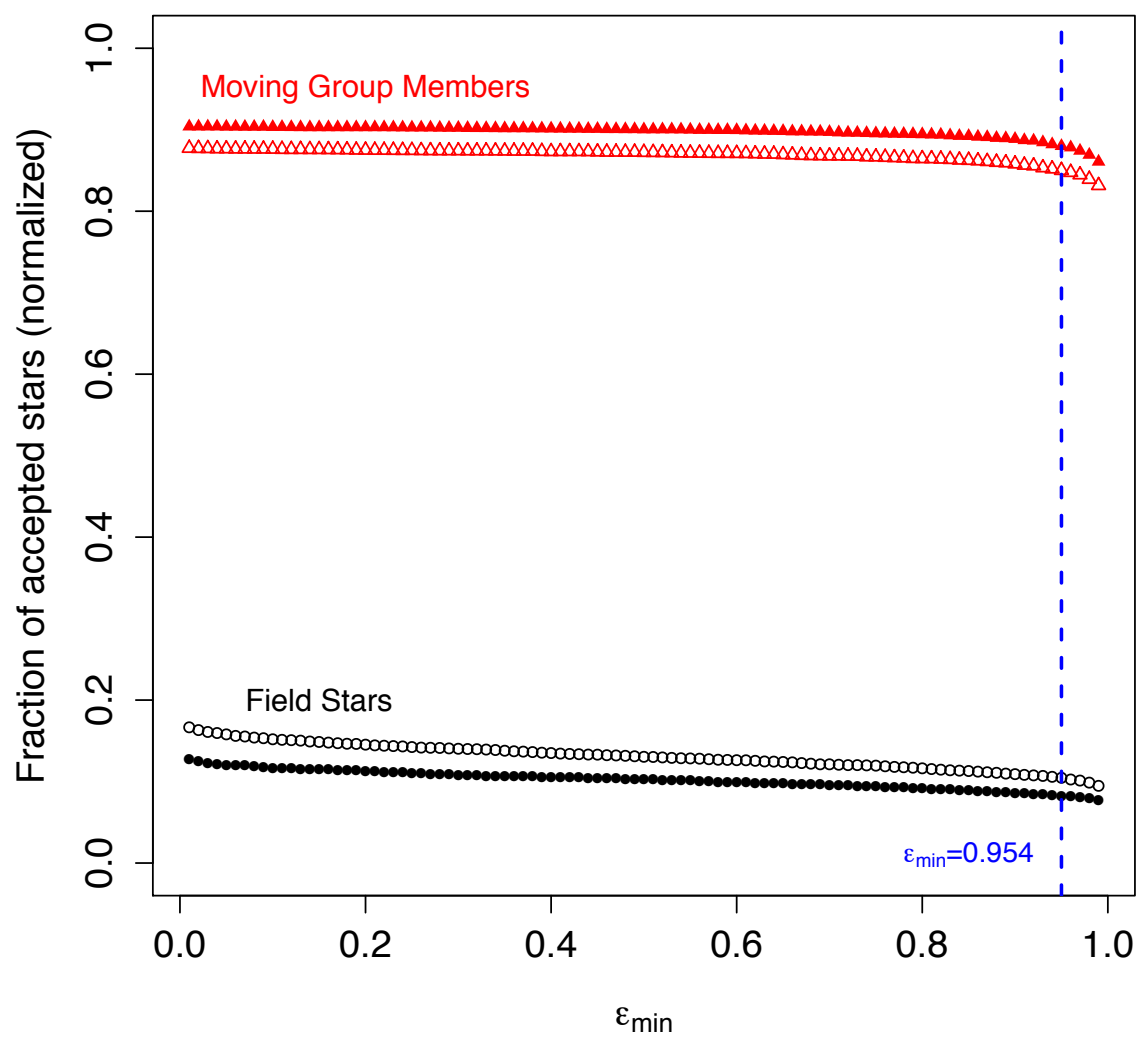

Figura 2.10: Fração de membros do MG e estrelas de campo que são aceitos pelo CPSM em função do valor de $\epsilon_{\text {min }}$. Círculos e triângulos denotam, respectivamente, as estrelas de campo e os membros do MG. Símbolos abertos e fechados representam, respectivamente, os resultados do CPSM clássico e o novo CPSM. A linha azul tracejada indica o valor de $\epsilon_{\min }=0.954$ adotado neste trabalho. Cada ponto é uma média de 100 simulações Monte Carlo para um MG centrado em $\left(180^{\circ}, 0^{\circ}\right)$ a $100 \mathrm{pc}$.

Figura retirada de Galli et al. (2012). 


\subsubsection{Teste de normalidade da distribuição de $s$}

Na seção 2.3.2 assumimos que $s$ segue uma distribuição normal com média igual a zero e variância igual à unidade. Verificamos a validade dessa hipótese em simulações de MGs com diferentes configurações de posição, distância, tamanho angular e dispersão de velocidade. O teste de normalidade de Kolmogorov-Smirnov foi utilizado para investigar se a distribuição de $s$ é normal. Os resultados são apresentados na Figura 2.11 para um conjunto de 1000 simulações Monte Carlo. Para 99.6\% dos casos simulados o valor de $p$ fornecido pelo teste estatístico, é superior ao nível de significância de $5 \%$ ( $\alpha=0.05)$ o que nos permite concluir que a hipótese nula (distribuição normal em $s$ ) é válida.

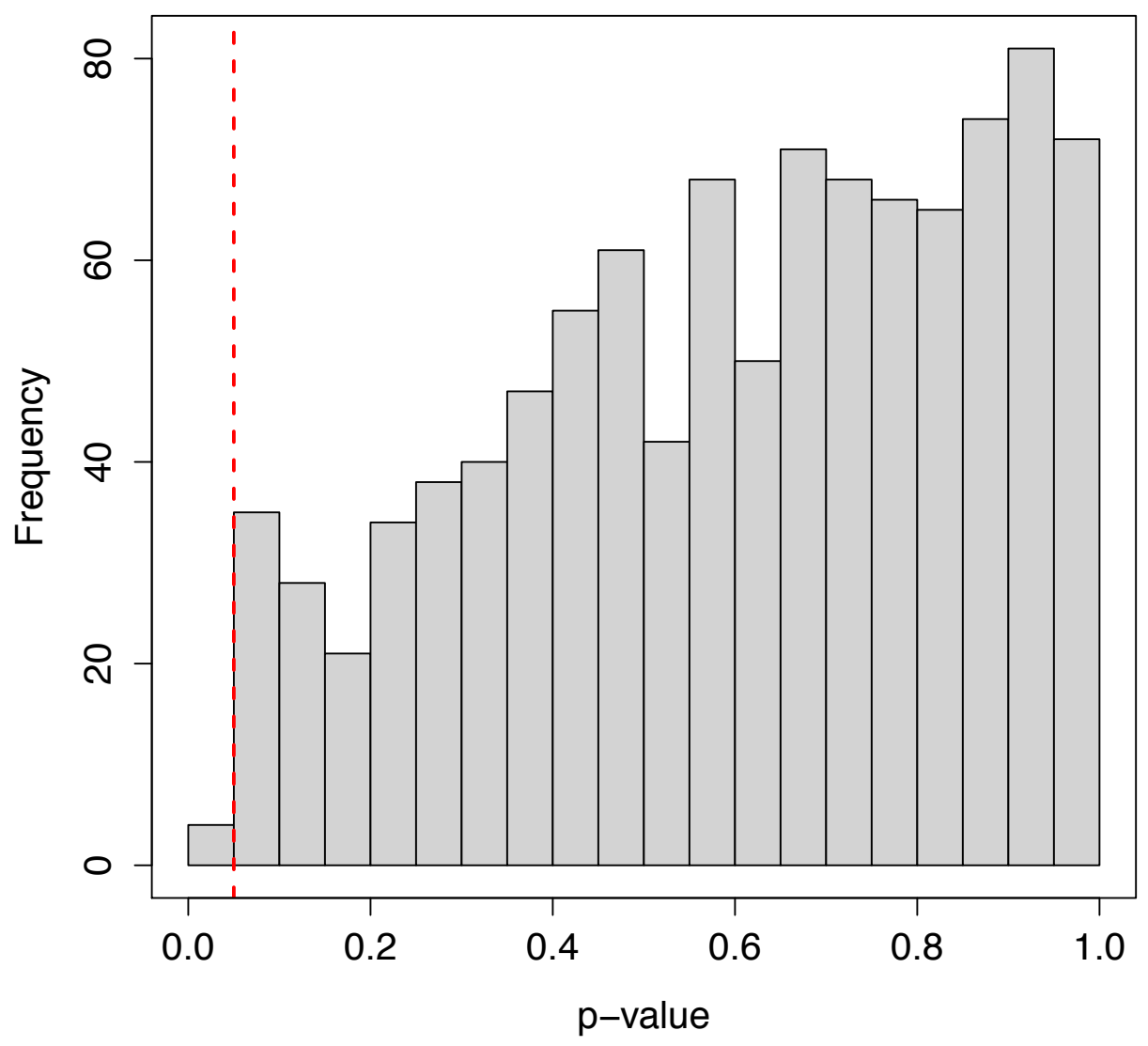

Figura 2.11: Valores de $p$ obtidos com o teste de Kolmogorov-Smirnov para um conjunto de 1000 simulações Monte Carlo de MGs com diferentes configurações. A linha vermelha tracejada indica o nível de significância adotado $(\alpha=0.05)$. Figura retirada de Galli et al. (2012). 


\subsection{Aplicação ao Aglomerado Aberto das Híades}

O aglomerado aberto das Híades é utilizado neste trabalho como uma primeira aplicação do novo CPSM em dados reais. Os dados utilizados para o estudo desse aglomerado são oriundos do catálogo Hipparcos.

\subsubsection{Matriz de covariância}

Os catálogos astrométricos fornecem geralmente apenas o erro em $1 \sigma$ das grandezas observadas (posição, movimento próprio e paralaxe). O catálogo HipPARCos fornece para cada estrela a matriz de covariância completa na forma $5 \times 5$ indicando explicitamente o coeficiente de correlação das variáveis envolvidas $\left(\alpha, \delta, \mu_{\alpha}^{*}, \mu_{\delta}, \pi\right)$. Nesse caso o uso da matriz de covariância completa na propagação de erros é obrigatório. O erro associado às coordenadas do pólo do grande círculo de cada estrela (vide seção 2.3.2) deve considerar os termos de segunda ordem. A expressão completa para a propagação de erros é dada por

$$
\begin{aligned}
\sigma_{\alpha^{p}}^{2} & =\left(\frac{\partial \alpha^{p}}{\partial \alpha}\right)^{2} \sigma_{\alpha}^{2}+\left(\frac{\partial \alpha^{p}}{\partial \delta}\right)^{2} \sigma_{\delta}^{2}+\left(\frac{\partial \alpha^{p}}{\partial \mu_{\alpha}^{*}}\right)^{2} \sigma_{\mu_{\alpha^{*}}}^{2}+\left(\frac{\partial \alpha^{p}}{\partial \mu_{\delta}}\right)^{2} \sigma_{\mu_{\delta}}^{2}+ \\
& +2\left(\frac{\partial \alpha^{p}}{\partial \alpha}\right)\left(\frac{\partial \alpha^{p}}{\partial \delta}\right) \rho_{\alpha \delta} \sigma_{\alpha} \sigma_{\delta}+2\left(\frac{\partial \alpha^{p}}{\partial \alpha}\right)\left(\frac{\partial \alpha^{p}}{\partial \mu_{\alpha}^{*}}\right) \rho_{\alpha \mu_{\alpha}^{*}} \sigma_{\alpha} \sigma_{\mu_{\alpha}^{*}}+ \\
+ & 2\left(\frac{\partial \alpha^{p}}{\partial \alpha}\right)\left(\frac{\partial \alpha^{p}}{\partial \mu_{\delta}}\right) \rho_{\alpha \mu_{\delta}} \sigma_{\alpha} \sigma_{\mu_{\delta}}+2\left(\frac{\partial \alpha^{p}}{\partial \delta}\right)\left(\frac{\partial \alpha^{p}}{\partial \mu_{\alpha}^{*}}\right) \rho_{\delta \mu_{\alpha}^{*}} \sigma_{\delta} \sigma_{\mu_{\alpha}^{*}}+ \\
+ & 2\left(\frac{\partial \alpha^{p}}{\partial \delta}\right)\left(\frac{\partial \alpha^{p}}{\partial \mu_{\delta}}\right) \rho_{\delta \mu_{\delta}} \sigma_{\delta} \sigma_{\mu_{\delta}}+2\left(\frac{\partial \alpha^{p}}{\partial \mu_{\alpha}^{*}}\right)\left(\frac{\partial \alpha^{p}}{\partial \mu_{\delta}}\right) \rho_{\mu_{\alpha}^{*} \mu_{\delta}} \sigma_{\mu_{\alpha}^{*}} \sigma_{\mu_{\delta}}, \\
\sigma_{\delta^{p}}^{2} & =\left(\frac{\partial \delta^{p}}{\partial \alpha}\right)^{2} \sigma_{\alpha}^{2}+\left(\frac{\partial \delta^{p}}{\partial \delta}\right)^{2} \sigma_{\delta}^{2}+\left(\frac{\partial \delta^{p}}{\partial \mu_{\alpha}^{*}}\right)^{2} \sigma_{\mu_{\alpha^{*}}}^{2}\left(\frac{\partial \delta^{p}}{\partial \mu_{\delta}}\right)^{2} \sigma_{\mu_{\delta}}^{2}+ \\
& +2\left(\frac{\partial \delta^{p}}{\partial \alpha}\right)\left(\frac{\partial \delta^{p}}{\partial \delta}\right) \rho_{\alpha \delta} \sigma_{\alpha} \sigma_{\delta}+2\left(\frac{\partial \delta^{p}}{\partial \alpha}\right)\left(\frac{\partial \delta^{p}}{\partial \mu_{\alpha}^{*}}\right) \rho_{\alpha \mu_{\alpha}^{*}} \sigma_{\alpha} \sigma_{\mu_{\alpha}^{*}}+ \\
+ & 2\left(\frac{\partial \delta^{p}}{\partial \alpha}\right)\left(\frac{\partial \delta^{p}}{\partial \mu_{\delta}}\right) \rho_{\alpha \mu_{\delta}} \sigma_{\alpha} \sigma_{\mu_{\delta}}+2\left(\frac{\partial \delta^{p}}{\partial \delta}\right)\left(\frac{\partial \delta^{p}}{\partial \mu_{\alpha}^{*}}\right) \rho_{\delta \mu_{\alpha}^{*}} \sigma_{\delta} \sigma_{\mu_{\alpha}^{*}}+ \\
+ & 2\left(\frac{\partial \delta^{p}}{\partial \delta}\right)\left(\frac{\partial \delta^{p}}{\partial \mu_{\delta}}\right) \rho_{\delta \mu_{\delta}} \sigma_{\delta} \sigma_{\mu_{\delta}}+2\left(\frac{\partial \delta^{p}}{\partial \mu_{\alpha}^{*}}\right)\left(\frac{\partial \delta^{p}}{\partial \mu_{\delta}}\right) \rho_{\mu_{\alpha}^{*} \mu_{\delta}} \sigma_{\mu_{\alpha}^{*}} \sigma_{\mu_{\delta}},
\end{aligned}
$$

onde $\rho$ representa o coeficiente de correlação entre duas variáveis. O uso das equações (2.36) e (2.37) deve ser preferível sempre que possível. Esse mesmo procedimento poderá ser empregado nos dados da futura missão espacial GAIA que também terá o seu catálogo final publicado com a matriz de covariância completa (Holl \& Lindegren 2012). 


\subsubsection{Análise do ponto de convergência}

A amostra em estudo é formada por uma lista de 218 estrelas do catálogo Hipparcos investigadas por Perryman et al. (1998). A pertinência ao aglomerado aberto das Híades foi estabelecida com base no movimento espacial das estrelas. Os dados de posição e movimento próprio são do catálogo Hipparcos, enquanto as velocidades radiais utilizadas por Perryman et al. (1998) foram obtidas de diversas fontes na literatura.

Uma análise posterior do CP desse aglomerado foi realizada por de Bruijne (1999a) e o resultado encontrado foi $\left(\alpha_{c p}, \delta_{c p}\right)=\left(97.81^{\circ}, 6.74^{\circ}\right) \pm\left(0.52^{\circ}, 0.21^{\circ}\right)$ com 213 estrelas identificadas como membro do aglomerado. Essa solução foi obtida usando a dispersão de velocidade $\sigma_{v}=2 \mathrm{~km} / \mathrm{s}$. Conforme discutido por de Bruijne (1999a) um valor superior a $1.5 \mathrm{~km} / \mathrm{s}$ é necessário para recuperar todos os membros do aglomerado. Adotando a mesma dispersão de velocidade $\left(\sigma_{v}=2 \mathrm{~km} / \mathrm{s}\right)$ e a distância média de 46 pc fornecida por Perryman et al. (1998) determinamos o CP das Híades utilizando o novo CPSM. O resultado obtido é $\left(\alpha_{c p}, \delta_{c p}\right)=\left(97.69^{\circ}, 6.30^{\circ}\right) \pm\left(0.89^{\circ}, 0.36^{\circ}\right)$ com 217 estrelas identificadas como membro do aglomerado. Conclui-se que as duas soluções são compatíveis entre si e o número de membros identificados pelo novo CPSM é maior. Uma determinação independente do CP das Híades foi obtida por Schwan (1991) partindo de uma amostra menor com 145 estrelas e movimentos próprios dos catálogos FK5 (Fricke et al. 1988) e PPM (Roeser \& Bastian 1988). O resultado obtido, $\left(\alpha_{c p}, \delta_{c p}\right)=\left(97.68^{\circ}, 5.98^{\circ}\right) \pm\left(0.42^{\circ}, 0.18^{\circ}\right)$, é também consistente com a solução do novo CPSM. A Figura 2.12 ilustra os movimentos próprios das estrelas do aglomerado aberto das Híades e as soluções de CP em cada trabalho.

O novo CPSM eliminou somente uma estrela (Hip 28774) do conjunto inicial de 218 estrelas utilizadas no cálculo do CP. O movimento próprio dessa estrela é $\left(\mu_{\alpha} \cos \delta, \mu_{\delta}\right)=$ $(-2.46,-7.00) \pm(18.43,9.99) \mathrm{mas} / \mathrm{yr}$ na primeira versão do catálogo HippARCos (ESA 1997, HIP97) e $\left(\mu_{\alpha} \cos \delta, \mu_{\delta}\right)=(42.95,-2.16) \pm(14.68,8.04)$ mas/yr na nova versão do catálogo (van Leeuwen 2007, HIP07). A sua paralaxe também varia bastante nas duas versões do catálogo: $\pi_{H I P 97}=(12.81 \pm 12.80)$ mas e $\pi_{H I P 07}=(39.18 \pm 11.54)$ mas. O grau de incerteza e a baixa qualidade dos dados para essa estrela justificam a sua eliminação. 


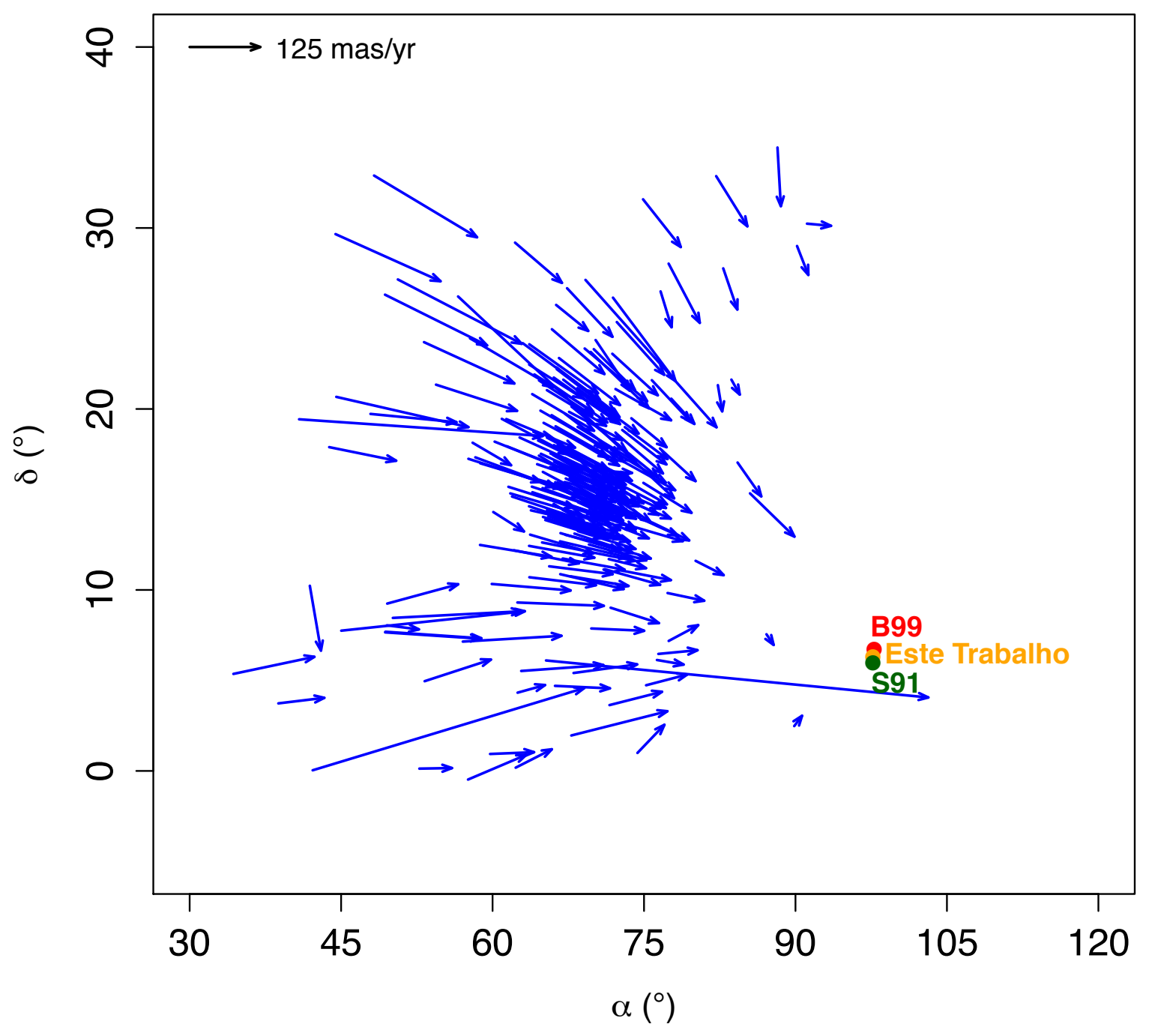

Figura 2.12: Diagrama dos vetores movimento próprio para o conjunto de 218 estrelas do aglomerado aberto das Híades identificadas por Perryman et al. (1998) e os CPs obtidos por de Bruijne (1999a, B99), Schwan (1991, S91) e neste trabalho. As barras de erro no CP são muito pequenas para serem visualizadas. 


\subsubsection{Paralaxes cinemáticas}

Uma vez conhecida a posição do CP calculamos a paralaxe individual de cada membro do aglomerado das Híades com velocidade radial conhecida usando a equação (2.5). A velocidade radial dessas estrelas é dada na Tabela 2 de Perryman et al. (1998). A incerteza na paralaxe leva em conta os erros no movimento próprio e na velocidade radial das estrelas.

As paralaxes calculadas neste trabalho são comparadas com dois conjuntos de dados independentes: paralaxes trigonométricas do catálogo Hipparcos e paralaxes seculares de de Bruijne et al. (2001). A comparação com as paralaxes trigonométricas é apresentada na Figura 2.13. O desvio quadrático médio das diferenças (r.m.s., do inglês root mean square) é 3.62 mas (HIP97) e 3.34 mas (HIP07). O desvio médio é 1.18 mas (HIP97) e 0.02 mas (HIP07). Como podemos observar, existe um pequeno desvio sistemático com a primeira versão do catálogo enquanto o acordo é melhor para a nova versão do HiPPARCos.

As paralaxes seculares obtidas por de Bruijne et al. (2001) foram calculadas com movimentos próprios dos catálogos Hipparcos e TychO2 (Høg et al. 2000). Na Figura 2.14 apresentamos a comparação com cada conjunto de paralaxes. O r.m.s. das diferenças é 1.55 mas (Hipparcos) e 1.68 mas (TyCHO2). O desvio médio é 0.23 mas (Hipparcos) e -0.27 mas (Tycho2). Notamos que exite um bom acordo com os dois conjuntos de dados cujas paralaxes são visivelmente mais precisas que as paralaxes trigonométricas do catálogo Hipparcos. Conclui-se que os resultados obtidos neste trabalho com o novo CPSM aplicado ao aglomerado aberto das Híades são consistentes e compatíveis com aqueles encontrados na literatura. 
Hipparcos (ESA 1997)

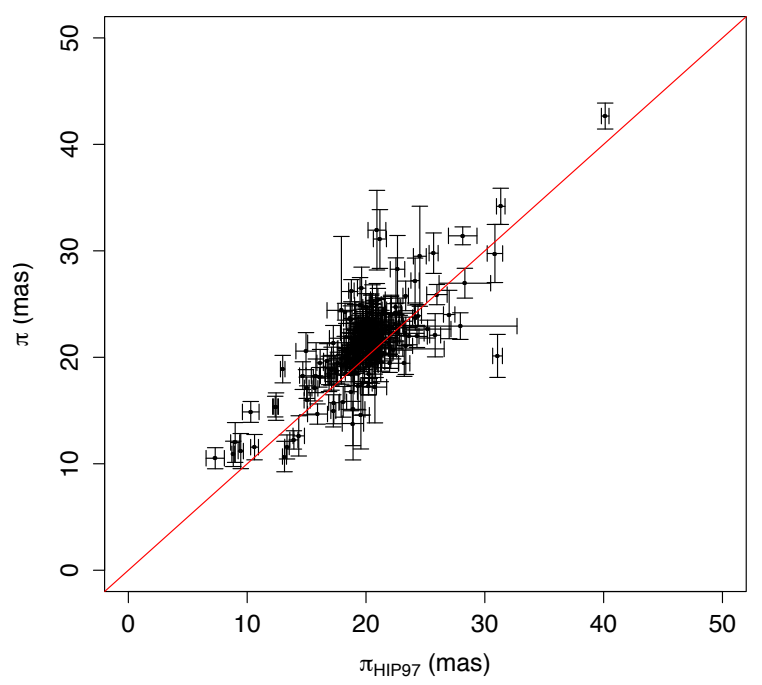

Hipparcos (van Leeuwen 2007)

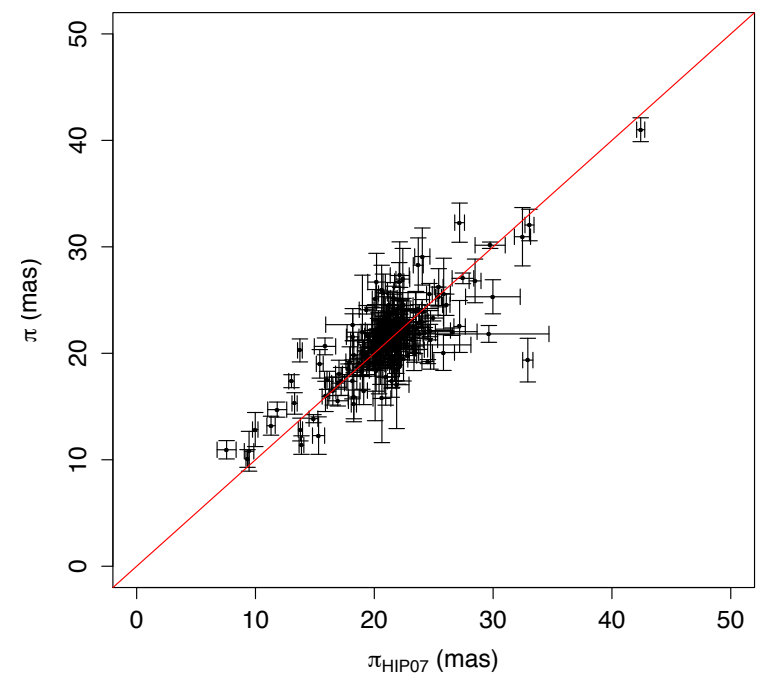

Figura 2.13: Comparação com as paralaxes trigonométricas do catálogo HipPARCos: HIP97 (esquerda) e HIP07 (direita). A linha vermelha contínua representa o resultado esperado para valores iguais.
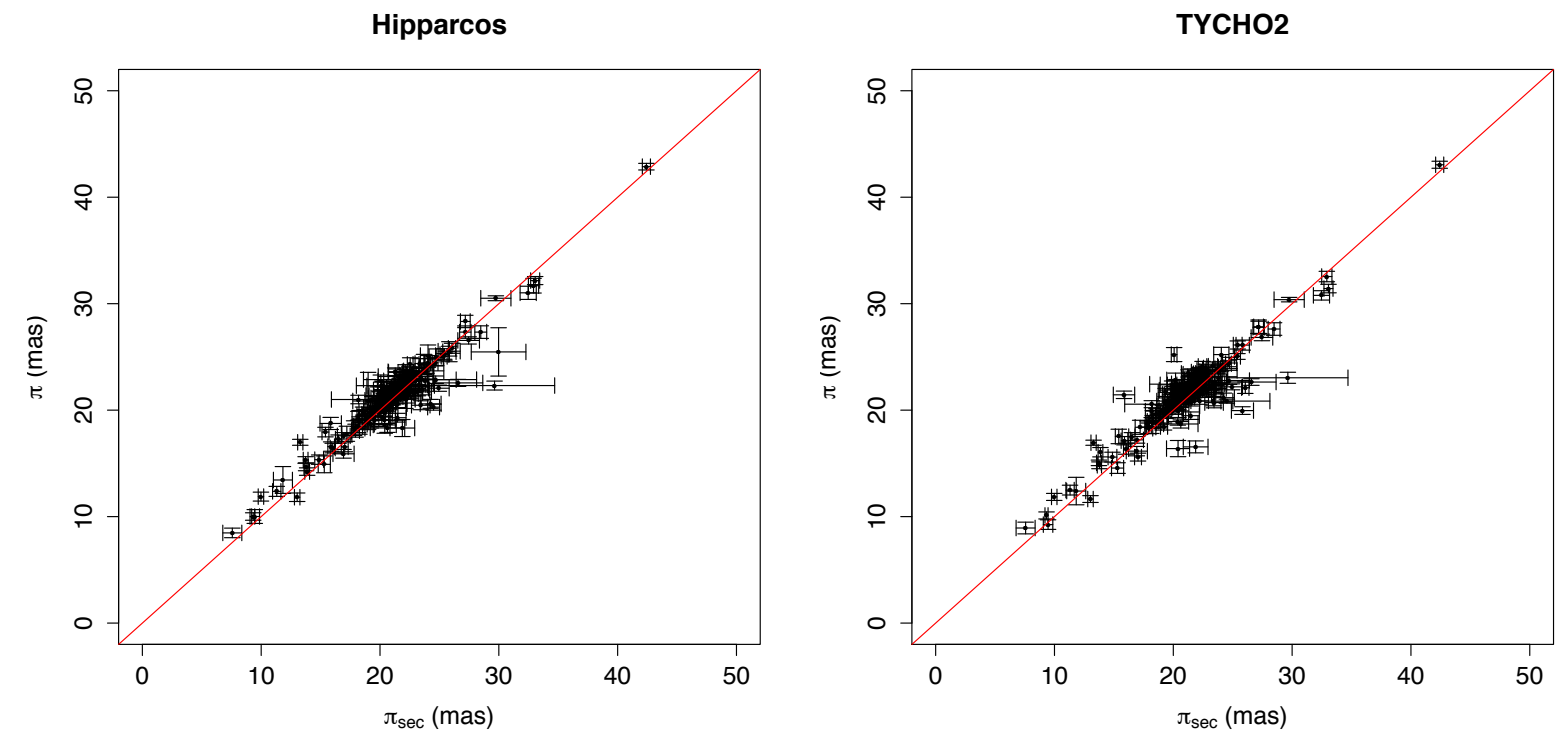

Figura 2.14: Comparação com as paralaxes seculares de de Bruijne et al. (2001) para os dois conjuntos de dados: Hipparcos (esquerda) e TyCHO2 (direita). A linha vermelha contínua representa o resultado esperado para valores iguais. 


\subsection{Conclusão}

Desenvolvemos uma nova versão do CPSM baseada na idéia de representar o movimento próprio das estrelas por um grande círculo na esfera celeste. O novo CPSM combina (i) o método de máxima verossimilhança para a determinação simultânea da posição aproximada do CP e seleção de membros do MG com (ii) uma rotina de minimização que retorna uma solução de CP mais refinada e a sua incerteza. A nova estratégia inclui o efeito da dispersão de velocidade do MG no cálculo do CP aplicando uma correção individual para cada estrela que depende do seu movimento próprio. Esse procedimento considera que as estrelas do grupo não estão todas à mesma distância.

Os dois CPSMs foram testados e comparados com simulações Monte Carlo no que diz respeito à seleção de membros, precisão e exatidão da solução de CP. Investigamos os efeitos devido (a) à dispersão de velocidade do MG, (b) erros nos movimentos próprios, (c) distância média ao MG, (d) número de membros e (e) posição relativa entre as estrelas e o CP. O novo CPSM retorna uma solução de CP mas precisa que o CPSM clássico para $95 \%$ dos casos simulados. Verificamos também que o novo CPSM é mais eficiente na eliminação de estrelas de campo. Esse ponto é particularmente importante na presença de estrelas de fundo que em geral apresentam movimentos próprios menores. Uma explicação para isso vem do princípio de cada estratégia. No CPSM clássico o movimento próprio é decomposto em duas componentes e a minimização de $X^{2}$ baseia-se na amplitude de uma componente direcional. A representação por grandes círculos no novo CPSM nos permite considerar a amplitude e a direção do vetor movimento próprio em conjunto no processo de minimização. O novo CPSM identifica aproximadamente $80 \%$ dos membros do grupo com uma contaminação estimada em $20 \%$ do número total de estrelas de campo na amostra (até 200 pc). À medida que a distância aumenta, a eficiência em eliminar estrelas de campo diminui, mas ainda é superior àquela observada no CPSM clássico. A eliminação das estrelas de campo restantes na amostra exige o conhecimento de outros parâmetros da estrela (por exemplo, paralaxe e velocidade radial). Verificamos que na ausência dos efeitos devido à dispersão de velocidade, rotação Galáctica e contaminação da amostra os dois CPSMs retornam a mesma solução de CP. 
O novo CPSM foi aplicado ao aglomerado aberto das Híades e os resultados obtidos estão em bom acordo com determinações anteriores do CP. As paralaxes calculadas neste trabalho são consistentes com as paralaxes trigonométricas do catálogo HipPARCos. Observa-se um acordo melhor com a nova versão do catálogo. Os resultados aqui obtidos também estão em perfeito acordo com dois conjuntos de paralaxes seculares encontrados na literatura cuja precisão é em geral melhor que as paralaxes trigonométricas do HiPPARCOs.

A implementação original do novo CPSM foi adaptada para considerar a matriz de covariância completa do catálogo Hipparcos na propagação de erros. O mesmo procedimento poderá ser aplicado aos dados da futura missão espacial GAIA. O novo CPSM aqui apresentado será aplicado nos próximos capítulos para investigar as propriedades cinemáticas de estrelas jovens em regiões de formação estelar próximas. 
Capítulo 3

\section{OBSERVAÇÕES}

"A astronomia compele a alma a olhar para cima e leva-nos deste mundo para outro."

Platão

\section{Conteúdo}

3.1 Programa Observacional . . . . . . . . . . . 62

3.2 Velocidade Radial . . . . . . . . . . . . . . 63

3.3 Classificação do Perfil $\mathrm{H} \alpha \ldots \ldots \ldots$. . . . . . . . . 66

3.4 Largura Equivalente do Li e $\mathrm{H} \alpha \ldots \ldots \ldots$. . . . . . . 68

3.5 Binárias Espectroscópicas . . . . . . . . . . 69 69

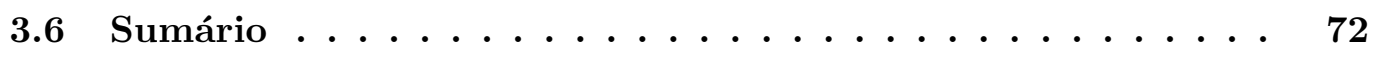

A pequena quantidade de estrelas pré-sequência principal com velocidade radial conhecida na literatura é a principal limitação para o cálculo de distâncias. Neste capítulo apresentamos a medida dessa grandeza para algumas estrelas da amostra em estudo e outros parâmetros resultantes das nossas observações. 


\subsection{Programa Observacional}

As observações foram realizadas em modo visitante com o espectrógrafo FEROS (Kaufer et al. 1999) de alta resolução $(\mathrm{R}=48000)$ montado no telescópio de 2.2m MPG/ESO (La Silla, Chile). A cobertura espectral do instrumento abrange a região do óptico e infravermelho próximo no intervalo de $3500 \AA$ a $9200 \AA$ ao longo de 39 ordens espectrais. O FEROS é alimentado simultaneamente por duas fibras óticas fornecendo (i) o espectro do objeto e do céu ou (ii) o espectro do objeto e da lâmpada de calibração (ThAr). Embora esse instrumento não tenha sido desenvolvido especificamente para a medida da velocidade radial, a sua alta estabilidade permite atingir uma precisão de até $\sim 10 \mathrm{~m} / \mathrm{s}$ no modo objeto-calibração (Setiawan et al. 2000). Uma rotina de redução automática dos espectros (FEROS Data Reduction Software) desenvolvida em ambiente MIDAS acompanha o instrumento e permite obter os espectros reduzidos em tempo real. Essa rotina é responsável pelas correções de bias e flat-field, extração das ordens, correção devido à velocidade baricêntrica, reconstrução do espectro e calibração em comprimento de onda.

Observamos uma amostra com 52 estrelas PMS nas regiões de formação estelar de Lupus e Ophiuchus durante o período de 29/04/2011 a 05/05/2011 (7 noites). O tempo de exposição para cada estrela variou entre 5-60 min dependendo da magnitude da estrela e de tal modo a obter uma relação de sinal-ruído (S/N) entre 20 e 30 em todas as regiões do espectro. As observações foram feitas no modo objeto-calibração para atingir uma melhor precisão na velocidade radial. Os espectros foram re-reduzidos com a rotina automática de redução do FEROS e o resultado obtido em tempo real foi utilizado somente para uma inspeção visual e a tomada de decisões ao longo da observação. Ao invés de analisar um único espectro reconstruído a partir dos espectros individuais de cada ordem, optamos por trabalhar com os 39 espectros (um por ordem) em separado. Esse procedimento nos permite eliminar as ordens no vermelho (ordem $\leq 7$ ) e no azul (ordem $\geq 30$ ) onde a contribuição dos erros é maior (Setiawan et al. 2003). 


\subsection{Velocidade Radial}

A velocidade radial de uma estrela é calculada pela correlação cruzada do espectro da estrela de programa com um espectro de referência que pode ser de dois tipos: (i) espectro de uma estrela com velocidade radial conhecida ou (ii) espectro sintético com velocidade radial nula. A posição do pico da função de correlação fornece a velocidade radial relativa entre o espectro de programa e o de referência nos dois casos.

No primeiro caso utilizamos como referência a estrela padrão HD 82106 (K3V, V=7.2 mag) que foi observada em todas as noites da campanha observacional e cuja velocidade radial é conhecida com boa precisão $\left(V_{r}=29.75 \pm 0.05 \mathrm{~km} / \mathrm{s}\right.$, Udry et al. 1999). Calculamos a velocidade radial das estrelas de programa fazendo a correlação cruzada com um espectro de referência de HD 82106 (obtido em 04/05/2011) utilizando a rotina fxcor do IRAF que retorna a velocidade radial relativa entre os dois espectros. Esse procedimento é repetido para todas as ordens do espectro. A velocidade radial final e sua incerteza são dadas, respectivamente, pela média e o desvio padrão do conjunto de valores considerados (descontando a velocidade radial da estrela padrão). As ordens espectrais com velocidade radial discrepante são eliminadas no processo. Ao invés de utilizar a velocidade radial de HD 82106 que encontramos na literatura, optamos por recalculá-la diretamente das nossas observações o que também nos permite avaliar os resultados obtidos. Para tanto, fazemos uma correlação cruzada entre o espectro de referência de HD 82106 e o espectro solar. A média dos valores obtidos para todos espectros de HD 81206 coletados ao longo do programa observacional é $V_{r}=29.70 \pm 0.06 \mathrm{~km} / \mathrm{s}$ o que mostra um bom acordo com o valor da literatura.

O segundo método consiste em fazer a correlação cruzada do espectro de programa com uma máscara binária construída para um determinado tipo espectral e em repouso (velocidade radial nula). Utilizamos o código MACS (Weise 2010; Weise et al. 2010), gentilmente fornecido pelo Dr. Johny Setiawan (MPIA/Heidelberg), que implementa o procedimento descrito por Baranne et al. (1996) ajustando uma função de correlação para cada ordem espectral. O programa oferece três máscaras binárias para os tipos espectrais F0V, G2V e K0V. Para melhor comparar as duas técnicas em questão utilizamos a máscara G2V (tipo espectral solar) no cálculo da velocidade radial de HD 82106. Para as estrelas de programa usamos a máscara K0V que é mais representativa das estrelas na amostra. A 
velocidade radial média de HD 82106 obtida com essa técnica é $V_{r}=29.74 \pm 0.04 \mathrm{~km} / \mathrm{s}$, o que mostra um excelente acordo com o valores obtidos anteriormente e na literatura. Na Figura 3.1 apresentamos a velocidade radial de HD 82106 obtida com cada técnica.

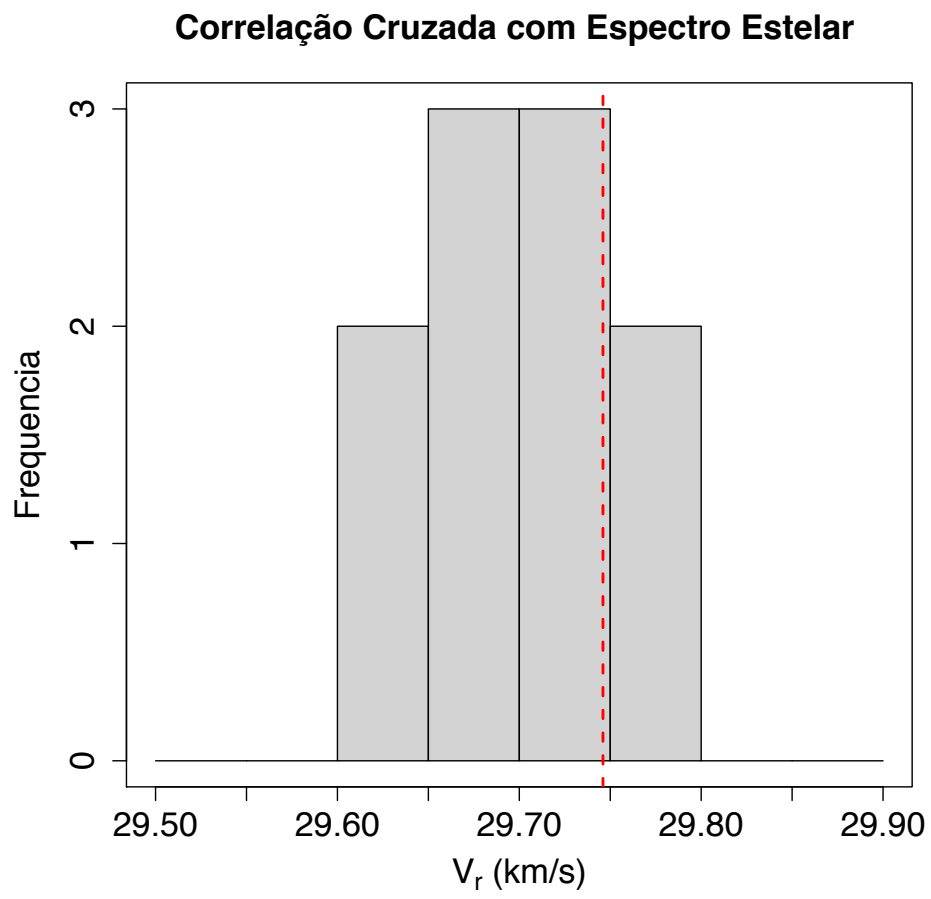

Correlação Cruzada com Espectro Sintético

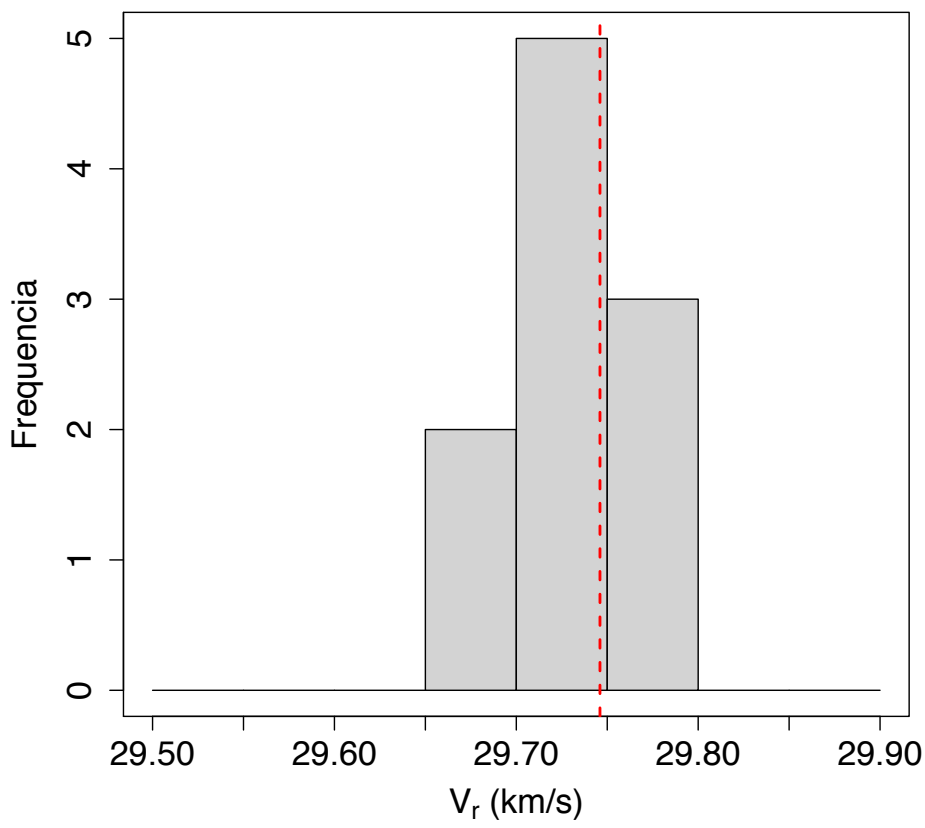

Figura 3.1: Histograma das velocidades radiais obtidas com cada técnica para a estrela HD 82106 . A linha vermelha tracejada indica o valor da literatura $V_{r}=29.75 \pm 0.05 \mathrm{~km} / \mathrm{s}$ (Udry et al. 1999). 
O uso de duas técnicas para o cálculo da velocidade radial nos permite melhor avaliar o resultado obtido para cada estrela. O procedimento adotado neste trabalho consiste em calcular a velocidade radial para as estrelas de programa com as duas técnicas usando somente as ordens espectrais em comum (ordens não rejeitadas). A velocidade radial final do alvo é a média de dois valores independentes (um para cada técnica) e a incerteza é obtida por propagação de erros. Na Tabela 3.1 apresentamos a comparação dos resultados deste trabalho para um conjunto controle de 3 estrelas PMS com velocidade radial determinada por diversos autores. As velocidades radiais obtidas são compatíveis com os valores encontrados na literatura e o r.m.s. das diferenças é $500 \mathrm{~m} / \mathrm{s}$. Os resultados de velocidade radial para as estrelas de programa são apresentados na Tabela 3.2 (no final do capítulo) juntamente com outras informações extraídas das observações. Para algumas estrelas mais fracas da amostra a velocidade radial é oriunda de um único método (aquele com menor dispersão nas ordens espectrais). O resultado aqui apresentado nesses casos é apenas indicativo e deverá ser confirmado em outro momento. Essas estrelas estão assinaladas com o símbolo ":" na Tabela 3.2.

Tabela 3.1 - Comparação das velocidades radiais obtidas neste trabalho com aquelas publicadas na literatura para o conjunto controle com 3 estrelas PMS.

\begin{tabular}{cccc} 
& Este Trabalho & Literatura & \\
\hline \hline Estrela & $V_{r} \pm \sigma_{V_{r}}$ & $V_{r} \pm \sigma_{V_{r}}$ & Referência \\
& $(\mathrm{km} / \mathrm{s})$ & $(\mathrm{km} / \mathrm{s})$ & \\
\hline \hline RXJ1524.5-3652 & $3.75 \pm 0.33$ & $4.20 \pm 1.00$ & Wichmann et al. (1999) \\
& & $4.10 \pm 0.35$ & Torres et al. (2006) \\
& & $4.40 \pm 0.40$ & James et al. (2006) \\
& & $4.60 \pm 0.40$ & James et al. (2006) \\
\hline RXJ1547.6-4018 & $2.85 \pm 0.37$ & $2.80 \pm 1.00$ & Wichmann et al. (1999) \\
& & $3.20 \pm 0.07$ & Torres et al. (2006) \\
& & $3.10 \pm 0.40$ & James et al. (2006) \\
& & $3.20 \pm 0.40$ & James et al. (2006) \\
\hline RXJ1538.0-3807 & $2.46 \pm 0.28$ & $3.40 \pm 1.00$ & Wichmann et al. (1999) \\
& & $3.00 \pm 0.20$ & Guenther et al. (2007) \\
\hline
\end{tabular}




\subsection{Classificação do Perfil $\mathrm{H} \alpha$}

A linha $\mathrm{H} \alpha$ é uma das características mais proeminentes no espectro das CTTSs e pode assumir diferentes formas (Joy 1945). Analisamos o perfil da linha $\mathrm{H} \alpha\left(P_{H \alpha}\right)$ segundo a classificação de Reipurth et al. (1996) descrita abaixo:

- Tipo I: perfil simétrico (único pico) sem ou com pouca absorção;

- Tipo II: perfil com dois picos onde a intensidade do pico secundário é superior à metade da intensidade do pico primário. A classificação é acrescida das letras $B$ ou $R$ se o pico secundário está deslocado, respectivamente, para o azul ou vermelho;

- Tipo III: perfil com dois picos onde a intensidade do pico secundário é inferior à metade da intensidade do pico primário. A classificação é acrescida das letras $B$ ou $R$ se o pico secundário está deslocado, respectivamente, para o azul ou vermelho;

- Tipo IV: perfil P Cygni ou P Cygni inverso.

Posteriormente, Wichmann et al. (1999) incluiu ainda duas subclasses no esquema de classificação:

(i) Tipo Ia: perfil do tipo I em absorção;

(ii) Tipo II-C: perfil com absorção central.

Na Figura 3.2 apresentamos um exemplo de cada perfil da linha $\mathrm{H} \alpha$ encontrado na amostra. A classificação obtida para cada estrela de programa é apresentada na Tabela 3.2. 

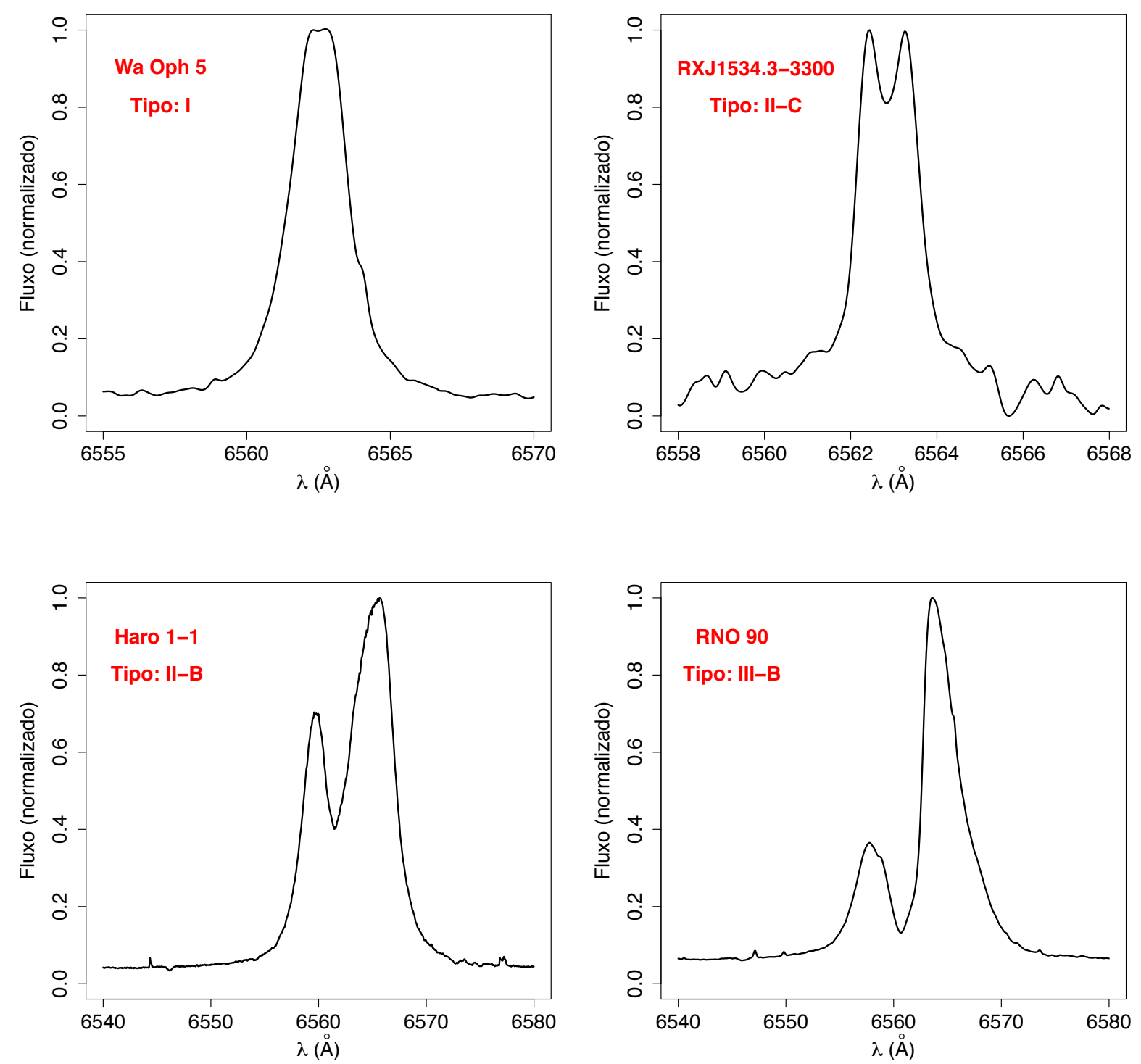

Figura 3.2: Representação do perfil H $\alpha$ para 4 estrelas de programa da amostra. 


\subsection{Largura Equivalente do Li e $\mathbf{H} \alpha$}

A presença do Li é a principal característica de estrelas jovens. A linha de ressonância do Li I $(\lambda 6708 \AA)$ é na verdade um blend das linhas do Fe I $(\lambda 6707.44 \AA)$, Fe I+Sm II $(\lambda 6707.50 \AA)$, V I $\left(\lambda 6708.11 \AA\right.$ ) e do isótopo ${ }^{6} \mathrm{Li}$ (Basri et al. 1991). Na resolução espectral do FEROS $(\mathrm{R}=48000)$ não é possível separar essas linhas e a contribuição de cada componente no cálculo da largura equivalente é pequena (da ordem de grandeza do ruído). Medimos a largura equivalente $W_{\lambda}$ da linha de Li com a rotina splot do IRAF a partir do ajuste gaussiano no perfil observado e por integração direta. A diferença nos valores obtidos pelas duas técnicas é inferior a $25 \mathrm{mÅ}$. A incerteza é obtida variando-se o nível do contínuo. O resultado apresentado na Tabela 3.2 é a média dos valores obtidos pelas duas técnicas. As larguras equivalentes do Li não estão corrigidas pelo efeito de velamento e os valores reais podem ser superiores aos indicados (principalmente para as CTTSs).

Para a medida da largura equivalente da linha $\mathrm{H} \alpha$ utilizamos a técnica de integração direta. Nos casos em que a linha $\mathrm{H} \alpha$ observada é do tipo I ou Ia ajustamos também um perfil de Voigt. A diferença média nos valores obtidos pelas duas técnicas é $10 \%$. A incerteza é calculada a partir de medidas sucessivas variando a posição do contínuo. Utilizamos a largura equivalente da linha $\mathrm{H} \alpha$ para classificar as estrelas da amostra em CTTS ou WTTS segundo o critério abaixo (Appenzeller \& Mundt 1989):

- WTTS, se $W_{\lambda}(H \alpha)<10 \AA$,

- CTTS, se $W_{\lambda}(H \alpha) \geq 10 \AA$.

Verificamos que existem 10 CTTS e 42 WTTS na amostra de estrelas observadas. Os resultados desta seção são apresentados na Tabela 3.2. Em alguns casos, onde não foi possível medir a largura equivalente com boa precisão, fornecemos um limite superior. Adotamos a convenção de valor negativo para as larguras equivalentes das linhas em emissão. 


\subsection{Binárias Espectroscópicas}

As estrelas pertencentes a sistemas binários e múltiplos foram excluídas do programa observacional, pois nesses casos a velocidade radial não pode ser determinada com uma única observação. No entanto, identificamos duas binárias espectroscópicas com linha dupla (SB2) para as quais não havia sido encontrado indício de binariedade na literatura. Verificamos que a linha de Li está presente nas duas componentes desses sistemas e que $W_{\lambda}(H \alpha) \leq 10 \AA$ o que nos permite classificá-las como WTTSs. O estudo de sistemas binários é importante na determinação da massa das estrelas que geralmente são obtidas por modelos evolutivos no caso de estrelas jovens. Essas estrelas poderão ser investigadas futuramente com essa finalidade, mas no momento isso está muito além dos objetivos deste trabalho.
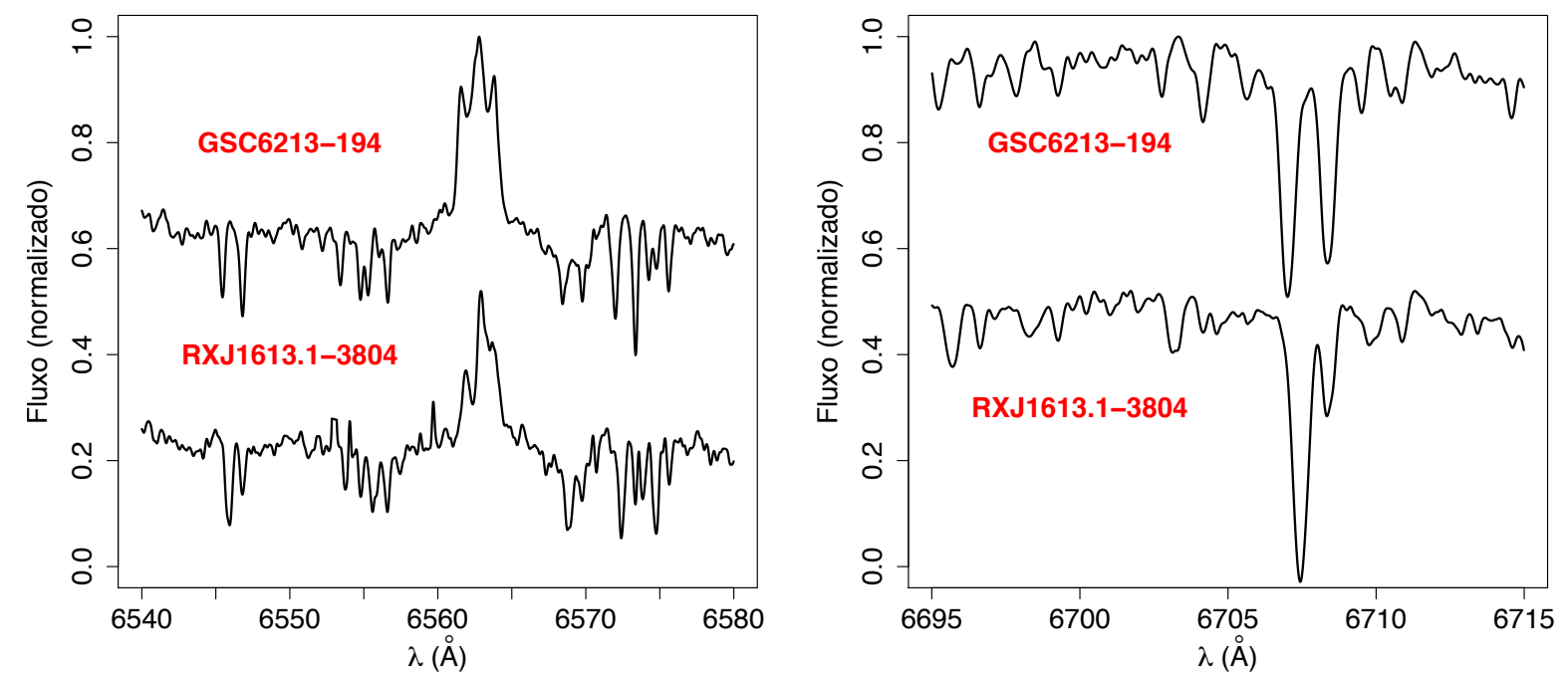

Figura 3.3: Região do espectro com as linhas $\mathrm{H} \alpha$ (esquerda) e Li (direita) para as estrelas identificadas como binárias espectroscópicas. 
Tabela 3.2 - Para cada estrela observada fornecemos o identificador, posição (época 2000), velocidade radial, largura equivalente das linhas de $L i$ e $H \alpha$, classificação do perfil $H \alpha$ e subclasse TTSs.

\begin{tabular}{|c|c|c|c|c|c|c|c|}
\hline ID & $\begin{array}{c}\alpha \\
(\mathrm{h}: \mathrm{m}: \mathrm{s})\end{array}$ & $\begin{array}{c}\delta \\
(\circ \quad \prime \prime \prime)\end{array}$ & $\begin{array}{c}V_{r} \\
(\mathrm{~km} / \mathrm{s})\end{array}$ & $\begin{array}{c}W_{\lambda}(L i) \\
(\AA)\end{array}$ & $\begin{array}{c}W_{\lambda}(H \alpha) \\
(\AA)\end{array}$ & $P_{H \alpha}$ & Tipo \\
\hline RXJ1448.2-4103 & 144813.3 & -410258 & $+5.95 \pm 0.17$ & $0.336 \pm 0.017$ & $\lesssim 0.1$ & & WTTS \\
\hline RXJ1452.4-3740 & 145226.2 & -374008 & $+5.00 \pm 0.40$ & $0.402 \pm 0.003$ & $\lesssim 0.1$ & & WTTS \\
\hline RXJ1454.2-3955 & 145411.3 & -395523 & $+3.83 \pm 0.38$ & $0.382 \pm 0.002$ & $-0.68 \pm 0.04$ & II-B & WTTS \\
\hline RXJ1502.4-3405 & 150226.0 & -340513 & $+3.88 \pm 0.17$ & $0.437 \pm 0.001$ & $-0.27 \pm 0.08$ & II-R & WTTS \\
\hline RXJ1505.4-3857 & 150525.9 & -385703 & $+4.92 \pm 0.33$ & $0.416 \pm 0.004$ & $-0.92 \pm 0.08$ & II-R & WTTS \\
\hline RXJ1505.9-4311 & 150556.9 & -431202 & $+3.99 \pm 0.27$ & $0.465 \pm 0.001$ & $-0.99 \pm 0.05$ & II-R & WTTS \\
\hline RXJ1506.7-3047 & 150642.6 & -304733 & $+0.81 \pm 0.24$ & $0.480 \pm 0.002$ & $-1.82 \pm 0.13$ & II-C & WTTS \\
\hline RXJ1508.0-3338 & 150805.1 & -333755 & $+1.65 \pm 0.23$ & $0.447 \pm 0.010$ & $-0.55 \pm 0.02$ & II-R & WTTS \\
\hline RXJ1508.4-3338 & 150825.0 & -333755 & $+1.85 \pm 0.43$ & $0.438 \pm 0.011$ & $-1.29 \pm 0.11$ & II-B & WTTS \\
\hline RXJ1508.8-3715 & 150853.8 & -371546 & $+4.51 \pm 0.28$ & $0.421 \pm 0.012$ & $-0.50 \pm 0.06$ & II-C & WTTS \\
\hline RXJ1515.1-4438 & 151509.3 & -443836 & $+6.34 \pm 0.34$ & $0.367 \pm 0.012$ & $-1.11 \pm 0.05$ & II-C & WTTS \\
\hline RXJ1518.0-4445 & 151801.3 & -444426 & $+5.97 \pm 0.15$ & $0.099 \pm 0.007$ & $0.81 \pm 0.05$ & Ia & WTTS \\
\hline RXJ1524.5-3652 & 152432.4 & -365202 & $+3.75 \pm 0.33$ & $0.344 \pm 0.001$ & $\lesssim 0.1$ & & WTTS \\
\hline RXJ1526.8-3721 & 152652.6 & -372206 & $+1.92 \pm 0.21$ & $0.505 \pm 0.002$ & $-2.25 \pm 0.15$ & II-B & WTTS \\
\hline RXJ1529.8-4523 & 152948.9 & -452245 & $+4.64 \pm 0.41$ & $0.442 \pm 0.008$ & $-0.75 \pm 0.03$ & II-B & WTTS \\
\hline RXJ1534.3-3300 & 153423.2 & -330009 & $+1.40 \pm 0.20$ & $0.525 \pm 0.008$ & $-1.11 \pm 0.08$ & II-C & WTTS \\
\hline RXJ1538.0-3807 & 153802.7 & -380723 & $+2.46 \pm 0.28$ & $0.423 \pm 0.021$ & $-1.93 \pm 0.44$ & I & WTTS \\
\hline RXJ1539.7-3450 & 153946.4 & -345102 & $+5.39 \pm 0.29$ & $0.284 \pm 0.013$ & $-0.32 \pm 0.02$ & II-C & WTTS \\
\hline RXJ1542.0-3601 & 154205.2 & -360132 & $-0.05 \pm 0.26$ & $0.492 \pm 0.011$ & $-0.81 \pm 0.03$ & II-C & WTTS \\
\hline RXJ1544.5-3521 & 154435.3 & -352149 & $+2.47 \pm 0.23$ & $0.491 \pm 0.008$ & $-1.37 \pm 0.07$ & II-C & WTTS \\
\hline RXJ1547.1-3540 & 154708.4 & -354019 & $+0.82 \pm 4.90:$ & $0.472 \pm 0.016$ & $-1.09 \pm 0.39$ & I & WTTS \\
\hline RXJ1547.6-4018 & 154741.8 & -401826 & $+2.85 \pm 0.37$ & $0.387 \pm 0.012$ & $0.17 \pm 0.01$ & Ia & WTTS \\
\hline RXJ1548.0-4004 & 154802.1 & -400428 & $+2.07 \pm 0.35$ & $0.433 \pm 0.014$ & $-2.01 \pm 0.06$ & II-C & WTTS \\
\hline RXJ1548.9-3513 & 154854.1 & -351318 & $+0.62 \pm 0.16$ & $0.372 \pm 0.002$ & $-0.41 \pm 0.03$ & II-R & WTTS \\
\hline RXJ1601.8-4026 & 160149.5 & -402619 & $+2.87 \pm 0.46$ & $0.371 \pm 0.011$ & $-1.39 \pm 0.08$ & I & WTTS \\
\hline RXJ1606.3-4447 & 160623.4 & -444735 & $+4.68 \pm 0.54$ & $0.455 \pm 0.007$ & $-0.48 \pm 0.06$ & II-R & WTTS \\
\hline GSC6780-1061 & 160654.4 & -241611 & $-5.43 \pm 0.19$ & $0.561 \pm 0.001$ & $-1.55 \pm 0.09$ & II-C & WTTS \\
\hline RXJ1608.0-3857 & 160800.0 & -385751 & $-2.42 \pm 0.84:$ & $0.638 \pm 0.002$ & $-1.91 \pm 0.14$ & III-B & WTTS \\
\hline F304 & 160811.0 & -39 1046 & $+2.76 \pm 0.11$ & $0.449 \pm 0.001$ & $-0.66 \pm 0.02$ & II-R & WTTS \\
\hline V908Sco & 160901.9 & -390512 & $-0.77 \pm 0.82:$ & $0.570 \pm 0.004$ & $-51.24 \pm 4.20$ & I & CTTS \\
\hline RXJ1609.9-3923 & 160954.0 & -39 2327 & $-0.21 \pm 0.46$ & $0.550 \pm 0.003$ & $-22.33 \pm 0.93$ & I & CTTS \\
\hline RXJ1611.6-3841 & 161138.0 & -384135 & $+2.52 \pm 0.39$ & $0.438 \pm 0.007$ & $-3.93 \pm 0.31$ & II-C & WTTS \\
\hline GSC6793-994 & 161402.1 & -230102 & $-2.28 \pm 0.51$ & $0.356 \pm 0.013$ & $\lesssim 0.1$ & & WTTS \\
\hline PDS145 & 161420.9 & -190605 & $-7.67 \pm 10.85:$ & $0.261 \pm 0.007$ & $-65.94 \pm 2.30$ & II-B & CTTS \\
\hline RXJ1615.9-3947 & 161556.7 & -394716 & $+0.09 \pm 0.27$ & $0.456 \pm 0.011$ & $-2.37 \pm 0.23$ & II-C & WTTS \\
\hline RXJ1615.9-3241 & 161557.0 & -324124 & $-0.45 \pm 0.26$ & $0.458 \pm 0.010$ & $-0.68 \pm 0.06$ & II-C & WTTS \\
\hline RXJ1620.7-2348 & 162046.0 & -234821 & $-3.21 \pm 0.21$ & $0.465 \pm 0.002$ & $-0.37 \pm 0.07$ & II-C & WTTS \\
\hline RXJ1621.4-2312 & 162128.5 & -231211 & $-8.37 \pm 2.24$ & $0.588 \pm 0.011$ & $-1.27 \pm 0.09$ & II-B & WTTS \\
\hline Haro1-1 & 162134.7 & -261227 & $-4.29 \pm 0.50$ & $0.455 \pm 0.024$ & $-153.60 \pm 2.50$ & II-B & CTTS \\
\hline GSC6794-537 & 162307.8 & -230100 & $-10.05 \pm 1.41:$ & $0.491 \pm 0.011$ & $-0.52 \pm 0.10$ & II-B & WTTS \\
\hline HD147454 & 162332.3 & -343950 & $-0.10 \pm 0.41$ & $0.112 \pm 0.004$ & $2.32 \pm 0.15$ & Ia & WTTS \\
\hline SAO207620 & 162337.7 & -344021 & $-0.41 \pm 0.14$ & $0.195 \pm 0.005$ & $0.72 \pm 0.09$ & $\mathrm{Ia}$ & WTTS \\
\hline
\end{tabular}


Tabela 3.2 - continuação.

\begin{tabular}{lccccccc}
\hline ID & $\begin{array}{c}\alpha \\
(\mathrm{h}: \mathrm{m}: \mathrm{s})\end{array}$ & $\begin{array}{c}\delta \\
\left({ }^{\prime} \prime{ }^{\prime \prime}\right)\end{array}$ & $\begin{array}{c}V_{r} \\
(\mathrm{~km} / \mathrm{s})\end{array}$ & $\begin{array}{c}W_{\lambda}(\mathrm{Li}) \\
(\AA)\end{array}$ & $\begin{array}{c}W_{\lambda}(\mathrm{H \alpha}) \\
(\AA)\end{array}$ & $\begin{array}{c}P_{H \alpha} \\
\text { Tipo }\end{array}$ \\
\hline GSC6794-156 & 162451.4 & -223932 & $-5.27 \pm 1.72:$ & $0.338 \pm 0.001$ & $-0.90 \pm 0.18$ & II-R & WTTS \\
RXJ1625.4-2346 & 162528.6 & -234627 & $-10.38 \pm 0.64$ & $0.381 \pm 0.001$ & $0.24 \pm 0.01$ & Ia & WTTS \\
DoAr25 & 162623.7 & -244314 & $-8.25 \pm 0.59$ & $0.547 \pm 0.002$ & $-8.28 \pm 0.50$ & IV-R & CTTS \\
RNO90 & 163409.2 & -154817 & $-12.92 \pm 7.92:$ & $0.360 \pm 0.006$ & $-78.00 \pm 2.40$ & III-B & CTTS \\
He3-1254 & 164644.3 & -151438 & $-8.98 \pm 0.29$ & $0.444 \pm 0.008$ & $-97.14 \pm 4.40$ & III-B & CTTS \\
WaOph6 & 164845.6 & -141636 & $-10.09 \pm 0.54:$ & $0.520 \pm 0.009$ & $-24.19 \pm 0.95$ & III-B & CTTS \\
WaOph5 & 164900.8 & -141711 & $-9.54 \pm 3.04:$ & $0.668 \pm 0.001$ & $-54.07 \pm 7.31$ & I & CTTS \\
V1725Oph & 171613.9 & -205746 & $-11.77 \pm 0.84:$ & $0.636 \pm 0.007$ & $-22.51 \pm 1.60$ & III-B & CTTS \\
\hline GSC6213-194 & 160941.0 & -221759 & & & & & SB2, WTTS \\
RXJ1613.1-3804 & 161312.7 & -380351 & & & & SB2, WTTS \\
\hline
\end{tabular}




\subsection{Sumário}

Observamos 52 estrelas PMS nas regiões formação estelar de Lupus e Ophiuchus. As observações foram realizadas com o espectrógrafo FEROS (2.2m MPG/ESO) e os espectros foram reduzidos com a rotina automática de redução de dados do instrumento. Com os espectros obtidos determinamos a (i) velocidade radial das estrelas, (ii) classificação do perfil $\mathrm{H} \alpha$, (ii) largura equivalente das linhas de $\mathrm{Li}$ e $\mathrm{H} \alpha$ e (iv) subclasse TTS. As velocidades radiais foram calculadas com duas técnicas independentes mostrando um bom acordo com os resultados da literatura. Verificamos que a amostra é formada por 10 CTTS e 42 WTTS. Identificamos duas estrelas como binárias espectroscópicas (SB2) para as quais não havíamos encontrado indícios de binariedade na literatura. As velocidade radiais aqui obtidas serão utilizadas nos capítulos 4 e 5 para o cálculo da distância dessas estrelas. 
Capítulo 4

\section{Um Estudo Cinemático da Região DE FormaÇÃo Estelar DE Lupus}

"A maravilhosa disposição e harmonia do Universo só pode ter tido origem segundo o plano de um Ser que tudo sabe e tudo pode. Isso fica sendo a minha última e mais elevada descoberta."

Isaac Newton

\section{Conteúdo}

4.1 Introdução $\ldots \ldots \ldots \ldots \ldots \ldots \ldots \ldots$

4.2 Estrelas Pré-Sequência Principal em Lupus . . . . . . . . . . 76

4.3 Análise do Ponto de Convergência . . . . . . . . . . . 83

4.4 Distâncias . . . . . . . . . . . . . . . 90

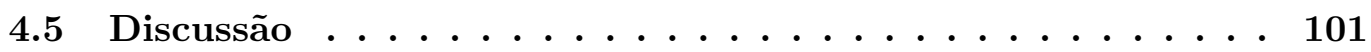

4.6 Conclusão . . . . . . . . . . . . . . . . . 110

A região de formação estelar de Lupus contém uma das associações mais ricas em estrelas T Tauri. Neste capítulo investigamos as propriedades cinemáticas e apresentamos a distância para as estrelas nessa região. Um artigo com os resultados deste capítulo foi submetido para publicação e se encontra em anexo (Apêndice C). 


\subsection{Introdução}

O complexo de Lupus é formado por diversas nuvens moleculares com formação estelar restrita às nuvens Lupus 1, Lupus 2, Lupus 3 e Lupus 4 (Hara et al. 1999; Tachihara et al. 2001). As primeiras estrelas PMS em Lupus foram descobertas por Henize (1954), The (1962) e Schwartz (1977). O catálogo de Schwartz inclui a maior parte das CTTSs conhecidas até o momento que foram descobertas pela linha $\mathrm{H} \alpha$ em emissão e associação com as nuvens moleculares da região. Um número grande de estrelas foi detectada como fontes de raios-X pelo satélite ROSAT e posteriormente confirmadas como WTTSs em levantamentos espectroscópicos (Krautter et al. 1997; Wichmann et al. 1997a,b). Os resultados desse levantamento mostram que Lupus é cercado por uma associação extensa de WTTSs enquanto as CTTSs aparecem em menor número e estão localizadas na vizinhança imediata das nuvens moleculares. A grande dispersão da população de WTTSs faz com que Lupus seja uma das maiores regiões de formação estelar próxima em termos de extensão angular no céu. Recentemente, a lista de estrelas jovens nessa região foi expandida com a detecção de novas fontes no infravermelho pelo telescópio espacial Spitzer (Merín et al. 2008). A análise de pertinência dessas estrelas com base nos seus movimentos próprios foi realizada por López Martí et al. (2011).

O alvo de muitos trabalhos ao longo das últimas décadas não foi a região de Lupus, mas a associação Scorpius-Centaurus (Sco-Cen) que contém a associação de estrelas OB mais próxima do Sol. Ela é dividida em três subgrupos: Scorpius Superior (US, do inglês Upper Scorpius), Centaurus Superior-Lupus (UCL, do inglês Upper Centaurus Lupus) e Centaurus Inferior-Crux (LCC, do inglês Lower Centaurus Crux). O complexo de Lupus se situa entre US e UCL representando muito provavelmente um episódio mais recente do processo de formação estelar das estrelas de baixa massa nessa região. A cinemática e pertinência das estrelas da Sco-Cen foi investigada por de Zeeuw et al. (1999) com base nos movimentos próprios e paralaxes do catálogo HiPPARCos utilizando uma combinação do método "Spaghetti" (Hoogerwerf \& Aguilar 1999) e o CPSM (de Bruijne 1999a). A distância média obtida nesse estudo para o subgrupo UCL é de 140 pc. A distância de Lupus foi estimada inicialmente como sendo igual à distância de UCL (Hughes et al. 1993). A maior proximidade da região de Lupus com UCL dificulta a separação das estrelas entre os dois grupos. Mamajek et al. (2002) conduziram um levantamento espectroscópico e 
identificaram 56 estrelas jovens como membros de UCL. Uma lista mais completa com 81 estrelas que pertencem a esse grupo foi apresentada por Preibisch \& Mamajek (2008) em um artigo de revisão recente sobre a Sco-Cen. Um ponto importante é que algumas estrelas identificadas nesse trabalho como membros de UCL foram também classificadas como estrelas de Lupus por Krautter et al. (1997) e Wichmann et al. (1997a,b). Um estudo da pertinência dessas estrelas é necessário e nos permitirá melhor definir cada grupo.

A distância ao complexo de Lupus é um assunto ainda em aberto com valores variando entre 100 pc a 360 pc (Franco 1990; Hughes et al. 1993; Knude \& Hog 1998; Crawford 2000; Knude \& Nielsen 2001; Franco 2002; Alves \& Franco 2006; Lombardi et al. 2008b). Esses resultados de distância são em geral obtidos pela combinação de distâncias de estrelas individuais com estimativas de extinção o que fornece uma distância aproximada ao complexo. Uma outra técnica utilizada é o cálculo da paralaxe média das estrelas com paralaxe Hipparcos identificadas nas proximidades das nuvens moleculares do complexo (Wichmann et al. 1998; Bertout et al. 1999). Essas estimativas de distância dependem fortemente da região do complexo que é investigada e são limitadas pela pequena quantidade de estrelas HipParCos existentes na região de Lupus. Existe um consentimento geral que cada nuvem do complexo de Lupus se situa a uma distância diferente o que explica o grande intervalo de distâncias calculadas (Knude 2009; Knude 2010). Com base em mapas de extinção obtidos com fotometria 2MASS, Lombardi et al. (2008b) estimaram a profundidade da região de Lupus em $51_{-35}^{+61}$ pc. Comerón (2008) discute em um artigo de revisão recente de Lupus que a distância de 150 pc é representativa para as nuvens Lupus 1, Lupus 2 e Lupus 4 enquanto o valor de 200 pc deve ser usado para Lupus 3. Embora a distância média ao complexo de nuvens em Lupus seja bastante discutida na literatura, pouco progresso foi feito no que diz respeito à determinação de distâncias individuais das estrelas nessa região. 


\subsection{Estrelas Pré-Sequência Principal em Lupus}

\subsubsection{Levantamento das estrelas candidatas}

O ponto de partida para o estudo da região de formação estelar de Lupus foi o levantamento das estrelas PMS (e candidatas). No momento consideramos somente as estrelas com movimento próprio conhecido visto que essa grandeza é a base da análise que será desenvolvida. Os limites considerados neste trabalho para a região de Lupus se estendem entre $325^{\circ} \leq l \leq 342^{\circ}$ e $0^{\circ} \leq b \leq 25^{\circ}$ em coordenadas Galácticas incluindo a região das nuvens e proximidades. Uma compilação parcial das estrelas foi realizada na construção do catálogo de movimentos próprios de estrelas PMS de Ducourant et al. (2005). Existem 197 estrelas nessa região que são procedentes do catálogo Herbig \& Bell (1988), levantamento de estrelas jovens do Pico dos Dias (Gregorio-Hetem et al. 1992; Torres et al. 1995) e demais trabalhos citados anteriormente (vide seção 4.1). Incluimos ainda 37 estrelas de Comerón (2008) e outras 24 estrelas de López Martí et al. (2011) que não foram consideradas no catálogo de Ducourant et al. (2005). Portanto, partimos de uma amostra inicial com

258 estrelas PMS na região de Lupus. É importante mencionar que a proximidade dessas estrelas ao complexo de formação estelar não garante que elas estejam de fato associadas à região. A pertinência dessas estrelas será investigada na seção 4.3 com o CPSM.

\subsubsection{Movimentos próprios}

O principal catálogo de movimentos próprios utilizado neste trabalho é o SPM4 (Girard et al. 2011), pois apresenta o melhor compromisso entre a qualidade e quantidade de movimentos próprios medidos para as estrelas em Lupus. O SPM4 é completo até $V=17.5$ fornecendo movimentos próprios e fotometria $B V$ de 103 milhões de estrelas. Encontramos uma medida do movimento próprio no SPM4 para 222 estrelas da amostra. Para as estrelas mais brilhantes da amostra $(V \leq 12)$ utilizamos também o catálogo TyCHO2 e nos demais casos os movimentos próprios de Ducourant et al. (2005). Para duas estrelas usamos os catálogos PPMXL (Roeser et al. 2010) e UCAC4 (Zacharias et al. 2012), pois apresentam as únicas medidas do movimento próprio que encontramos na literatura para essas estrelas. O grande número de sistemas binários ou múltiplos é um fator importante em Lupus. Estima-se que até $40 \%$ das estrelas nessa região sejam binárias (Ghez et al. 
1997; Merín et al. 2008). As estrelas binárias estão claramente identificadas com o sufixo "AB" no catálogo Ducourant et al. (2005) indicando que o movimento próprio medido é representativo do sistema binário. Nesses casos optamos por utilizar o movimento próprio deste catálogo ao invés do SPM4, onde a existência de sistemas binários não é informada.

Eliminamos da amostra 32 estrelas com movimento próprio não significativo em cada componente (isto é, $\sigma_{\mu} \geq \mu$ ) e outras 9 estrelas com movimento próprio discrepante após um corte em $3 \sigma$ na distribuição dos movimentos próprios. O movimento próprio médio das 217 estrelas restantes na amostra é $\left(\mu_{\alpha} \cos \delta, \mu_{\delta}\right)=(-16,-21)$ mas/yr e o erro médio em cada componente é 2 mas/yr. Essas estrelas serão utilizadas na análise que segue para investigar as propriedades cinemáticas da região de Lupus. Na Figura 4.1 apresentamos os histogramas do movimento próprio para essas estrelas. Os movimentos próprios com os respectivos erros associados são mostrados na Figura 4.2. Como podemos notar existe um grupo de estrelas com movimento próprio pequeno (próximo de zero) possivelmente associado a uma população de fundo. Isso será discutido mais adiante (vide seção 4.5).

\subsubsection{Divisão da amostra}

A distribuição das estrelas de Lupus na amostra é ilustrada na Figura 4.3. Incluímos nesta figura a posição das 81 estrelas de Preibisch \& Mamajek (2008) que pertencem ao subgrupo UCL da Sco-Cen. Devido à proximidade e sobreposição com as estrelas de UCL levantamos a suspeita de uma possível contaminação da amostra de estrelas em Lupus (em particular, para as estrelas mais dispersas). Isso nos motivou a dividir a amostra em dois grupos. Como podemos notar pela Figura 4.3, existe uma população de estrelas concentrada na vizinhança imediata das nuvens moleculares que será designada neste trabalho como população dentro das nuvens ou na vizinhança das nuvens. Existe ainda uma outra população de estrelas mais afastadas das nuvens que será denominada população fora das nuvens ou dispersa. Essa população é formada pelas WTTSs identificadas nos levantamentos espectroscópicos de Krautter et al. (1997) e Wichmann et al. (1997a,b). A caixa desenhada na Figura 4.3 estabelece os limites de cada população. Embora esses limites sejam arbritários à primeira vista, essa separação é necessária para a análise desenvolvida neste trabalho. A região dentro das nuvens se estende entre $334^{\circ} \leq l \leq 342^{\circ}, 5^{\circ} \leq b \leq 25^{\circ}$ e contém 159 estrelas enquanto a população fora das nuvens, $l<334^{\circ}$, tem 58 estrelas. 

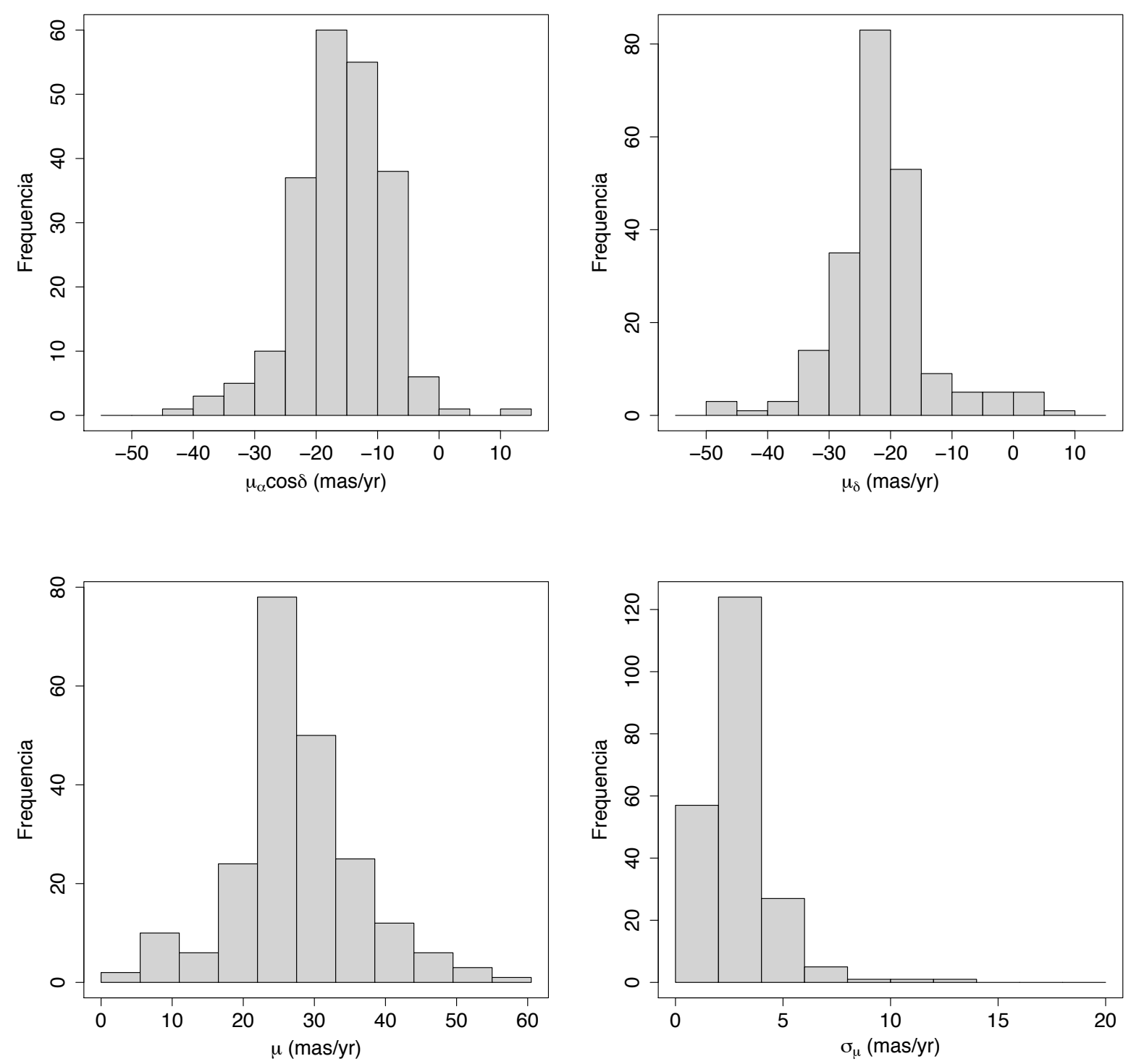

Figura 4.1: Distribuição do movimento próprio em cada componente (painel superior), movimento próprio total e incerteza (painel inferior) das 217 estrelas PMS em Lupus. 
Utilizamos o teste estatístico de Kolmogorov-Smirnov para investigar se as estrelas de Lupus e UCL dentro e fora das nuvens seguem a mesma distribuição de movimentos próprios. Para tanto, utilizamos os movimentos próprios do catálogo TYCHO2 para as 81 estrelas de UCL que são dadas por Preibisch \& Mamajek (2008). Os resultados são apresentados na Figura 4.4. Adotando o nível de significância em 5\% ( $\alpha=0.05)$ concluímos que a distribuição dos movimentos próprios das estrelas de Lupus (dentro das nuvens) e UCL são diferentes. No entanto, na comparação dos movimentos próprios de Lupus (fora das nuvens) e UCL verificamos que a hipótese nula não pode ser rejeitada. Isso confirma a suspeita inicial de uma contaminação na amostra das estrelas candidatas de Lupus por parte do subgrupo UCL da Sco-Cen.

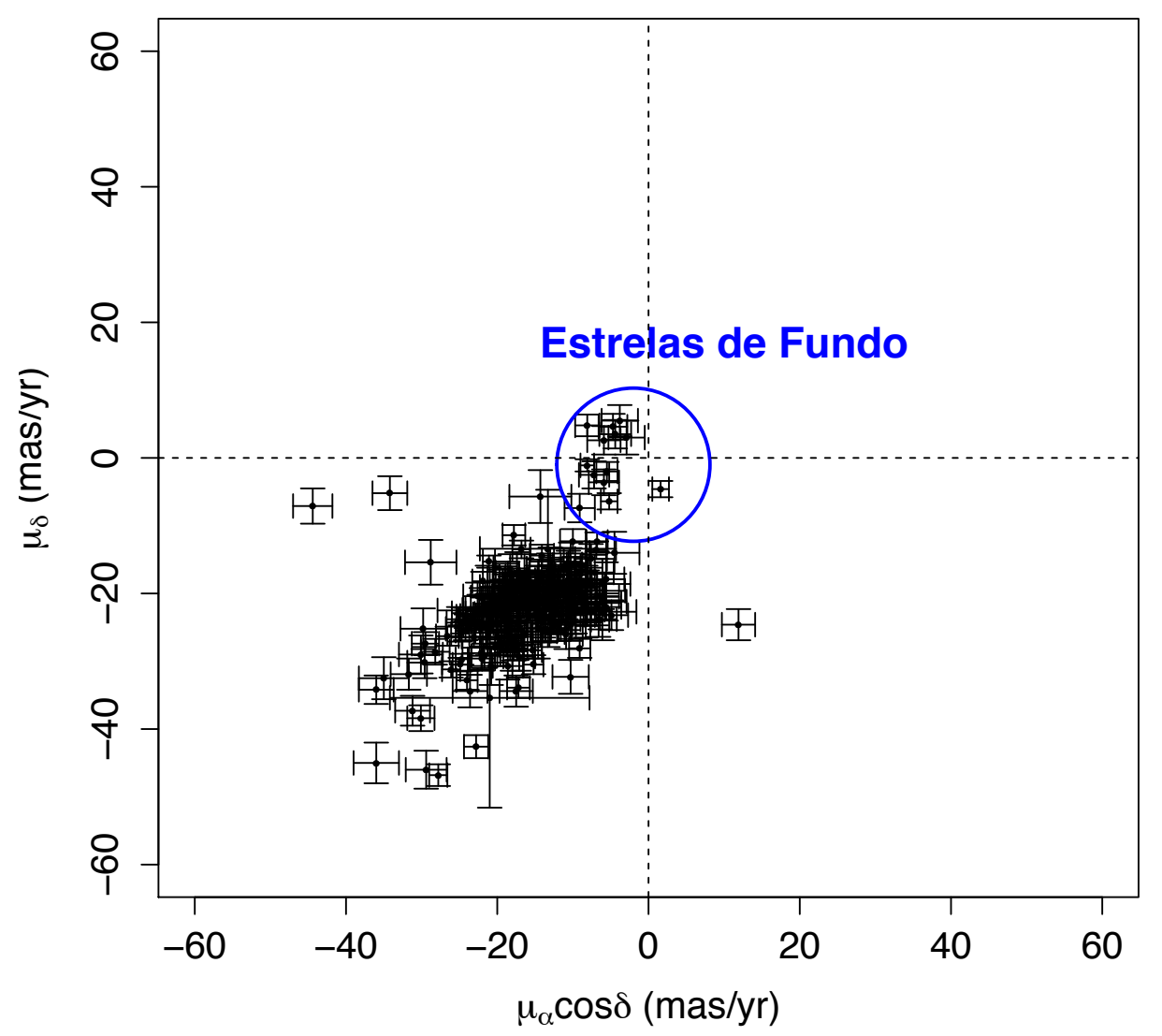

Figura 4.2: Movimentos próprios e os erros associados para as 217 estrelas PMS em Lupus. 

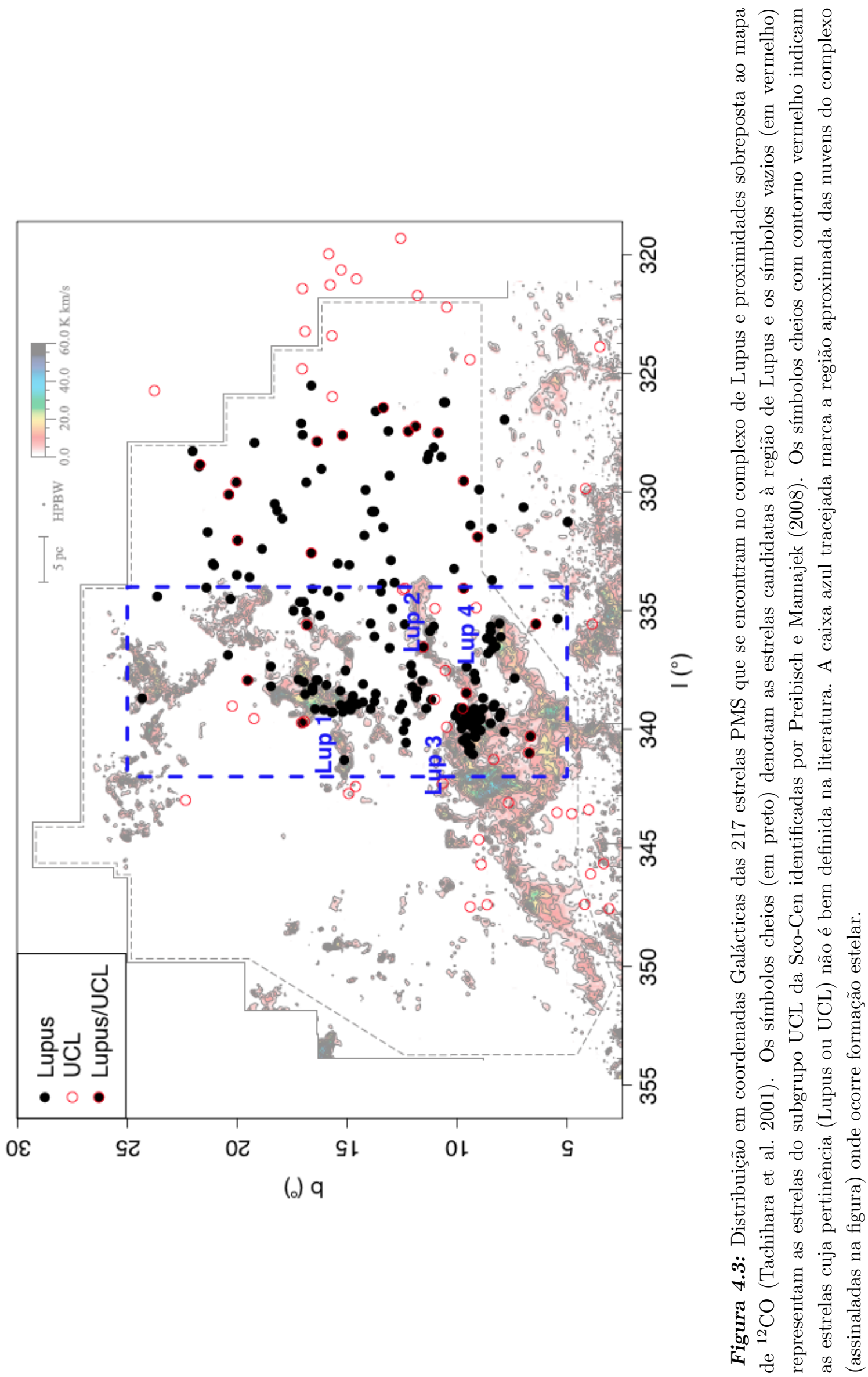
Lupus ("dentro das nuvens") vs. UCL

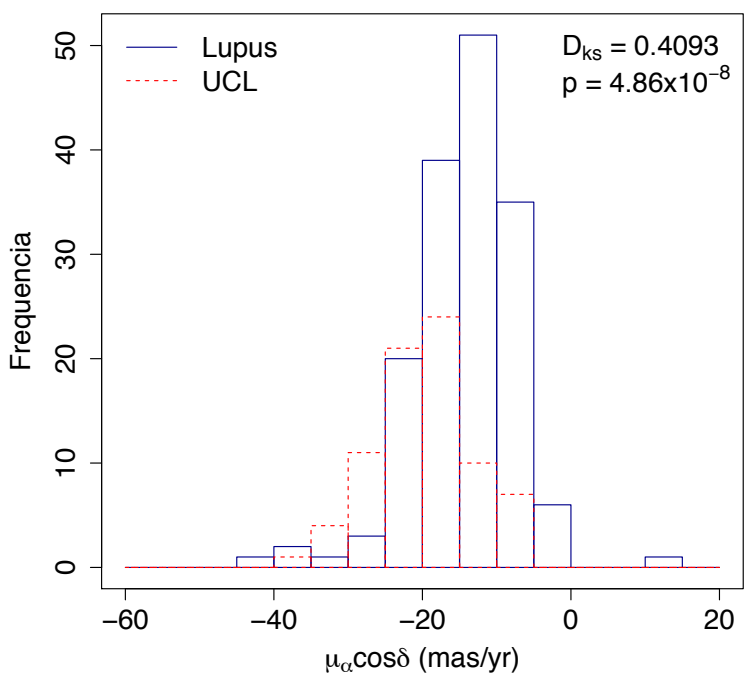

Lupus ("fora das nuvens") vs. UCL

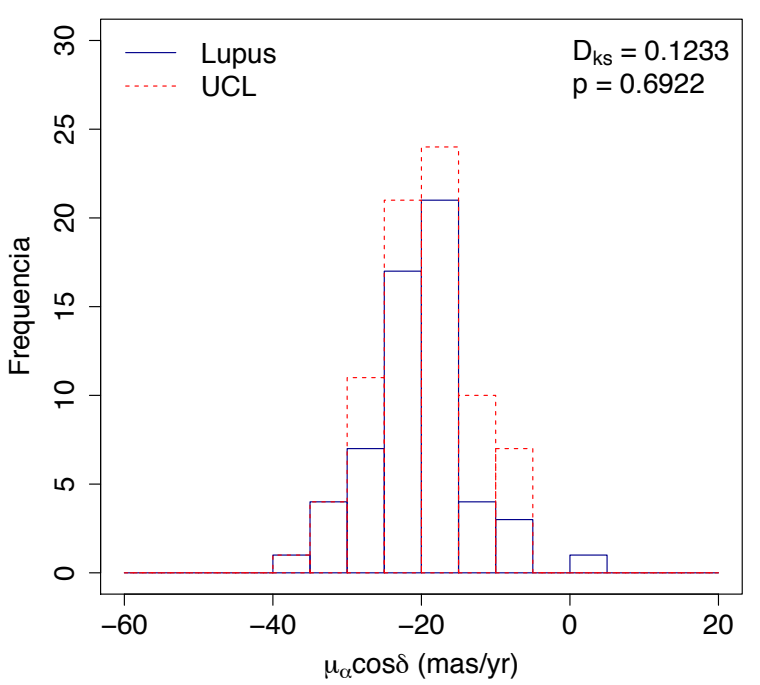

Lupus ("dentro das nuvens") vs. UCL

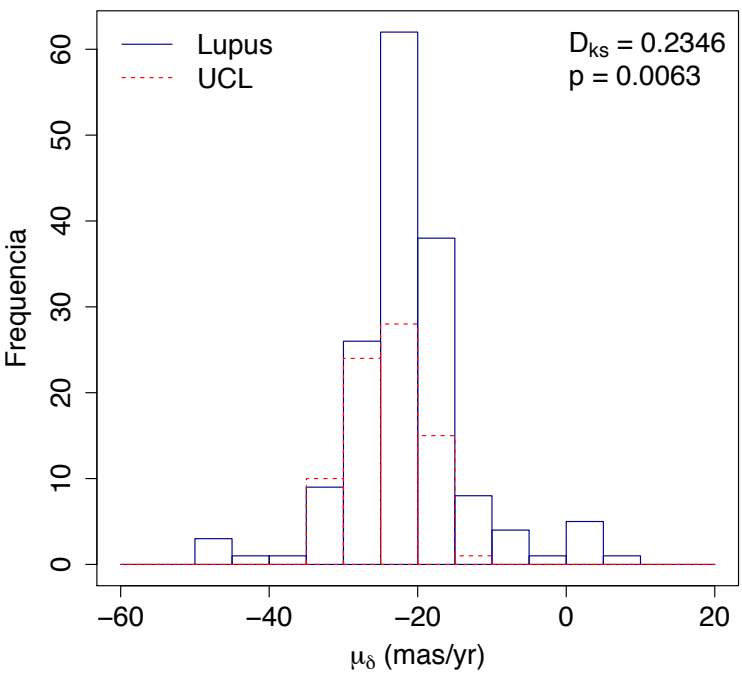

Lupus ("fora das nuvens") vs. UCL

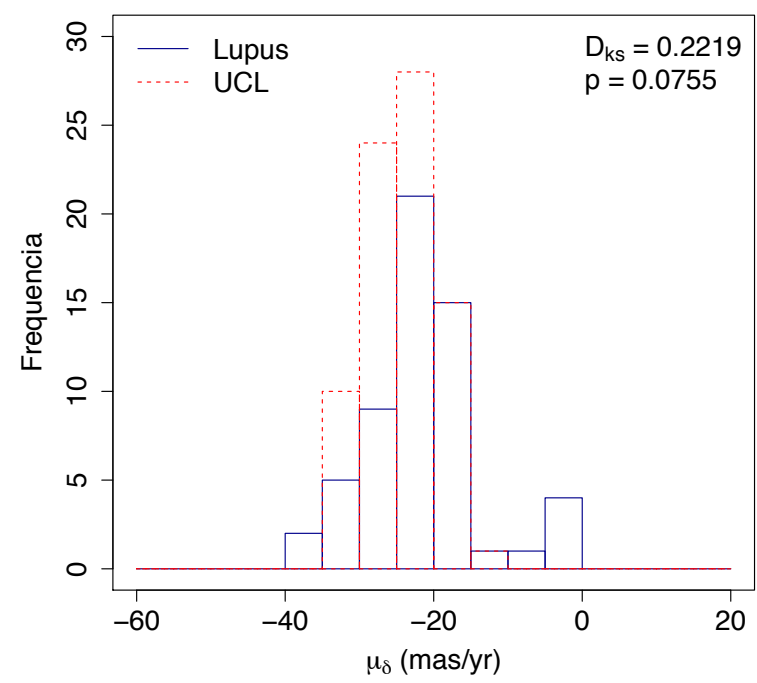

Figura 4.4: Distribuição dos movimentos próprios das estrelas de Lupus dentro e fora da região das nuvens moleculares comparada aos movimentos próprios das estrelas do subgrupo UCL da Sco-Cen. Em cada figura indicamos o resultado do teste de Kolmogorov-Smirnov, onde o valor de $p$ está normalizado de 0 a 1 e $D_{k s}$ indica a discrepância máxima entre as duas distribuições. 


\subsubsection{Velocidades radiais}

Realizamos um levantamento na literatura das velocidades radiais medidas para as estrelas da amostra. As fontes utilizadas neste trabalho são: Herbig \& Bell (1988), GregorioHetem et al. (1992), Barbier-Brossat et al. (1994), Duflot et al. (1995), Dubath et al. (1996), Grenier et al. (1999), Wichmann et al. (1999), Barbier-Brossat \& Figon (2000), Madsen et al. (2002), Melo (2003), Nordström et al. (2004), Bobylev (2006), Gontcharov (2006), James et al. (2006), Malaroda et al. (2006), Torres et al. (2006), Guenther et al. (2007), Kharchenko et al. (2007) e White et al. (2007).

Encontramos uma medida da velocidade radial para 108 estrelas. Na Tabela 3.2 (capítulo 3) apresentamos o valor dessa grandeza para outras 30 estrelas que foram calculadas neste trabalho o que resulta em 138 estrelas com velocidade radial conhecida. $\mathrm{Na}$ Figura 4.5 apresentamos a distribuição das velocidades radias. As estrelas fora das nuvens têm velocidades radiais sensivelmente maiores que as estrelas dentro das nuvens.
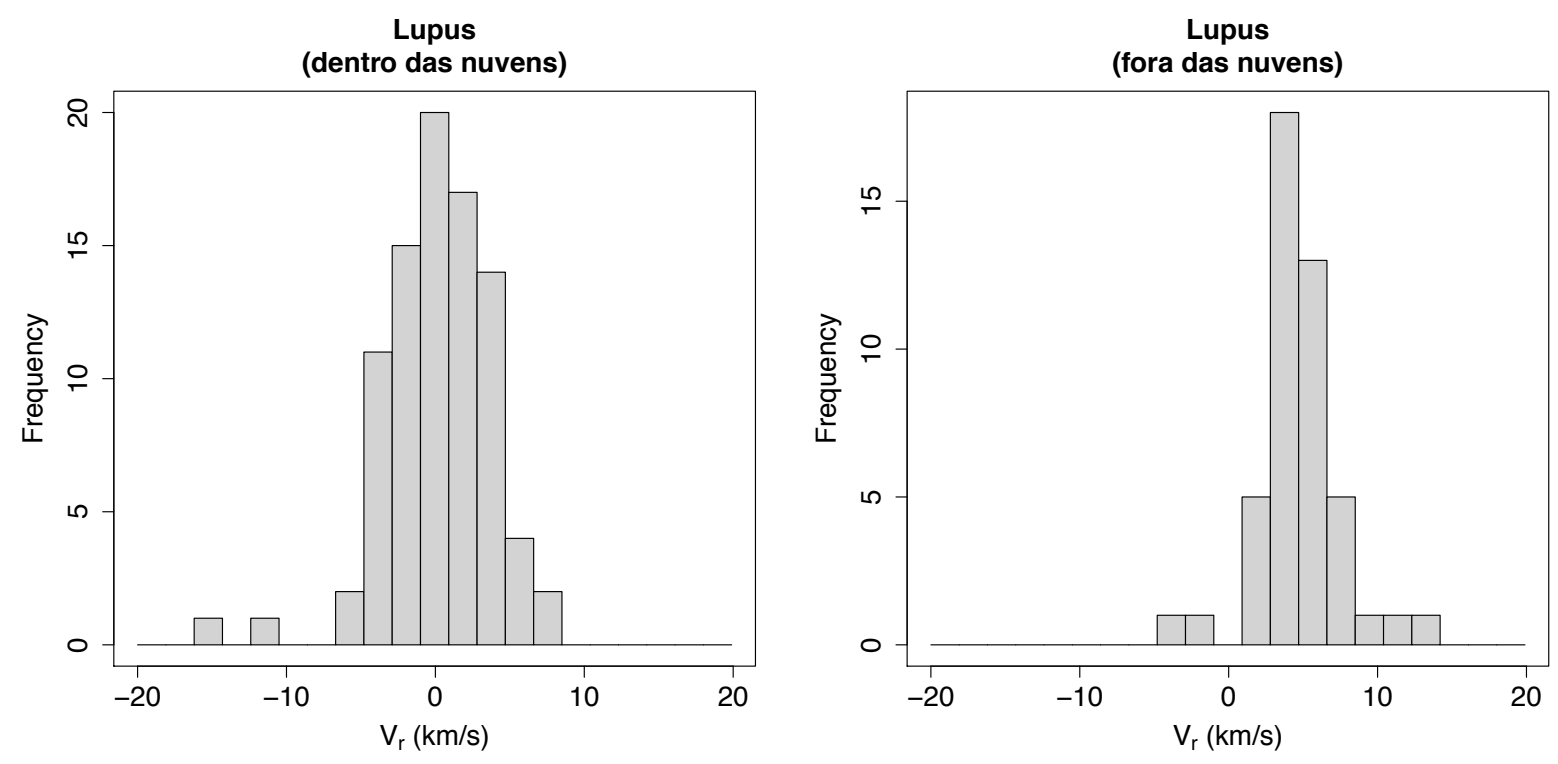

Figura 4.5: Distribuição da velocidade radial das estrelas PMS na região de Lupus. 


\subsection{Análise do Ponto de Convergência}

As estrelas PMS na região de Lupus formam um MG onde os vetores de movimento próprio exibem uma direção preferencial conforme ilustrado na Figura 4.6. Utilizamos o novo CPSM (Galli et al. 2012) para o cálculo da posição do CP e seleção dos membros do MG de Lupus. Investigamos a solução de $\mathrm{CP}$ obtida com diferentes subconjuntos da amostra e discutimos a pertinência das estrelas na região com base nesse método.

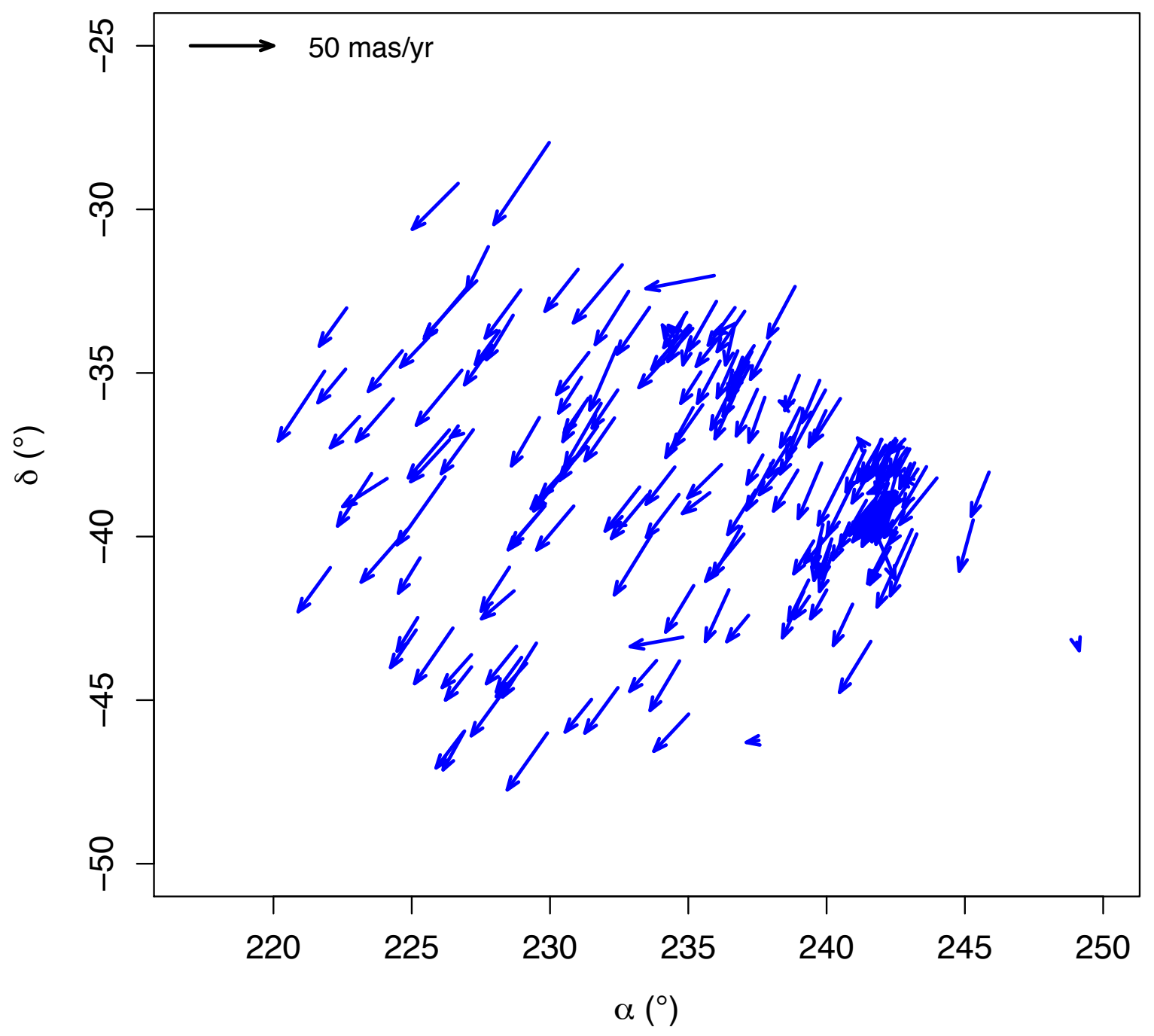

Figura 4.6: Diagrama dos vetores de movimento próprio para as 217 estrelas PMS na região de Lupus. 


\subsubsection{Resultados preliminares}

Utilizamos no cálculo do CP uma estimativa da dispersão de velocidade e a distância média ao MG (vide seção 2.2.3). A dispersão de velocidade observada em MGs de estrelas jovens é de poucos km/s (Mathieu 1986). Os valores observados nas regiões de formação estelar próximas são 1-2 km/s (Jones \& Herbig 1979, Dubath et al. 1996, Makarov 2007b). Adotamos $\sigma_{v}=2 \mathrm{~km} / \mathrm{s}$ (esse valor será justificado na seção 4.4). Como estimativa de distância consideramos $d=150$ pc que é representativo para a maior parte das nuvens do complexo de Lupus (Comerón 2008). Os resultados obtidos não dependem do valor exato desses parâmetros.

Quando aplicamos o CPSM ao conjunto de 217 estrelas da amostra encontramos uma solução com 153 estrelas e o CP com coordenadas

$$
\left(\alpha_{c p}, \delta_{c p}\right)=\left(93.3^{\circ},-25.0^{\circ}\right) \pm\left(2.4^{\circ}, 3.6^{\circ}\right)
$$

Em um trabalho recente, Makarov (2007b) investigou as propriedades cinemáticas da região de Lupus baseado em uma amostra com 93 TTSs de Krautter et al. (1997) e os movimentos próprios do UCAC2 (Zacharias et al. 2004). Ele obteve como solução de CP

$$
\left(\alpha_{c p}, \delta_{c p}\right)=\left(92.8^{\circ},-28.1^{\circ}\right) \pm\left(3.1^{\circ}, 5.0^{\circ}\right)
$$

Notamos que existe um bom acordo entre os dois resultados. As diferenças são explicadas pelo fato que cada solução foi obtida com uma amostra diferente de estrelas, movimentos próprios de outros catálogos e estratégias distintas para o cálculo do CP.

Apesar da boa concordância deste primeiro resultado é importante investigar se as estrelas dentro e fora das nuvens compartilham as mesmas propriedades cinemáticas, isto é, definem o mesmo CP na esfera celeste. Além disso, a provável contaminação da amostra por estrelas do UCL (vide seção 4.2.3) deve ser analisada cuidadosamente, pois a inclusão dessas estrelas na solução afeta a posição do CP. 


\subsubsection{Centaurus Superior-Lupus (UCL)}

O subgrupo UCL da Sco-Cen foi investigado por de Bruijne (1999b) no que diz respeito à determinação da velocidade espacial das estrelas, dispersão de velocidade do grupo e distâncias individuais. Os resultados desse estudo sugerem que o grupo se encontra a $140 \mathrm{pc}$ e tem dispersão de velocidade $\sigma_{v} \leq 1.5 \mathrm{~km} / \mathrm{s}$. Calculamos o CP de UCL usando a lista de $\mathbf{8 1}$ estrelas candidatas de Preibisch \& Mamajek (2008) e os movimentos próprios do catálogo TYCHO2 para essas estrelas. O CPSM retorna uma solução com $\mathbf{5 5}$ estrelas e CP com coordenadas

$$
\left(\alpha_{c p}, \delta_{c p}\right)=\left(105.0^{\circ},-38.3^{\circ}\right) \pm\left(2.8^{\circ}, 2.7^{\circ}\right)
$$

A eliminação de algumas estrelas desta lista é justificada, pois os critérios de seleção utilizados por Preibisch \& Mamajek (2008) são diferentes daqueles impostos pelo CPSM. O resultado obtido com as 81 estrelas da lista é $\left(\alpha_{c p}, \delta_{c p}\right)=\left(99.1^{\circ},-31.7^{\circ}\right) \pm\left(2.0^{\circ}, 2.4^{\circ}\right)$. Consideramos neste trabalho que as 55 estrelas selecionadas pelo CPSM são membros verdadeiros (confirmados) e definem as propriedades cinemáticas do grupo UCL.

\subsubsection{Lupus (dentro das nuvens)}

Conforme observado na Figura 4.3 existe um conflito na literatura para 25 estrelas da amostra cuja pertinência à região de Lupus ou UCL não é bem esclarecida. Essas estrelas foram primeiramente classificadas como WTTSs de Lupus por Krautter et al. (1997), Wichmann et al. (1997a,b) e posteriormente identificadas por Preibisch \& Mamajek (2008) como membros de UCL. O procedimento que adotamos consiste em não excluir essas estrelas da amostra, mas deixar que a pertinência delas (membro ou não-membro) seja definida pelo CPSM. Desse modo, calculamos o CP partindo das 159 estrelas na vizinhança das nuvens moleculares conforme os limites impostos para essa região na Figura 4.3. Encontramos uma solução com 101 estrelas selecionadas e CP com coordenadas

$$
\left(\alpha_{c p}, \delta_{c p}\right)=\left(111.2^{\circ},-47.4^{\circ}\right) \pm\left(6.7^{\circ}, 5.5^{\circ}\right)
$$

Como podemos notar, esta solução difere consideravelmente daquela obtida na seção 4.3.1. 


\subsubsection{Lupus (fora das nuvens)}

Quando aplicamos o CPSM no conjunto de 58 estrelas na região fora das nuvens identificamos um grupo com 15 estrelas e CP com coordenadas

$$
\left(\alpha_{c p}, \delta_{c p}\right)=\left(105.4^{\circ},-34.4^{\circ}\right) \pm\left(11.1^{\circ}, 10.4^{\circ}\right)
$$

Esse resultado se aproxima mais daquele apresentado na seção 4.3.2 para o grupo UCL. Calculamos a probabilidade de pertinência dessas estrelas ao UCL e à região das nuvens de Lupus usando a solução de CP obtida em cada caso. Os resultados são apresentados na Tabela 4.1 onde verificamos que a probabilidade de pertinência ao grupo UCL é maior para todas estrelas. Concluímos que essas estrelas são membros prováveis de UCL e estão contaminando a amostra de estrelas candidatas em Lupus.

É possível afirmar que as demais estrelas da população fora das nuvens são membros de Lupus? Para responder essa pergunta eliminamos as 15 estrelas que são membros prováveis de UCL e recalculamos o CP com as outras estrelas. Desse modo, encontramos um outro grupo com 15 estrelas que definem um CP com coordenadas

$$
\left(\alpha_{c p}, \delta_{c p}\right)=\left(112.6^{\circ},-48.7^{\circ}\right) \pm\left(17.8^{\circ}, 11.9^{\circ}\right)
$$

Essa solução está em bom acordo com aquela obtida para a região das nuvens de Lupus (seção 4.3.3) o que sugere que essas estrelas pertencem ao mesmo MG.

\subsubsection{Lupus (solução final)}

O procedimento descrito nas seções anteriores nos permitiu separar 15 estrelas da amostra que são membros prováveis de UCL. Nossa última análise consiste em calcular o CP para as estrelas dentro e fora das nuvens na mesma solução eliminando as estrelas de UCL. Assim, encontramos um conjunto com 114 estrelas e CP com coordenadas

$$
\left(\alpha_{c p}, \delta_{c p}\right)=\left(112.3^{\circ},-48.8^{\circ}\right) \pm\left(5.4^{\circ}, 4.0^{\circ}\right)
$$

Verificamos que a exclusão de estrelas com probabilidade de pertinência maior ao grupo UCL alterou significativamente a posição do CP quando comparada à solução da seção 4.3.1. Dentro desse contexto, confirmamos que a amostra inicial de estrelas estava contaminada com estrelas do UCL. 
Os resultados de CP discutidos até o momento incluem apenas as estrelas com probabilidade de pertinência maior que 50\%. Embora esse corte seja arbritário, ele nos permite considerar somente as estrelas com probabilidade maior de pertencer ao MG de Lupus. As 114 estrelas selecionadas pelo CPSM são consideradas membros do MG de Lupus e o CP obtido nesta seção será utilizado no cálculo da distância dessas estrelas (vide seção 4.4). Essas estrelas serão apresentadas mais adiante nas Tabelas 4.2 e $4.3 \mathrm{com}$ as respectivas paralaxes. Entre as 25 estrelas com pertinência conflitante na literatura (vide seção 4.3.3), verificamos que 12 estrelas foram confirmadas como membros do MG de Lupus nesta análise de CP. Essas estrelas estão assinaladas com ":" nas Tabelas 4.2 e 4.3. As demais 13 estrelas foram rejeitadas pelo CPSM e são provavelmente membros do grupo UCL conforme discutido por Preibisch \& Mamajek (2008).

\subsubsection{Simulações Monte Carlo}

Para confirmar a solução de CP que foi obtida na seção 4.3.5 realizamos simulações Monte Carlo de re-amostragem do movimento próprio. O movimento próprio de cada estrela é obtido de uma distribuição gaussiana centrada no próprio valor e com largura igual à incerteza da medida. Foram realizadas 1000 simulações e para cada conjunto das 114 estrelas simuladas calculamos o CP. Os resultados dessa análise são apresentados na Figura 4.7. Como podemos observar, o resultado obtido por Makarov (2007b) se encontra mais afastado da distribuição dos CPs simulados e reflete muito provavelmente uma solução mista que contém estrelas de Lupus e UCL. A solução obtida na seção 4.3.5 está em perfeito acordo com o centro da distribuição que tem coordenadas

$$
\left(\alpha_{c p}, \delta_{c p}\right)=\left(112.9^{\circ},-49.1^{\circ}\right) \pm\left(4.5^{\circ}, 3.4^{\circ}\right)
$$

Este resultado concorda também com os CPs calculados para as estrelas de Lupus dentro das nuvens (vide seção 4.3.3) e fora das nuvens (vide seção 4.3.4) em separado. Portanto, concluimos que o CP obtido neste trabalho é representativo das estrelas de Lupus. 


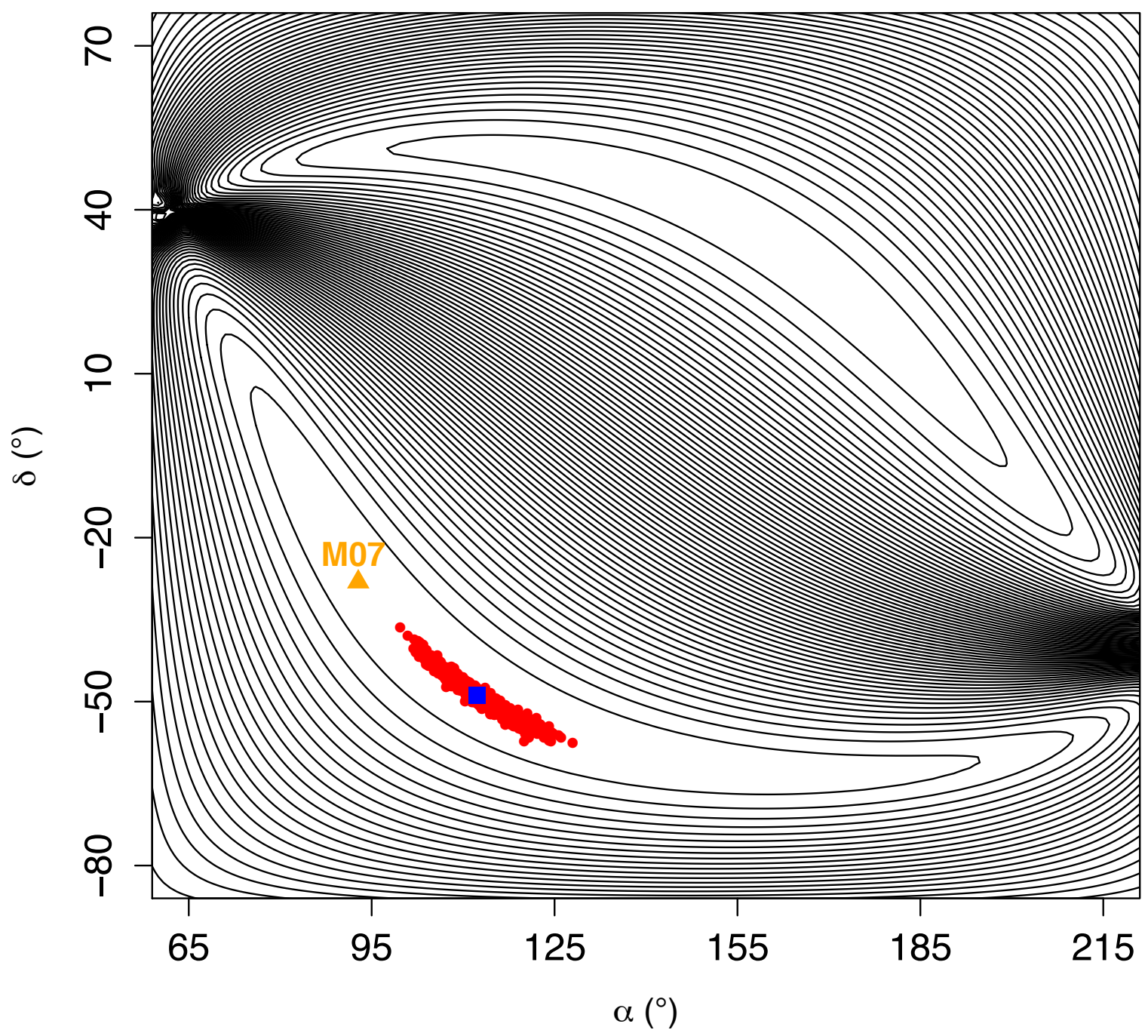

Figura 4.7: Soluções de CP obtidas para 1000 simulações Monte Carlo (pontos em vermelho) sobrepostas às curvas de $X^{2}$ constante. O quadrado azul representa a solução obtida neste trabalho (vide seção 4.3.5) enquanto o triângulo laranja indica a solução obtida por Makarov (2007b, M07). 


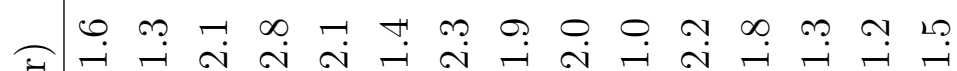

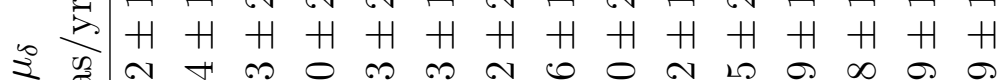
₹ घ 


\subsection{Distâncias}

\subsubsection{Comentário sobre as velocidades radiais}

Um ponto particular em Lupus é que a velocidade radial observada das estrelas é pequena. O valor médio e o desvio padrão das velocidades radiais medidas neste trabalho são $\bar{V}_{r}=(2.7 \pm 1.9) \mathrm{km} / \mathrm{s}$ (vide Tabela 3.2 ). A incerteza média nas velocidades radiais é $\sigma_{V_{r}}=0.5 \mathrm{~km} / \mathrm{s}$. Investigamos o impacto no cálculo da paralaxe e velocidade espacial causado por uma medida falsa da velocidade radial da estrela. Um desvio $\Delta V_{r}$ nas velocidades radiais é responsável pela variação $\Delta \pi$ na paralaxe que é dada por

$$
\Delta \pi \simeq \frac{A \mu_{\|}}{\tan \lambda}\left(\frac{\Delta V_{r}}{V_{r}^{2}}\right) .
$$

Esse efeito desloca a velocidade espacial da estrela por $\Delta V_{\text {space }}$ que é dado por

$$
\Delta V_{\text {space }} \simeq \frac{A \mu_{\|}}{\sin \lambda}\left(\frac{\Delta \pi}{\pi^{2}}\right) .
$$

Estimamos a magnitude desses erros usando valores médios de posição $(\alpha, \delta)=\left(237^{\circ},-38^{\circ}\right)$, movimento próprio $\left(\mu_{\alpha}^{*}, \mu_{\delta}\right)=(-15,-23) \operatorname{mas} / \mathrm{yr}$, velocidade radial $\left(V_{r}=2.7 \mathrm{~km} / \mathrm{s}\right)$, distância $(d=150$ pc ou $\pi=6.7$ mas) e a solução de CP obtida na seção 4.3.5. Verificamos que um pequeno desvio $\Delta V_{r}=0.5 \mathrm{~km} / \mathrm{s}$ na medida da velocidade radial desloca a paralaxe em $\Delta \pi \simeq 2$ mas e a velocidade espacial em $\Delta V_{\text {space }} \simeq 5 \mathrm{~km} / \mathrm{s}$.

O efeito discutido é muito menor nas regiões de formação estelar onde as estrelas apresentam velocidades radiais maiores. Esse é o caso da região de Taurus-Auriga que foi investigada por Bertout \& Genova (2006). Usando valores médios do movimento próprio, velocidade radial, distância e o $\mathrm{CP}$ obtido por esses autores calculamos $\Delta \pi \simeq 0.2$ mas e $\Delta V_{\text {space }} \simeq 0.7 \mathrm{~km} / \mathrm{s}$ para o mesmo desvio de $\Delta V_{r}=0.5 \mathrm{~km} / \mathrm{s}$. A discussão apresentada nesta seção mostra que é necessário um cuidado maior ao utilizar as velocidades radias encontradas na literatura e ressalta a importância da medida precisa dessa grandeza no cálculo da paralaxe e velocidade espacial das estrelas de Lupus. 


\subsubsection{Paralaxe calculada a partir da velocidade radial}

Uma vez definido o MG calculamos a paralaxe individual das estrelas com velocidade radial conhecida usando a equação (2.5). A incerteza na paralaxe é obtida da propagação dos erros no movimento próprio e velocidade radial.

Encontramos uma medida da velocidade radial para 55 estrelas entre as 114 estrelas que pertencem ao MG de Lupus. Eliminamos desta análise as estrelas com velocidade radial não significativa e as binárias, pois a velocidade radial varia. Para contornar os problemas discutidos na seção 4.4.1 estabelecemos limites para o valor da velocidade espacial das estrelas de Lupus. Para tanto, partimos de um conjunto com 8 estrelas $^{1}$ na amostra que estão catalogadas no Hipparcos e que pertencem ao MG de Lupus. Usamos a paralaxe trigonométrica do catálogo HipPARCos e a equação (2.6) para estimar a velocidade espacial dessas estrelas. Verificamos que a velocidade espacial das estrelas obtida desse modo varia entre $\left(V_{\text {inf }} \simeq 17 \mathrm{~km} / \mathrm{s}\right)$ e $\left(V_{\text {sup }} \simeq 33 \mathrm{~km} / \mathrm{s}\right)$ o que define os limites inferior e superior para a velocidade espacial das estrelas de Lupus neste trabalho. Eliminamos da análise as estrelas cuja paralaxe calculada resulta em uma velocidade espacial que se encontra fora desse intervalo. Após esse processo de seleção chegamos a um conjunto de $\mathbf{2 6}$ estrelas para as quais calculamos a paralaxe individual a partir da velocidade radial. Esses resultados são apresentados na Tabela 4.2. A média e o desvio padrão das paralaxes obtidas são $\bar{\pi}=7.1 \pm 1.8$ mas. A incerteza média é $\sigma_{\pi}=1.3$ mas.

Em seguida calculamos as componentes $(\mathrm{U}, \mathrm{V}, \mathrm{W})$ da velocidade Galáctica das estrelas usando o procedimento descrito por Johnson \& Soderblom (1987). Na Figura 4.8 apresentamos os histogramas da velocidade espacial. O valor médio e o desvio padrão obtidos para cada componente da velocidade são ${ }^{2}$

$$
\begin{gathered}
U=-4.4 \pm 2.4 \mathrm{~km} / \mathrm{s}, \quad V=-20.6 \pm 4.1 \mathrm{~km} / \mathrm{s}, \quad W=-5.8 \pm 1.6 \mathrm{~km} / \mathrm{s} \\
V_{\text {space }}=22.0 \pm 4.3 \mathrm{~km} / \mathrm{s} .
\end{gathered}
$$

\footnotetext{
${ }^{1} \mathrm{Sz} 120$ também está catalogada no Hipparcos, mas não foi considerada nesta análise. Trata-se de um sistema binário (Correia et al. 2006) cuja paralaxe nas duas versões do HipPARCos difere por um fator igual a dois.

${ }^{2}$ A velocidade espacial das estrelas será designada por $V_{\text {space }}$ daqui em diante para diferenciar da componente $V$ da velocidade Galáctica.
} 

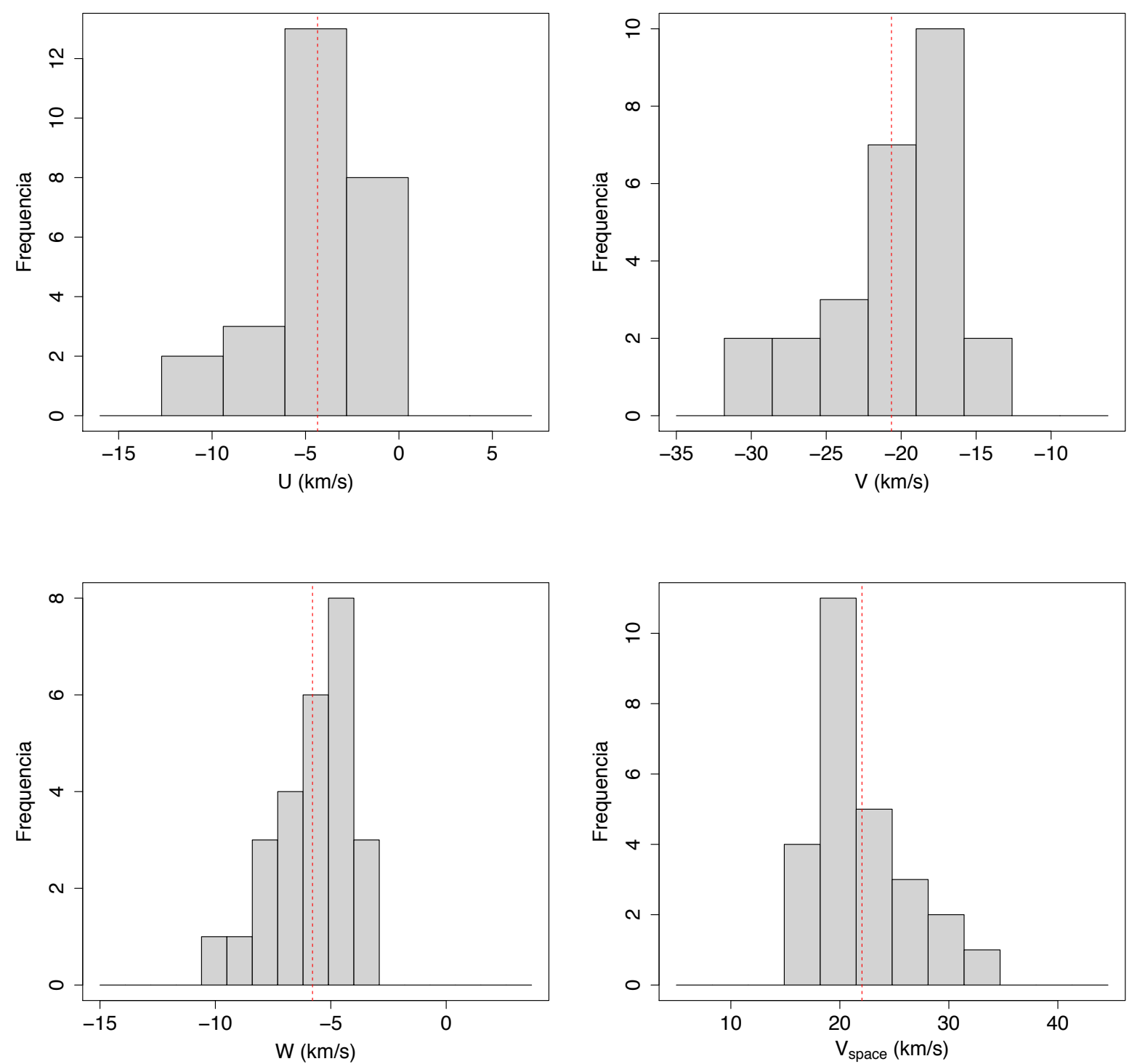

Figura 4.8: Distribuição da velocidade espacial e suas componentes para as 26 estrelas de Lupus com paralaxe calculada a partir da velocidade radial. A linha vermelha tracejada indica o valor médio em cada distribuição. 
Calculamos a média e o desvio padrão das duas componentes da velocidade tangencial

$$
V_{\alpha}=-12.2 \pm 2.3 \mathrm{~km} / \mathrm{s}, \quad V_{\delta}=-17.6 \pm 4.0 \mathrm{~km} / \mathrm{s}
$$

A dispersão de velocidade na componente $V_{\alpha}$ confirma o valor estimado de $\sigma_{v}=2 \mathrm{~km} / \mathrm{s}$ que foi utilizado no cálculo do CP (vide seção 4.3.1). No entanto, como a dispersão em $V_{\delta}$ é maior, concluímos que identificamos um MG mínimo que pode não apresentar todos os membros que estão cinematicamente associados à região de Lupus. Optamos por manter a solução obtida com $\sigma_{v}=2 \mathrm{~km} / \mathrm{s}$ (ao invés de recalcular o CP com $\sigma_{v}=4 \mathrm{~km} / \mathrm{s}$ ) e trabalhar com um número reduzido de estrelas PMS em Lupus para evitar uma contaminação maior por parte das estrelas de UCL.

\subsubsection{Paralaxe calculada a partir da velocidade espacial}

A hipótese do movimento espacial comum para as estrelas que pertencem ao mesmo MG nos permite calcular a paralaxe das estrelas cuja velocidade radial não é conhecida. Utilizamos a equação (2.6), onde a velocidade do MG é obtida da média das velocidades espaciais das 26 estrelas com velocidade radial conhecida. Nesse caso a incerteza na paralaxe leva em conta o erro da velocidade espacial na propagação de erros. Na Figura 4.9 comparamos as paralaxes calculadas a partir da velocidade radial e velocidade espacial para as 26 estrelas da seção 4.4.2. Verificamos que as paralaxes obtidas pelas duas estratégias são compatíveis dentro das incertezas consideradas o que nos permite adotar a hipótese do movimento espacial comum. O r.m.s. das diferenças é 1.3 mas enquanto a diferença média é 0.2 mas. Em seguida calculamos a paralaxe a partir da velocidade espacial média do MG para as outras $\mathbf{8 8}$ estrelas cuja velocidade radial não é conhecida.

A dispersão de velocidade do MG impede que as estrelas tenham exatamente a mesma velocidade espacial enquanto o uso da equação (2.6) considera um único valor (igual) para todas estrelas. Para contornar esse problema adotamos um procedimento de reamostragem onde a velocidade espacial é obtida de uma distribuição gaussiana centrada na velocidade média do MG e com largura igual à dispersão de velocidade medida. Foram realizadas 1000 simulações para as 88 estrelas. Para cada velocidade espacial obtida calculamos a paralaxe pela equação (2.6). O resultado final é a média desses valores. 
Esse procedimento considera que em cada iteração as estrelas do MG têm velocidades diferentes e nos permite reproduzir o efeito da dispersão de velocidade. Além disso, o resultado de paralaxe fica menos dependente do valor exato da velocidade espacial média do MG obtida na seção 4.4.2. As paralaxes calculadas a partir da velocidade espacial são apresentadas na Tabela 4.3.

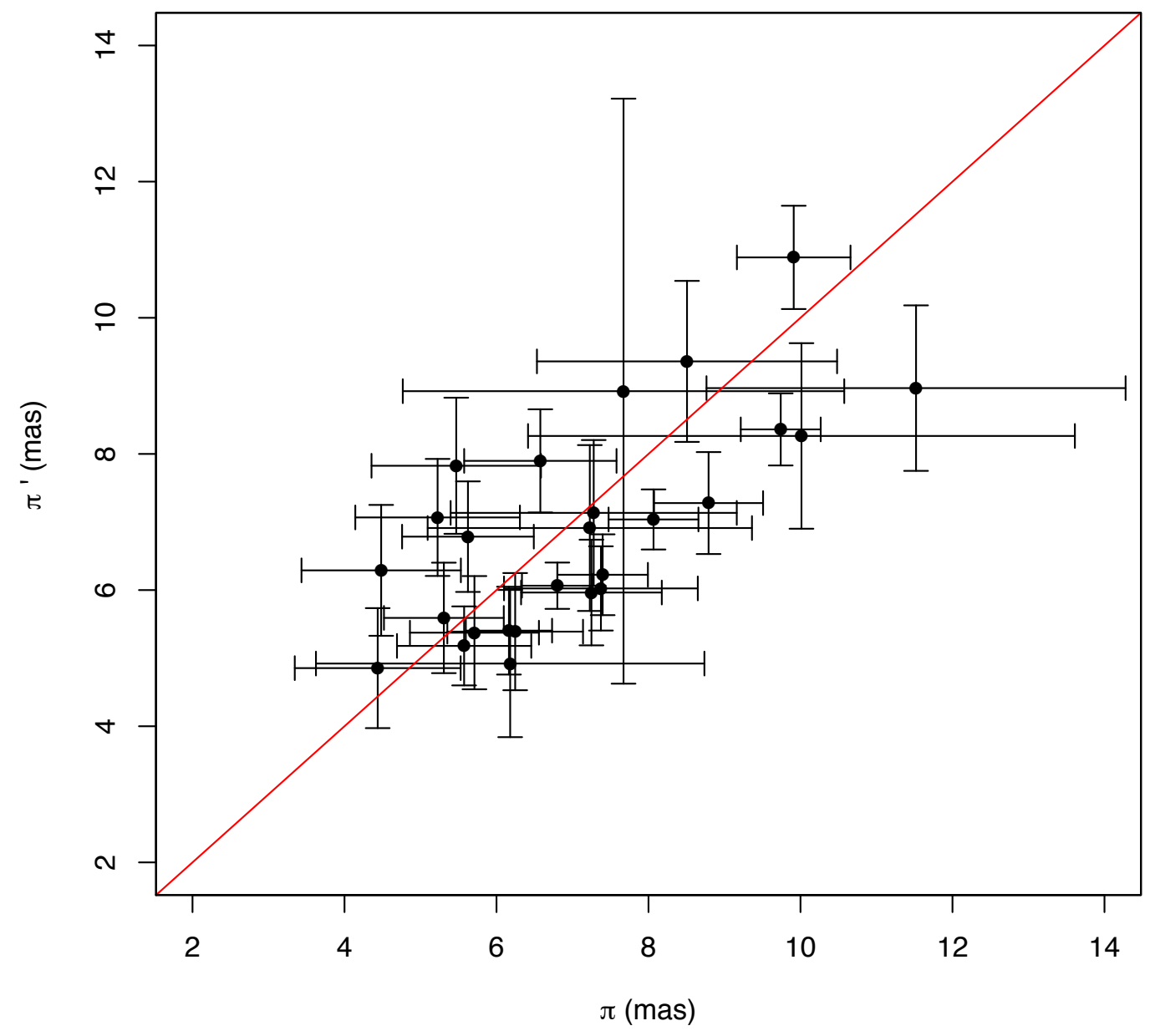

Figura 4.9: Comparação entre as paralaxes calculadas a partir da velocidade radial $(\pi)$ e velocidade espacial média do MG $\left(\pi^{\prime}\right)$ para as 26 estrelas com velocidade radial conhecida. A linha vermelha contínua representa o resultado esperado para valores iguais. 


\subsubsection{Comparação com o catálogo Hipparcos}

Encontramos uma medida da paralaxe trigonométrica no catálogo HiPPARCos para 9 estrelas que pertencem ao MG de Lupus. Na Figura 4.10 apresentamos a comparação das paralaxes obtidas neste trabalho com aquelas do catálogo Hipparcos. Calculamos a paralaxe a partir da velocidade radial para duas estrelas (HD134974 e RXJ1504.8-3950) com velocidade radial conhecida. Para as demais estrelas utilizamos a velocidade espacial do MG e o procedimento descrito na seção 4.4.3. O r.m.s. das diferenças é 2.4 mas (HIP97) e 1.9 mas (HIP07), e a diferença média é -0.7 mas (HIP97) e +0.3 mas (HIP07).

Embora exista um acordo geral melhor com o HIP07 verificamos que para algumas estrelas a paralaxe obtida neste trabalho se aproxima mais da medida do HIP97. Para investigar esse efeito comparamos as paralaxes das duas versões do catálogo HiPPARCos para essas estrelas. Os resultados desta análise são apresentados na Figura 4.11 e indicam que existe um desvio sistemático de 1 mas entre os dois catálogos. O grau de incerteza dos dados Hipparcos na região de Lupus pode ser justificada pela distância em que as estrelas se encontram e também pela magnitude das estrelas que se aproxima do limite de detecção do satélite. Isso ressalta a importância e a necessidade da determinação de distâncias nesta região de formação estelar. 
Hipparcos (ESA 1997)

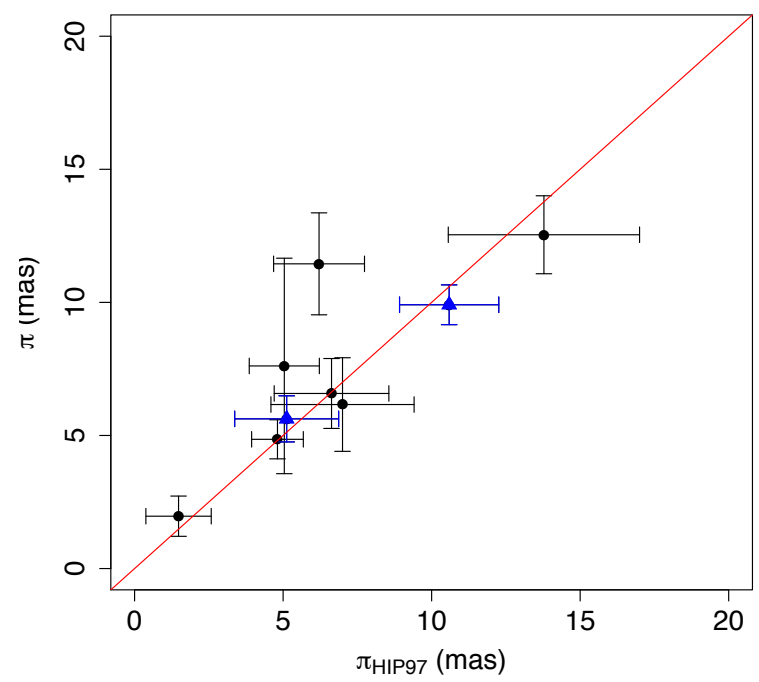

Hipparcos (van Leeuwen 2007)

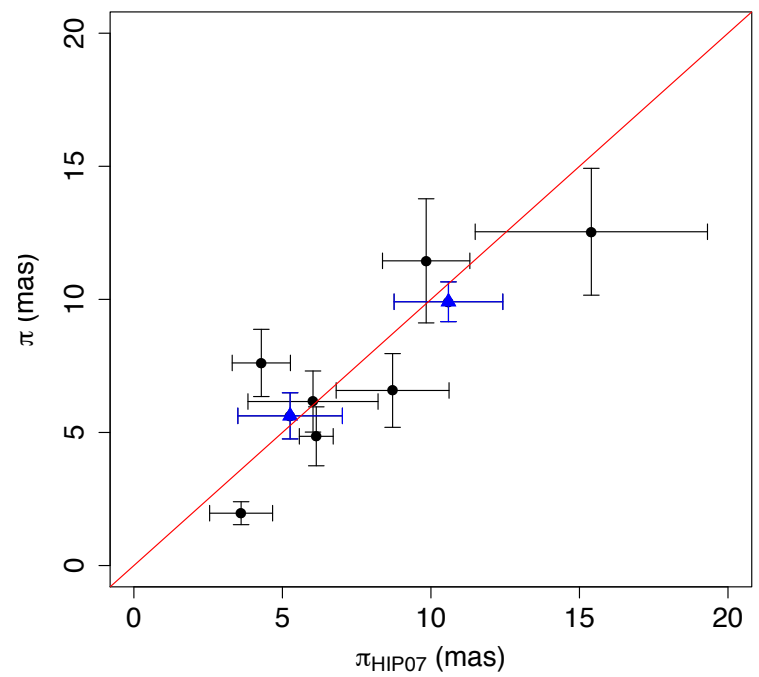

Figura 4.10: Comparação com as paralaxes trigonométricas do catálogo HipPARCos. A linha vermelha contínua representa o resultado esperado para valores iguais. Os pontos em azul representam as 2 estrelas (HD134974 e RXJ1504.8-3950) cuja paralaxe foi determinada a partir da velocidade radial.

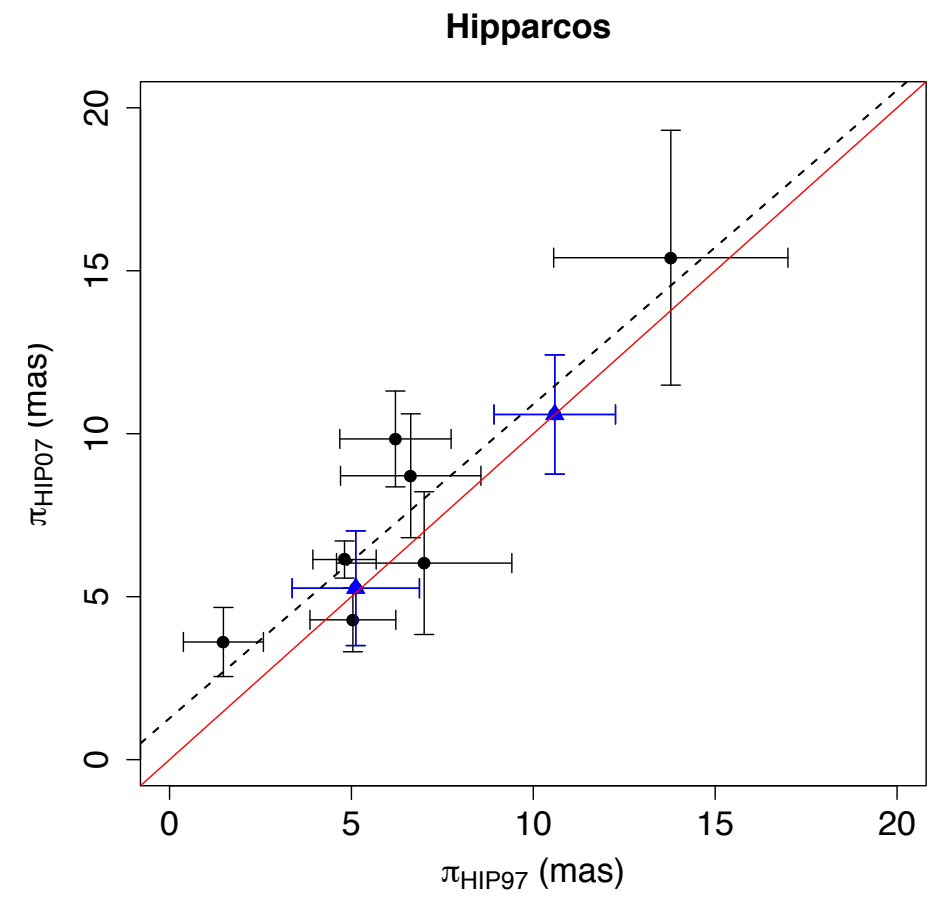

Figura 4.11: Comparação entre as paralaxes trigonométricas das duas versões do catálogo HiPPARCOS para as estrelas 9 estrelas PMS que pertencem ao MG de Lupus. A linha vermelha contínua representa o resultado esperado para valores iguais e a linha preta tracejada indica o ajuste linear aos dados. Os pontos em azul representam as 2 estrelas (HD134974 e RXJ1504.8-3950) discutidas na seção 4.4.4. 


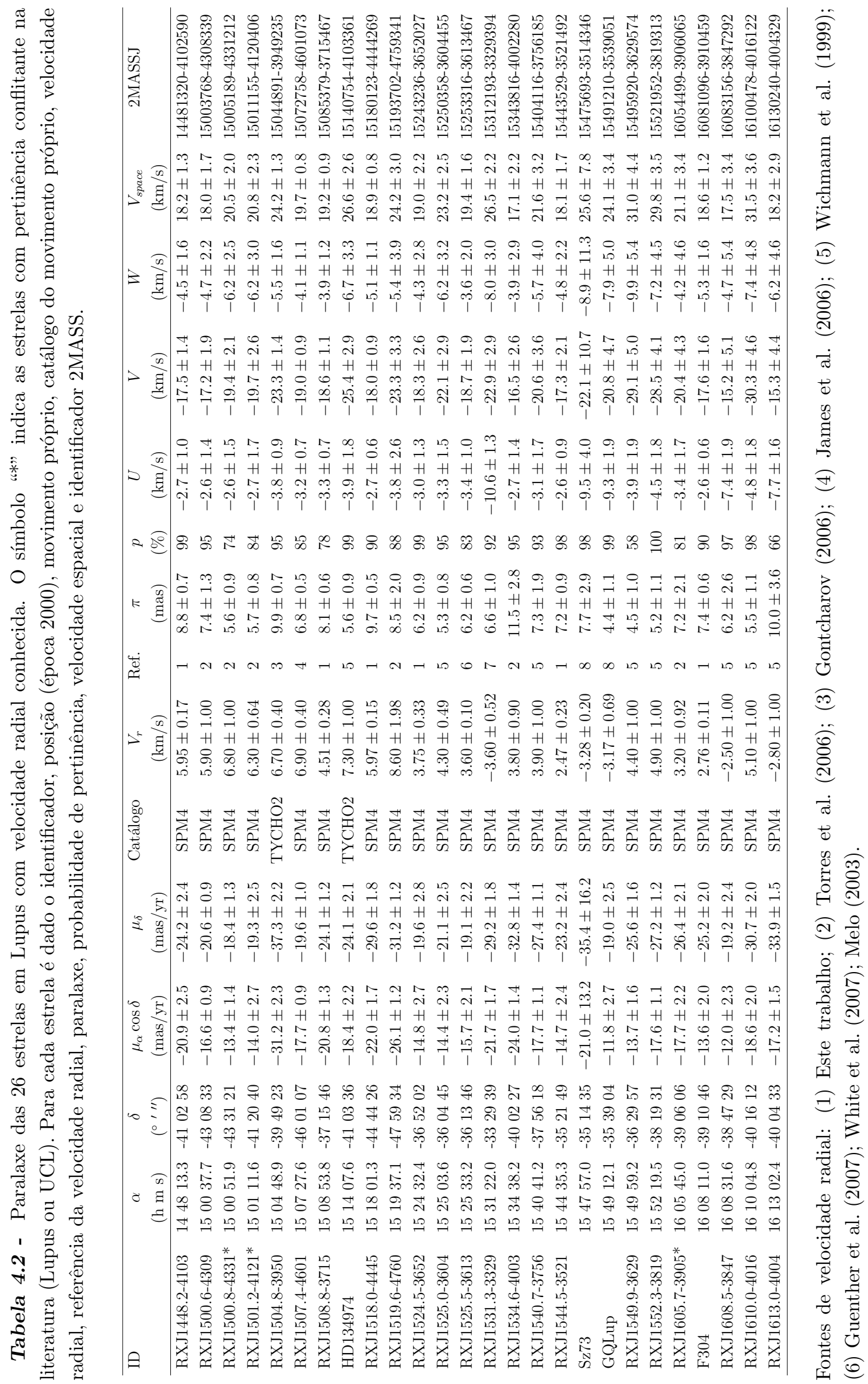


Tabela 4.3 - Paralaxe calculada a partir da velocidade espacial do MG para as 88 estrelas em Lupus sem velocidade radial. O símbolo "*” indica as estrelas com pertinência conflitante na literatura (Lupus ou UCL). Para cada estrela é dado o identificador, posição (época 2000), movimento próprio, catálogo do movimento próprio, probabilidade de pertinência, paralaxe e identificador 2MASS.

\begin{tabular}{|c|c|c|c|c|c|c|c|c|}
\hline ID & $\begin{array}{c}\alpha \\
(\mathrm{h} \mathrm{m} \mathrm{s})\end{array}$ & $\begin{array}{c}\delta \\
\left({ }^{\circ} \quad{ }^{\prime \prime} \prime \prime\right)\end{array}$ & $\begin{array}{c}\mu_{\alpha} \cos \delta \\
(\mathrm{mas} / \mathrm{yr})\end{array}$ & $\begin{array}{c}\mu_{\delta} \\
(\mathrm{mas} / \mathrm{yr})\end{array}$ & Catálogo & $\begin{array}{c}p \\
(\%)\end{array}$ & $\begin{array}{c}\pi \\
(\mathrm{mas})\end{array}$ & 2MASSJ \\
\hline RXJ1447.3-3503 & 144723.5 & -350313 & $-30.1 \pm 1.8$ & $-38.4 \pm 1.9$ & SPM4 & 72 & $11.4 \pm 2.7$ & $14472343-3503134$ \\
\hline RXJ1450.4-3507* & 145025.8 & -350648 & $-18.0 \pm 1.3$ & $-18.4 \pm 1.3$ & SPM4 & 65 & $6.0 \pm 1.5$ & $14502581-3506486$ \\
\hline RXJ1450.5-3459 & 145035.1 & -345905 & $-17.8 \pm 1.3$ & $-20.9 \pm 1.3$ & SPM4 & 100 & $6.4 \pm 1.6$ & $14503508-3459056$ \\
\hline RXJ1454.2-3955 & 145411.3 & -395523 & $-22.1 \pm 2.5$ & $-28.8 \pm 2.4$ & SPM4 & 78 & $8.5 \pm 2.0$ & $14541121-3955233$ \\
\hline RXJ1505.9-4311 & 150556.9 & -431202 & $-24.9 \pm 1.0$ & $-30.5 \pm 1.0$ & SPM4 & 100 & $9.3 \pm 2.1$ & $15055685-4312031$ \\
\hline RXJ1511.0-3252AB & 151104.5 & -325130 & $-14.0 \pm 3.0$ & $-24.0 \pm 3.0$ & D05 & 52 & $6.3 \pm 1.7$ & $15110450-3251304$ \\
\hline RXJ1511.6-3550 & 151137.0 & -355042 & $-17.9 \pm 1.6$ & $-21.7 \pm 1.6$ & SPM4 & 92 & $6.5 \pm 1.6$ & $15113696-3550417$ \\
\hline RXJ1512.6-3417 & 151239.8 & -341659 & $-15.6 \pm 1.8$ & $-18.3 \pm 1.7$ & SPM4 & 86 & $5.5 \pm 1.4$ & 15123981-3416591 \\
\hline RXJ1512.8-4508A* & 151250.2 & -450805 & $-19.0 \pm 2.0$ & $-22.0 \pm 2.0$ & D05 & 91 & $7.0 \pm 1.7$ & $15125018-4508044$ \\
\hline HD135127 & 151439.6 & -344541 & $-17.3 \pm 1.3$ & $-24.3 \pm 1.3$ & TYCHO2 & 92 & $6.9 \pm 1.7$ & $15143959-3445412$ \\
\hline RXJ1515.7-3332 & 151545.4 & -333159 & $-23.1 \pm 1.6$ & $-26.4 \pm 1.6$ & SPM4 & 52 & $8.0 \pm 1.8$ & $15154537-3331597$ \\
\hline RXJ1518.4-3738* & 151826.9 & -373802 & $-18.1 \pm 1.3$ & $-26.5 \pm 1.2$ & SPM4 & 78 & $7.3 \pm 1.6$ & $15182692-3738021$ \\
\hline GSC6770-655 & 151952.9 & -280227 & $-36.0 \pm 3.0$ & $-45.0 \pm 3.0$ & D05 & 73 & $13.0 \pm 2.9$ & $15195295-2802266$ \\
\hline RXJ1527.3-3603 & 152722.9 & -360409 & $-23.6 \pm 2.3$ & $-34.4 \pm 2.4$ & SPM4 & 93 & $9.6 \pm 2.2$ & $15272286-3604087$ \\
\hline RXJ1529.3-3737 & 152919.0 & -373720 & $-19.2 \pm 0.9$ & $-23.5 \pm 0.9$ & SPM4 & 64 & $6.9 \pm 1.5$ & $15291901-3737205$ \\
\hline RXJ1529.7-3628 & 152947.3 & -362837 & $-16.6 \pm 1.6$ & $-20.8 \pm 1.7$ & SPM4 & 82 & $6.1 \pm 1.5$ & $15294727-3628374$ \\
\hline RXJ1538.7-4411* & 153843.1 & -441147 & $-19.1 \pm 1.9$ & $-27.2 \pm 1.9$ & SPM4 & 100 & $7.7 \pm 1.8$ & $15384306-4411474$ \\
\hline RXJ1539.7-3450 & 153946.4 & -345102 & $-14.9 \pm 2.0$ & $-19.3 \pm 2.1$ & SPM4 & 83 & $5.5 \pm 1.4$ & $15394637-3451027$ \\
\hline RXJ1540.3-3426A & 154018.5 & -342615 & $-14.7 \pm 3.7$ & $-19.5 \pm 3.6$ & $\mathrm{SPM} 4$ & 93 & $5.5 \pm 1.7$ & $15401850-3426146$ \\
\hline PDS395 & 154046.4 & -422953 & $-18.2 \pm 1.3$ & $-25.5 \pm 1.1$ & TYCHO2 & 98 & $7.4 \pm 1.8$ & $15404638-4229536$ \\
\hline SSTc2dJ154148.3-350145 & 154148.3 & -350146 & $-13.0 \pm 4.1$ & $-17.2 \pm 4.0$ & SPM4 & 94 & $4.9 \pm 1.7$ & $15414827-3501458$ \\
\hline RXJ1544.0-3311* & 154403.8 & -331111 & $-18.1 \pm 1.3$ & $-26.9 \pm 1.4$ & SPM4 & 100 & $7.4 \pm 1.7$ & $15440376-3311110$ \\
\hline RXJ1546.6-3618 & 154641.2 & -361847 & $-12.7 \pm 1.9$ & $-23.8 \pm 2.0$ & SPM4 & 60 & $6.1 \pm 1.5$ & $15464121-3618472$ \\
\hline RXJ1546.7-3459 & 154645.1 & -345947 & $-17.4 \pm 3.6$ & $-20.6 \pm 3.4$ & SPM4 & 57 & $6.1 \pm 1.8$ & $15464506-3459473$ \\
\hline RXJ1547.1-3540 & 154708.4 & -354019 & $-13.3 \pm 2.2$ & $-25.7 \pm 2.2$ & SPM4 & 51 & $6.5 \pm 1.6$ & 15470841-3540195 \\
\hline RXJ1547.6-4018 & 154741.8 & -401826 & $-18.7 \pm 1.1$ & $-27.6 \pm 1.1$ & SPM4 & 99 & $7.6 \pm 1.8$ & $15474176-4018267$ \\
\hline HMLup & 154750.6 & -352835 & $-9.7 \pm 4.1$ & $-22.0 \pm 3.9$ & SPM4 & 55 & $5.4 \pm 1.8$ & $15475062-3528353$ \\
\hline HNLup & 154805.2 & -351553 & $-10.3 \pm 7.2$ & $-19.6 \pm 7.1$ & SPM4 & 93 & $5.0 \pm 2.5$ & $15480523-3515526$ \\
\hline RXJ1548.7-3520 & 154842.5 & -352007 & $-11.2 \pm 2.9$ & $-16.1 \pm 2.8$ & SPM4 & 98 & $4.4 \pm 1.4$ & $15484253-3520066$ \\
\hline RXJ1548.9-3513 & 154854.1 & -351318 & $-17.4 \pm 2.1$ & $-27.7 \pm 2.1$ & SPM4 & 98 & $7.4 \pm 1.8$ & $15485411-3513186$ \\
\hline Sz76 & 154930.7 & -354951 & $-16.1 \pm 2.8$ & $-20.8 \pm 2.7$ & SPM4 & 72 & $6.0 \pm 1.6$ & $15493074-3549514$ \\
\hline HD141277* & 154945.0 & -392509 & $-18.2 \pm 2.3$ & $-24.4 \pm 2.2$ & TYCHO2 & 74 & $7.0 \pm 1.8$ & 15494499-3925089 \\
\hline RXJ1550.7-3828 & 155046.7 & -382927 & $-10.3 \pm 1.3$ & $-16.0 \pm 1.3$ & SPM4 & 100 & $4.4 \pm 1.0$ & $15504672-3829267$ \\
\hline Sz77 & 155147.0 & -355643 & $-12.5 \pm 2.2$ & $-20.5 \pm 2.1$ & SPM4 & 98 & $5.4 \pm 1.4$ & $15514695-3556440$ \\
\hline RXJ1555.4-3338 & 155526.3 & -333822 & $-17.8 \pm 1.8$ & $-28.1 \pm 1.8$ & SPM4 & 100 & $7.5 \pm 1.9$ & 15552621-3338232 \\
\hline Sz81 & 155550.3 & -380133 & $-15.8 \pm 1.4$ & $-22.4 \pm 1.5$ & SPM4 & 81 & $6.2 \pm 1.4$ & $15555030-3801329$ \\
\hline RXJ1556.0-3655 & 155602.1 & -365528 & $-9.3 \pm 2.5$ & $-19.2 \pm 2.4$ & SPM4 & 68 & $4.8 \pm 1.3$ & $15560210-3655282$ \\
\hline Sz82 & 155609.2 & -375606 & $-12.7 \pm 3.9$ & $-21.5 \pm 4.0$ & SPM4 & 98 & $5.6 \pm 1.8$ & $15560921-3756057$ \\
\hline
\end{tabular}


Tabela 4.3 - continuação.

\begin{tabular}{|c|c|c|c|c|c|c|c|c|}
\hline ID & $\begin{array}{c}\alpha \\
(\mathrm{h} \mathrm{m} \mathrm{s})\end{array}$ & $\begin{array}{c}\delta \\
\left({ }^{\circ},{ }^{\prime \prime}\right)\end{array}$ & $\begin{array}{c}\mu_{\alpha} \cos \delta \\
(\mathrm{mas} / \mathrm{yr})\end{array}$ & $\begin{array}{c}\mu_{\delta} \\
(\mathrm{mas} / \mathrm{yr})\end{array}$ & Catálogo & $\begin{array}{c}p \\
(\%)\end{array}$ & $\begin{array}{c}\pi \\
\text { (mas) }\end{array}$ & 2MASSJ \\
\hline Hip78092 & 155641.9 & -421923 & $-13.9 \pm 1.0$ & $-25.5 \pm 1.0$ & TYCHO2 & 64 & $6.6 \pm 1.5$ & $15564188-4219232$ \\
\hline Sz126 & 155724.0 & -424004 & $-13.1 \pm 1.9$ & $-22.3 \pm 2.1$ & SPM4 & 93 & $5.9 \pm 1.5$ & $15572401-4240044$ \\
\hline Sz127 & 155730.4 & -421032 & $-10.0 \pm 1.7$ & $-12.3 \pm 1.8$ & SPM4 & 69 & $3.6 \pm 1.0$ & $15573035-4210324$ \\
\hline $\mathrm{Sz} 128$ & 155807.3 & -415148 & $-12.9 \pm 3.3$ & $-18.0 \pm 3.3$ & SPM4 & 90 & $5.1 \pm 1.6$ & $15580732-4151479$ \\
\hline RXJ1558.9-3646 & 155859.8 & -364620 & $-11.6 \pm 3.0$ & $-23.1 \pm 2.9$ & SPM4 & 74 & $5.9 \pm 1.6$ & $15585980-3646206$ \\
\hline CD-3610569 & 155949.5 & -362828 & $-29.4 \pm 2.7$ & $-46.0 \pm 2.8$ & TYCHO2 & 95 & $12.3 \pm 2.9$ & $15594951-3628279$ \\
\hline RXJ1559.9-3750 & 155954.2 & -375047 & $-11.0 \pm 1.3$ & $-19.8 \pm 1.3$ & SPM4 & 89 & $5.2 \pm 1.2$ & $15595416-3750468$ \\
\hline SSTc2dJ160000.6-422158 & 160000.6 & -422157 & $-10.4 \pm 2.3$ & $-15.8 \pm 2.5$ & SPM4 & 98 & $4.3 \pm 1.3$ & $16000060-4221567$ \\
\hline Sz131 & 160049.4 & -413004 & $-8.3 \pm 5.4$ & $-21.2 \pm 5.3$ & SPM4 & 63 & $5.2 \pm 2.1$ & $16004943-4130038$ \\
\hline RXJ1601.9-3613 & 160159.2 & -361255 & $-18.6 \pm 2.6$ & $-24.7 \pm 2.6$ & SPM4 & 51 & $7.0 \pm 1.8$ & $16015918-3612555$ \\
\hline EXLup & 160305.5 & -401825 & $-9.8 \pm 2.3$ & $-18.2 \pm 2.4$ & SPM4 & 91 & $4.7 \pm 1.3$ & $16030548-4018254$ \\
\hline HD143677* & 160345.4 & -435549 & $-12.5 \pm 1.1$ & $-22.6 \pm 1.4$ & TYCHO2 & 85 & $5.9 \pm 1.4$ & $16034536-4355492$ \\
\hline RXJ1603.8-3938* & 160352.5 & -393901 & $-17.1 \pm 2.1$ & $-29.3 \pm 2.1$ & SPM4 & 97 & $7.7 \pm 1.8$ & $16035250-3939013$ \\
\hline HD143978 & 160457.1 & -385715 & $-27.8 \pm 1.2$ & $-46.8 \pm 1.6$ & TYCHO2 & 99 & $12.3 \pm 2.8$ & $16045707-3857157$ \\
\hline RXJ1605.5-3837 & 160533.3 & -383745 & $-12.4 \pm 2.5$ & $-22.7 \pm 2.5$ & SPM4 & 93 & $5.8 \pm 1.6$ & $16053329-3837451$ \\
\hline HOLup & 160700.6 & $\begin{array}{lll}-39 & 02 & 19\end{array}$ & $-10.1 \pm 2.5$ & $-18.1 \pm 2.5$ & SPM4 & 98 & $4.7 \pm 1.4$ & 16070061-3902194 \\
\hline RXJ1607.2-3839 & 160713.7 & -383924 & $-12.9 \pm 2.1$ & $-18.0 \pm 2.2$ & SPM4 & 73 & $5.0 \pm 1.3$ & $16071370-3839238$ \\
\hline RXJ1608.0-3857 & 160800.0 & -385751 & $-13.4 \pm 2.1$ & $-20.3 \pm 2.1$ & SPM4 & 89 & $5.6 \pm 1.5$ & $16075996-3857510$ \\
\hline Sz96 & 160812.6 & -390833 & $-8.7 \pm 2.2$ & $-20.1 \pm 2.3$ & SPM4 & 56 & $4.9 \pm 1.3$ & $16081263-3908334$ \\
\hline RXJ1608.3-3843 & 160818.3 & -384405 & $-20.8 \pm 2.8$ & $-30.7 \pm 2.8$ & SPM4 & 70 & $8.4 \pm 2.1$ & $16081824-3844052$ \\
\hline Sz97 & 160821.8 & -390421 & $-10.3 \pm 2.7$ & $-19.8 \pm 2.7$ & SPM4 & 91 & $5.0 \pm 1.4$ & $16082180-3904214$ \\
\hline Sz99 & 160824.0 & -390549 & $-14.6 \pm 4.5$ & $-25.1 \pm 4.5$ & SPM4 & 100 & $6.6 \pm 2.1$ & 16082404-3905494 \\
\hline RXJ1608.4-3840 & 160825.2 & -384056 & $-13.2 \pm 2.5$ & $-16.6 \pm 2.5$ & SPM4 & 51 & $4.8 \pm 1.3$ & $16082519-3840558$ \\
\hline Sz102 & 160829.7 & -390311 & $-12.7 \pm 4.3$ & $-19.7 \pm 4.4$ & SPM4 & 97 & $5.4 \pm 1.9$ & $16082972-3903110$ \\
\hline V856Sco & 160834.3 & $\begin{array}{lll}-39 & 06 & 18\end{array}$ & $-12.5 \pm 1.2$ & $-21.6 \pm 1.6$ & TYCHO2 & 99 & $5.7 \pm 1.4$ & $16083427-3906181$ \\
\hline RXJ1608.6-3922 & 160836.2 & -392302 & $-10.6 \pm 2.2$ & $-23.2 \pm 2.2$ & SPM4 & 57 & $5.8 \pm 1.6$ & $16083617-3923024$ \\
\hline SSTc2dJ160839.8- & 160839.7 & -392923 & $-18.0 \pm 3.9$ & $-27.5 \pm 3.9$ & SPM4 & 90 & $7.5 \pm 2.0$ & $16083974-3929228$ \\
\hline RXJ1608.9-3905 & 160854.3 & -390606 & $-8.4 \pm 2.5$ & $-20.4 \pm 2.5$ & SPM4 & 51 & $5.0 \pm 1.3$ & $16085427-3906057$ \\
\hline Sz111 & 160854.7 & -393743 & $-8.1 \pm 2.3$ & $-18.9 \pm 2.3$ & SPM4 & 59 & $4.6 \pm 1.3$ & $16085468-3937431$ \\
\hline Sz112 & 160855.5 & -390234 & $-9.6 \pm 2.8$ & $-18.4 \pm 2.9$ & SPM4 & 93 & $4.7 \pm 1.4$ & $16085553-3902339$ \\
\hline Sz113 & 160857.8 & -390223 & $-12.5 \pm 3.5$ & $-19.8 \pm 3.5$ & SPM4 & 97 & $5.3 \pm 1.6$ & $16085780-3902227$ \\
\hline V908Sco & 160901.9 & -390512 & $-7.7 \pm 2.1$ & $-18.4 \pm 2.2$ & SPM4 & 56 & $4.6 \pm 1.2$ & $16090185-3905124$ \\
\hline SSTc2dJ160904.6-392112 & 160904.5 & -392113 & $-10.6 \pm 2.6$ & $-16.4 \pm 2.7$ & SPM4 & 96 & $4.4 \pm 1.3$ & $16090452-3921125$ \\
\hline Sz115 & 160906.2 & -390852 & $-12.9 \pm 3.4$ & $-18.4 \pm 3.4$ & SPM4 & 84 & $5.1 \pm 1.6$ & $16090621-3908518$ \\
\hline Sz134 & 160912.3 & -414025 & $-13.3 \pm 2.1$ & $-20.4 \pm 2.2$ & SPM4 & 91 & $5.5 \pm 1.5$ & $16091226-4140249$ \\
\hline RXJ1609.4-3850 & 160927.4 & -385019 & $-10.3 \pm 2.0$ & $-17.3 \pm 2.0$ & SPM4 & 100 & $4.6 \pm 1.2$ & $16092739-3850186$ \\
\hline Sz116 & 160942.6 & -391941 & $-16.4 \pm 2.1$ & $-26.1 \pm 2.2$ & SPM4 & 93 & $7.0 \pm 1.7$ & 16094258-3919407 \\
\hline Sz117 & 160944.4 & -391330 & $-12.7 \pm 2.5$ & $-20.1 \pm 2.5$ & SPM4 & 96 & $5.4 \pm 1.6$ & 16094434-3913301 \\
\hline Sz118 & 160948.6 & $\begin{array}{lll}-3911 & 17\end{array}$ & $-13.3 \pm 9.0$ & $-13.4 \pm 8.7$ & SPM4 & 73 & $4.1 \pm 2.9$ & 16094864-391116s \\
\hline RXJ1609.9-3923 & 160954.0 & -392327 & $-7.6 \pm 2.1$ & $-14.9 \pm 2.2$ & SPM4 & 92 & $3.8 \pm 1.1$ & $16095399-3923275$ \\
\hline Sz119 & 160957.1 & -385948 & $-11.9 \pm 2.3$ & $-24.6 \pm 2.4$ & SPM4 & 70 & $6.2 \pm 1.6$ & $16095707-3859479$ \\
\hline Sz120 & 161010.6 & -400744 & $-5.2 \pm 1.1$ & $-6.4 \pm 1.2$ & TYCHO2 & 83 & $1.9 \pm 0.6$ & $16101054-4007437$ \\
\hline Sz122 & 161016.4 & -390805 & $-15.9 \pm 2.4$ & $-21.9 \pm 2.4$ & SPM4 & 57 & $6.2 \pm 1.6$ & $16101642-3908050$ \\
\hline
\end{tabular}


Tabela 4.3 - continuação.

\begin{tabular}{lcccccccc}
\hline ID & $\begin{array}{c}\alpha \\
(\mathrm{h} \mathrm{m} \mathrm{s})\end{array}$ & $\begin{array}{c}\delta \\
\left({ }^{\prime}{ }^{\prime \prime}{ }^{\prime \prime}\right)\end{array}$ & $\begin{array}{c}\mu_{\alpha} \cos \delta \\
(\mathrm{mas} / \mathrm{yr})\end{array}$ & $\begin{array}{c}\mu_{\delta} \\
(\mathrm{mas} / \mathrm{yr})\end{array}$ & $\begin{array}{c}\text { Catálogo } \\
\text { Sz123 }\end{array}$ & $\begin{array}{c}p \\
(\%)\end{array}$ & $\begin{array}{c}\pi \\
(\mathrm{mas})\end{array}$ & 2MASSJ \\
RXJ1612.0-3840 & 161251.6 & -385314 & $-7.1 \pm 2.5$ & $-16.9 \pm 2.6$ & SPM4 & 68 & $4.1 \pm 1.2$ & $16105158-3853137$ \\
SSTc2dJ161207.6-381324 & 161207.6 & -384027 & $-9.8 \pm 1.0$ & $-15.7 \pm 1.1$ & SPM4 & 96 & $4.2 \pm 1.0$ & $16120140-3840276$ \\
RXJ1612.3-4012 & 161222.1 & -401252 & $-22.8 \pm 1.6$ & $-42.6 \pm 1.7$ & SPM4 & 77 & $10.9 \pm 2.4$ & $16122204-4012522$ \\
SSTc2dJ161243.8-381503 & 161243.7 & -381503 & $-6.8 \pm 1.4$ & $-12.4 \pm 1.4$ & SPM4 & 99 & $3.2 \pm 0.9$ & $16124373-3815031$ \\
RXJ1614.4-3808 & 161426.4 & -380800 & $-15.2 \pm 0.7$ & $-22.2 \pm 0.7$ & SPM4 & 54 & $6.1 \pm 1.3$ & $16142637-3807597$ \\
HD147402* & 162329.6 & -395800 & $-11.6 \pm 1.0$ & $-24.2 \pm 1.2$ & SPM4 & 82 & $6.1 \pm 1.4$ & $16232955-3958008$ \\
\hline
\end{tabular}




\subsection{Discussão}

\subsubsection{Paralaxe das subclasses de estrelas pré-sequência principal}

Entre as 114 estrelas que pertencem ao MG de Lupus existem 31 CTTSs, 79 WTTSs e 4 HAeBes. No que diz respeito à população de WTTSs, 44 estrelas se encontram na vizinhança das nuvens e 35 estrelas estão na região fora das nuvens. Na Figura 4.12 apresentamos a distribuição das estrelas PMS que pertencem ao MG de Lupus. Como podemos notar, a população fora das nuvens é formada por WTTSs (e uma HAeBe). Verificamos que em Lupus 4 existe uma fração maior de CTTSs enquanto que nas demais nuvens encontramos um equilíbrio maior entre CTTSs e WTTSs. Isso está relacionado a um efeito de seleção da amostra inicial uma vez que a maior parte das estrelas de Lupus 4 são provenientes de Comerón (2008) que discute somente as CTTSs nesta nuvem.

Um ponto interessante nesta análise é a distância e dispersão das TTSs em Lupus. Na Tabela 4.4 comparamos a paralaxe (distância) média de cada subclasse. Os resultados obtidos mostram que as WTTSs fora das nuvens são mais próximas enquanto as CTTSs na vizinhança das nuvens formam uma população mais distante. Como podemos notar, as CTTSs estão mais concentradas enquanto as WTTSs são mais dispersas. A maior dispersão das distâncias (paralaxes) calculadas para as WTTSs é um indicativo dos efeitos de profundidade nesta região de formação estelar.

Tabela 4.4 - Propriedades das TTSs em Lupus. Para cada subclasse fornecemos o número de estrelas e os valores médios com o desvio padrão das paralaxes e distâncias.

\begin{tabular}{lccc}
\hline Amostra & Estrelas & $\begin{array}{c}\pi \pm \sigma_{\pi} \\
(\mathrm{mas})\end{array}$ & $\begin{array}{c}d \pm \sigma_{d} \\
(\mathrm{pc})\end{array}$ \\
\hline CTTS & 31 & $5.1 \pm 0.9$ & $196_{-29}^{+42}$ \\
WTTS (dentro das nuvens) & 44 & $6.3 \pm 1.8$ & $159_{-35}^{+63}$ \\
WTTS (fora das nuvens) & 35 & $7.7 \pm 2.0$ & $130_{-27}^{+46}$ \\
\hline
\end{tabular}



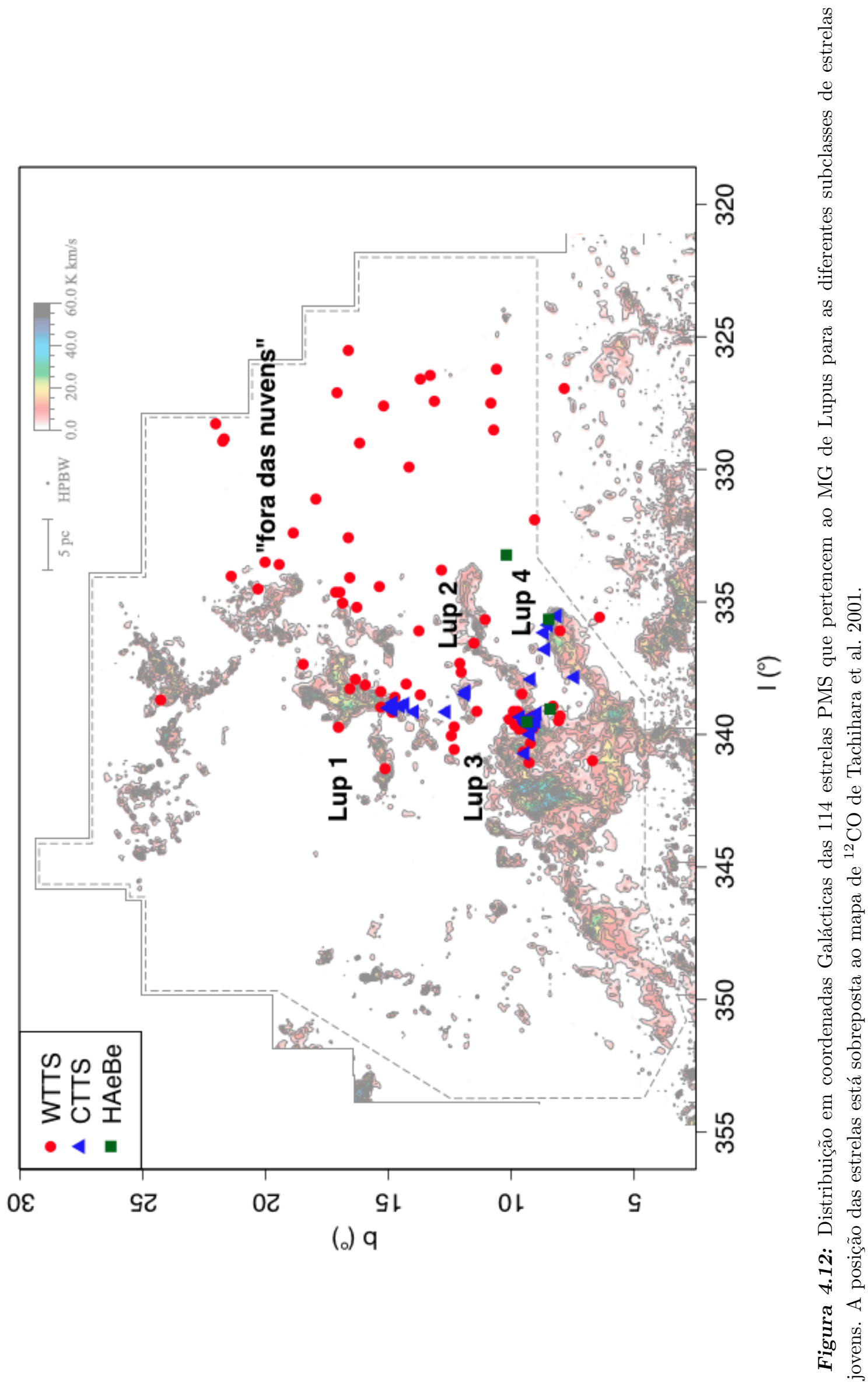


\subsubsection{Paralaxe dos subgrupos de Lupus}

Os resultados obtidos neste trabalho nos permitem investigar a distância de cada subgrupo no complexo de nuvens em Lupus. Na Figura 4.13 apresentamos a distribuição das estrelas em cada nuvem. Em seguida discutimos os resultados de distância para cada subgrupo com base na Tabela 4.5. A distribuição das paralaxes calculadas neste trabalho é apresentada na Figura 4.14.

Tabela 4.5 - Propriedades dos subgrupos em Lupus. Em cada caso fornecemos o número de estrelas e os valores médios com o desvio padrão das paralaxes e distâncias.

\begin{tabular}{lccc}
\hline Amostra & $\begin{array}{c}\text { Estrelas } \\
(\mathrm{mas})\end{array}$ & $\begin{array}{c}d \pm \sigma_{d} \\
(\mathrm{pc})\end{array}$ \\
\hline Lupus 1 & 19 & $6.0 \pm 1.1$ & $167_{-26}^{+37}$ \\
Lupus 2 & 10 & $5.9 \pm 1.1$ & $169_{-26}^{+39}$ \\
Lupus 3 & 42 & $5.8 \pm 1.9$ & $172_{-43}^{+84}$ \\
Lupus 4 & 7 & $5.2 \pm 1.0$ & $192_{-31}^{+46}$ \\
Lupus (fora das nuvens) & 36 & $7.5 \pm 1.9$ & $133_{-27}^{+45}$ \\
\hline Lupus (todas estrelas) & 114 & $6.4 \pm 1.9$ & $156_{-36}^{+66}$ \\
\hline
\end{tabular}

Lupus (todas estrelas): a média e o desvio padrão das paralaxes é $\bar{\pi}=6.4 \pm 1.9$ mas. O erro médio nas paralaxes é $\bar{\sigma}_{\pi}=1.6$ mas o que corresponde à precisão média de $25 \%$ nas paralaxes obtidas neste trabalho. Tanto a distribuição das paralaxes observada na Figura 4.14 quanto os resultados da Tabela 4.5 sugerem a existência de estrelas de fundo e de frente (conforme antecipado na seção 4.2.2) o que nos permite concluir que a região de Lupus ocupa um grande volume no espaço.

Lupus (fora das nuvens): esta população é formada em grande parte por WTTSs que estão dispersas em extensão angular e profundidade. Calculamos a paralaxe a partir da velocidade radial para 15 estrelas nesta região. A estrela mais próxima, RXJ1534.6-4003, se encontra a $87_{-17}^{+27}$ pc, enquanto que a mais distante, RXJ1525.0-3604, está a $188_{-24}^{+33}$ pc. 


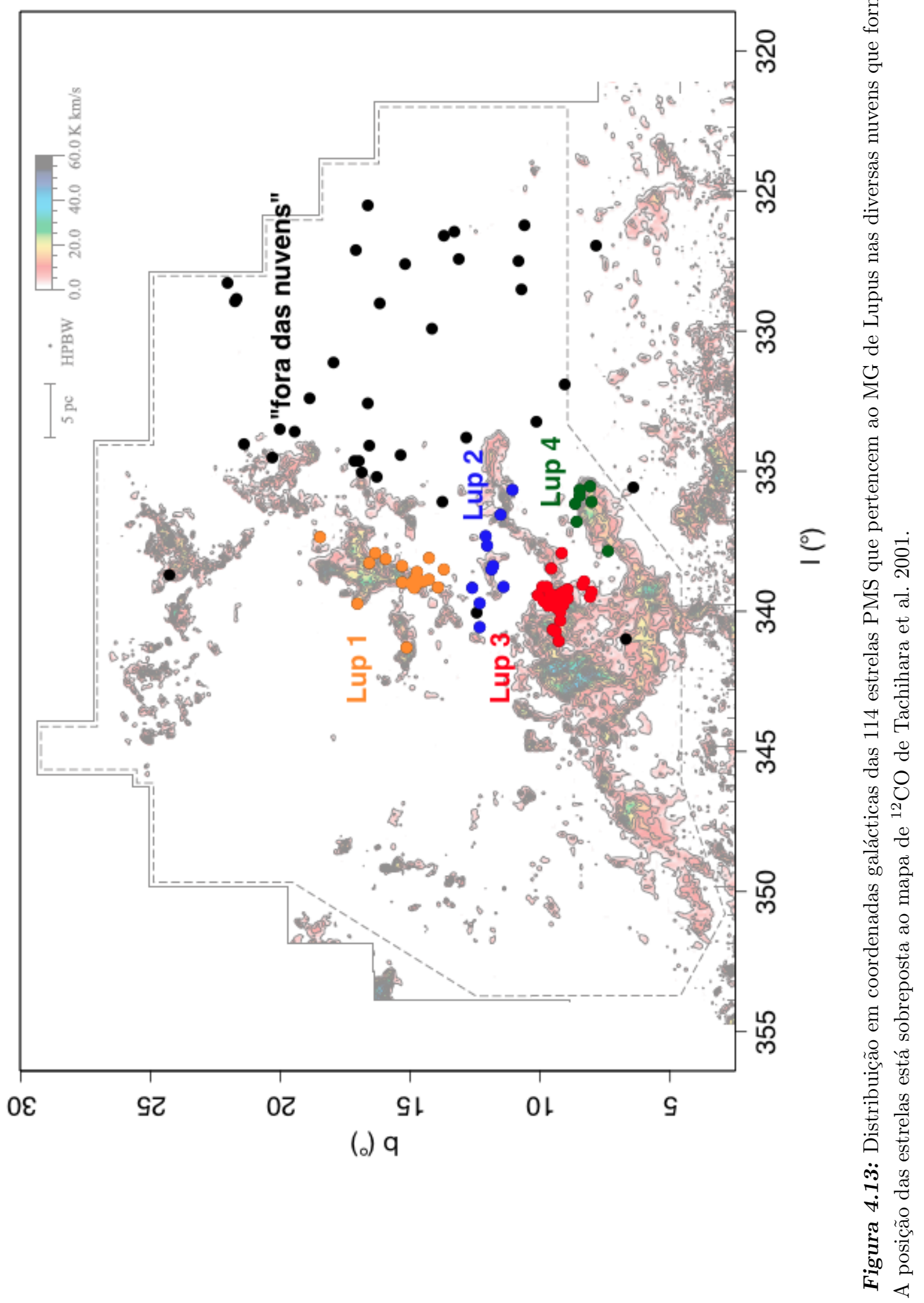


Lupus 1, 2 e 4: as estrelas dessas nuvens representam em conjunto $32 \%$ do MG de Lupus. Enquanto Lupus 1 e Lupus 2 têm aproximadamente a mesma distância média e profundidade, Lupus 4 é mais distante e as estrelas estão mais dispersas. Calculamos a paralaxe a partir da velocidade radial para 5 estrelas em Lupus 1 e uma estrela em Lupus 2. Dentro desse contexto, os resultados obtidos para Lupus 4 são menos precisos, pois as paralaxes de todas estrelas foram obtidas a partir da velocidade espacial média do MG. No entanto, Hip 78092 é uma HAeBe que se encontra na direção de Lupus 4 e a paralaxe Hipparcos para essa estrela $\left(\pi_{H I P 97}=5.04 \pm 1.18\right.$ mas e $\pi_{H I P 07}=4.29 \pm 0.98$ mas $)$ confirma a paralaxe média aproximada que foi obtida neste trabalho (vide Tabela 4.5).

Lupus 3: notamos a existência de efeitos de profundidade importantes nesta nuvem quando comparado às demais nuvens do complexo. Uma possível explicação para esse resultado é a existência de subgrupos ao longo da linha de visada que se encontram em distâncias diferentes. Calculando a paralaxe média e o desvio padrão para as CTTSs (16 estrelas) e WTTSs (24 estrelas) de Lupus 3 obtemos

$$
\begin{aligned}
& \bar{\pi}_{C T T S}=4.8 \pm 0.8 \mathrm{mas}, \\
& \bar{\pi}_{W T T S}=6.5 \pm 2.2 \mathrm{mas} .
\end{aligned}
$$

Como podemos notar, o desvio padrão nas paralaxes calculadas para as WTTSs é maior que o valor observado para as CTTSs. Isso sugere que as WTTSs estão muito mais dispersas que as CTTSs e que existem efeitos de profundidade importantes nesta nuvem (voltamos a esse ponto na seção 4.5.3). 

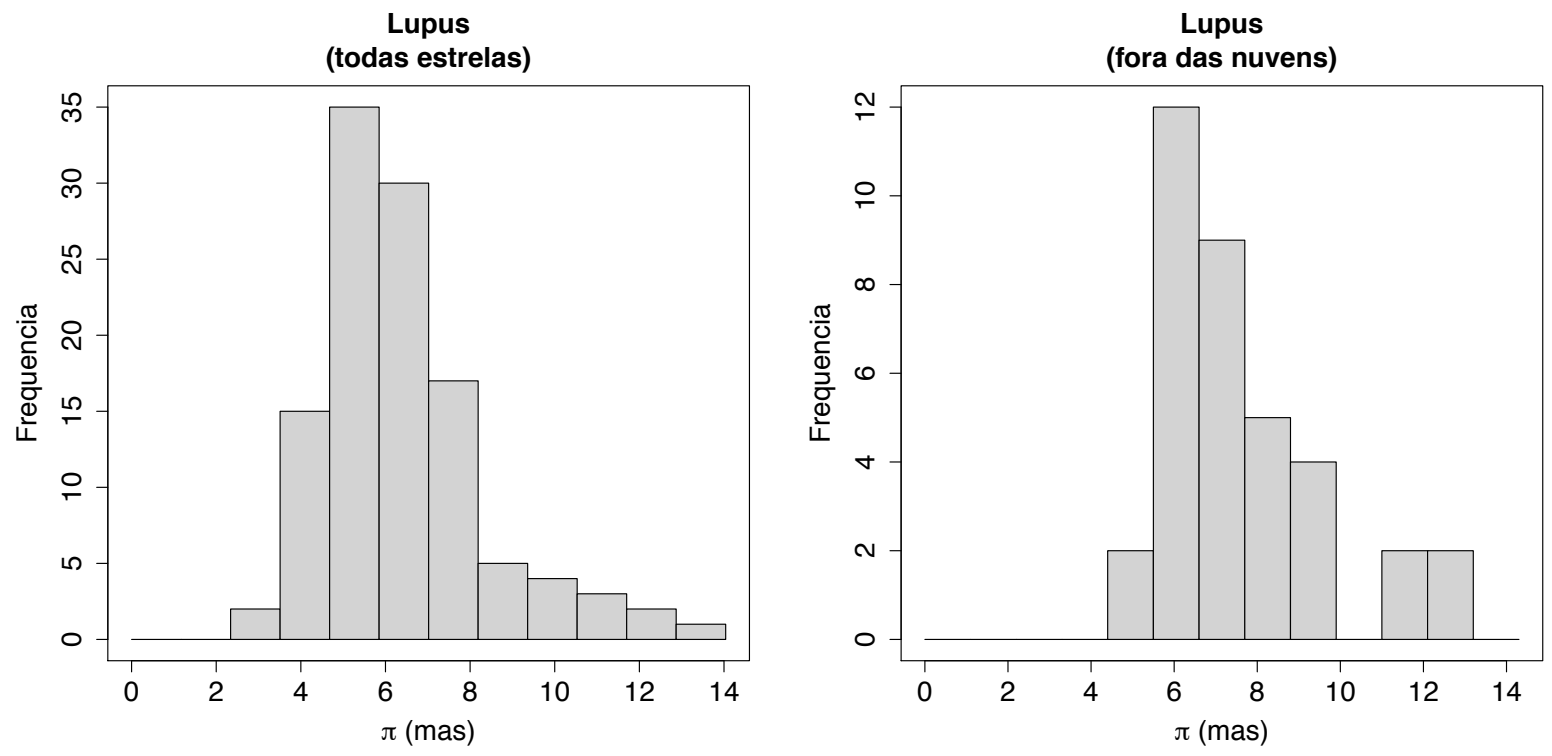

Lupus 1

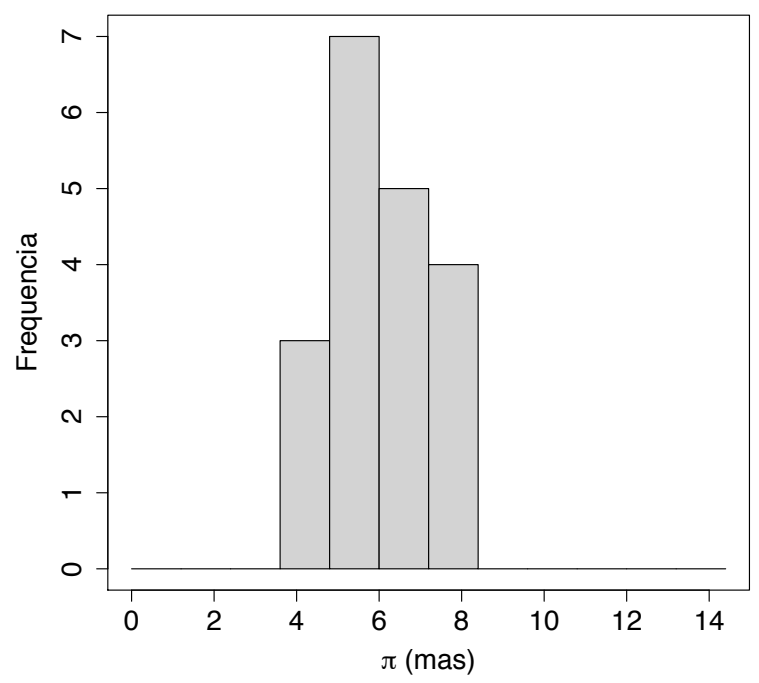

Lupus 2

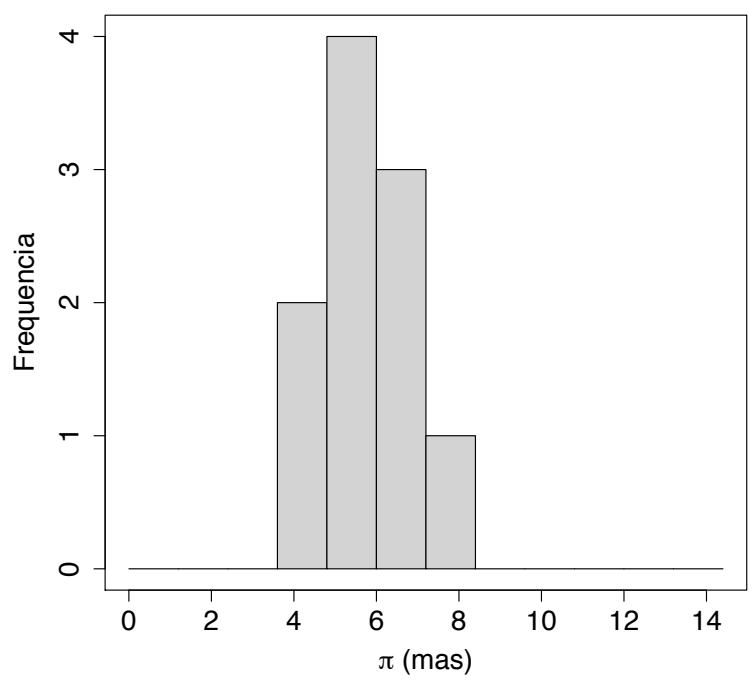

Lupus 3

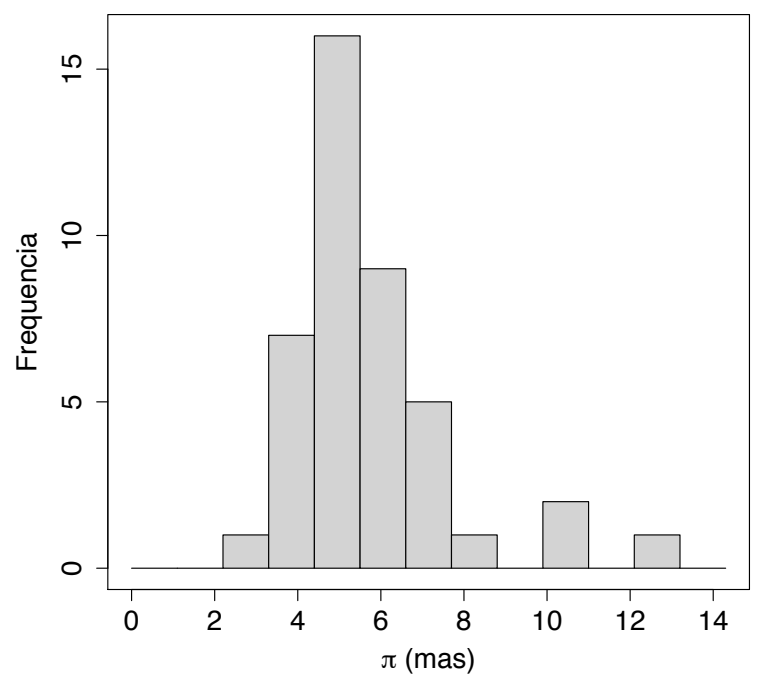

Lupus 4

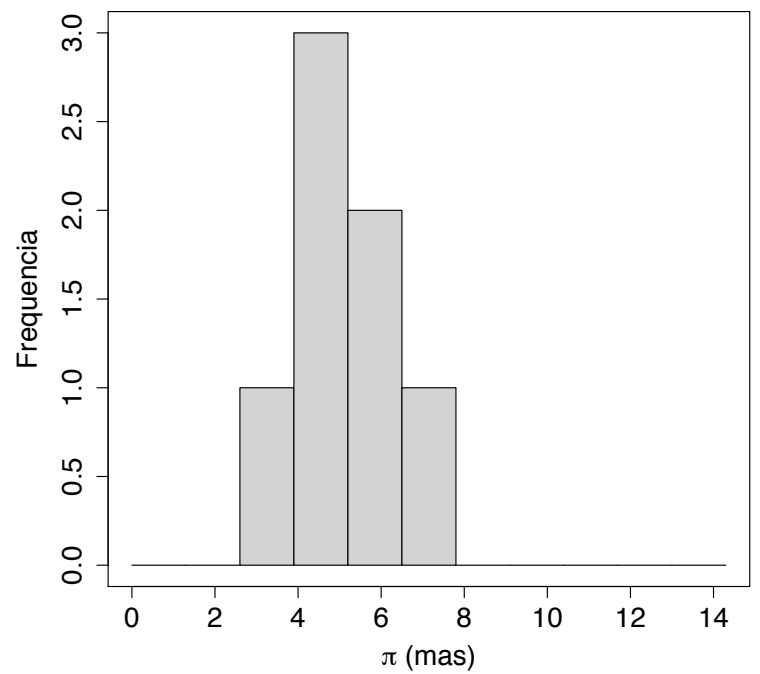

Figura 4.14: Distribuição das paralaxes calculadas neste trabalho para cada subgrupo da região de formação estelar de Lupus. 


\subsubsection{Distribuição espacial das estrelas}

Um resultado imediato do cálculo de distâncias é a distribuição espacial das estrelas. A posição Galáctica $(\mathrm{X}, \mathrm{Y}, \mathrm{Z})$ é dada em um sistema tridimensional de coordenadas cartesianas com origem no Sol onde X aponta para o centro da Galáxia, Y na direção da rotação Galáctica e Z para o pólo norte Galáctico. Na Figura 4.15 investigamos a posição espacial das 114 estrelas que pertencem ao MG de Lupus.

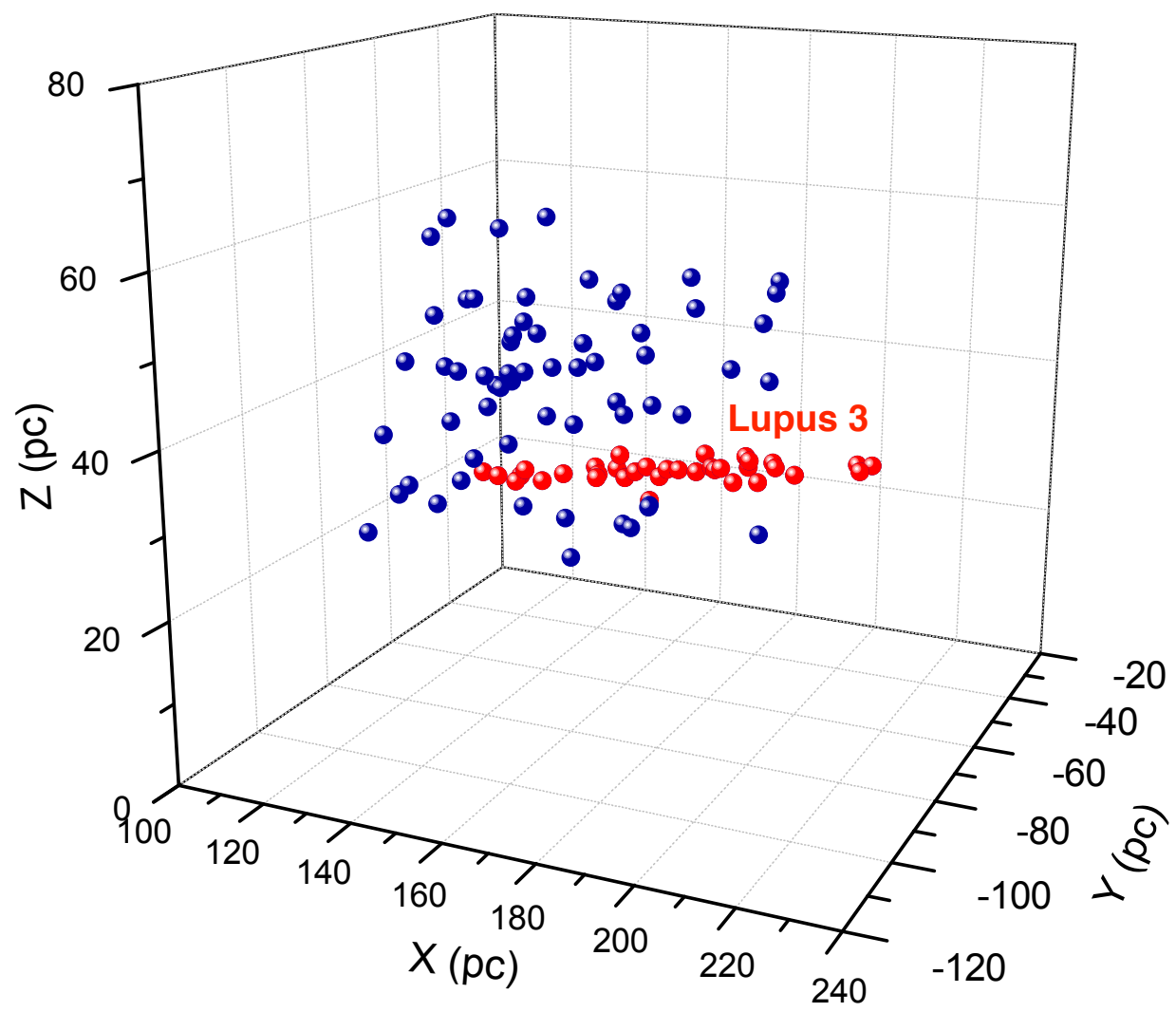

Figura 4.15: Distribuição espacial das 114 estrelas que pertencem ao MG de Lupus. As estrelas da nuvem Lupus 3 são colocadas em destaque (vermelho).

Como mencionado anteriormente confirmamos que o MG de Lupus ocupa um grande volume no espaço. Em particular, notamos que Lupus 3 apresenta uma estrutura filamentar que se estende por cerca de 100 pc em profundidade. Partindo das distâncias calculadas por mapas de extinção Knude (2010) sugere a existência de três componentes em Lupus 3 que se encontram em distâncias diferentes ( $155 \pm 3$ pc, $205 \pm 5$ pc e $230 \pm 21$ pc). No entanto, 
a incerteza nas distâncias das duas componentes mais distantes sugere que existe uma sobreposição desses subgrupos. Com os resultados obtidos neste trabalho não é possível separar as estrelas de Lupus 3 em três componentes. No entanto, notamos que existe uma componente mais próxima $(d \sim 150$ pc) formada por WTTSs e outra mais afastada $(d \sim 200$ pc) que contém CTTSs e WTTSs conforme ilustrado na Figura 4.16. Este primeiro resultado deverá ser confirmado com a medida da velocidade radial de um número maior de estrelas seguido do cálculo da distância a partir dessa grandeza.

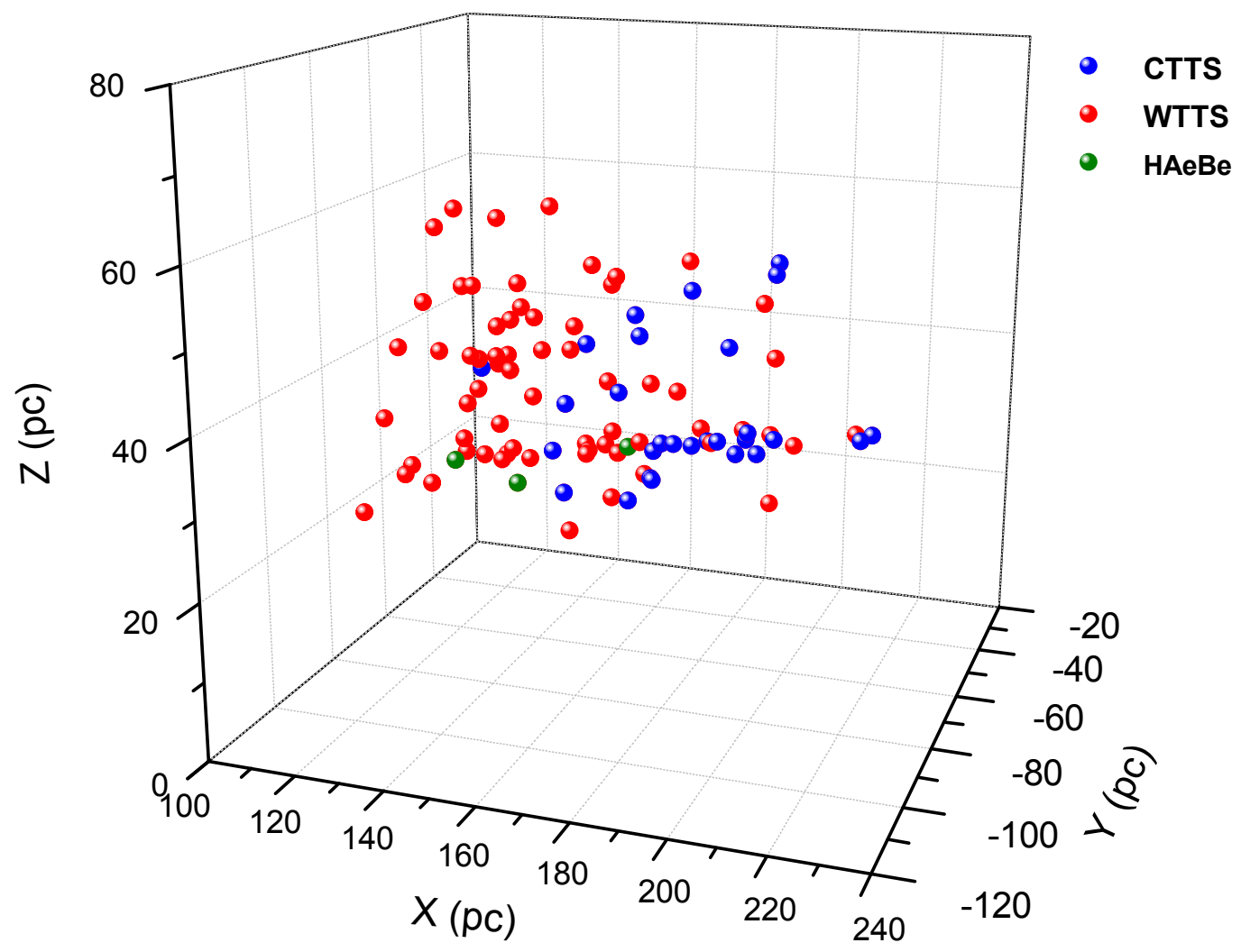

Figura 4.16: Distribuição espacial das 114 estrelas que pertencem ao MG de Lupus para cada subclasse de estrela PMS. 


\subsubsection{Velocidade espacial das estrelas}

A projeção do vetor velocidade espacial no sistema de coordenadas (X,Y,Z) da seção 4.5.3 define as componentes $(\mathrm{U}, \mathrm{V}, \mathrm{W})$ da velocidade. Na Figura 4.17 apresentamos o vetor de velocidade para as 26 estrelas com paralaxe calculada a partir da velocidade radial. Conforme esperado pela hipótese de movimento espacial comum, verificamos que os vetores velocidade espacial são paralelos com uma pequena dispersão de velocidade.
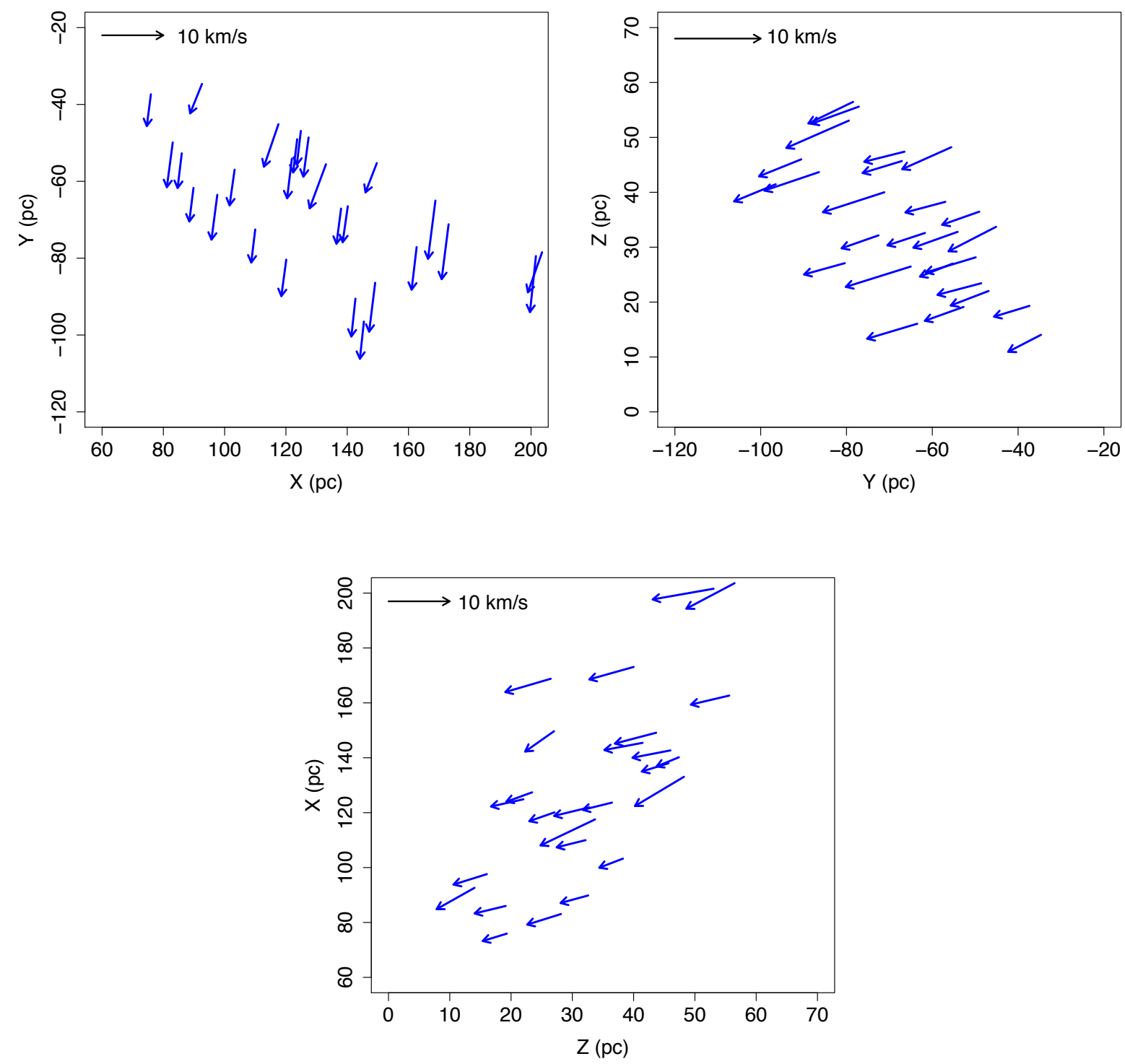

Figura 4.17: Posição (X,Y,Z) e vetor velocidade espacial para as 26 estrelas com paralaxe calculada a partir da velocidade radial. 


\subsection{Conclusão}

Investigamos as propriedades cinemáticas das estrelas PMS na região de formação estelar de Lupus partindo dos movimentos próprios publicados em diversos catálogos (SPM4, TYCHO2 e Ducourant et al. 2005). O novo CPSM foi aplicado em diversos subconjuntos da amostra inicial de estrelas candidatas e permitiu separar aquelas que pertencem ao subgrupo UCL da Sco-Cen. Identificamos um MG com 114 estrelas na região de Lupus. A solução de CP calculada neste trabalho foi testada com simulações Monte Carlo confirmando o resultado obtido. Esta nova determinação do CP das estrelas de Lupus difere daquela obtida por Makarov (2007b) que representa muito provavelmente uma solução mista que contém estrelas de Lupus e UCL.

Partindo das velocidades radiais encontradas na literatura e aquelas obtidas neste trabalho (vide capítulo 3) calculamos a paralaxe de 26 estrelas. Para as outras 88 estrelas utilizamos a velocidade espacial média do MG e um procedimento de re-amostragem desse valor para obter a paralaxe. De posse dos resultados de distância das 114 estrelas que pertencem ao MG discutimos as propriedades dos diversos subgrupos nesta região. Verificamos que as CTTSs estão mais concentradas na vizinhança das nuvens moleculares do complexo e representam uma população mais distante. Por outro lado, as WTTSs são em média mais próximas e estão mais dispersas em profundidade e extensão angular no céu. Observamos que o MG de Lupus ocupa um grande volume no espaço com algumas estrelas mais próximas que 100 pc e outras além de 200 pc. Em particular, encontramos evidências de uma estrutura filamentar que se estende por dezenas de parsec em profundidade na

direção da nuvem Lupus 3. A natureza dessa estrutura ainda não é conhecida e deverá ser investigada e confirmada com a medida da velocidade radial para um número maior de estrelas nessa região. Os resultados de distância obtidos neste capítulo serão utilizados mais adiante (capítulo 6) para investigar as propriedades físicas dessas estrelas. 
Capítulo 5

\section{Cinemática das Estrelas PrÉ-Sequência PRINCIPAL EM OPHIUCHUS}

"Reconheça o que está ao alcance dos seus olhos, e o que está oculto tornar-se-á claro para você."

Jesus Cristo

\section{Conteúdo}

5.1 Introdução . . . . . . . . . . . . . . . . 112

5.2 Estrelas Pré-Sequência Principal em Ophiuchus . . . . . . . 114

5.3 Análise do Ponto de Convergência . . . . . . . . . . . . 119

5.4 Distâncias . . . . . . . . . . . . . 123

5.5 Discussão ....................... 130

5.6 Conclusão . . . . . . . . . . . . . . . 136

O complexo de nuvens em Ophiuchus é uma das regiões mais próximas com formação estelar intensa e recente. Neste capítulo investigamos as propriedades cinemáticas das estrelas jovens nessa região com dados de movimento próprio e o método do ponto de convergência. Apresentamos os resultados de distância dessas estrelas que serão utilizados posteriormente na determinação das propriedades físicas. 


\subsection{Introdução}

A região de formação estelar de Ophiuchus é um complexo de nuvens que contém um dos aglomerados embebidos mais ricos da vizinhança solar (Porras et al. 2003). A estrutura da região revelada por mapas de ${ }^{12} \mathrm{CO}$ (Nozawa et al. 1991; Ridge et al. 2006) e mapas de extinção (Cambrésy 1999; Lombardi et al. 2008a) mostra a existência de diversas nuvens com subestruturas e filamentos de gás que se estendem por dezenas de pc (de Geus et al. 1990). Lynds 1688 (L1688) é a nuvem mais investigada na literatura por apresentar formação estelar recente e uma população jovem com idade de 1 Myr (Luhman \& Rieke 1999). Um ponto particular em Ophiuchus é a alta extinção visual em algumas regiões do complexo (por exemplo, em L1688) o que dificulta as observações no óptico (Wilking \& Lada 1983). Uma grande parte das estrelas jovens nessa região foram detectadas em outros comprimentos de onda, por exemplo, em raios-X e no infravermelho. As estrelas identificadas como fontes de raios-X são provenientes dos levantamentos Einstein (Montmerle et al. 1983), ROSAT (Casanova et al. 1995; Grosso et al. 2000; Martin et al. 1998), ASCA (Kamata et al. 1997), Chandra (Imanishi et al. 2001; Gagné et al. 2004) e XMMNewton (Ozawa et al. 2005). No que diz respeito às estrelas detectadas no infravermelho inclui-se os levantamentos no infravermelho próximo (Vrba et al. 1975, Greene \& Young 1992; Comeron et al. 1993; Allen et al. 2002) e infravermelho distante (Grasdalen et al. 1973; Elias 1978; Wilking et al. 1989; Bontemps et al. 2001). No óptico os levantamentos espectroscópicos realizados (Struve \& Rudkjøbing 1949; Wilking et al. 1987; Bouvier \& Appenzeller 1992; Wilking et al. 2005) estão restritos às regiões do complexo com baixa extinção visual.

A formação de estrelas na região de Ophiuchus está ligada à história da formação estelar na associação Sco-Cen. Um cenário possível sugere que a formação estelar nessa região tenha sido induzida por uma frente de choque (Vrba et al. 1977; Loren \& Wootten 1986). Preibisch \& Zinnecker 1999 propõem um cenário no qual uma supernova no subgrupo US da Sco-Cen explodiu e induziu a formação estelar em Ophiuchus com a chegada da onda de choque na região que ocorreu há 1 Myr atrás. Esse evento concorda com a idade média de 0.3 Myr observada para as estrelas em L1688 (Luhman \& Rieke 1999). No entanto, a descoberta de outras estrelas na vizinhança de L1688 com idades estimadas entre 2 Myr e 5 Myr se opõe a esse modelo (Bouvier \& Appenzeller 1992; Martin et al. 1998; Wilking 
et al. 2005). A formação dessas estrelas é contemporânea à formação estelar em US e pode ser explicada pela passagem de uma onda de choque que se originou no subgrupo UCL e induziu a formação estelar nessas regiões (Wilking et al. 2008). Uma evidência possível desse cenário é a existência de um "anel" de H I em expansão que envolve toda Sco-Cen (de Geus 1992). Tachihara et al. (1996) afirma que o mesmo processo que iniciou a formação estelar em Ophiuchus pode também ter sido responsável pela formação estelar nas nuvens do complexo de Lupus. Isso sugere uma origem comum para as estrelas nessas regiões.

As primeiras determinações de distância em Ophiuchus foram obtidas por fotometria das estrelas nesta região e os resultados obtidos variam de $125 \pm 25$ pc (de Geus et al. 1989) a $165 \pm 20$ pc (Chini 1981). O estudo da extinção das estrelas com dados do catálogo Hipparcos realizado por Knude \& Hog (1998) sugere uma distância média entre 120 pc e 150 pc. Mais recentemente, Lombardi et al. (2008b) estimaram a distância em $119 \pm 6$ pc com mapas de extinção e fotometria 2MASS. Uma outra determinação da distância obtida por Mamajek (2008) coloca a região de Ophiuchus a $131 \pm 3$ pc. Esse resultado foi obtido pela média das paralaxes HipPARCos de 8 estrelas brilhantes associadas às nebulosas de reflexão na vizinhança de L1688. A melhor determinação da distância obtida até o momento em Ophiuchus é oriunda da paralaxe medida com VLBA por Loinard et al. (2008) para duas estrelas: DoAr $21\left(d=121.9_{-5.3}^{+5.8}\right.$ pc) e S1 $\left(d=116.9_{-6.4}^{+7.2}\right.$ pc $)$. Os resultados encontrados na literatura sugerem que as estrelas na região de Ophiuchus (em particular, L1688) se estendem por algumas dezenas de parsecs em profundidade. O cenário da formação estelar e a carência de medidas de distância das estrelas torna essa região ao mesmo tempo interessante e difícil de ser estudada. Evidentemente, mais do que em outros casos, a distância individual das estrelas em Ophiuchus é uma informação fundamental para investigar a história da formação estelar dessa região e será tratada neste capítulo. 


\subsection{Estrelas Pré-Sequência Principal em Ophiuchus}

\subsubsection{Levantamento das estrelas candidatas}

O complexo de Ophiuchus se estende em coordenadas Galácticas entre $-12^{\circ} \leq l \leq 8^{\circ}$ e $7^{\circ} \leq b \leq 24^{\circ}$ (Lombardi et al. 2008b). Existem 113 estrelas PMS no catálogo de movimentos próprios de Ducourant et al. (2005) que se encontram nessa região. Completamos nossa amostra com outras 290 estrelas em L1688 que são provenientes dos levantamentos de estrelas jovens mencionados na seção 5.1 e que foram compiladas em um artigo de revisão recente (Wilking et al. 2008). Portanto, estamos partindo de uma amostra com 403 estrelas cuja distribuição no céu é apresentada na Figura 5.1. A pertinência dessas estrelas à região de Ophiuchus é baseada em diversos critérios como a presença de Li, linha $\mathrm{H} \alpha$ em emissão, fontes de raios-X, excesso de emissão no infravermelho e posição estimada no diagrama-HR. Existem 22 estrelas na amostra que foram identificadas por Preibisch \& Mamajek (2008) como membros de baixa massa do subgrupo US da Sco-Cen. Dentro desse contexto, pretendemos também investigar a pertinência dessas estrelas com os dados de movimento próprio disponíveis na literatura e o CPSM.

\subsubsection{Movimentos Próprios}

O catálogo de movimentos próprios SPM4 utilizado na região de Lupus é restrito às estrelas com $\delta<-20^{\circ}$ e não cobre toda região de Ophiuchus que se estende além desse limite. Completamos os dados de movimento próprio com o catálogo UCAC4 (Zacharias et al. 2012) que foi recentemente publicado e cobre o céu todo. ${ }^{1}$ Incluímos também nesta análise o catálogo PPMXL para as estrelas que não estão catalogadas no SPM4 e UCAC4. Para as estrelas mais brilhantes da amostra $(V \leq 12)$ usamos ainda o catálogo TyCHO2 e no caso das binárias utilizamos os movimentos próprios de Ducourant et al. (2005) conforme discutido na seção 4.2.2.

\footnotetext{
${ }^{1}$ Uma primeira análise do catálogo UCAC4 foi realizada em conjunto pelas equipes de astrometria do IAG/USP e do Observatório de Bordeaux. O artigo publicado (Teixeira et al. 2011) se encontra em anexo no Apêndice D. Os resultados desse estudo indicam que existe uma boa concordância entre os movimentos próprios do UCAC4 e aqueles obtidos com o círculo meridiano CCD do Observatório Abrahão de Moraes (Valinhos, SP) para as estrelas mais brilhantes.
} 


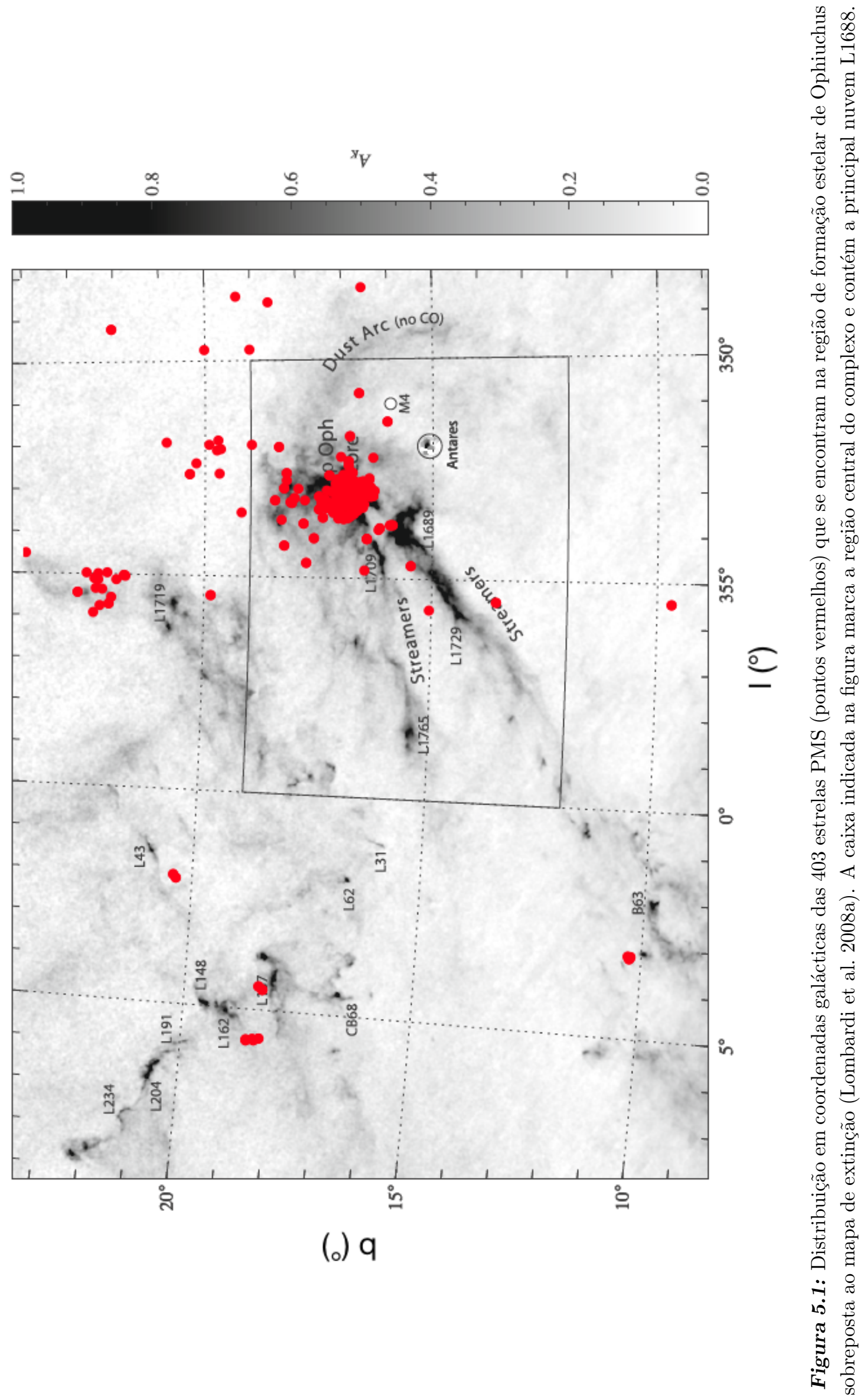


Encontramos pelo menos uma medida do movimento próprio para 221 estrelas da amostra. Para as estrelas com duas ou mais medidas de movimento próprio consideramos aquela com a menor incerteza. Eliminamos 63 estrelas com movimento próprio não significativo em cada componente (isto é, $\sigma_{\mu} \geq \mu$ ) e outras 15 estrelas com movimento próprio discrepante após um corte em $3 \sigma$ na distribuição dos movimentos próprios. Isso resulta em uma amostra com 143 estrelas. A proporção dessas estrelas em cada catálogo é a seguinte: 57 estrelas do SPM4, 55 estrelas do PPMXL, 20 estrelas de Ducourant et al. (2005), 7 estrelas do TyCHO2, 3 estrelas do UCAC4 e uma estrela (DoAr 21) de Loinard et al. (2008) cujo movimento próprio foi medido com VLBA. O movimento próprio médio é $\left(\mu_{\alpha} \cos \delta, \mu_{\delta}\right)=(-11,-25)$ mas/yr e o erro médio em cada componente é 4 mas/yr.

Na Figura 5.2 apresentamos os histogramas do movimento próprio onde se observa que a distribuição dos erros nos movimentos próprios apresenta dois picos bem definidos. Isso é justificado pela utilização de diversos catálogos. O erro médio das estrelas com movimentos próprios do SPM4 e PPMXL é, respectivamente, 3 mas/yr e 8 mas/yr, o que coincide com a posição aproximada dos picos. Tendo em vista a importância dos dados de movimento próprio neste trabalho, colocamos um filtro que consiste em considerar somente as estrelas cuja incerteza no movimento próprio é menor que 5 mas/yr. Esse limite corresponde aproximadamente ao erro médio no movimento próprio total das 143 estrelas da amostra e nos permite separar as duas populações de estrelas na Figura 5.2. As 95 estrelas que satisfazem essa condição serão utilizadas na análise que segue com o intuito de melhor definir o CP e as propriedades cinemáticas dessa região de formação estelar.

Após o levantamento dos dados de movimento próprio disponíveis na literatura para as estrelas em Ophiuchus concluímos que essa grandeza ainda não foi muito explorada. Verificamos que o movimento próprio não é conhecido para quase metade das estrelas jovens identificadas até hoje e que em muitos casos esses dados são de baixa qualidade. Uma possível explicação para isso é o fato das estrelas apresentarem magnitudes mais elevadas e ainda estarem embebidas na nuvem. Tendo em vista a importância desses dados, iniciamos uma campanha observacional no Observatório Abrahão de Moraes (Valinhos, SP) e Observatório de Bordeaux para medir o movimento próprio dessas estrelas. No entanto, a conclusão desse programa observacional se encontra além das propostas deste trabalho e será tratada em outro momento. Portanto, estamos partindo de um número reduzido 
de estrelas em Ophiuchus que pode não apresentar todos os membros cinematicamente associados à esta região de formação estelar.
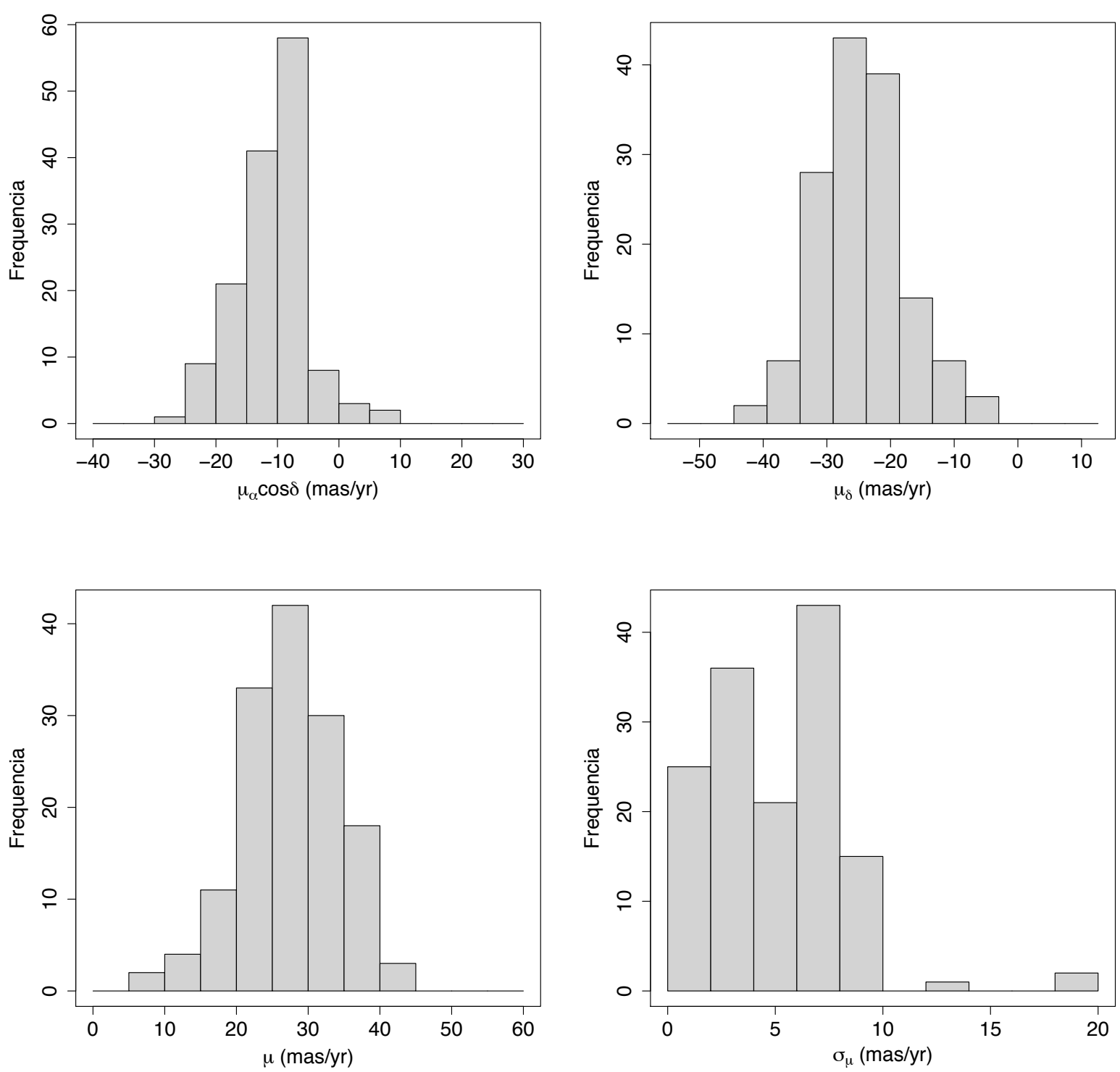

Figura 5.2: Distribuição dos movimentos próprios em cada componente (painel superior), movimento próprio total e incerteza (painel inferior) das 143 estrelas PMS em Ophiuchus. 


\subsubsection{Velocidades Radiais}

Realizamos um levantamento das estrelas de Ophiuchus com velocidade radial conhecida na literatura. As fontes de velocidade radial utilizadas neste trabalho são: Herbig \& Bell (1988), Gregorio-Hetem et al. (1992), Barbier-Brossat et al. (1994), Walter et al. (1994), Duflot et al. (1995), Dubath et al. (1996), Grenier et al. (1999), Wichmann et al. (1999), Barbier-Brossat \& Figon (2000), Madsen et al. (2002), Melo (2003), Nordström et al. (2004), Valenti \& Fischer (2005), Bobylev (2006), Gontcharov (2006), James et al. (2006), Malaroda et al. (2006), Torres et al. (2006), Guenther et al. (2007), Kharchenko et al. (2007), White et al. (2007), Prato (2007).

Com os dados da Tabela 3.2 existem ao todo 49 estrelas na região de Ophiuchus cuja velocidade radial é conhecida. Na Figura 5.3 apresentamos a distribuição das velocidades radiais nesta região. A velocidade radial média e o desvio padrão são $V_{r}=-5.8 \pm 1.2 \mathrm{~km} / \mathrm{s}$.

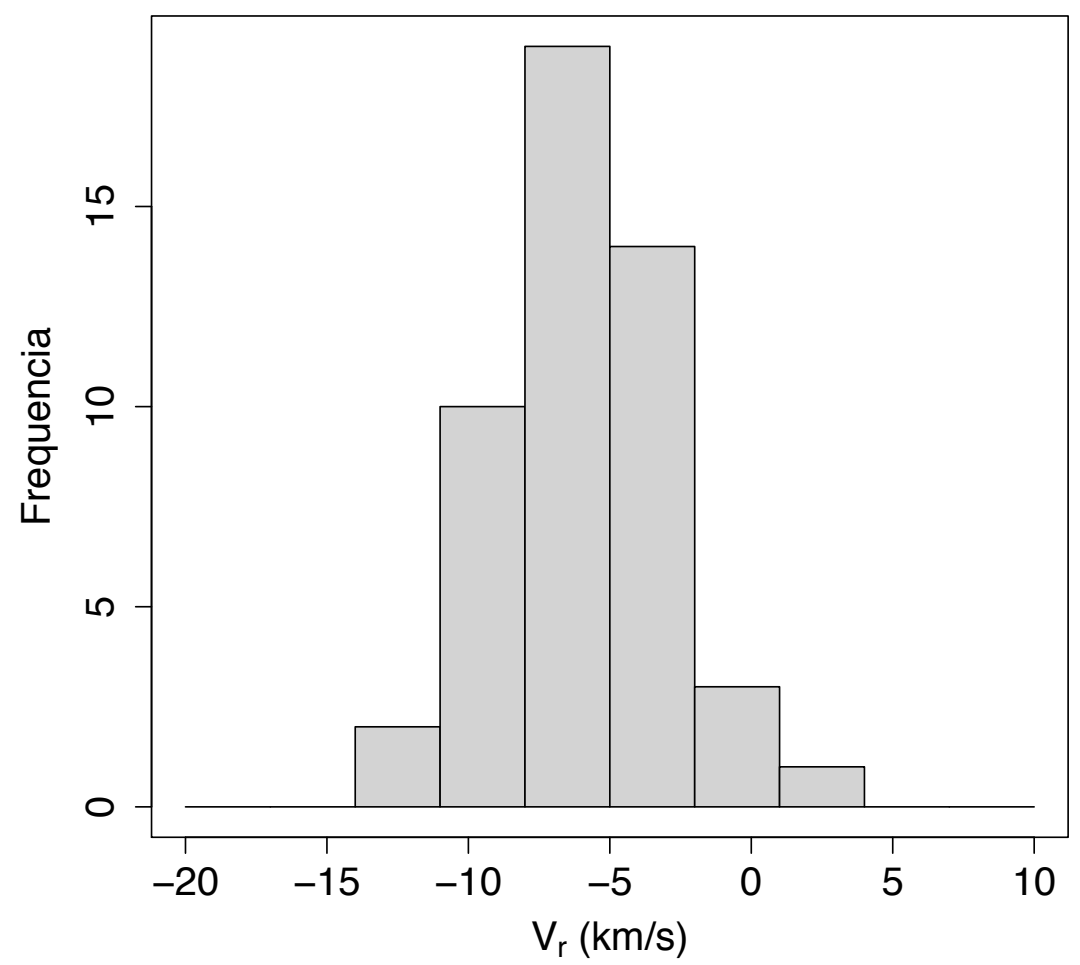

Figura 5.3: Distribuição da velocidade radial das estrelas PMS na região de Ophiuchus. 


\subsection{Análise do Ponto de Convergência}

As estrelas PMS na região de Ophiuchus formam um MG onde os vetores de movimento próprio exibem uma direção preferencial conforme ilustrado na Figura 5.4. O novo CPSM é utilizado para obter a posição do CP e selecionar as estrelas que pertencem ao MG.

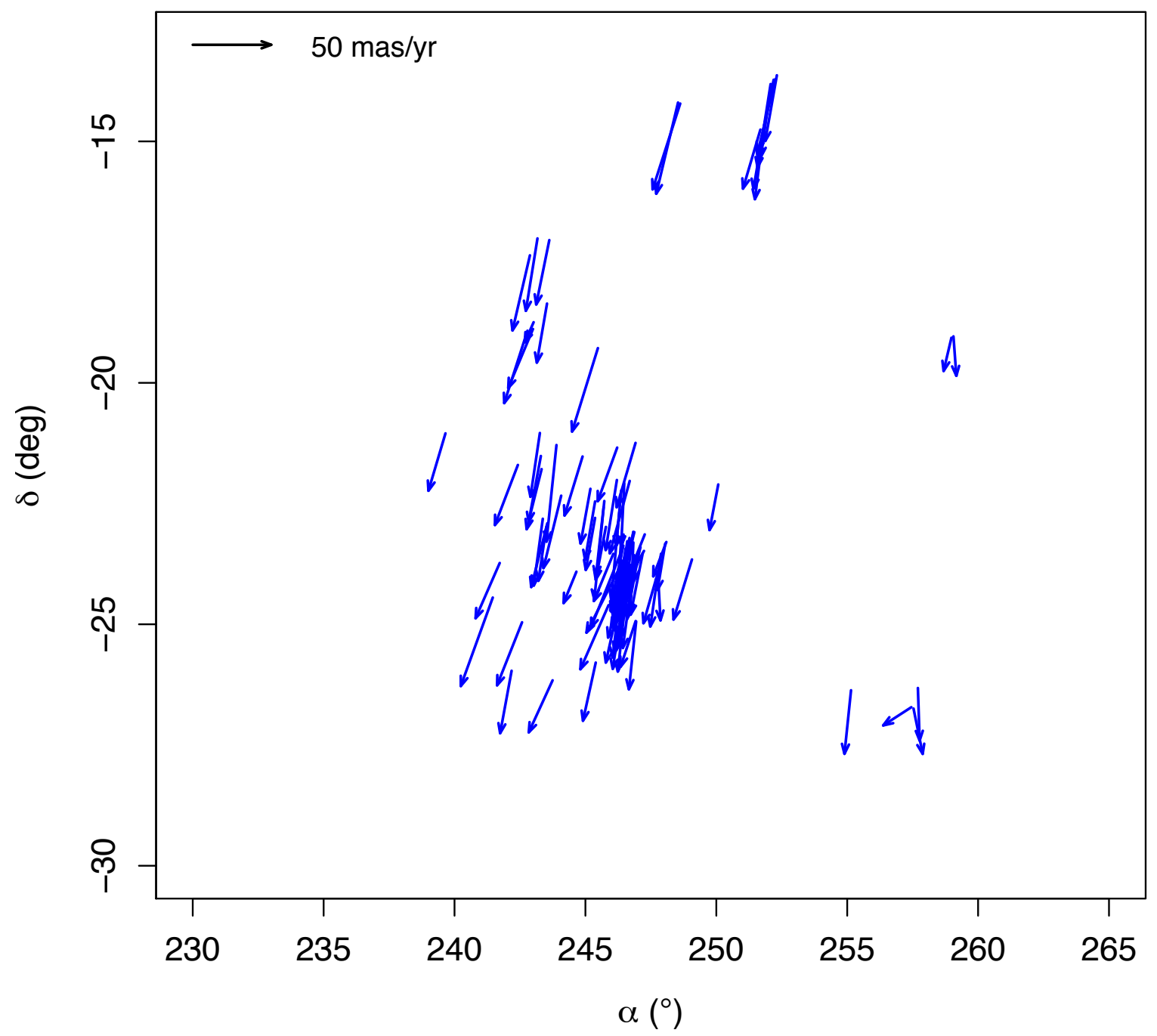

Figura 5.4: Diagrama dos vetores de movimento próprio para as 95 estrelas PMS na região de Ophiuchus. 


\subsubsection{Scorpius Superior (US)}

Inicialmente calculamos o CP das estrelas do subgrupo US da Sco-Cen devido à proximidade com a região de Ophiuchus. Partimos de uma amostra com 137 estrelas jovens nessa região que foram compiladas por Preibisch \& Mamajek (2008) e que têm movimentos próprios no catálogo TYCHO2. A distância média ao subgrupo US é 145 pc e a dispersão de velocidade é $\sigma_{v} \leq 1.5 \mathrm{~km} / \mathrm{s}$ (de Bruijne 1999b). O CPSM quando aplicado a esse conjunto de estrelas retorna uma solução com 113 estrelas e CP com coordenadas

$$
\left(\alpha_{c p}, \delta_{c p}\right)=\left(120^{\circ},-59^{\circ}\right) \pm\left(10^{\circ}, 4^{\circ}\right)
$$

Esse resultado concorda com aquele obtido por de Zeeuw et al. (1999) para as estrelas OB em US: $\left(\alpha_{c p}, \delta_{c p}\right)=\left(116^{\circ},-57^{\circ}\right)$. A diferença nas coordenadas do CP aqui calculadas é justificada pelo fato que a amostra não é a mesma e inclui as estrelas de baixa massa identificadas posteriormente por Preibisch \& Zinnecker (1999).

\subsubsection{Ophiuchus}

Para o cálculo do CP das 95 estrelas em Ophiuchus utilizamos como parâmetros de entrada no CPSM a distância média de 130 pc (Wilking et al. 2008) e a dispersão de velocidade $\sigma_{v}=2 \mathrm{~km} / \mathrm{s}$ (Makarov 2007a). O novo CPSM retorna uma solução com 44 estrelas e CP com coordenadas

$$
\left(\alpha_{c p}, \delta_{c p}\right)=\left(90^{\circ},-45^{\circ}\right) \pm\left(11^{\circ}, 18^{\circ}\right)
$$

Ao contrário do que se observa em Lupus e UCL, as regiões de Ophiuchus e US se encontram mais separadas em um diagrama de posição (vide Figura 1.1). Optamos por recalcular o CP com um valor maior da dispersão de velocidade o que nos permite incluir mais estrelas na solução. A nova solução obtida com $\sigma_{v}=3 \mathrm{~km} / \mathrm{s}$ tem 55 estrelas e o CP com coordenadas

$$
\left(\alpha_{c p}, \delta_{c p}\right)=\left(90^{\circ},-43^{\circ}\right) \pm\left(12^{\circ}, 21^{\circ}\right)
$$

Verificamos que a inclusão de outras 11 estrelas não deslocou significativamente a posição do CP. Essa solução é obtida considerando-se somente os membros com probabilidade de pertinência maior que 50\%. O corte em probabilidades, embora seja arbritrário, tem como objetivo evitar que os membros de baixa probabilidade perturbem a solução do 
conjunto. A maior incerteza associada à posição do $\mathrm{CP}$ das estrelas de Ophiuchus quando comparada àquela obtida em Lupus (vide seção 4.3) é consequência dos fatores discutidos na seção 2.4.3.2 (qualidade dos movimentos próprios, concentração das estrelas no céu e número de membros).

A cinemática das estrelas em Ophiuchus foi também investigada por Makarov (2007a) com uma amostra de 58 estrelas PMS e movimentos próprios do UCAC2. O resultado obtido naquele trabalho é

$$
\left(\alpha_{c p}, \delta_{c p}\right)=\left(100^{\circ},-42^{\circ}\right) \pm\left(25^{\circ}, 29^{\circ}\right)
$$

Conclui-se que as duas soluções de CP são compatíveis dentro dos erros envolvidos e que o resultado aqui obtido é mais preciso.

\subsubsection{Simulações Monte Carlo}

Investigamos a solução de CP obtida para o MG em Ophiuchus com simulações de re-amostragem do movimento próprio das 55 estrelas selecionadas. Utilizamos o mesmo procedimento adotado na região de Lupus e que está descrito na seção 4.3.6. Os resultados são apresentados na Figura 5.5. Os CPs simulados se distribuem ao longo da banda central com $X^{2}$ mínimo que é bastante alongada. O maior alongamento das curvas de $X^{2}$ decorre da maior concentração das estrelas no céu e explica a maior incerteza nas coordenadas do CP. A média das soluções obtidas é

$$
\left(\alpha_{c p}, \delta_{c p}\right)=\left(96^{\circ},-47^{\circ}\right) \pm\left(16^{\circ}, 10^{\circ}\right) .
$$

Eliminando as soluções mais distantes (e menos prováveis) que se estendem além de $\alpha \simeq$ $100^{\circ}$ o centro da distribuição se desloca para

$$
\left(\alpha_{c p}, \delta_{c p}\right)=\left(90.9^{\circ},-43.4^{\circ}\right) \pm\left(3.6^{\circ}, 6.0^{\circ}\right)
$$

e se aproxima do resultado obtido neste trabalho (vide seção 5.3.2). Conforme observado na Figura 5.5 a solução de CP obtida por Makarov (2007a) se encontra mais deslocada dos CPs simulados. Verificamos que a amostra de estrelas investigada naquele trabalho, a fonte dos movimentos próprios e a estratégia de cálculo do CP utilizadas são diferentes o que explica a diferença entre as duas soluções. 


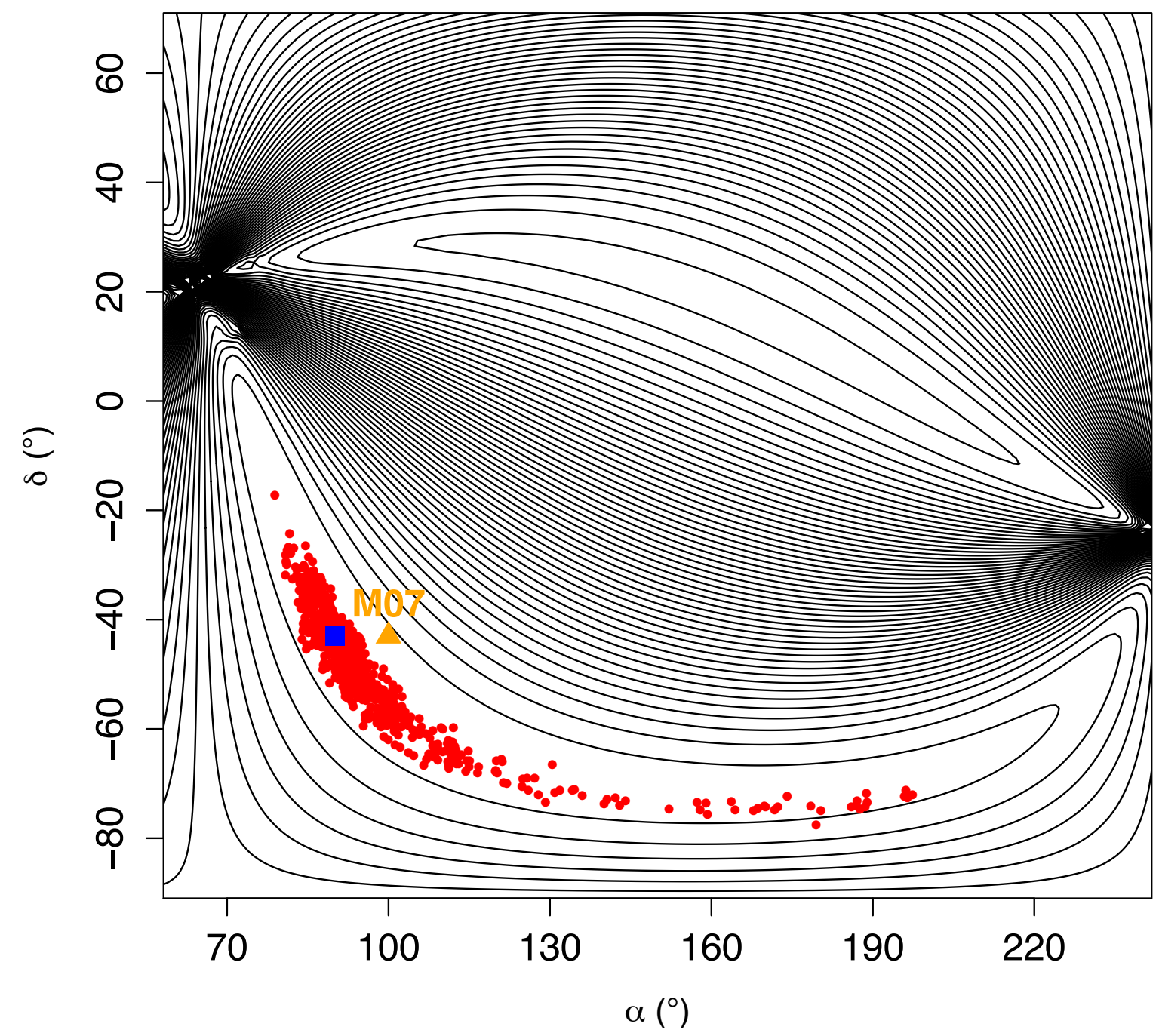

Figura 5.5: Soluções de CP obtidas para 1000 simulações Monte Carlo (pontos em vermelho) sobrepostas às curvas de $X^{2}$ constante. O quadrado azul representa a solução obtida neste trabalho (vide seção 5.3.2) enquanto o triângulo laranja indica a solução obtida por Makarov (2007a, M07). 


\subsection{Distâncias}

\subsubsection{Paralaxe calculada a partir da velocidade radial}

Entre as 55 estrelas que pertencem ao MG de Ophiuchus existem 33 estrelas com velocidade radial conhecida. Partindo dessa amostra eliminamos as estrelas com velocidade radial não significativa e as binárias. Adotamos o mesmo procedimento utilizado para a região de Lupus e descrito na seção 4.4.2 que consiste em estabelecer limites para a velocidade espacial das estrelas. Verificamos que existem 4 estrelas Hipparcos que pertencem ao MG de Ophiuchus. Usamos a paralaxe trigonométrica do catálogo HipPARCos para calcular a velocidade espacial dessas estrelas e verificamos que elas se encontram entre $V_{\text {inf }} \simeq 15 \mathrm{~km} / \mathrm{s}$ e $V_{\text {sup }} \simeq 28 \mathrm{~km} / \mathrm{s}$. Eliminamos as estrelas cuja paralaxe calculada a partir da velocidade radial implica em uma velocidade espacial fora desse intervalo. Esse processo de seleção retorna uma amostra 14 estrelas cujas paralaxes são obtidas pela equação (2.5). Esses resultados são apresentados na Tabela 5.1. A média e o desvio padrão das paralaxes calculadas são $\bar{\pi}=7.4 \pm 1.9$ mas. Verificamos que esse valor é consistente com a estimativa de distância utilizada como parâmetro de entrada no CPSM (vide seção 5.3.2). A incerteza média nas paralaxes é $\bar{\sigma}_{\pi}=0.9$ mas.

Calculamos as componentes $(\mathrm{U}, \mathrm{V}, \mathrm{W})$ da velocidade espacial das estrelas usando o procedimento descrito por Johnson \& Soderblom (1987). A distribuição das velocidades em cada componente é apresentada na Figura 5.6. A média e o desvio padrão obtidos em cada componente e na velocidade espacial são:

$$
\begin{gathered}
U=-5.4 \pm 1.0 \mathrm{~km} / \mathrm{s}, \quad V=-15.4 \pm 2.9 \mathrm{~km} / \mathrm{s}, \quad W=-9.2 \pm 2.4 \mathrm{~km} / \mathrm{s} \\
V_{\text {space }}=18.8 \pm 3.7 \mathrm{~km} / \mathrm{s} .
\end{gathered}
$$

Em seguida determinamos a média e o desvio padrão dos valores obtidos para as duas componentes da velocidade tangencial:

$$
V_{\alpha}=-4.7 \pm 1.4 \mathrm{~km} / \mathrm{s}, \quad V_{\delta}=-17.0 \pm 3.5 \mathrm{~km} / \mathrm{s}
$$

Confirmamos que a estimativa de $\sigma_{v}=3 \mathrm{~km} / \mathrm{s}$ utilizada no CPSM é consistente com o limite superior da dispersão de velocidade na componente tangencial da velocidade das estrelas. 

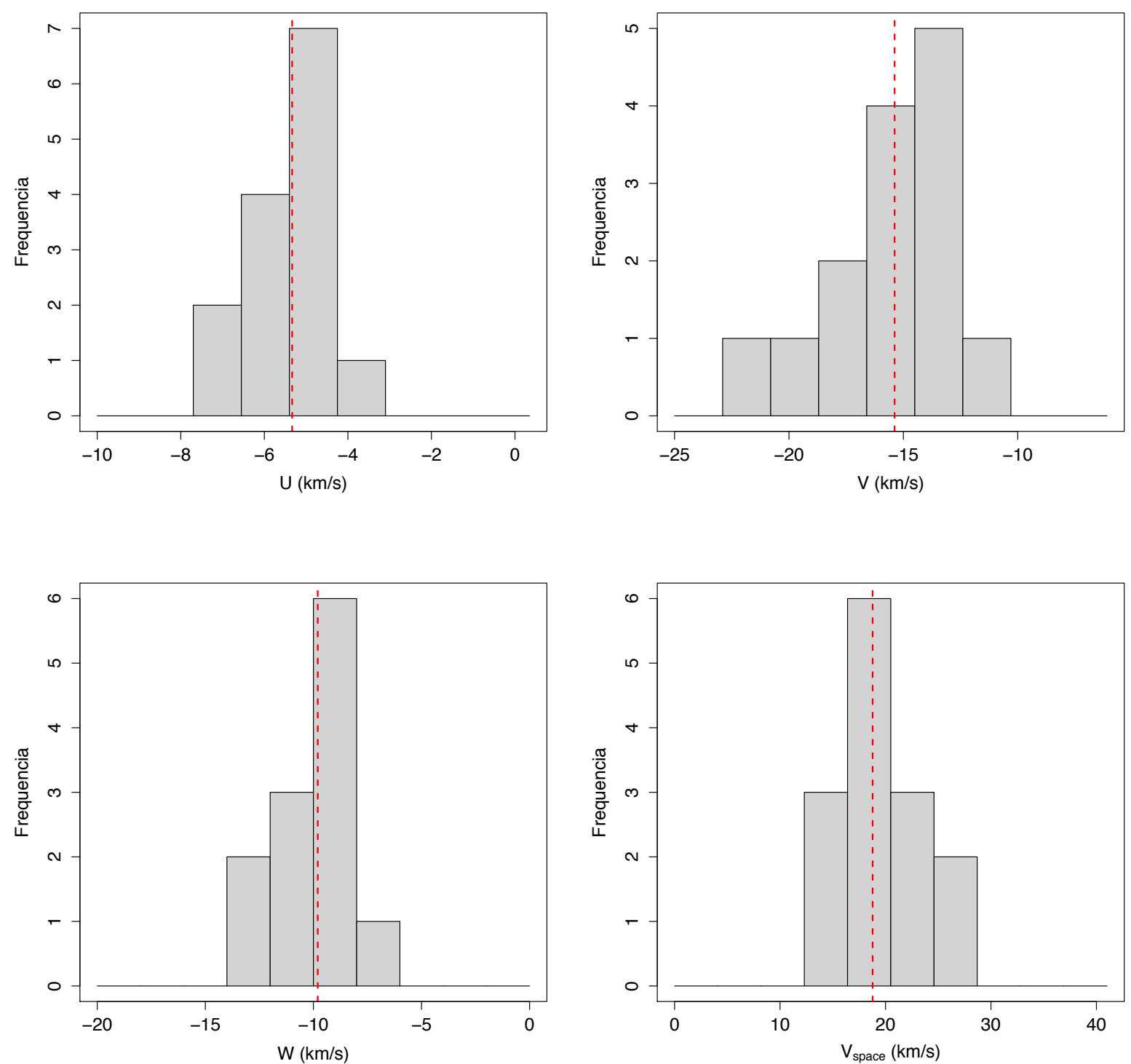

Figura 5.6: Distribuição da velocidade espacial e suas componentes para as 14 estrelas de Ophiuchus com paralaxe calculada a partir da velocidade radial. A linha vermelha tracejada indica o valor médio em cada distribuição.

A velocidade espacial média aqui calculada para as estrelas de Ophiuchus é consistente com o valor de $19.0 \mathrm{~km} / \mathrm{s}$ obtido por Mamajek (2008) com base nas paralaxes trigonométricas do catálogo Hipparcos de 8 estrelas na vizinhança de L1688. A velocidade espacial média das estrelas do subgrupo US da Sco-Cen obtida por Sartori et al. (2003) é $(U, V, W)=(-6.7,-16.0,-8.0) \pm(5.9,3.5,2.7) \mathrm{km} / \mathrm{s}$. Como podemos notar, a diferença entre a velocidade espacial das estrelas de Ophiuchus e US é pequena e indistinguível 
dentro dos erros envolvidos e a dispersão de velocidade de cada grupo.

Existem 5 estrelas com velocidade radial medida neste trabalho (vide Tabela 3.2) e que foram rejeitadas na análise, pois a velocidade espacial se encontra além dos limites estabelecidos. Isso é explicado pela velocidade radial das estrelas que é diferente das demais. Elas são apresentadas na Tabela 5.1 em separado. Calculamos a probabilidade de pertinência dessas estrelas aos MGs de Lupus, Ophiuchus, UCL e US com base na solução de CP aqui obtida em cada caso. Concluímos que de fato essas estrelas têm uma probabilidade maior de pertencer ao MG de Ophiuchus. No entanto, verificamos que para GSC6793-994 e RXJ1620.7-2348 as distâncias e velocidades estão subestimadas enquanto que para GSC6794-537, RXJ1625.4-2346 e V1725Oph essas grandezas estão superestimadas com relação às outras estrelas da Tabela 5.1. Devido à pequena quantidade de estrelas com velocidade radial conhecida nessa região não é possível afirmar no momento que a distribuição de velocidades (e distâncias) se estende para esses extremos. Essa hipótese deverá ser investigada futuramente com a medida da velocidade radial para um número maior de estrelas.

\subsubsection{Paralaxe calculada a partir da velocidade espacial}

Na Figura 5.7 comparamos as paralaxes das 14 estrelas da seção 5.4 .1 calculadas a partir da velocidade radial e da velocidade espacial média do MG. O desvio médio das paralaxes obtidas pelas duas estratégias é 0.3 mas enquanto o r.m.s. é 1.3 mas. Os resultados obtidos são compatíveis dentro dos erros envolvidos o que nos permite adotar a hipótese do movimento espacial comum para as estrelas do MG de Ophiuchus. Assim, utilizamos a equação (2.6) para calcular a paralaxe das estrelas cuja velocidade radial não é conhecida. Entre as 55 estrelas que pertencem ao MG, 36 estrelas não têm velocidade radial medida. Calculamos a paralaxe dessas estrelas utilizando o mesmo procedimento de re-amostragem da velocidade espacial do MG descrito na seção 4.4.3 para as estrelas de Lupus. Os resultados obtidos são apresentados na Tabela 5.2. 


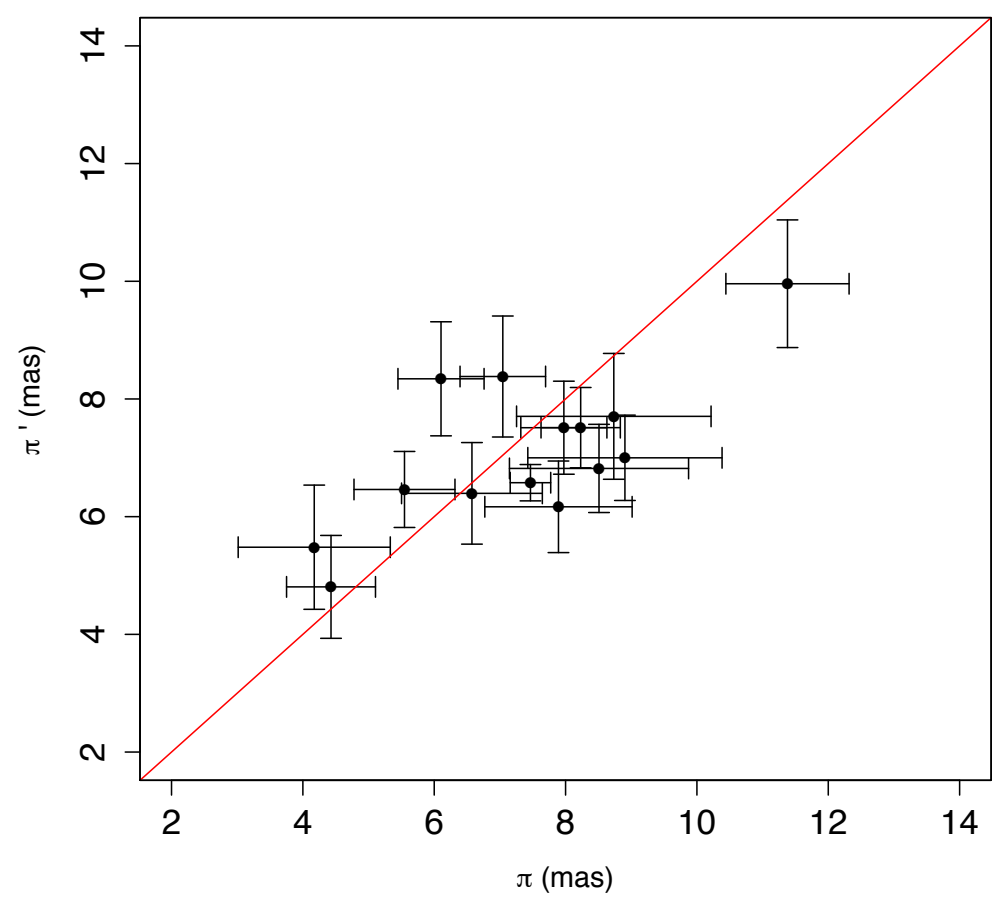

Figura 5.7: Comparação entre as paralaxes calculadas a partir da velocidade radial $(\pi)$ e velocidade espacial média do MG $\left(\pi^{\prime}\right)$ para as 14 estrelas com velocidade radial conhecida. A linha vermelha contínua representa o resultado esperado para valores iguais.

\subsubsection{Comparação com o catálogo Hipparcos}

Encontramos uma medida da paralaxe trigonométrica no catálogo HipPARCos para 4 estrelas PMS que pertencem ao MG de Ophiuchus. Calculamos a paralaxe a partir da velocidade radial para duas estrelas (AS216 e V1121Oph) desse conjunto. Na Figura 5.8 comparamos as paralaxes obtidas neste trabalho com as paralaxes trigonométricas do catálogo Hipparcos. O r.m.s. das diferenças é 2.6 mas e 1.0 mas, respectivamente, com o HIP97 e HIP07. Na Figura 5.9 comparamos as duas versões do Hipparcos na região de Ophiuchus. O desvio médio é 0.1 mas e o r.m.s. das diferenças é 3.0 mas. Analogamente ao que observamos na região de Lupus existe uma suspeita dos dados HipPARCos na região de Ophiuchus. Isso reflete a dificuldade em medir distâncias nessas regiões de formação estelar e justifica a pequena quantidade de estrelas com distância conhecida na literatura. 
Hipparcos (ESA 1997)

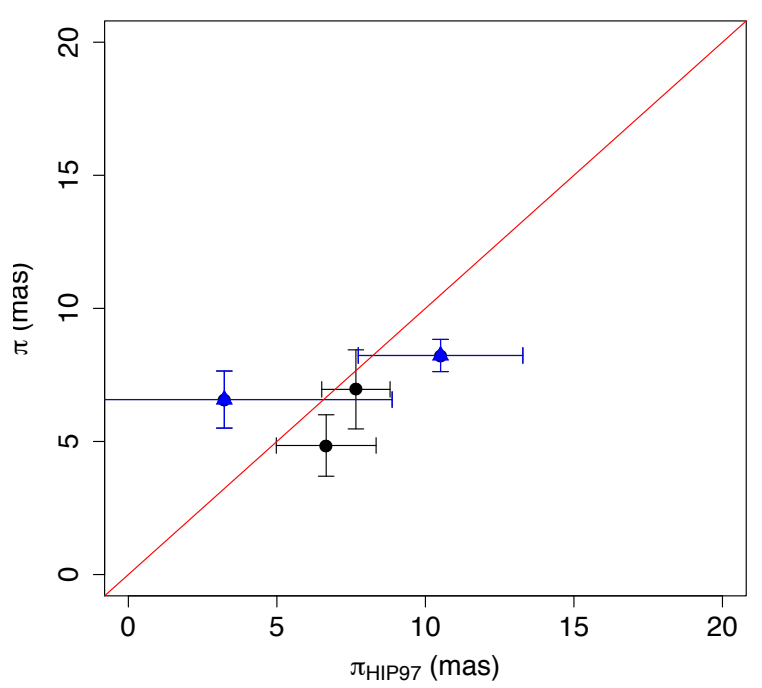

Hipparcos (van Leeuwen 2007)

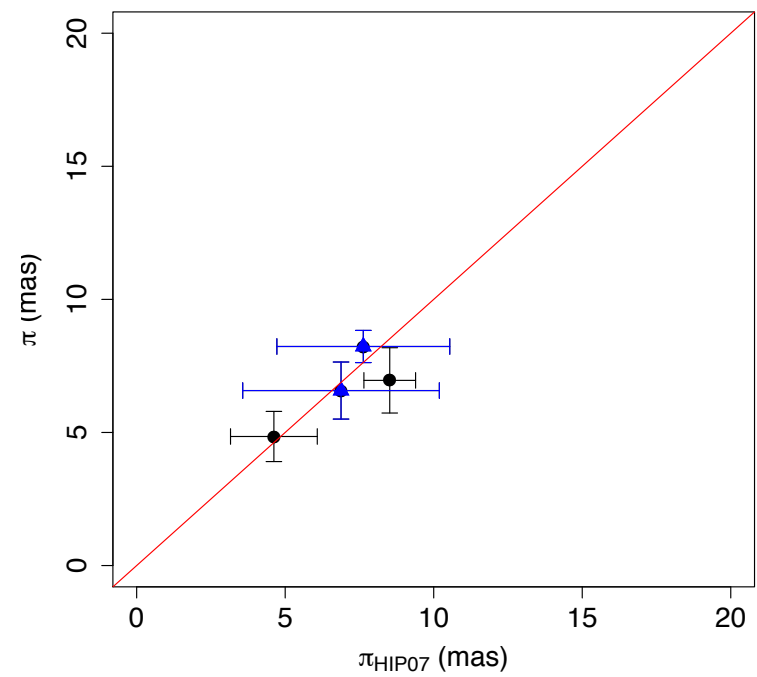

Figura 5.8: Comparação com as paralaxes trigonométricas do catálogo HipPARCos. A linha vermelha contínua representa o resultado esperado para valores iguais. Os pontos em azul representam as duas estrelas (AS216 e V1121Oph) com paralaxe determinada a partir da velocidade radial.

\section{Hipparcos}

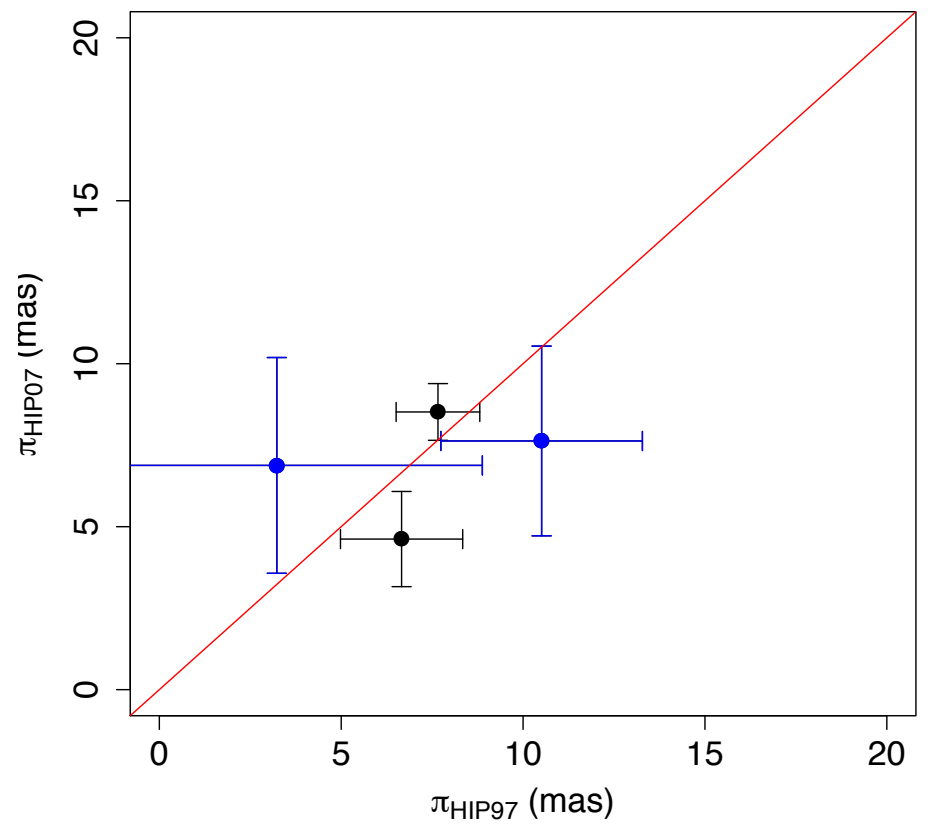

Figura 5.9: Comparação entre as paralaxes trigonométricas das duas versões do catálogo HiPPARCOS para as 4 estrelas PMS que pertencem ao MG de Ophiuchus. A linha vermelha contínua representa o resultado esperado para valores iguais. Os pontos em azul representam as duas estrelas (AS216 e V1121Oph) com paralaxe determinada a partir da velocidade radial. 


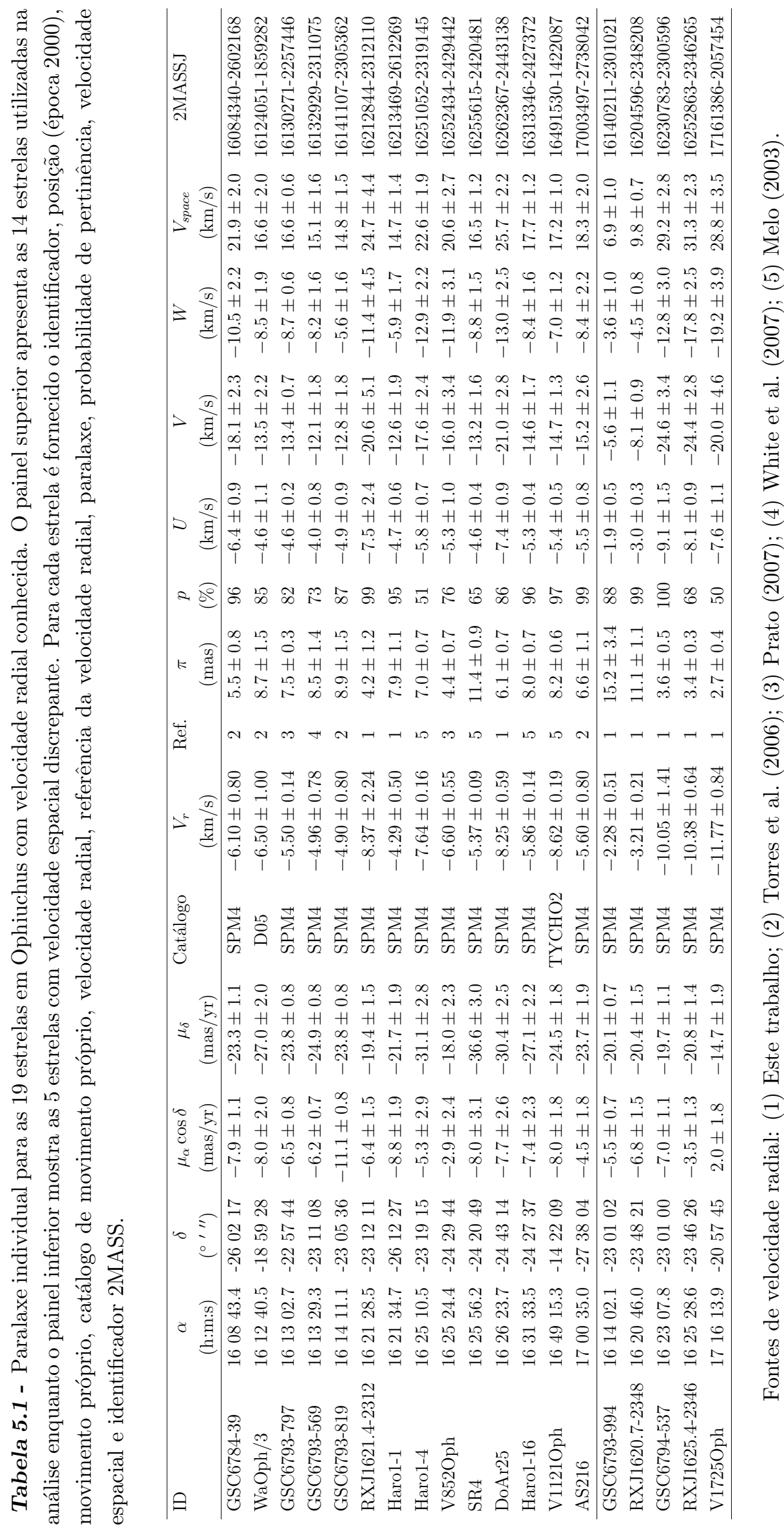


Tabela 5.2 - Paralaxe calculada a partir da velocidade espacial do MG para as 36 estrelas em Ophiuchus sem velocidade radial. Para cada estrela é fornecido o identificador, posição (época 2000), movimento próprio, catálogo do movimento próprio, probabilidade de pertinência, paralaxe e identificador 2MASS.

\begin{tabular}{|c|c|c|c|c|c|c|c|c|}
\hline ID & $\begin{array}{c}\alpha \\
(\mathrm{h}: \mathrm{m}: \mathrm{s})\end{array}$ & $\begin{array}{c}\delta \\
\left({ }^{\circ} \quad \prime \prime \prime\right)\end{array}$ & $\begin{array}{c}\mu_{\alpha} \cos \delta \\
(\mathrm{mas} / \mathrm{yr})\end{array}$ & $\begin{array}{c}\mu_{\delta} \\
(\mathrm{mas} / \mathrm{yr})\end{array}$ & Catálogo & $\begin{array}{c}p \\
(\%)\end{array}$ & $\begin{array}{c}\pi \\
\text { (mas) }\end{array}$ & 2MASSJ \\
\hline He3-1126 & 155836.9 & -225715 & $-11.7 \pm 0.8$ & $-21.4 \pm 0.8$ & SPM4 & 82 & $6.8 \pm 1.6$ & $15583692-2257153$ \\
\hline V866ScoA & 161131.4 & -183825 & $-12.0 \pm 2.0$ & $-28.0 \pm 2.0$ & D05 & 98 & $8.7 \pm 2.1$ & $16113134-1838259$ \\
\hline PDS080 & 161311.6 & -222907 & $-8.4 \pm 0.5$ & $-24.6 \pm 0.5$ & SPM4 & 97 & $7.3 \pm 1.6$ & $16131158-2229066$ \\
\hline GSC6213-306 & 161318.6 & -221249 & $-10.3 \pm 0.4$ & $-22.4 \pm 0.4$ & SPM4 & 91 & $6.8 \pm 1.6$ & $16131858-2212489$ \\
\hline PDS81 & 161408.0 & -193828 & $-7.0 \pm 2.0$ & $-22.0 \pm 2.0$ & D05 & 95 & $6.7 \pm 1.7$ & 16140792-1938292 \\
\hline GSC6214-2384 & 161934.0 & -222829 & $-12.4 \pm 1.0$ & $-22.0 \pm 0.9$ & SPM4 & 52 & $7.0 \pm 1.8$ & 16193396-2228294 \\
\hline RXJ1621.4-2332AB & 162128.8 & -233239 & $-6.9 \pm 1.3$ & $-21.8 \pm 1.4$ & SPM4 & 98 & $6.4 \pm 1.6$ & $16212881-2332389$ \\
\hline RXJ1622.7-2325AB & 162246.8 & -232533 & $-5.3 \pm 3.1$ & $-25.7 \pm 2.9$ & SPM4 & 74 & $7.3 \pm 2.1$ & $16224680-2325331$ \\
\hline RXJ1622.8-2333 & 162253.3 & -233311 & $-6.9 \pm 4.0$ & $-36.7 \pm 3.9$ & SPM4 & 52 & $10.5 \pm 2.9$ & 16225336-2333101 \\
\hline RXJ1624.8-2359 & 162448.4 & -235916 & $-7.7 \pm 2.8$ & $-26.1 \pm 2.7$ & SPM4 & 97 & $7.6 \pm 1.9$ & $16244839-2359160$ \\
\hline ROXR1-4 & 162526.9 & -244309 & $-3.1 \pm 1.8$ & $-23.6 \pm 1.8$ & SPM4 & 50 & $6.5 \pm 1.6$ & $16252686-2443089$ \\
\hline ROXR1-7 & 162547.7 & -243740 & $-8.2 \pm 4.0$ & $-21.0 \pm 4.0$ & PPMXL & 97 & $6.3 \pm 2.1$ & $16254767-2437394$ \\
\hline ROX3 & 162549.7 & -245132 & $-5.5 \pm 1.6$ & $-24.0 \pm 1.6$ & SPM4 & 82 & $6.9 \pm 2.0$ & $16254964-2451318$ \\
\hline ROXR1-14 & 162603.3 & -241746 & $-4.8 \pm 3.7$ & $-6.3 \pm 3.6$ & SPM4 & 82 & $2.1 \pm 1.4$ & $16260329-2417464$ \\
\hline ISO-Oph16 & 162609.3 & -243412 & $-8.2 \pm 1.3$ & $-26.8 \pm 1.3$ & SPM4 & 98 & $7.7 \pm 1.8$ & $16260931-2434121$ \\
\hline DoAr24 & 162617.1 & -242022 & $-5.3 \pm 3.2$ & $-30.8 \pm 3.1$ & SPM4 & 56 & $8.6 \pm 2.2$ & $16261706-2420216$ \\
\hline ROXRA16 & 162621.0 & -240852 & $-13.0 \pm 3.4$ & $-25.6 \pm 3.3$ & SPM4 & 63 & $7.9 \pm 2.2$ & $16262097-2408518$ \\
\hline WMR2005 4-25 & 162625.7 & -241428 & $-14.9 \pm 4.9$ & $-36.6 \pm 4.9$ & PPMXL & 89 & $11.0 \pm 3.3$ & $16262568-2414278$ \\
\hline WMR2005 2-36 & 162633.0 & -240017 & $-7.2 \pm 3.9$ & $-35.7 \pm 3.9$ & PPMXL & 64 & $10.1 \pm 2.7$ & $16263297-2400168$ \\
\hline ROXN6 & 162644.4 & -244714 & $-9.8 \pm 3.9$ & $-24.4 \pm 3.9$ & PPMXL & 94 & $7.2 \pm 2.3$ & $16264441-2447138$ \\
\hline ROX20-2se & 162715.1 & -245139 & $-9.6 \pm 2.0$ & $-22.2 \pm 2.0$ & SPM4 & 86 & $6.7 \pm 1.6$ & $16271513-2451388$ \\
\hline WMR2005 4-10 & 162717.6 & -240514 & $-7.1 \pm 3.9$ & $-28.4 \pm 3.9$ & PPMXL & 90 & $8.1 \pm 2.3$ & $16271758-2405136$ \\
\hline WMR2005 1-6 & 162718.4 & -245454 & $-6.1 \pm 3.9$ & $-25.9 \pm 3.9$ & PPMXL & 89 & $7.3 \pm 2.2$ & $16271836-2454537$ \\
\hline GY92 264 & 162726.6 & -242554 & $-9.6 \pm 3.9$ & $-30.5 \pm 3.9$ & PPMXL & 100 & $8.9 \pm 2.5$ & $16272658-2425543$ \\
\hline ROXN47 & 162728.7 & -245432 & $-14.7 \pm 3.9$ & $-31.7 \pm 3.9$ & PPMXL & 68 & $9.6 \pm 2.7$ & $16272873-2454317$ \\
\hline GSC6794-337 & 162739.5 & -224523 & $-13.0 \pm 3.0$ & $-24.0 \pm 3.0$ & D05 & 53 & $7.6 \pm 2.1$ & $16273956-2245230$ \\
\hline SR9 & 162740.3 & -242204 & $-13.0 \pm 2.0$ & $-34.0 \pm 2.0$ & D05 & 91 & $10.1 \pm 2.4$ & $16274028-2422040$ \\
\hline WMR2005 3-21 & 162745.2 & -250333 & $-5.0 \pm 3.9$ & $-25.3 \pm 3.9$ & PPMXL & 80 & $7.2 \pm 2.2$ & $16274522-2503328$ \\
\hline SR10 & 162755.6 & -242618 & $-7.1 \pm 1.9$ & $-24.0 \pm 1.9$ & SPM4 & 98 & $6.9 \pm 1.7$ & $16275558-2426179$ \\
\hline WMR2005 3-39 & 162824.8 & -243544 & $-16.6 \pm 3.9$ & $-33.5 \pm 3.9$ & PPMXL & 50 & $10.2 \pm 2.7$ & $16282479-2435434$ \\
\hline V853Oph & 162845.3 & -242819 & $-8.3 \pm 1.6$ & $-22.9 \pm 1.7$ & SPM4 & 98 & $6.8 \pm 1.7$ & $16284527-2428190$ \\
\hline Haro1-14 & 163105.2 & -240440 & $-10.0 \pm 2.6$ & $-19.0 \pm 2.5$ & SPM4 & 65 & $5.9 \pm 1.6$ & 16310516-2404401 \\
\hline DoAr51 & 163211.8 & -244022 & $-5.3 \pm 1.4$ & $-17.3 \pm 1.5$ & SPM4 & 100 & $5.0 \pm 1.2$ & $16321179-2440213$ \\
\hline HD150193 & 164017.9 & -235345 & $-6.0 \pm 1.4$ & $-17.0 \pm 1.6$ & TYCHO2 & 96 & $5.0 \pm 1.2$ & $16401792-2353452$ \\
\hline PDS89 & 164713.6 & -151428 & $-7.0 \pm 2.0$ & $-22.0 \pm 2.0$ & D05 & 99 & $6.9 \pm 1.8$ & $16471358-1514275$ \\
\hline RNO92 & 171555.7 & -205603 & $-5.4 \pm 1.8$ & $-12.4 \pm 1.9$ & SPM4 & 71 & $3.8 \pm 1.1$ & $17155567-2056029$ \\
\hline
\end{tabular}




\subsection{Discussão}

\subsubsection{Paralaxe das subclasses de estrelas pré-sequência principal}

Entre as 55 estrelas que pertencem ao MG de Ophiuchus existem 19 CTTSs, 32 WTTSs, 3 HAeBes e 1 anã marrom (BD, do inglês brown dwarf). A estrela GY92 264 foi incluída na lista de YSOs compilada por Wilking et al. (2008) e posteriormente identificada como BD por Geers et al. (2011). Em se tratando de um único objeto, com 100\% de probabilidade de pertinência, optamos por mantê-la na amostra. A distribuição das estrelas que pertencem ao MG de Ophiuchus é mostrada na Figura 5.10.

Tabela 5.3 - Propriedades das TTSs em Ophiuchus. Para cada subclasse fornecemos o número de estrelas e os valores médios com desvio padrão das paralaxes e distâncias.

\begin{tabular}{lccc}
\hline Amostra & Estrelas & $\begin{array}{c}\pi \pm \sigma_{\pi} \\
(\mathrm{mas})\end{array}$ & $\begin{array}{c}d \pm \sigma_{d} \\
(\mathrm{pc})\end{array}$ \\
\hline CTTS & 19 & $7.6 \pm 1.8$ & $132_{-25}^{+41}$ \\
WTTS & 32 & $7.2 \pm 2.6$ & $139_{-37}^{+79}$ \\
\hline
\end{tabular}

Na Tabela 5.3 comparamos a paralaxe (distância) de cada subclasse de TTS. Como podemos notar, as CTTSs e WTTSs em Ophiuchus se encontram aproximadamente à mesma distância média (ao contrário daquilo que observamos na região de Lupus). No entanto, observamos também em Ophiuchus que as WTTSs estão mais dispersas em profundidade que as CTTSs.

Um resultado importante da análise que foi desenvolvida em Ophiuchus é a existência de TTSs mais afastadas da região central do complexo e que se movem em conjunto com as demais estrelas. Esse é o caso, por exemplo, da estrela AS216 que tem probabilidade de pertinência alta $(p=99 \%)$. Embora as TTSs sejam em geral encontradas nas proximidades de nuvens moleculares ou em associações de estrelas, AS216 é uma CTTS isolada (Quast et al. 1987). A paralaxe aqui calculada para $\operatorname{AS} 216$ ( $\pi=6.6 \pm 1.1$ mas) é compatível com a paralaxe trigonométrica do catálogo HipPARCOS $\left(\pi_{H I P 07}=6.88 \pm 3.31\right.$ mas $)$ e apresenta uma incerteza menor. Enfatizamos que para essa estrela em particular o resultado mais relevante da nossa análise é a pertinência ao MG de Ophiuchus (com alta probabilidade). 


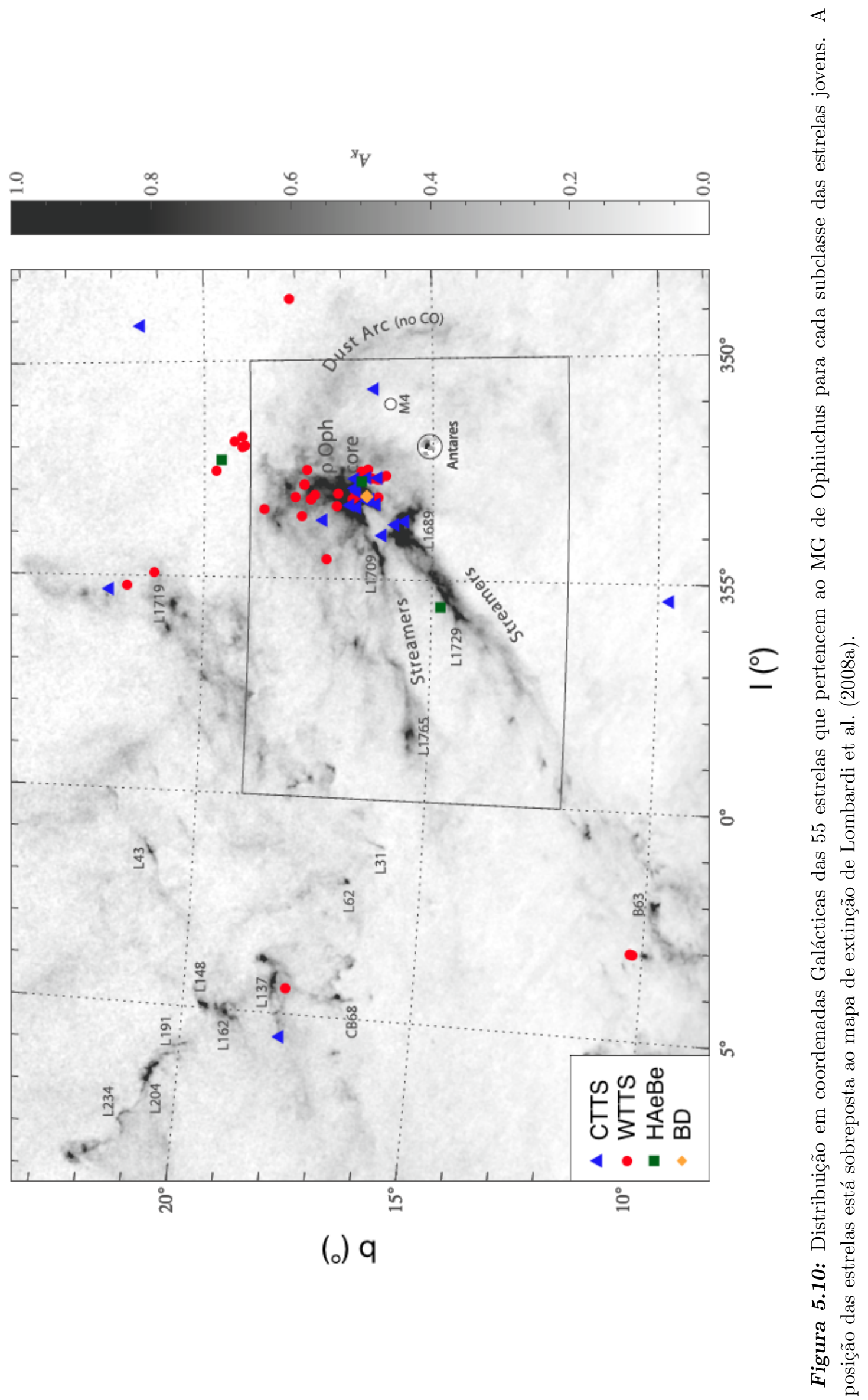




\subsubsection{Distribuição espacial das estrelas}

Partindo das distâncias calculadas neste trabalho, determinamos a posição $(\mathrm{X}, \mathrm{Y}, \mathrm{Z})$ das estrelas. Na Figura 5.11 apresentamos a distribuição espacial das 55 estrelas que pertencem ao MG de Ophiuchus. Como podemos observar, L1688 se estende por algumas dezenas de parsec em profundidade apresentando possíveis subestruturas. A estrela mais afastada do grupo é uma entre as cinco estrelas discutidas na seção 5.4.1 que apresenta velocidade radial diferente das demais e cuja paralaxe está sob suspeita. As estrelas mais dispersas nesse diagrama de posição correspondem aos membros do MG que se encontram mais afastados da região central do complexo de formação estelar. Concluímos que também existem efeitos de profundidade importantes em Ophiuchus que são desconsiderados quando utilizamos a distância média das estrelas como estimativa de distância para a região.

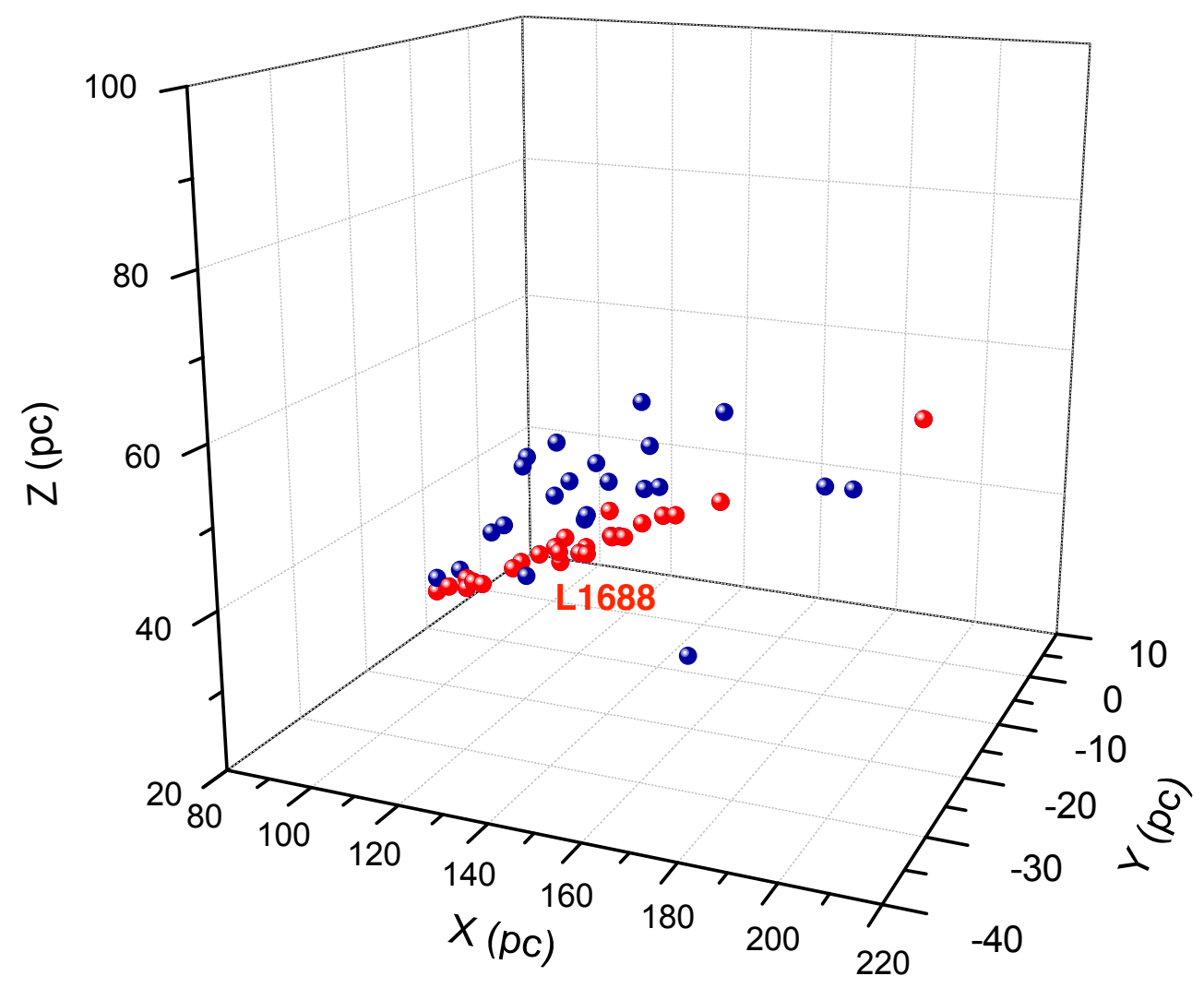

Figura 5.11: Distribuição espacial das 55 estrelas que pertencem ao MG de Ophiuchus. As estrelas da nuvem L1688 são colocadas em destaque (vermelho). 


\subsubsection{Lynds 1688 (L1688)}

Na região central do complexo de Ophiuchus (dentro da caixa assinalada na Figura 5.10) se encontram as principais nuvens com formação estelar (L1688 e L1689) além dos filamentos de gás (L1709 e L1729) que se estendem além das nuvens. Nesta seção investigamos a estrutura de L1688, principal nuvem do complexo, que foi apresentada em destaque na Figura 5.11. Na Tabela 5.4 comparamos a distância das 26 TTSs em L1688 que pertencem ao MG de Ophiuchus. Como podemos notar, as CTTSs são em média mais próximas e estão menos dispersas em profundidade que as WTTSs. No entanto, a dispersão observada para as duas populações de TTSs em L1688 explica a extensão desta nuvem na Figura 5.11.

Tabela 5.4 - Propriedades das TTSs em L1688. Para cada subclasse fornecemos o número de estrelas e os valores médios com desvio padrão das paralaxes e distâncias.

\begin{tabular}{lccc}
\hline Amostra & Estrelas & $\begin{array}{c}\pi \pm \sigma_{\pi} \\
(\mathrm{mas})\end{array}$ & $\begin{array}{c}d \pm \sigma_{d} \\
(\mathrm{pc})\end{array}$ \\
\hline CTTS & 13 & $7.8 \pm 1.9$ & $128_{-25}^{+41}$ \\
WTTS & 13 & $7.1 \pm 2.4$ & $141_{-36}^{+72}$ \\
\hline
\end{tabular}

Na Figura 5.12 investigamos a posição das estrelas em L1688 e na sua vizinhança. Um ponto interessante é que as estrelas que estamos analisando se encontram preferencialmente na periferia da nuvem. Esse efeito de seleção é justificado, pois as estrelas mais embebidas na nuvem são em geral mais fracas, não apresentam dados de movimento próprio na literatura (ou dados de baixa qualidade) e, portanto, não foram investigadas neste trabalho. Conforme observado na Figura 5.12 identificamos uma componente de L1688 ao sul de HD 147889 que está mais distante $(d>135$ pc) e outra, ao norte de HD 147889, que está mais próxima $(d<135$ pc). Esse resultado sugere a existência de subestruturas em L1688 que deverão ser confirmadas com mais dados de movimento próprio e velocidade radial para outras estrelas nessa região. 


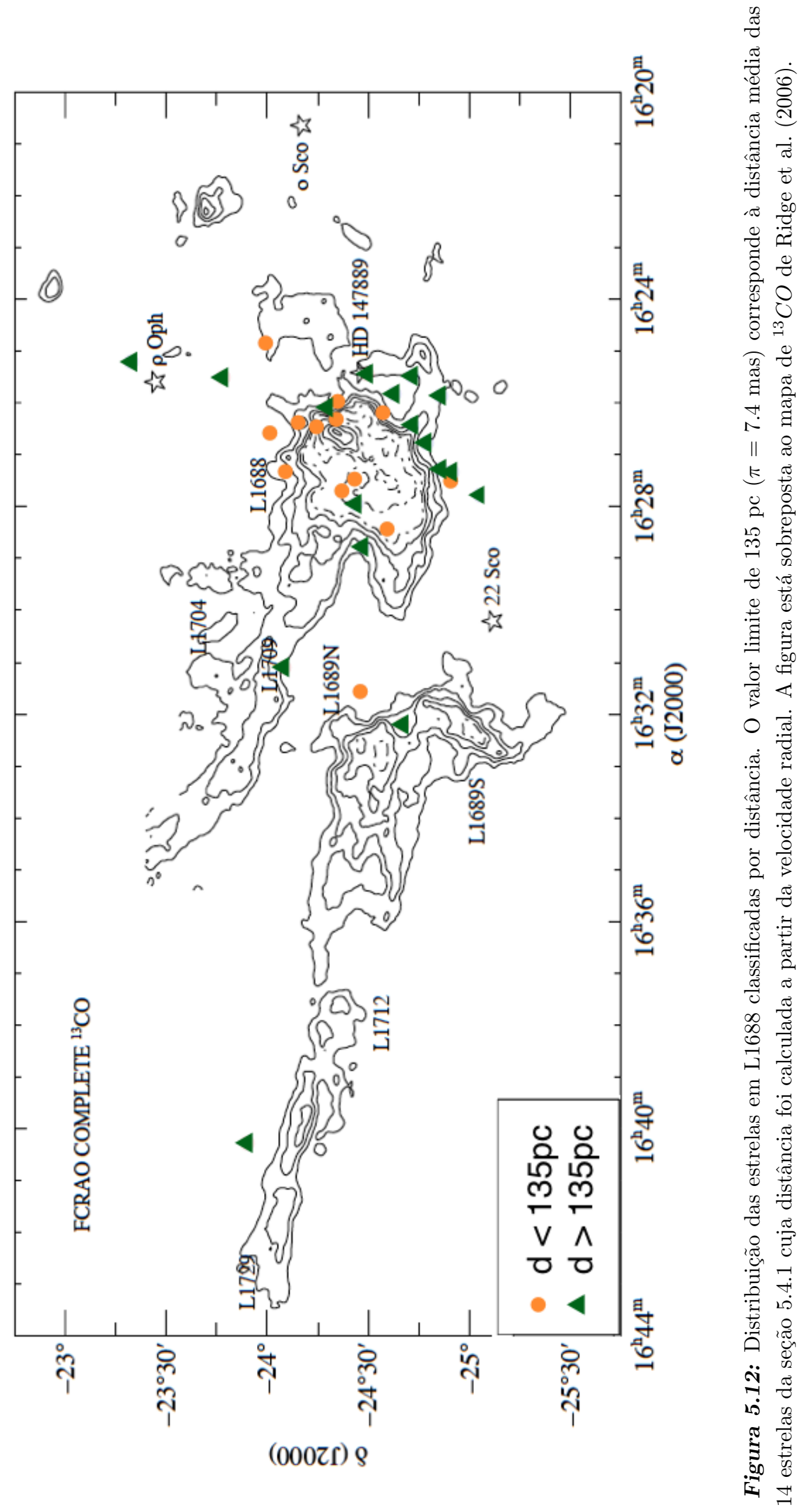




\subsubsection{Velocidade espacial das estrelas}

Na Figura 5.13 apresentamos a velocidade espacial projetada nos planos XY, YZ e ZX para as 14 estrelas com paralaxe calculada a partir da velocidade radial (vide Tabela 5.1). Conforme esperado, confirmamos que as estrelas do MG de Ophiuchus apresentam vetores de velocdidade espacial paralelos com uma pequena dispersão de velocidade.
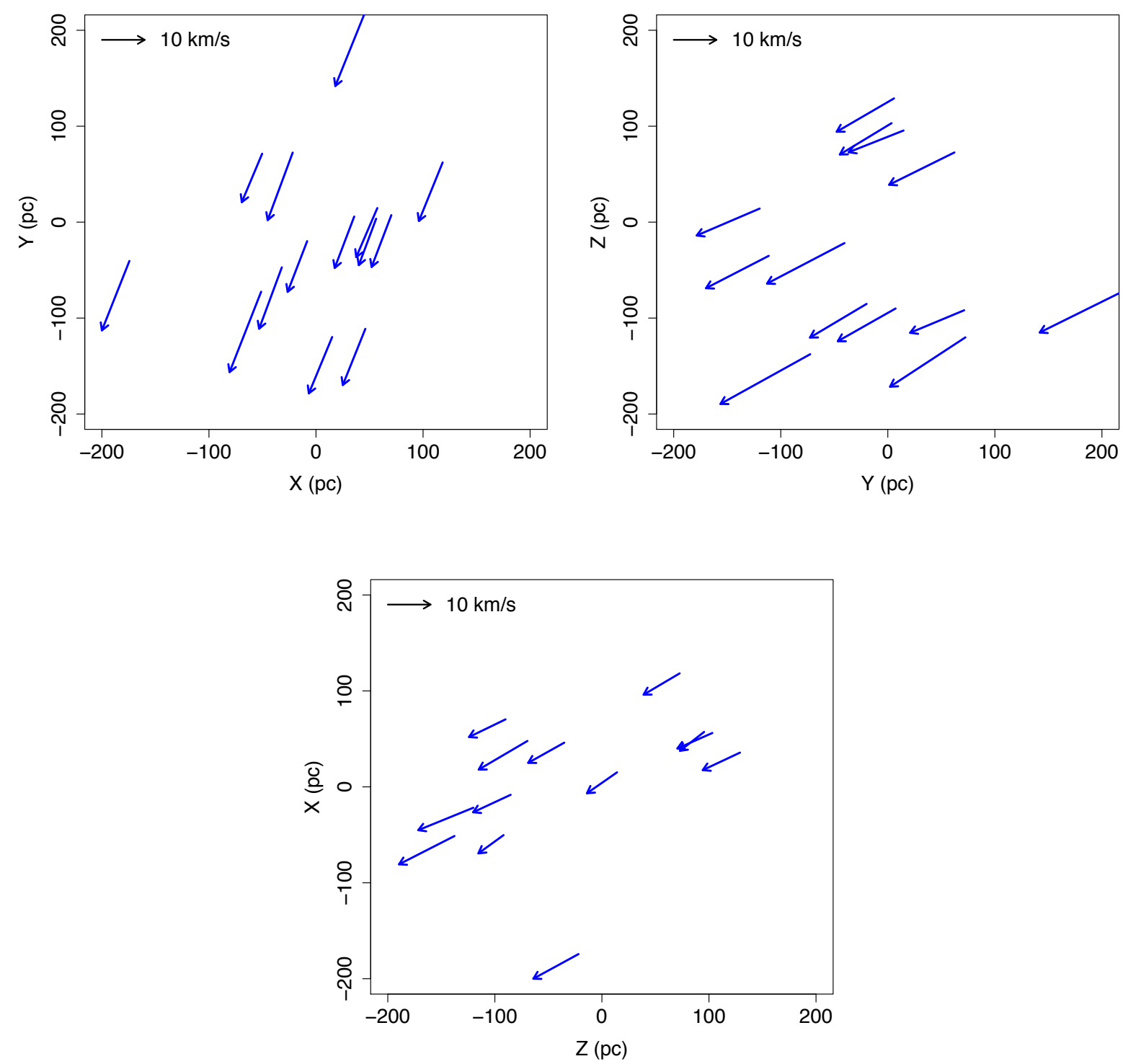

Figura 5.13: Posição (X,Y,Z) e vetor velocidade espacial para as 14 estrelas com paralaxe calculada a partir da velocidade radial. 


\subsection{Conclusão}

Partindo dos dados de movimento próprio disponíveis em diversos catálogos (SPM4, UCAC4, PPMXL, Tycho2 e Ducourant et al. 2005) identificamos um MG com 55 estrelas na região de formação estelar de Ophiuchus utilizando o novo CPSM. Comparamos o CP das estrelas em Ophiuchus e US, e concluímos que elas exibem propriedades cinemáticas distintas apesar da proximidade desses grupos. Calculamos a paralaxe para 14 estrelas do MG a partir das velocidades radiais medidas neste trabalho e outras que encontramos na literatura. Eliminamos 5 estrelas com velocidade radial conhecida, pois a velocidade espacial assim calculada difere das demais. Com a pequena quantidade de velocidades radiais disponíveis não é possível afirmar que a distribuição de velocidade espacial em Ophiuchus se estende para esses extremos. A continuação do programa observacional para medir velocidades radiais é necessária e poderá esclarecer esse ponto. Para as outras 36 estrelas calculamos a paralaxe a partir da velocidade espacial média do MG. Comparando as paralaxes aqui obtidas com as paralaxes trigonométricas do HipPARCos verificamos que existe um acordo melhor com os resultados da nova redução do catálogo.

Os resultados de distância obtidos indicam que as CTTSs e WTTSs se encontram aproximadamente à mesma distância média (ao contrário daquilo que foi observado na região de Lupus) sendo as WTTSs mais dispersas em profundidade. Um ponto interessante na análise desenvolvida é a existência de TTSs isoladas da região central do complexo, mas que se movimentam em conjunto com as demais estrelas (por exemplo, AS216). A origem dessas TTSs dispersas (isoladas) é um assunto ainda em aberto e pretendemos investigá-lo em outro momento. Ao investigar a distribuição espacial das estrelas que pertencem ao MG de Ophiuchus notamos que as estrelas em L1688 se estendem por dezenas de parsec em profundidade. Investigando a estrutura dessa nuvem identificamos uma componente mais próxima e outra mais afastada que 135 pc (distância média). As distâncias aqui obtidas são utilizadas no capítulo 6 para a determinação das propriedades físicas dessas estrelas. 


\section{Propriedades FísicAs dAs Estrelas Jovens}

\section{EM Lupus E Ophiuchus}

"Os caminhos que conduzem o homem ao saber são tão maravilhosos quanto o próprio saber."

Johannes Kepler

\section{Conteúdo}

6.1 Introdução . . . . . . . . . . . . . . . 138

6.2 Luminosidade . . . . . . . . . . . . . . . 141

6.3 Diagrama-HR . . . . . . . . . . . . . . . 142

6.4 Massa e Idade . . . . . . . . . . . . . . . . 146

6.5 Comparação com Taurus-Auriga . . . . . . . . . 153

6.6 Simulação do Diagrama-HR . . . . . . . . . . . . 158

6.7 Distância Individual vs. Distância Média . . . . . . . . . 161

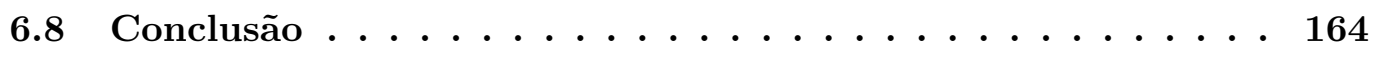

A distância é uma grandeza fundamental na astronomia, pois nos permite obter a luminosidade, massa e idade das estrelas. Neste capítulo apresentamos uma primeira aplicação dos resultados de distância, foco desta tese de doutorado, na determinação das propriedades físicas das estrelas e construção do diagrama-HR da região de Lupus e Ophiuchus. Comparamos os resultados obtidos em cada região e ressaltamos a importância do uso da distância individual das estrelas na determinação dos parâmetros físicos. 


\subsection{Introdução}

A região de formação estelar em Lupus exibe uma população diversificada de estrelas jovens. A maior parte das CTTS conhecidas até o momento foram identificadas por Schwartz (1977) com base na linha de emissão $H \alpha$. A descoberta de uma grande quantidade de WTTSs detectadas em raios-X pelo satélite ROSAT (Krautter et al. 1997; Wichmann et al. 1997a,b) aumentou significativamente o número de estrelas PMS conhecidas nessa região e revelou a existência de uma população mais dispersa e afastada das nuvens do complexo. Diversos cenários de formação estelar têm sido propostos para explicar a origem das WTTSs dispersas: as estrelas se formaram nas nuvens e se afastaram devido à dispersão de velocidade do grupo (Wichmann et al. 1999), elas foram ejetadas com alta velocidade pela interação com outras estrelas (Sterzik \& Durisen 1995) ou se formaram em pequenas nuvens que logo se dissiparam (Feigelson 1996). Por outro lado, Briceno et al. (1997) argumentam que a maior parte das fontes identificadas em raios-X são na verdade estrelas mais evoluídas que já se encontram na sequência principal de idade zero (ZAMS, do inglês Zero Age Main Sequence). Uma outra alternativa foi proposta por Wichmann et al. (1997a) sugerindo a existência de uma mistura de populações com as estrelas do cinturão de Gould que se encontram na mesma direção e distância aproximada da região de Lupus. Após a análise de pertinência desenvolvida no capítulo 4 concluímos que de fato algumas estrelas mais dispersas compartilham as mesmas propriedades cinemáticas das estrelas concentradas nas nuvens o que nos permite classificá-las como estrelas de Lupus. Um parâmetro importante na discussão dos possíveis cenários da origem dessas estrelas dispersas é a idade delas que pode agora ser obtida com o conhecimento da distância.

A distribuição de massa e idade das estrelas PMS em Lupus foi obtida em diversos trabalhos na literatura. Os resultados encontrados dependem fortemente da amostra de estrelas e das isócronas utilizadas em cada caso. Hughes et al. (1994) verificaram que o pico das distribuições de massa e idade das estrelas em Lupus se encontra, respectivamente, em $0.3-0.4 M_{\odot}$ e 3 Myr partindo de uma amostra com 69 CTTSs e as isócronas de D'Antona \& Mazzitelli (1994). Com base nesses resultados os autores afirmam que as estrelas de Lupus são em média mais velhas e têm massa menor que as estrelas PMS na região de Taurus-Auriga (Strom et al. 1989). Posteriormente, Wichmann et al. (1997a) realizaram um estudo mais detalhado visando uma comparação entre as CTTSs e WTTSs. Os autores 
concluíram que as WTTSs fora das nuvens $\left(t \sim 7.8 \mathrm{Myr}, M=1.04 M_{\odot}\right)$ são mais velhas e têm massa maior que as WTTSs dentro das nuvens $\left(t \sim 2.5 \mathrm{Myr}, M=0.69 M_{\odot}\right)$ e CTTSs $\left(t \sim 1.4 \mathrm{Myr}, M=0.30 M_{\odot}\right)$ sendo as distribuições de massa e idade de cada subgrupo significativamente diferentes. Recentemente, Mortier et al. (2011) investigaram as propriedades físicas de uma amostra com 54 YSOs dominada por estrelas com tipo espectral tardio oriundas do levantamento Spitzer c2d. Eles calcularam a idade média de 3.6 Myr e 4.4 Myr utilizando, respectivamente, as isócronas de Baraffe et al. (1998) e Siess et al. (2000) e verificaram que a distribuição de massa se estende entre $0.1 M_{\odot}$ e $1.0 M_{\odot}$ com o pico em $0.3 M_{\odot}$ para os dois conjuntos de isócronas utilizadas.

A formação estelar na região de Ophiuchus também foi alvo de muitos estudos nos últimos anos. A maior proximidade e a acessibilidade aos observadores dos hemisférios norte e sul contribuíram para a maior quantidade de pesquisa desenvolvida nessa região. Greene \& Meyer (1995) investigaram as propriedades físicas de 19 YSOs em L1688 a partir de um levantamento espectroscópico no infravermelho e encontraram uma idade mediana de 0.3 Myr para as estrelas mais embebidas na nuvem. Além disso, os autores verificaram que a distribuição de massa dessas estrelas se estende entre $0.1 M_{\odot}$ e $2.5 M_{\odot}$ com valor mediano em $0.4-0.5 M_{\odot}$. Luhman \& Rieke (1999) confirmaram a existência dessa população mais jovem de estrelas com idades entre 0.1 Myr e 1 Myr partindo de uma amostra maior com 100 estrelas. Outros levantamentos que incluem também as estrelas mais afastadas do centro de L1688 revelam a existência de uma população um pouco mais velha. Bouvier \& Appenzeller (1992) relatam a descoberta de 30 estrelas PMS com idades entre 1-10 Myr e massas no intervalo de $0.3 M_{\odot}$ a $2.5 M_{\odot}$. Posteriormente, Martin et al. (1998) verificaram a existência de populações de estrelas jovens com idades diferentes na região: YSOs mais jovens que 1 Myr embebidos na região central de L1688, TTSs com idades de 1-10 Myr e outras estrelas mais evoluídas (10-30 Myr) e dispersas. Partindo de uma amostra com 139 estrelas na vizinhança de L1688, Wilking et al. (2005) calcularam a idade mediana de 2.1 Myr para essas estrelas. Eles discutem que a formação dessas estrelas é contemporânea àquelas de baixa massa no subgrupo US da Sco-Cen e teria como origem muito provavelmente o mesmo evento. 
Embora estimativas de massa e idade para as estrelas PMS em Lupus e Ophiuchus já existam na literatura, é importante mencionar que o cálculo desses parâmetros resulta da posição dessas estrelas no diagrama-HR. Para construir um diagrama-HR é necessário conhecer a luminosidade e a temperatura efetiva (ou tipo espectral) de cada estrela. A determinação da luminosidade de uma estrela depende do conhecimento da distância em que ela se encontra. Os trabalhos mencionados anteriormente utilizaram a distância média de cada região de formação estelar para obter os parâmetros individuais (massa e idade) de cada estrela. Conforme discutido nos capítulos 4 e 5, a distância média de um grupo de estrelas deixa de ser um parâmetro confiável quando o grupo em questão ocupa um grande volume no espaço e se estende por dezenas de parsec em profundidade. Esse é o caso dos MGs nas regiões de Lupus e Ophiuchus. Neste capítulo visamos refinar o diagramaHR dessas regiões de formação estelar e determinar as propriedades físicas (luminosidade, massa e idade) das estrelas PMS que pertencem ao MG de cada região. Esses resultados são comparados àqueles obtidos por Bertout et al. (2007) na região de Taurus-Auriga. 


\subsection{Luminosidade}

A luminosidade é o primeiro parâmetro físico que decorre do cálculo da distância e pode ser obtida conhecendo-se o fluxo da estrela. Realizamos um levantamento dos dados fotométricos $U B V R_{C} I_{C}$ existentes na literatura para as estrelas PMS que pertencem aos MGs de Lupus e Ophiuchus. No catálogo 2MASS (Cutri et al. 2003) obtemos JHK para todas estrelas. Encontramos o valor da extinção visual $A_{V}$ para a maior parte das estrelas e completamos esses dados para as demais. Calculamos a extinção visual para as CTTSs seguindo a proposta de Cieza et al. (2005) que consiste em utilizar o índice de cor $\left(R_{C}-I_{C}\right)$. A extinção visual é dada por:

$$
A_{V}=4.76 E\left(R_{C}-I_{C}\right)
$$

onde $E\left(R_{C}-I_{C}\right)=\left(R_{C}-I_{C}\right)-\left(R_{C}-I_{C}\right)_{0}$ é o excesso de cor e $\left(R_{C}-I_{C}\right)_{0}$ é a cor intrínseca da estrela. Para as WTTSs, onde o excesso no infravermelho é menor, utilizamos o procedimento descrito por Kenyon \& Hartmann (1995) que usa $\left(V-R_{C}\right),\left(V-I_{C}\right)$ ou $(J-K)$. Os autores confirmaram que os resultados obtidos com cada cor são equivalentes no caso das WTTSs. Nesse caso usamos a lei de extinção usual

$$
A_{V}=R E(B-V) \text {, }
$$

onde $R=3.1$ (Savage \& Mathis 1979). Utilizamos os índices de cor intrínsecos para cada tipo espectral que são dados por Kenyon \& Hartmann (1995) e as relações entre os excessos de cor que são dadas por Bessell \& Brett (1988).

Calculamos a luminosidade das CTTSs utilizando o fluxo em $I_{C}$, pois o excesso de emissão no infravermelho é menor nesse filtro. Conforme discutido por Cieza et al. (2005), o procedimento padrão que consiste em utilizar o fluxo em $J$ leva a resultados superestimados de luminosidade e consequentemente subestima a idade das CTTSs quando colocadas no diagrama-HR. No caso das WTTSs utilizamos os fluxos em $I_{C}$ ou $V$. Verificamos que os dois procedimentos retornam resultados equivalentes com um desvio médio de $6 \%$ nos valores obtidos. Para algumas poucas WTTSs da amostra onde o fluxo em $V$ e $I_{C}$ não é conhecido utilizamos $J$ (2MASS). 
Para cada estrela calculamos a magnitude absoluta bolométrica que é dada por

$$
M_{b o l}=M_{V}+B C(V)=-5 \log d+5+\left(V-A_{V}\right)+B C(V),
$$

onde $d$ é a distância de cada estrela em parsec e $B C(V)$ é a correção bolométrica. As distâncias são obtidas das paralaxes aqui calculadas (vide Tabelas 4.2, 4.3, 5.1 e 5.2). Finalmente, calculamos a luminosidade de cada estrela por

$$
\frac{L}{L_{\odot}}=10^{-0.4\left(M_{b o l}-M_{b o l}^{\odot}\right)}
$$

onde $L_{\odot}=3.83 \times 10^{33} \mathrm{erg} / \mathrm{s}$ e $M_{\text {bol }}^{\odot}=4.75$ são os valores da luminosidade e magnitude bolométrica solar (Cox 2000). Adotamos as correções bolométricas para cada tipo espectral que são dadas por Kenyon \& Hartmann (1995). Os resultados de luminosidade são apresentados na Tabela 6.1 com os demais parâmetros estelares que serão discutidos mais adiante. O cálculo da incerteza na luminosidade é dominado pelo erro na distância de tal modo que as demais fontes de erro podem ser desprezadas em primeira aproximação. Estimamos as luminosidades mínima e máxima para cada estrela variando o valor da distância dentro da sua incerteza.

\subsection{Diagrama-HR}

O diagrama-HR é um instrumento importante no estudo da evolução estelar e será utilizado neste trabalho para investigar as estrelas PMS em Lupus e Ophiuchus. Partindo das luminosidades calculadas e a temperatura efetiva (ou tipo espectral) de cada estrela que encontramos na literatura construímos o diagrama-HR de cada região de formação estelar. Assumimos um erro de $\pm 100 \mathrm{~K}$ na temperatura efetiva para construção do diagrama-HR. Eliminamos dessa análise as estrelas com $L<0.15 L_{\odot}$ e tipo espectral mais tardio que M4 conforme descrito por Bertout et al. (2007). Esses valores são obtidos dos limites estimados das distribuições de magnitude $(V \simeq 15 \mathrm{mag})$ e temperatura efetiva $\left(T_{\text {eff }} \simeq 3300 \mathrm{~K}\right)$. Esse corte nos permite melhor comparar os resultados de Lupus e Ophiuchus com aqueles obtidos em Taurus-Auriga por Bertout et al. (2007). As estrelas cujo erro relativo na paralaxe é maior que $50 \%$ foram excluídas do diagrama-HR, pois as luminosidades obtidas não são significativas. As CTTSs onde somente o fluxo em J (2MASS) é conhecido não foram consideradas na análise pelas razões discutidas anteriormente. Tendo em vista esses 
cortes e a disponibilidade de dados fotométricos para essas estrelas, a análise que segue fica restrita a 99 estrelas em Lupus (24 CTTSs, 71 WTTSs e 4 HAeBes) e 38 estrelas em Ophiuchus (15 CTTSs, 20 WTTSs e 3 HAeBes).

Apresentamos nos diagramas-HR as trajetórias evolutivas e isócronas de estrelas PMS de Siess et al. (2000) obtidas com metalicidade solar $Z=0.02$. As trajetórias evolutivas cobrem um intervalo de massas entre $0.1 M_{\odot}$ e $7.0 M_{\odot}$ até a chegada da estrela na ZAMS. Devido à pequena quantidade de HAeBes na amostra, optamos por apresentar os diagramas-HR somente na região de baixas massas (até $2 M_{\odot}$ ) o que nos permite melhor visualizar a distribuição das TTSs (população dominante).

Na Figura 6.1(a) apresentamos o diagrama-HR das TTSs em Lupus. Como podemos notar, as WTTSs existem em maior número e exibem duas distribuições: uma população mais jovem que se mistura com as CTTSs e outra mais evoluída que se aproxima da ZAMS. Isso pode ser explicado lembrando-se que a região de Lupus é formada por uma população de estrelas próxima às nuvens moleculares e outra mais dispersa. Na Figura 6.1(b) reproduzimos o diagrama-HR destacando as estrelas de cada população (dentro e fora das nuvens). Concluímos que as WTTSs mais evoluídas se encontram na região fora das nuvens do complexo de Lupus enquanto a população mais jovem está concentrada nas nuvens.

O diagrama-HR das TTSs em Ophiuchus é mostrado na Figura 6.2(a). Verificamos que as CTTS se distribuem em torno da isócrona de 3 Myr. A maior parte das WTTSs se distribui em idades maiores embora algumas delas, mais jovens e com massa mais baixa, se misturem com as CTTSs (como observado também no diagrama-HR de Lupus). As estrelas que pertencem à principal nuvem da região de Ophiuchus, L1688, são colocadas em destaque na Figura 6.2(b). Como podemos notar, as TTSs em L1688 são em geral mais jovens que as estrelas nas demais nuvens (ou dispersas).

Comparando o diagrama-HR das duas regiões concluímos que a dispersão de idade é maior em Lupus. Essa região apresenta tanto estrelas mais jovens quanto outras mais evoluídas que já se aproximam da ZAMS. Um outro aspecto que chama atenção é que as CTTSs em Lupus apresentam tipo espectral mais tardio, enquanto em Ophiuchus elas cobrem um intervalo maior de temperatura efetiva. Voltamos a esse ponto na seção 6.6 com os resultados das simulações no diagrama-HR de cada região. 

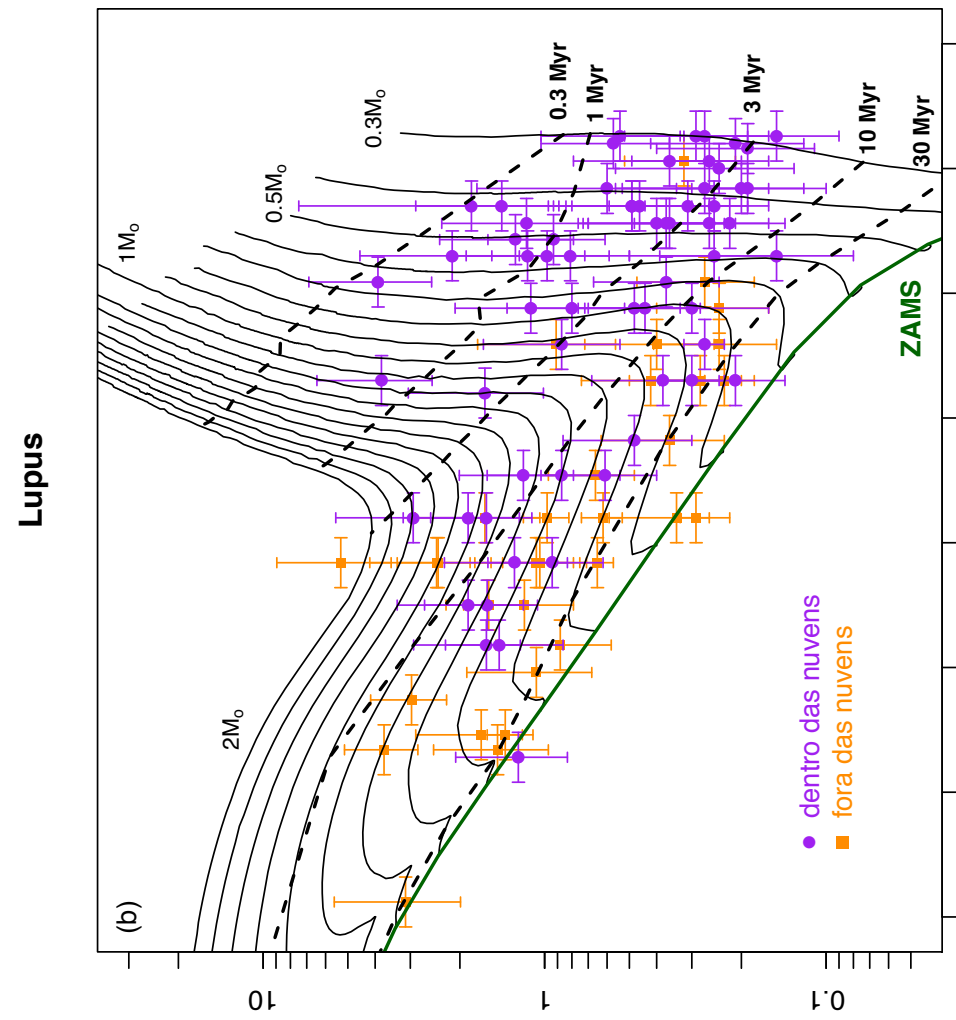

(ㄱำ

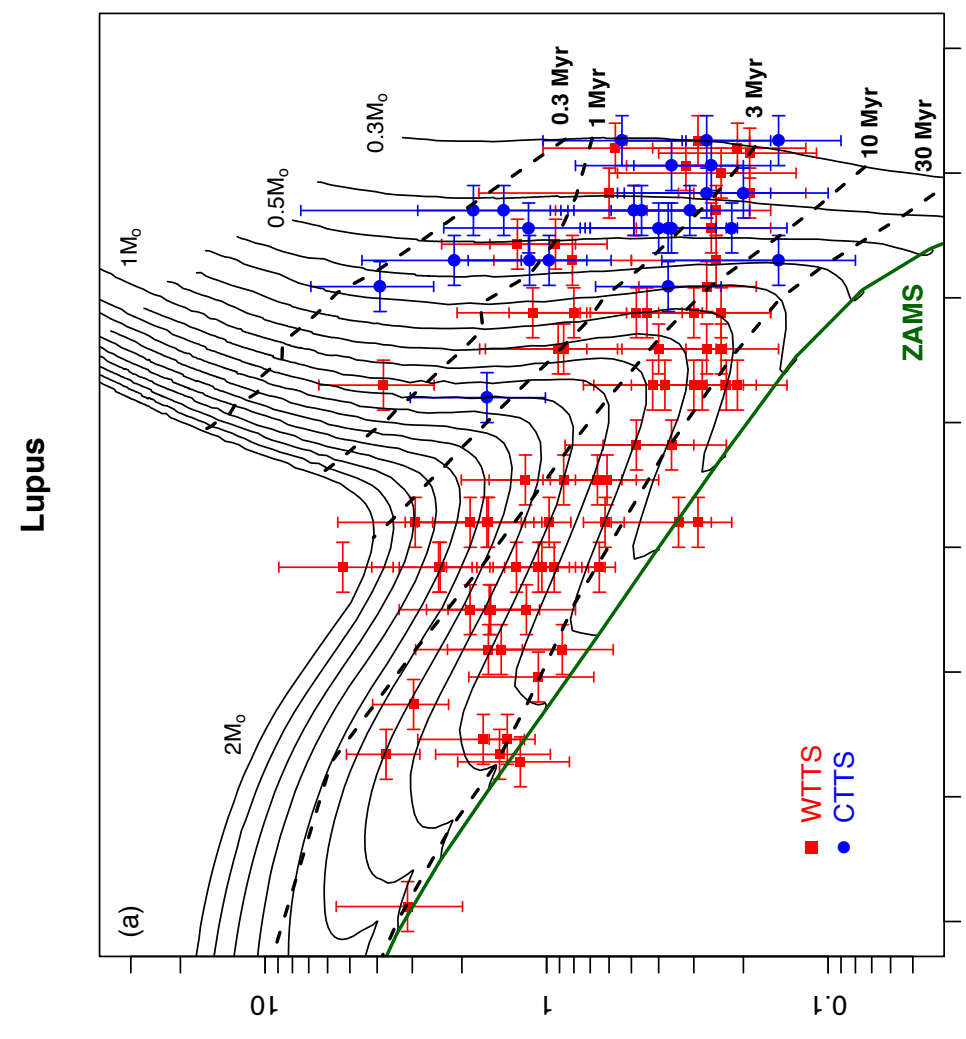

구

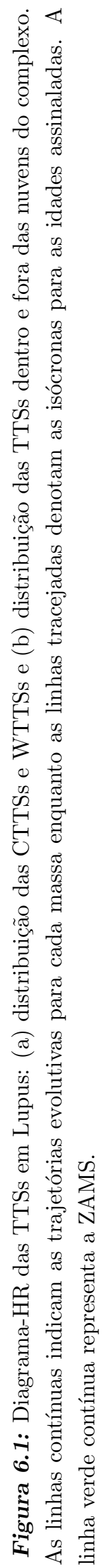




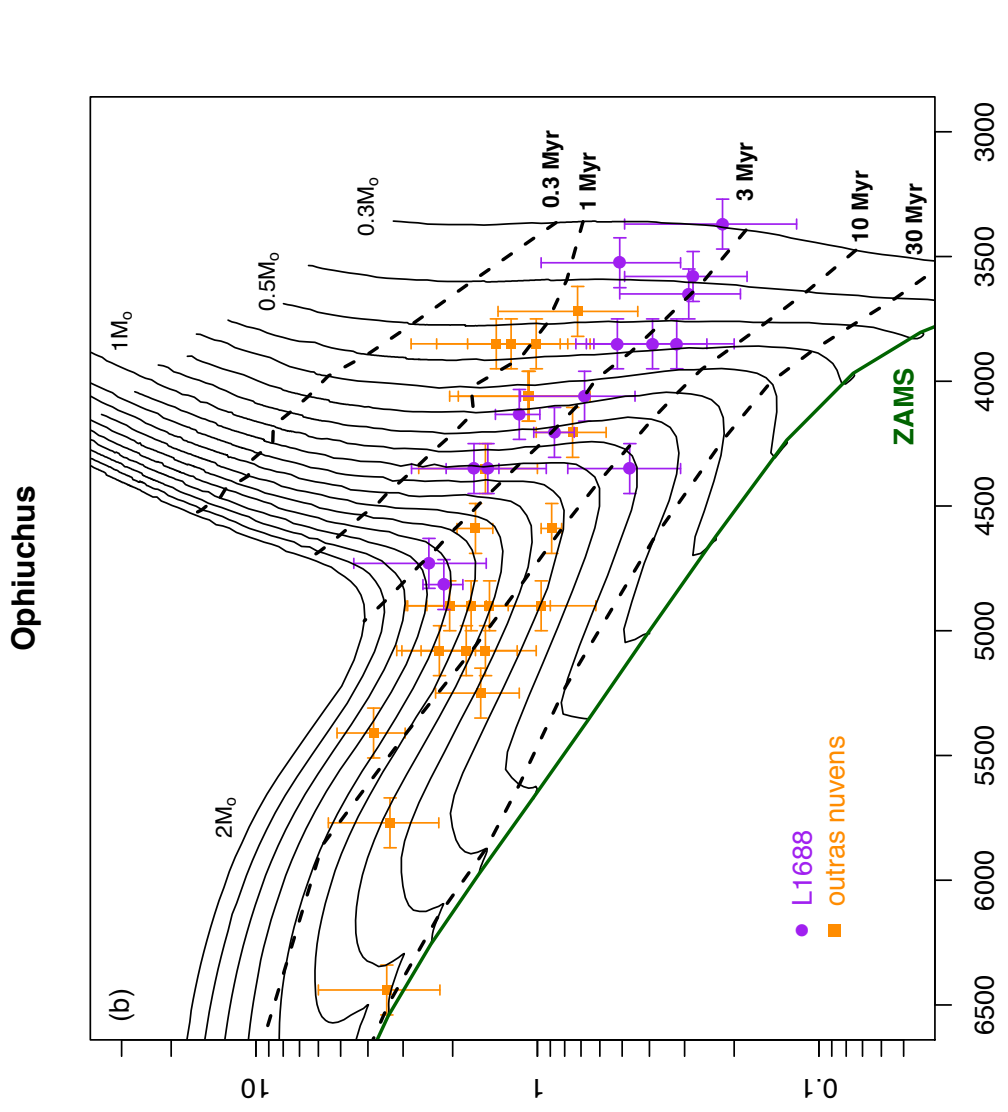

ซึ

(ำ 7

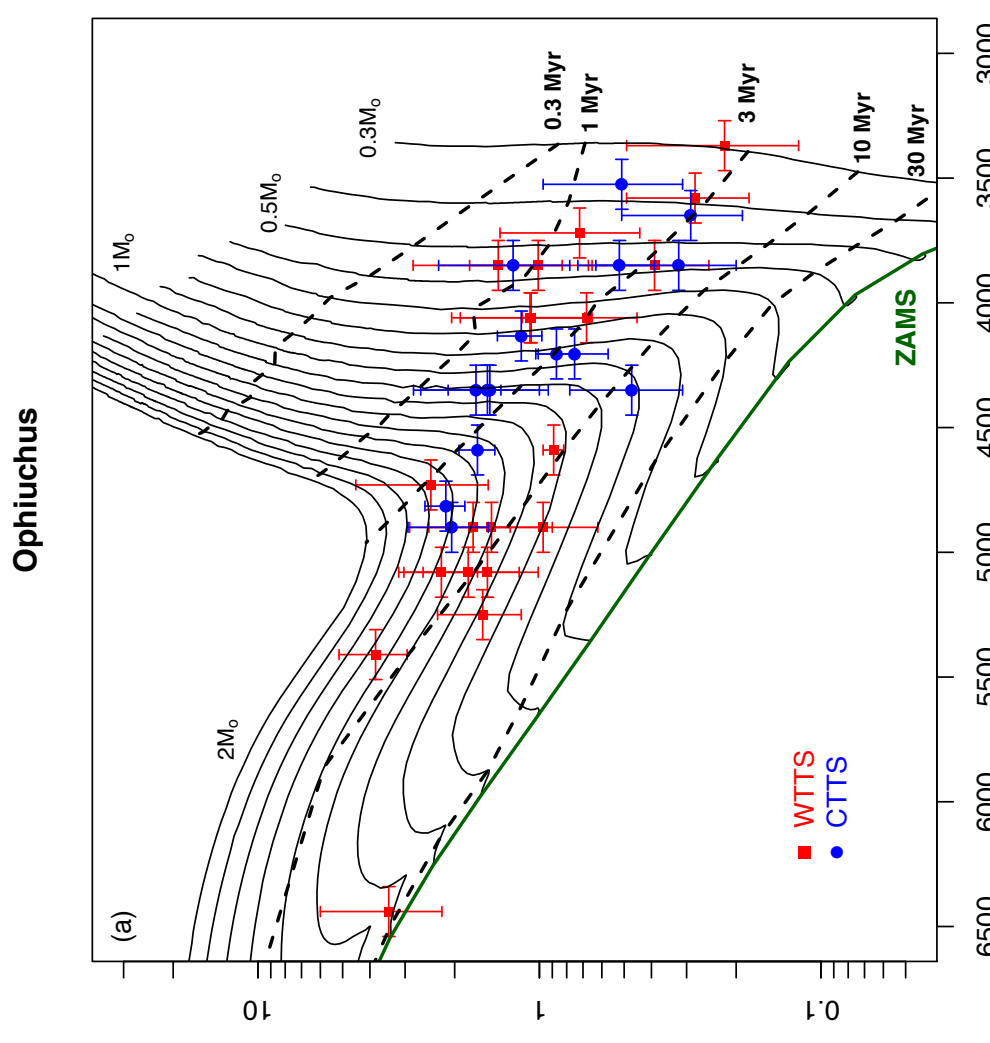

(ำ 7

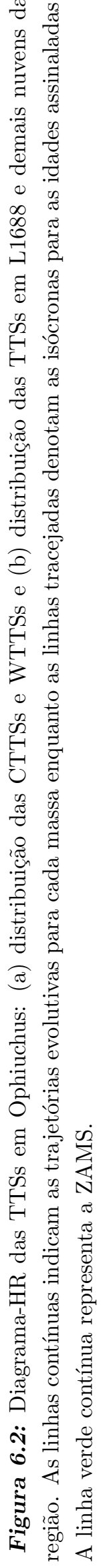




\subsection{Massa e Idade}

A massa e a idade das estrelas PMS aqui tratadas são estimadas a partir da posição de cada estrela no diagrama-HR usando a grade de trajetórias evolutivas e isócronas de Siess et al. (2000). Utilizamos o servidor de Siess et al. (2000) que recebe como parâmetros de entrada a luminosidade e a temperatura efetiva, e retorna a massa, a idade e o raio da estrela. Verificamos que o raio $R$ da estrela obtido desse modo concorda em média dentro de $5 \%$ com o resultado obtido pela relação

$$
L=4 \pi R^{2} \sigma T_{e f}^{4}
$$

onde $\sigma=5.67 \cdot 10^{-5} \mathrm{erg} \mathrm{cm}^{-2} \mathrm{~K}^{-4} \mathrm{~s}^{-1}$ é a constante de Stefan-Boltzmann (Cox 2000). A incerteza na massa, idade e raio é obtida variando-se a luminosidade da estrela dentro dos limites extremos definidos pelo erro dessa grandeza. Apresentamos os resultados obtidos para cada estrela na Tabela 6.1.

A distribuição de massa e idade das estrelas PMS em cada região de formação estelar é apresentada na Figura 6.3 (Lupus) e Figura 6.4 (Ophiuchus). Um ponto importante é a existência de duas populações claramente distintas em Lupus como podemos notar tanto na distribuição de massa quanto de idade. A origem dos dois picos na Figura 6.3 é atribuída às duas populações de TTSs nessa região (dentro e fora das nuvens). Voltando ao diagramaHR de Lupus (vide Figura 6.1) verificamos que as estrelas fora das nuvens são em média mais velhas e têm massa maior que as estrelas dentro das nuvens o que explica a distribuição de massa e idade observada. Por outro lado, observamos que essas distribuições são mais alargadas na região de Ophiuchus. Como podemos notar pela Figura 6.2 as estrelas em L1688 têm aproximadamente a mesma idade, no entanto elas cobrem um grande intervalo de massas $\left(0.3 M_{\odot}\right.$ até $\left.1.7 M_{\odot}\right)$. A separação do histograma de idades em dois picos (como observado em Lupus) é menos evidente, pois a diferença de idade entre as estrelas em L1688 e nas demais nuvens é menor que aquela observada na região de Lupus entre as estrelas dentro e fora das nuvens. Uma primeira análise da idade das estrelas em cada região de formação estelar nos permite concluir que as estrelas em Ophiuchus (4 Myr) são em média mais jovens que as estrelas em Lupus (6 Myr). 

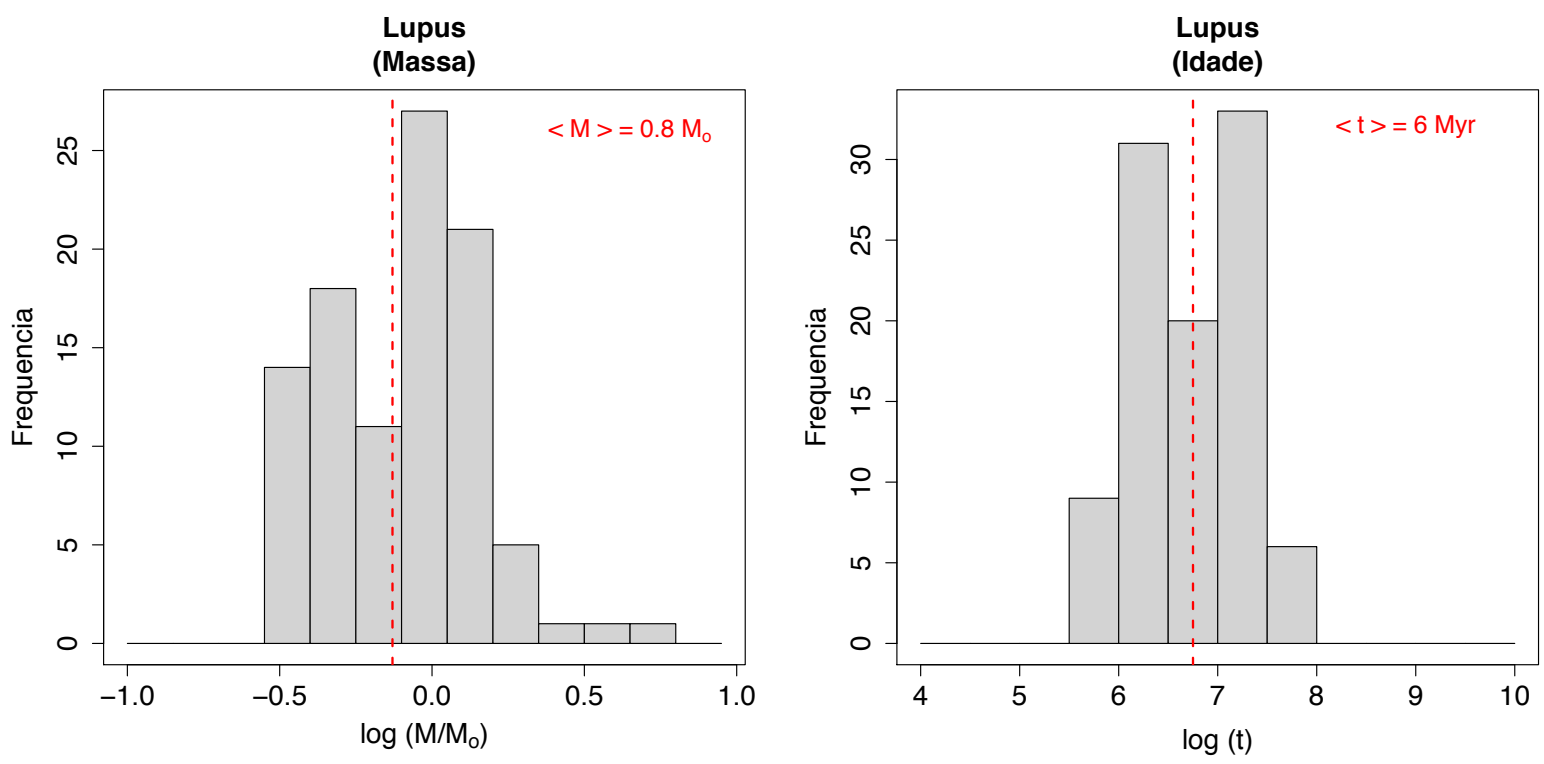

Figura 6.3: Distribuição de massa (esquerda) e idade (direita) das estrelas PMS em Lupus. A idade $t$ é dada em $\operatorname{Myr}\left(1 \mathrm{Myr}=10^{6}\right.$ anos). A linha vermelha tracejada representa a média de cada distribuição que está assinalada na figura.
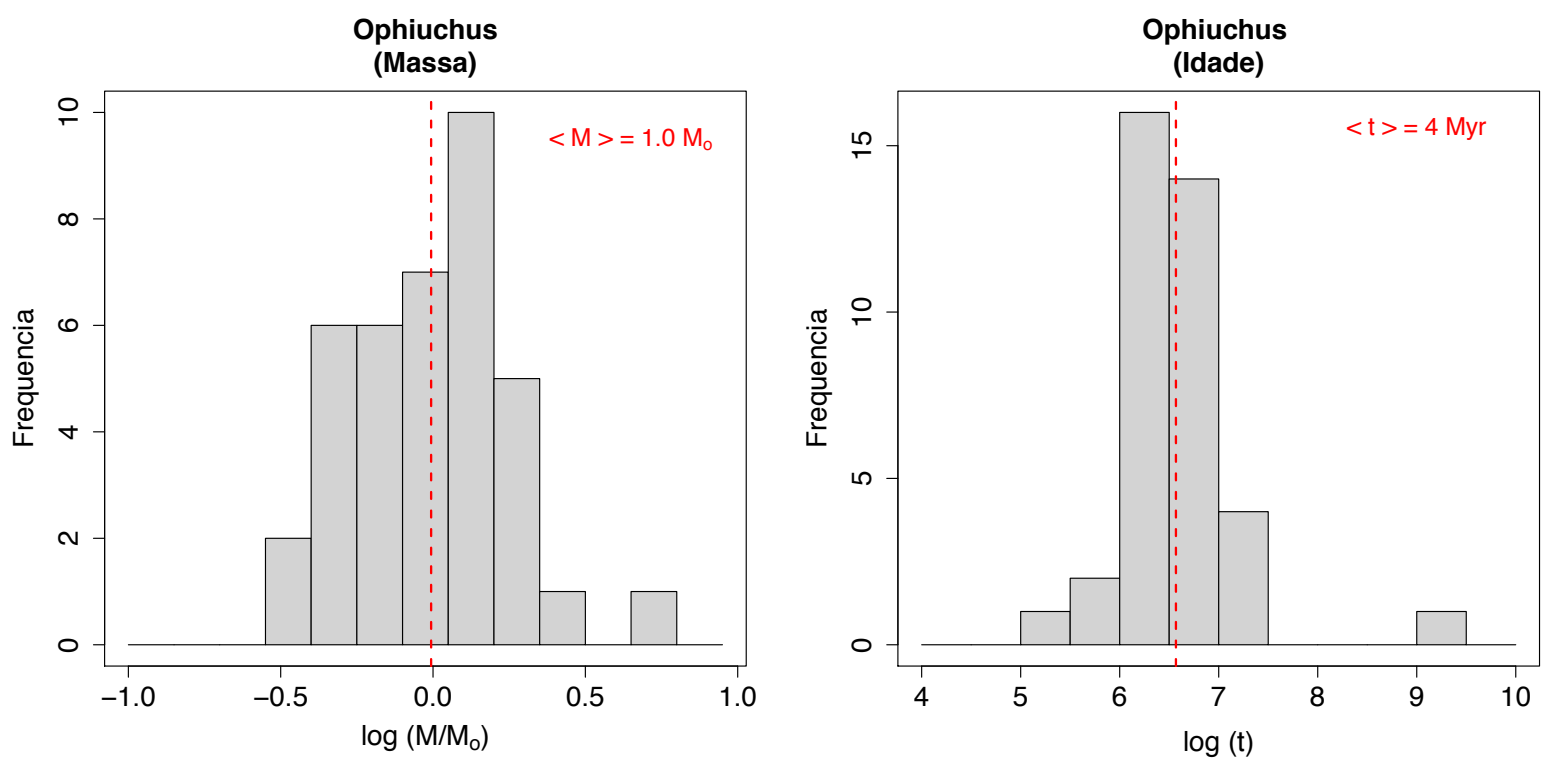

Figura 6.4: Distribuição de massa (esquerda) e idade (direita) das estrelas PMS em Ophiuchus. A idade $t$ é dada em $\operatorname{Myr}\left(1 \mathrm{Myr}=10^{6}\right.$ anos $)$. A linha vermelha tracejada representa a média de cada distribuição que está assinalada na figura. 
Tabela 6.1 - Propriedades físicas das estrelas PMS em Lupus e Ophiuchus. Para cada estrela fornecemos o identificador, tipo espectral, temperatura efetiva, extinção visual, luminosidade, massa, idade, raio, classificação TTS e as referências dos dados fotométricos (no final da tabela). Os valores assinalados com “:” são apenas indicativos.

\begin{tabular}{|c|c|c|c|c|c|c|c|c|c|}
\hline ID & $\mathrm{ST}$ & $\begin{array}{l}T_{e f} \\
(\mathrm{~K}) \\
\end{array}$ & $\begin{array}{c}A_{V} \\
(\mathrm{mag})\end{array}$ & $\begin{array}{c}L \pm \sigma_{L} \\
\left(L_{\odot}\right) \\
\end{array}$ & $\begin{array}{c}M \pm \sigma_{M} \\
\left(M_{\odot}\right) \\
\end{array}$ & $\begin{array}{c}\log t \pm \sigma_{\log t} \\
(\mathrm{t} \text { em Myr) }\end{array}$ & $\begin{array}{c}R \pm \sigma_{R} \\
\left(R_{\odot}\right) \\
\end{array}$ & Tipo & Ref. \\
\hline \multicolumn{10}{|c|}{ Lupus } \\
\hline RXJ1448.2-4103 & $\mathrm{K} 2$ & 4900 & 0.55 & $0.62_{-0.09}^{+0.12}$ & $1.00 \pm 0.06$ & $7.40 \pm 0.09$ & $1.03 \pm 0.09$ & WTTS & 1,2 \\
\hline RXJ1500.6-4309 & K3 & 4730 & 0.78 & $0.66_{-0.18}^{+0.31}$ & $1.09 \pm 0.13$ & $7.24 \pm 0.18$ & $1.17 \pm 0.18$ & WTTS & 3 \\
\hline RXJ1500.8-4331 & K0 & 5250 & 0.33 & $1.58_{-0.40}^{+0.65}$ & $1.28 \pm 0.16$ & $7.17 \pm 0.15$ & $1.43 \pm 0.23$ & WTTS & 3 \\
\hline RXJ1501.2-4121 & G3 & 5830 & 0.28 & $3.72_{-0.90}^{+1.41}$ & $1.39 \pm 0.15$ & $7.15 \pm 0.11$ & $1.80 \pm 0.28$ & WTTS & 3 \\
\hline RXJ1504.8-3950 & G5 & 5770 & 0.22 & $1.38_{-0.18}^{+0.22}$ & $1.10 \pm 0.01$ & $7.47 \pm 0.24$ & $1.14 \pm 0.06$ & WTTS & 4,5 \\
\hline RXJ1507.4-4601 & K1 & 5080 & 0.28 & $0.65_{-0.08}^{+0.10}$ & $0.95 \pm 0.05$ & $7.49 \pm 0.07$ & $0.99 \pm 0.07$ & WTTS & 2 \\
\hline RXJ1508.8-3715 & K5 & 4350 & 0.28 & $0.23_{-0.03}^{+0.04}$ & $0.80 \pm 0.04$ & $7.59 \pm 0.09$ & $0.79 \pm 0.06$ & WTTS & 6 \\
\hline HD134974 & G7 & 5630 & 0.66 & $2.97_{-0.74}^{+1.18}$ & $1.38 \pm 0.14$ & $7.13 \pm 0.12$ & $1.74 \pm 0.26$ & WTTS & 3 \\
\hline RXJ1518.0-4445 & $\mathrm{K} 2$ & 4900 & 0.00 & $0.29_{-0.03}^{+0.03}$ & $0.85:$ & $7.58:$ & $0.78:$ & WTTS & 2 \\
\hline RXJ1519.6-4760 & G9 & 5410 & 0.00 & $0.88_{-0.30}^{+0.61}$ & $1.02 \pm 0.17$ & $7.52 \pm 0.22$ & $1.01 \pm 0.33$ & WTTS & 3 \\
\hline RXJ1524.5-3652 & $\mathrm{K} 1$ & 5080 & 0.20 & $1.04_{-0.24}^{+0.37}$ & $1.16 \pm 0.13$ & $7.26 \pm 0.15$ & $1.25 \pm 0.18$ & WTTS & 7 \\
\hline RXJ1525.0-3604 & K1 & 5080 & 0.39 & $2.42_{-0.59}^{+0.92}$ & $1.61 \pm 0.18$ & $6.83 \pm 0.14$ & $1.90 \pm 0.30$ & WTTS & 7 \\
\hline RXJ1525.5-3613 & $\mathrm{K} 2$ & 4900 & 0.26 & $0.98_{-0.16}^{+0.21}$ & $1.20 \pm 0.07$ & $7.15 \pm 0.10$ & $1.30 \pm 0.09$ & WTTS & 4,7 \\
\hline RXJ1531.3-3329 & G9 & 5410 & 0.50 & $1.61_{-0.40}^{+0.63}$ & $1.21 \pm 0.13$ & $7.26 \pm 0.13$ & $1.36 \pm 0.22$ & WTTS & 3 \\
\hline RXJ1534.6-4003K & K6 & 4205 & 0.32 & $0.24_{-0.08}^{+0.17}$ & $0.79 \pm 0.11$ & $7.21 \pm 0.32$ & $0.97 \pm 0.34$ & WTTS & 7 \\
\hline RXJ1540.7-3756 & K6 & 4205 & 0.25 & $0.40_{-0.15}^{+0.32}$ & $0.82 \pm 0.10$ & $6.91 \pm 0.24$ & $1.57 \pm 0.29$ & WTTS & 7 \\
\hline RXJ1544.5-3521 & K5 & 4350 & 0.61 & $0.30_{-0.06}^{+0.09}$ & $0.88 \pm 0.10$ & $7.38 \pm 0.09$ & $1.11 \pm 0.04$ & WTTS & 6 \\
\hline Sz73 & M0 & 3850 & 2.75 & $0.15_{-0.07}^{+0.24}$ & $0.59 \pm 0.02$ & $7.18 \pm 0.53$ & $0.81 \pm 0.37$ & CTTS & 8 \\
\hline GQLup & K7-M0 & 3955 & 0.95 & $3.91_{-1.39}^{+2.97}$ & $0.66 \pm 0.04$ & $5.67 \pm 0.23$ & $3.78 \pm 0.49$ & CTTS & 8 \\
\hline RXJ1549.9-3629 & $\mathrm{K} 2$ & 4900 & 0.32 & $1.87_{-0.64}^{+1.31}$ & $1.50 \pm 0.26$ & $6.89 \pm 0.28$ & $1.73 \pm 0.52$ & WTTS & 7 \\
\hline RXJ1552.3-3819 & K7 & 4060 & 0.47 & $0.48_{-0.15}^{+0.28}$ & $0.80 \pm 0.02$ & $6.72 \pm 0.29$ & $1.23 \pm 0.16$ & WTTS & 7 \\
\hline RXJ1605.7-3905 & G9 & 5410 & 0.15 & $1.45_{-0.58}^{+1.47}$ & $1.18 \pm 0.24$ & $7.31 \pm 0.26$ & $1.29 \pm 0.43$ & WTTS & 7 \\
\hline F304 & K6 & 4205 & 0.35 & $0.27_{-0.04}^{+0.05}$ & $0.90 \pm 0.08$ & $6.30 \pm 0.02$ & $1.12 \pm 0.06$ & WTTS & 7 \\
\hline RXJ1608.5-3847 & M2 & 3580 & 0.74 & $0.60_{-0.30}^{+1.14}$ & $0.41 \pm 0.01$ & $6.09 \pm 0.29$ & $1.92 \pm 0.52$ & WTTS & 7 \\
\hline RXJ1610.0-4016 & $\mathrm{K} 2$ & 4900 & 0.27 & $1.61_{-0.50}^{+0.93}$ & $1.45 \pm 0.21$ & $6.88 \pm 0.18$ & $1.70 \pm 0.31$ & WTTS & 7 \\
\hline RXJ1613.0-4004 & $\mathrm{K} 7$ & 4060 & 1.68 & $0.30_{-0.14}^{+0.43}$ & $0.81 \pm 0.06$ & $7.06 \pm 0.40$ & $1.22 \pm 0.39$ & WTTS & 7 \\
\hline RXJ1450.4-3507 & $\mathrm{K} 1$ & 5080 & 0.44 & $2.39_{-0.85}^{+1.79}$ & $1.60 \pm 0.31$ & $6.84 \pm 0.24$ & $1.89 \pm 0.48$ & WTTS & 3 \\
\hline RXJ1450.5-3459 & $\mathrm{K} 4$ & 4590 & 0.44 & $0.36_{-0.13}^{+0.27}$ & $0.90 \pm 0.14$ & $7.48 \pm 0.27$ & $0.91 \pm 0.20$ & WTTS & 2 \\
\hline RXJ1454.2-3955 & K2 & 4900 & 0.61 & $0.34_{-0.12}^{+0.25}$ & $0.81 \pm 0.18$ & $7.66 \pm 0.23$ & $0.80 \pm 0.21$ & WTTS & 2 \\
\hline RXJ1505.9-4311 & $\mathrm{K} 7$ & 4060 & 0.33 & $0.24_{-0.08}^{+0.16}$ & $0.80 \pm 0.08$ & $7.22 \pm 0.19$ & $0.98 \pm 0.22$ & WTTS & 7 \\
\hline RXJ1511.0-3252AB & K6 & 4205 & 0.39 & $0.91_{-0.35}^{+0.82}$ & $0.93 \pm 0.03$ & $6.46 \pm 0.37$ & $1.69 \pm 0.68$ & WTTS & 7 \\
\hline RXJ1511.6-3550 & K5 & 4350 & 0.39 & $0.42_{-0.15}^{+0.32}$ & $1.00 \pm 0.09$ & $7.17 \pm 0.33$ & $1.15 \pm 0.23$ & WTTS & 7 \\
\hline RXJ1512.6-3417 & K5 & 4350 & 0.61 & $0.28_{-0.10}^{+0.22}$ & $0.85 \pm 0.24$ & $7.47 \pm 0.43$ & $0.95 \pm 0.45$ & WTTS & 2 \\
\hline RXJ1512.8-4508A & G8 & 5520 & 0.00 & $1.07_{-0.38}^{+0.82}$ & $1.01 \pm 0.22$ & $7.47 \pm 0.40$ & $1.06 \pm 0.38$ & WTTS & 3 \\
\hline HD135127 & F5 & 6440 & 0.17 & $3.12_{-1.13}^{+2.47}$ & $1.37 \pm 0.13$ & $7.51 \pm 0.37$ & $1.44 \pm 0.38$ & WTTS & 7 \\
\hline RXJ1515.7-3332K & K0 & 5250 & 0.30 & $1.18_{-0.39}^{+0.77}$ & $1.15 \pm 0.22$ & $7.31 \pm 0.21$ & $1.24 \pm 0.28$ & WTTS & 7 \\
\hline RXJ1518.4-3738 & $\mathrm{K} 1$ & 5080 & 0.20 & $1.07_{-0.35}^{+0.71}$ & $1.17 \pm 0.22$ & $7.24 \pm 0.23$ & $1.27 \pm 0.31$ & WTTS & 7 \\
\hline RXJ1527.3-3603 & K7-M0 & 3955 & 0.66 & $0.27_{-0.09}^{+0.19}$ & $0.70 \pm 0.01$ & $6.98 \pm 0.33$ & $0.97 \pm 0.25$ & WTTS & 6 \\
\hline
\end{tabular}


Tabela 6.1 - continuação.

\begin{tabular}{|c|c|c|c|c|c|c|c|c|c|}
\hline ID & ST & $\begin{array}{l}T_{e f} \\
(\mathrm{~K})\end{array}$ & $\begin{array}{c}A_{V} \\
(\mathrm{mag})\end{array}$ & $\begin{array}{c}L \pm \sigma_{L} \\
\left(L_{\odot}\right)\end{array}$ & $\begin{array}{c}M \pm \sigma_{M} \\
\left(M_{\odot}\right)\end{array}$ & $\begin{array}{r}\log t \pm \sigma_{\log t} \\
(\mathrm{t} \text { em Myr) }\end{array}$ & $\begin{array}{c}R \pm \sigma_{R} \\
\left(R_{\odot}\right)\end{array}$ & Tipo & Ref. \\
\hline RXJ1529.3-3737 & M3 & 3470 & 0.00 & $0.32_{-0.10}^{+0.20}$ & $0.35 \pm 0.03$ & $6.28 \pm 0.16$ & $1.50 \pm 0.24$ & WTTS & 7 \\
\hline RXJ1529.7-3628 & K2 & 4900 & 0.44 & $1.63_{-0.58}^{+1.25}$ & $1.45 \pm 0.28$ & $6.88 \pm 0.27$ & $1.68 \pm 0.47$ & WTTS & 3 \\
\hline RXJ1538.7-4411 & G5 & 5770 & 0.62 & $1.68_{-0.58}^{+1.19}$ & $1.10 \pm 0.10$ & $7.38 \pm 0.27$ & $1.19 \pm 0.29$ & WTTS & 7 \\
\hline RXJ1539.7-3450 & $\mathrm{K} 4$ & 4590 & 0.50 & $0.48_{-0.17}^{+0.39}$ & $1.00 \pm 0.17$ & $7.32 \pm 0.25$ & $1.05 \pm 0.21$ & WTTS & 6 \\
\hline RXJ1540.3-3426A & M3.5 & 3420 & 0.20 & $0.19_{-0.08}^{+0.21}$ & $0.31 \pm 0.02$ & $6.48 \pm 0.28$ & $1.19 \pm 0.42$ & WTTS & 6,10 \\
\hline PDS395 & A8 & 7580 & 4.43 & $73_{-26}^{+54}$ & $3.03 \pm 0.57$ & $6.24 \pm 0.23$ & $4.73 \pm 1.25$ & HAeBe & 4 \\
\hline SSTc2dJ154148.3-350145 & M3 & 3500 & 0.30 & $0.24_{-0.11}^{+0.32}$ & $0.36 \pm 0.03$ & $6.43 \pm 0.31$ & $1.36 \pm 0.45$ & WTTS & 10 \\
\hline RXJ1544.0-3311 & K0 & 5250 & 0.66 & $1.60_{-0.53}^{+1.07}$ & $1.29 \pm 0.24$ & $7.16 \pm 0.22$ & $1.44 \pm 0.35$ & WTTS & 3 \\
\hline RXJ1546.6-3618 & $\mathrm{K} 1$ & 5080 & 0.35 & $1.28_{-0.46}^{+0.98}$ & $1.25 \pm 0.26$ & $7.15 \pm 0.25$ & $1.39 \pm 0.37$ & WTTS & 7 \\
\hline RXJ1546.7-3459 & M0 & 3850 & 0.00 & $0.25_{-0.10}^{+0.25}$ & $0.59 \pm 0.01$ & $6.83 \pm 0.40$ & $0.83 \pm 0.31$ & WTTS & 6 \\
\hline RXJ1547.1-3540 & K5 & 4350 & 0.22 & $0.21_{-0.08}^{+0.16}$ & $0.79 \pm 0.16$ & $7.60 \pm 0.32$ & $0.82 \pm 0.15$ & WTTS & 6 \\
\hline RXJ1547.6-4018 & K1 & 5080 & 0.30 & $0.94_{-0.32}^{+0.65}$ & $1.11 \pm 0.21$ & $7.31 \pm 0.24$ & $1.19 \pm 0.29$ & WTTS & 7 \\
\hline HMLup & M3 & 3470 & 0.85 & $0.36_{-0.16}^{+0.44}$ & $0.35 \pm 0.01$ & $6.24 \pm 0.26$ & $1.54 \pm 0.59$ & CTTS & 8 \\
\hline HNLup & M1.5 & 3650 & 2.30 & $1.82_{-1.02}^{+5.65}$ & $0.52 \pm 0.04$ & $5.79 \pm 1.18$ & $2.93 \pm 2.10$ & CTTS & 8 \\
\hline Sz76 & M1 & 3720 & 1.90 & $0.37_{-0.14}^{+0.33}$ & $0.48 \pm 0.01$ & $6.41 \pm 0.30$ & $1.32 \pm 0.42$ & CTTS & 8 \\
\hline HD141277 & K0 & 5250 & 0.37 & $1.87_{-0.68}^{+1.47}$ & $1.36 \pm 0.28$ & $7.09 \pm 0.24$ & $1.56 \pm 0.45$ & WTTS & 7 \\
\hline RXJ1550.7-3828 & K7 & 4060 & 0.72 & $0.80_{-0.27}^{+0.56}$ & $0.76 \pm 0.03$ & $6.38 \pm 0.28$ & $1.76 \pm 0.46$ & WTTS & 6 \\
\hline Sz77 & M0 & 3850 & 0.79 & $1.15_{-0.43}^{+0.98}$ & $0.56 \pm 0.06$ & $5.96 \pm 0.15$ & $2.25 \pm 0.45$ & CTTS & 8 \\
\hline RXJ1555.4-3338 & K5 & 4350 & 0.44 & $0.38_{-0.14}^{+0.30}$ & $0.94 \pm 0.15$ & $7.28 \pm 0.36$ & $0.97 \pm 0.28$ & WTTS & 7 \\
\hline RXJ1556.0-3655 & M1 & 3720 & 0.07 & $0.40_{-0.15}^{+0.36}$ & $0.48 \pm 0.01$ & $6.37 \pm 0.29$ & $1.46 \pm 0.42$ & CTTS & 7 \\
\hline $\mathrm{Sz} 82$ & M0 & 3850 & 0.98 & $2.13_{-0.90}^{+2.40}$ & $0.68 \pm 0.02$ & $5.89 \pm 0.54$ & $2.70 \pm 1.48$ & CTTS & 8 \\
\hline Hip78092 & F6 & 6360 & 0.37 & $15_{-5}^{+10}$ & $1.95 \pm 0.33$ & $6.80 \pm 0.19$ & $2.98 \pm 0.72$ & HAeBe & 4 \\
\hline Sz126 & K7-M0 & 3955 & 0.55 & $0.37_{-0.13}^{+0.29}$ & $0.69 \pm 0.02$ & $6.76 \pm 0.35$ & $1.17 \pm 0.36$ & CTTS & 8 \\
\hline Sz128 & M1.5 & 3650 & 2.00 & $0.46_{-0.19}^{+0.52}$ & $0.43 \pm 0.01$ & $6.24 \pm 0.29$ & $1.56 \pm 0.59$ & CTTS & 8 \\
\hline RXJ1558.9-3646 & M1.5 & 3650 & 0.37 & $0.49_{-0.19}^{+0.44}$ & $0.43 \pm 0.01$ & $6.21 \pm 0.24$ & $1.65 \pm 0.47$ & WTTS & 7 \\
\hline CD-3610569 & K3 & 4730 & 0.69 & $0.61_{-0.21}^{+0.43}$ & $1.07 \pm 0.21$ & $7.29 \pm 0.27$ & $1.15 \pm 0.29$ & WTTS & 7 \\
\hline RXJ1559.9-3750 & M0.5 & 3785 & 0.42 & $0.93_{-0.32}^{+0.66}$ & $0.51 \pm 0.01$ & $6.01 \pm 0.20$ & $2.12 \pm 0.49$ & WTTS & 7 \\
\hline SSTc2dJ160000.6-422158 & M4 & 3400 & 0.80 & $0.21_{-0.08}^{+0.21}$ & $0.31 \pm 0.02$ & $6.42 \pm 0.26$ & $1.28 \pm 0.29$ & WTTS & 10,11 \\
\hline Sz131 & $\mathrm{M} 2$ & 3580 & 1.65 & $0.20_{-0.10}^{+0.36}$ & $0.39 \pm 0.02$ & $6.59 \pm 0.43$ & $1.08 \pm 0.50$ & CTTS & 8 \\
\hline RXJ1601.9-3613 & K3 & 4730 & 0.81 & $0.87_{-0.32}^{+0.73}$ & $1.20 \pm 0.24$ & $7.09 \pm 0.31$ & $1.31 \pm 0.39$ & WTTS & 7 \\
\hline EXLup & M0 & 3850 & 0.00 & $0.98_{-0.38}^{+0.92}$ & $0.56 \pm 0.06$ & $6.03 \pm 0.18$ & $2.05 \pm 0.55$ & CTTS & 8 \\
\hline HD143677 & $\mathrm{K} 1$ & 5080 & 0.22 & $5.30_{-1.80}^{+3.64}$ & $2.11 \pm 0.33$ & $6.48 \pm 0.20$ & $2.81 \pm 0.63$ & WTTS & 7 \\
\hline RXJ1603.8-3938 & $\mathrm{K} 3$ & 4730 & 0.42 & $1.19_{-0.40}^{+0.82}$ & $1.37 \pm 0.22$ & $6.87 \pm 0.26$ & $2.01 \pm 0.39$ & WTTS & 7 \\
\hline HD143978 & G2 & 5860 & 0.15 & $1.24_{-0.41}^{+0.83}$ & $1.22:$ & $7.35:$ & $1.40:$ & WTTS & 7 \\
\hline RXJ1605.5-3837 & M1 & 3720 & 0.57 & $0.26_{-0.10}^{+0.22}$ & $0.47 \pm 0.01$ & $6.61 \pm 0.32$ & $1.10 \pm 0.31$ & WTTS & 7 \\
\hline HOLup & M1 & 3720 & 1.25 & $1.16_{-0.47}^{+1.16}$ & $0.52 \pm 0.05$ & $5.93 \pm 0.16$ & $2.32 \pm 0.39$ & CTTS & 8 \\
\hline RXJ1607.2-3839 & $\mathrm{K} 7$ & 4060 & 0.89 & $1.12_{-0.42}^{+0.96}$ & $0.74 \pm 0.02$ & $6.19 \pm 0.27$ & $1.99 \pm 0.59$ & WTTS & 7 \\
\hline RXJ1608.0-3857 & M0 & 3850 & 0.27 & $0.81_{-0.31}^{+0.73}$ & $0.56 \pm 0.01$ & $6.12 \pm 0.24$ & $1.90 \pm 0.53$ & WTTS & 7 \\
\hline Sz96 & M1.5 & 3650 & 0.50 & $0.49_{-0.18}^{+0.40}$ & $0.43 \pm 0.01$ & $6.21 \pm 0.23$ & $1.65 \pm 0.46$ & CTTS & 8 \\
\hline RXJ1608.3-3843 & K7 & 4060 & 0.44 & $0.44_{-0.16}^{+0.35}$ & $0.80 \pm 0.01$ & $6.78 \pm 0.32$ & $1.23 \pm 0.35$ & WTTS & 7 \\
\hline Sz97 & M3 & 3470 & 1.00 & $0.26_{-0.10}^{+0.23}$ & $0.34 \pm 0.04$ & $6.37 \pm 0.22$ & $1.42 \pm 0.34$ & CTTS & 8 \\
\hline V856Sco & A7 & 7850 & 0.85 & $96_{-34}^{+70}$ & $3.30 \pm 0.59$ & $6.21 \pm 0.27$ & $5.20 \pm 1.25$ & HAeBe & 8 \\
\hline RXJ1608.6-3922 & K6 & 4205 & 1.75 & $0.87_{-0.33}^{+0.78}$ & $0.93 \pm 0.03$ & $6.49 \pm 0.37$ & $1.66 \pm 0.44$ & WTTS & 7 \\
\hline
\end{tabular}


Tabela 6.1 - continuação.

\begin{tabular}{|c|c|c|c|c|c|c|c|c|c|}
\hline ID & $\mathrm{ST}$ & $\begin{array}{l}T_{e f} \\
(\mathrm{~K})\end{array}$ & $\begin{array}{c}A_{V} \\
(\mathrm{mag})\end{array}$ & $\begin{array}{c}L \pm \sigma_{L} \\
\left(L_{\odot}\right)\end{array}$ & $\begin{array}{c}M \pm \sigma_{M} \\
\left(M_{\odot}\right)\end{array}$ & $\begin{array}{c}\log t \pm \sigma_{\log t} \\
(\mathrm{t} \text { em Myr) }\end{array}$ & $\begin{array}{c}R \pm \sigma_{R} \\
\left(R_{\odot}\right)\end{array}$ & Tipo & Ref. \\
\hline RXJ1608.9-3905 & $\mathrm{K} 2$ & 4900 & 0.39 & $2.93_{-1.12}^{+2.59}$ & $1.79 \pm 0.34$ & $6.58 \pm 0.31$ & $2.27 \pm 0.71$ & WTTS & 7 \\
\hline Sz111 & M1.5 & 3650 & 0.10 & $0.31_{-0.12}^{+0.28}$ & $0.43 \pm 0.01$ & $6.43 \pm 0.29$ & $1.30 \pm 0.44$ & CTTS & 8 \\
\hline Sz112 & M4 & 3370 & 0.85 & $0.27_{-0.11}^{+0.28}$ & $0.30 \pm 0.01$ & $6.30 \pm 0.21$ & $1.39 \pm 0.46$ & CTTS & 8 \\
\hline V908Sco & M4 & 3370 & 0.52 & $0.54_{-0.21}^{+0.48}$ & $0.30 \pm 0.01$ & $6.09 \pm 0.60$ & $2.04 \pm 0.73$ & CTTS & 8 \\
\hline Sz115 & M4 & 3370 & 0.20 & $0.15_{-0.06}^{+0.17}$ & $0.29 \pm 0.02$ & $6.55 \pm 0.27$ & $1.12 \pm 0.38$ & CTTS & 8 \\
\hline Sz134 & M1 & 3720 & 0.00 & $0.22_{-0.08}^{+0.18}$ & $0.47 \pm 0.03$ & $6.71 \pm 0.38$ & $1.10 \pm 0.35$ & CTTS & 8 \\
\hline RXJ1609.4-3850 & M0.5 & 3785 & 0.20 & $1.27_{-0.48}^{+1.09}$ & $0.51 \pm 0.01$ & $5.89 \pm 0.19$ & $2.47 \pm 0.44$ & WTTS & 7 \\
\hline Sz116 & M1.5 & 3650 & 0.30 & $0.25_{-0.09}^{+0.19}$ & $0.43 \pm 0.01$ & $6.55 \pm 0.27$ & $1.15 \pm 0.27$ & WTTS & 8 \\
\hline Sz117 & M2 & 3580 & 0.45 & $0.27_{-0.11}^{+0.26}$ & $0.39 \pm 0.01$ & $6.44 \pm 0.29$ & $1.39 \pm 0.37$ & CTTS & 8 \\
\hline RXJ1609.9-3923 & M1.5 & 3650 & 1.38 & $1.42_{-0.58}^{+1.46}$ & $0.52 \pm 0.01$ & $5.87 \pm 0.60$ & $2.55 \pm 0.87$ & CTTS & 7 \\
\hline Sz119 & M4 & 3370 & 0.21 & $0.29_{-0.11}^{+0.23}$ & $0.30 \pm 0.01$ & $6.27 \pm 0.18$ & $1.44 \pm 0.27$ & WTTS & 8 \\
\hline Sz120 & B5 & 15400 & 1.55 & $1150_{-463}^{+1154}$ & $5.03 \pm 0.95$ & $5.67 \pm 0.22$ & $3.42 \pm 1.54$ & HAeBe & 8 \\
\hline Sz122 & M2 & 3580 & 0.00 & $0.19_{-0.07}^{+0.16}$ & $0.39 \pm 0.01$ & $6.62 \pm 0.27$ & $1.08 \pm 0.29$ & WTTS & 8 \\
\hline Sz123 & M1 & 3720 & 0.90 & $0.36_{-0.15}^{+0.37}$ & $0.48 \pm 0.04$ & $6.43 \pm 0.40$ & $1.31 \pm 0.52$ & CTTS & 8 \\
\hline RXJ1612.0-3840 & K5 & 4350 & 0.94 & $3.80_{-1.30}^{+2.64}$ & $1.16 \pm 0.05$ & $5.84 \pm 0.20$ & $3.16 \pm 0.74$ & WTTS & 7 \\
\hline SSTc2dJ161207.6-381324 & M4 & 3400 & 0.60 & $0.57_{-0.21}^{+0.46}$ & $0.32 \pm 0.02$ & $6.06 \pm 0.59$ & $2.14 \pm 0.73$ & WTTS & 10 \\
\hline SSTc2dJ161243.8-381503 & K5 & 4400 & 1.00 & $1.63_{-0.62}^{+1.42}$ & $1.20 \pm 0.01$ & $6.35 \pm 0.33$ & $2.00 \pm 0.12$ & CTTS & 10,12 \\
\hline HD147402 & G3 & 5830 & 0.25 & $1.47_{-0.50}^{+1.01}$ & $1.11 \pm 0.09$ & $7.47 \pm 0.20$ & $1.20 \pm 0.29$ & WTTS & 7 \\
\hline \multicolumn{10}{|c|}{ Ophiuchus } \\
\hline GSC6784-39 & G9 & 5410 & 0.60 & $3.82_{-0.87}^{+1.33}$ & $1.66 \pm 0.17$ & $6.89 \pm 0.12$ & $2.13 \pm 0.31$ & WTTS & 3 \\
\hline $\mathrm{WaOph} / 3$ & K0 & 5250 & 0.74 & $1.59_{-0.43}^{+0.72}$ & $1.28 \pm 0.17$ & $6.17 \pm 0.16$ & $1.43 \pm 0.25$ & WTTS & 4 \\
\hline GSC6793-797 & K4 & 4590 & 1.04 & $0.89_{-0.07}^{+0.08}$ & $1.20 \pm 0.04$ & $6.75 \pm 0.05$ & $2.08 \pm 0.02$ & WTTS & 13,14 \\
\hline GSC6793-569 & K1 & 5080 & 2.00 & $2.23_{-0.57}^{+0.93}$ & $1.56 \pm 0.19$ & $6.87 \pm 0.16$ & $1.83 \pm 0.30$ & WTTS & 13,14 \\
\hline GSC6793-819 & $\mathrm{K} 2$ & 4900 & 0.84 & $1.72_{-0.46}^{+0.75}$ & $1.49 \pm 0.19$ & $6.85 \pm 0.18$ & $1.72 \pm 0.29$ & WTTS & 3 \\
\hline RXJ1621.4-2312 & K7 & 4060 & 1.11 & $1.07_{-0.41}^{+0.98}$ & $0.75 \pm 0.02$ & $6.22 \pm 0.29$ & $1.90 \pm 0.60$ & WTTS & 15,16 \\
\hline Haro1-1 & K5-K7 & 4205 & 2.67 & $0.75_{-0.17}^{+0.27}$ & $0.95 \pm 0.02$ & $6.60 \pm 0.20$ & $1.58 \pm 0.23$ & CTTS & 4,17 \\
\hline Haro1-4 & K6-K7 & 4133 & 2.11 & $1.16_{-0.19}^{+0.25}$ & $0.82 \pm 0.01$ & $6.22 \pm 0.11$ & $1.90 \pm 0.16$ & CTTS & 4 \\
\hline V852Oph & M0 & 3850 & 0.90 & $0.52_{-0.13}^{+0.20}$ & $0.57 \pm 0.01$ & $6.37 \pm 0.18$ & $1.55 \pm 0.26$ & CTTS & 4 \\
\hline SR4 & K6 & 4205 & 2.86 & $0.87_{-0.13}^{+0.16}$ & $0.93 \pm 0.01$ & $6.49 \pm 0.11$ & $1.66 \pm 0.11$ & CTTS & 4,17 \\
\hline DoAr25 & K5 & 4350 & 3.40 & $1.68_{-0.31}^{+0.43}$ & $1.11 \pm 0.01$ & $6.27 \pm 0.13$ & $2.15 \pm 0.19$ & CTTS & 18,19 \\
\hline Haro1-16 & $\mathrm{K} 2-\mathrm{K} 3$ & 4815 & 3.30 & $2.15_{-0.31}^{+0.40}$ & $1.60 \pm 0.11$ & $6.66 \pm 0.17$ & $2.04 \pm 0.22$ & CTTS & 17,19 \\
\hline V1121Oph & K4 & 4590 & 1.08 & $1.66_{-0.22}^{+0.27}$ & $1.44 \pm 0.08$ & $6.57 \pm 0.04$ & $1.94 \pm 0.17$ & CTTS & 3,4 \\
\hline AS216 & $\mathrm{K} 2$ & 4900 & 0.67 & $2.05_{-0.53}^{+0.88}$ & $1.59 \pm 0.19$ & $6.76 \pm 0.17$ & $1.87 \pm 0.33$ & CTTS & 3,20 \\
\hline V866ScoA & K5 & 4350 & 1.09 & $1.53_{-0.53}^{+1.11}$ & $1.11 \pm 0.02$ & $6.32 \pm 0.29$ & $2.07 \pm 0.37$ & CTTS & 4 \\
\hline PDS080 & $\mathrm{F} 1$ & 7050 & 0.51 & $7_{-2}^{+5}$ & $1.68 \pm 0.14$ & $7.12 \pm 0.23$ & $1.69 \pm 0.29$ & HAeBe & 4 \\
\hline GSC6213-306 & K1 & 5080 & 0.67 & $1.79_{-0.61}^{+1.24}$ & $1.43 \pm 0.27$ & $6.98 \pm 0.23$ & $1.66 \pm 0.41$ & WTTS & 3 \\
\hline PDS81 & $\mathrm{K} 2$ & 4900 & 0.73 & $0.97_{-0.35}^{+0.78}$ & $1.20 \pm 0.23$ & $7.15 \pm 0.24$ & $1.30 \pm 0.33$ & WTTS & 4 \\
\hline GSC6214-2384 & $\mathrm{K} 1$ & 5080 & 0.67 & $1.53_{-0.52}^{+1.06}$ & $1.36 \pm 0.26$ & $7.05 \pm 0.24$ & $1.54 \pm 0.39$ & WTTS & 3 \\
\hline RXJ1621.4-2332A & F5 & 6440 & 4.63 & $3.43_{-1.21}^{+2.57}$ & $1.37 \pm 0.14$ & $9.03 \pm 0.21$ & $1.48 \pm 0.37$ & WTTS & 15 \\
\hline RXJ1621.4-2332B & M0 & 3850 & 0.00 & $1.01_{-0.36}^{+0.76}$ & $0.56 \pm 0.06$ & $6.02 \pm 0.14$ & $2.08 \pm 0.15$ & WTTS & 15 \\
\hline RXJ1622.7-2325A & M1 & 3720 & 3.19 & $0.72_{-0.28}^{+0.66}$ & $0.48 \pm 0.01$ & $6.08 \pm 0.23$ & $1.93 \pm 0.60$ & WTTS & 15 \\
\hline RXJ1624.8-2359 & K3 & 4730 & 5.22 & $2.43_{-0.91}^{+2.06}$ & $1.66 \pm 0.24$ & $6.51 \pm 0.36$ & $2.22 \pm 0.72$ & WTTS & 13,15 \\
\hline ROXR1-4 & K7 & 4060 & 1.78 & $0.68_{-0.23}^{+0.47}$ & $0.77 \pm 0.03$ & $6.48 \pm 0.29$ & $1.56 \pm 0.47$ & WTTS & 15,16 \\
\hline
\end{tabular}


Tabela 6.1 - continuação.

\begin{tabular}{|c|c|c|c|c|c|c|c|c|c|}
\hline ID & ST & $\begin{array}{l}T_{e f} \\
(\mathrm{~K})\end{array}$ & $\begin{array}{c}A_{V} \\
\text { (mag) }\end{array}$ & $\begin{array}{c}L \pm \sigma_{L} \\
\left(L_{\odot}\right)\end{array}$ & $\begin{array}{c}M \pm \sigma_{M} \\
\left(M_{\odot}\right)\end{array}$ & $\begin{array}{l}\log t \pm \sigma_{\log t} \\
(\mathrm{t} \text { em Myr) }\end{array}$ & $\begin{array}{c}R \pm \sigma_{R} \\
\left(R_{\odot}\right)\end{array}$ & Tipo & Ref. \\
\hline ROXR1-7 & M4 & 3370 & 1.57 & $0.22_{-0.09}^{+0.27}$ & $0.30 \pm 0.02$ & $6.38 \pm 0.61$ & $1.33 \pm 0.61$ & WTTS & 15 \\
\hline ROX3 & M0 & 3850 & 0.24 & $0.39_{-0.13}^{+0.28}$ & $0.58 \pm 0.01$ & $6.54 \pm 0.31$ & $1.29 \pm 0.45$ & WTTS & 4 \\
\hline ISO-Oph16 & $\mathrm{A} 0$ & 9520 & 6.51 & $782_{-254}^{+495}$ & $6.06 \pm 0.76$ & $5.47 \pm 0.17$ & $10.11 \pm 1.63$ & HAeBe & 23 \\
\hline DoAr24 & K5 & 4350 & 4.62 & $\begin{array}{l}1.50_{-0.57}^{+1.30} \\
\text { - }\end{array}$ & $1.11 \pm 0.03$ & $6.33 \pm 0.33$ & $2.07 \pm 0.50$ & CTTS & 4,17 \\
\hline ROX20-2se & M2 & 3580 & 1.52 & $0.28_{-0.10}^{+0.21}$ & $0.39 \pm 0.01$ & $6.42 \pm 0.24$ & $1.40 \pm 0.32$ & WTTS & 15 \\
\hline GSC6794-337 & $\mathrm{K} 2$ & 4900 & 1.34 & $1.48_{-0.58}^{+1.41}$ & $1.41 \pm 0.32$ & $6.93 \pm 0.31$ & $1.59 \pm 0.54$ & WTTS & 3 \\
\hline SR9 & K5 & 4350 & 2.30 & $0.47_{-0.16}^{+0.31}$ & $1.08 \pm 0.11$ & $7.10 \pm 0.29$ & $1.43 \pm 0.24$ & CTTS & 18,24 \\
\hline SR10 & M1.5 & 3650 & 0.51 & $0.29_{-0.10}^{+0.22}$ & $0.43 \pm 0.01$ & $6.47 \pm 0.26$ & $1.28 \pm 0.38$ & CTTS & 4 \\
\hline V853Oph & M2.5 & 3525 & 0.14 & $0.51_{-0.20}^{+0.46}$ & $0.40 \pm 0.01$ & $6.15 \pm 0.20$ & $1.79 \pm 0.54$ & CTTS & 4 \\
\hline Haro1-14 & M0 & 3850 & 0.57 & $0.32_{-0.13}^{+0.31}$ & $0.58 \pm 0.04$ & $6.67 \pm 0.41$ & $1.17 \pm 0.42$ & CTTS & 4 \\
\hline DoAr51 & M0 & 3850 & 1.21 & $1.24_{-0.46}^{+1.04}$ & $0.56 \pm 0.11$ & $5.93 \pm 0.14$ & $2.39 \pm 0.32$ & CTTS & $4,15,17$ \\
\hline HD150193 & A1 & 9230 & 1.15 & $36_{-13}^{+28}$ & $2.45 \pm 0.19$ & $6.66 \pm 0.11$ & $2.30 \pm 0.82$ & HAeBe & 4 \\
\hline PDS89 & $\mathrm{K} 7$ & 4060 & 0.74 & $1.08_{-0.39}^{+0.83}$ & $0.74 \pm 0.02$ & $6.21 \pm 0.26$ & $1.90 \pm 0.62$ & WTTS & 4 \\
\hline RNO92 & M0 & 3850 & 3.47 & $1.40_{-0.56}^{+1.41}$ & $0.55 \pm 0.05$ & $5.89 \pm 0.59$ & $2.47 \pm 0.96$ & WTTS & 13,17 \\
\hline
\end{tabular}

Referências: (1) Høg et al. (2000); (2) Wichmann et al. (1997b); (3) Torres et al. (2006); (4) Sartori et al. (2003); (5) Fabricius et al. (2002); (6) Krautter et al. (1997); (7) Wichmann et al. (1997a); (8) Hughes et al. (1994); (9) Preibisch et al. (1998); (10) Comerón et al. (2009); (11) Merín et al. (2008); (12) López Martí et al. (2011); (13) Ducourant et al. (2005); (14) Carpenter et al. (2009); (15) Cieza et al. (2007); (16) Wahhaj et al. (2010); (17) Herbig \& Bell (1988); (18) Wilking et al. (2005); (19) McClure et al. (2010); (20) Batalha et al. (1998); (21) Gras-Velázquez e Ray (2005); (22) Grankin et al. (2007); (23) Gudennavar et al. (2012); (24) Evans et al. (2009). 
A existência de subestruturas e estrelas dispersas exige uma análise mais detalhada de cada região de formação estelar. Na Tabela 6.2 comparamos as propriedades físicas de cada subgrupo em Lupus e Ophiuchus. Conforme esperado, as estrelas mais dispersas do complexo de nuvens em cada região formam a população de estrelas PMS mais velha e com maior massa. As estrelas fora das nuvens de Lupus têm idade média de $\sim 15$ Myr enquanto as estrelas nas nuvens do complexo apresentam idade média de $\sim 3$ Myr. Lupus 3 é o subgrupo mais jovem da região de Lupus. Verificamos que a maior dispersão de massa nessa nuvem deve-se à maior quantidade de CTTSs, que têm massa baixa $\left(\leq 0.5 M_{\odot}\right)$, e WTTSs que cobrem um intervalo maior de massa entre $0.3 M_{\odot}$ e $1.8 M_{\odot}$. Além disso, inclui-se no cálculo da massa média as HAeBe que têm massa maior. Lupus 1 e Lupus 2 apresentam idades médias próximas sendo que as estrelas em Lupus 2 têm massa sensivelmente maior. A pequena quantidade de estrelas em Lupus 4 torna a comparação com as demais nuvens mais difícil. As poucas estrelas nesse subgrupo (dominado por CTTSs) exibem massas mais baixas que nas demais nuvens. No entanto, como mencionado na seção 4.5.1 esse resultado pode ser consequência de um efeito de seleção da amostra. A idade média das estrelas em L1688 obtida neste trabalho é $\sim 2$ Myr. Esse resultado está em bom acordo com o valor de 2.1 Myr obtido por Wilking et al. (2005) em L1688 e na vizinhança da nuvem. No entanto, notamos que as estrelas mais jovens de L1688 (idade $<1$ Myr, conforme discutido na seção 6.1) e também mais embebidas na nuvem não estão presente na amostra pelos motivos discutidos no capítulo 5 (carência de movimentos próprios).

Tabela 6.2 - Massa e idade das estrelas PMS em cada subgrupo das regiões de formação estelar em Lupus e Ophiuchus. Para cada subgrupo fornecemos o número de CTTSs, WTTSs, HAeBes, o valor médio (com desvio padrão) e a mediana dos resultados de massa e idade.

\begin{tabular}{|c|c|c|c|c|c|c|c|}
\hline & CTTS & WTTS & HAeBe & $\begin{array}{c}\overline{\bar{M}} \pm \sigma_{\bar{M}} \\
\left(M_{\odot}\right)\end{array}$ & $\begin{array}{c}\text { Med. }(M) \\
\left(M_{\odot}\right)\end{array}$ & $\begin{array}{c}\overline{\overline{\log t}} \pm \sigma_{\overline{\log t}} \\
(t \text { em Myr })\end{array}$ & $\begin{array}{c}\text { Med. }(\log t) \\
(t \text { em Myr })\end{array}$ \\
\hline Lupus 1 & 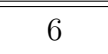 & 11 & 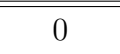 & $0.78 \pm 0.37$ & 0.66 & $6.77 \pm 0.60$ & 6.89 \\
\hline Lupus 2 & 2 & 8 & 0 & $0.84 \pm 0.33$ & 0.78 & $6.64 \pm 0.53$ & 6.55 \\
\hline Lupus 3 & 12 & 19 & 2 & $0.90 \pm 0.95$ & 0.52 & $6.39 \pm 0.40$ & 6.32 \\
\hline Lupus 4 & 4 & 1 & 1 & $0.71 \pm 0.62$ & 0.45 & $6.59 \pm 0.22$ & 6.65 \\
\hline Lupus (fora das nuvens) & 0 & 32 & 1 & $1.10 \pm 0.47$ & 1.10 & $7.17 \pm 0.38$ & 7.24 \\
\hline L1688 & 10 & 5 & 1 & $1.15 \pm 1.37$ & 0.80 & $6.41 \pm 0.33$ & 6.45 \\
\hline Ophiuchus (sem L1688) & 5 & 15 & 2 & $1.22 \pm 0.48$ & 1.30 & $6.68 \pm 0.66$ & 6.71 \\
\hline
\end{tabular}




\subsection{Comparação com Taurus-Auriga}

Iniciamos a comparação dos resultados aqui obtidos em Lupus e Ophiuchus com a região de Taurus-Auriga (Bertout et al. 2007) investigando o tipo espectral das TTSs em cada região na Figura 6.5. Como podemos notar, Lupus é a região que exibe a maior quantidade de estrelas com tipo espectral tardio. Verificamos que $42 \%$ das estrelas nessa região têm tipo espectral $M$ (ou mais tardio).
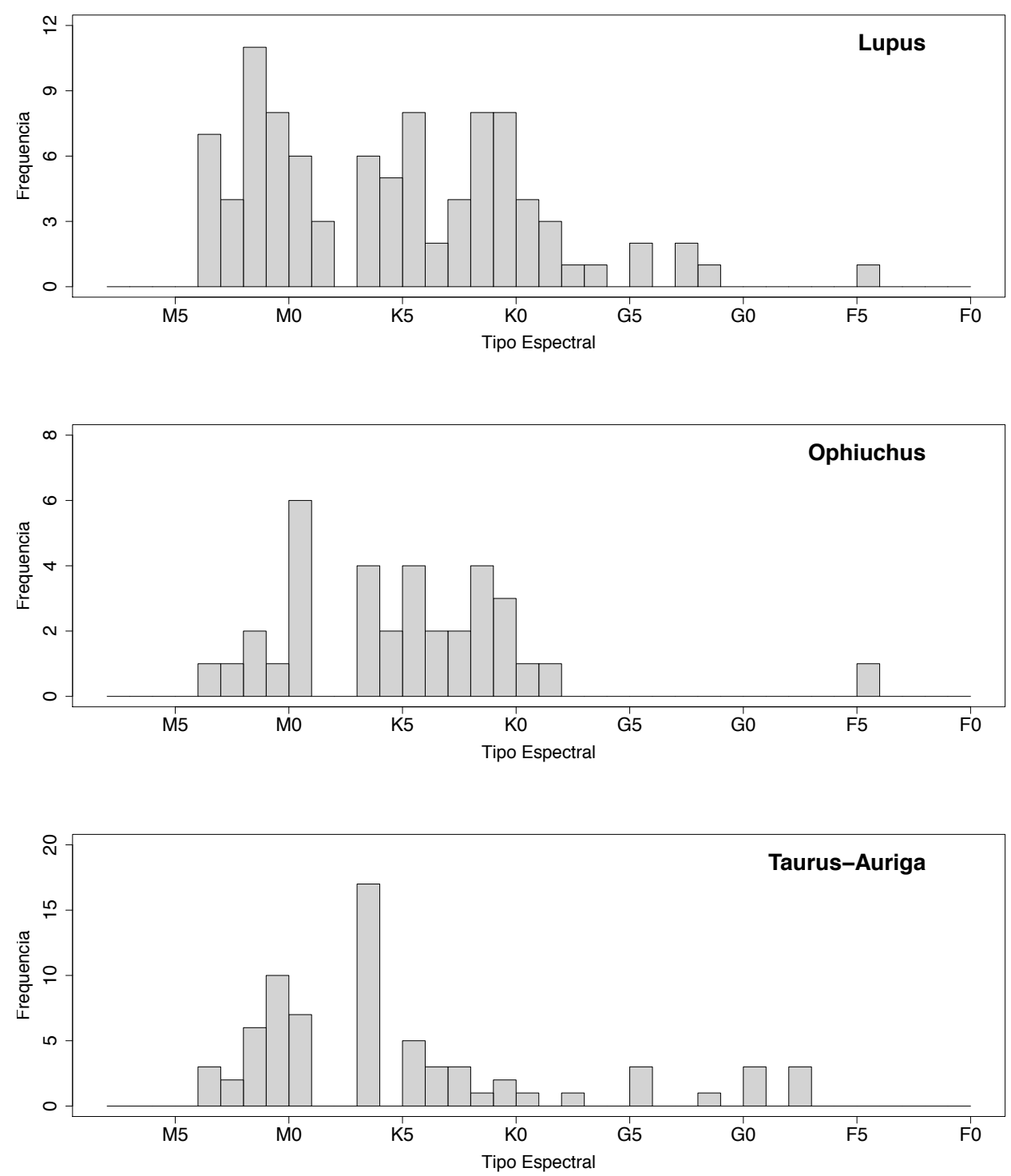

Figura 6.5: Tipo espectral das TTSs em Lupus, Ophiuchus e Taurus-Auriga. 
O diagrama-HR de Taurus-Auriga ${ }^{1}$ é apresentado na Figura 6.6. Como podemos notar pela comparação com os diagramas-HR de Lupus e Ophiuchus (vide Figuras 6.1 e 6.2) a separação entre CTTSs e WTTSs em Taurus-Auriga é mais evidente enquanto que em Lupus e Ophiuchus existe uma sobreposição maior entre as duas populações. A resposta para esse problema está muito além dos objetivos deste trabalho, mas será certamente investigada em outro momento. Consideramos a possibilidade que algumas estrelas tenham sido mal classificadas ou que existam discos de transição como identificados na região de Taurus-Auriga (vide Figura 6.6). A classificação das TTSs em CTTSs e WTTSs é um assunto ainda debatido na literatura. Alguns autores impõem outros critérios além do valor padrão de $10 \AA$ na largura equivalente da linha $H \alpha$ para diferenciar entre as duas subclasses (Hughes et al. 1994; Martin et al. 1998) o que poderia também explicar o resultado observado. Uma outra hipótese que pretendemos investigar é o tempo de vida do disco de acresção das estrelas em cada região. A interação com sistemas vizinhos, ventos e explosões de supernovae na Sco-Cen podem diminuir o tempo de vida dos discos circunestelares das estrelas em Lupus e Ophiuchus (Martin et al. 1998). Segundo o cenário proposto por Bertout et al. (2007) as CTTSs evoluem em WTTSs à medida que o disco é "acretado" pela estrela. O maior número de WTTSs (em Lupus) e a sobreposição entre as duas populações de TTSs no diagrama-HR poderia ser explicada dentro desse contexto pelo tempo de vida dos discos de acresção.

\footnotetext{
${ }^{1}$ Estamos reproduzindo a Figura 1 de Bertout et al. (2007) com os dados da Tabela 4 desse artigo com o objetivo de apresentar o diagrama-HR de Lupus, Ophiuchus e Taurus-Auriga no mesmo formato.
} 


\section{Taurus-Auriga}

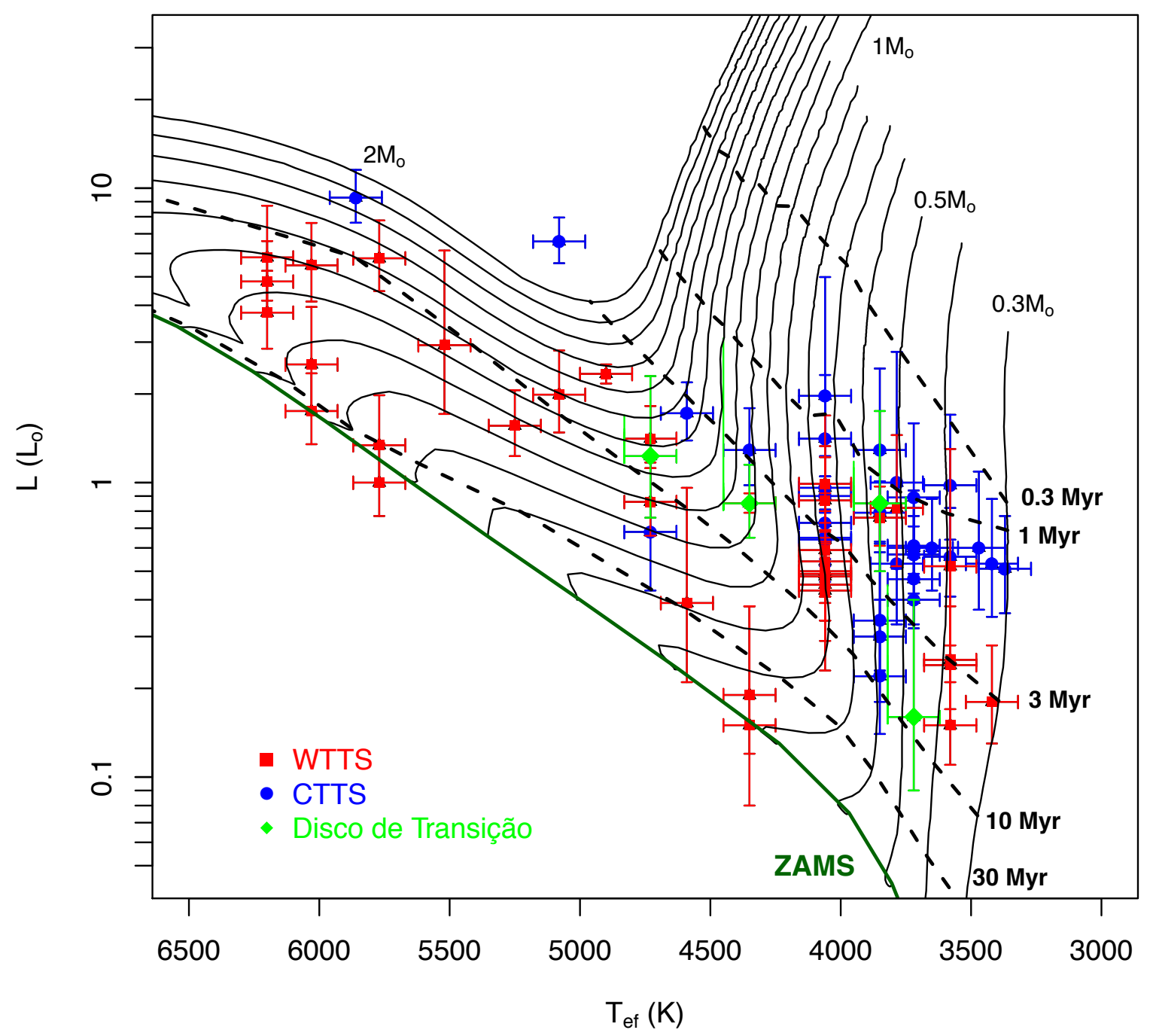

Figura 6.6: Diagrama-HR das TTSs em Taurus-Auriga (Bertout et al. 2007). As linhas contínuas indicam as trajetórias evolutivas para cada massa enquanto as linhas tracejadas denotam as isócronas para as idades assinaladas. A linha verde contínua representa a ZAMS. 
Na Figura 6.7 mostramos a distribuição de massa e idade das estrelas PMS em TaurusAuriga. Notamos a existência de dois picos na distribuição de massas o que é explicado pelo fato das CTTSs apresentarem em média massas menores que as WTTSs nessa região. O mesmo comportamento é observado na região de Lupus (vide Figura 6.3). Verificamos que a idade média das estrelas em Taurus-Auriga (4 Myr) se aproxima do valor observado para as estrelas dentro das nuvens de Lupus (3 Myr) e Ophiuchus (4 Myr). Por outro lado, as estrelas fora das nuvens de Lupus formam a população mais velha (15 Myr) que é discutida neste trabalho.
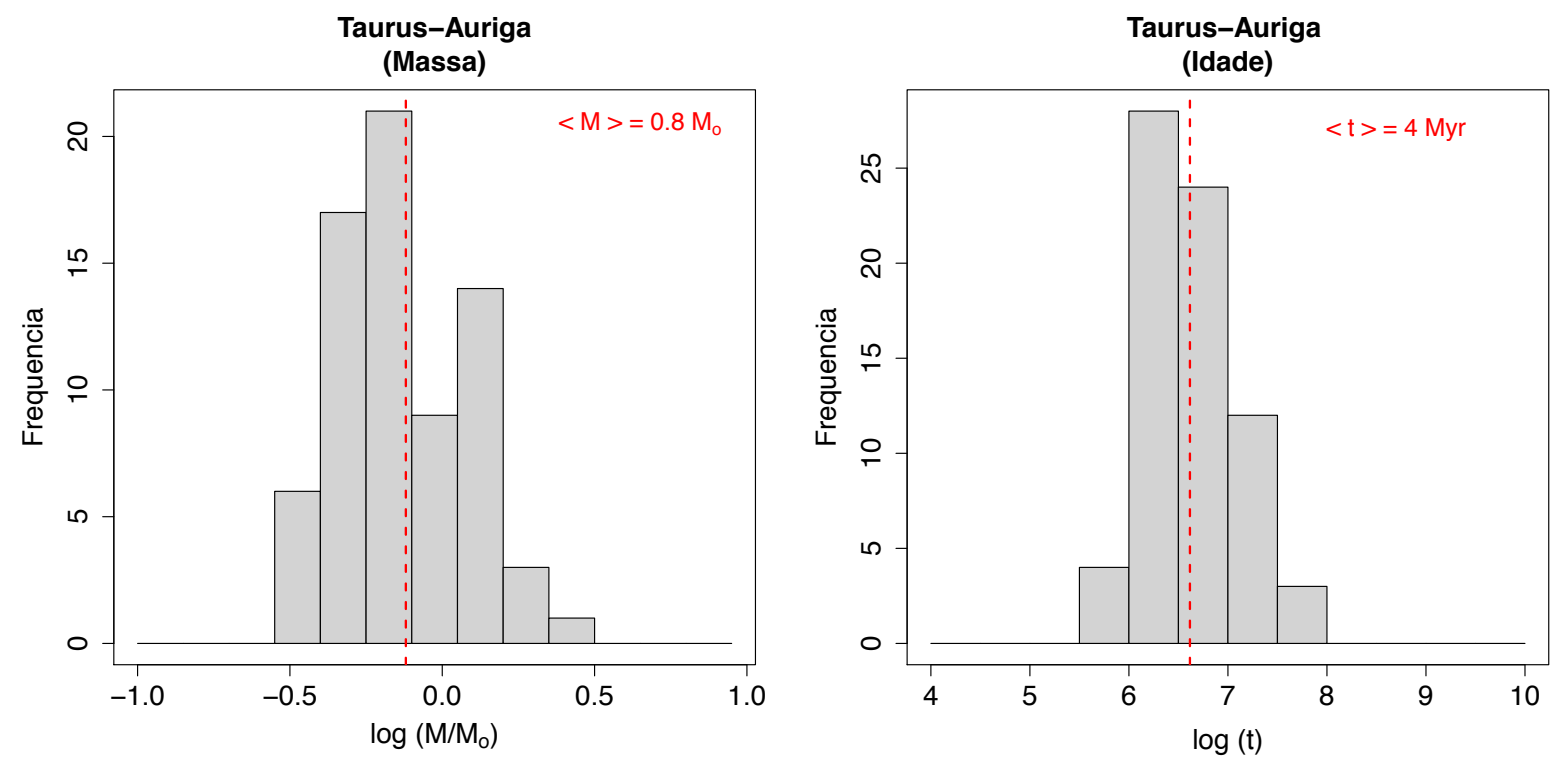

Figura 6.7: Distribuição de massa (esquerda) e idade (direita) das estrelas PMS em Taurus-Auriga (Bertout et al. 2007). A idade $t$ é dada em $\operatorname{Myr}\left(1 \mathrm{Myr}=10^{6}\right.$ anos). A linha vermelha tracejada representa a média de cada distribuição que está assinalada na figura.

Na Tabela 6.3 comparamos os valores médios e medianos de massa e idade das TTSs em cada região de formação estelar. Como podemos observar, as WTTS dentro das nuvens são mais jovens e têm massa menor que as WTTSs fora das nuvens. As duas populações de WTTSs, embora distintas, são mais velhas e têm massa maior que as CTTSs nessa região. Notamos que os valores medianos de massa e idade das CTTSs e WTTS (dentro das nuvens) em Lupus são próximos daqueles obtidos em Taurus-Auriga. Hughes et al. (1994) afirmam que as estrelas de Lupus são mais velhas que as estrelas de Taurus-Auriga partindo da distância média para construir o diagrama-HR e estimar a idade das estrelas. 
Excluindo a população fora das nuvens o que observamos neste trabalho é que existe uma diferença pequena de idade entre as CTTSs e WTTSs nas duas regiões sendo que a idade média das TTSs em Taurus-Auriga é sensivelmente maior. No que diz respeito à população de WTTSs fora das nuvens de Lupus, podemos afirmar que essas estrelas são significativamente mais velhas que as estrelas em Taurus-Auriga.

A região de Ophiuchus difere das demais tanto no número de estrelas utilizada nesta análise quanto nas propriedades físicas das TTSs. Com base nesta amostra observamos que as CTTS em Ophiuchus têm massas maiores que as CTTSs em Lupus e Taurus-Auriga. Notamos ainda que a diferença de idade entre as CTTSs e WTTSs nessa região é menor quando comparada às demais regiões. Esse primeiro resultado deverá ser confirmado com a análise de uma amostra maior de TTSs nessa região.

Tabela 6.3 - Massa e idade das TTSs em Lupus, Ophiuchus e Taurus-Auriga (Bertout et al. 2007). Para cada região apresentamos o número de estrelas, o valor médio (com desvio padrão) e a mediana dos resultados de massa e idade.

\begin{tabular}{lccccc}
\hline \hline & Estrelas & $\begin{array}{c}\bar{M} \pm \sigma_{\bar{M}} \\
\left(M_{\odot}\right)\end{array}$ & $\begin{array}{c}\text { Med. }(M) \\
\left(M_{\odot}\right)\end{array}$ & $\begin{array}{c}\overline{\log t} \pm \sigma_{\overline{\log t} t} \\
(t \text { em Myr })\end{array}$ & $\begin{array}{c}\text { Med. (log } t) \\
(t \text { em Myr })\end{array}$ \\
\hline \hline CTTS & 24 & $0.50 \pm 0.19$ & 0.48 & $6.28 \pm 0.35$ & 6.32 \\
WTTS (dentro das nuvens) & 39 & $0.86 \pm 0.40$ & 0.81 & $6.72 \pm 0.49$ & 6.72 \\
WTTS (fora das nuvens) & 32 & $1.09 \pm 0.33$ & 1.10 & $7.20 \pm 0.34$ & 7.24 \\
\hline \multicolumn{5}{c}{ Ophiuchus } \\
\hline CTTS & 15 & $0.95 \pm 0.39$ & 0.95 & $6.46 \pm 0.28$ & 6.47 \\
WTTS & 20 & $1.04 \pm 0.46$ & 1.20 & $6.67 \pm 0.67$ & 6.53 \\
\hline \multicolumn{5}{c}{ Taurus-Auriga (Bertout et al. 2007) } \\
\hline WTTS 33 & $0.72 \pm 0.44$ & 0.57 & $6.37 \pm 0.37$ & 6.28 \\
WTTS & 38 & $0.97 \pm 0.40$ & 0.80 & $6.82 \pm 0.44$ & 6.74 \\
\hline
\end{tabular}




\subsection{Simulação do Diagrama-HR}

Um ponto em comum nas regiões de Lupus, Ophiuchus e Taurus-Auriga é que as CTTSs são em média mais jovens que as WTTSs conforme observado na Tabela 6.3. Nesta seção investigamos a distribuição das CTTSs e WTTSs no diagrama-HR de cada região de formação estelar. Realizamos um conjunto de 1000 simulações Monte Carlo para cada estrela variando a sua luminosidade e temperatura efetiva dentro da incerteza dessas medidas. Os resultados obtidos são apresentados na Figura 6.8.

Como podemos notar pela Figura 6.8(a) as CTTSs e WTTSs em Lupus se separam em massa e idade no diagrama-HR. Utilizando a isócrona de 3 Myr como referência verificamos que $81 \%$ das CTTSs e 26\% das WTTSs simuladas são mais jovens que 3 Myr. Observamos ainda que $78 \%$ das CTTSs e $18 \%$ das WTTSs simuladas têm massa inferior a $0.5 M_{\odot}$. Com base nesses resultados e na Figura 6.8(a) concluímos que a maior parte das CTTSs em Lupus são mais jovens, exibem tipo espectral mais tardio (temperatura efetiva mais baixa) e têm massa menor que as WTTSs nessa região.

Na Figura 6.8(b) apresentamos o diagrama-HR simulado das TTSs em Ophiuchus. A menor quantidade de pontos deve-se evidentemente ao menor número de estrelas que estamos analisando nesta região. Verificamos que 57\% das CTTSs e 36\% das WTTSs simuladas em Ophiuchus são mais jovens que 3 Myr. Observamos que existe uma mistura maior entre as duas populações de TTSs. No entanto, como estamos investigando as propriedades de uma amostra pequena de estrelas nessa região, esse efeito ainda deverá ser confirmado. Verificamos também que 51\% das WTTSs em Lupus e somente $24 \%$ das WTTSs simuladas em Ophiuchus são mais velhas que $10 \mathrm{Myr}$. Com base na amostra estudada concluímos que as CTTSs em Ophiuchus são mais velhas que as CTTSs em Lupus e cobrem um intervalo maior de massa e tipo espectral. Além disso, constatamos que a região de Lupus tem uma população de TTSs mais jovem e outra mais evoluída quando comparada às TTSs em Ophiuchus. 


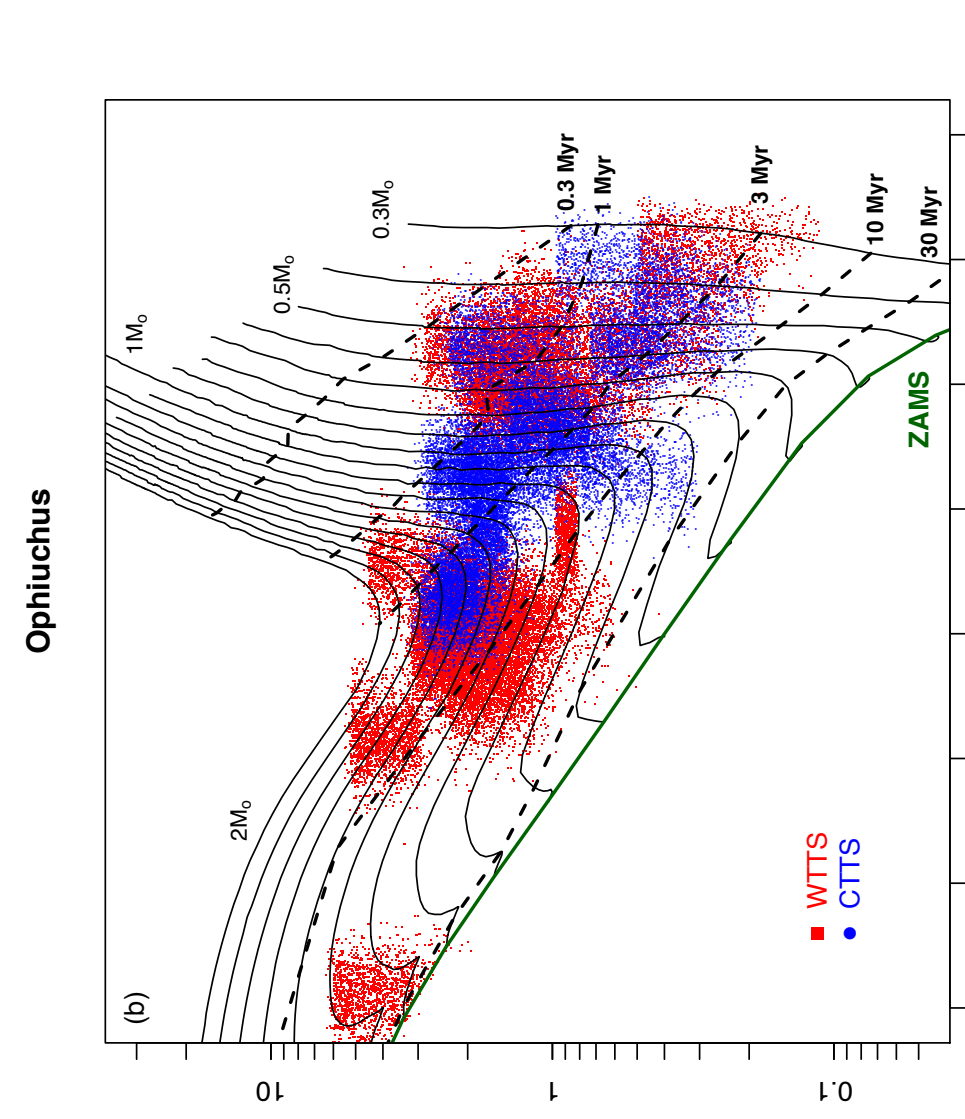

ఫ

(ำ 7

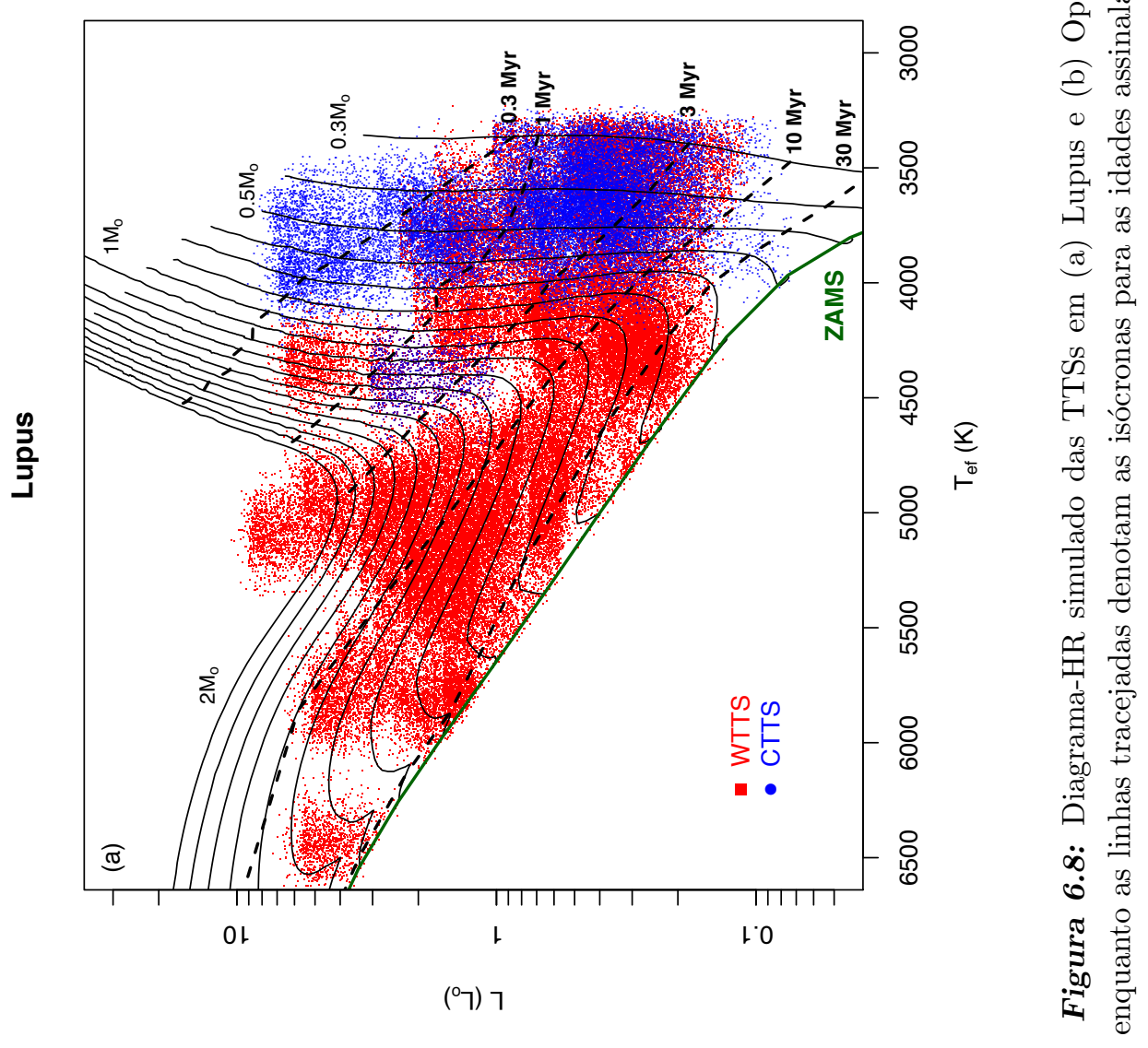


Comparamos a distribuição de idade das TTSs em Lupus e Ophiuchus a partir das simulações realizadas no diagrama-HR. Para cada conjunto de estrelas simuladas (1000 simulações no total) investigamos se as CTTSs e WTTSs exibem a mesma distribuição de idade usando o teste de Kolmogorov-Smirnov. Na Figura 6.9 apresentamos a distribuição do valor de $p$ que resulta desse teste estatístico em cada região. Como podemos notar, a probabilidade que as CTTSs e WTTSs tenham a mesma distribuição de idade é muito baixa, visto que as CTTSs são mais jovens. Esse resultado sustenta o cenário evolutivo (CTTS $\rightarrow$ WTTS) proposto por Bertout et al. (2007) na região de Taurus-Auriga.

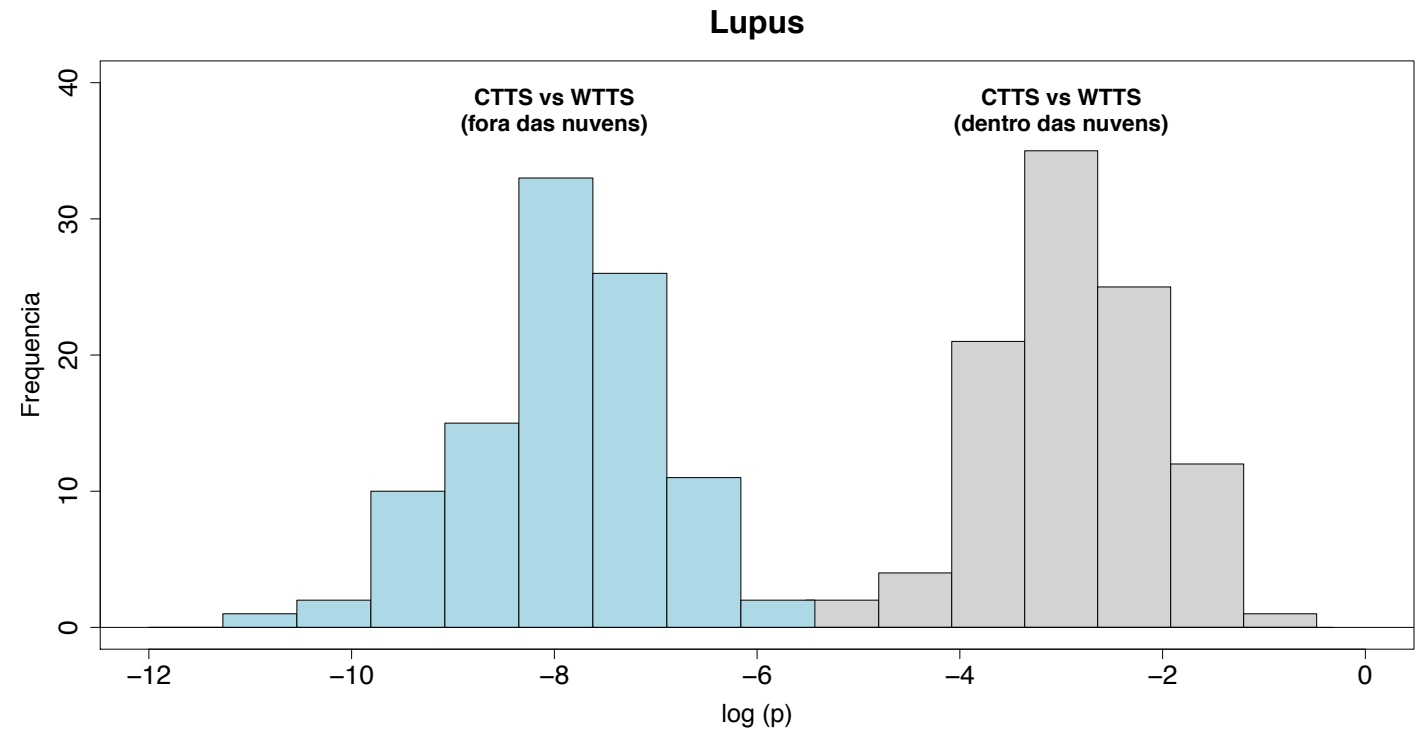

Ophiuchus

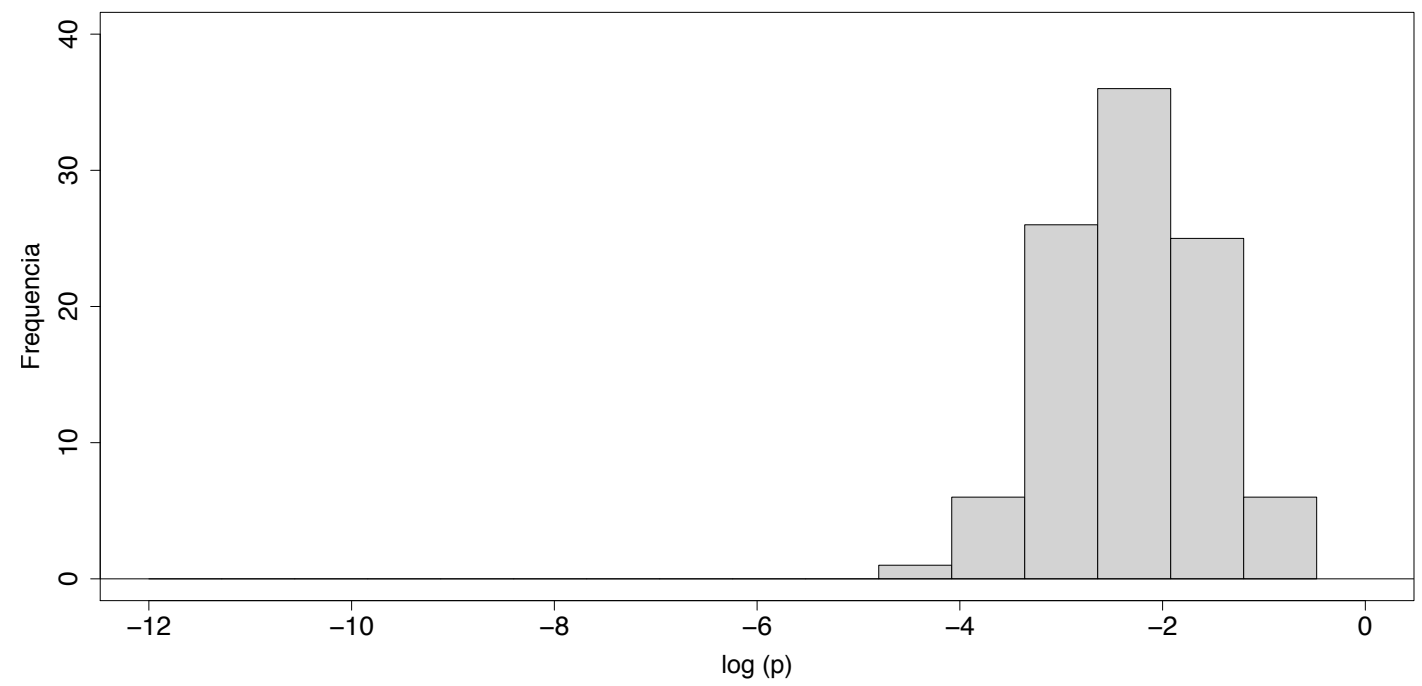

Figura 6.9: Distribuição do valor de $p$ obtido do teste de Kolmogorov-Smirnov da distribuição de idade das CTTSs e WTTSs em Lupus (painel superior) e Ophiuchus (painel inferior). 


\subsection{Distância Individual vs. Distância Média}

Concluímos este capítulo enfatizando a importância do uso da distância individual de cada estrela no cálculo de suas propriedades físicas. Para tanto, construímos uma amostra de controle onde a luminosidade, massa, idade e raio das estrelas são obtidas a partir da distância média de cada região de formação estelar. Comparamos esses resultados com aqueles discutidos até o momento. Utilizamos os valores de distância média mais recentes na literatura para cada região: $155 \pm 8$ pc em Lupus e $119 \pm 6$ pc em Ophiuchus (Lombardi et al. 2008a). Embora a luminosidade assim obtida seja mais precisa, ela é menos exata, pois as estrelas não se encontram todas à mesma distância. O erro de 8 pc (em Lupus) e 6 pc (em Ophiuchus) no valor da distância média não reflete a profundidade de cada complexo de formação estelar, mas o erro atribuído ao método de determinação da distância média. Por exemplo, Lombardi et al. (2008a) apresentam a distância média de $155 \pm 8$ pc, mas estimam a profundidade do complexo de Lupus em $51_{-35}^{+61} \mathrm{pc}$.

Como mencionado anteriormente, a massa e a idade das estrelas PMS é estimada pela posição da estrela no diagrama-HR que é dada em função da temperatura efetiva e luminosidade. Considerando que a temperatura efetiva (tipo espectral) da estrela seja bem determinada, o efeito da distância no diagrama-HR consiste em deslocar a estrela na vertical variando a luminosidade. Qual é o efeito desse deslocamento no diagrama-HR nas estimativas de massa e idade das estrelas ? Para responder essa pergunta apresentamos na Tabela 6.4 as propriedades físicas de algumas estrelas da amostra que foram obtidas pela distância individual (calculada a partir da velocidade radial) e a distância média da região de formação estelar correspondente. Consideramos somente as estrelas cuja distância difere em algumas dezenas de parsec da distância média. Não se trata aqui de discutir se os resultados são compatíveis entre si, mas em analisar o desvio causado pela variação na distância. Notamos que para as estrelas mais quentes da Tabela 6.4, o uso da distância média implica em um desvio de 20\% nas massas calculadas. Quanto à idade dessas estrelas verificamos que existe uma variação maior, como é o caso de GSC6784-39, cuja idade calculada pela distância média é quase duas vezes maior que o valor obtido pela distância individual. Para as estrelas com tipo espectral mais tardio, por exemplo V852Oph, notamos que o desvio na massa da estrela é pequeno. Como podemos observar na Figura 6.8, a trajetória evolutiva das estrelas com baixa massa $\left(M \leq 0.5 M_{\odot}\right)$ é quase 
vertical e o deslocamento da estrela no diagrama-HR devido à variação da distância não altera significativamente a sua massa. Por outro lado, a idade de V852Oph é cerca de cinco vezes maior quando obtida pela distância média e o raio é quase duas vezes menor. Quando a distância da estrela é superestimada, a luminosidade calculada é maior e a idade fica subestimada.

Tabela 6.4 - Comparação das propriedades físicas das estrelas calculadas a partir da distância individual de cada estrela $\left(d_{i n d}\right)$ e a distância média $\left(d_{m e d}\right)$ da região de formação estelar. Fornecemos para cada estrela o seu tipo espectral, distância, luminosidade, massa, idade, raio e a região de formação estelar.

\begin{tabular}{lcccccccccccc}
\hline ID & $\mathrm{ST}$ & $d_{\text {ind }}(p c)$ & $L / L_{\odot}$ & $M / M_{\odot}$ & $t(M y r)$ & $R / R_{\odot}$ & $d_{\text {med }}(p c)$ & $L / L_{\odot}$ & $M / M_{\odot}$ & $t(M y r)$ & $R / R_{\odot}$ & Região \\
\hline RXJ1519.6-4760 & $\mathrm{G} 9$ & 118 & 0.88 & 1.02 & 33 & 1.01 & 155 & 1.53 & 1.18 & 21 & 1.29 & Lup \\
RXJ1448.2-4103 & K2 & 114 & 0.62 & 1.00 & 25 & 1.03 & 155 & 1.15 & 1.25 & 12 & 1.38 & Lup \\
RXJ1504.8-3950 & G5 & 101 & 1.38 & 1.10 & 30 & 1.14 & 155 & 3.25 & 1.34 & 15 & 1.67 & Lup \\
RXJ1613.0-4004 & K7 & 100 & 0.30 & 0.81 & 12 & 1.22 & 155 & 0.72 & 0.77 & 3 & 1.56 & Lup \\
GSC6784-39 & G9 & 180 & 3.82 & 1.66 & 8 & 2.13 & 119 & 1.66 & 1.31 & 14 & 1.52 & Oph \\
V852Oph & M0 & 226 & 0.52 & 0.57 & 2 & 1.55 & 119 & 0.14 & 0.59 & 11 & 0.87 & Oph \\
RXJ1621.4-2312 & K7 & 240 & 1.07 & 0.75 & 2 & 1.90 & 119 & 0.26 & 0.81 & 10 & 1.22 & Oph \\
SR4 & K6 & 88 & 0.87 & 0.93 & 3 & 1.66 & 119 & 1.59 & 0.90 & 1 & 2.72 & Oph \\
\hline
\end{tabular}

Na Figura 6.10 comparamos o diagrama-HR das TTSs em Lupus e Ophiuchus obtido com a distância individual de cada estrela e a distância média da região de formação estelar. Apresentamos o diagrama-HR que resulta das simulações (conforme descrito na seção 6.6). Aplicamos o mesmo procedimento para o diagrama-HR obtido a partir da distância média de cada região. Como podemos observar, neste último caso as TTSs estão menos dispersas o que se deve ao erro da distância média que é menor, mas não representativo de cada estrela ou da profundidade da região conforme discutido anteriormente. Um ponto importante é que o uso da distância média tende a concentrar as estrelas no diagrama-HR ocultando tanto as estrelas mais jovens quanto as mais velhas da amostra. Em particular notamos que a idade das CTTSs é superestimada com o uso da distância média.

Concluímos da Tabela 6.4 e Figura 6.10 que existem desvios significativos na determinação das propriedades físicas das estrelas e diagrama-HR quando a distância média da região de formação estelar é utilizada para essa finalidade. 


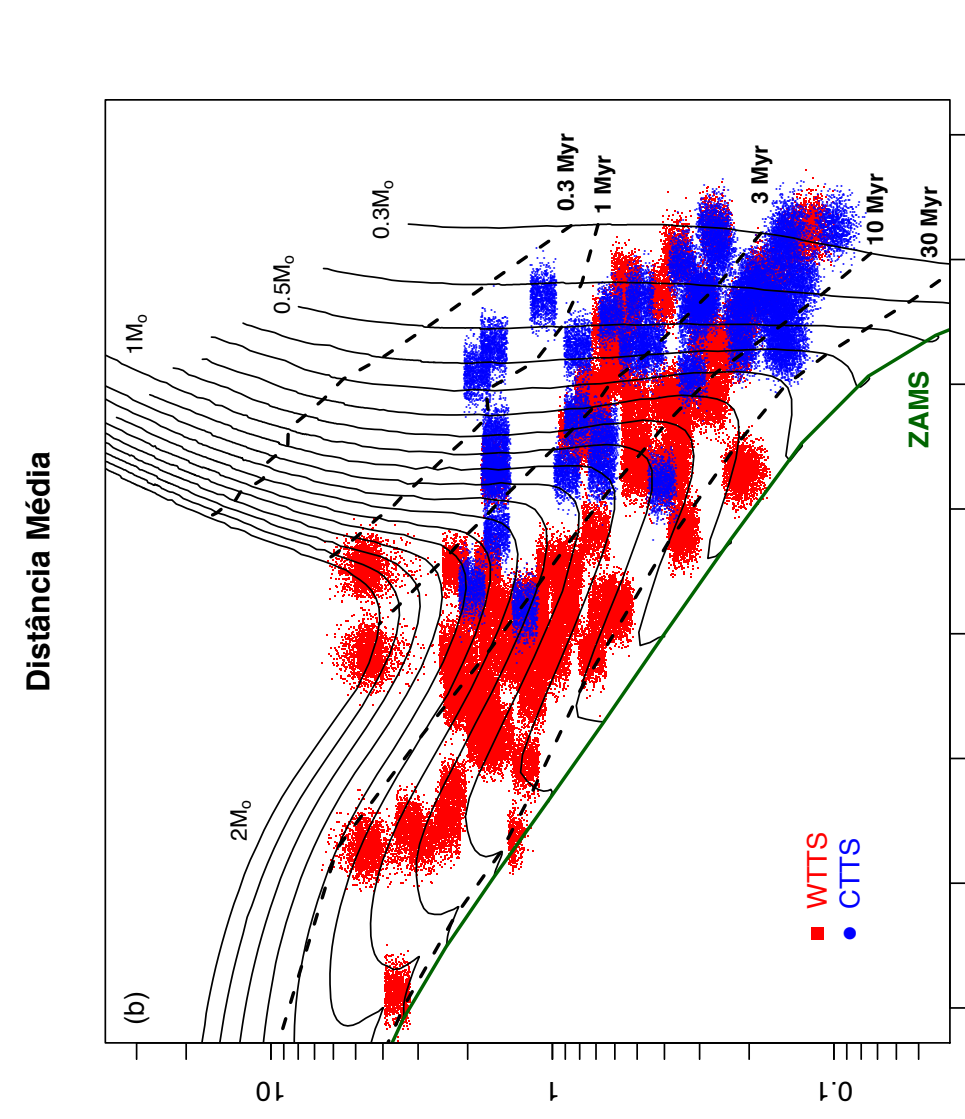

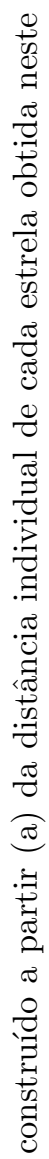

(ㄱ) 7

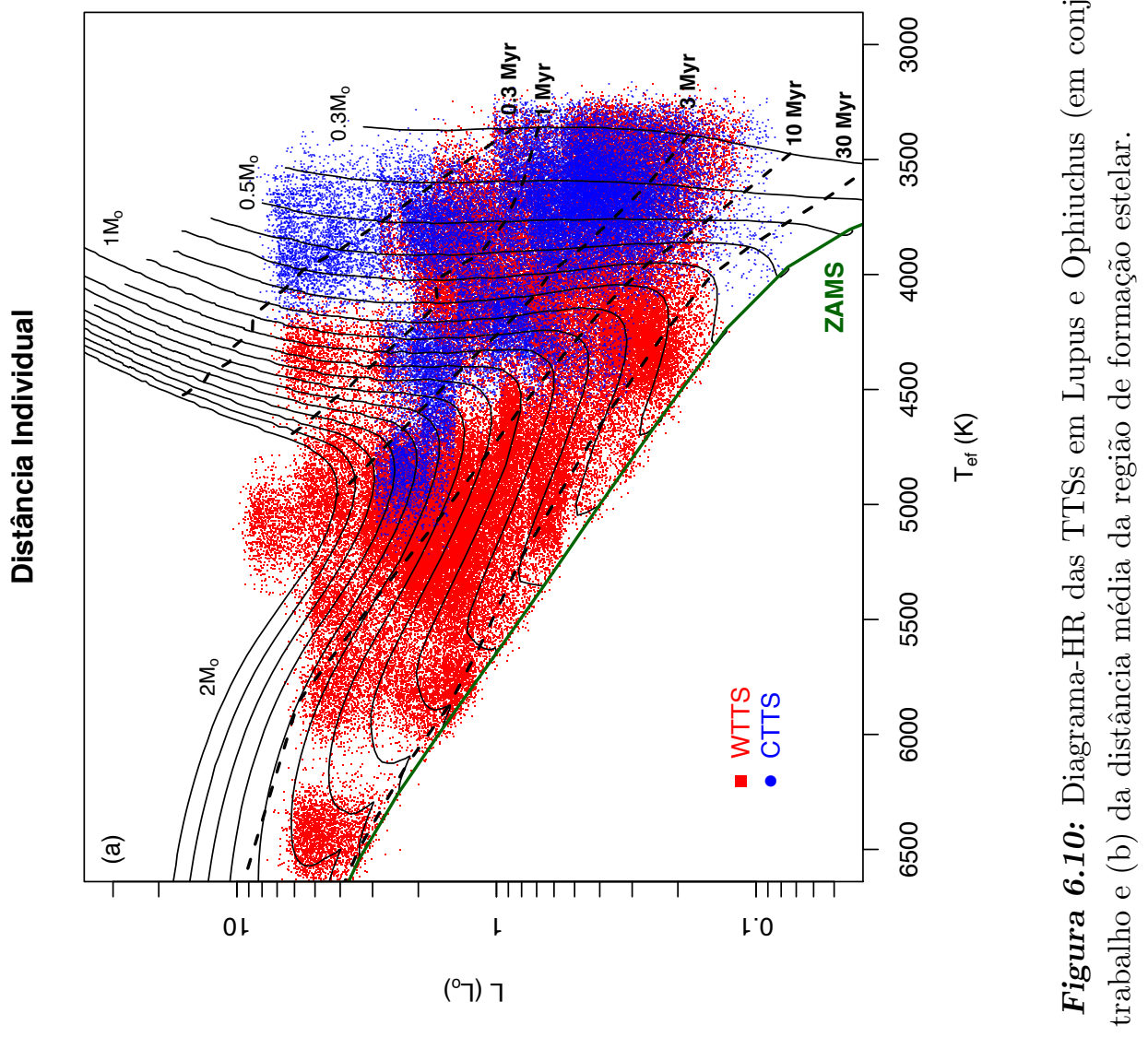




\subsection{Conclusão}

Neste capítulo discutimos as propriedades físicas das estrelas PMS em Lupus e Ophiuchus. Partindo das distâncias aqui obtidas e dos dados fotométricos disponíveis na literatura determinamos a luminosidade, massa, idade e raio de 99 estrelas em Lupus (24 CTTSs, 71 WTTSs, 4 HAeBes) e 38 estrelas em Ophiuchus (15 CTTSs, 20 WTTSs, 3 HAeBes). Confirmamos que as estrelas mais dispersas ( $15 \mathrm{Myr})$ na região de Lupus são mais velhas que as estrelas dentro das nuvens $(\sim 3 \mathrm{Myr})$ e verificamos que Lupus 3 é o subgrupo mais jovem nessa região. Na região de Ophiuchus encontramos uma população jovem $(\sim 2 \mathrm{Myr})$ que se distribui nas bordas da nuvem L1688 (conforme discutido no capítulo 5).

Comparando as propriedades físicas das TTSs em cada região de formação estelar concluímos que as CTTSs em Lupus são preferencialmente de baixa massa $\left(M \leq 0.5 M_{\odot}\right)$ e apresentam tipo espectral tardio. Em Ophiuchus observamos que as CTTSs se distribuem em um intervalo maior de massa e temperatura efetiva. Verificamos que tanto a distribuição de massa quanto de idade das estrelas em Lupus apresenta dois picos o que reflete bem a existência das duas populações (dentro e fora das nuvens) nesta região. Notamos que no diagrama-HR de Taurus-Auriga a separação entre CTTSs e WTTSs é mais evidente enquanto que em Lupus e Ophiuchus existe uma sobreposição maior que será investigada com mais detalhes em outro momento. Realizamos simulações no diagrama-HR de Lupus e Ophiuchus o que nos permitiu confirmar que a distribuição de idade das CTTSs e WTTSs é diferente, sendo as CTTSs mais jovens. Esse resultado concorda com o cenário evolutivo das TTSs proposto por Bertout et al. (2007) em Taurus-Auriga.

Finalizamos o capítulo ressaltando a importância do uso da distância individual de cada estrela no cálculo das propriedades físicas. Comparamos o diagrama-HR construído a partir da distância individual de cada estrela e distância média da região de formação estelar. Verificamos que existem desvios significativos, principalmente na idade das estrelas, quando utilizamos a distância média para o cálculo dos parâmetros físicos. O diagrama-HR obtido a partir da distância média oculta as estrelas mais jovens e mais velhas da amostra. 
Capítulo 7

\section{CONSIDERAÇÕes Finais}

"Você nunca sabe que resultados virão da sua ação. Mas se você não fizer nada, não existirão resultados."

Mahatma Gandhi

O plano inicial desta tese de doutorado previa a determinação da distância de estrelas pré-sequência principal que não foram contempladas durante a missão espacial HiPPARCOS e cuja medida da paralaxe trigonométrica a partir do solo não é acessível. A importância, carência e necessidade imediata dessa informação nos motivou a desenvolver a tese sobre esse assunto. Em diversos momentos eu me questionei se seria possível atingir esse objetivo sabendo da dificuldade que existe no cálculo da distância e a minha (pouca) experiência no início do doutorado direto. Hoje vejo que o plano inicial, embora ambicioso em alguns aspectos, foi concretizado e ampliado além das nossas perspectivas. Uma grande parte da tese foi dedicada ao cálculo do ponto de convergência, análise de pertinência e determinação da distância das estrelas. No entanto, uma primeira exploração desses resultados na obtenção das propriedades físicas das estrelas revelou a importância e aplicação do conhecimento da distância ampliando os horizontes do trabalho. 
Embora as conclusões de todas etapas do trabalho já tenham sido discutidas em cada capítulo, destacamos aqui alguns pontos que marcaram esta tese de doutorado.

\section{- Um novo método para o cálculo do ponto de convergência}

Uma parte importante desta tese foi o desenvolvimento de uma nova estratégia que nos permite simultaneamente calcular o $\mathrm{CP}$ e selecionar os membros do MG. O novo CPSM tem como base a representação do movimento próprio das estrelas por um grande círculo na esfera celeste. A intersecção dos grandes círculos das estrelas define o CP. Quando comparado ao CPSM clássico da literatura com base nas simulções de MGs com diferentes configurações, concluímos que a nova estratégia retorna em muitos casos uma solução mais precisa e é mais eficiente na seleção dos membros e eliminação das estrelas de campo. Uma aplicação ao aglomerado aberto das Híades retornou resultados compatíveis com aqueles já conhecidos e permitiu validar a nova estratégia aqui desenvolvida.

\section{- Observações e a medida da velocidade radial}

As observações foram realizadas com o espectrógrafo FEROS (ESO, La Silla) e os espectros coletados foram utilizados na medida da velocidade radial. Calculamos a velocidade radial de 52 estrelas PMS em Lupus e Ophiuchus pela correlação cruzada do espectro da estrela de programa com um espectro de referência. As velocidades radiais aqui obtidas foram utilizadas no cálculo da distância dessas estrelas.

\section{- Cinemática e distância das estrelas em Lupus e Ophiuchus}

Investigamos a cinemática das estrelas PMS em Lupus e Ophiuchus partindo dos movimentos próprios disponíveis em diversos catálogos (SPM4, UCAC4, PPMXL, TyCHO2 e Ducourant et al. 2005) e o CPSM desenvolvido neste trabalho. Utilizamos o CPSM na região de Lupus para separar as estrelas do subgrupo UCL da Sco-Cen que contaminavam a amostra. Identificamos um MG com 114 estrelas PMS na região de Lupus e verificamos que algumas estrelas mais dispersas compartilham as mesmas propriedades cinemáticas das estrelas concentradas nas nuvens moleculares. Com o conhecimento da distância individual das estrelas confirmamos que o complexo de Lupus ocupa um grande volume no espaço. Em particular, encontramos evidências de uma estrutura filamentar que se estende por dezenas de parsec 
na direção da nuvem Lupus 3. A natureza dessa estrutura ainda não é conhecida e deverá ser investigada com mais detalhes a partir da medida da velocidade radial (seguida do cálculo da distância) para um número maior de estrelas. Em Ophiuchus identificamos um MG com 55 estrelas PMS, sendo que a maior parte dos membros está concentrada na nuvem L1688. No entanto, notamos que algumas TTSs dispersas, como por exemplo AS216, se movem em conjunto com as demais estrelas na região central deste complexo de formação estelar. Partindo das distâncias calculadas investigamos a estrutura de L1688 que também se estende por dezenas de parsec em profundidade. As estrelas de L1688 consideradas nesta análise se encontram preferencialmente na periferia da nuvem. Como podemos notar, o conhecimento da distância individual das estrelas revela a existência de efeitos de profundidade importantes que são desprezados quando consideramos somente a distância média ou aproximada de cada região de formação estelar. O erro obtido na distância média em diversos trabalhos é, em geral, pequeno e não reflete a dimensão de cada região de formação estelar.

\section{- Propriedades físicas das estrelas em Lupus e Ophiuchus}

Partindo das distâncias aqui obtidas calculamos a luminosidade, massa, idade e raio das estrelas PMS em Lupus e Ophiuchus assim como o diagrama-HR de cada região. Verificamos que as WTTSs dispersas em Lupus são mais velhas que as TTSs concentradas nas nuvens moleculares do complexo. Notamos que as CTTSs com massa superior a $0.5 M_{\odot}$ são menos frequentes em Lupus. Em Ophiuchus as CTTSs cobrem um intervalo maior de massa e temperatura efetiva. Uma comparação com as TTSs em Taurus-Auriga mostra que existe uma sobreposição maior das WTTSs mais jovens com as CTTSs no diagrama-HR das regiões de Lupus e Ophiuchus. Com base nas simulações realizadas no diagrama-HR de Lupus e Ophiuchus confirmamos que a distribuição de idade das CTTSs e WTTSs é diferente sendo as CTTSs mais jovens. Esse resultado sustenta o cenário evolutivo das TTSs proposto por Bertout et al. (2007) em Taurus-Auriga. Comparamos os resultados obtidos usando a distância individual de cada estrela e a distância média da região de formação estelar. Concluímos que existem desvios importantes quando a distância média (igual para todas estrelas) é utilizada para calcular os parâmetros físicos individuais das estrelas. 
Com o andamento da tese algumas questões surgiram e ficaram em aberto. Em seguida levantamos alguns pontos como perspectivas de continuação deste trabalho que serão certamente desenvolvidas em outro momento.

\section{- Campanha observacional: movimento próprio e velocidade radial}

Existe um grande número de estrelas jovens cujo movimento próprio não é conhecido ou é de baixa qualidade principalmente na região de Ophiuchus. A medida do movimento próprio das estrelas mais fracas e ainda embebidas nas nuvens moleculares progenitoras é um trabalho desafiador que exige um cuidado maior. No entanto, ele deverá contribuir para a análise cinemática e o cálculo da distância de uma quantidade maior de estrelas nessa região. Por outro lado, observamos que outras estrelas que já foram investigadas neste trabalho ainda carecem da velocidade radial. Pretendemos confirmar a natureza filamentar observada em Lupus 3 com a medida da velocidade radial de um número maior de estrelas.

\section{- Cenário evolutivo das estrelas T Tauri}

Qual é o tempo de vida dos discos de acresção das TTSs em Lupus e Ophiuchus ? Existe alguma influência do ambiente na evolução dos discos ? Como isso afeta a escala de tempo da formação de planetas e evolução das estrelas ? Por que não encontramos CTTSs com massa mais alta em Lupus ? Como é a função de massa inicial em cada região de formação estelar ? Essas são algumas das questões que pretendemos investigar partindo das distâncias individuais e as propriedades físicas das TTSs obtidas neste trabalho de tese. 


\section{- História da formação estelar na vizinhança solar}

As regiões de formação estelar de Lupus e Ophiuchus pertencem a um complexo muito maior de nuvens situadas até cerca de 200 pc. Nesse complexo inclui-se ainda Scorpius-Centaurus, Corona-Australis e Chamaeleon. A direção preferencial do movimento próprio das estrelas nessas regiões para longitudes e latitudes decrescentes (conforme observado na Figura 7.1) sugere uma relação entre essas estruturas. Diversos cenários da formação estelar têm sido propostos para explicar a origem desse complexo: formação estelar sequencial (Elmegreen \& Lada 1977), formação pelo impacto de nuvens em alta velocidade no plano galáctico (Lepine \& Duvert 1994) e a formação em um braço espiral que passa próximo ao Sol (Sartori et al. 2003). Entendemos que as distribuições de idade e velocidade das estrelas são pertinentes nesta discussão. Essas grandezas só podem ser obtidas com o conhecimento da distância. Embora a associação Scorpius-Centaurus já tenha sido mais investigada na literatura, as regiões de Chamaeleon e Corona-Australis foram menos abordadas. A estratégia que foi desenvolvida ao longo desta tese de doutorado poderá ser estendida para essas e outras regiões de formação estelar.

Uma outra questão ainda em aberto é a relação das estrelas nessas regiões de formação estelar ( 200 pc) com as associações de estrelas jovens próximas (até 100 pc). Nesse raio inclui-se, por exemplo, TW Hydrae, $\beta$ Pictoris, AB Doradus, Tucana/Hologorium e $\eta$ Chamaeleontis. A formação desses grupos nas proximidades da Sco-Cen foi proposta em alguns trabalhos usando a técnica de traceback (Mamajek et al. 2000; de la Reza et al. 2006). O novo CPSM poderá ser aplicado a esses grupos para uma análise da cinemática das estrelas, estudo de pertinência entre cada associação e a identificação de novos membros ou outros grupos de estrelas que compartilham as mesmas propriedades cinemáticas. Dentro desse contexto, pretendemos ampliar aquilo foi desenvolvido ao longo do doutorado visando fornecer subsídios que contribuam para explicar o cenário da formação estelar na vizinhança solar.

"O importante é não parar de questionar." 


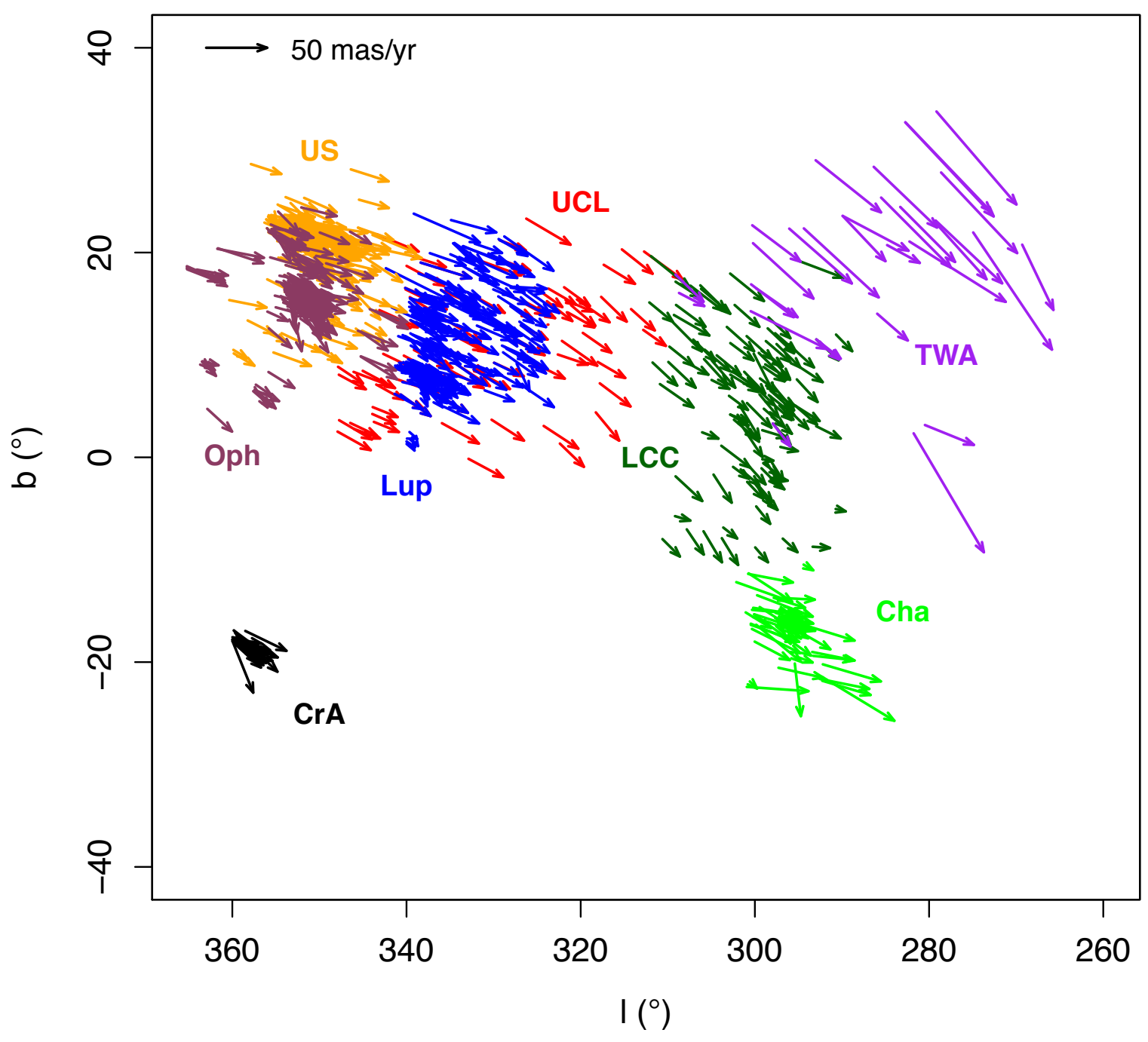

Figura 7.1: Posição e movimento próprio no sistema de coordenadas Galácticas das estrelas PMS em Ophiuchus (Oph), Upper Scorpius (US), Lupus (Lup), Upper Centaurus-Lupus (UCL), Lower CentaurusCrux (LCC), Chamaeleon (Cha), TW Hydrae (TWA) e Corona-Australis (CrA). 


\section{Referências Bibliográficas}

Abad C., Vieira K., Systematic motions in the galactic plane found in the Hipparcos catalogue using Herschel's Method, A\&A, 2005, vol. 442, p. 745

Abad C., Vieira K., Bongiovanni A., Romero L., Vicente B., An extension of Herschel's method for dense and extensive catalogues. Application to the determination of solar motion, A\&A, 2003, vol. 397, p. 345

Allen L. E., Myers P. C., Di Francesco J., Mathieu R., Chen H., Young E., Hubble Space Telescope/NICMOS Imaging Survey of the Ophiuchus (Lynds 1688) Cluster, ApJ, 2002, vol. 566 , p. 993

Alves F. O., Franco G. A. P., The infrared void in the Lupus dark clouds revisited: a polarimetric approach, MNRAS, 2006, vol. 366, p. 238

Antoja T., Figueras F., Fernández D., Torra J., Origin and evolution of moving groups. I. Characterization in the observational kinematic-age-metallicity space, A\&A, 2008, vol. 490 , p. 135

Appenzeller I., Mundt R., T Tauri stars, A\&A Rev., 1989, vol. 1, p. 291

Baraffe I., Chabrier G., Allard F., Hauschildt P. H., Evolutionary models for solar metallicity low-mass stars: mass-magnitude relationships and color-magnitude diagrams, A\&A, 1998, vol. 337, p. 403

Baranne A., Queloz D., Mayor M., Adrianzyk G., Knispel G., Kohler D., Lacroix D., Meunier J.-P., Rimbaud G., Vin A., ELODIE: A spectrograph for accurate radial velocity measurements., A\&AS, 1996, vol. 119, p. 373 
Barbier-Brossat M., Figon P., Catalogue général de vitesses radiales moyennes pour les étoiles galactiques. Mean radial velocities catalog of galactic stars, A\&AS, 2000, vol. 142, p. 217

Barbier-Brossat M., Petit M., Figon P., Third bibliographic catalogue of stellar radial velocities (Text in French)., A\&AS, 1994, vol. 108, p. 603

Basri G., Martin E. L., Bertout C., The lithium resonance line in T Tauri stars, A\&A, 1991, vol. 252, p. 625

Batalha C. C., Quast G. R., Torres C. A. O., Pereira P. C. R., Terra M. A. O., Jablonski F., Schiavon R. P., de La Reza J. R., Sartori M. J., Photometric variability of southern T Tauri stars, A\&AS, 1998, vol. 128, p. 561

Bertiau F. C., Absolute Magnitudes of Stars in the Scorpo-Centaurus Association., ApJ, 1958 , vol. 128 , p. 533

Bertout C., T Tauri stars - Wild as dust, ARA\&A, 1989, vol. 27, p. 351

Bertout C., Genova F., A kinematic study of the Taurus-Auriga T association, A\&A, 2006, vol. 460, p. 499

Bertout C., Robichon N., Arenou F., Revisiting Hipparcos data for pre-main sequence stars, A\&A, 1999, vol. 352, p. 574

Bertout C., Siess L., Cabrit S., The evolution of stars in the Taurus-Auriga T association, A\&A, 2007, vol. 473, p. L21

Bessell M. S., Brett J. M., JHKLM photometry - Standard systems, passbands, and intrinsic colors, PASP, 1988, vol. 100, p. 1134

Blaauw A., The O Associations in the Solar Neighborhood, ARA\&A, 1964, vol. 2, p. 213

Bobylev V. V., Kinematics of the Gould Belt based on open clusters, Astronomy Letters, 2006, vol. 32 , p. 816

Bontemps S., Andre P., Kaas A. A., Nordh L., Olofsson G., Huldtgren M., Abergel A., Blommaert J., ISOCAM observations of the rho Ophiuchi cloud: Luminosity and mass functions of the pre-main sequence embedded cluster, A\&A, 2001, vol. 372, p. 173 
Bouvier J., Appenzeller I., A magnitude-limited spectroscopic and photometric survey of Rho Ophiuchus X-ray sources, A\&AS, 1992, vol. 92, p. 481

Briceno C., Hartmann L. W., Stauffer J. R., Gagne M., Stern R. A., Caillault J.-P., X-Rays Surveys and the Post-T Tauri Problem, AJ, 1997, vol. 113, p. 740

Brown A., On the Determination of the Convergent Point of a Moving Cluster from Proper Motions., ApJ, 1950, vol. 112, p. 225

Cambrésy L., Mapping of the extinction in giant molecular clouds using optical star counts, A\&A, 1999, vol. 345, p. 965

Carpenter J. M., Mamajek E. E., Hillenbrand L. A., Meyer M. R., Debris Disks in the Upper Scorpius OB Association, ApJ, 2009, vol. 705, p. 1646

Casanova S., Montmerle T., Feigelson E. D., Andre P., ROSAT X-ray sources embedded in the rho Ophiuchi cloud core, ApJ, 1995, vol. 439, p. 752

Chini R., Multicolour photometry of stars in the Ophiuchus dark cloud region, A\&A, 1981, vol. 99 , p. 346

Cieza L., Padgett D. L., Stapelfeldt K. R., Augereau J.-C., Harvey P., Evans II N. J., Merín B., Koerner D., Sargent A., van Dishoeck E. F., Allen L., The Spitzer c2d Survey of Weak-Line T Tauri Stars. II. New Constraints on the Timescale for Planet Building, ApJ, 2007, vol. 667, p. 308

Cieza L. A., Kessler-Silacci J. E., Jaffe D. T., Harvey P. M., Evans II N. J., Evidence for Jand H-Band Excess in Classical T Tauri Stars and the Implications for Disk Structure and Estimated Ages, ApJ, 2005, vol. 635, p. 422

Comerón F., , 2008 The Lupus Clouds. p. 295

Comeron F., Rieke G. H., Burrows A., Rieke M. J., The Stellar Population in the rho Ophiuchi Cluster, ApJ, 1993, vol. 416, p. 185

Comerón F., Spezzi L., López Martí B., A new population of cool stars and brown dwarfs in the Lupus clouds, A\&A, 2009, vol. 500, p. 1045 
Correia S., Zinnecker H., Ratzka T., Sterzik M. F., A VLT/NACO survey for triple and quadruple systems among visual pre-main sequence binaries, A\&A, 2006, vol. 459, p. 909

Cox A. N., Allen's astrophysical quantities, 2000

Crawford I. A., A study of interstellar Nai D absorption lines towards the Lupus molecular clouds, MNRAS, 2000, vol. 317, p. 996

Cutri R. M., Skrutskie M. F., van Dyk S., Beichman C. A., Carpenter J. M., Chester T., Cambresy L., Evans T., Fowler J., Gizis J., Howard E., Huchra J., Jarrett T., Kopan E. L., Kirkpatrick J. D., Light R. M., Marsh K. A., 2MASS All-Sky Catalog of Point Sources (Cutri+ 2003), VizieR Online Data Catalog, 2003, vol. 2246, p. 0

D'Antona F., Mazzitelli I., New pre-main-sequence tracks for M less than or equal to 2.5 solar mass as tests of opacities and convection model, ApJS, 1994, vol. 90, p. 467

de Bruijne J. H. J., A refurbished convergent-point method for finding moving groups in the HIPPARCOS Catalogue, MNRAS, 1999a, vol. 306, p. 381

de Bruijne J. H. J., Structure and colour-magnitude diagrams of Scorpius OB2 based on kinematic modelling of Hipparcos data, MNRAS, 1999b, vol. 310, p. 585

de Bruijne J. H. J., Science performance of Gaia, ESA's space-astrometry mission, Ap\&SS, 2012, vol. 341 , p. 31

de Bruijne J. H. J., Hoogerwerf R., de Zeeuw P. T., A Hipparcos study of the Hyades open cluster. Improved colour-absolute magnitude and Hertzsprung-Russell diagrams, A\&A, 2001, vol. 367 , p. 111

de Geus E. J., Interactions of stars and interstellar matter in Scorpio Centaurus, A\&A, 1992, vol. 262 , p. 258

de Geus E. J., Bronfman L., Thaddeus P., A CO survey of the dark clouds in Ophiuchus, A\&A, 1990, vol. 231, p. 137

de Geus E. J., de Zeeuw P. T., Lub J., Physical parameters of stars in the Scorpio-Centaurus OB association, A\&A, 1989, vol. 216, p. 44 
de la Reza R., Jilinski E., Ortega V. G., Dynamical Evolution of the TW Hydrae Association, AJ, 2006, vol. 131, p. 2609

de Zeeuw P. T., Hoogerwerf R., de Bruijne J. H. J., Brown A. G. A., Blaauw A., A HIPPARCOS Census of the Nearby OB Associations, AJ, 1999, vol. 117, p. 354

Dehnen W., The Effect of the Outer Lindblad Resonance of the Galactic Bar on the Local Stellar Velocity Distribution, AJ, 2000, vol. 119, p. 800

Dehnen W., Binney J. J., Local stellar kinematics from HIPPARCOS data, MNRAS, 1998, vol. 298 , p. 387

Dubath P., Reipurth B., Mayor M., Radial and rotational velocities of young stars in Chamaeleon and Lupus., A\&A, 1996, vol. 308, p. 107

Ducourant C., Teixeira R., Hambly N. C., Oppenheimer B. R., Hawkins M. R. S., Rapaport M., Modolo J., Lecampion J. F., Trigonometric parallaxes of high velocity halo white dwarf candidates, A\&A, 2007, vol. 470, p. 387

Ducourant C., Teixeira R., Périé J. P., Lecampion J. F., Guibert J., Sartori M. J., Pre-main sequence star Proper Motion Catalogue, A\&A, 2005, vol. 438, p. 769

Duflot M., Figon P., Meyssonnier N., Vitesses radiales. Catalogue WEB: Wilson Evans Batten. Subtittle: Radial velocities: The Wilson-Evans-Batten catalogue., A\&AS, 1995, vol. 114, p. 269

Dzib S., Loinard L., Mioduszewski A. J., Boden A. F., Rodríguez L. F., Torres R. M., VLBA Determination of the Distance to Nearby Star-forming Regions. IV. A Preliminary Distance to the Proto-Herbig AeBe Star EC 95 in the Serpens Core, ApJ, 2010, vol. 718, p. 610

Dzib S., Loinard L., Rodríguez L. F., Mioduszewski A. J., Torres R. M., VLBA Determination of the Distance to Nearby Star-forming Regions. VI. The Distance to the Young Stellar Object HW 9 in Cepheus A, ApJ, 2011, vol. 733, p. 71

Eggen O. J., Star Streams and Galactic Structure, AJ, 1996, vol. 112, p. 1595

Elias J. H., An infrared study of the Ophiuchus dark cloud, ApJ, 1978, vol. 224, p. 453 
Elmegreen B. G., Lada C. J., Sequential formation of subgroups in OB associations, ApJ, 1977, vol. 214 , p. 725

ESA The Hipparcos and Tycho Catalogues (ESA 1997), VizieR Online Data Catalog, 1997, vol. 1239, p. 0

Evans II N. J., Dunham M. M., Jørgensen J. K., Enoch M. L., Merín B., van Dishoeck E. F., Alcalá J. M., Myers P. C., Stapelfeldt K. R., Huard T. L., Allen L. E., Harvey P. M., van Kempen T., Blake G. A., Koerner D. W., The Spitzer c2d Legacy Results: Star-Formation Rates and Efficiencies; Evolution and Lifetimes, ApJS, 2009, vol. 181, p. 321

Fabricius C., Makarov V. V., Knude J., Wycoff G. L., Henry Draper catalogue identifications for Tycho-2 stars, A\&A, 2002, vol. 386, p. 709

Feast M., Whitelock P., Galactic kinematics of Cepheids from HIPPARCOS proper motions, MNRAS, 1997, vol. 291, p. 683

Feigelson E. D., Dispersed T Tauri Stars and Galactic Star Formation, ApJ, 1996, vol. 468, p. 306

Franco G. A. P., The interstellar medium in the solar vicinity - Analysis of the distribution of colour excesses E(b-y) towards 10 selected areas, A\&A, 1990, vol. 227, p. 499

Franco G. A. P., On the infrared void in the Lupus dark clouds, MNRAS, 2002, vol. 331, p. 474

Fricke W., Schwan H., Lederle T., Bastian U., Bien R., Burkhardt G., Du Mont B., Hering R., Jährling R., Jahreiß H., Röser S., Schwerdtfeger H.-M., Walter H. G., Fifth fundamental catalogue (FK5). Part 1: The basic fundamental stars, Veroeffentlichungen des Astronomischen Rechen-Instituts Heidelberg, 1988, vol. 32, p. 1

Fux R., Order and chaos in the local disc stellar kinematics induced by the Galactic bar, A\&A, 2001, vol. 373, p. 511

Gagné M., Skinner S. L., Daniel K. J., Simultaneous Chandra and Very Large Array Observations of Young Stars and Protostars in $\rho$ Ophiuchus Cloud Core A, ApJ, 2004, vol. 613 , p. 393 
Galli P. A. B., Teixeira R., Ducourant C., Bertout C., Benevides-Soares P., A new method for calculating the convergent point of a moving group, A\&A, 2012, vol. 538, p. A23

Geers V., Scholz A., Jayawardhana R., Lee E., Lafrenière D., Tamura M., Substellar Objects in Nearby Young Clusters (SONYC). II. The Brown Dwarf Population of $\rho$ Ophiuchi, ApJ, 2011, vol. 726, p. 23

Ghez A. M., McCarthy D. W., Patience J. L., Beck T. L., The Multiplicity of Pre-MainSequence Stars in Southern Star-forming Regions, ApJ, 1997, vol. 481, p. 378

Girard T. M., van Altena W. F., Zacharias N., Vieira K., Casetti-Dinescu D. I., Castillo D., Herrera D., Lee Y. S., Beers T. C., Monet D. G., López C. E., The Southern Proper Motion Program. IV. The SPM4 Catalog, AJ, 2011, vol. 142, p. 15

Gontcharov G. A., Pulkovo Compilation of Radial Velocities for 35495 Hipparcos stars in a common system, Astronomy Letters, 2006, vol. 32, p. 759

Grankin K. N., Melnikov S. Y., Bouvier J., Herbst W., Shevchenko V. S., Results of the ROTOR-program. I. The long-term photometric variability of classical T Tauri stars, A\&A, 2007, vol. 461, p. 183

Gras-Velázquez À., Ray T. P., Weak-line T Tauri stars: circumstellar disks and companions. I. Spectral energy distributions and infrared excesses, A\&A, 2005, vol. 443, p. 541

Grasdalen G. L., Strom K. M., Strom S. E., A 2-MICRON Map of the Ophiuchus DarkCloud Region, ApJ, 1973, vol. 184, p. L53

Green R. M., Spherical astronomy, 1985

Greene T. P., Meyer M. R., An Infrared Spectroscopic Survey of the rho Ophiuchi Young Stellar Cluster: Masses and Ages from the H-R Diagram, ApJ, 1995, vol. 450, p. 233

Greene T. P., Young E. T., Near-infrared observations of young stellar objects in the Rho Ophiuchi dark cloud, ApJ, 1992, vol. 395, p. 516

Gregorio-Hetem J., Lepine J. R. D., Quast G. R., Torres C. A. O., de La Reza R., A search for T Tauri stars based on the IRAS point source catalog., AJ, 1992, vol. 103, p. 549 
Grenier S., Burnage R., Faraggiana R., Gerbaldi M., Delmas F., Gómez A. E., Sabas V., Sharif L., Radial velocities of HIPPARCOS southern B8-F2 type stars, A\&AS, 1999, vol. 135 , p. 503

Grosso N., Montmerle T., Bontemps S., André P., Feigelson E. D., X-rays and regions of star formation: a combined ROSAT-HRI/near-to-mid IR study of the rho Oph dark cloud, A\&A, 2000, vol. 359, p. 113

Gudennavar S. B., Bubbly S. G., Preethi K., Murthy J., A Compilation of Interstellar Column Densities, ApJS, 2012, vol. 199, p. 8

Guenther E. W., Esposito M., Mundt R., Covino E., Alcalá J. M., Cusano F., Stecklum B., Pre-main sequence spectroscopic binaries suitable for VLTI observations, A\&A, 2007, vol. 467, p. 1147

Hara A., Tachihara K., Mizuno A., Onishi T., Kawamura A., Obayashi A., Fukui Y., A Study of Dense Cloud Cores and Star Formation in Lupus: $\mathrm{C}^{18} \mathrm{O} \mathrm{J}=1-0$ Observations with NANTEN, PASJ, 1999, vol. 51, p. 895

Hartmann L., Accretion Processes in Star Formation: Second Edition. Cambridge University Press, 2009

Henize K. G., H $\alpha$-EMISSION Stars Associated with a Dark Lane in Lupus., ApJ, 1954, vol. 119 , p. 459

Herbig G. H., The Spectra of Be- and Ae-TYPE Stars Associated with Nebulosity, ApJS, 1960, vol. 4, p. 337

Herbig G. H., Bell K. R., Third Catalog of Emission-Line Stars of the Orion Population : $3: 1988,1988$

Høg E., Fabricius C., Makarov V. V., Urban S., Corbin T., Wycoff G., Bastian U., Schwekendiek P., Wicenec A., The Tycho-2 catalogue of the 2.5 million brightest stars, A\&A, 2000, vol. 355, p. L27

Holl B., Lindegren L., Error characterization of the Gaia astrometric solution. I. Mathematical basis of the covariance expansion model, A\&A, 2012, vol. 543, p. A14 
Hoogerwerf R., Aguilar L. A., Identification of moving groups and member selection using HIPPARCOS data, MNRAS, 1999, vol. 306, p. 394

Hughes J., Hartigan P., Clampitt L., The distance to the Lupus star formation region, AJ, 1993, vol. 105 , p. 571

Hughes J., Hartigan P., Krautter J., Kelemen J., The stellar population of the Lupus clouds, AJ, 1994, vol. 108, p. 1071

Imanishi K., Tsujimoto M., Koyama K., X-Ray Detection from Bona Fide and Candidate Brown Dwarfs in the $\rho$ Ophiuchi Cloud with Chandra, ApJ, 2001, vol. 563, p. 361

James D. J., Melo C., Santos N. C., Bouvier J., Fundamental properties of pre-main sequence stars in young, southern star forming regions: metallicities, A\&A, 2006, vol. 446, p. 971

Johnson D. R. H., Soderblom D. R., Calculating galactic space velocities and their uncertainties, with an application to the Ursa Major group, AJ, 1987, vol. 93, p. 864

Jones B. F., Herbig G. H., Proper motions of T Tauri variables and other stars associated with the Taurus-Auriga dark clouds, AJ, 1979, vol. 84, p. 1872

Jones D. H. P., The kinematics of the Scorpio-Centaurus association and Gould's belt., MNRAS, 1971, vol. 152, p. 231

Joy A. H., T Tauri Variable Stars., ApJ, 1945, vol. 102, p. 168

Kamata Y., Koyama K., Tsuboi Y., Yamauchi S., X-Ray Analysis of the rho Ophiuchi Dark Cloud with ASCA: Source Identification, X-Ray Spectra, and Temporal Variability, PASJ, 1997, vol. 49, p. 461

Kaufer A., Stahl O., Tubbesing S., Nørregaard P., Avila G., Francois P., Pasquini L., Pizzella A., Commissioning FEROS, the new high-resolution spectrograph at La-Silla., The Messenger, 1999, vol. 95, p. 8

Kenyon S. J., Hartmann L., Pre-Main-Sequence Evolution in the Taurus-Auriga Molecular Cloud, ApJS, 1995, vol. 101, p. 117 
Kharchenko N. V., Scholz R.-D., Piskunov A. E., Röser S., Schilbach E., Astrophysical supplements to the ASCC-2.5: Ia. Radial velocities of 55000 stars and mean radial velocities of 516 Galactic open clusters and associations, Astronomische Nachrichten, 2007, vol. 328 , p. 889

Knude J., Depth of the Lupus I - VI complex. In IAU Symposium , vol. 254 of IAU Symposium, 2009, p. 35P

Knude J., Distances to nearby molecular clouds and star forming regions.III. Localizing extinction jumps with a Hipparcos calibration of 2mass photometry, ArXiv e-prints, 2010

Knude J., Hog E., Interstellar reddening from the HIPPARCOS and TYCHO catalogues. I. Distances to nearby molecular clouds and star forming regions, A\&A, 1998, vol. 338, p. 897

Knude J., Nielsen A. S., V - (V-I) distance to Lupus 2, A\&A, 2001, vol. 373, p. 714

Krautter J., Wichmann R., Schmitt J. H. M. M., Alcala J. M., Neuhauser R., Terranegra L., New "weak-line"-T Tauri stars in Lupus, A\&AS, 1997, vol. 123, p. 329

Lepine J. R. D., Duvert G., Star formation by infall of high velocity clouds on the galactic disk, A\&A, 1994, vol. 286, p. 60

Loinard L., Torres R. M., Mioduszewski A. J., Rodríguez L. F., A Preliminary VLBA Distance to the Core of Ophiuchus, with an Accuracy of 4\%, ApJ, 2008, vol. 675, p. L29

Lombardi M., Lada C. J., Alves J., 2MASS wide field extinction maps. II. The Ophiuchus and the Lupus cloud complexes, A\&A, 2008a, vol. 489, p. 143

Lombardi M., Lada C. J., Alves J., Hipparcos distance estimates of the Ophiuchus and the Lupus cloud complexes, A\&A, 2008b, vol. 480, p. 785

López Martí B., Jiménez-Esteban F., Solano E., A proper motion study of the Lupus clouds using Virtual Observatory tools, A\&A, 2011, vol. 529, p. A108

Loren R. B., Wootten A., A massive prestellar molecular core and adjacent compression front in the Rho Ophiuchi cloud, ApJ, 1986, vol. 306, p. 142 
Luhman K. L., Rieke G. H., Low-Mass Star Formation and the Initial Mass Function in the $\rho$ Ophiuchi Cloud Core, ApJ, 1999, vol. 525, p. 440

Madsen K., Nielsen H., Tingleff O., Methods for non-linear least squares problems, 2nd edition, 2004

Madsen S., Dravins D., Lindegren L., Astrometric radial velocities. III. Hipparcos measurements of nearby star clusters and associations, A\&A, 2002, vol. 381, p. 446

Makarov V. V., Signatures of Dynamical Star Formation in the Ophiuchus Association of Pre-Main-Sequence Stars, ApJ, 2007a, vol. 670, p. 1225

Makarov V. V., The Lupus Association of Pre-Main-Sequence Stars: Clues to Star Formation Scattered in Space and Time, ApJ, 2007b, vol. 658, p. 480

Malaroda S., Levato H., Galliani S., Stellar radial velocities bibliographic catalog (Malaroda+, 2006), VizieR Online Data Catalog, 2006, vol. 3249

Mamajek E. E., On the distance to the Ophiuchus star-forming region, Astronomische Nachrichten, 2008, vol. 329, p. 10

Mamajek E. E., Lawson W. A., Feigelson E. D., The $\eta$ Chamaeleontis Cluster: Origin in the Sco-Cen OB Association, ApJ, 2000, vol. 544, p. 356

Mamajek E. E., Meyer M. R., Liebert J., Post-T Tauri Stars in the Nearest OB Association, AJ, 2002, vol. 124, p. 1670

Martin E. L., Montmerle T., Gregorio-Hetem J., Casanova S., Spectroscopic classification of X-ray selected stars in the rho Ophiuchi star-forming region and vicinity, MNRAS, 1998, vol. 300, p. 733

Mathieu R. D., The dynamical evolution of young clusters and associations, Highlights of Astronomy, 1986, vol. 7, p. 481

McClure M. K., Furlan E., Manoj P., Luhman K. L., Watson D. M., Forrest W. J., Espaillat C., Calvet N., D’Alessio P., Sargent B., Tobin J. J., Chiang H.-F., The Evolutionary State of the Pre-main Sequence Population in Ophiuchus: A Large Infrared Spectrograph Survey, ApJS, 2010, vol. 188, p. 75 
Melo C. H. F., The short period multiplicity among T Tauri stars, A\&A, 2003, vol. 410, p. 269

Merín B., Jørgensen J., Spezzi L., Alcalá J. M., Evans II N. J., Harvey P. M., Prusti T., Chapman N., Huard T., van Dishoeck E. F., Comerón F., The Spitzer c2d Survey of Large, Nearby, Interstellar Clouds. XI. Lupus Observed with IRAC and MIPS, ApJS, 2008, vol. 177 , p. 551

Montmerle T., Koch-Miramond L., Falgarone E., Grindlay J. E., Einstein observations of the Rho Ophiuchi dark cloud - an X-ray Christmas tree, ApJ, 1983, vol. 269, p. 182

Mortier A., Oliveira I., van Dishoeck E. F., Spectroscopic properties of young stellar objects in the Lupus molecular clouds, MNRAS, 2011, vol. 418, p. 1194

Navarro J. F., Helmi A., Freeman K. C., The Extragalactic Origin of the Arcturus Group, ApJ, 2004, vol. 601, p. L43

Nordström B., Mayor M., Andersen J., Holmberg J., Pont F., Jørgensen B. R., Olsen E. H., Udry S., Mowlavi N., The Geneva-Copenhagen survey of the Solar neighbourhood. Ages, metallicities, and kinematic properties of $14000 \mathrm{~F}$ and G dwarfs, A\&A, 2004, vol. 418, p. 989

Nozawa S., Mizuno A., Teshima Y., Ogawa H., Fukui Y., A study of (C-13)O cloud cores in Ophiuchus, ApJS, 1991, vol. 77, p. 647

Ozawa H., Grosso N., Montmerle T., The X-ray emission from Young Stellar Objects in the $\rho$ Ophiuchi cloud core as seen by XMM-Newton., A\&A, 2005, vol. 429, p. 963

Perryman M. A. C., Brown A. G. A., Lebreton Y., Gomez A., Turon C., Cayrel de Strobel G., Mermilliod J. C., Robichon N., Kovalevsky J., Crifo F., The Hyades: distance, structure, dynamics, and age, A\&A, 1998, vol. 331, p. 81

Petrie R. M., The Calculation of the Convergent of a moving Cluster of Small apparent diameter, MNRAS, 1949, vol. 109, p. 693

Porras A., Christopher M., Allen L., Di Francesco J., Megeath S. T., Myers P. C., A Catalog of Young Stellar Groups and Clusters within 1 Kiloparsec of the Sun, AJ, 2003, vol. 126 , p. 1916 
Prato L., A Survey for Young Spectroscopic Binary K7-M4 Stars in Ophiuchus, ApJ, 2007, vol. 657 , p. 338

Preibisch T., The reliability of age measurements for Young Stellar Objects from Hertzsprung-Russell or color-magnitude diagrams, Research in Astronomy and Astrophysics, 2012, vol. 12, p. 1

Preibisch T., Guenther E., Zinnecker H., Sterzik M., Frink S., Roeser S., A lithium-survey for pre-main sequence stars in the Upper Scorpius OB association, A\&A, 1998, vol. 333, p. 619

Preibisch T., Mamajek E., , 2008 The Nearest OB Association: Scorpius-Centaurus (Sco OB2). p. 235

Preibisch T., Zinnecker H., The History of Low-Mass Star Formation in the Upper Scorpius OB Association, AJ, 1999, vol. 117, p. 2381

Press W. H., Teukolsky S. A., Vetterling W. T., Flannery B. P., Numerical recipes in C. The art of scientific computing, 1992

Quast G. R., Torres C. A. P. C. O., de La Reza R., Mello G. F. P., Isolated post-T Tauri stars., Rev. Mexicana Astron. Astrofis., 1987, vol. 14, p. 360

Reipurth B., Pedrosa A., Lago M. T. V. T., H $\alpha$ emission in pre-main sequence stars. I. an atlas of line profiles., A\&AS, 1996, vol. 120, p. 229

Ridge N. A., Di Francesco J., Kirk H., Li D., Goodman A. A., Alves J. F., Arce H. G., Borkin M. A., Caselli P., Foster J. B., Heyer M. H., Johnstone D., Kosslyn D. A., Lombardi M., Pineda J. E., Schnee S. L., Tafalla M., The COMPLETE Survey of StarForming Regions: Phase I Data, AJ, 2006, vol. 131, p. 2921

Roeser S., Bastian U., A new star catalogue of SAO type, A\&AS, 1988, vol. 74, p. 449

Roeser S., Demleitner M., Schilbach E., The PPMXL Catalog of Positions and Proper Motions on the ICRS. Combining USNO-B1.0 and the Two Micron All Sky Survey (2MASS), AJ, 2010, vol. 139, p. 2440

Roman N. G., The Ursa Major Group., ApJ, 1949, vol. 110, p. 205 
Sartori M. J., Lépine J. R. D., Dias W. S., Formation scenarios for the young stellar associations between galactic longitudes $1=280 \mathrm{degr}-360 \mathrm{degr}$, A\&A, 2003, vol. 404, p. 913

Sartori M. J., Lepine J. R. D., Dias W. S., Kinematics and HR Diagrams of Southern Young Stars (Sartori+ 2003), VizieR Online Data Catalog, 2003, vol. 340, p. 40913

Savage B. D., Mathis J. S., Observed properties of interstellar dust, ARA\&A, 1979, vol. 17, p. 73

Schwan H., The distance and main sequence of the Hyades cluster based on 145 stars with highly accurate proper motions obtained from work on the catalogues FK 5 and PPM, A\&A, 1991, vol. 243, p. 386

Schwartz R. D., A survey of southern dark clouds for Herbig-Haro objects and H-alpha emission stars, ApJS, 1977, vol. 35, p. 161

Seares F. H., Regression Lines and the Functional Relation. II. Charlier's Formulae for a Moving Cluster., ApJ, 1945, vol. 102, p. 366

Setiawan J., Pasquini L., da Silva L., Hatzes A., von der Luhe O., Kaufer A., Girardi L., de La R. R., de Medeiros J. R., A study of the activity of G and K giants through their precise radial velocity. Breaking the 10-m/sec accuracy with FEROS., The Messenger, 2000, vol. 102, p. 13

Setiawan J., Pasquini L., da Silva L., von der Lühe O., Hatzes A., Precise radial velocity measurements of $\mathrm{G}$ and $\mathrm{K}$ giants. First results, A\&A, 2003, vol. 397, p. 1151

Siess L., Dufour E., Forestini M., An internet server for pre-main sequence tracks of lowand intermediate-mass stars, A\&A, 2000, vol. 358, p. 593

Smart W. M., Stellar kinematics, 1968

Sterzik M. F., Durisen R. H., Escape of T Tauri stars from young stellar systems., A\&A, 1995, vol. 304, p. L9 
Strom K. M., Strom S. E., Edwards S., Cabrit S., Skrutskie M. F., Circumstellar material associated with solar-type pre-main-sequence stars - A possible constraint on the timescale for planet building, AJ, 1989, vol. 97, p. 1451

Strom S. E., Optical and Infrared Observations of Young Stellar Objects - an Informal Review, PASP, 1972, vol. 84, p. 745

Struve O., Rudkjøbing M., Stellar Spectra with Emission Lines in the Obscuring Clouds of Ophiuchus and Scorpius., ApJ, 1949, vol. 109, p. 92

Tachihara K., Dobashi K., Mizuno A., Ogawa H., Fukui Y., 13CO (J=1-0) Observations of the Lupus Molecular Clouds, PASJ, 1996, vol. 48, p. 489

Tachihara K., Toyoda S., Onishi T., Mizuno A., Fukui Y., Neuhäuser R., ${ }^{12}$ CO Molecular Cloud Survey and Global Star Formation in Lupus, PASJ, 2001, vol. 53, p. 1081

Teixeira R., Galli P. A. B., Benevides-Soares P., Le Campion J. F., Fidêncio M., KroneMartins A., Ducourant C., Sinachopoulos D., Proper motion and densification of the International Celestial Reference Frame in the direction of the Galactic bulge, A\&A, 2011, vol. 534, p. A91

The P.-S., On faint H-alpha emission stars in Lupus and Scorpius., Contributions from the Bosscha Observervatory, 1962, vol. 15, p. 0

Torres C. A. O., Quast G., de La Reza R., Gregorio-Hetem J., Lepine J. R. D., Search for T Tauri Stars Based on the IRAS Point Source Catalog. II., AJ, 1995, vol. 109, p. 2146

Torres C. A. O., Quast G. R., da Silva L., de La Reza R., Melo C. H. F., Sterzik M., Search for associations containing young stars (SACY). I. Sample and searching method, A\&A, 2006, vol. 460, p. 695

Torres R. M., Loinard L., Mioduszewski A. J., Boden A. F., Franco-Hernández R., Vlemmings W. H. T., Rodríguez L. F., VLBA Determination of the Distance to nearby Star-forming Regions. V. Dynamical Mass, Distance, and Radio Structure of V773 Tau A, ApJ, 2012, vol. 747, p. 18 
Torres R. M., Loinard L., Mioduszewski A. J., Rodríguez L. F., VLBA Determination of the Distance to Nearby Star-Forming Regions. III. HP TAU/G2 and the Three-Dimensional Structure of Taurus, ApJ, 2009, vol. 698, p. 242

Trumpler R. J., Weaver H. F., Statistical astronomy, 1953

Turon C., Luri X., Masana E., Building the cosmic distance scale: from Hipparcos to Gaia, Ap\&SS, 2012, p. 71

Udry S., Mayor M., Queloz D., Towards a New Set of High-Precision Radial-Velocity Standard Stars. In IAU Colloq. 170: Precise Stellar Radial Velocities, vol. 185 of Astronomical Society of the Pacific Conference Series, 1999, p. 367

Valenti J. A., Fischer D. A., Spectroscopic Properties of Cool Stars (SPOCS). I. 1040 F, G, and K Dwarfs from Keck, Lick, and AAT Planet Search Programs, ApJS, 2005, vol. 159, p. 141

van Leeuwen F., Validation of the new Hipparcos reduction, A\&A, 2007, vol. 474, p. 653

Vrba F. J., Schmidt G. D., Burke Jr. E. W., The infrared development of Nova Aquilae 1975., ApJ, 1977, vol. 211, p. 480

Vrba F. J., Strom K. M., Strom S. E., Grasdalen G. L., Further study of the stellar cluster embedded in the Ophiuchus dark cloud complex, ApJ, 1975, vol. 197, p. 77

Wahhaj Z., Cieza L., Koerner D. W., Stapelfeldt K. R., Padgett D. L., Case A., Keller J. R., Merín B., Evans II N. J., Harvey P., Sargent A., van Dishoeck E. F., Allen L., Blake G., Brooke T., Chapman N., Mundy L., Myers P. C., The Spitzer c2d Survey of Weak-line T Tauri Stars. III. The Transition from Primordial Disks to Debris Disks, ApJ, 2010, vol. 724, p. 835

Walter F. M., Vrba F. J., Mathieu R. D., Brown A., Myers P. C., X-ray sources in regions of star formation. 5: The low mass stars of the Upper Scorpius association, AJ, 1994, vol. 107 , p. 692

Weise P., Search for planets around young stars with the radial velocity technique, University of Heidelberg, 2010, Tese de Doutorado 
Weise P., Launhardt R., Setiawan J., Henning T., Rotational velocities of nearby young stars, A\&A, 2010, vol. 517, p. A88

White R. J., Gabor J. M., Hillenbrand L. A., High-Dispersion Optical Spectra of Nearby Stars Younger Than the Sun, AJ, 2007, vol. 133, p. 2524

Wichmann R., Bastian U., Krautter J., Jankovics I., Rucinski S. M., HIPPARCOS observations of pre-main-sequence stars, MNRAS, 1998, vol. 301, p. L39

Wichmann R., Covino E., Alcalá J. M., Krautter J., Allain S., Hauschildt P. H., Highresolution spectroscopy of ROSAT-discovered weak-line T Tauri stars near Lupus, MNRAS, 1999, vol. 307, p. 909

Wichmann R., Krautter J., Covino E., Alcala J. M., Neuhaeuser R., Schmitt J. H. M. M., The T Tauri star population in the Lupus star forming region., A\&A, 1997, vol. 320, p. 185

Wichmann R., Sterzik M., Krautter J., Metanomski A., Voges W., T Tauri stars and the Gould Belt near Lupus., A\&A, 1997, vol. 326, p. 211

Wilking B. A., Gagné M., Allen L. E., , 2008 Star Formation in the $\rho$ Ophiuchi Molecular Cloud. p. 351

Wilking B. A., Lada C. J., The discovery of new embedded sources in the centrally condensed core of the Rho Ophiuchi dark cloud - The formation of a bound cluster, ApJ, 1983, vol. 274, p. 698

Wilking B. A., Lada C. J., Young E. T., IRAS observations of the Rho Ophiuchi infrared cluster - Spectral energy distributions and luminosity function, ApJ, 1989, vol. 340, p. 823

Wilking B. A., Meyer M. R., Robinson J. G., Greene T. P., Optical Spectroscopy of the Surface Population of the $\rho$ Ophiuchi Molecular Cloud: The First Wave of Star Formation, AJ, 2005, vol. 130, p. 1733

Wilking B. A., Schwartz R. D., Blackwell J. H., An H-alpha emission-line survey of the rho Ophiuchi dark cloud complex, AJ, 1987, vol. 94, p. 106 
Zacharias N., Finch C. T., Girard T. M., Henden A., Bartlett J. L., Monet D. G., Zacharias M. I., UCAC4 Catalogue (Zacharias+, 2012), VizieR Online Data Catalog, 2012, vol. 1322, p. 0

Zacharias N., Urban S. E., Zacharias M. I., Wycoff G. L., Hall D. M., Monet D. G., Rafferty T. J., The Second US Naval Observatory CCD Astrograph Catalog (UCAC2), AJ, 2004, vol. 127, p. 3043 
ApÊNDICE 



\section{Apêndice A}

\section{LISTA DE ACRÔNIMOS}

Para simplificar a leitura deste documento introduzimos acrônimos ao longo do texto. Nos títulos de capítulos, seções e parágrafos utilizamos o nome por extenso. Visando uma proximidade maior com os trabalhos publicados na literatura (em particular, aqueles que resultaram da tese de doutorado) optamos por utilizar os acrônimos e as unidades de cada grandeza estudada em inglês. Abaixo apresentamos uma lista dos acrônimos utilizados:

- CP: ponto de convergência (do inglês, convergent point)

- CPSM: método do ponto de convergência (do inglês, convergent point search method)

- CTTS: estrela T Tauri clássica (do inglês, classical T Tauri star)

- DP: ponto de divergência (do inglês, divergent point)

- BD: anã marrom (do inglês, brown dwarf)

- HAeBe: estrela Ae/Be de Herbig (do inglês, Herbig Ae/Be star)

- LCC: Centaurus-Crux Inferior (do inglês, Lower Centaurus Crux)

- MG: grupo de estrelas em movimento (do inglês, moving group)

- Myr: um milhão de anos (1 Myr = $10^{6}$ anos $)$

- PMS: pré-sequência principal (do inglês, pre-main sequence)

- r.m.s.: valor eficaz (do inglês, root mean square)

- Sco-Cen: associação Scorpius-Centaurus 
- TTS: estrela T Tauri (do inglês, T Tauri star)

- UCL: Centaurus-Lupus Superior (do inglês, Upper Centaurus Lupus)

- US: Scorpius Superior (do inglês, Upper Scorpius)

- WTTS: estrela T Tauri fraca (do inglês, weak-emission line T Tauri star)

- YSO: objeto estelar jovem (do inglês, young stellar object)

- ZAMS: sequência principal de idade zero (do inglês, zero age main sequence) 
Apêndice B

A new method for calculating the convergent point of a moving group

Phillip Andreas Brenner Galli, Ramachrisna Teixeira, Christine Ducourant, Claude Bertout e Paulo Benevides-Soares.

Publicado no periódico Astronomy \& Astrophyiscs, vol. 538, p. A23 (2012) 


\title{
A new method for calculating the convergent point of a moving group
}

\author{
P. A. B. Galli ${ }^{1}$, R. Teixeira ${ }^{1}$, C. Ducourant ${ }^{2}$, C. Bertout ${ }^{3}$, and P. Benevides-Soares ${ }^{1}$ \\ 1 Instituto de Astronomia, Geofísica e Ciências Atmosféricas, Universidade de São Paulo, Rua do Matão 1226, Cidade Universitária, \\ 05508-900 São Paulo, SP, Brazil \\ e-mail: galli@astro.iag.usp.br \\ 2 Observatoire Aquitain des Sciences de l'Univers, CNRS-UMR 5804, BP 89, Floirac, France \\ ${ }^{3}$ Institut d'Astrophysique, 98bis Bd. Arago, 75014 Paris, France
}

Received 14 October 2011 / Accepted 23 November 2011

\begin{abstract}
Context. Convergent point (CP) search methods are important tools for studying the kinematic properties of open clusters and young associations whose members share the same spatial motion.

Aims. We present a new CP search strategy based on proper motion data. We test the new algorithm on synthetic data and compare it with previous versions of the $\mathrm{CP}$ search method. As an illustration and validation of the new method we also present an application to the Hyades open cluster and a comparison with independent results.

Methods. The new algorithm rests on the idea of representing the stellar proper motions by great circles over the celestial sphere and visualizing their intersections as the $\mathrm{CP}$ of the moving group. The new strategy combines a maximum-likelihood analysis for simultaneously determining the $\mathrm{CP}$ and selecting the most likely group members and a minimization procedure that returns a refined $\mathrm{CP}$ position and its uncertainties. The method allows one to correct for internal motions within the group and takes into account that the stars in the group lie at different distances.

Results. Based on Monte Carlo simulations, we find that the new CP search method in many cases returns a more precise solution than its previous versions. The new method is able to find and eliminate more field stars in the sample and is not biased towards distant stars. The CP solution for the Hyades open cluster is in excellent agreement with previous determinations.
\end{abstract}

Key words. methods: statistical - open clusters and associations: general - astrometry - methods: analytical - proper motions open clusters and associations: individual: Hyades

\section{Introduction}

Ever since their discovery, the existence of stellar groups with common space motion in the solar neighborhood has been an intriguing issue whose understanding is still far from complete. The origin and evolution of these comoving groups of stars, usually referred to simply as moving groups, is explained by different scenarios including cluster disruption, dynamical effects, and accretion events (Eggen 1996; Dehnen 2000; Fux 2001; Navarro et al. 2004). Moving groups, which are observed as overdensities in the velocity space and exhibit a low internal velocity dispersion, typically a few $\mathrm{km} \mathrm{s}^{-1}$ or less (Mathieu 1986), allow study of the large-scale structure and dynamics of the Milky Way (Antoja et al. 2008). Because of perspective effects, the proper motions of comoving stars ${ }^{1}$ appear to converge to a single point in the celestial sphere referred to as the convergent point (CP) of the moving cluster. The $\mathrm{CP}$ is important not only for determining which stars are actual members of a moving cluster, but also for deriving individual kinematic distances of moving group members, provided that their radial velocities are known. This is very valuable when the trigonometric parallax from the ground is not

\footnotetext{
1 In fact, the proper motions of a group of stars converge to a vertex either if their space motions are parallel or if they are expanding uniformly from a moving point. These two dynamical states are strictly equivalent as far as proper motions are concerned and radial velocity information is needed to distinguish between them (Blaauw 1964).
}

accessible and HIPPARCos parallaxes are not available (for recent applications of this strategy see Mamajek 2005; Bertout \& Genova 2006).

The first algorithms implementing a method for calculating the CP coordinates come from Charlier and Bohlin in 1916 (see Smart 1968). Each of them derived an equation independently using the position angle of stars to determine the CP position on the celestial sphere. However, the constant least-square coefficients involved in solving these equations were subject to measurement errors, thus leading to systematic errors in the derived CP coordinates. Later, Seares (1945), Petrie (1949) and Roman (1949) proposed different ways of correcting the Charlier and Bohlin equations. Their strategies represent, in a first approximation, different ways of using the position angle of stars to determine the $\mathrm{CP}$ solution.

Another approach was proposed by Brown (1950). He introduced a reference frame with the origin approximately at the center of the cluster and a fundamental plane defined by drawing a great circle through the origin in the direction of the average proper motion of the cluster. The proper motion of each star is then resolved into two components, one parallel to the reference plane and the other one perpendicular to the same plane, with the former expected to be much larger than the latter. The coordinates of the $\mathrm{CP}$ were then derived by applying the method of maximum likelihood. 
Based on this method, Jones (1971, hereafter J71) presented a twofold algorithm dedicated to simultaneously selecting the moving group members and calculating the $\mathrm{CP}$ position. This method was later improved and reformulated by de Bruijne (1999, hereafter B99) to make full use of the HIPPARCos data and allow for internal motions within the moving group.

There are many methods of finding the $\mathrm{CP}$ coordinates of moving groups. Several of them use, as J71 and B99 do, the observed proper motions of stars and differ in the details of the search strategy (see for example Makarov \& Robichon 2001; Makarov 2007a,b). Other methods use different observed quantities, such as parallaxes and radial velocities (Chen et al. 1997; Chereul et al. 1999; Hoogerwerf \& Aguilar 1999; Asiain et al. 1999). The choice of which method to use depends essentially on the observational information that is available. The new CP search method that we propose in this article uses the observed proper motions to select the stars that belong to the moving group and determine the $\mathrm{CP}$ position. It builds on the works of J71 and B99, so we give in Sect. 2 some details on these methods that will be useful in presenting our own work.

In the rest of this paper we present and test a new version of the CP search method. Section 3 describes our new algorithm while Sect. 4 deals with the construction of synthetic data from moving group simulations dedicated to testing and investigating the performance of our algorithm in comparison with previous ones. Section 5 presents an application of our method to the Hyades open cluster using HIPPARCos data, and finally Sect. 6 summarizes the results of this work.

\section{The classic CP search method}

\subsection{Jones' CP search method}

In J71, Jones proposed a maximum-likelihood method for simultaneously determining the $\mathrm{CP}$ position and the members of the moving group. To begin with, we consider a moving group of stars with known positions $(\alpha, \delta)$ and proper motions $\left(\mu_{\alpha} \cos \delta, \mu_{\delta}\right)$, which are the two components of the proper motion vector $\boldsymbol{\mu}$. The tangential velocity $\boldsymbol{V}_{\mathrm{t}}\left[\mathrm{km} \mathrm{s}^{-1}\right]$ is written in terms of the parallax $\pi$ [mas] of the star and its proper motion $\mu$ [mas/yr] as

$\left|V_{\mathrm{t}}\right|=\frac{A|\mu|}{\pi}$

where $A=4.74047 \mathrm{~km} \mathrm{yr} / \mathrm{s}$ is the ratio of one astronomical unit in $\mathrm{km}$ to the number of seconds in one Julian year. Furthermore, the radial velocity $\boldsymbol{V}_{\mathrm{r}}\left[\mathrm{km} \mathrm{s}^{-1}\right]$ of a given star is given as a function of the stream space velocity $\boldsymbol{V}\left[\mathrm{km} \mathrm{s}^{-1}\right]$ by

$\left|\boldsymbol{V}_{\mathrm{r}}\right|=|\boldsymbol{V}| \cos \lambda$,

where $\lambda$, the angular distance between the $\mathrm{CP}$ coordinates $\left(\alpha_{\mathrm{cp}}, \delta_{\mathrm{cp}}\right)$ and a given star of the moving group, is computed from

$\cos \lambda=\sin \delta \sin \delta_{\mathrm{cp}}+\cos \delta \cos \delta_{\mathrm{cp}} \cos \left(\alpha_{\mathrm{cp}}-\alpha\right)$.

We recall that $\lambda$ is also the angle between $\boldsymbol{V}$ and $\boldsymbol{V}_{\mathrm{r}}$ for parallel motions, which allows us to calculate the tangential and radial components of the space velocity. The two components of the proper motion $\mu_{\|}$, directed parallel to the great circle that joins the star and the CP, while $\mu_{\perp}$, directed perpendicular to the same great circle (see Fig. 1), can be expressed as

$\left(\begin{array}{c}\mu_{\|} \\ \mu_{\perp}\end{array}\right)=\left(\begin{array}{cc}\sin \theta & \cos \theta \\ -\cos \theta & \sin \theta\end{array}\right)\left(\begin{array}{c}\mu_{\alpha} \cos \delta \\ \mu_{\delta}\end{array}\right)$

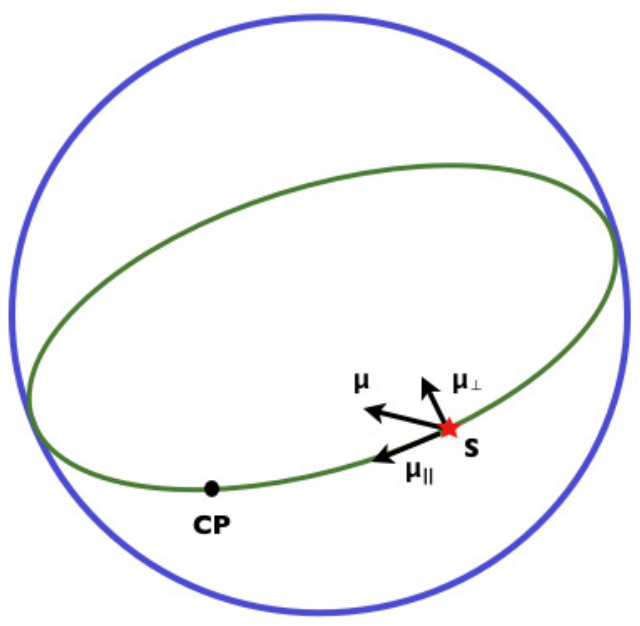

Fig. 1. Schematic representation of the proper motion components parallel and perpendicular to the great circle that contains the star $S$ and the $\mathrm{CP}$ of the moving group.

where the position angle $\theta$ of the $\mathrm{CP}$ is given by

$\tan \theta=\frac{\sin \left(\alpha_{\mathrm{cp}}-\alpha\right)}{\cos \delta \tan \delta_{\mathrm{cp}}-\sin \delta \cos \left(\alpha_{\mathrm{cp}}-\alpha\right)}$.

When strict convergence occurs, i.e., when the proper motion vector is directed exactly towards the $\mathrm{CP}$, we have $\mu_{\perp}=0$ and $\mu_{\|}=\mu$. In practice, strict convergence is not attained because of measurement errors and the internal velocity dispersion within the comoving group of stars.

The basic idea of Jones' method is to determine the CP of the moving group by comparing $\mu_{\perp}$ for each star with its expectation value of zero. The procedure for finding the $\mathrm{CP}$ of a moving group with $N$ stellar candidates starts by overlaying the sky with a grid. Each grid point is a CP candidate whose coordinates are denoted by $\left(\alpha_{\mathrm{cp}}, \delta_{\mathrm{cp}}\right)$. At each grid point one computes the $X^{2}$ value

$X^{2}=\sum_{j=1}^{N} t_{\perp_{j}}^{2}$

where $t_{\perp}=\mu_{\perp} / \sigma_{\perp}$ is the error-weighted value of $\mu_{\perp}$ for each star in the group. Assuming that $t_{\perp}$ is normally distributed, $X^{2}$ is distributed as $\chi^{2}$ with $N-2$ degrees of freedom. Minimizing $X^{2}$ is equivalent to maximizing the likelihood function of the computed $t_{\perp}$ values. Thus, the most likely CP is the grid point with the lowest $X^{2}$ value. However, the lowest $X^{2}$ could still occur by chance, so one should evaluate the probability $\epsilon$ that $X^{2}$ is higher than its observed value. It is given by

$\epsilon=\frac{1}{\Gamma\left[\frac{1}{2}(N-2)\right]} \int_{X^{2}}^{\infty} x^{\frac{1}{2}[N-2]-1} \mathrm{e}^{-x} \mathrm{~d} x$.

If the computed probability is too low, the star with the highest $\left|t_{\perp}\right|$ is rejected. The number of stars in the sample is corrected and one goes back to the first step. This procedure is repeated until $\epsilon$ has reached a preset value (to be discussed later). When this is done, the grid point in the last iteration is defined as the $\mathrm{CP}$ of the moving group, and all remaining stars in the sample are considered to be group members. Thus, determining the $\mathrm{CP}$ of a moving group is linked to the selection of its members. Before 
doing the analysis discussed previously, it is necessary to eliminate stars with low $t_{\perp}$ values, because these stars are not rejected by the method. The rejection criterion is defined as

$$
t=\frac{\mu}{\sigma}=\frac{\sqrt{\mu_{\alpha}^{2} \cos ^{2} \delta+\mu_{\delta}^{2}}}{\sqrt{\sigma_{\mu_{\alpha} \cos \delta}^{2}+\sigma_{\mu_{\delta}}^{2}}} \leq t_{\min },
$$

where $t_{\min }$ is the rejection threshold (see below).

Searching for the CP on a grid of trial points defined on the sky was made necessary because of the limited computer power available in the early 70s. However, the grid-based search represents a robust method of determining the $\mathrm{CP}$ position of a moving group, because it avoids the sometimes complicated topology of the $X^{2}$ function. One disadvantage of this implementation is that it only returns the coordinates of the $\mathrm{CP}$ and gives no information about their errors.

\subsection{De Bruijne's CP search method}

Unlike the Jones method, which does not consider any possible internal motions within the moving group, the "refurbished" CP method developed by B99 also handles this and takes advantage of the much more powerful computing facilities available today. Jones's version of the CP method is modified in three ways and extended to include the determination of individual membership probabilities for each moving group star as explained below.

First, the method is adapted to take advantage of the HIPPARCOS data regarding the propagation of errors. Second, the definition of $t_{\perp}$ is modified to include the velocity dispersion in the moving group. The selection of stars with $t_{\perp}=0$ does not allow one to identify all moving group members, since the proper motions of some are not directed exactly towards the CP because of their velocity dispersion. De Bruijne's method assumes that the velocity dispersion $\sigma_{\mathrm{v}}\left[\mathrm{km} \mathrm{s}^{-1}\right]$ and the mean distance $d$ [pc] of the group are known in advance. Thus, the one-dimensional velocity dispersion $\sigma_{\text {int }}$ [mas/yr] of the group, in proper motion units, is given by

$\sigma_{\mathrm{int}}=\frac{1000 \sigma_{\mathrm{v}}}{A d}$

and the new definition of $t_{\perp}$ becomes

$t_{\perp}=\frac{\mu_{\perp}}{\sqrt{\sigma_{\perp}^{2}+\sigma_{\mathrm{int}}^{2}}}$.

The definition of $t_{\min }$ is also modified to account for $\sigma_{\text {int }}$. It is written

$t_{\min }=\frac{\mu}{\sqrt{\sigma_{\mu}^{2}+\sigma_{\text {int }}^{2}}}$.

Furthermore, the grid-based approach used by $\mathrm{J} 71$ is replaced by a direct minimization routine that returns the $\mathrm{CP}$ position, as well as its uncertainty. Finally, a membership probability $p$ is assigned to each moving group member and takes the velocity dispersion into account. It is given by

$p=\exp \left[-\frac{1}{2}\left(\frac{\mu_{\perp}^{2}}{\sigma_{\perp}^{2}+\sigma_{\text {int }}^{2}}\right)\right]$.

As discussed by B99, the rejection threshold for a given star depends not only on its individual membership probability, but also on the membership probability of all stars considered. Including the velocity dispersion term in the definition of $t_{\perp}$ tends to raise the individual membership probabilities, thus allowing the method to include stars that are not members of the moving group. Therefore, stars with very low membership probability, even if not rejected by the method, should be carefully analyzed. On the other hand, stars with high membership probability (i.e. low $t_{\perp}$ ) should also be verified, because the selection of moving group members is biased towards distant stars that generally have smaller proper motions, thus smaller $\mu_{\perp}$ components.

\section{A new CP search method}

The original implementation of the CP method considers the stellar proper motion vector in two separate steps: a directional decomposition of the proper motion into the components $\mu_{\|}, \mu_{\perp}$ followed by a minimization routine based on the amplitude of $\mu_{\perp}$. Our algorithm differs from previous ones by considering both direction and amplitude of the stellar proper motions at once in the minimization routine. As a result, we will see that the new method is less biased towards more distant stars. We also take into account the internal motions of group members by introducing an individual correction for each star that depends on its proper motion and accounts for the fact that stars within the group lie at different distances. Our method makes an initial guess of the CP position using a grid-based approach that is similar to J71. Once an approximate CP position is found, it is then refined by an analytical minimization routine to return a more accurate solution. This procedure not only guarantees the solution convergence but also returns both the $\mathrm{CP}$ coordinates and their uncertainties. We present below the details of our algorithm.

The apparent motion of cluster members over the celestial sphere takes place along the arcs of great circles. The idea of visualizing the proper motion of a star over a great circle was first introduced by Herschel in 1783 (see Trumpler \& Weaver 1953) and has been used more recently by Abad et al. (2003) and Abad $\&$ Vieira (2005). Let $\boldsymbol{r}=(x, y, z)=(\cos \alpha \cos \delta, \sin \alpha \cos \delta, \sin \delta)$ denote the position of a star with coordinates $(\alpha, \delta)$ and proper motion $\left(\mu_{\alpha}, \mu_{\delta}\right)$, in the usual orthogonal equatorial coordinate system, in which the unit vectors $\hat{\boldsymbol{x}}, \hat{\boldsymbol{y}}, \hat{z}$ point respectively to the vernal equinox, the point on the equator with $\alpha=90^{\circ}$, and the northern equatorial pole. We compute the angular velocity of the star as the time derivative of the position vector

$\dot{\boldsymbol{r}}=\mu_{\alpha} \cos \delta\left(\begin{array}{c}-\sin \alpha \\ \cos \alpha \\ 0\end{array}\right)+\mu_{\delta}\left(\begin{array}{c}-\cos \alpha \sin \delta \\ -\sin \alpha \sin \delta \\ \cos \delta\end{array}\right)$

The great circle representing the motion of a given star is the intersection of the celestial sphere with the plane defined by $r$ and $\dot{\boldsymbol{r}}$ (see Fig. 2). Whenever a group of stars with parallel motions exists, their great circles should intersect at two opposite points on the celestial sphere: the $\mathrm{CP}$ and its mirror point. We denote the mirror point of the $\mathrm{CP}$ in this work as the divergent point (DP) of the moving group. Assuming that $\left(\alpha_{\mathrm{cp}}, \delta_{\mathrm{cp}}\right)$ are the coordinates of the $\mathrm{CP}$, the coordinates of the DP are $\left(\alpha_{\mathrm{dp}}, \delta_{\mathrm{dp}}\right)=\left(\alpha_{\mathrm{cp}}+180^{\circ},-\delta_{\mathrm{cp}}\right)$. From this visualization of the proper motions over the entire sphere one sees that a search for the group vertex will in fact return two geometrically equivalent solutions. The distinction between both solutions depends on the direction of the proper motion vectors. 


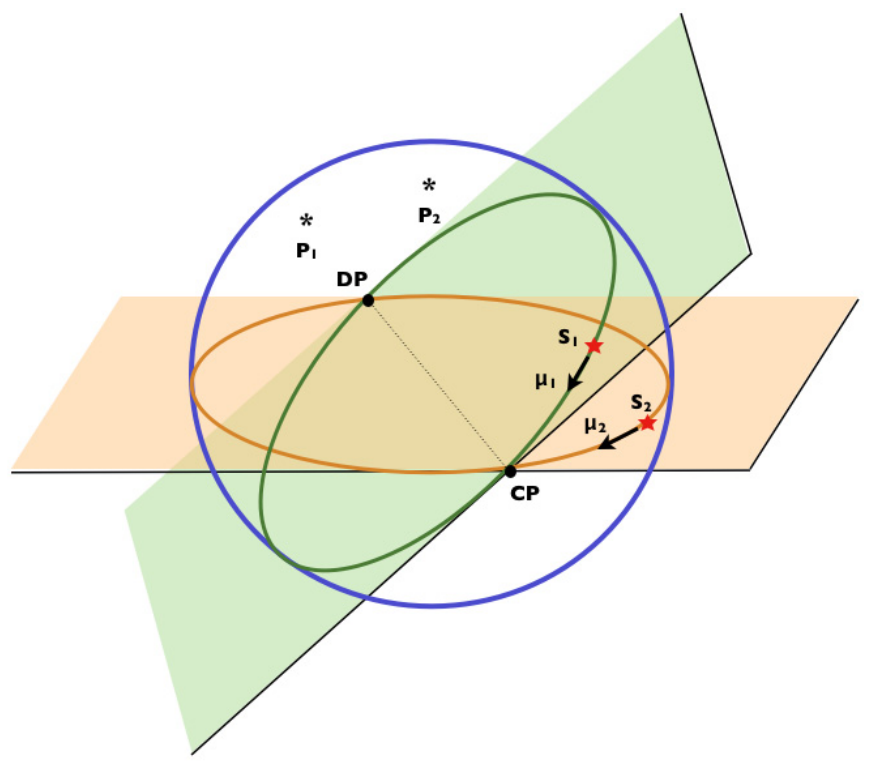

Fig. 2. Schematic representation of the motion over a great circle for two stars $S_{1}$ and $S_{2}$ of the moving group with proper motions $\mu_{1}$ and $\mu_{2}$. The poles of the corresponding great circles are given by $P_{1}$ and $P_{2}$.

The great circle of each moving group member contains the star, the CP (DP) and the direction of motion. It can be described by the vector $\boldsymbol{p}$ normal to the plane defined by $\boldsymbol{r}$ and $\boldsymbol{r}$ :

$\boldsymbol{p}=\boldsymbol{r} \times \dot{\boldsymbol{r}}=\mu_{\alpha} \cos \delta\left(\begin{array}{c}-\cos \alpha \sin \delta \\ -\sin \alpha \sin \delta \\ \cos \delta\end{array}\right)-\mu_{\delta}\left(\begin{array}{c}-\sin \alpha \\ \cos \alpha \\ 0\end{array}\right)$

For each group member, the vector $\boldsymbol{p}$ defines the pole of its great circle on the celestial sphere. The pole representation of each star is unique and includes its position plus its motion. Whenever a group of comoving stars exists, the poles of the great circles of its members are located in a common plane, and the intersection of this plane with the celestial sphere forms another great circle. The poles of this polar great circle are the apex and antapex of the moving group, i.e., its CP and DP. In practice these two points are the intersections of the individual stellar great circles (see Fig. 2). They are calculated by forming the cross products of all poles of members between themselves, $\boldsymbol{p}_{\boldsymbol{i}} \times \boldsymbol{p}_{\boldsymbol{j}}$, when $i$ and $j$ are group members. The distribution of intersection points between the polar great circles of a group of stars can highlight preferred directions of motion and may be used to search for moving group members (see Abad et al. 2003).

\subsection{The new CP search algorithm}

The basic idea of our procedure is to determine the polar great circle that best interpolates the individual great circle poles of the moving group stars. The poles of the interpolated great circle return the $\mathrm{CP}$ and DP solutions of the moving group. To derive a first approximation of the $\mathrm{CP}$ coordinates we follow the grid-based approach developed by $\mathrm{J} 71$, which is adapted to our strategy. For simplicity we describe the algorithm in the equatorial coordinate system, but it is independent of the chosen coordinate system.
Given a sample of $N$ stars with known positions $(\alpha, \delta)$, proper motions $\left(\mu_{\alpha} \cos \delta, \mu_{\delta}\right)$, and corresponding errors, we perform the following steps:

1. Compute the pole $\left(\alpha^{p}, \delta^{p}\right)$ of the great circle for each star in the sample and the corresponding errors $\left(\sigma_{\alpha^{p}}, \sigma_{\delta^{p}}\right)$. The derived expressions are given in Appendix A.

2. Define a grid of trial CPs on the plane of the sky and number each grid point $\left(i=1,2,3, \ldots, N_{\text {grid }}\right)$.

3. Start at grid point $i=1$ and assume that this point is the CP. The coordinates of this point are referred to as $\left(\alpha_{\mathrm{cp}}, \delta_{\mathrm{cp}}\right)$.

4. Calculate for each star the error-weighted value $s$ of the orthogonality error $\kappa$

$$
s=\frac{\kappa}{\sigma_{\kappa}} .
$$

The orthogonality error $\kappa$ is defined as

$\kappa_{j}=\sin \delta_{\mathrm{cp}} \sin \delta_{j}^{p}+\cos \delta_{\mathrm{cp}} \cos \delta_{j}^{p} \cos \left(\alpha_{\mathrm{cp}}-\alpha_{j}^{p}\right)$,

where $j$ runs over all stars in the sample $(j=1,2,3, \ldots, N)$. The derived expressions for $\kappa$ and $\sigma_{\kappa}$ are given in Appendix B. We assume that $s$ is normally distributed with zero mean and unit variance (the validity of this hypothesis will be discussed in Sect. 4.5). The probability distribution for the $\operatorname{star} j$ with a given $s$ is then

$$
p_{j}=\frac{1}{\sqrt{2 \pi}} \exp \left(-\frac{1}{2} s_{j}^{2}\right) .
$$

5. At the given grid point compute $X^{2}$ as

$$
X^{2}=\sum_{j=1}^{N} s_{j}^{2}
$$

6. Determine $X^{2}$ at each grid point by repeating steps 3-5.

7. The total probability $P$ for the set of calculated values is given by

$P=\prod_{j=1}^{N} p_{j}=\frac{1}{(2 \pi)^{N / 2}} \mathrm{e}^{-\frac{1}{2} X^{2}}$

and defines the likelihood function. With $s$ distributed normally, $X^{2}$ is distributed as $\chi^{2}$ with $N-2$ degrees of freedom. Maximizing the likelihood function is equivalent to minimizing $X^{2}$. Define the grid point with the lowest $X^{2}$ as the most likely CP.

8. The lowest $X^{2}$ could still occur by chance rather than by a good fit between the observations and the model. As in J71, evaluate the probability $\epsilon$ that $X^{2}$ will exceed the observed value of $X^{2}$ by chance. It is given by

$$
\epsilon=\frac{1}{\Gamma\left[\frac{1}{2}(N-2)\right]} \int_{X^{2}}^{\infty} x^{\frac{1}{2}[N-2]-1} \mathrm{e}^{-x} \mathrm{~d} x,
$$

where $\Gamma(x)$ denotes the Gamma function for $x>0$.

9. If $\epsilon<\epsilon_{\min }$, that is to say, if the computed value of $X^{2}$ is unacceptably high, reject the star with the highest $|s|$ value, correct the number of stars in the sample $N \rightarrow N-1$, and go back to step 3. Otherwise continue to the next step.

10. When $\epsilon$ has reached an acceptable value (to be discussed in Sect. 4.4), choose the grid point considered in the last iteration as the maximum-likelihood CP and all nonrejected stars in the sample are identified as moving group members. 
The algorithm presented above simultaneously selects moving group members and calculates the CP position. It is based only on position and proper motion data. As in J71, before starting the procedure, it is necessary to reject all stars with proper motion data that carry poor information because of measurement errors.

\subsection{Correction for internal velocity dispersion}

The new CP search algorithm described above selects those stars in the sample as moving group members whose orthogonality error $\kappa$ approaches zero. When $\kappa$ approaches its expected value of zero, the plane defined by the individual poles of stars and the one that passes through the CP and DP solutions are orthogonal. The poles of group members are located on the same plane when the stars in the group have parallel motions. However, perfect parallelism will not necessarily be achieved by some moving group members. Therefore, selecting only those stars with $s=0$ will not identify all group members. Consequently, a small amount of deviation of $s$ due to the velocity dispersion in the group should be permitted.

The procedure that we use to allow for internal motions within the moving group is similar to the one proposed by B99. The definition of $s$ is changed to

$s=\frac{\kappa}{\sqrt{\sigma_{\kappa}^{2}+\Delta \kappa^{2}}}$,

where $\Delta \kappa$ is an estimate of the one-dimensional velocity dispersion in the group that is translated into the proper motion scatter $\left(\Delta \mu_{\alpha}, \Delta \mu_{\delta}\right)$ of group stars. It is given by

$\Delta \kappa=\sqrt{\left(\left|\frac{\partial \kappa}{\partial \mu_{\alpha}^{*}}\right| \Delta \mu_{\alpha}^{*}\right)^{2}+\left(\left|\frac{\partial \kappa}{\partial \mu_{\delta}}\right| \Delta \mu_{\delta}\right)^{2}}$

where $\mu_{\alpha}^{*}=\mu_{\alpha} \cos \delta$. The proper motion scatter in each component is estimated by using Eq. (9) and assuming that the velocity dispersion and the mean distance to the group are known in advance. We constructed synthetic samples of moving groups (as discussed in Sect. 4) with different velocity dispersions and concluded that the scatter observed in the proper motion components is consistent with this estimate given by Eq. (9) and similar in both components. One should note that the term $\Delta \kappa$ proposed in this work to take the internal motions within the group into account differs from the one introduced by B99, because the former is an individual correction applied to each star. Although average group parameters (velocity dispersion and distance) are used, the partial derivatives in Eq. (22) depend on the stellar position and proper motion of each group member. Because we use the proper motions, we account for the various distances of group members. This point is particularly important when dealing with moving groups that occupy a large volume in space.

Following the procedure developed by B99, we then attribute a membership probability to each group member, defined as

$p_{j}=\exp \left[-\frac{1}{2}\left(\frac{\kappa_{j}^{2}}{\sigma_{\kappa_{j}}^{2}+\Delta \kappa_{j}^{2}}\right)\right]$.

\subsection{Refining the CP coordinates}

Once we have found an approximated CP position $\left(\alpha_{\mathrm{cp}}, \delta_{\mathrm{cp}}\right)$ of the moving group as explained above, we refine it by implementing a direct minimization routine in two dimensions that leads to the following nonlinear least square equations that must be solved. We have

$$
\begin{aligned}
& \frac{\partial X^{2}}{\partial \alpha_{\mathrm{cp}}}=0 \rightarrow \sum_{j=1}^{N} s_{j} \frac{\partial s_{j}}{\partial \alpha_{\mathrm{cp}}}=0 \\
& \frac{\partial X^{2}}{\partial \delta_{\mathrm{cp}}}=0 \rightarrow \sum_{j=1}^{N} s_{j} \frac{\partial s_{j}}{\partial \delta_{\mathrm{cp}}}=0 .
\end{aligned}
$$

We follow the Levenberg-Marquardt method to solve these equations as described in Madsen et al. (2004) and Press et al. (1992). The model is approximated at each iteration by a first order Taylor series expansion, and we use the initial guess $\left(\alpha_{\mathrm{cp}}, \delta_{\mathrm{cp}}\right)$ of the CP position to obtain the final coordinates of the $\mathrm{CP}$ iteratively by successive approximations. This method returns the $\mathrm{CP}$ solution and its uncertainty in the form of a $2 \times 2$ covariance matrix. The stopping criterion for our routine is defined by the magnitude of the CP uncertainties in the covariance matrix since iterating to machine accuracy is generally unnecessary.

To simplify the following discussion, we denote the two CP search methods (CPSMs) discussed in Sect. 2 (J71 and B99) collectively by the name classic $C P$ search method, or classic CPSM, and the new method described in Sect. 3 by the name new CP search method, or new CPSM. We emphasize that the definition of the $X^{2}$ function is not the same in the classic and new CPSMs. We used the appropriate definition of $X^{2}$ in the various tests of the classic and new CPSMs that we discuss below. Our minimization routine using the analytical derivatives $\partial X^{2} / \partial \alpha_{\mathrm{cp}}$ and $\partial X^{2} / \partial \delta_{\mathrm{cp}}$ differs from the one used by B99.

\section{Monte Carlo simulations of moving groups}

CPSMs handle two tasks: the search for the most likely moving group members and determination of the $\mathrm{CP}$ position. Detection of group members requires the moving group to be distinguished from the field population by its kinematic properties, whereas the position of the $\mathrm{CP}$ is influenced by several parameters. To investigate both abilities of the CP method, we applied the classic and new CPSMs to synthetic samples of moving groups with different configurations. In our simulations we focused mainly on the differences and advantages of both methods.

\subsection{Construction of synthetic datasets}

We construct our synthetic data using the Galactic coordinates system, which is more convenient, particularly when dealing with Galactic rotation. That is, we define a rectangular coordinate system in which the unit vectors $\hat{\boldsymbol{u}}, \hat{\boldsymbol{v}}, \hat{\boldsymbol{w}}$ point, respectively, to the Galactic center, to the direction of Galactic rotation, and to the Galactic north pole. The Galactic coordinates $(l, b)$ of the stars are randomly drawn in the sky region occupied by the assumed moving group. We vary the Galactic longitude $l$ of the cluster center from $0^{\circ}$ to $360^{\circ}$ in steps of $60^{\circ}$ and assume a constant latitude of $b=0^{\circ}$. Our choice of $b$ is motivated by the fact that young clusters and star-forming regions lie very close to the Galactic plane. The individual distances are drawn from a Gaussian distribution where the mean distance depends on the cluster simulation. The stars are distributed in a distance range of $30 \mathrm{pc}$ projected along the line of sight. We assume three different configurations of distance and vary the projected angular size of the group. Table 1 summarizes the characteristics of our synthetic samples.

The stellar velocity components $\boldsymbol{V}=(u, v, w)$ are randomly drawn from a sphere in velocity space with radius of $20 \mathrm{~km} \mathrm{~s}^{-1}$. 
Table 1. Mean distance, field size, and number of moving group and field stars in the synthetic samples for each configuration $(A, B, C)$ considered in this work.

\begin{tabular}{ccccc}
\hline \hline$\#$ & Distance $(\mathrm{pc})$ & Field size $l \times b\left(\mathrm{deg}^{2}\right)$ & $N_{\mathrm{MG}}$ & $N_{\text {field }}$ \\
\hline $\mathrm{A}$ & 100 & $20 \times 20$ & 200 & 1000 \\
$\mathrm{~B}$ & 200 & $15 \times 15$ & 100 & 500 \\
$\mathrm{C}$ & 400 & $10 \times 10$ & 50 & 250 \\
\hline
\end{tabular}

The three velocity components and position are translated into proper motion $\left(\mu_{l} \cos b, \mu_{b}\right)$ and radial velocity $V_{\mathrm{r}}$ for each star, after which observational errors are added. Position and proper motion errors are also drawn from Gaussian distributions with means of 1 mas and $1 \mathrm{mas} / \mathrm{yr}$, respectively. We then add to the streaming motion of the group three components representing the velocity dispersion, the reflex of the solar motion with respect to the local standard of rest, and the Galactic rotation, in the following manner. Each of the dispersion velocity components $(\delta u, \delta v, \delta w)$ is independently drawn from a Gaussian distribution with an isotropic velocity dispersion of $\sigma_{\mathrm{v}}=1 \mathrm{~km} \mathrm{~s}^{-1}$. Our choice of $\sigma_{\mathrm{v}}$ is motivated by the fact that young stellar groups often exhibit a low-velocity dispersion. For the solar motion we adopt $(U, V, W)_{\odot}=(10.00,5.23,7.17) \mathrm{km} \mathrm{s}^{-1}$ (Dehnen \& Binney 1998) $)^{2}$ and $A=14.82 \mathrm{~km} \mathrm{~s}^{-1} \mathrm{kpc}^{-1}$ and $B=$ $-12.37 \mathrm{~km} \mathrm{~s}^{-1} \mathrm{kpc}^{-1}$ for the Oort constants (Feast \& Whitelock 1997). We introduce Galactic rotation in the simulated stellar proper motions by using the first-order formulae given in Green (1985).

Our synthetic samples of stars include both the moving group stars and a field population. The density of stars in each field corresponds approximately to that of the HIPPARCos cata$\log \left(\sim 3 \mathrm{stars} / \mathrm{deg}^{2}\right)$. Field stars are located in the same field of view and have the same observational errors as moving groups stars. They are unrelated to the moving group and have random motions in amplitude and direction.

Each run consists of 100 Monte Carlo cluster simulations for each Galactic position and distance of the cluster. We follow the procedure adopted by B99 to correct for internal motions by taking the value for $\sigma_{\mathrm{v}}$ that was actually used to construct our synthetic data samples. For both CPSMs, we assume the stop value of the probability $\epsilon$ defined in Eq. (7) to be $\epsilon_{\min }=0.954$, as suggested by B99 (we come back to this in Sect. 4.4). In the following, we investigate and compare both CPSMs using the synthetic data sets. We first investigate the ability of each CPSM to find group members and reject field stars (see Sect. 4.2), then we come to a more detailed analysis of the $\mathrm{CP}$ itself as a function of several parameters (see Sect. 4.3).

\subsection{Selection of cluster members and rejection of field stars}

The CP method selects moving group members based on their proper motions and reject field stars that are not in the same proper motion range. A distinction must be made between field stars at the same distance as the moving group and background stars. To begin with, we construct samples as described in Sect. 4.1 and consider the case where the field population is at the same average distance as the moving group. The results of these simulations are shown in Fig. 3. On the whole, both

\footnotetext{
2 Although a more recent value of the solar motion given by Schönrich et al. (2010) exists, we adopted the value of Dehnen \& Binney (1998), which has been widely used in the literature. The results and conclusions of this paper do not depend on the specific value used for the solar motion.
}

CPSMs exhibit a similar performance even if the fraction of rejected field stars by the new CPSM is higher in some specific cases. Our simulations show that at the shorter distances of Table 1 the fraction of cluster members that can be retrieved for both CPSMs is higher than $\sim 80 \%$ and the contamination of field stars amounts to $\sim 20 \%$ of the total number of field stars. At greater distances, the ability to find and eliminate field stars decreases for both CPSMs, as expected, since the quality of proper motions also diminishes.

We now consider the case where the field population consists of background stars located two times farther than the moving group. We perform the same simulations as before and present the results in Fig. 4. We find that the performance of the two methods differ; the new CPSM is likely to eliminate more background stars than the classic CPSM. The difference between both CPSMs is more evident at greater distances (e.g. at $d=400 \mathrm{pc}$ ) where the distributions of rejected field stars for each CPSM are clearly separated. As already discussed by B99, the classic CPSM is biased toward distant stars. The definition of the $X^{2}$ function to be minimized in the classic CPSM considers only one directional component of the proper motion vector, which leads to a biased selection of stars with small $\mu_{\perp}$ components. However, not all stars with low $\mu_{\perp}$ are necessarily members, because stars at farther distances, such as background stars, generally have smaller proper motions (i.e. small $\mu_{\perp}$ ). On the other hand, the definition of the $X^{2}$ function using great circle poles in the new CPSM considers both direction and amplitude of the proper motion vector at the same time. By considering the full proper motion vector instead of only one directional component (as in the classic CPSM) in the definition of the minimizing function $X^{2}$ leads to a membership selection that is not biased towards distant stars.

\subsection{Convergent point analysis}

As discussed in Sect. 4.2 the CP method is able to identify the moving group with a contamination of field stars that amounts to $\sim 20 \%$ of the total field population in the sample. Although the method retrieves a high fraction of cluster members, the $\mathrm{CP}$ is shifted from its true position due to the contribution of the remaining field stars in the sample. To better investigate the $\mathrm{CP}$ solution itself it is necessary to work on a sample where the moving group is the dominating population. In the following, we set $N_{\mathrm{MG}}=80$ and $N_{\text {field }}=20$ in all configurations $(\mathrm{A}, \mathrm{B}, \mathrm{C})$ of Table 1, construct our samples as described in Sect. 4.1, and present the results of $\mathrm{CP}$ analysis.

\subsubsection{The effect of velocity dispersion on CP positions}

As explained earlier, the velocity dispersion of the cluster prevents the proper motion vectors from pointing exactly towards the CP. Here we investigate how internal velocity dispersion affects both CPSMs by comparing the CP positional accuracy found for moving groups with different values of velocity dispersion. To do so, we construct three sets of synthetic data as described above, with $\sigma_{\mathrm{v}}=1.0,2.0$, and $3.0 \mathrm{~km} \mathrm{~s}^{-1}$. We display in Fig. 5 the CP positions obtained with both CPSMs, overlaid on contour lines of constant $X^{2}$ values for a cluster with no internal velocity dispersion, centered on the same position and distance as the synthetic groups with nonzero internal dispersion velocities. The results shown in Fig. 5 are for a moving group with $(l, b)=\left(180^{\circ}, 0^{\circ}\right)$ and $d=100 \mathrm{pc}$. We find that the CP positions derived by both CPSMs are distributed along an elongated 
P. A. B. Galli et al.: A new method for calculating the convergent point of a moving group

$\mathrm{d}=100 \mathrm{pc}$

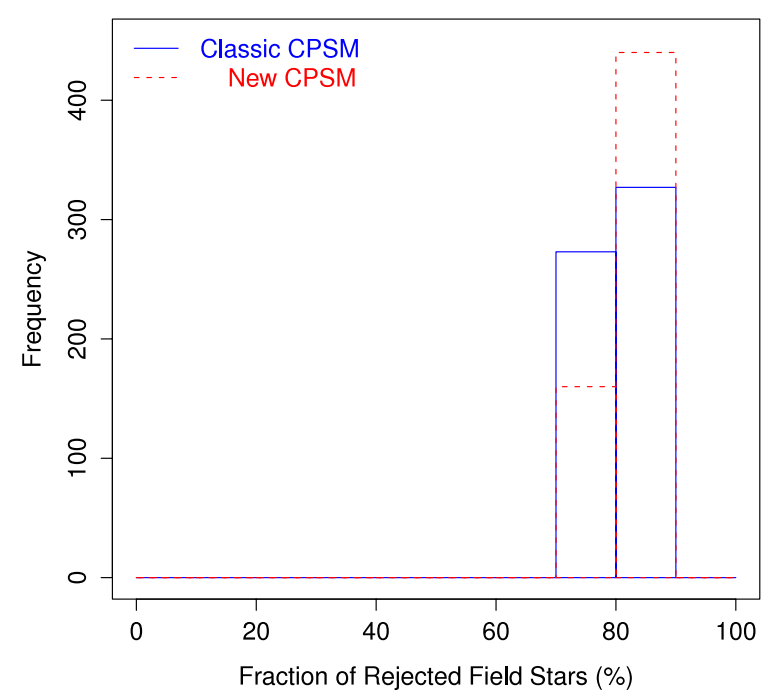

$\mathbf{d}=200 \mathrm{pc}$

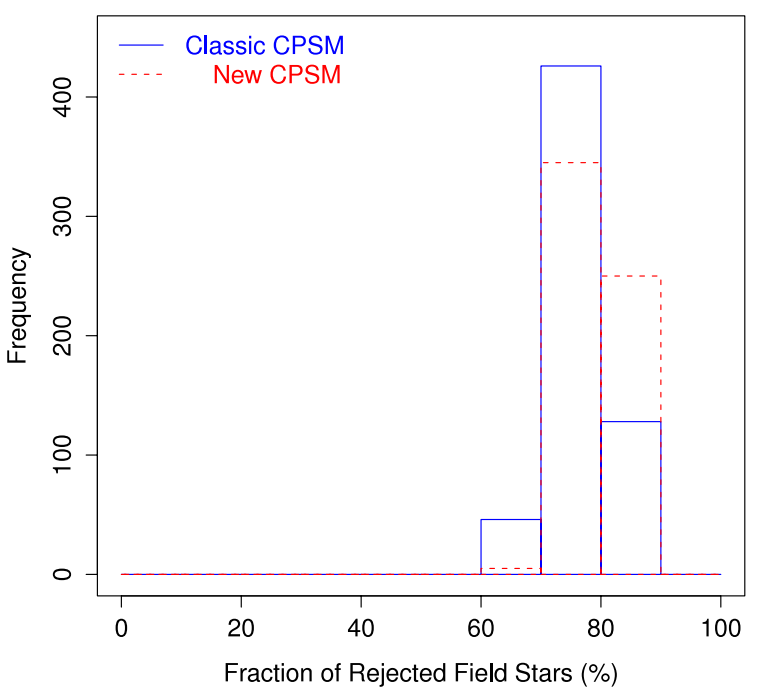

$\mathrm{d}=400 \mathrm{pc}$

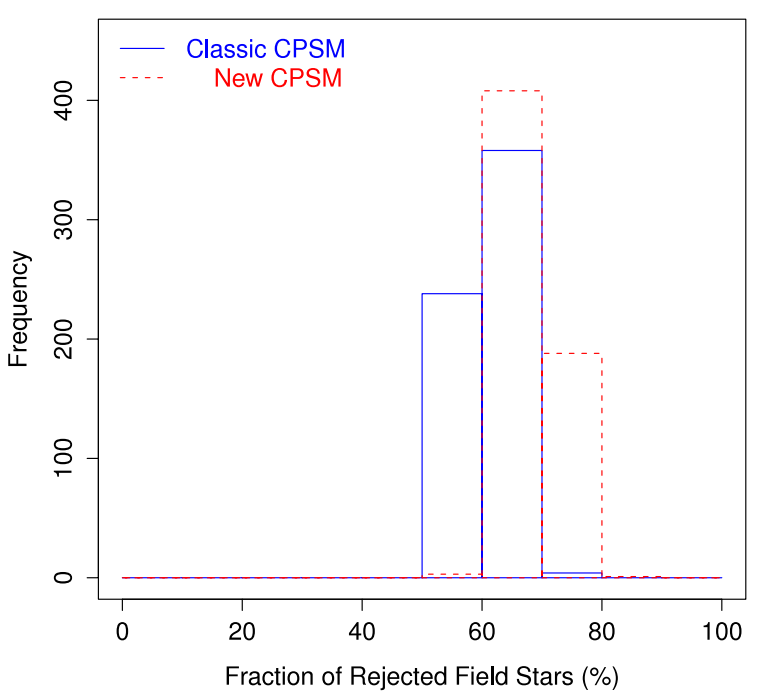

Fig. 3. Fraction of rejected field stars for both CPSMs in the $1800 \mathrm{MC}$ simulations. The field population is assumed to be at the same distance as the moving group. $d=100 p c$

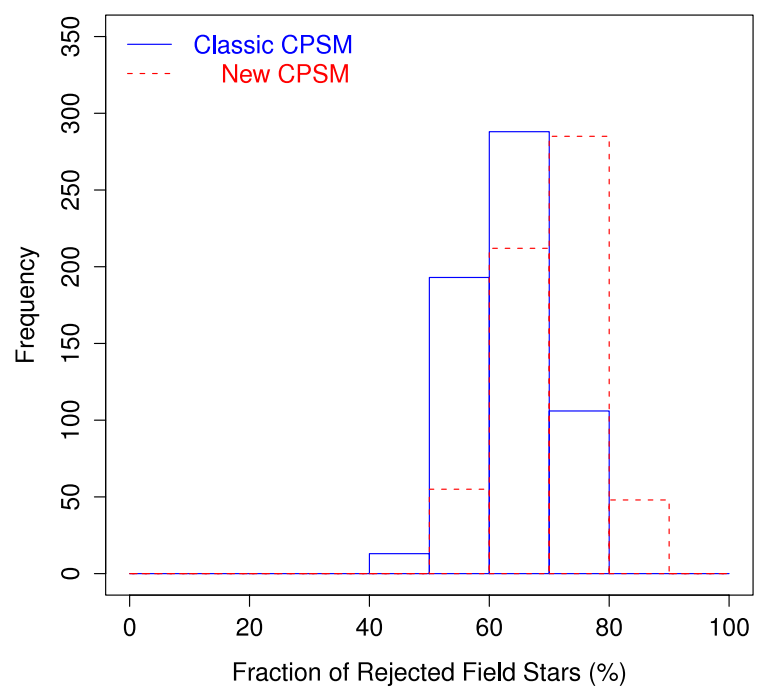

$d=200 p c$

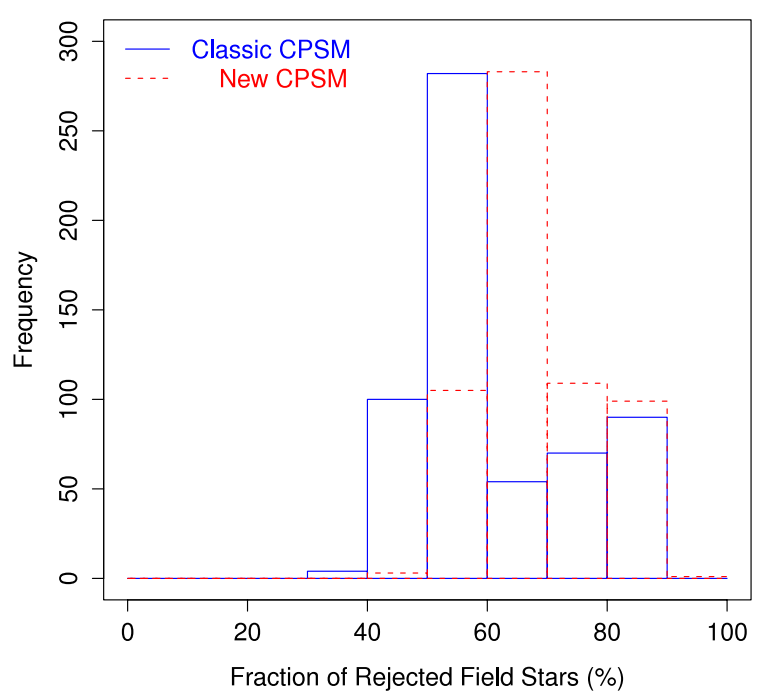

$\mathrm{d}=400 \mathrm{pc}$

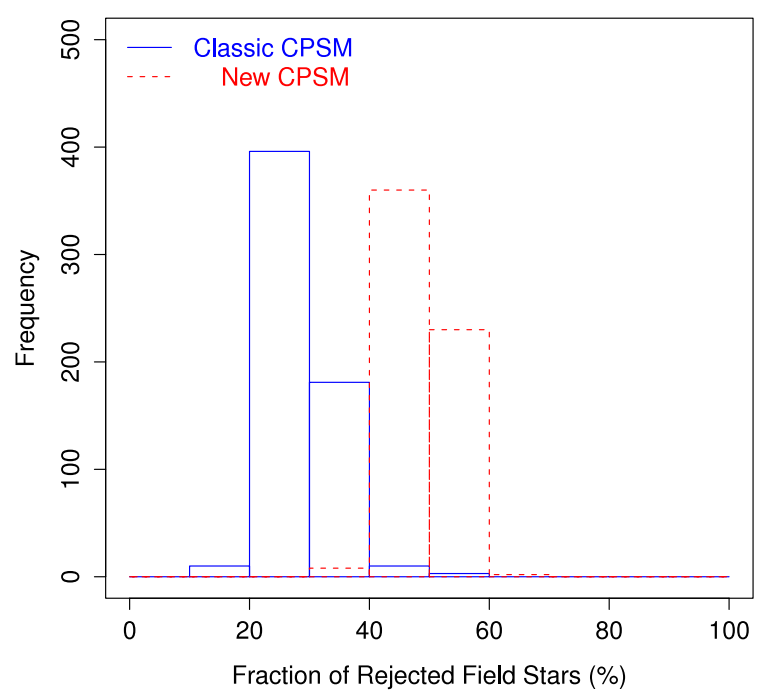

Fig. 4. Fraction of rejected field stars for both CPSMs. The results summarize 1800 MC simulations. The field population consists of background stars at twice the distance of the moving group. 
structure. The great circle on which the CP lies is defined by the proper motion vectors of group members, however the precise location of the $\mathrm{CP}$ over this great circle appears uncertain. As seen in Sect. 3, when strict convergence to the CP occurs, the individual poles of group members lie on a common plane that defines the polar great circle in the celestial sphere. Because of internal motions within the group, the poles are scattered around the polar great circle, and the precise position of the $\mathrm{CP}$ is not well defined, which explains the elongated structure observed in Fig. 5 (see also Bertiau 1958). When comparing both CPSMs, we find that they perform similarly and that the dispersion of $\mathrm{CP}$ positions always grows with the increasing velocity dispersion within the group.

\subsubsection{Precision of the CP position derived by each CPSM}

We now discuss the $\mathrm{CP}$ position uncertainties as derived by the covariance matrix. We compare the CP precision of both CPSMs in terms of the quantity $\Delta \sigma_{\mathrm{cp}}=\sigma_{\mathrm{cp}}^{\text {classic }}-\sigma_{\mathrm{cp}}^{\text {new }}$, where $\sigma_{\mathrm{cp}}$, the combined uncertainty of the $\mathrm{CP}$ position $\left(l_{\mathrm{cp}}, b_{\mathrm{cp}}\right)$ for each method, is given by

$\sigma_{\mathrm{cp}}=\sqrt{\sigma_{l_{\mathrm{cp}}}^{2}+\sigma_{b_{\mathrm{cp}}}^{2}}$

The results of $1800 \mathrm{MC}$ simulations for six values of the Galactic longitude (see above) and three different distances are shown in Fig. 6. When $\Delta \sigma_{\mathrm{cp}}$ (normalized in order to represent only relative values between both CPSMs) is positive, the CP position uncertainties derived by the classic CPSM are higher than the ones obtained with the new CPSM. We find that the new CPSM returns a more precise $\mathrm{CP}$ solution for $95 \%$ of the 1800 simulations. On the other hand, we also observe that the number of cases where $\Delta \sigma_{\mathrm{cp}}<0$, (i.e., where uncertainties in the classic CPSM are lower than in the new CPSM) grows with increasing distance of the moving group. In other words, the new CPSM is less precise than the classic CPSM at large distances, where the higher degree of concentration of the stars affects the determination of the $\mathrm{CP}$ position, defined as the intersection of the individual great circles of group members.

\subsubsection{Role of moving group parameters on the solution accuracy of the new CPSM}

The precision of the $\mathrm{CP}$ position is also influenced by several other parameters such as observational errors on proper motions, number of moving group members, and angular distance from the moving group to the $\mathrm{CP}$. The most efficient way to investigate the effect of each parameter is to consider an ideal cluster model in which the stellar velocity components only result from the streaming motion of the cluster (i.e., the internal velocity dispersion is zero and the effects of Galactic rotation are not considered). We find, as expected, that for an ideal moving group both CPSMs return the same solution, with $X^{2}=0$ and $\epsilon=1$. In the following, we focus on the role of each parameter for the results obtained with the new CPSM. We emphasize that the qualitative results of this investigation, obtained by using an ideal synthetic moving group, depend neither on the specific cluster position $\left(l, 0^{\circ}\right)$ nor on its distance. However, the amplitude of the CP uncertainties as given in Fig. 7 are specific to configuration \#A of Table 1 with the cluster centered at $\left(180^{\circ}, 0^{\circ}\right)$, which we present here as an example to show the relative importance of each moving group parameter in the $\mathrm{CP}$ solution.
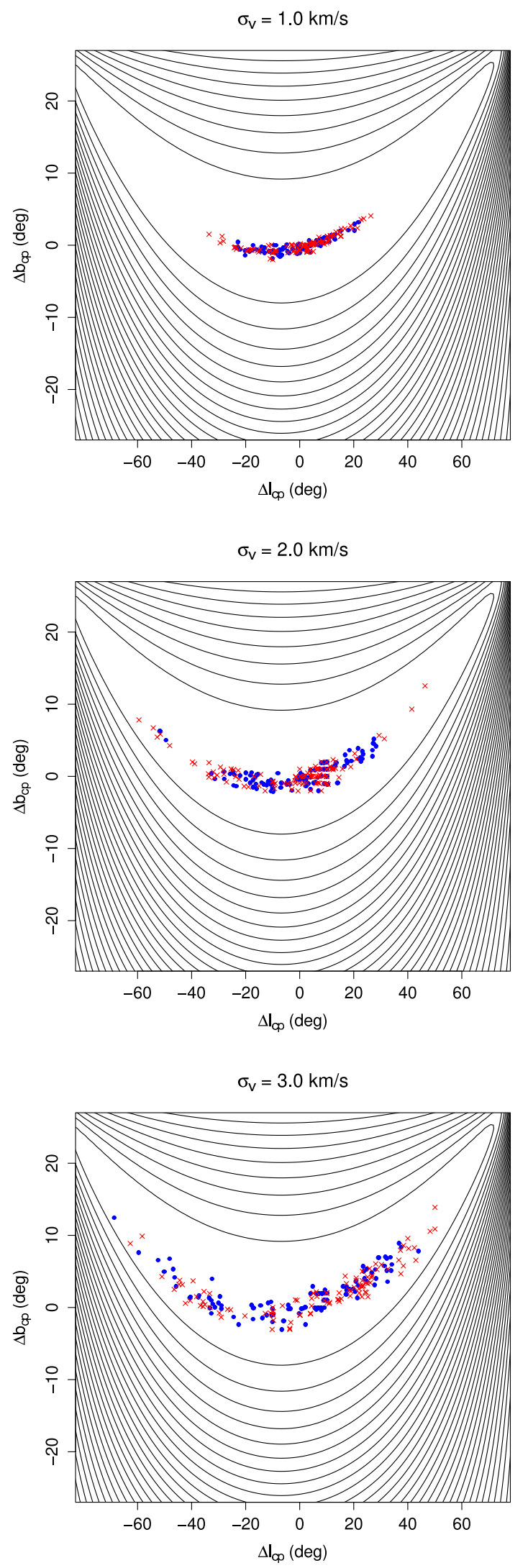

Fig. 5. Relative position $\left(\Delta l_{\mathrm{cp}}, \Delta b_{\mathrm{cp}}\right)$ of the $\mathrm{CP}$ for 100 simulations and three different velocity dispersions. Blue dots and red crosses denote, respectively, the results of the classic and new CPSMs. The contours indicate $X^{2}$ levels linearly spaced by $\Delta X^{2}=2000$ for a cluster with the properties given in the text. The relative position of the computed $\mathrm{CP}$ $\left(l_{\mathrm{cp}}^{\mathrm{sim}}, b_{\mathrm{cp}}^{\mathrm{sim}}\right)$ for each simulation is given by $\Delta l_{\mathrm{cp}}=l_{\mathrm{cp}}^{\mathrm{sim}}-l_{\mathrm{cp}}$ and $\Delta b_{\mathrm{cp}}=$ $b_{\mathrm{cp}}^{\mathrm{sim}}-b_{\mathrm{cp}}$. 
$\mathrm{d}=100 \mathrm{pc}$

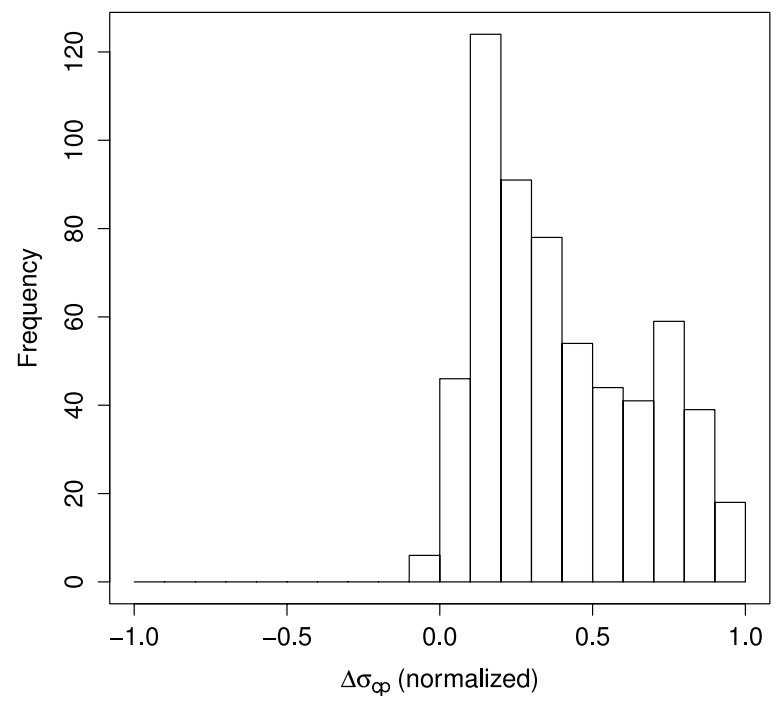

$\mathrm{d}=200 \mathrm{pc}$

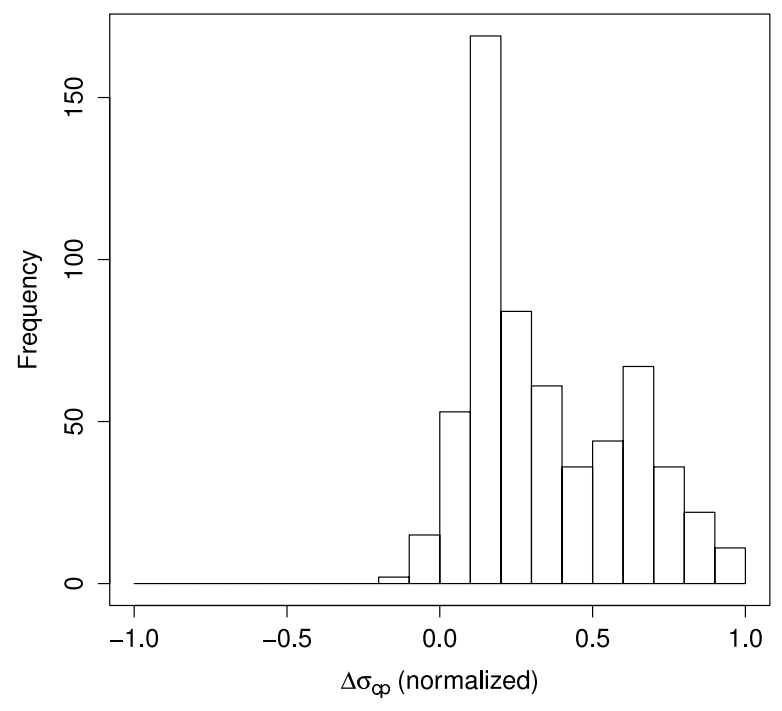

$\mathrm{d}=400 \mathrm{pc}$

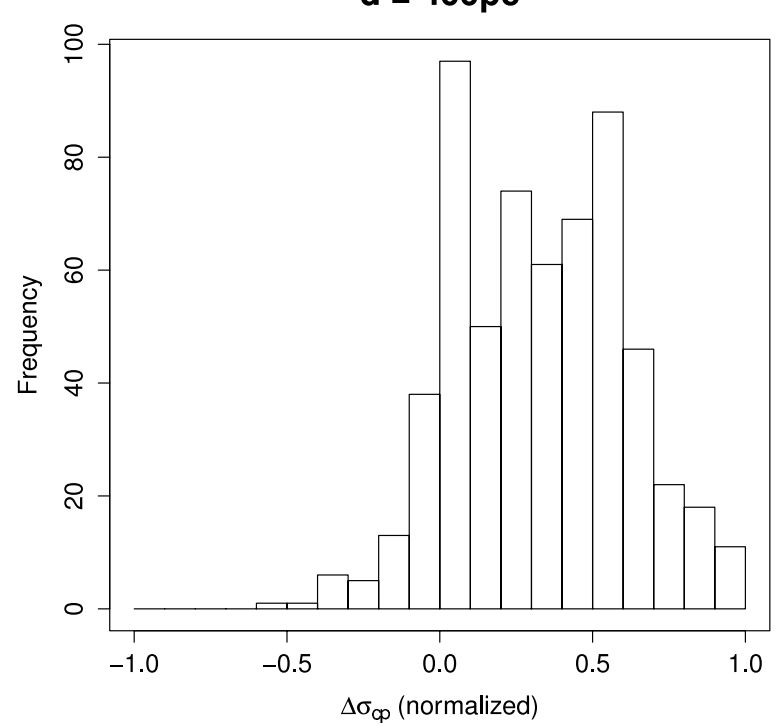

Fig. 6. Comparison of the $\mathrm{CP}$ errors between both methods in the $1800 \mathrm{MC}$ simulations of clusters located at three different distances.
We first investigated the influence of proper motion errors on the $\mathrm{CP}$ position precision. We constructed synthetic samples of an ideal moving group (as defined above) by varying the relative error on proper motion data (in each component) from 5\% to $100 \%$. The results are presented in Fig. $7 \mathrm{a}$ and show that the errors on the $\mathrm{CP}$ position grow linearly with the increasing errors on proper motions.

We now come back to the distance of the moving group and its effect on the $\mathrm{CP}$ position precision. As mentioned above, we expect the distance to influence the $\mathrm{CP}$ position errors since stars at greater distances have smaller proper motions, and the angular size of the group changes with respect to the distance. The question arises whether the precision of the $\mathrm{CP}$ is more affected by the deterioration of proper motion data at larger distances or by cluster concentration. Our numerical simulations make it possible to separate both effects. To do so, we simulate different moving groups varying one of these quantities while the other remains fixed. The results are shown in Figs. $7 b$ and c. We find that cluster concentration is the dominant source of error on the $\mathrm{CP}$ position at large distances.

We investigated the effect of the number of group members used for the $\mathrm{CP}$ position determination. Figure $7 \mathrm{~d}$ shows the results of this computation, where we varied the number of moving group members from 10 to 300 . We find that above $N=200$ no significant improvement in the $\mathrm{CP}$ position precision is achieved.

Finally, we seek to determine the effect of the angular distance from the moving group to the $\mathrm{CP}$. To do so, we construct synthetic data sets by varying the Galactic longitude of the cluster center from $0^{\circ}$ to $360^{\circ}$ in steps of $0.15^{\circ}$. We consider the stellar three-dimensional velocity components to be isotropic, which fixes the $\mathrm{CP}$ position at $\left(45.00^{\circ}, 35.26^{\circ}\right)$. Our results are shown in Figs. 7e and $\mathrm{f}$. We find that moving groups displaced by $180^{\circ}$ have the same $\mathrm{CP}$ errors. This is because their relative position to the nearest solution (CP or DP) is the same. Those groups whose angular distance to the $\mathrm{CP}$ exceed $90^{\circ}$ are closer to the DP than to the CP. We recall that both solutions are always obtained with the same probability, so that the $X^{2}$ values are the same. We conclude that the angular distance between the moving group and the $\mathrm{CP}$ influences the precision of the solution. The least precise $\mathrm{CP}$ solution is obtained for moving groups displaced by $90^{\circ}$ with respect to the $\mathrm{CP}$ (or DP).

\subsection{Choice of $\epsilon_{\min }$}

The final selection of moving group members depends mainly on the choice of $\epsilon_{\min }$, the stop parameter that should allow finding the largest number of moving group members with the least contamination by field stars. So far, we have adopted in our new CP strategy the value of $\epsilon_{\min }=0.954$, which was used by B99 in his implementation of the classic CPSM. This choice allows one to compare the performance of both CPSMs under the same conditions. To do so, we investigated whether the adopted value of $\epsilon_{\min }$ by $\mathrm{B} 99$ is truly the optimum one for our new strategy. To do so, we ran the new CPSM on our synthetic data sets and varied the value of $\epsilon_{\min }$ from 0.00 to 0.99 . We then computed the fraction of true cluster members and field stars in the sample of stars selected by the new CPSM. The results are presented in Fig. 8 for a moving group with $(l, b)=\left(180^{\circ}, 0^{\circ}\right)$ at $d=100 \mathrm{pc}$. Similar conclusions apply to all cluster configurations considered in this work. As already expected, the relative fraction of group members in the sample of nonrejected stars is lower for the classic CPSM. This is because more field stars are accepted by this method. We verified that the number of rejected group members is equivalent for both $\mathrm{CP}$ methods. We find that for 

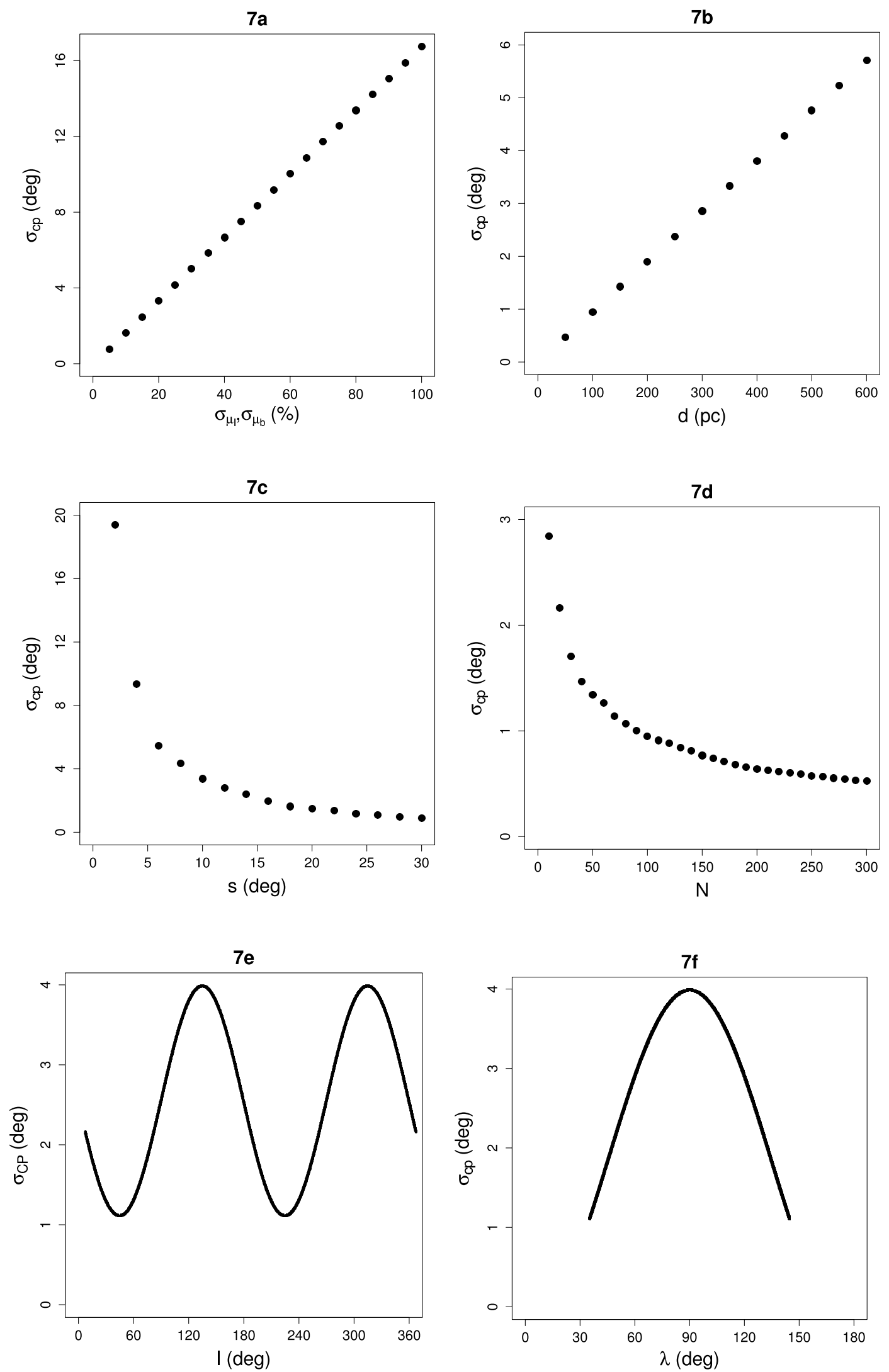

Fig. 7. Accuracy of the $\mathrm{CP}$ position as a function of a) observational errors on proper motions, b) distance with field size fixed, c) field size of the group $(s \times s)$ with fixed distance, d) number of group members, e) galactic longitude of the cluster with isotropic stellar velocity, and f) angular distance $\lambda$ from the $\mathrm{CP}$ to the moving group. 
P. A. B. Galli et al.: A new method for calculating the convergent point of a moving group

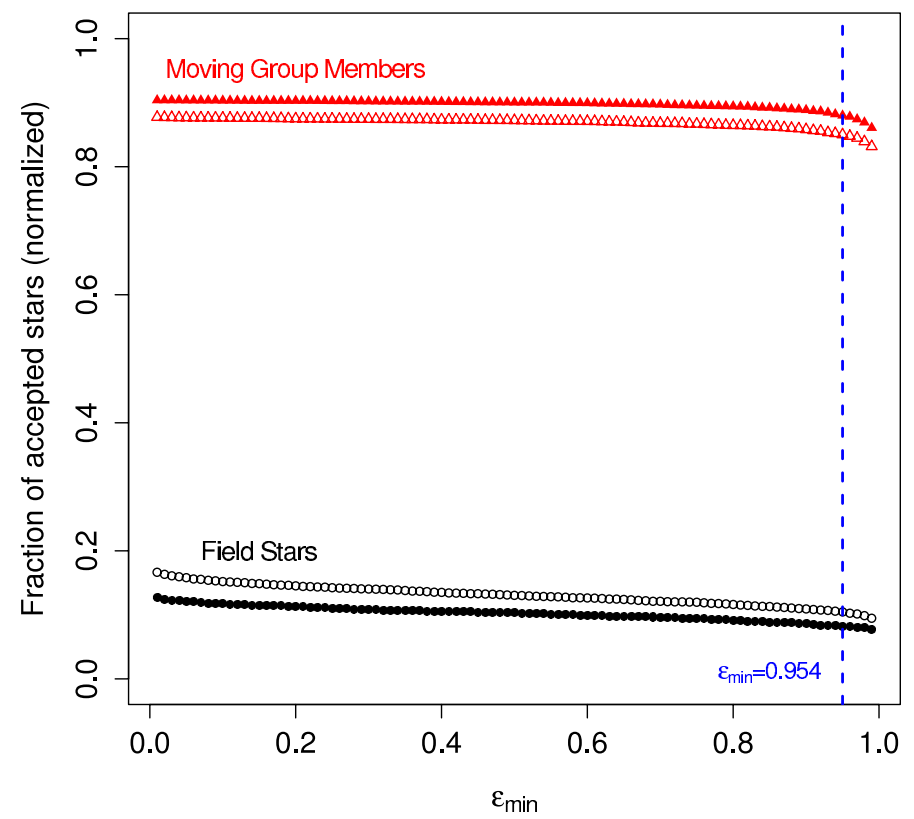

Fig. 8. Fraction of accepted group members and field stars in the sample as a function of the adopted value for $\epsilon_{\min }$. Circles denote field stars and triangles cluster members. Open and filled symbols represent the results obtained with the classic and new CPSMs, respectively. The vertical dashed line marks $\epsilon_{\min }=0.954$. Each point represents the average value of 100 Monte Carlo simulations for a cluster with $(l, b)=\left(180^{\circ}, 0^{\circ}\right)$ and $d=100 \mathrm{pc}$.

$\epsilon_{\min }>0.954$ the number of moving group stars decreases while the fraction of field stars in the sample remains practically constant. Thus, we conclude that the value of $\epsilon_{\min }=0.954$ can also be used in the new CPSM, since it offers the best compromise between the number of accepted group members and rejected field stars.

\subsection{Normality tests applied to the $s$ distribution}

In Sect. 3.1 we assumed that $s$ was normally distributed with zero mean and unit variance. Coming back to this point, we discuss the validity of this hypothesis. We carried out another set of Monte Carlo simulations with different configurations of cluster position, distance, angular size, and velocity dispersion in order to investigate whether the observed $s$ distribution is normal with zero mean and unit variance. The Kolmogorov-Smirnov normality test applied to the synthetic datasets reveals no significant differences between the simulations and the model. We present in Fig. 9 the results of this analysis for 1000 Monte Carlo simulations with different cluster configurations. We observe that the Kolmogorov-Smirnov test gives a $p$-value that is higher than the adopted significance level of $5 \%$ for $99.6 \%$ for our simulated moving groups. Thus, we conclude that the assumption of a normal distribution of $s$ is valid.

\section{Application to the Hyades}

Ever since the discovery that it is a nearby moving group, the Hyades open cluster has played a central role in astronomy in studies of Galactic structure, chemical evolution, distance calibration and stellar evolution models (see for example de Bruijne et al. 2001, and references therein). Here we use the Hyades open cluster as a first application of our new CPSM to real data. We

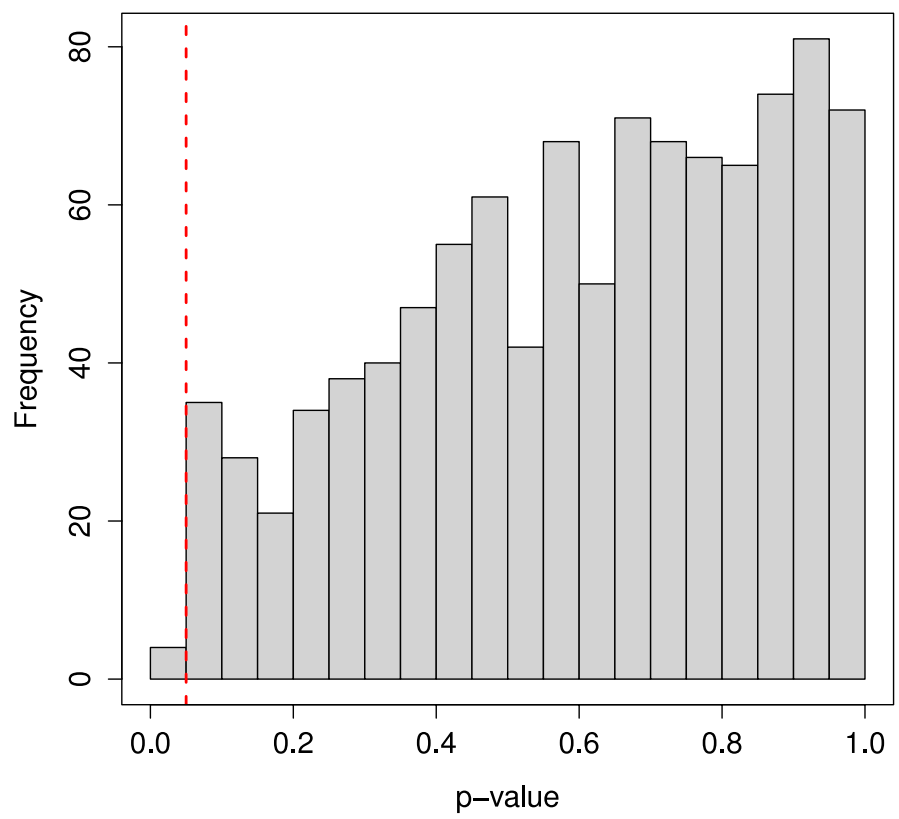

Fig. 9. Results of the Kolmogorov-Smirnov normality test applied to 1000 Monte Carlo simulations of moving groups with different configurations. The red dashed line marks the 5\% level of significance.

present the $\mathrm{CP}$ search using data from the HIPPARCos catalog and compare our results with previous ones.

\subsection{Covariance matrix}

In general astrometric catalogs quote only standard errors on the observables. However, the HIPPARCOS catalog provides for each star a $5 \times 5$ covariance matrix for the measured astrometric parameters (position, proper motion, and parallax) and proper use of the full covariance matrix is mandatory. The new CPSM is adapted to consider the full covariance matrix of the HIPPARCOS data, and the specific formalism used for the propagation of errors is described in Appendix C.

\subsection{CP analysis of the Hyades open cluster}

The sample of Hyades members that we use here consists of 218 stars from HIPPARCos as given in Perryman et al. (1998, hereafter P98), whose membership analysis was based on the three-dimensional velocity of the stars and the structure of the cluster.

As already discussed by B99, a nonzero velocity dispersion is required in order to retrieve all cluster members. We adopt the value $\sigma_{\mathrm{v}}=2.0 \mathrm{~km} \mathrm{~s}^{-1}$ used by B99 and the mean distance of $d=46 \mathrm{pc}$ as given in P98. After applying the new CPSM to the 218 members as the starting sample, the position of the $\mathrm{CP}$ is $\left(\alpha_{\mathrm{cp}}, \delta_{\mathrm{cp}}\right)=\left(97.69^{\circ}, 6.30^{\circ}\right) \pm\left(0.89^{\circ}, 0.36^{\circ}\right)$, and we retrieve 217 cluster members with a correlation coefficient of $\rho=-0.75$. Our solution is in good agreement with B99, $\left(\alpha_{\mathrm{cp}}, \delta_{\mathrm{cp}}\right)=\left(97.81^{\circ}, 6.74^{\circ}\right) \pm\left(0.52^{\circ}, 0.21^{\circ}\right)$ with 213 cluster members and $\rho=-0.84$. Another $\mathrm{CP}$ solution for the Hyades open cluster was found by Schwan (1991, hereafter S91), who obtained $\left(\alpha_{\mathrm{cp}}, \delta_{\mathrm{cp}}\right)=\left(97.68^{\circ}, 5.98^{\circ}\right) \pm\left(0.42^{\circ}, 0.18^{\circ}\right)$ using only 145 stars and proper motions from the FK5 and PPM catalogs. Figure 10 illustrates the proper motion vectors of the Hyades members and the derived CP coordinates. We find 


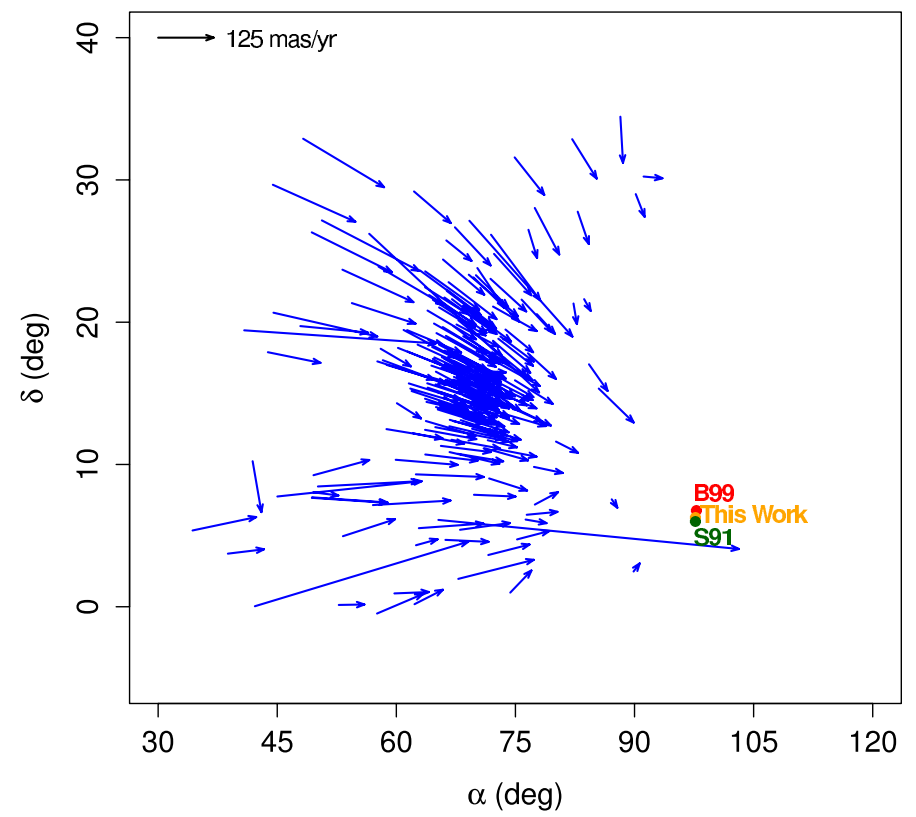

Fig. 10. Proper motion vectors of the Hyades members and the CP coordinates derived in different works. The $\mathrm{CP}$ error bars are too small to be seen.

that only one star, HIP 28774, was rejected in our analysis as a nonmember of the Hyades open cluster. Its proper motion is given as $\left(\mu_{\alpha} \cos \delta, \mu_{\delta}\right)=(-2.46,-7.00) \pm(18.43,9.99) \mathrm{mas} / \mathrm{yr}$ in the original HIPPARCOS catalog (ESA 1997, hereafter HIP97) and as $\left(\mu_{\alpha} \cos \delta, \mu_{\delta}\right)=(42.95,-2.16) \pm(14.68,8.04) \mathrm{mas} / \mathrm{yr}$ in the new HIPPARCos reduction (van Leeuwen 2007, hereafter HIP07). These two values are different with low quality, as shown by the large uncertainties. The mean uncertainty on the proper motions of other cluster members is $\leq 2 \mathrm{mas} / \mathrm{yr}$ in each component. Furthermore, the parallax changes from $\pi_{\mathrm{HIP} 97}=$ $(12.81 \pm 12.80)$ mas to $\pi_{\mathrm{HIP} 07}=(39.18 \pm 11.54)$ mas, which reflects the uncertainties of the HIPPARCos data for this star. That the new CPSM considered that HIP 28774 is not a Hyades member thus appears justified.

\subsection{Determination of individual parallaxes}

Once the CP coordinates of the Hyades have been determined, a final check and further extension of our CPSM lies in computing individual parallaxes and comparing it with HIP97 and HIP07. The determination of individual parallaxes in this work is restricted to group members whose radial velocity is known and given in Table 2 of P98. The individual parallaxes of group members are given by

$\pi=\frac{A \mu_{\|}}{V_{\mathrm{r}} \tan \lambda}$,

where the corresponding quantities and units are defined in Sect. 2. This equation is also valid for linear expansion of the group mentioned before (see Blaauw 1964). The parallax uncertainty is derived by error propagation of this equation and takes the error budget of proper motions and radial velocities into account.

We present in Fig. 11 a comparison of the parallaxes derived in this work with the ones given in HIP97 and HIP07. We find a slight bias when comparing our results with HIP97, which disappears when we compare our parallaxes with the presumably more accurate ones given in HIP07. Another dataset that we used to compare the individual parallaxes of this work are the secular parallaxes of Hyades cluster members given by de Bruijne et al. (2001). These secular parallaxes were calculated using proper motion data from the HIPPARCOS and TYCHO 2 catalogs. The results of this comparison for each data set are shown in Fig. 12. We conclude that our results agree well with the more precise secular parallaxes of the Hyades open cluster.

\section{Summary}

We presented a new CPSM based on the idea of representing the stellar proper motions by great circles on the celestial sphere. The new CP method combines (i) maximum-likelihood analysis to simultaneously determine the $\mathrm{CP}$ and select moving group members with (ii) a direct minimization routine used to return a more refined CP position and its uncertainties. Our new method allows us to correct for internal motions within the group by applying an individual correction for each star that depends on its proper motion. This procedure takes into account that the stars in the group are not located at the same distance.

We performed extensive Monte Carlo simulations to test and compare our new CPSM with the classic CPSM regarding the selection of moving members and the accuracy of the CP solution. We investigated the effects of (a) the velocity dispersion of the cluster, (b) observational errors on proper motion, (c) cluster distance, (d) number of group members, and (e) angular distance to the $\mathrm{CP}$ on the $\mathrm{CP}$ solution. Our new CPSM returned a more precise CP solution than the classic CPSM for $95 \%$ of the simulations. We verified that in the absence of velocity dispersion (ideal moving group) both methods exhibit the same performance. We also found that the new CPSM finds and eliminates more field stars than the classic CPSM. This situation is even more evident in the presence of background stars that generally have smaller proper motions. The new CPSM is able to retrieve more than $80 \%$ of all cluster members with a contamination around $20 \%$ of the total number of field stars at distances of $100 \mathrm{pc}$ and $200 \mathrm{pc}$. At larger distances the efficiency of rejecting field stars decreases, but it is still higher than the efficiency of the classic CPSM. The definition of the $X^{2}$ function in the new CP method considers both the amplitude and direction of the stellar proper motion vector instead of only one directional component that removes the bias towards distant stars that is observed in the classic CPSM. Additional information (e.g. parallaxes and radial velocities) is required to eliminate the remaining field stars in the sample that were not rejected by the CPSM.

The new CPSM is shown to work well when applied to the Hyades open cluster, and its results agree well with previous determinations of the $\mathrm{CP}$ position. The individual parallaxes derived using our $\mathrm{CP}$ solution are fully consistent with the trigonometric parallaxes given in the HIPPARCos catalog. Although our results agree well with data from both reductions, we find that our external precision is slightly better for HIP07. Our results are also in good agreement with the secular parallaxes found in the literature, which are more precise than the trigonometric parallaxes given in the HIPPARCos catalog.

The original implementation of the new CPSM was adapted to take the full covariance matrix of the HIPPARCos catalog into account. The same procedure can also be applied to the future data of the Gaia mission, which will also be published with the full covariance matrix. The new CPSM will be used in forthcoming papers to investigate the kinematic properties of several young associations. 
P. A. B. Galli et al.: A new method for calculating the convergent point of a moving group
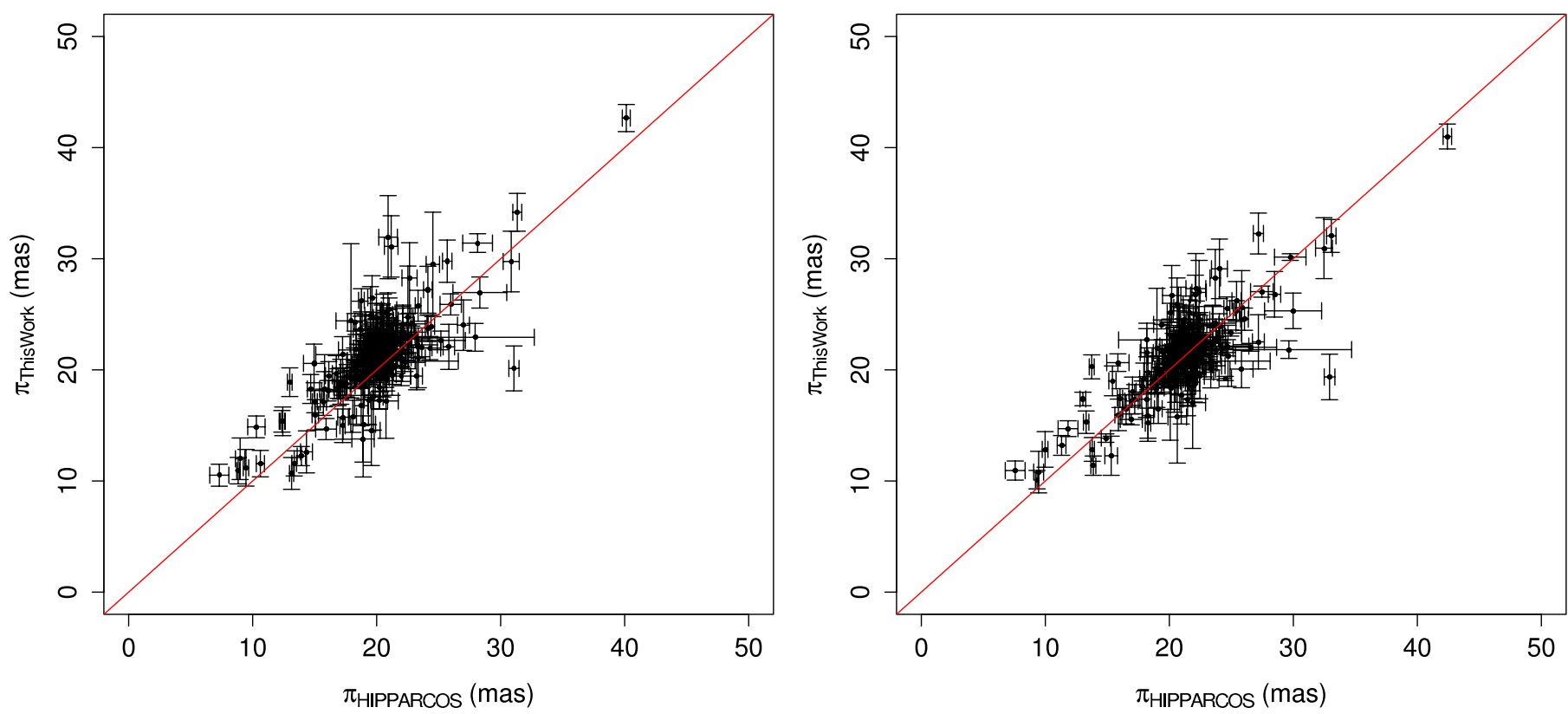

Fig. 11. Individual parallaxes of Hyades members derived in this work compared with their counterparts in HIP97 (left panel) and HIP07 (right panel). The red line represents the expected distribution for equal results. The rms with respect to HIP97 is 3.62 mas and 3.34 mas for HIP07. The mean difference between the parallaxes derived in this work, and the ones in HIP97 and HIP07 are 1.18 mas and 0.02 mas, respectively.
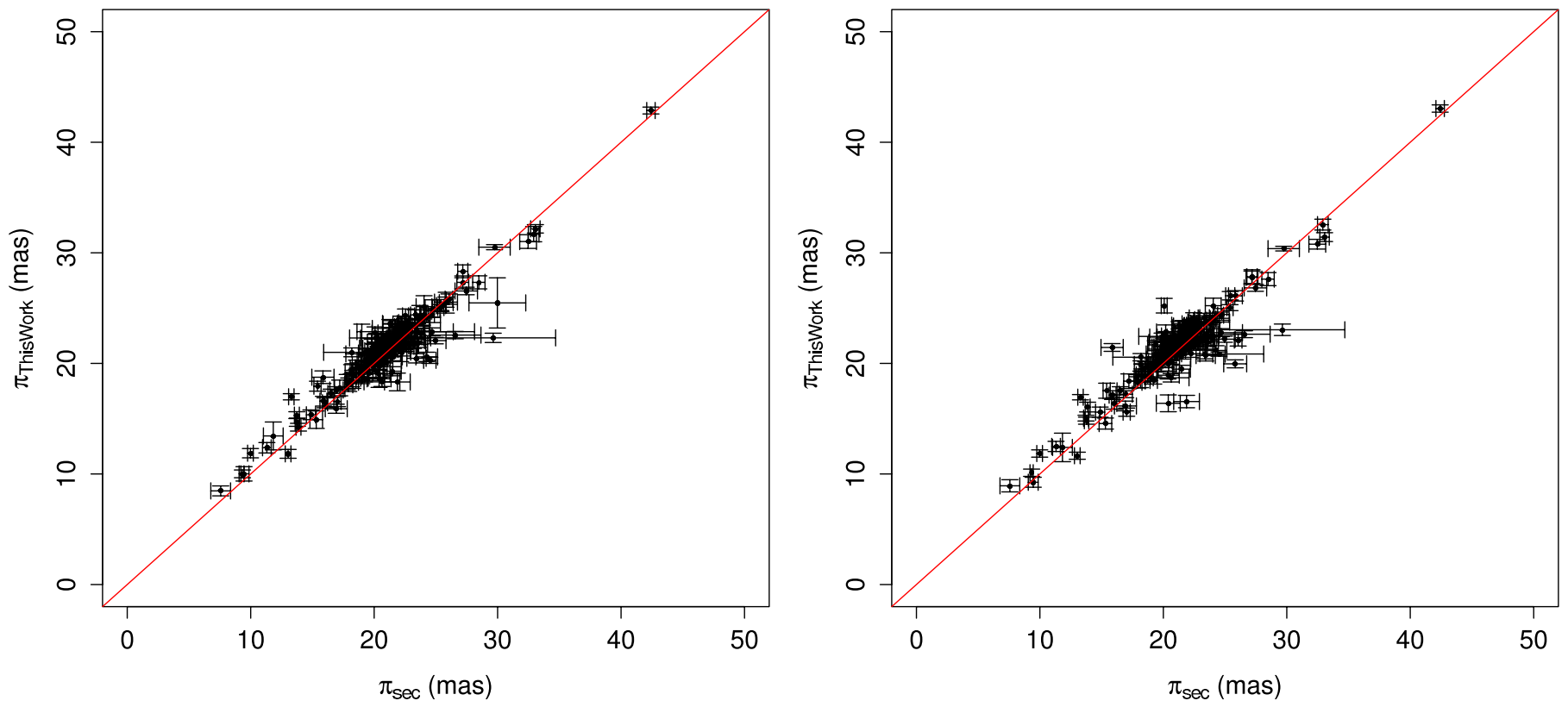

Fig. 12. Individual parallaxes of Hyades members derived in this work compared to the secular parallaxes of de Bruijne et al. (2001) derived using proper motion data from the HIPPARCOS (left panel) and TYCHO2 (right panel) catalogs. The red line represents the expected distribution for equal results. The rms with respect to the HIPPARCos secular parallaxes is 1.55 mas and 1.68 mas for TYCHO 2 secular parallaxes, and the mean difference between the parallaxes is 0.23 mas and -0.27 mas.

Acknowledgements. It is pleasure to thank Alberto Krone Martins and Michel Rapaport for helpful and stimulating discussions. We are also grateful to the referee for constructive comments that helped us to improve the manuscript. This research was funded by a doctoral fellowship from FAPESP and made use of the SIMBAD database operated at the CDS, Strasbourg, France.

\section{References}

Abad, C., \& Vieira, K. 2005, A\&A, 442, 745

Abad, C., Vieira, K., Bongiovanni, A., Romero, L., \& Vicente, B. 2003, A\&A, 397,345

Antoja, T., Figueras, F., Fernández, D., \& Torra, J. 2008, A\&A, 490, 135
Asiain, R., Figueras, F., Torra, J., \& Chen, B. 1999, A\&A, 341, 427

Bertiau, F. C. 1958, ApJ, 128, 533

Bertout, C., \& Genova, F. 2006, A\&A, 460, 499

Blaauw, A. 1964, ARA\&A, 2, 213

Brown, A. 1950, ApJ, 112, 225

Chen, B., Asiain, R., Figueras, F., \& Torra, J. 1997, A\&A, 318, 29

Chereul, E., Crézé, M., \& Bienaymé, O. 1999, A\&AS, 135, 5

de Bruijne, J. H. J. 1999, MNRAS, 306, 381

de Bruijne, J. H. J., Hoogerwerf, R., \& de Zeeuw, P. T. 2001, A\&A, 367, 111

Dehnen, W. 2000, AJ, 119, 800

Dehnen, W., \& Binney, J. J. 1998, MNRAS, 298, 387

Eggen, O. J. 1996, AJ, 112, 1595

ESA 1997, VizieR Online Data Catalog, 1239, 0 
Feast, M., \& Whitelock, P. 1997, MNRAS, 291, 683

Fux, R. 2001, A\&A, 373, 511

Green, R. M. 1985, Spherical astronomy (CUP)

Hoogerwerf, R., \& Aguilar, L. A. 1999, MNRAS, 306, 394

Jones, D. H. P. 1971, MNRAS, 152, 231

Madsen, K., Nielsen, H., \& Tingleff, O. 2004, Methods for non-linear least squares problems, 2nd edition, ed. K. Madsen, H. B. Nielsen, \& O. Tingleff Makarov, V. V. 2007a, ApJ, 670, 1225

Makarov, V. V. 2007b, ApJ, 658, 480

Makarov, V. V., \& Robichon, N. 2001, A\&A, 368, 873

Mamajek, E. E. 2005, ApJ, 634, 1385

Mathieu, R. D. 1986, in Highlights of Astronomy 7, Proc. XIX IAU gen. Assembly (Dordrecht: D. Reidel Publ.), 481
Navarro, J. F., Helmi, A., \& Freeman, K. C. 2004, ApJ, 601, L43 Perryman, M. A. C., Brown, A. G. A., Lebreton, Y., et al. 1998, A\&A, 331, 81 Petrie, R. M. 1949, MNRAS, 109, 693

Press, W. H., Teukolsky, S. A., Vetterling, W. T., \& Flannery, B. P. 1992, Numerical recipes in $\mathrm{C}$, The art of scientific computing

Roman, N. G. 1949, ApJ, 110, 205

Schönrich, R., Binney, J., \& Dehnen, W. 2010, MNRAS, 403, 1829

Schwan, H. 1991, A\&A, 243, 386

Seares, F. H. 1945, ApJ, 102, 366

Smart, W. M. 1968, Stellar kinematics

Trumpler, R. J., \& Weaver, H. F. 1953, Statistical astronomy

van Leeuwen, F. 2007, A\&A, 474, 653

\section{Appendix A: Pole coordinates $\left(\alpha^{p}, \delta^{p}\right)$}

The rectangular coordinates of the normalized polar vector $\boldsymbol{p}$ are given as

$\boldsymbol{p}=\frac{\mu_{\alpha}^{*}}{\sqrt{\mu_{\alpha^{*}}^{2}+\mu_{\delta}^{2}}}\left(\begin{array}{c}-\cos \alpha \sin \delta \\ -\sin \alpha \sin \delta \\ \cos \delta\end{array}\right)-\frac{\mu_{\delta}}{\sqrt{\mu_{\alpha^{*}}^{2}+\mu_{\delta}^{2}}}\left(\begin{array}{c}-\sin \alpha \\ \cos \alpha \\ 0\end{array}\right)$,

where $\mu_{\alpha^{*}}=\mu_{\alpha} \cos \delta$. The determination of the celestial coordinates $\left(\alpha^{p}, \delta^{p}\right)$ of the pole requires transformation to an equatorial polar coordinate system. This yields

$\alpha^{p}=\arctan \left(\frac{-\mu_{\alpha} \cos \delta \sin \delta \sin \alpha-\mu_{\delta} \cos \alpha}{-\mu_{\alpha} \cos \delta \sin \delta \cos \alpha+\mu_{\delta} \sin \alpha}\right)$

$\delta^{p}=\arctan \left(\frac{\mu_{\alpha} \cos ^{2} \delta}{\sqrt{\left(\mu_{\alpha} \cos \delta \sin \delta\right)^{2}+\mu_{\delta}^{2}}}\right)$.

The corresponding errors $\left(\sigma_{\alpha^{p}}, \sigma_{\delta^{p}}\right)$ are obtained by error propagation of Eqs. (A.2) and (A.3). We have to first order

$\sigma_{\alpha^{p}}^{2}=\left(\frac{\partial \alpha^{p}}{\partial \alpha}\right)^{2} \sigma_{\alpha}^{2}+\left(\frac{\partial \alpha^{p}}{\partial \delta}\right)^{2} \sigma_{\delta}^{2}+\left(\frac{\partial \alpha^{p}}{\partial \mu_{\alpha^{*}}}\right)^{2} \sigma_{\mu_{\alpha^{*}}}^{2}+\left(\frac{\partial \alpha^{p}}{\partial \mu_{\delta}}\right)^{2} \sigma_{\mu_{\delta}}^{2}$

and

$\sigma_{\delta^{p}}^{2}=\left(\frac{\partial \delta^{p}}{\partial \alpha}\right)^{2} \sigma_{\alpha}^{2}+\left(\frac{\partial \delta^{p}}{\partial \delta}\right)^{2} \sigma_{\delta}^{2}+\left(\frac{\partial \delta^{p}}{\partial \mu_{\alpha^{*}}}\right)^{2} \sigma_{\mu_{\alpha^{*}}}^{2}+\left(\frac{\partial \delta^{p}}{\partial \mu_{\delta}}\right)^{2} \sigma_{\mu_{\delta}}^{2}$.

The partial derivatives are given as

$\frac{\partial \alpha^{p}}{\partial \alpha}=\frac{1-\frac{\left(-\mu_{\alpha}^{*} \sin \delta \sin \alpha-\mu_{\delta} \cos \alpha\right)\left(\mu_{\alpha}^{*} \sin \delta \sin \alpha+\mu_{\delta} \cos \alpha\right)}{\left(-\mu_{\alpha}^{*} \sin \delta \cos \alpha+\mu_{\delta} \sin \alpha\right)^{2}}}{1+\left(\frac{-\mu_{\alpha}^{*} \sin \delta \sin \alpha-\mu_{\delta} \cos \alpha}{-\mu_{\alpha}^{*} \sin \delta \cos \alpha+\mu_{\delta} \sin \alpha}\right)^{2}}$,

$\frac{\partial \alpha^{p}}{\partial \delta}=-\frac{\frac{\mu_{\alpha}^{*} \cos \delta \sin \alpha}{-\mu_{\alpha}^{*} \sin \delta \cos \alpha+\mu_{\delta} \sin \alpha}}{1+\left(\frac{-\mu_{\alpha}^{*} \sin \delta \sin \alpha-\mu_{\delta} \cos \alpha}{-\mu_{\alpha}^{*} \sin \delta \cos \alpha+\mu_{\delta} \sin \alpha}\right)^{2}}+\frac{\frac{\left(-\mu_{\alpha}^{*} \sin \delta \sin \alpha-\mu_{\delta} \cos \alpha \mu_{\alpha}^{*} \cos \delta \cos \alpha\right.}{\left(-\mu_{\alpha}^{*} \sin \delta \cos \alpha+\mu_{\delta} \sin \alpha\right)^{2}}}{1+\left(\frac{-\mu_{\alpha}^{*} \sin \delta \sin \alpha-\mu_{\delta} \cos \alpha}{-\mu_{\alpha}^{*} \sin \delta \cos \alpha+\mu_{\delta} \sin \alpha}\right)^{2}}$,

$\frac{\partial \alpha^{p}}{\partial \mu_{\alpha}^{*}}=-\frac{\frac{\sin \delta \sin \alpha}{-\mu_{\alpha}^{*} \sin \delta \cos \alpha+\mu_{\delta} \sin \alpha}}{1+\left(\frac{-\mu_{\alpha}^{*} \sin \delta \sin \alpha-\mu_{\delta} \cos \alpha}{-\mu_{\alpha}^{*} \sin \delta \cos \alpha+\mu_{\delta} \sin \alpha}\right)^{2}}+\frac{\frac{\left(-\mu_{\alpha}^{*} \sin \delta \sin \alpha-\mu_{\delta} \cos \alpha\right) \sin \delta \cos \alpha}{\left(-\mu_{\alpha}^{*} \sin \delta \cos \alpha+\mu_{\delta} \sin \alpha\right)^{2}}}{1+\left(\frac{-\mu_{\alpha}^{*} \sin \delta \sin \alpha-\mu_{\delta} \cos \alpha}{-\mu_{\alpha}^{*} \sin \delta \cos \alpha+\mu_{\delta} \sin \alpha}\right)^{2}}$

$\frac{\partial \alpha^{p}}{\partial \mu_{\delta}}=-\frac{\frac{\cos \alpha}{-\mu_{\alpha}^{*} \sin \delta \cos \alpha+\mu_{\delta} \sin \alpha}}{1+\left(\frac{-\mu_{\alpha}^{*} \sin \delta \sin \alpha-\mu_{\delta} \cos \alpha}{-\mu_{\alpha}^{*} \sin \delta \cos \alpha+\mu_{\delta} \sin \alpha}\right)^{2}}-\frac{\frac{\left(-\mu_{\alpha}^{*} \sin \delta \sin \alpha-\mu_{\delta} \cos \alpha\right) \sin \alpha}{\left(-\mu_{\alpha}^{2} \sin \delta \cos \alpha+\mu_{\delta} \sin \alpha\right)^{2}}}{1+\left(\frac{-\mu_{\alpha}^{*} \sin \delta \sin \alpha-\mu_{\delta} \cos \alpha}{-\mu_{\alpha}^{*} \sin \delta \cos \alpha+\mu_{\delta} \sin \alpha}\right)^{2}}$ 
$\frac{\partial \delta^{p}}{\partial \alpha}=0$

$\frac{\partial \delta^{p}}{\partial \delta}=\frac{-\frac{\mu_{\alpha}^{*} \sin \delta}{\sqrt{\left(\mu_{\alpha}^{*} \sin \delta\right)^{2}+\mu_{\delta}^{2}}}-\frac{\left(\mu_{\alpha}^{*} \cos \delta\right)^{2} \mu_{\alpha}^{*} \sin \delta}{\left(\left(\mu_{\alpha}^{*} \sin \delta\right)^{2}+\mu_{\delta}^{2}\right)^{3 / 2}}}{1+\frac{\left(\mu_{\alpha}^{*} \cos \delta\right)^{2}}{\left(\mu_{\alpha}^{*} \sin \delta\right)^{2}+\mu_{\delta}^{2}}}$,

$\frac{\partial \delta^{p}}{\partial \mu_{\alpha}^{*}}=\frac{\frac{\cos \delta}{\sqrt{\left(\mu_{\alpha}^{*} \sin \delta\right)^{2}+\mu_{\delta}^{2}}}-\frac{\left(\mu_{\alpha}^{*} \sin \delta\right)^{2} \cos \delta}{\left(\left(\mu_{\alpha}^{*} \sin \delta\right)^{2}+\mu_{\delta}^{2}\right)^{3 / 2}}}{1+\frac{\left(\mu_{\alpha}^{*} \cos \delta\right)^{2}}{\left(\mu_{\alpha}^{*} \sin \delta\right)^{2}+\mu_{\delta}^{2}}}$

$\frac{\partial \delta^{p}}{\partial \mu_{\delta}}=\frac{-\mu_{\alpha}^{*} \mu_{\delta} \cos \delta}{\left(\left(\mu_{\alpha}^{*} \sin \delta\right)^{2}+\mu_{\delta}^{2}\right)^{3 / 2}\left(1+\frac{\left(\mu_{\alpha}^{*} \cos \delta\right)^{2}}{\left(\mu_{\alpha}^{*} \sin \delta\right)^{2}+\mu_{\delta}^{2}}\right)}$.

\section{Appendix B: Orthogonality error}

Let us assume that $\hat{\boldsymbol{e}}_{\mathrm{cp}}$ is a unit vector that defines the direction of the CP $\left(\alpha_{\mathrm{cp}}, \delta_{\mathrm{cp}}\right)$ in the celestial sphere, and $\boldsymbol{p}$, the normalized polar vector as given by Eq. (A.1), defines the pole $\left(\alpha^{p}, \delta^{p}\right)$ of the great circle for a given star in the group. We define $\boldsymbol{\kappa}=\kappa \hat{\boldsymbol{e}}_{\mathbf{c p}}$ where $\kappa$ is the orthogonality error and denotes the amount of $\boldsymbol{p}$ that is projected in the $\hat{\boldsymbol{e}}_{\text {cp }}$ direction. It is written as

$\boldsymbol{\kappa}=\left(\boldsymbol{p} \cdot \hat{\boldsymbol{e}}_{\mathrm{cp}}\right) \hat{\boldsymbol{e}}_{\mathrm{cp}}=\underbrace{(\cos \theta)}_{\kappa} \hat{\boldsymbol{e}}_{\mathrm{cp}}$

where $\theta$ is the angle between $\boldsymbol{p}$ and $\hat{\boldsymbol{e}}_{\text {cp }}$. However, $\theta$ is also the angle between the planes that contain $\boldsymbol{p}$ and $\hat{\boldsymbol{e}}_{\text {cp }}$, which defines it as a spherical angle. It is given by the cosine formula in the spherical triangle that contains the $\mathrm{CP}\left(\alpha_{\mathrm{cp}}, \delta_{\mathrm{cp}}\right)$ and the pole $\left(\alpha^{p}, \delta^{p}\right)$ as

$\kappa \equiv \cos \theta=\sin \delta_{\mathrm{cp}} \sin \delta^{p}+\cos \delta_{\mathrm{cp}} \cos \delta^{p} \cos \left(\alpha_{\mathrm{cp}}-\alpha^{p}\right)$.

Whenever perfect parallelism of the stellar motions is achieved, $\boldsymbol{p}$ and $\hat{\boldsymbol{e}}_{\text {cp }}$ are orthogonal and $\kappa=0$. In practice, one needs to minimize $\kappa$ to search for the CP and moving group members. The corresponding error of $\kappa$ is given by error propagation and takes position and proper motion errors into account. It is given as

$\sigma_{\kappa}^{2}=\left[-\cos \delta_{\mathrm{cp}} \cos \delta^{p} \sin \left(\alpha^{p}-\alpha_{\mathrm{cp}}\right)\right]^{2} \sigma_{\alpha^{p}}^{2}+\left[\sin \delta_{\mathrm{cp}} \cos \delta^{p}-\cos \delta_{\mathrm{cp}} \sin \delta^{p} \cos \left(\alpha^{p}-\alpha_{\mathrm{cp}}\right)\right]^{2} \sigma_{\delta^{p}}^{2}$.

\section{Appendix C: Error propagation}

The HIPPARCos catalog provides the five astrometric parameters $\left(\alpha, \delta, \mu_{\alpha}^{*}, \mu_{\delta}, \pi\right)$ together with the full covariance matrix. Thus, the propagation of errors must consider the covariances between all observables. The complete expression for the error propagation is given as

$$
\begin{aligned}
\sigma_{\alpha^{p}}^{2}= & \left(\frac{\partial \alpha^{p}}{\partial \alpha}\right)^{2} \sigma_{\alpha}^{2}+\left(\frac{\partial \alpha^{p}}{\partial \delta}\right)^{2} \sigma_{\delta}^{2}+\left(\frac{\partial \alpha^{p}}{\partial \mu_{\alpha}^{*}}\right)^{2} \sigma_{\mu_{\alpha^{*}}}^{2}+\left(\frac{\partial \alpha^{p}}{\partial \mu_{\delta}}\right)^{2} \sigma_{\mu_{\delta}}^{2}+2\left(\frac{\partial \alpha^{p}}{\partial \alpha}\right)\left(\frac{\partial \alpha^{p}}{\partial \delta}\right) \rho_{\alpha \delta} \sigma_{\alpha} \sigma_{\delta}+2\left(\frac{\partial \alpha^{p}}{\partial \alpha}\right)\left(\frac{\partial \alpha^{p}}{\partial \mu_{\alpha}^{*}}\right) \rho_{\alpha \mu_{\alpha}^{*}} \sigma_{\alpha} \sigma_{\mu_{\alpha}^{*}} \\
& +2\left(\frac{\partial \alpha^{p}}{\partial \alpha}\right)\left(\frac{\partial \alpha^{p}}{\partial \mu_{\delta}}\right) \rho_{\alpha \mu_{\delta}} \sigma_{\alpha} \sigma_{\mu_{\delta}}+2\left(\frac{\partial \alpha^{p}}{\partial \delta}\right)\left(\frac{\partial \alpha^{p}}{\partial \mu_{\alpha}^{*}}\right) \rho_{\delta \mu_{\alpha}^{*}} \sigma_{\delta} \sigma_{\mu_{\alpha}^{*}}+2\left(\frac{\partial \alpha^{p}}{\partial \delta}\right)\left(\frac{\partial \alpha^{p}}{\partial \mu_{\delta}}\right) \rho_{\delta \mu_{\delta}} \sigma_{\delta} \sigma_{\mu_{\delta}}+2\left(\frac{\partial \alpha^{p}}{\partial \mu_{\alpha}^{*}}\right)\left(\frac{\partial \alpha^{p}}{\partial \mu_{\delta}}\right) \rho_{\mu_{\alpha}^{*} \mu_{\delta}} \sigma_{\mu_{\alpha}^{*}} \sigma_{\mu_{\delta}}
\end{aligned}
$$

and

$$
\begin{aligned}
\sigma_{\delta^{p}}^{2}= & \left(\frac{\partial \delta^{p}}{\partial \alpha}\right)^{2} \sigma_{\alpha}^{2}+\left(\frac{\partial \delta^{p}}{\partial \delta}\right)^{2} \sigma_{\delta}^{2}+\left(\frac{\partial \delta^{p}}{\partial \mu_{\alpha}^{*}}\right)^{2} \sigma_{\mu_{\alpha^{*}}}^{2}+\left(\frac{\partial \delta^{p}}{\partial \mu_{\delta}}\right)^{2} \sigma_{\mu_{\delta}}^{2}+2\left(\frac{\partial \delta^{p}}{\partial \alpha}\right)\left(\frac{\partial \delta^{p}}{\partial \delta}\right) \rho_{\alpha \delta} \sigma_{\alpha} \sigma_{\delta}+2\left(\frac{\partial \delta^{p}}{\partial \alpha}\right)\left(\frac{\partial \delta^{p}}{\partial \mu_{\alpha}^{*}}\right) \rho_{\alpha \mu_{\alpha}^{*}} \sigma_{\alpha} \sigma_{\mu_{\alpha}^{*}} \\
& +2\left(\frac{\partial \delta^{p}}{\partial \alpha}\right)\left(\frac{\partial \delta^{p}}{\partial \mu_{\delta}}\right) \rho_{\alpha \mu_{\delta}} \sigma_{\alpha} \sigma_{\mu_{\delta}}+2\left(\frac{\partial \delta^{p}}{\partial \delta}\right)\left(\frac{\partial \delta^{p}}{\partial \mu_{\alpha}^{*}}\right) \rho_{\delta \mu_{\alpha}^{*}} \sigma_{\delta} \sigma_{\mu_{\alpha}^{*}}+2\left(\frac{\partial \delta^{p}}{\partial \delta}\right)\left(\frac{\partial \delta^{p}}{\partial \mu_{\delta}}\right) \rho_{\delta \mu_{\delta}} \sigma_{\delta} \sigma_{\mu_{\delta}}+2\left(\frac{\partial \delta^{p}}{\partial \mu_{\alpha}^{*}}\right)\left(\frac{\partial \delta^{p}}{\partial \mu_{\delta}}\right) \rho_{\mu_{\alpha}^{*} \mu_{\delta}} \sigma_{\mu_{\alpha}^{*}} \sigma_{\mu_{\delta}},
\end{aligned}
$$

where $\rho_{x y}$ denotes the correlation coefficient of the observables $x$ and $y$. The partial derivatives are given in Appendix A. 
Apêndice C

\section{A kinematic study and membership analysis of the Lupus star-forming region}

Phillip Andreas Brenner Galli, Claude Bertout, Ramachrisna Teixeira e Christine Ducourant.

Submetido para publicação no periódico Astronomy \& Astrophyiscs (2012) 


\title{
A kinematic study and membership analysis of the Lupus star-forming region ${ }^{\star}$
}

\author{
P.A.B. Galli ${ }^{1}$, C. Bertout ${ }^{2}$, R. Teixeira ${ }^{1}$, and C. Ducourant ${ }^{3}$ \\ ${ }^{1}$ Instituto de Astronomia, Geofísica e Ciências Atmosféricas, Universidade de São Paulo, Rua do Matão, 1226 - Cidade Universitária, \\ 05508-900, São Paulo - SP, Brazil \\ e-mail: galli@astro.iag.usp.br \\ 2 Institut d'Astrophysique, 98bis, Bd. Arago, 75014 Paris, France \\ 3 Observatoire Aquitain des Sciences de 1 'Univers, CNRS-UMR 5804, BP 89, Floirac, France
}

Received / Accepted

ABSTRACT

\begin{abstract}
Aims. To reliably determine the fundamental parameters (mass and age) of young stellar objects a precise determination of the distance to individual stars is required. This paper is dedicated to investigating the kinematic properties of the Lupus moving group of young stars with the primary objective of deriving individual parallaxes for each group member.

Methods. We identify those stars in the Lupus star-forming region that define the co-moving association of young stars by utilizing our new, improved convergent point search method that allows us to derive from the stars' proper motions the precise position of the convergent point and the probability that stars found in the region are members of the co-moving association. We used published proper motion catalogues and searched the literature for radial velocities, which are needed to compute individual parallaxes. We supplemented the radial velocity data with new measurements from spectroscopic observations performed with the FEROS spectrograph mounted on the MPG/ESO 2.2m telescope at La Silla.

Results. We identify a co-moving group with 114 pre-main sequence stars and candidates that define the kinematic properties of the Lupus low-mass star-forming region. We derive individual parallaxes for stars with known radial velocity, and tentative parallaxes for the remaining group members by assuming that all stars share the same space motion. The convergent point method makes it possible to distinguish the Lupus and Upper Centaurus Lupus stars from the adjacent Scorpius-Centaurus association. We find significant depth effects in this region and show that the classical T Tauri stars, located in the close vicinity of the Lupus molecular clouds, form a background population while the weak-emission line T Tauri stars are more dispersed in angular extent and depth.

Conclusions. The newly derived individual parallaxes will be used in a forthcoming paper to refine the masses and ages of Lupus $\mathrm{T}$ Tauri stars, with the aim to better constrain the lifetime of their circumstellar, protoplanetary disks.
\end{abstract}

Key words. methods: data analysis - technique: radial velocities - astrometry - parallaxes - proper motions - stars: distances - stars: kinematics and dynamics - open clusters and associations: individual: Lupus

\section{Introduction}

The inferred fundamental parameters of T Tauri stars (TTSs), the young solar-type pre-main sequence stars first discovered by Joy (1945), depend sensitively on their assumed distance. TTSs are usually associated with molecular clouds, the distances of which can be estimated, e.g., from the photometry of a few bright stars enshrouded in reflection nebulosity (see, for example, Racine 1968), or from 2MASS extinction maps combined with known stellar parallaxes (Lombardi et al. 2008). The distances to the nearby star-forming regions (SFRs) are thus known to relatively good accuracy, providing a first estimate of the distances to the members of the stellar population associated with them. Although these average parallaxes provide valuable information, we must know more precisely the distances to the individual members of the young association to better constrain their ages and masses by comparing observed stellar properties to evolutionary models.

TTSs are late-type objects and usually faint in the visible, suffering typically from 1 to 2 magnitudes of extinction in that range, while the nearby SFRs to which they belong are typically

\footnotetext{
* Based on observations collected at the European Southern Observatory, Chile (ESO Programme 087.C-0315).
}

in the distance range $0.1-1 \mathrm{kpc}$. Parallax determination from the ground is usually impossible for these objects, and they even presented a challenge for the HIPPARcos mission (ESA 1997), which observed only a few of them. Bertout et al. (1999) computed new astrometric solutions using the HIPPARcos data for groups of TTSs in various SFRs, thus providing post-HIPPARCos average distances to these groups.

Some progress in determining the distance of individual TTSs has been done in recent years, using two different methods. Loinard and collaborators used the Very Long Baseline Array (VLBA) to determine parallaxes of a few selected objects, mainly in the Taurus-Auriga SFR (Loinard et al. 2007; Torres et al. 2007, 2009; Dzib et al. 2010, 2011; Torres et al. 2012). The VLBA data allow for a very precise parallax determination but the method is observationally intensive, limiting its application to a few remarkable stars. Another approach rests on the fact that the members of young associations share the same spatial motion and uses their proper motions to determine individual parallaxes for members of some nearby associations. This was done, e.g., for the TW Hydrae association by Mamajek (2005) and for the Taurus-Auriga SFR by Bertout \& Genova (2006). The derived parallaxes using this method are not as precise as those obtained by VLBA observations, but it yields usable par- 
allaxes for all members of the moving group that have measured radial velocities. Thus, while we have progressed, we are still far from knowing the distances to the large sample of pre-main sequence stars (PMSs) that is required to study such timely topics as the effect of differing protostellar environments on disk lifetimes and the timescales of planet formation. The situation will change considerably with the launch of the Gaia mission, as its instruments will measure the parallaxes and proper motions of millions of faint stars. Although the satellite's launch date is 2013 , its catalog will be published several years later, so one would appreciate making some progress in determining the distances of TTSs in the meantime. Also, the astrometric methods that are developed and tested for this purpose will certainly be useful in the Gaia era.

In this paper, we study the largest southern SFR, located in Lupus, using the recently developed new version of the convergent point (CP) method presented by Galli et al. (2012). Section 2 is devoted to a brief presentation of the Lupus SFR and its previous distance determinations, while Sect. 3 discusses the sample of Lupus candidate stars found in recent catalogues and proper motion information needed for the CP analysis. Sect. 4 discusses our search for radial velocity data, including observations carried out for a number of stars in our sample with FEROS mounted on the $2.2 \mathrm{~m} \mathrm{MPG/ESO} \mathrm{telescope} \mathrm{at} \mathrm{La} \mathrm{Silla,} \mathrm{Chile.}$ The CP and membership analysis are discussed in Sect. 5, while Sect. 6 presents the parallax computations for the moving group members. We discuss the results of this investigation in Sect. 7. Finally, our conclusions are given in Sect. 8.

\section{The Lupus association of young stars}

The Lupus dark cloud complex is a low-mass star-forming region that contains four main star-forming clouds (Lupus 1 to 4) and constitutes one of the richest associations of TTSs. Many of the Lupus TTSs have been identified from ROSAT X-ray observations and from the spectroscopic surveys conducted by Krautter et al. (1997) and Wichmann et al. (1997a,b). These surveys showed that the Lupus molecular clouds are surrounded by an extended halo of late-type, X-ray active stars, which bear resemblance to the so-called weak emission-line TTSs (WTTSs), the X-ray active TTSs without circumstellar accretion disks (Walter 1986). If the ROSAT-detected objects were all WTTSs belonging to the Lupus association, then they would greatly exceed the number of so-called classical TTSs (CTTSs), the TTS subgroup showing evidence for circumstellar accretion disks (Bertout 1987; Bertout et al. 1988). Lupus CTTSs were identified by Schwartz (1977) on the basis of their association with the molecular clouds and their $\mathrm{H} \alpha$ emission. This early census of Lupus young stars was recently expanded by infrared observations with the Spitzer Space Observatory (Merín et al. 2008) and a proper motion study of these objects was performed by López Martí et al. (2011).

The focus of most kinematic studies over the past century was not the Lupus clouds, but the adjacent Scorpius-Centaurus (Sco-Cen) association that contains the nearest OB association. It is divided in the three subgroups Upper Scorpius (US), Upper Centaurus-Lupus (UCL) and Lower Centaurus-Crux (LCC). The Lupus molecular clouds, which are the probable signpost of a more recent episode of star formation in that region (see Comerón 2008), occupy a gap between US and UCL. The distance of the Sco-Cen subgroups was investigated by de Zeeuw et al. (1999), who re-assessed association membership using the Hipparcos data and derived a mean distance of $140 \mathrm{pc}$ for the UCL subgroup. The Lupus SFR was first estimated to be at the same distance as UCL (Hughes et al. 1993). The close proximity of the UCL subgroup makes it difficult to distinguish between members of the Lupus association and of the UCL association. Mamajek et al. (2002) conducted a spectroscopic survey of UCL candidate members and selected 56 stars that had the same proper motion as UCL association members. A more complete list containing 81 UCL candidate stars is given in the recent review on the Sco-Cen association by Preibisch \& Mamajek (2008). As it turned out, many of these stars were previously reported as WTTS members of the Lupus association by Krautter et al. (1997) and Wichmann et al. (1997a,b). Since the membership status of these objects remains unclear, a more detailed study is clearly necessary.

In the past decade, several works have cast doubt on the distance to the Lupus star-forming region. From the angular extent of the molecular clouds (about $27 \mathrm{pc}$ ), and assuming that the molecular region is nearly spherical, the distances of individual association members are expected to range from about $110 \mathrm{pc}$ to 190 pc. Two bright TTSs observed by Hipparcos, RY Lup and V856 Sco, are apparently associated with clouds of the Lupus star-forming region, and their parallaxes are compatible with the above range, but distances reported in the literature range from 100 pc (Knude \& Hog 1998) to 360 pc for Lupus 2 (Knude \& Nielsen 2001).

Bertout et al. (1999) used five stars connected with the Lupus complex that had been observed by HipParcos to compute the average parallax of the Lupus SFR. They found a distance equal to $206_{-20}^{+34} \mathrm{pc}$, a large value compared to the previous estimates, but noticed that the group parallax determination was dominated by the brightest stars HIP 79080 and 79081, two components of a Herbig Ae/Be (HAeBe) system located in Lupus 3. Considering only the three fainter stars HIP 77157, 78094 and 78317, located in Lupus 1, 2 and 4 respectively, They obtained a distance of $147_{-27}^{+42} \mathrm{pc}$, in agreement with previous determinations of the Lupus association distance, but with a large uncertainty, and a value of $228_{-30}^{+42} \mathrm{pc}$ for HIP 79080 and 79081 . Bertout et al. (1999) concluded that either HIP 79080 and 79081 are not members of Lupus 3, which appears unlikely, or this cloud is farther away than the other subgroups. Lombardi et al. (2008) concluded from their detailed study of 2MASS extinction maps that Lupus has a depth of $51_{-35}^{+61} \mathrm{pc}$, and that this "might be the result of different Lupus subclouds being at different distances". The kinematic study presented hereafter aims at shedding light on the structure of the Lupus clouds as well as on the membership of the Lupus association of PMS stars.

\section{Sample of Lupus candidate members with known proper motions}

\subsection{Proper motions properties}

The Ducourant et al. (2005, hereafter D05) proper motion catalogue for PMS stars contains 197 stars in the general area of the Lupus SFR, which approximately ranges from $325^{\circ} \leq l \leq 342^{\circ}$ in Galactic longitude and $0^{\circ} \leq b \leq 25^{\circ}$ in Galactic latitude. The stars listed in this catalogue were mostly identified by Herbig \& Bell (1988), Krautter et al. (1997) and Wichmann et al. $(1997 \mathrm{a}, \mathrm{b})$. We then include in the sample 37 stars of the comprehensive review performed by Comerón (2008) that were not considered in D05, and another 24 stars from the $c 2 d$ Spitzer Legacy Program detected in the recent paper of López Martí et al. (2011, only group A sources of their study). Our first list of presumed members of the Lupus association therefore consists of 258 stars. 
In order to access the more recent measurements, we searched for proper motion data in the PPMXL (Roeser et al. 2010), SPM4 (Girard et al. 2011) and UCAC4 (Zacharias et al. 2012) catalogues for all the 258 stars in our sample. The main source of proper motions is the SPM4 catalogue, which represents the best present-day compromise between proper motion precision and target coverage for the stars in our sample. For the brightest stars $(V \leq 12)$ in the sample we also searched the Түсно 2 (Høg et al. 2000) catalogue and used these values when the proper motions given in SPM4 was of lower precision. Doing so, we found proper motion information for 241 stars of our initial sample and for the remaining ones we kept the proper motions given in D05. We used the proper motions of the PPMXL and UCAC4 catalogues for two stars, since these are the only values available in the literature.

Whenever dealing with the kinematics of SFRs the high fraction of binaries and multiple systems plays an important role. The overall binary fraction in Lupus is expected to reach about $30 \%$ to $40 \%$ (Ghez et al. 1997; Merín et al. 2008). An advantage of the D05 catalogue is that these systems are clearly identified with a mention $\mathrm{AB}$ indicating that the given proper motion is representative of the binary system. In these cases we decided to use the proper motion given in D05 for both resolved and unresolved binaries rather than taking the value provided by SPM4, where the existence of close companions is not mentioned.

The CP search method selects cluster members and defines the $\mathrm{CP}$ of a moving group based on proper motion data. Therefore, before starting our analysis, it is necessary to reject all stars with proper motion that carry poor information because of measurement errors. We reject from the sample 32 stars whose proper motion is dominated by errors (i.e., $\sigma_{\mu_{\alpha, \delta}} \geq \mu_{\alpha, \delta}$ ) in both components. After a $3 \sigma$ elimination in both proper motion components we reject another 9 stars that are in obvious disagreement with the common streaming motion of the Lupus moving group. The remaining 217 PMS stars are considered bona fide Lupus association candidate members and will be used in this paper to investigate the kinematic properties of this SFR and discuss their membership status based on the $\mathrm{CP}$ analysis. Their average proper motion is $\left(\mu_{\alpha} \cos \delta, \mu_{\delta}\right)=(-16,-21) \mathrm{mas} / \mathrm{yr}$ with an average precision of about 2 mas/yr in each component.

Figure 1 presents histograms of the proper motion components for this sample, while Fig. 2 displays all proper motion values and their associated uncertainties. We note that a small group of these stars displays small proper motions. Because we considered only stars related to the Lupus SFR whose PMS status had already been established in previous works, it appears unlikely that field stars pollute our sample. We therefore assume for the moment that these stars form a background population belonging to the Lupus complex and we will come back to this point in Sect. 7. Figure 3 shows the proper motion vectors for our sample of Lupus stars. Although the position of the CP is not clearly apparent in this plot, one notices that the proper motion vectors point towards a common direction. The precise coordinates of the CP will be derived in Sect. 5.

\subsection{Refining the Lupus pre-main sequence star sample}

While the majority of the stars in our sample lie very close to the Lupus clouds, a fraction of the stars is scattered over a wider region (see Fig. 4). Most of these stars were identified and classified as WTTSs by Krautter et al. (1997) and Wichmann et al. (1997a,b) from ROSAT X-ray pointed observations and all skysurvey. However, we mentioned that the Lupus SFR is located near the UCL subgroup of the Sco-Cen association, so one sus-
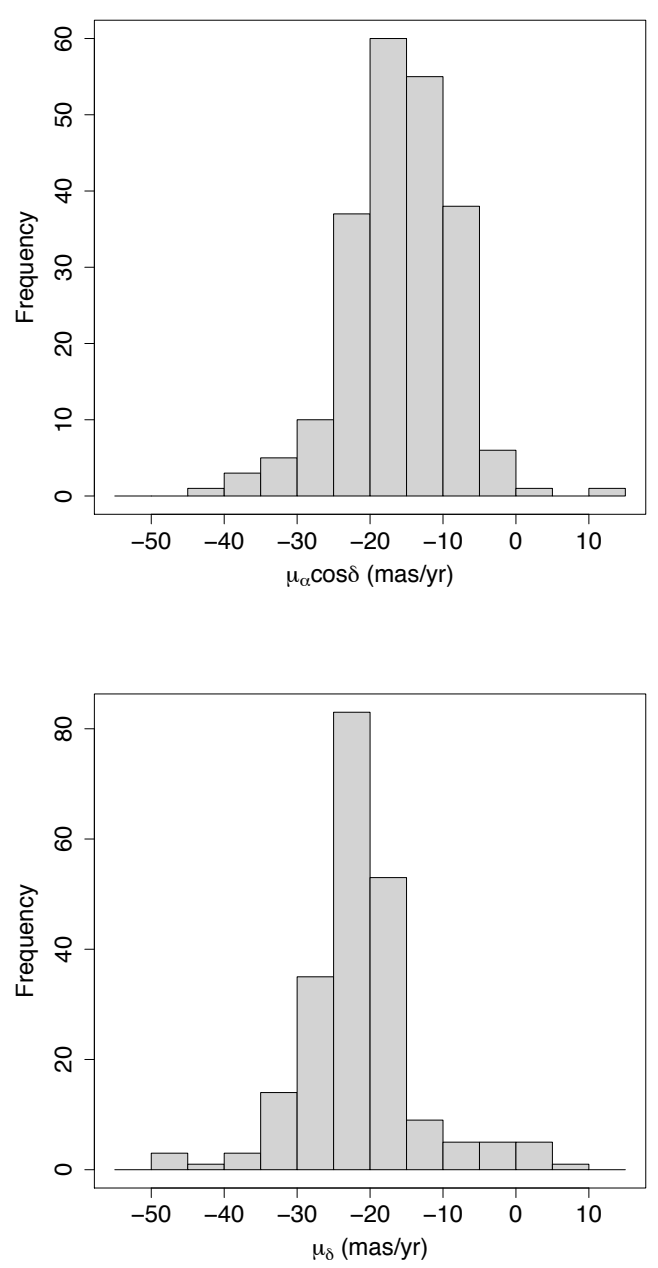

Fig. 1. Proper motion histogram for the 217 PMS stars of the Lupus.

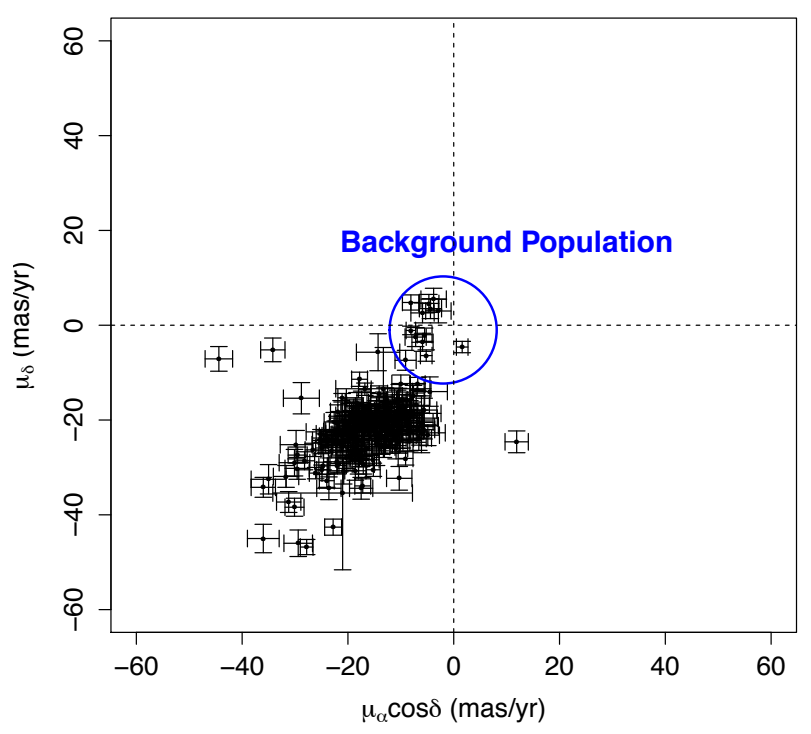

Fig. 2. Proper motion and associated errors for the 217 stars considered in this work. 


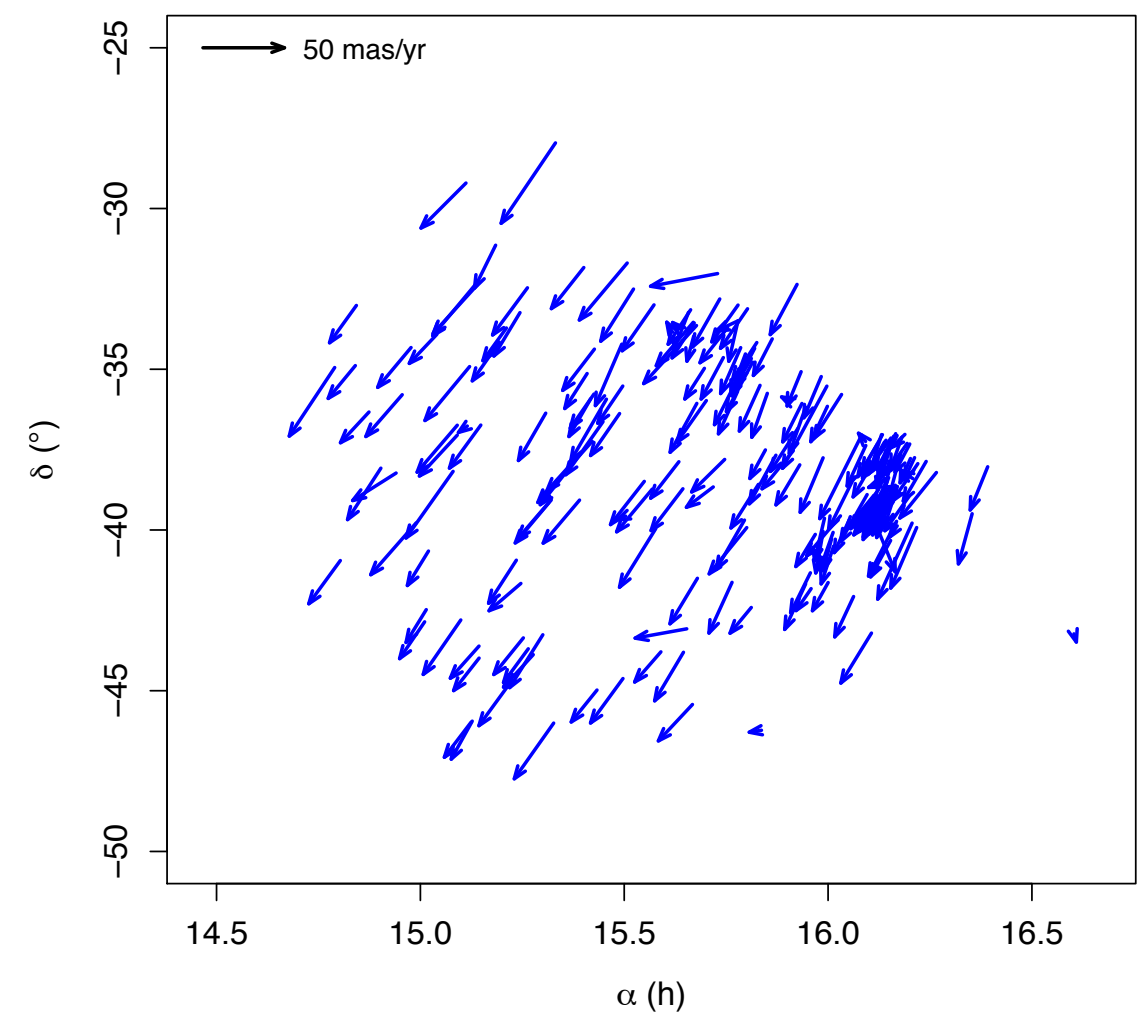

Fig. 3. Proper motion vectors of the 217 Lupus candidate stars.

pects that some of these stars might not be related to the Lupus SFR but might be part instead of the older Sco-Cen association. Indeed, a list of 81 low-mass candidate members of the UCL subgroup is given in the recent review by Preibisch \& Mamajek (2008), and our sample of Lupus association candidate members includes 25 stars of their list. Preibisch \& Mamajek (2008) also claim that other stars previously identified as Lupus PMS stars by Krautter et al. (1997) and Wichmann et al. (1997b) appear to be UCL members because of their proper motions and position in the HR-diagram.

It appears (Preibisch \& Mamajek 2008) that the UCL stars are spread over a large extent on the sky while the spatial distribution of Lupus stars exhibits two components: (i) an "oncloud" population concentrated in the immediate vicinity of the molecular clouds and (ii) a more dispersed "off-cloud" population surrounding the clouds. The dashed-line box drawn in Fig. 4 encloses the on-cloud population. The corresponding region spans the range of Galactic coordinates $334^{\circ} \leq l \leq 342^{\circ}$ and $5^{\circ} \leq b \leq 25^{\circ}$ and contains 159 stars while the off-cloud population in our sample is located in the region with $l<334^{\circ}$ which contains 58 stars. There is some arbitrariness involved in defining these regions but the idea of separating our sample into two groups turned out to be necessary to our analysis, as will be seen below, and has also been suggested by Preibisch \& Mamajek (2008), who wrote: “... it is probably wise for astrophysical studies to separate the on-or near-cloud Lupus members from the off-cloud UCL/US members.". We will show in Sect. 5 that the off-cloud population is indeed a mix of Lupus and UCL stars.

\section{Radial velocities}

We mentioned that stellar radial velocities (RVs) are needed to determine individual parallaxes. We summarize here our search for RVs in the literature and some additional observations for stars with unknown RVs.

\subsection{Radial velocities from the literature}

We searched the CDS databases to access RV information for the stars in our sample. The search made use of the data mining tools available on the CDS site. We also looked for published RV data that are not available via the Web-based CDS service. Our search for RVs, made as exhaustive as possible, is based on: Herbig \& Bell (1988), Gregorio-Hetem et al. (1992), BarbierBrossat et al. (1994), Duflot et al. (1995), Dubath et al. (1996), Grenier et al. (1999), Wichmann et al. (1999), Barbier-Brossat \& Figon (2000), Madsen et al. (2002), Melo (2003), Nordström et al. (2004), Bobylev (2006), Gontcharov (2006), James et al. (2006), Malaroda et al. (2006), Torres et al. (2006), Guenther et al. (2007), Kharchenko et al. (2007) and White et al. (2007).

We found RVs for only 108 stars of the full sample (oncloud and off-cloud populations), which reflects the scarcity of this measurement in the literature. This is because previous spectroscopic investigations in Lupus have often focused on the few bright stars of this region. Figure 5 displays the RV distribution in our sample. We note that stars in the off-cloud region exhibit $\mathrm{RV}$ s that are slightly higher than the values observed for the oncloud population. 

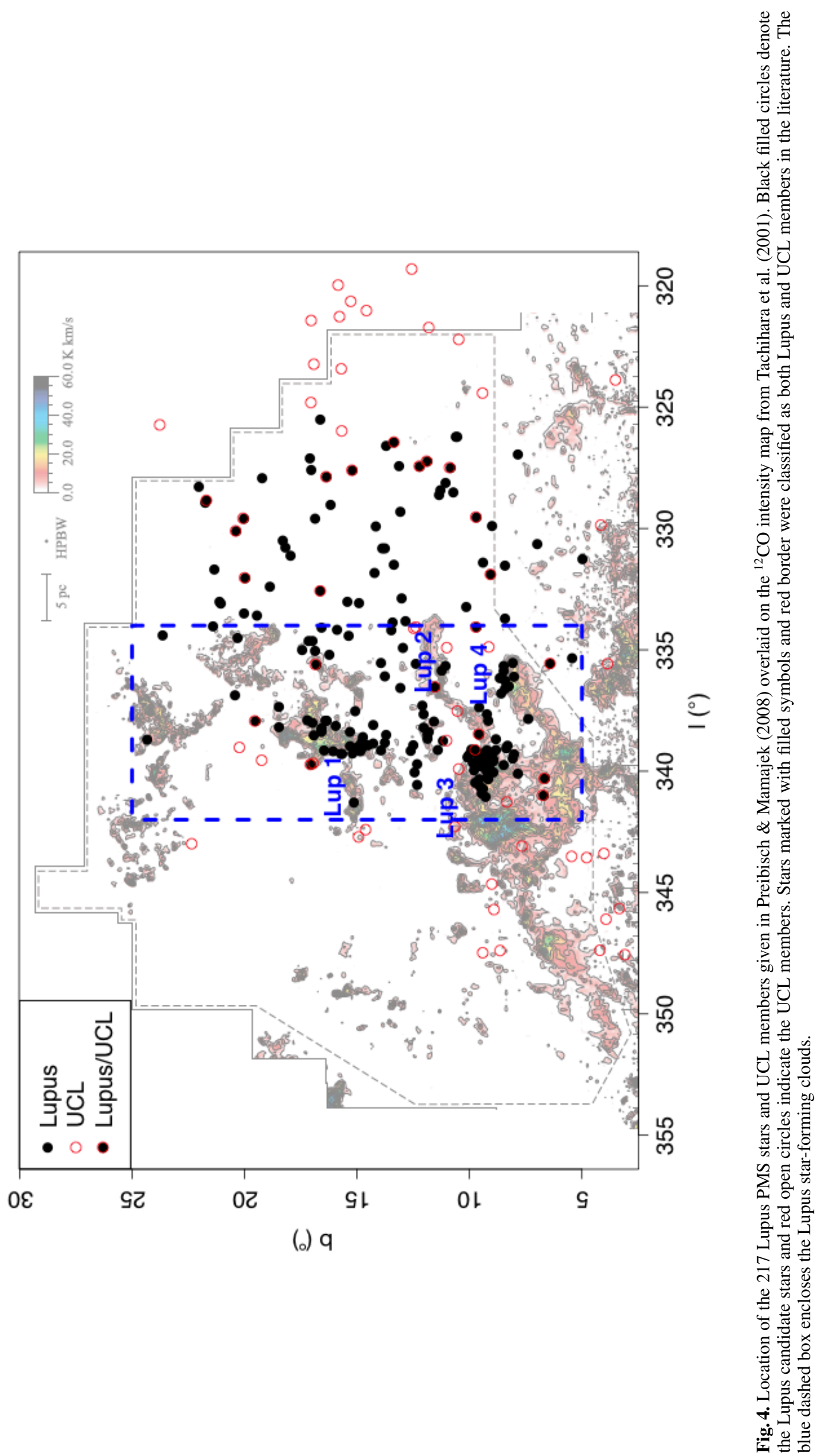


\subsection{Additional radial velocity observations}

The scarcity of measured RVs is the main limitation in deriving individual distances in this work. To increase the number of Lupus stars with known RV information, we thus performed spectroscopic observations with the high-resolution $(R=48000)$ FEROS (Kaufer et al. 1999) échelle spectrograph mounted at the ESO/MPG 2.2m telescope operated at La Silla (Chile). In addition to its high performance, FEROS provides a full wavelength coverage in the optical region (between $3500 \AA$ and $9200 \AA$ ) over 39 spectral orders. The observations were taken in object calibration mode, which allows acquiring simultaneous spectra of the object and of the ThAr cell. Exposure times ranged from $5 \mathrm{~min}$ to $60 \mathrm{~min}$, so that a $\mathrm{S} / \mathrm{N}$ of about 20-30 was achieved. We observed 52 stars spread over the Lupus and Ophiuchus ${ }^{1}$ SFRs during the nights of April 29 to May 05, 2011. Binaries and stars belonging to multiple systems as given in the literature were not included in our list of targets. The observed spectra were reduced with the standard FEROS data reduction pipeline, which performs bias subtraction, flat-fielding, scattered-light removal, échelleorder extraction, barycentric velocity correction and wavelength calibration of the spectra. The extracted wavelength-calibrated spectra for each spectral order were not merged into a single spectrum, but used as 39 separate spectra (one per order) to determine the RV of the target. This procedure enabled us to eliminate the largest noise contributions that come from the orders in the red (orders $\leq 7$ ) and blue (orders $\geq 30$ ) regions of the spectra (see Setiawan et al. 2003).

We derived RVs by cross-correlating the reduced spectra of program stars with template spectra. To maximize the quality of our RV measurements we use both a standard-star spectrum and a numerical mask as template spectrum.

The cross-correlation with a stellar template utilized the standard star HD $82106(V=7.2 \mathrm{mag}, \mathrm{K} 3 \mathrm{~V})$, which was observed every night and whose RV is known to a high accuracy $\left(V_{r}=\right.$ $+29.75 \pm 0.05 \mathrm{~km} / \mathrm{s}$, Udry et al. 1999). We computed the RVs of program stars by cross-correlating their spectrograms orderby-order with one reference spectrogram of HD 82106 (taken on May 04, 2011) using the IRAF task fxcor. We obtained the RV for each order separately and then averaged these values. The errors of our RV measurements were determined from the variance of all orders considered. Orders with discrepant values (due to a lower $\mathrm{S} / \mathrm{N}$ ) were obviously not considered. To investigate the accuracy of our results we calculated the RV of HD 82106 as derived from our observations. We cross-correlated the reference spectrogram with all spectrograms of HD 82106 spread over the whole observing campaign. The absolute RV of the reference spectrum was determined by cross-correlating it with the solar spectrum. The mean RV derived from our observations is $V_{r}=+29.71 \pm 0.05 \mathrm{~km} / \mathrm{s}$, in good accordance with Udry et al. (1999).

The other alternative to compute RVs consisted of a crosscorrelation with a box-shaped binary template. The procedure follows the method outlined in Baranne et al. (1996) that fits a Gaussian to the cross-correlation function of each order. The center of the Gaussian then gives the RV of the target (see Weise et al. 2010, for more details). To better compare the RVs derived for HD 82106 using both techniques, we used a binary template of a G2V star. Our choice of the template is motivated by the use of the solar spectrum to derive the absolute RV of HD 82106 in

\footnotetext{
1 The kinematic properties of the Ophiuchus SFR will be presented in a companion paper (Galli et al., in preparation). However, the results of our observations are presented in this paper since only a few stars of that region were observed in our program.
}

our first approach (see above). For program stars, we used a K0V star template that is more representative of our targets' spectral types. The mean RV derived with this alternative strategy is $V_{r}=$ $+29.74 \pm 0.04 \mathrm{~km} / \mathrm{s}$, which is perfectly consistent with the values mentioned above.

Using both a standard-star spectrum and a numerical template is valuable for gaining confidence in the derived results. In order to spot possible errors, the RVs of program stars were computed using only the common (not rejected) orders in both procedures. The final RV of our targets is the average of two independent values (one for each method). The uncertainties are calculated by propagating the individual errors and they are on the order of a few hundreds $\mathrm{m} / \mathrm{s}$. When one of the methods failed to return a $\mathrm{RV}$ value (due to low $\mathrm{S} / \mathrm{N}$ in some orders) we considered only the result as derived by the other method. A more realistic idea of our external precision is given in Table 1 by comparing the RVs derived in this work with published results for a control group of 3 stars in our sample with previously known RVs. We conclude that our results are fully compatible with the values found in the literature, with a rms uncertainty of about $500 \mathrm{~m} / \mathrm{s}$. We present in Table 2 the RVs of 52 stars belonging to the Lupus and Ophiuchus SFRs, together with the Li I and $\mathrm{H} \alpha$ equivalent widths (EWs) derived from our observations.

Table 1. Comparison of RVs derived in this paper with those published in the literature.

\begin{tabular}{cccc} 
& This Work & Literature & \\
\hline \hline ID & $\begin{array}{c}V_{r} \pm \sigma_{V_{r}} \\
(\mathrm{~km} / \mathrm{s})\end{array}$ & $\begin{array}{c}V_{r} \pm \sigma_{V_{r}} \\
(\mathrm{~km} / \mathrm{s})\end{array}$ & Reference \\
& $3.75 \pm 0.33$ & $4.20 \pm 1.00$ & Wichmann et al. (1999) \\
\hline RXJ1524.5-3652 & & $4.10 \pm 0.35$ & Torres et al. (2006) \\
& & $4.40 \pm 0.40$ & James et al. (2006) \\
& & $4.60 \pm 0.40$ & James et al. (2006) \\
\hline RXJ1547.6-4018 & $2.85 \pm 0.37$ & $2.80 \pm 1.00$ & Wichmann et al. (1999) \\
& & $3.20 \pm 0.07$ & Torres et al. (2006) \\
& & $3.10 \pm 0.40$ & James et al. (2006) \\
& & $3.20 \pm 0.40$ & James et al. (2006) \\
\hline RXJ1538.0-3807 & $2.46 \pm 0.28$ & $3.40 \pm 1.00$ & Wichmann et al. (1999) \\
& & $3.00 \pm 0.20$ & Guenther et al. (2007) \\
\hline
\end{tabular}

Equivalent widths of the $\mathrm{Li}$ I and $\mathrm{H} \alpha$ lines were measured from our spectra using IRAF splot routine. The presence of $\mathrm{Li}$ absorption in late-type stars is one of the primary criteria for stellar youth (see Basri et al. 1991). The Li I $\lambda 6708 \AA$ resonance doublet is blended by Fe lines and the lithium isotope ${ }^{6} \mathrm{Li}$. It is not possible to separate individual lines at our spectral resolution $(\sim 48000)$ and we thus see no evidence of these features. The lithium EWs were measured using both a Gaussian fit and direct integration. The difference between these values is smaller than $25 \mathrm{m \AA}$, which we consider to be the upper limit of our measurement errors. The contribution from the neighboring blending lines is expected to be smaller than the uncertainty of our results which is estimated by varying the location of the continuum adjacent to the line.

The $\mathrm{H} \alpha$ emission line is one the most prominent spectroscopic features in the visible spectra of CTTSs (Joy 1945; Herbig 1962). The line profile is often complex and takes different shapes, which were studied and classified by Reipurth et al. (1996). We measured the $\mathrm{H} \alpha$ EWs by direct integration and by fitting a Voigt profile (when the target exhibited a $\mathrm{H} \alpha$ profile with a single peak). Our measurement errors are mainly caused by the uncertainty on the continuum level in the vicinity of the line. We use the standard limit of $10 \AA$ (see, e.g. Appenzeller \& 

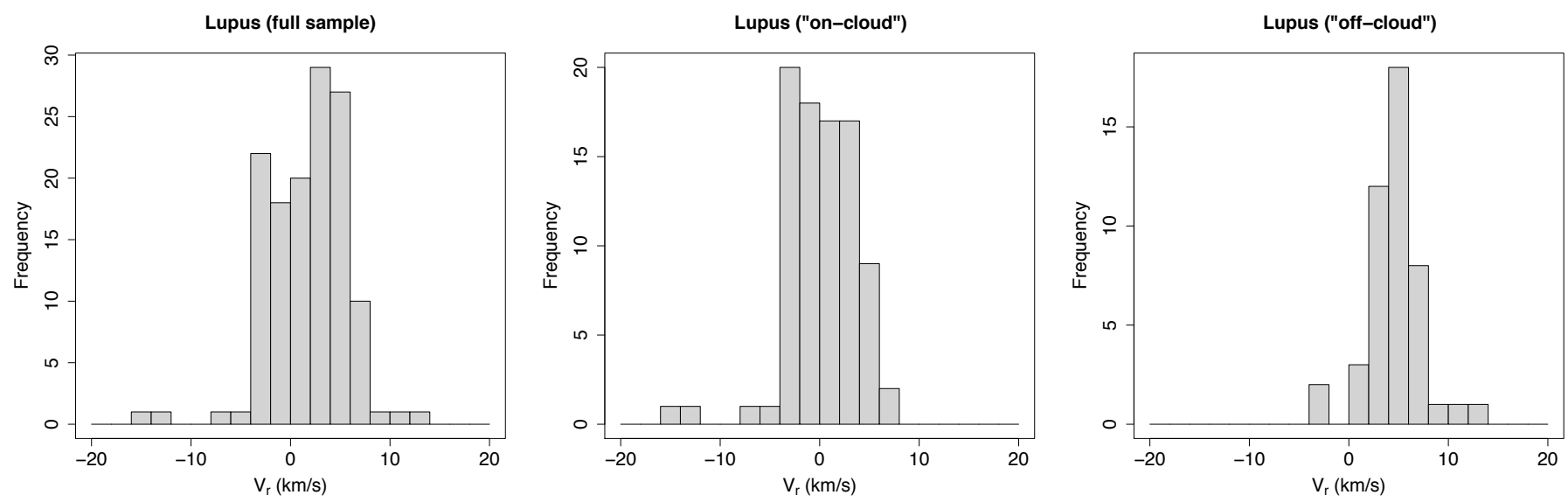

Fig. 5. Histogram of RVs found in the literature for the 108 stars in Lupus.

Mundt 1989) to distinguish between CTTSs (EW(H $\alpha) \geq 10 \AA)$ and WTTSs $(\mathrm{EW}(\mathrm{H} \alpha)<10 \AA)$ and find that our sample contains 10 CTTSs and 42 WTTSs. A more detailed inspection of the spectra in our sample revealed the existence of 2 PMS spectroscopic binaries (SB2) for which no evidence of binarity could be found in the literature prior to our observations. We present the results of our observations in Table 2.

\section{Analysis and results}

In the following we derive the $\mathrm{CP}$ of the co-moving group of Lupus stars considered in this paper. We discuss the various $\mathrm{CP}$ positions derived from different subsets of our initial sample and perform a membership analysis to distinguish between Lupus and UCL stars. The technique that we use to find the CP position of the Lupus moving group is the new $\mathrm{CP}$ search method that was recently developed by our team. We refer the reader to the original paper (Galli et al. 2012) for more details on the implementation of this method.

\subsection{Preliminary analysis}

As explained above, we suspect that the Lupus off-cloud population is a mix of Lupus and UCL stars. To better investigate this point we compare the proper motion distribution in both on-cloud and off-cloud components of the Lupus sample with the proper motions of the UCL members given in Preibisch \& Mamajek (2008). The UCL members are sufficient bright and we found proper motion data in the Түсно 2 catalogue for all but two stars.

We apply the Kolmogorov-Smirnov normality test to the proper motion distributions to test whether Lupus and UCL stars are drawn from the same parent distribution. The results of this analysis are presented in Fig. 6. Adopting the significance level of $5 \%$ we conclude that the proper motion distribution in both components of the Lupus on-cloud population and UCL stars are significantly different. On the other hand, the results for the off-cloud population show that the null hypothesis cannot be rejected, confirming our suspicion that the Lupus off-cloud population is contaminated by UCL stars.

The velocity dispersion in Lupus is a first input parameter that we must determine for performing the $\mathrm{CP}$ analysis. The velocity dispersion of young moving groups is expected to be low, only a few $\mathrm{km} / \mathrm{s}$ (Mathieu 1986). Typical values of velocity dispersion in nearby SFRs are 1-2 km/s (Jones \& Herbig 1979; Dubath et al. 1996; Makarov 2007). We adopt $\sigma_{v}=2.0 \mathrm{~km} / \mathrm{s}$ as the one-dimensional velocity dispersion of the Lupus moving group and we will come back to this point in Sect. 6. Another input parameter in the CP method is the mean distance to the moving group. As discussed in Sect. 2, the distance to the Lupus SFR has undergone substantial revision in the literature over the last decade. Here we use the value of $d=150 \mathrm{pc}$ that seems to be adequate for most of the clouds in the complex (Comerón 2008). The CP search method is rather insensitive to small variations in both parameters.

When we apply the CP search method to the 217 stars in the sample, we end up with a $\mathrm{CP}$ position given by

$$
\left(\alpha_{c p}, \delta_{c p}\right)=\left(93.3^{\circ},-25.0^{\circ}\right) \pm\left(2.4^{\circ}, 3.6^{\circ}\right)
$$

with 153 moving group members. In a recent paper Makarov (2007) investigated the kinematics of the Lupus stellar sample of 93 PMS stars identified by Krautter et al. (1997). The CP position derived in his work is

$$
\left(\alpha_{c p}, \delta_{c p}\right)=\left(92.8^{\circ},-28.1^{\circ}\right) \pm\left(3.1^{\circ}, 5.0^{\circ}\right) .
$$

There is good agreement between both solutions and it is probable that the small differences are caused by the different samples of Lupus stars, the different proper motion sources and the different CP search methods.

\subsection{Convergent point analysis of the Lupus subgroups}

Whether the above result is the most appropriate for the sample of the TTSs associated with the Lupus molecular clouds is questionable. One must clarify whether the two on-cloud and off-cloud subsamples have the same CP on the celestial sphere, since the CP solution found above might be affected by UCL stars misidentified as Lupus WTTSs. We investigate this question below.

\subsubsection{Convergent point of the UCL subgroup}

Jos de Bruijne (1999) derived the cluster centroid space motion, the internal velocity dispersion, and individual parallaxes for the UCL subgroup from Monte Carlo simulations. Assuming that the group is located at $140 \mathrm{pc}$, he found that that the velocity dispersion of the cluster is $\sigma_{v} \leq 1.5 \mathrm{~km} / \mathrm{s}$. We apply 
Table 2. Results from our observations. The upper panel presents the RVs derived in this paper together with EWs for the Li and $H \alpha$ lines. We also provide the TTS subclass based on $E W(H \alpha)$. The symbol ":" indicates uncertain values of RVs where one of the cross-correlation techniques failed to return a result. Negative and positive values of $E W(H \alpha)$ denote that the line is, respectively, in emission and absorption. The lower panel presents those stars that show evidence of spectroscopic binarity (SB2).

\begin{tabular}{|c|c|c|c|c|c|c|}
\hline ID & $\begin{array}{c}\alpha \\
\text { (h:m:s) }\end{array}$ & $\begin{array}{c}\delta \\
\left({ }^{\circ},{ }^{\prime \prime}\right)\end{array}$ & $\begin{array}{c}V_{r} \\
(\mathrm{~km} / \mathrm{s})\end{array}$ & $\begin{array}{c}E W(L i) \\
(\AA)\end{array}$ & $\begin{array}{c}E W(H \alpha) \\
(\AA)\end{array}$ & Remarks \\
\hline RXJ1448.2-4103 & 144813.3 & -410258 & $+5.95 \pm 0.17$ & $0.336 \pm 0.017$ & $\lesssim 0.1$ & WTTS \\
\hline RXJ1452.4-3740 & 145226.2 & -374008 & $+5.00 \pm 0.40$ & $0.402 \pm 0.003$ & $\lesssim 0.1$ & WTTS \\
\hline RXJ1454.2-3955 & 145411.3 & -395523 & $+3.83 \pm 0.38$ & $0.382 \pm 0.002$ & $-0.68 \pm 0.04$ & WTTS \\
\hline RXJ1502.4-3405 & 150226.0 & -340513 & $+3.88 \pm 0.17$ & $0.437 \pm 0.001$ & $-0.27 \pm 0.08$ & WTTS \\
\hline RXJ1505.4-3857 & 150525.9 & -385703 & $+4.92 \pm 0.33$ & $0.416 \pm 0.004$ & $-0.92 \pm 0.08$ & WTTS \\
\hline RXJ1505.9-4311 & 150556.9 & -431202 & $+3.99 \pm 0.27$ & $0.465 \pm 0.001$ & $-0.99 \pm 0.05$ & WTTS \\
\hline RXJ1506.7-3047 & 150642.6 & -304733 & $+0.81 \pm 0.24$ & $0.480 \pm 0.002$ & $-1.82 \pm 0.13$ & WTTS \\
\hline RXJ1508.0-3338 & 150805.1 & -333755 & $+1.65 \pm 0.23$ & $0.447 \pm 0.010$ & $-0.55 \pm 0.02$ & WTTS \\
\hline RXJ1508.4-3338 & 150825.0 & -333755 & $+1.85 \pm 0.43$ & $0.438 \pm 0.011$ & $-1.29 \pm 0.11$ & WTTS \\
\hline RXJ1508.8-3715 & 150853.8 & -371546 & $+4.51 \pm 0.28$ & $0.421 \pm 0.012$ & $-0.50 \pm 0.06$ & WTTS \\
\hline RXJ1515.1-4438 & 151509.3 & -443836 & $+6.34 \pm 0.34$ & $0.367 \pm 0.012$ & $-1.11 \pm 0.05$ & WTTS \\
\hline RXJ1518.0-4445 & 151801.3 & -444426 & $+5.97 \pm 0.15$ & $0.099 \pm 0.007$ & $0.81 \pm 0.05$ & WTTS \\
\hline RXJ1524.5-3652 & 152432.4 & -365202 & $+3.75 \pm 0.33$ & $0.344 \pm 0.001$ & $\lesssim 0.1$ & WTTS \\
\hline RXJ1526.8-3721 & 152652.6 & -372206 & $+1.92 \pm 0.21$ & $0.505 \pm 0.002$ & $-2.25 \pm 0.15$ & WTTS \\
\hline RXJ1529.8-4523 & 152948.9 & -452245 & $+4.64 \pm 0.41$ & $0.442 \pm 0.008$ & $-0.75 \pm 0.03$ & WTTS \\
\hline RXJ1534.3-3300 & 153423.2 & -330009 & $+1.40 \pm 0.20$ & $0.525 \pm 0.008$ & $-1.11 \pm 0.08$ & WTTS \\
\hline RXJ1538.0-3807 & 153802.7 & -380723 & $+2.46 \pm 0.28$ & $0.423 \pm 0.021$ & $-1.93 \pm 0.44$ & WTTS \\
\hline RXJ1539.7-3450 & 153946.4 & -345102 & $+5.39 \pm 0.29$ & $0.284 \pm 0.013$ & $-0.32 \pm 0.02$ & WTTS \\
\hline RXJ1542.0-3601 & 154205.2 & -360132 & $-0.05 \pm 0.26$ & $0.492 \pm 0.011$ & $-0.81 \pm 0.03$ & WTTS \\
\hline RXJ1544.5-3521 & 154435.3 & -352149 & $+2.47 \pm 0.23$ & $0.491 \pm 0.008$ & $-1.37 \pm 0.07$ & WTTS \\
\hline RXJ1547.1-3540 & 154708.4 & -354019 & $+0.82 \pm 4.90$ & $0.472 \pm 0.016$ & $-1.09 \pm 0.39$ & WTTS \\
\hline RXJ1547.6-4018 & 154741.8 & -401826 & $+2.85 \pm 0.37$ & $0.387 \pm 0.012$ & $0.17 \pm 0.01$ & WTTS \\
\hline RXJ1548.0-4004 & 154802.1 & -400428 & $+2.07 \pm 0.35$ & $0.433 \pm 0.014$ & $-2.01 \pm 0.06$ & WTTS \\
\hline RXJ1548.9-3513 & 154854.1 & -351318 & $+0.62 \pm 0.16$ & $0.372 \pm 0.002$ & $-0.41 \pm 0.03$ & WTTS \\
\hline RXJ1601.8-4026 & 160149.5 & -402619 & $+2.87 \pm 0.46$ & $0.371 \pm 0.011$ & $-1.39 \pm 0.08$ & WTTS \\
\hline RXJ1606.3-4447 & 160623.4 & -444735 & $+4.68 \pm 0.54$ & $0.455 \pm 0.007$ & $-0.48 \pm 0.06$ & WTTS \\
\hline GSC6780-1061 & 160654.4 & -241611 & $-5.43 \pm 0.19$ & $0.561 \pm 0.001$ & $-1.55 \pm 0.09$ & WTTS \\
\hline RXJ1608.0-3857 & 160800.0 & -385751 & $-2.42 \pm 0.84:$ & $0.638 \pm 0.002$ & $-1.91 \pm 0.14$ & WTTS \\
\hline F304 & 160811.0 & -391046 & $+2.76 \pm 0.11$ & $0.449 \pm 0.001$ & $-0.66 \pm 0.02$ & WTTS \\
\hline V908Sco & 160901.9 & -390512 & $-0.77 \pm 0.82:$ & $0.570 \pm 0.004$ & $-51.24 \pm 4.20$ & CTTS \\
\hline RXJ1609.9-3923 & 160954.0 & $\begin{array}{ll}-39 & 2327\end{array}$ & $-0.21 \pm 0.46$ & $0.550 \pm 0.003$ & $-22.33 \pm 0.93$ & CTTS \\
\hline RXJ1611.6-3841 & 161138.0 & -384135 & $+2.52 \pm 0.39$ & $0.438 \pm 0.007$ & $-3.93 \pm 0.31$ & WTTS \\
\hline GSC6793-994 & 161402.1 & -230102 & $-2.28 \pm 0.51$ & $0.356 \pm 0.013$ & $\lesssim 0.1$ & WTTS \\
\hline PDS145 & 161420.9 & -190605 & $-7.67 \pm 10.85$ & $0.261 \pm 0.007$ & $-65.94 \pm 2.30$ & CTTS \\
\hline RXJ1615.9-3947 & 161556.7 & -394716 & $+0.09 \pm 0.27$ & $0.456 \pm 0.011$ & $-2.37 \pm 0.23$ & WTTS \\
\hline RXJ1615.9-3241 & 161557.0 & -324124 & $-0.45 \pm 0.26$ & $0.458 \pm 0.010$ & $-0.68 \pm 0.06$ & WTTS \\
\hline RXJ1620.7-2348 & 162046.0 & -234821 & $-3.21 \pm 0.21$ & $0.465 \pm 0.002$ & $-0.37 \pm 0.07$ & WTTS \\
\hline RXJ1621.4-2312 & 162128.5 & $\begin{array}{lll}-23 & 12 & 11\end{array}$ & $-8.37 \pm 2.24$ & $0.588 \pm 0.011$ & $-1.27 \pm 0.09$ & WTTS \\
\hline Haro1-1 & 162134.7 & -261227 & $-4.29 \pm 0.50$ & $0.455 \pm 0.024$ & $-153.60 \pm 2.50$ & CTTS \\
\hline GSC6794-537 & 162307.8 & -230100 & $-10.05 \pm 1.41:$ & $0.491 \pm 0.011$ & $-0.52 \pm 0.10$ & WTTS \\
\hline HD147454 & 162332.3 & -343950 & $-0.10 \pm 0.41$ & $0.112 \pm 0.004$ & $2.32 \pm 0.15$ & WTTS \\
\hline SAO207620 & 162337.7 & -344021 & $-0.41 \pm 0.14$ & $0.195 \pm 0.005$ & $0.72 \pm 0.09$ & WTTS \\
\hline GSC6794-156 & 162451.4 & $\begin{array}{lll}-22 & 39 & 32\end{array}$ & $-5.27 \pm 1.72:$ & $0.338 \pm 0.001$ & $-0.90 \pm 0.18$ & WTTS \\
\hline RXJ1625.4-2346 & 162528.6 & -234627 & $-10.38 \pm 0.64$ & $0.381 \pm 0.001$ & $0.24 \pm 0.01$ & WTTS \\
\hline DoAr25 & 162623.7 & -244314 & $-8.25 \pm 0.59$ & $0.547 \pm 0.002$ & $-8.28 \pm 0.50$ & CTTS \\
\hline RNO90 & 163409.2 & -154817 & $-12.92 \pm 7.92:$ & $0.360 \pm 0.006$ & $-78.00 \pm 2.40$ & CTTS \\
\hline He3-1254 & 164644.3 & -151438 & $-8.98 \pm 0.29$ & $0.444 \pm 0.008$ & $-97.14 \pm 4.40$ & CTTS \\
\hline WaOph6 & 164845.6 & -141636 & $-10.09 \pm 0.54$ & $0.520 \pm 0.009$ & $-24.19 \pm 0.95$ & CTTS \\
\hline WaOph5 & 164900.8 & -141711 & $-9.54 \pm 3.04:$ & $0.668 \pm 0.001$ & $-54.07 \pm 7.31$ & CTTS \\
\hline V1725Oph & 171613.9 & -205746 & $-11.77 \pm 0.84:$ & $0.636 \pm 0.007$ & $-22.51 \pm 1.60$ & CTTS \\
\hline GSC6213-194 & 160941.0 & -221759 & & & & SB2, WTTS \\
\hline RXJ1613.1-3804 & 161312.7 & -380351 & & & & SB2, WTTS \\
\hline
\end{tabular}


Lupus ("on-coud") x UCL

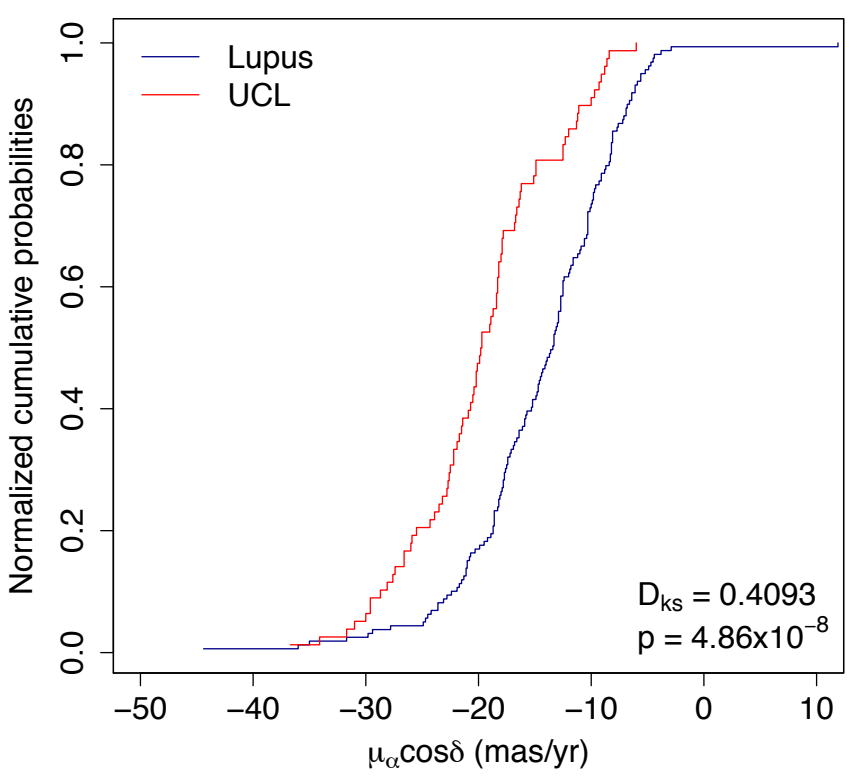

Lupus ("off-coud") x UCL

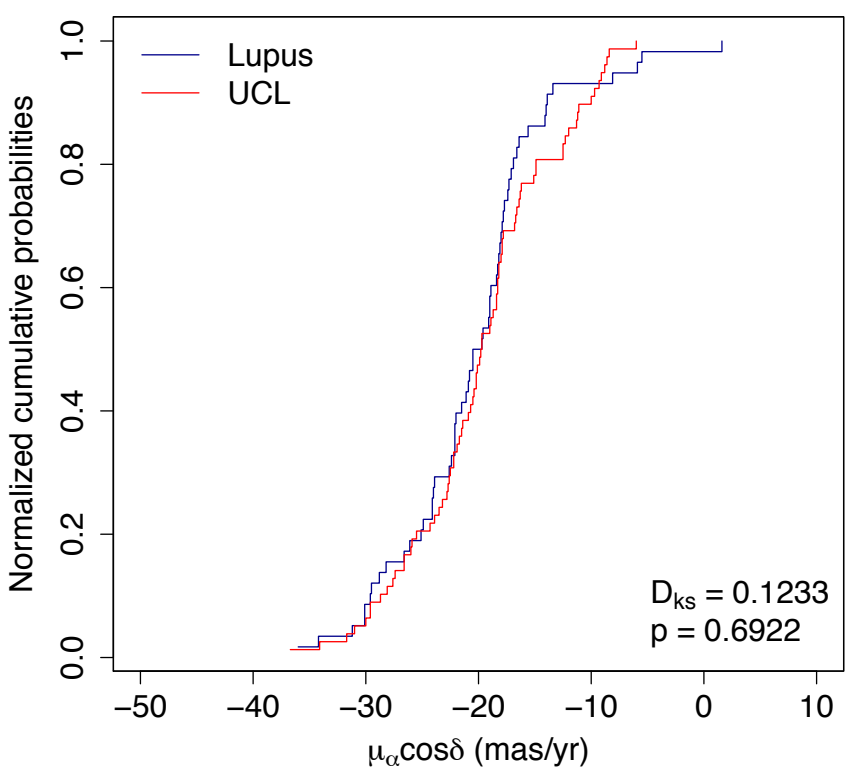

Lupus ("on-coud") x UCL

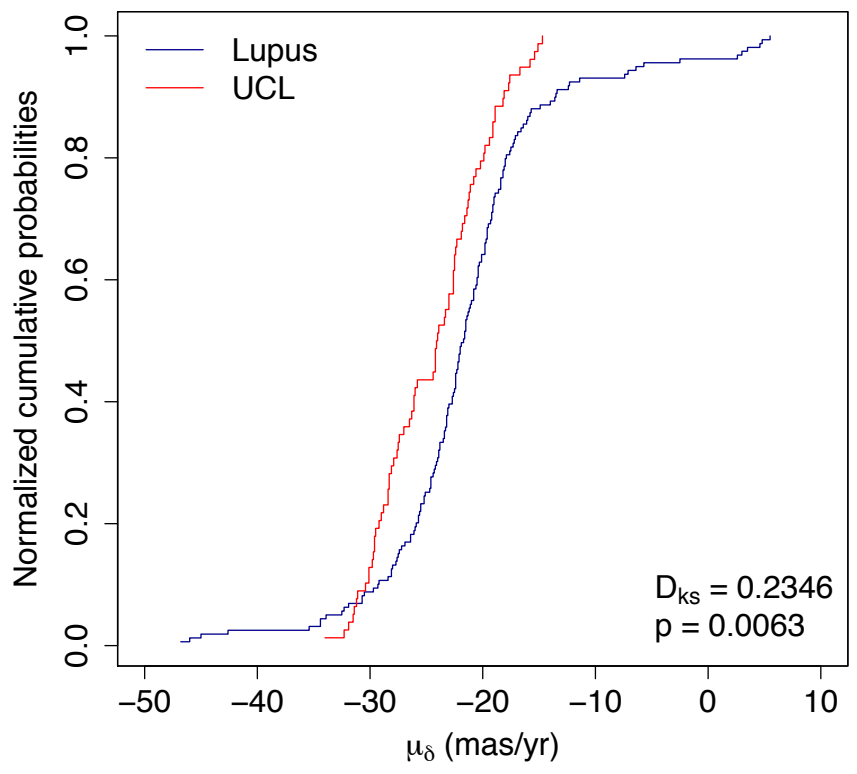

Lupus ("off-coud") x UCL

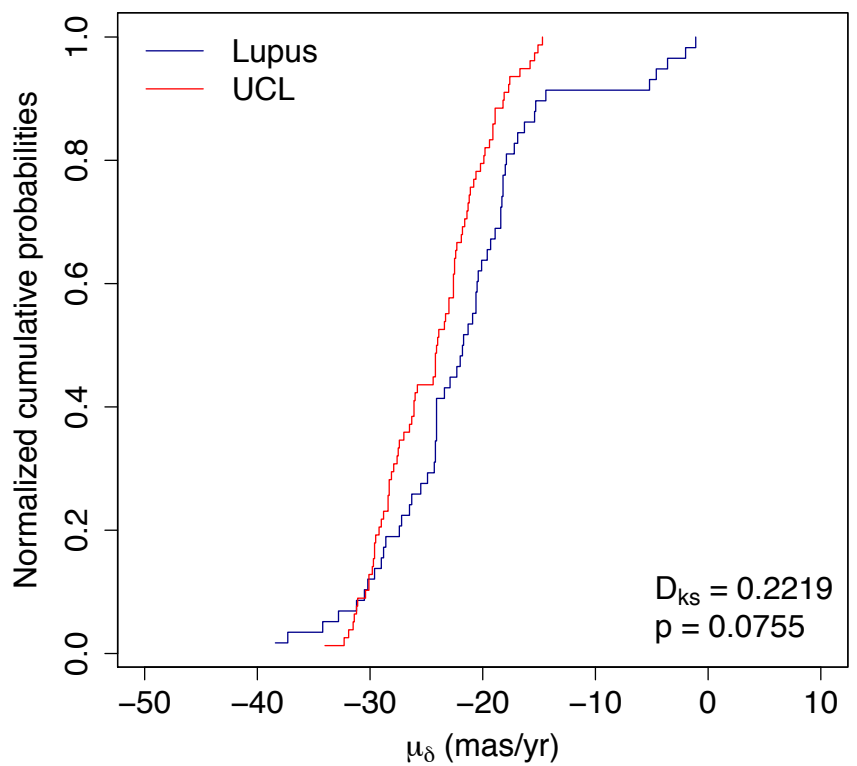

Fig. 6. Cumulative proper motion distribution in both components $\left(\mu_{\alpha} \cos \delta, \mu_{\delta}\right)$ of the Lupus on-cloud (upper panels) and off-cloud (lower panels) populations compared to the proper motion distribution of the UCL subgroup. We also provide in each panel the results for the KolmogorovSmirnov tests on the distributions of proper motions. $D_{k s}$ denotes the maximum discrepancy of both distributions and the $p$-value gives the probability (normalized from 0 to 1 ) for both distributions being drawn from the same parent distribution.

the CP search method to the sample of 81 UCL members from Preibisch \& Mamajek (2008) with the proper motions given in the Түсно 2 catalogue, and the average distance and velocity dispersion derived by de Bruijne (1999). We find a solution with 55 co-moving stars and the $\mathrm{CP}$ at

$$
\left(\alpha_{c p}, \delta_{c p}\right)=\left(105.0^{\circ},-38.3^{\circ}\right) \pm\left(2.8^{\circ}, 2.7^{\circ}\right) .
$$

That the CP method did not select all stars given in Preibisch $\&$ Mamajek (2008) as cluster members is not surprising because the membership analysis used in each case is not the same. When we include the rejected stars in our solution the $\mathrm{CP}$ position shifts to $\left(\alpha_{c p}, \delta_{c p}\right)=\left(99.1^{\circ},-31.7^{\circ}\right) \pm\left(2.0^{\circ}, 2.4^{\circ}\right)$. We conclude for the moment that the 55 stars selected by the CP method are confirmed members of the UCL subgroup and that this solution defines the CP of the UCL subgroup. 


\subsubsection{Convergent point of the Lupus on-cloud population}

Many of the stars that are presented in Preibisch \& Mamajek (2008) as UCL members come from the Mamajek et al. (2002) survey. They selected UCL candidate stars among the X-ray sources of the ROSAT All-Sky Survey Bright Catalog (Voges et al. 1999) based on proper motion and color-magnitude constraints. As shown in Fig. 4 some of these stars lie very close to the Lupus clouds and have also been considered as Lupus candidate members in the literature (Krautter et al. 1997; Wichmann et al. 1997a,b). We do not exclude these probable UCL members from our on-cloud sample of 159 stars but let the CP search method assign their membership status. Applying this strategy to the Lupus on-cloud population we find a co-moving group of 101 stars that defines a CP located at

$$
\left(\alpha_{c p}, \delta_{c p}\right)=\left(111.2^{\circ},-47.4^{\circ}\right) \pm\left(6.7^{\circ}, 5.5^{\circ}\right)
$$

We note that this solution is significantly different from the one presented in Sect. 5.1 with the complete sample.

\subsubsection{Convergent point of the Lupus off-cloud population}

If we now apply our CP search method to the Lupus off-cloud population of 58 stars, we find a group of 15 stars that defines the $\mathrm{CP}$ located at

$$
\left(\alpha_{c p}, \delta_{c p}\right)=\left(105.4^{\circ},-34.4^{\circ}\right) \pm\left(11.1^{\circ}, 10.4^{\circ}\right) .
$$

We then compute for each star the membership probability to the UCL subgroup and Lupus clouds using the CP solutions given, respectively, in Sect. 5.2.1 and Sect. 5.2.2. The results of this analysis are presented in Table 3 . We conclude that these stars are more likely to be members of the UCL subgroup than of the Lupus SFR.

Should the rejected stars be considered as Lupus members? To answer this question, we exclude from the sample the 15 stars suspected to be UCL members and run the CP analysis. We find another group of 15 stars defining a $\mathrm{CP}$ at

$$
\left(\alpha_{c p}, \delta_{c p}\right)=\left(112.6^{\circ},-48.7^{\circ}\right) \pm\left(17.8^{\circ}, 11.9^{\circ}\right) .
$$

This solution is in good agreement with the CP derived in Sect. 5.2.2 for the Lupus on-cloud population, and we thus conclude that they belong to the same moving group.

\subsubsection{Final convergent point for the Lupus moving group}

The procedure described in the previous sections made it possible to separate some UCL members included in our initial sample from the bona fide Lupus PMS population. Our final analysis consists in running the $\mathrm{CP}$ search method with both on-cloud and off-cloud populations together, but rejecting the 15 stars from Table 3 that are likely to be UCL members. We identify a moving group with 114 members and CP located at

$$
\left(\alpha_{c p}, \delta_{c p}\right)=\left(112.3^{\circ},-48.8^{\circ}\right) \pm\left(5.4^{\circ}, 4.0^{\circ}\right) .
$$

We note that the rejection of some likely UCL members in our sample dramatically changed the $\mathrm{CP}$ position of the moving group as compared to our first solution in Sect. 5.1, which now appears to be a mixed CP solution of Lupus and UCL stars, and is therefore not valid for either moving group. Tables 4 and 5 present the 114 Lupus moving group members selected by the $\mathrm{CP}$ search method together with their parallaxes (to be discussed in Sect. 6). We note that 12 stars that were classified as UCL members by Preibisch \& Mamajek (2008) and previously regarded as Lupus stars (Krautter et al. 1997; Wichmann et al. 1997a,b) have been accepted as Lupus members in our CP analysis. These stars are marked with the symbol "*”. On the other hand, we found that 13 stars previously catalogued as WTTSs following their discovery in X-ray have been rejected in this analysis and they are instead probable UCL members as discussed by Preibisch \& Mamajek (2008).

\subsubsection{Result check via Monte Carlo simulations}

To assess the validity of the CP location presented above, we performed Monte Carlo simulations of the 114 Lupus moving group members. We constructed 1000 samples of moving groups by resampling the stellar proper motions from a Gaussian distribution where the mean and variance are equal to the individual stellar proper motions and its uncertainty (in both components). We run the CP search method for each set of simulated stars and compute the $\mathrm{CP}$ of each moving group. The results of this study are presented in Fig. 7. The CP solution derived in this paper is fully consistent with the centroid distribution of the Monte Carlo realizations, located at

$$
\left(\alpha_{c p}, \delta_{c p}\right)=\left(112.9^{\circ},-49.1^{\circ}\right) \pm\left(4.5^{\circ}, 3.4^{\circ}\right)
$$

and we therefore conclude that it is representative of the Lupus moving group.

\section{Kinematic parallaxes}

\subsection{Parallaxes and space velocities of group members with known radial velocity}

Once the moving group is defined, it is possible to derive individual parallaxes $\pi_{\text {ind }}$ for group members if their RVs $V_{r}$ are known. Individual parallaxes are given by

$\pi_{\text {ind }}=\frac{A \mu_{\|}}{V_{r} \tan \lambda}$

where $A=4.74047 \mathrm{~km} \mathrm{yr} / \mathrm{s}$ is the ratio of one astronomical unit in $\mathrm{km}$ to the number of seconds in one Julian year, $\lambda$ is the angular distance from the $\mathrm{CP}$ position to a given star in the moving group and $\mu_{\|}$is the stellar proper motion component that points towards the CP (see Galli et al. 2012, for more details). The parallax uncertainty is derived by error propagation of this equation and takes the error budget of proper motions and RVs into account.

We found RVs for only 55 stars in the sample of 114 moving group members. We reject (resolved) binaries since it will not be possible to derive their parallaxes from a single RV measurement. Stars that exhibit poor RVs because of their errors are also excluded from this analysis. The observed RV for Lupus stars is expected to be low and a small variation accounts for a more significant shift in the parallax and space velocity (see Appendix A for more details). In order to spot possible errors on parallaxes and velocities, we define lower and upper limits for the space velocity of Lupus stars. To do so, we use $8 \mathrm{stars}^{2}$ with known trigonometric parallax in the Hipparcos catalogue that have been selected as moving group members in our CP analysis. The space velocities derived using HiPPARcos parallaxes range from $V_{\text {lower }} \simeq 17 \mathrm{~km} / \mathrm{s}$ and $V_{\text {upper }} \simeq 33 \mathrm{~km} / \mathrm{s}$ which define the

${ }^{2} \mathrm{Sz} 120$ is not considered in this analysis because it is a binary HAeBe star (Correia et al. 2006). 
P.A.B. Galli et al.: A kinematic study and membership analysis of the Lupus star-forming region

Table 3. Sample of 15 stars that are more likely to be associated to the UCL subgroup. We provide for each star the most usual identifier, position (epoch 2000), proper motion, source of proper motion, 2MASS identifier and the membership probabilities to the UCL subgroup and Lupus clouds.

\begin{tabular}{lcccccccc}
\hline ID & $\begin{array}{c}\alpha \\
(\mathrm{h}: \mathrm{m}: \mathrm{s})\end{array}$ & $\begin{array}{c}\delta \\
\left({ }^{\circ}{ }^{\prime \prime}\right)\end{array}$ & $\begin{array}{c}\mu_{\alpha} \cos \delta \\
(\mathrm{mas} / \mathrm{yr})\end{array}$ & $\begin{array}{c}\mu_{\delta} \\
(\mathrm{mas} / \mathrm{yr})\end{array}$ & Source & $\begin{array}{c}p_{U C L} \\
(\%)\end{array}$ & $\begin{array}{c}p_{L U P} \\
(\%)\end{array}$ & 2MASS \\
\hline RXJ1452.4-3740 & 145226.2 & -374008 & $-18.9 \pm 1.7$ & $-17.2 \pm 1.6$ & SPM4 & 70 & 22 & $14522619-3740088$ \\
RXJ1457.3-3613 & 145719.7 & -361227 & $-24.1 \pm 1.3$ & $-23.4 \pm 1.3$ & SPM4 & 72 & 13 & $14571962-3612274$ \\
RXJ1458.6-3541 & 145837.7 & -354030 & $-22.1 \pm 2.1$ & $-22.3 \pm 2.1$ & SPM4 & 91 & 37 & $14583769-3540302$ \\
RXJ1459.3-4013 & 145922.8 & -401312 & $-30.1 \pm 2.9$ & $-29.0 \pm 2.8$ & SPM4 & 68 & 16 & $14592275-4013120$ \\
RXJ1502.4-3405 & 150226.0 & -340513 & $-18.3 \pm 2.2$ & $-16.3 \pm 2.1$ & SPM4 & 51 & 15 & $15022600-3405131$ \\
RXJ1505.4-3716 & 150525.8 & -371533 & $-26.6 \pm 1.5$ & $-26.3 \pm 1.4$ & SPM4 & 56 & 7 & $15052583-3715325$ \\
RXJ1507.2-3505 & 150714.8 & -350459 & $-29.6 \pm 2.3$ & $-30.2 \pm 2.3$ & SPM4 & 70 & 12 & $15071481-3504595$ \\
RXJ1508.0-3338K & 150805.1 & -333755 & $-28.2 \pm 1.9$ & $-28.6 \pm 1.9$ & SPM4 & 64 & 8 & $15080509-3337556$ \\
RXJ1508.6-4423AB & 150837.8 & -442317 & $-19.0 \pm 2.0$ & $-18.0 \pm 2.0$ & D05 & 60 & 28 & $15083773-4423170$ \\
HD133938 & 150838.5 & -440052 & $-16.9 \pm 0.9$ & $-18.2 \pm 1.0$ & SPM4 & 98 & 68 & $15083849-4400519$ \\
RXJ1515.1-4438 & 151509.3 & -443836 & $-19.6 \pm 2.0$ & $-20.5 \pm 2.2$ & SPM4 & 83 & 48 & $15150933-4438362$ \\
RXJ1515.8-4418K & 151552.8 & -441817 & $-16.4 \pm 1.6$ & $-18.9 \pm 1.8$ & SPM4 & 100 & 85 & $15155274-4418173$ \\
RXJ1522.2-3959 & 152211.7 & -395950 & $-20.5 \pm 1.3$ & $-21.8 \pm 1.3$ & SPM4 & 63 & 22 & $15221162-3959509$ \\
RXJ1526.0-4501 & 152559.7 & -450116 & $-17.1 \pm 1.2$ & $-17.9 \pm 1.2$ & SPM4 & 61 & 33 & $15255964-4501157$ \\
RXJ1529.8-4523K & 152948.9 & -452245 & $-21.5 \pm 1.4$ & $-24.9 \pm 1.5$ & SPM4 & 84 & 48 & $15294888-4522457$ \\
\hline
\end{tabular}

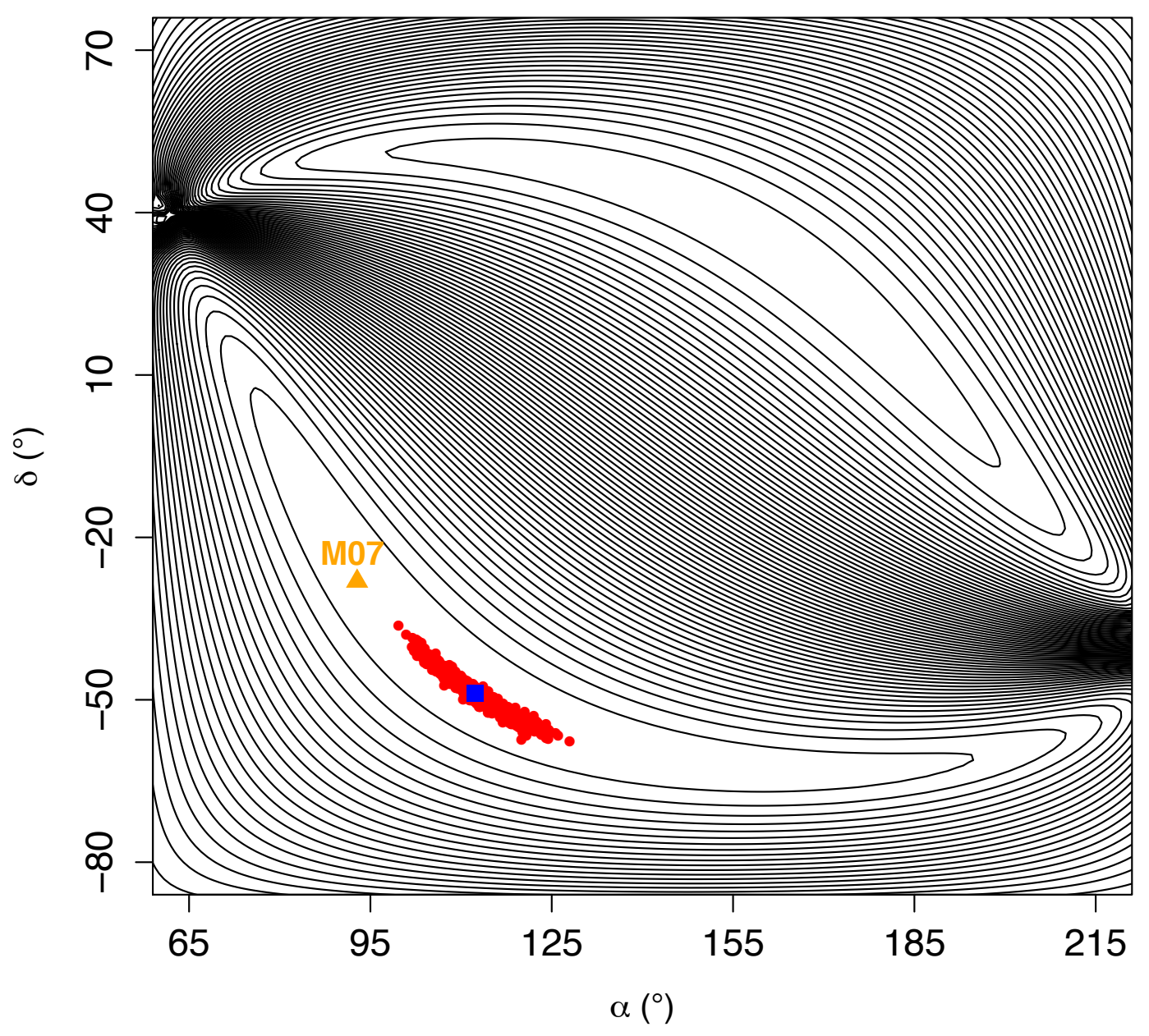

Fig. 7. Realizations of CP coordinates for 1000 Monte Carlo simulations (red dots) overlaid on the $X^{2}$ contours for the CP solution derived in Sect. 5.2.4. The blue square denotes the $\mathrm{CP}$ coordinates for the Lupus moving group derived in that section and the orange triangle denotes the $\mathrm{CP}$ solution given in Makarov (2007, M07). 
limits for the space velocity of Lupus stars. This leaves us with a sample of 26 stars that we define here as the Lupus core moving group. The mean parallax is $\bar{\pi}=(7.1 \pm 1.8)$ mas, which agrees well with the estimated distance of $150 \mathrm{pc}$ assumed in the $\mathrm{CP}$ analysis (see Sect. 5.1).

We compute the Galactic velocities for each star (see Fig. 8) using the procedure described in Johnson \& Soderblom (1987). Their average and standard deviation values are

$$
\begin{gathered}
U=(-4.4 \pm 2.4) \mathrm{km} / \mathrm{s} \\
V=(-20.6 \pm 4.1) \mathrm{km} / \mathrm{s} \\
W=(-5.8 \pm 1.6) \mathrm{km} / \mathrm{s} \\
V_{\text {space }}=(22.0 \pm 4.3) \mathrm{km} / \mathrm{s} .
\end{gathered}
$$

The space velocity for UCL stars is given by Sartori et al. (2003) as $(U, V, W)=(-6.7,-19.3,-5.7) \mathrm{km} / \mathrm{s}$. These results suggest that Lupus and UCL have statistically indistinguishable motions assuming a velocity dispersion of a few $\mathrm{km} / \mathrm{s}$. Although their relative motion $(\Delta U, \Delta V, \Delta W)=(2.3,-1.3,-0.1) \mathrm{km} / \mathrm{s}$ is small, we confirm from our CP analysis in Sect. 5 that Lupus and UCL have different kinematic properties and they define a different $\mathrm{CP}$ on the celestial sphere.

As a "by-product" of the parallax computation we derive the two components $V_{\alpha}$ and $V_{\delta}$ of the tangential velocity. Their average value and standard deviation are

$$
\begin{aligned}
& V_{\alpha}=(-12.2 \pm 2.3) \mathrm{km} / \mathrm{s} \\
& V_{\delta}=(-17.6 \pm 4.0) \mathrm{km} / \mathrm{s} .
\end{aligned}
$$

We note that the velocity dispersion of $\sigma_{v}=2 \mathrm{~km} / \mathrm{s}$ used in our CP analysis (see Sect. 5.1) corresponds approximately to the lowest dispersion in the tangential velocity components. We argue that we have detected a minimum moving group in our $\mathrm{CP}$ analysis that may not contain all stars kinematically associated with the Lupus SFR. This was done to avoid contamination of UCL stars in our sample of Lupus members. We present in Table 4 the parallaxes derived for the Lupus core moving group.

\subsection{Approximate parallaxes for other moving group members}

The hypothesis that all members of a moving group share the same space motion allows us to compute tentative parallaxes for group members with unknown RVs. First we derive the average spatial velocity $V_{\text {space }}$ from the Galactic velocity of the stars with known RVs using the 26 stars that define the Lupus core moving group (see Sect. 6.1). Then we compute an approximate parallax $\pi_{a p p}$ as

$\pi_{\text {app }}=\frac{A \mu_{\|}}{V_{\text {space }} \sin \lambda}$.

The uncertainty on this tentative parallax is again derived by error propagation and considers in this case the error budget of proper motions and the space velocity. We present in Fig. 9 a comparison between individual parallaxes (see Table 4) and approximate parallaxes for the Lupus core moving group. Both procedures return similar results within the admittedly large error bars, which tends to justify the assumption of a common space motion.

The velocity dispersion of the cluster prevents all stars from having exactly the same space velocity. However, the procedure described above to derive approximate parallaxes considers a single value of the space velocity for all stars in the group. We performed Monte Carlo simulations by resampling the spatial velocity from a Gaussian distribution where the mean and variance correspond to the values given in Sect. 6.1 for the average space velocity of the Lupus core moving group. We constructed a total of 1000 realizations. In each run we assign a different value of space velocity and derive the approximate parallax for each star by using Eq. 2. The average and standard deviation of the computed parallaxes give our final result. This strategy not only allows us to reproduce the effect of velocity dispersion, but also makes our parallax results less dependent on a single value used for the spatial velocity of the group. We present in Table 5 the approximate parallaxes derived for the remaining 88 Lupus stars with unknown RVs.

Let us stress, however, that the individual approximate parallaxes derived in this way should only be seen as tentative values that will be useful for preliminary statistical analyses of the Lupus association. They should be superseded by more precise values when RV measurements become available for these association members.

\subsection{Comparison with Hipparcos results}

As a final check of our results we compare the parallaxes derived in this work with HiPPARcos trigonometric parallaxes. We consider both versions of the catalogue: the original catalogue (ESA 1997, hereafter HIP97) and the new reduction of HipPARcos data (van Leeuwen 2007, hereafter HIP07). We found only 9 HiPPARcos stars among the 114 moving group members and derived individual parallaxes for two of them (HD134974 and RXJ1504.8-3950). For the remaining stars we used the group spatial velocity to compute approximate parallaxes. The results of this comparison are presented in Fig. 10. The rms with respect to HIP97 is 2.28 mas and 1.92 mas for HIP07. The mean difference between the parallaxes derived in this work and, the ones in HIP97 and HIP07 are -0.68 mas and 0.35 mas, respectively. We conclude that our results are in good agreement with the trigonometric parallaxes given in HipPaRcos. 
P.A.B. Galli et al.: A kinematic study and membership analysis of the Lupus star-forming region
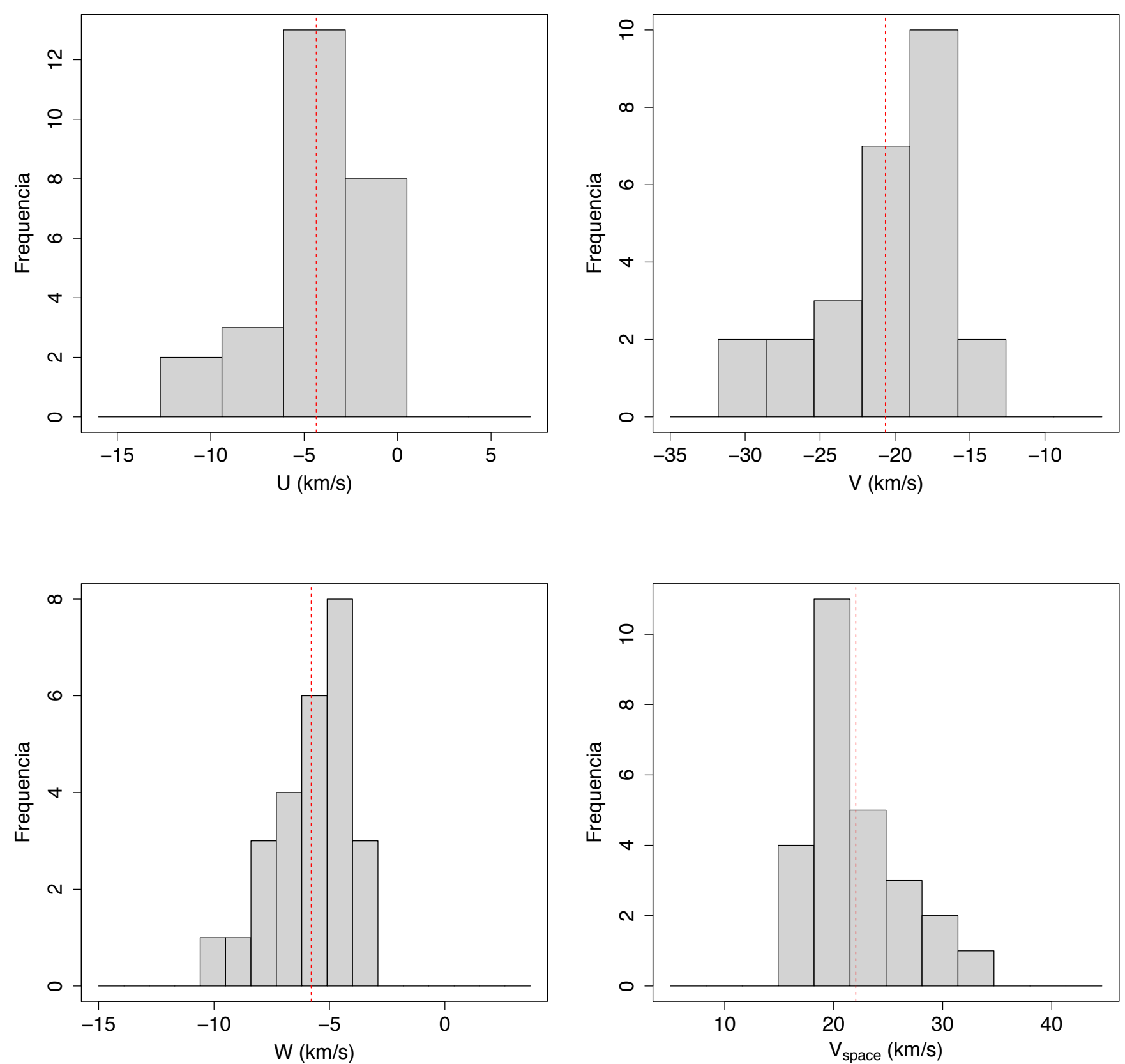

Fig. 8. Histograms of the Galactic velocity components for members of the Lupus moving group with known RVs. The red dashed line denotes the average values given in Sect. 6.1 


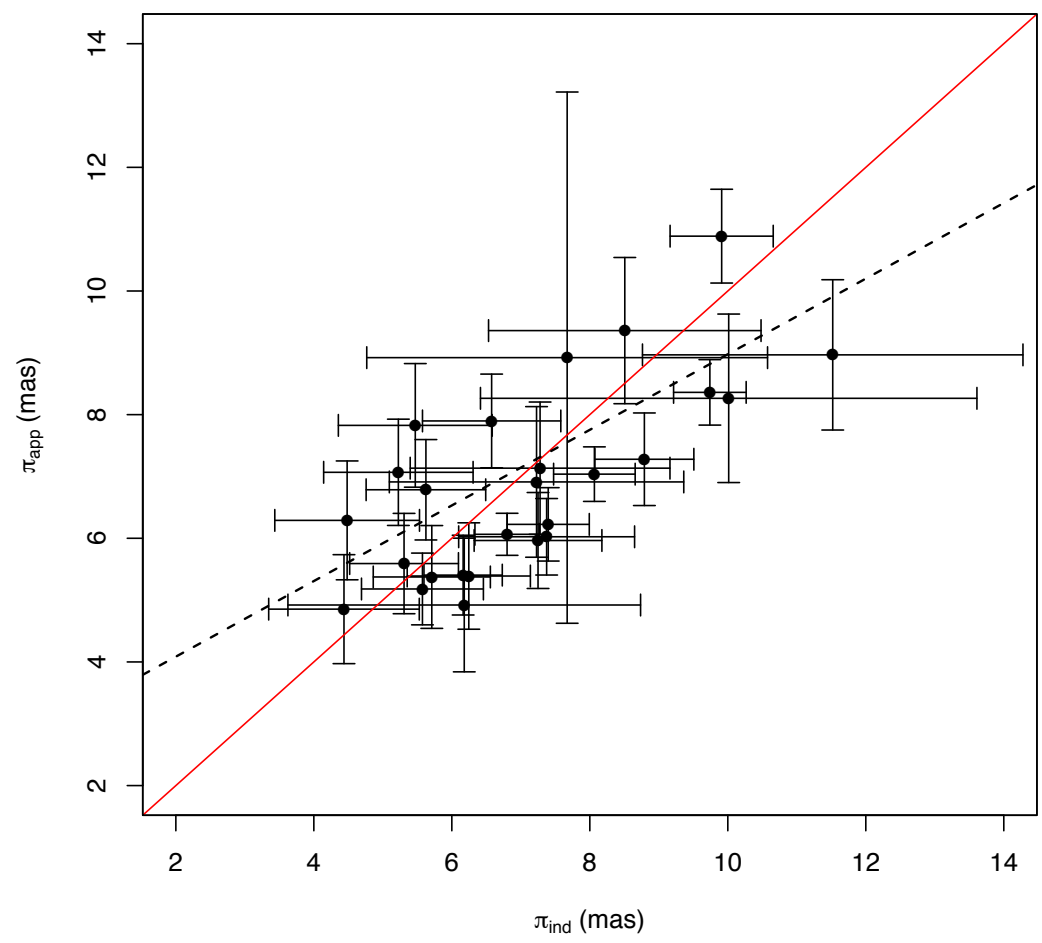

Fig. 9. Comparison of parallaxes computed with RVs $\left(\pi_{i n d}\right)$ and the spatial velocity $\left(\pi_{a p p}\right)$ for the Lupus core moving group of 26 stars. The red solid line indicates perfect correlation and the dashed line represents the weighted fit to the data. The mean difference between parallaxes computed with both strategies is 0.2 mas and the rms is 1.3 mas.

Hipparcos (ESA 1997)

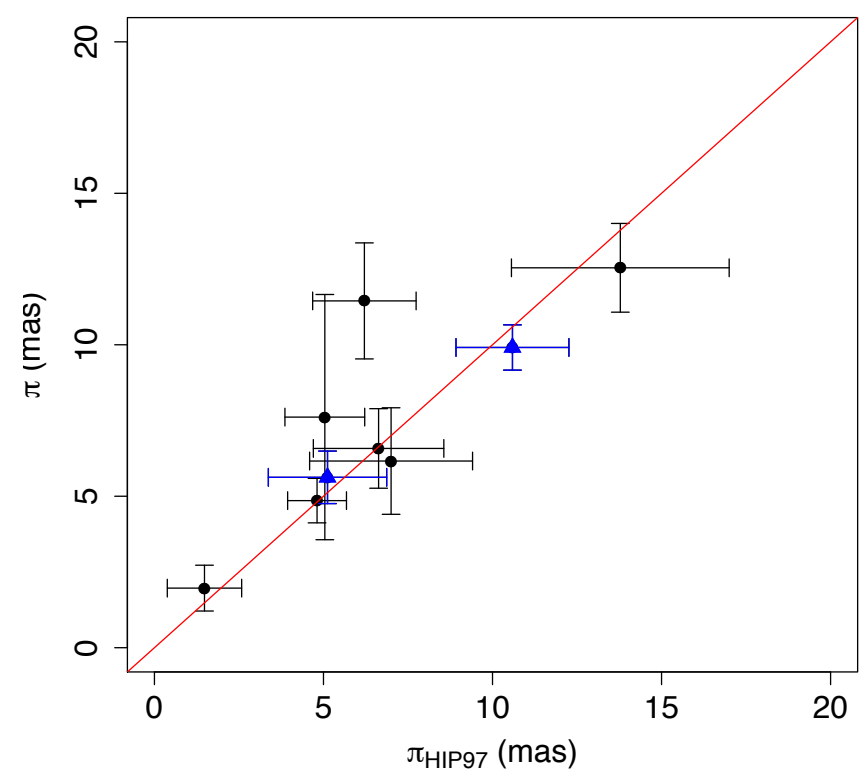

Hipparcos (van Leeuwen 2007)

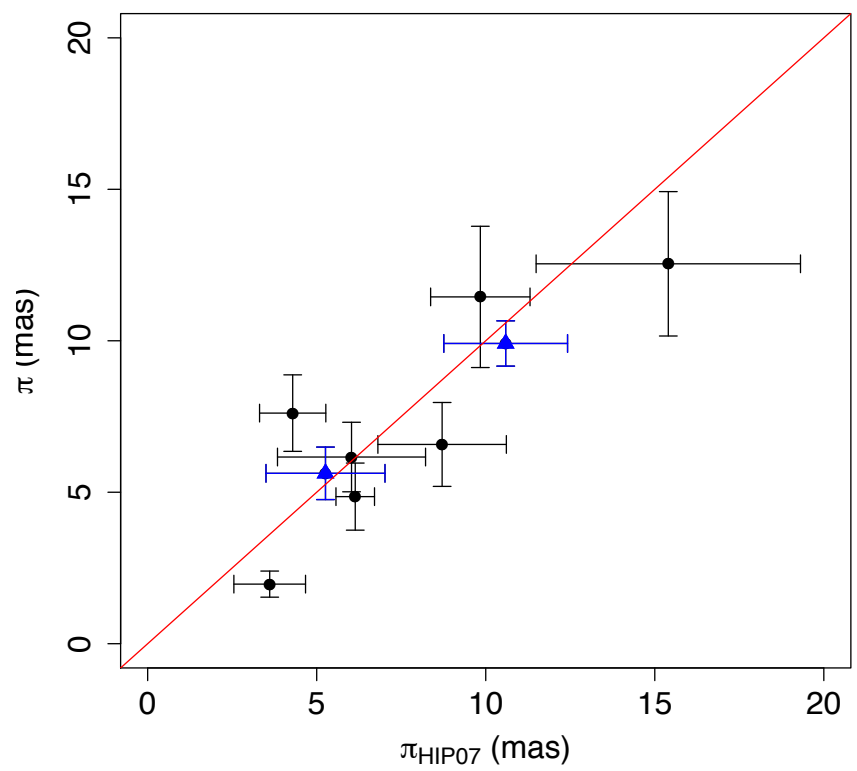

Fig. 10. Parallaxes derived in this paper compared with the trigonometric parallaxes given in HIP97 (left panel) and HIP07 (right panel). Blue triangles and black dots denote, respectively, parallaxes computed with RV and the group spatial velocity. The red solid line represents the expected distribution for equal results. 


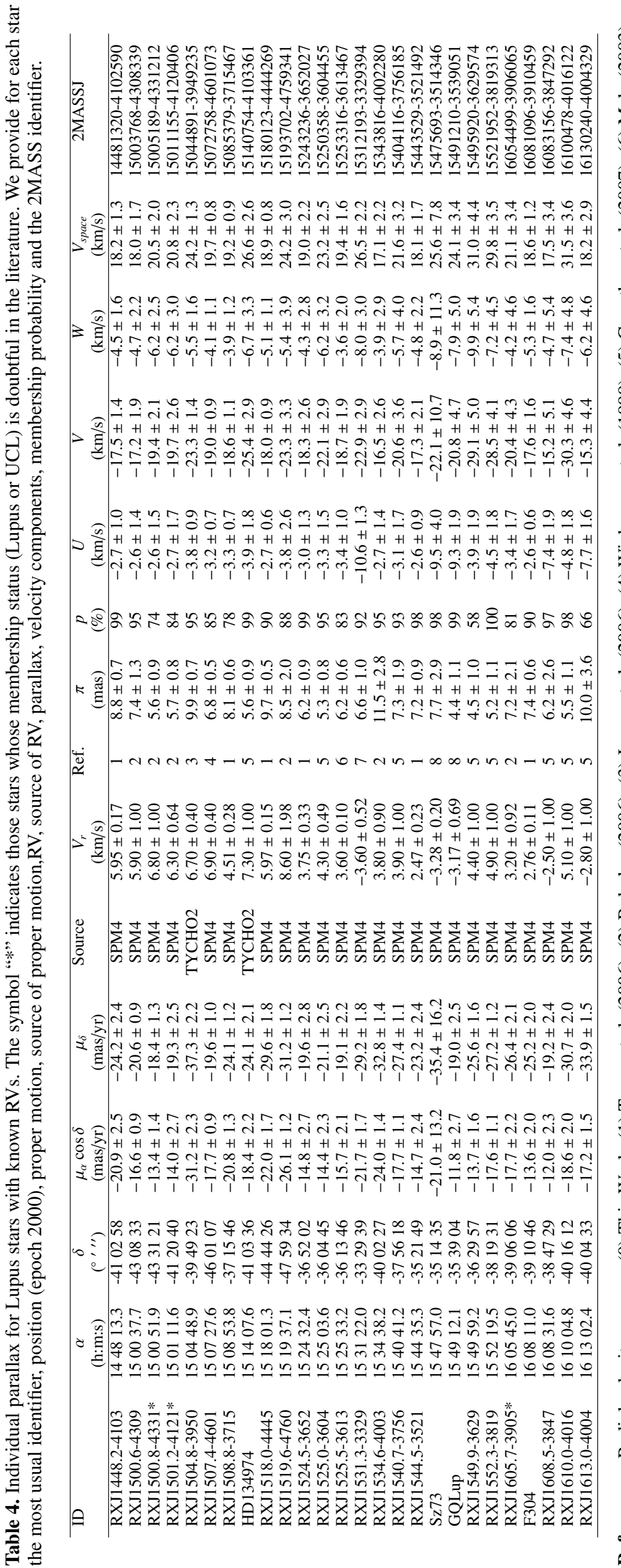


P.A.B. Galli et al.: A kinematic study and membership analysis of the Lupus star-forming region 
Table 5. Approximate parallax derived in this paper for Lupus stars with unknown RVs. The symbol “*” indicates those stars whose membership status (Lupus or UCL) is doubtful in the literature. We provide for each star the most usual identifier, position (epoch 2000), proper motion, source of proper motion, membership probability, parallax and the 2MASS identifier.

\begin{tabular}{|c|c|c|c|c|c|c|c|c|}
\hline ID & $\begin{array}{c}\alpha \\
\text { (h:m:s) }\end{array}$ & $\begin{array}{c}\delta \\
\left({ }^{\circ},{ }^{\prime \prime}\right)\end{array}$ & $\begin{array}{l}\mu_{\alpha} \cos \delta \\
(\mathrm{mas} / \mathrm{yr})\end{array}$ & $\begin{array}{c}\mu_{\delta} \\
\text { (mas/yr) }\end{array}$ & Source & $\begin{array}{c}p \\
(\%)\end{array}$ & $\begin{array}{c}\pi \\
\text { (mas) }\end{array}$ & 2MASSJ \\
\hline RXJ1447.3-3503 & 144723.5 & -350313 & $-30.1 \pm 1.8$ & $-38.4 \pm 1.9$ & SPM4 & 72 & $11.4 \pm 2.7$ & $14472343-3503134$ \\
\hline RXJ1450.4-3507* & 145025.8 & -350648 & $-18.0 \pm 1.3$ & $-18.4 \pm 1.3$ & SPM4 & 65 & $6.0 \pm 1.5$ & $14502581-3506486$ \\
\hline RXJ1450.5-3459 & 145035.1 & -345905 & $-17.8 \pm 1.3$ & $-20.9 \pm 1.3$ & SPM4 & 100 & $6.4 \pm 1.6$ & $14503508-3459056$ \\
\hline RXJ1454.2-3955 & 145411.3 & -395523 & $-22.1 \pm 2.5$ & $-28.8 \pm 2.4$ & SPM4 & 78 & $8.5 \pm 2.0$ & $14541121-3955233$ \\
\hline RXJ1505.9-4311 & 150556.9 & -431202 & $-24.9 \pm 1.0$ & $-30.5 \pm 1.0$ & SPM4 & 100 & $9.3 \pm 2.1$ & $15055685-4312031$ \\
\hline RXJ1511.0-3252AB & 151104.5 & -325130 & $-14.0 \pm 3.0$ & $-24.0 \pm 3.0$ & D05 & 52 & $6.3 \pm 1.7$ & $15110450-3251304$ \\
\hline RXJ1511.6-3550 & 151137.0 & -355042 & $-17.9 \pm 1.6$ & $-21.7 \pm 1.6$ & SPM4 & 92 & $6.5 \pm 1.6$ & $15113696-3550417$ \\
\hline RXJ1512.6-3417 & 151239.8 & -341659 & \pm 1.8 & $-18.3 \pm 1.7$ & SPM4 & 86 & $5.5 \pm 1.4$ & $15123981-3416591$ \\
\hline RXJ1512.8-4508A* & 151250.2 & -450805 & $-19.0 \pm 2.0$ & $-22.0 \pm 2.0$ & D05 & 91 & $7.0 \pm 1.7$ & $15125018-4508044$ \\
\hline HD135127 & 151439.6 & -344541 & $-17.3 \pm 1.3$ & $-24.3 \pm 1.3$ & TYCHO2 & 92 & $6.9 \pm 1.7$ & $15143959-3445412$ \\
\hline RXJ1515.7-3332 & 151545.4 & -333159 & $-23.1 \pm 1.6$ & $-26.4 \pm 1.6$ & SPM4 & 52 & $8.0 \pm 1.8$ & $15154537-3331597$ \\
\hline RXJ1518.4-3738* & 151826.9 & -373802 & $-18.1 \pm 1.3$ & $-26.5 \pm 1.2$ & SPM4 & 78 & $7.3 \pm 1.6$ & $15182692-3738021$ \\
\hline GSC6770-655 & 151952.9 & -280227 & $-36.0 \pm 3.0$ & $-45.0 \pm 3.0$ & D05 & 73 & $13.0 \pm 2.9$ & $15195295-2802266$ \\
\hline RXJ1527.3-3603 & 152722.9 & -360409 & $-23.6 \pm 2.3$ & $-34.4 \pm 2.4$ & SPM4 & 93 & $9.6 \pm 2.2$ & $15272286-3604087$ \\
\hline RXJ1529.3-3737 & 152919.0 & -373720 & $-19.2 \pm 0.9$ & $-23.5 \pm 0.9$ & SPM4 & 64 & $6.9 \pm 1.5$ & $15291901-3737205$ \\
\hline RXJ1529.7-3628 & 152947.3 & -362837 & $-16.6 \pm 1.6$ & $-20.8 \pm 1.7$ & SPM4 & 82 & $6.1 \pm 1.5$ & $15294727-3628374$ \\
\hline RXJ1538.7-4411* & 153843.1 & -441147 & $-19.1 \pm 1.9$ & $-27.2 \pm 1.9$ & SPM4 & 100 & $7.7 \pm 1.8$ & $15384306-4411474$ \\
\hline RXJ1539.7-3450 & 153946.4 & -345102 & $-14.9 \pm 2.0$ & $-19.3 \pm 2.1$ & SPM4 & 83 & $5.5 \pm 1.4$ & $15394637-3451027$ \\
\hline RXJ1540.3-3426A & 154018.5 & -342615 & $-14.7 \pm 3.7$ & $-19.5 \pm 3.6$ & SPM4 & 93 & $5.5 \pm 1.7$ & $15401850-3426146$ \\
\hline PDS395 & 154046.4 & -422953 & $-18.2 \pm 1.3$ & $-25.5 \pm 1.1$ & TYCHO2 & 98 & $7.4 \pm 1.8$ & $15404638-4229536$ \\
\hline $\operatorname{SSTc} 2 \mathrm{~d} J 1$ & 154148.3 & -350146 & $-13.0 \pm 4.1$ & $-17.2 \pm 4.0$ & SPM4 & 94 & $4.9 \pm 1.7$ & $15414827-3501458$ \\
\hline RXJ1544.0 & 154403.8 & -331111 & \pm 1.3 & $-26.9 \pm 1.4$ & SPM4 & 100 & $7.4 \pm 1.7$ & $15440376-3311110$ \\
\hline RXJ15 & 154641.2 & -361847 & $-12.7 \pm 1.9$ & $-23.8 \pm 2.0$ & SPM4 & 60 & $6.1 \pm 1.5$ & $15464121-3618472$ \\
\hline RXJ15 & 154645.1 & -345947 & $-17.4 \pm 3.6$ & $-20.6 \pm 3.4$ & SPM4 & 57 & $6.1 \pm 1.8$ & $15464506-3459473$ \\
\hline RXJ1547.1-3540 & 154708.4 & -354019 & $-13.3 \pm 2.2$ & $-25.7 \pm 2.2$ & SPM4 & 51 & $6.5 \pm 1.6$ & $15470841-3540195$ \\
\hline RXJ1547.6-4018 & 154741.8 & -401826 & $-18.7 \pm 1.1$ & $-27.6 \pm 1.1$ & SPM4 & 99 & $7.6 \pm 1.8$ & $15474176-4018267$ \\
\hline HMLup & 154750.6 & -352835 & $-9.7 \pm 4.1$ & $-22.0 \pm 3.9$ & SPM4 & 55 & $5.4 \pm 1.8$ & $15475062-3528353$ \\
\hline HNLup & 154805.2 & -351553 & $-10.3 \pm 7.2$ & $-19.6 \pm 7.1$ & SPM4 & 93 & $5.0 \pm 2.5$ & $15480523-3515526$ \\
\hline RXJ1548.7-3520 & 154842.5 & -352007 & $-11.2 \pm 2.9$ & $-16.1 \pm 2.8$ & SPM4 & 98 & $4.4 \pm 1.4$ & $15484253-3520066$ \\
\hline RXJ1548.9-3513 & 154854.1 & -351318 & $-17.4 \pm 2.1$ & $-27.7 \pm 2.1$ & SPM4 & 98 & $7.4 \pm 1.8$ & $15485411-3513186$ \\
\hline Sz76 & 154930.7 & -354951 & $-16.1 \pm 2.8$ & $-20.8 \pm 2.7$ & SPM4 & 72 & $6.0 \pm 1.6$ & $15493074-3549514$ \\
\hline HD141277* & 154945.0 & -392509 & $-18.2 \pm 2.3$ & $-24.4 \pm 2.2$ & TYCHO2 & 74 & $7.0 \pm 1.8$ & $15494499-3925089$ \\
\hline RXJ1550.7-3828 & 155046.7 & -382927 & $-10.3 \pm 1.3$ & $-16.0 \pm 1.3$ & SPM4 & 100 & $4.4 \pm 1.0$ & $15504672-3829267$ \\
\hline Sz77 & 155147.0 & -355643 & $-12.5 \pm 2.2$ & $-20.5 \pm 2.1$ & SPM4 & 98 & $5.4 \pm 1.4$ & $15514695-3556440$ \\
\hline RXJ1555.4-3338 & 155526.3 & -333822 & \pm 1.8 & $-28.1 \pm 1.8$ & SPM4 & 100 & 1.9 & -3338232 \\
\hline Sz81 & 155550.3 & -380133 & 1.4 & $-22.4 \pm 1.5$ & 14 & 81 & 1.4 & 3801329 \\
\hline RXJ15 & 155602.1 & -365528 & \pm 2.5 & $-19.2 \pm 2.4$ & SP & 68 & 4.8 & 155602 \\
\hline Sz82 & 155609.2 & -375606 & $-12.7 \pm 3.9$ & $-21.5 \pm 4.0$ & SPM4 & 98 & $5.6 \pm 1.8$ & 1556092 \\
\hline Hip78092 & 155641.9 & -421923 & $-13.9 \pm 1.0$ & $-25.5 \pm 1.0$ & TYCHO2 & 64 & $6.6 \pm 1.5$ & $15564188-4219232$ \\
\hline Sz126 & 155724.0 & -424004 & $-13.1 \pm 1.9$ & $-22.3 \pm 2.1$ & SPM4 & 93 & $5.9 \pm 1.5$ & $15572401-4240044$ \\
\hline Sz127 & 155730.4 & -421032 & $-10.0 \pm 1.7$ & $-12.3 \pm 1.8$ & SPM4 & 69 & $3.6 \pm 1.0$ & $15573035-4210324$ \\
\hline Sz128 & 155807.3 & -415148 & $-12.9 \pm 3.3$ & $-18.0 \pm 3.3$ & SPM4 & 90 & $5.1 \pm 1.6$ & $15580732-4151479$ \\
\hline RXJ1558.9-3646 & 155859.8 & -364620 & $-11.6 \pm 3.0$ & $-23.1 \pm 2.9$ & SPM4 & 74 & $5.9 \pm 1.6$ & $15585980-3646206$ \\
\hline CD-3610569 & 155949.5 & -362828 & $-29.4 \pm 2.7$ & $-46.0 \pm 2.8$ & TYCHO2 & 95 & $12.3 \pm 2.9$ & $15594951-3628279$ \\
\hline RXJ1559.9-3750 & 155954.2 & -375047 & $-11.0 \pm 1.3$ & $-19.8 \pm 1.3$ & SPM4 & 89 & $5.2 \pm 1.2$ & $15595416-3750469$ \\
\hline SSTc2dJ160000.6-422158 & 160000.6 & -422157 & $-10.4 \pm 2.3$ & $-15.8 \pm 2.5$ & SPM4 & 98 & $4.3 \pm 1.3$ & $16000060-4221567$ \\
\hline Sz131 & 160049.4 & -413004 & $-8.3 \pm 5.4$ & $-21.2 \pm 5.3$ & SPM4 & 63 & $5.2 \pm 2.1$ & $16004943-4130038$ \\
\hline RXJ1601.9-3613 & 160159.2 & -361255 & $-18.6 \pm 2.6$ & $-24.7 \pm 2.6$ & SPM4 & 51 & $7.0 \pm 1.8$ & $16015918-3612555$ \\
\hline EXLup & 160305.5 & -401825 & $-9.8 \pm 2.3$ & $-18.2 \pm 2.4$ & SPM4 & 91 & $4.7 \pm 1.3$ & $16030548-4018254$ \\
\hline HD1436 & 160345.4 & -435549 & $-12.5 \pm 1.1$ & $-22.6 \pm 1.4$ & TYCHO2 & 85 & $5.9 \pm 1.4$ & $16034536-4355492$ \\
\hline RXJ1603.8-3938* & 160352.5 & -393901 & $-17.1 \pm 2.1$ & $-29.3 \pm 2.1$ & SP & 97 & $7.7 \pm 1.8$ & $16035250-3939013$ \\
\hline HD143978 & 160457.1 & -385715 & $-27.8 \pm 1.2$ & $-46.8 \pm 1.6$ & TYCHO2 & 99 & $12.3 \pm 2.8$ & $16045707-3857157$ \\
\hline RXJ1605.5-3837 & 160533.3 & -383745 & $-12.4 \pm 2.5$ & $-22.7 \pm 2.5$ & SPM4 & 93 & $5.8 \pm 1.6$ & $16053329-3837451$ \\
\hline HOLup & 160700.6 & -390219 & $-10.1 \pm 2.5$ & $-18.1 \pm 2.5$ & SPM4 & 98 & $4.7 \pm 1.4$ & $16070061-3902194$ \\
\hline RXJ1607.2-3839 & 160713.7 & -383924 & $-12.9 \pm 2.1$ & $-18.0 \pm 2.2$ & SPM4 & 73 & $5.0 \pm 1.3$ & $16071370-3839238$ \\
\hline RXJ1608.0-3857 & 160800.0 & -385751 & $-13.4 \pm 2.1$ & $-20.3 \pm 2.1$ & SPM4 & 89 & $5.6 \pm 1.5$ & $16075996-3857510$ \\
\hline Sz96 & 160812.6 & -390833 & $-8.7 \pm 2.2$ & $-20.1 \pm 2.3$ & SPM4 & 56 & $4.9 \pm 1.3$ & $16081263-3908334$ \\
\hline RXJ1608.3-3843 & 160818.3 & -384405 & $-20.8 \pm 2.8$ & $-30.7 \pm 2.8$ & SPM4 & 70 & $8.4 \pm 2.1$ & $16081824-3844052$ \\
\hline Sz97 & 160821.8 & -390421 & $-10.3 \pm 2.7$ & $-19.8 \pm 2.7$ & SPM4 & 91 & $5.0 \pm 1.4$ & $16082180-3904214$ \\
\hline Sz99 & 160824.0 & -390549 & $-14.6 \pm 4.5$ & $-25.1 \pm 4.5$ & SPM4 & 100 & $6.6 \pm 2.1$ & $16082404-3905494$ \\
\hline RXJ1608.4-3840 & 160825.2 & -384056 & $-13.2 \pm 2.5$ & $-16.6 \pm 2.5$ & SPM4 & 51 & $4.8 \pm 1.3$ & $16082519-3840558$ \\
\hline Sz102 & 160829.7 & -390311 & $-12.7 \pm 4.3$ & $-19.7 \pm 4.4$ & SPM4 & 97 & $5.4 \pm 1.9$ & $16082972-3903110$ \\
\hline V856Sco & 160834.3 & -390618 & $-12.5 \pm 1.2$ & $-21.6 \pm 1.6$ & TYCHO2 & 99 & $5.7 \pm 1.4$ & $16083427-3906181$ \\
\hline RXJ1608.6-3922 & 160836.2 & -392302 & $-10.6 \pm 2.2$ & $-23.2 \pm 2.2$ & SPM4 & 57 & $5.8 \pm 1.6$ & $16083617-3923024$ \\
\hline
\end{tabular}


P.A.B. Galli et al.: A kinematic study and membership analysis of the Lupus star-forming region

Table 5. continued.

\begin{tabular}{|c|c|c|c|c|c|c|c|c|}
\hline ID & $\begin{array}{c}\alpha \\
(\mathrm{h}: \mathrm{m}: \mathrm{s})\end{array}$ & $\begin{array}{c}\delta \\
\left({ }^{\circ}, \prime \prime\right) \\
\end{array}$ & $\begin{array}{l}\mu_{\alpha} \cos \delta \\
(\mathrm{mas} / \mathrm{yr})\end{array}$ & $\begin{array}{c}\mu_{\delta} \\
\text { (mas/yr) }\end{array}$ & Source & $\begin{array}{c}p \\
(\%)\end{array}$ & $\begin{array}{c}\pi \\
\text { (mas) }\end{array}$ & 2MASSJ \\
\hline SSTc2dJ160839.8-392922 & 160839.7 & -392923 & $-18.0 \pm 3.9$ & $-27.5 \pm 3.9$ & SPM4 & 90 & $7.5 \pm 2.0$ & $16083974-3929228$ \\
\hline RXJ1608.9-3905 & 160854.3 & -390606 & $-8.4 \pm 2.5$ & $-20.4 \pm 2.5$ & SPM4 & 51 & $5.0 \pm 1.3$ & $16085427-3906057$ \\
\hline Sz111 & 160854.7 & -393743 & $-8.1 \pm 2.3$ & $-18.9 \pm 2.3$ & SPM4 & 59 & $4.6 \pm 1.3$ & $16085468-3937431$ \\
\hline Sz112 & 160855.5 & -390234 & $-9.6 \pm 2.8$ & $-18.4 \pm 2.9$ & SPM4 & 93 & $4.7 \pm 1.4$ & $16085553-3902339$ \\
\hline Sz113 & 160857.8 & -390223 & $-12.5 \pm 3.5$ & $-19.8 \pm 3.5$ & SPM4 & 97 & $5.3 \pm 1.6$ & $16085780-3902227$ \\
\hline V908Sco & 160901.9 & -390512 & $-7.7 \pm 2.1$ & $-18.4 \pm 2.2$ & SPM4 & 56 & $4.6 \pm 1.2$ & $16090185-3905124$ \\
\hline SSTc2dJ160904.6-392112 & 160904.5 & -392113 & $-10.6 \pm 2.6$ & $-16.4 \pm 2.7$ & SPM4 & 96 & $4.4 \pm 1.3$ & $16090452-3921125$ \\
\hline Sz115 & 160906.2 & -390852 & $-12.9 \pm 3.4$ & $-18.4 \pm 3.4$ & SPM4 & 84 & $5.1 \pm 1.6$ & $16090621-3908518$ \\
\hline Sz134 & 160912.3 & -414025 & $-13.3 \pm 2.1$ & $-20.4 \pm 2.2$ & SPM4 & 91 & $5.5 \pm 1.5$ & $16091226-4140249$ \\
\hline RXJ1609.4-3850 & 160927.4 & -385019 & $-10.3 \pm 2.0$ & $-17.3 \pm 2.0$ & SPM4 & 100 & $4.6 \pm 1.2$ & $16092739-3850186$ \\
\hline Sz116 & 160942.6 & -391941 & $-16.4 \pm 2.1$ & $-26.1 \pm 2.2$ & SPM4 & 93 & $7.0 \pm 1.7$ & $16094258-3919407$ \\
\hline Sz117 & 160944.4 & -391330 & $-12.7 \pm 2.5$ & $-20.1 \pm 2.5$ & SPM4 & 96 & $5.4 \pm 1.6$ & $16094434-3913301$ \\
\hline Sz118 & 160948.6 & $\begin{array}{lll}-39 & 11 & 17\end{array}$ & $-13.3 \pm 9.0$ & $-13.4 \pm 8.7$ & SPM4 & 73 & $4.1 \pm 2.9$ & 16094864-3911169 \\
\hline RXJ1609.9-3923 & 160954.0 & -392327 & $-7.6 \pm 2.1$ & $-14.9 \pm 2.2$ & SPM4 & 92 & $3.8 \pm 1.1$ & $16095399-3923275$ \\
\hline Sz119 & 160957.1 & -385948 & $-11.9 \pm 2.3$ & $-24.6 \pm 2.4$ & SPM4 & 70 & $6.2 \pm 1.6$ & $16095707-3859479$ \\
\hline Sz120 & 161010.6 & -400744 & $-5.2 \pm 1.1$ & $-6.4 \pm 1.2$ & TYCHO2 & 83 & $1.9 \pm 0.6$ & $16101054-4007437$ \\
\hline Sz122 & 161016.4 & -390805 & $-15.9 \pm 2.4$ & $-21.9 \pm 2.4$ & SPM4 & 57 & $6.2 \pm 1.6$ & $16101642-3908050$ \\
\hline Sz123 & 161051.6 & -385314 & $-7.1 \pm 2.5$ & $-16.9 \pm 2.6$ & SPM4 & 68 & $4.1 \pm 1.2$ & $16105158-3853137$ \\
\hline RXJ1612.0-3840 & 161201.4 & -384027 & $-9.8 \pm 1.0$ & $-15.7 \pm 1.1$ & SPM4 & 96 & $4.2 \pm 1.0$ & $16120140-3840276$ \\
\hline SSTc2dJ161207.6-381324 & 161207.6 & $\begin{array}{lll}-38 & 1324\end{array}$ & $-6.9 \pm 1.3$ & $-13.6 \pm 1.3$ & SPM4 & 93 & $3.4 \pm 0.9$ & $16120761-3813242$ \\
\hline RXJ1612.3-4012 & 161222.1 & -401252 & $-22.8 \pm 1.6$ & $-42.6 \pm 1.7$ & SPM4 & 77 & $10.9 \pm 2.4$ & $16122204-4012522$ \\
\hline SSTc2dJ161243.8-381503 & 161243.7 & -381503 & $-6.8 \pm 1.4$ & $-12.4 \pm 1.4$ & SPM4 & 99 & $3.2 \pm 0.9$ & $16124373-3815031$ \\
\hline RXJ1614.4-3808 & 161426.4 & -380800 & $-15.2 \pm 0.7$ & $-22.2 \pm 0.7$ & SPM4 & 54 & $6.1 \pm 1.3$ & $16142637-3807597$ \\
\hline HD147402* & 162329.6 & -395800 & $-11.6 \pm 1.0$ & $-24.2 \pm 1.2$ & SPM4 & 82 & $6.1 \pm 1.4$ & $16232955-3958008$ \\
\hline
\end{tabular}




\section{Discussion}

\subsection{Positions and parallaxes for pre-main sequence subclasses}

The Lupus moving group identified in this paper contains 31 CTTSs, 79 WTTSs and 4 HAeBes. Among the WTTSs we have 44 on-cloud and 35 off-cloud stars. Figure 11 displays the location of the various PMS sub-classes. We note that the Lupus 4 population consists mainly of CTTSs while in the remaining clouds we find both CTTSs and WTTSs, which may represent a selection effect of our input list of Lupus candidate stars (see Sect. 3). As already predicted, the off-cloud population contains only WTTSs (and one HAeBe) while the CTTSs are located in the immediate vicinity of the molecular clouds.

An interesting point arises when we compare the distances of these various TTS populations. We present in Table 6 the average parallaxes (distances) and standard deviations for each subgroup in the Lupus SFR. Our results show that the off-cloud WTTSs tend to be closer to us while the CTTSs are more distant. The large standard deviation of the on-cloud WTTSs is indicative of depth effects along the line of sight.

Table 6. Properties of the TTSs in Lupus. We provide for each TTS subclass the number of stars, average parallax and average distance with the corresponding standard deviations.

\begin{tabular}{lccc}
\hline Sample & Stars & $\begin{array}{c}\pi \pm \sigma_{\pi} \\
(\mathrm{mas})\end{array}$ & $\begin{array}{c}d \pm \sigma_{d} \\
(\mathrm{pc})\end{array}$ \\
\hline CTTS & 31 & $5.1 \pm 0.9$ & $196_{-29}^{+42}$ \\
WTTS (on-cloud) & 44 & $6.3 \pm 1.8$ & $159_{-35}^{+63}$ \\
WTTS (off-cloud) & 35 & $7.7 \pm 2.0$ & $130_{-27}^{+46}$ \\
\hline
\end{tabular}

\subsection{Notes on the parallaxes of Lupus subgroups}

The parallaxes derived in this paper allow us to investigate the properties of the various subgroups in this cloud complex. Figure 12 displays the location of Lupus members and the corresponding clouds. The histogram of parallaxes is presented in Fig. 13. In the following we discuss the parallaxes derived for these subgroups based on the results presented in Table 7 .

Table 7. Properties of the various subgroups in Lupus. We provide for each subgroup the number of stars, average parallax and average distance together with the corresponding standard deviations.

\begin{tabular}{lccc}
\hline Sample & Stars & $\begin{array}{c}\pi \pm \sigma_{\pi} \\
(\mathrm{mas})\end{array}$ & $\begin{array}{c}d \pm \sigma_{d} \\
(\mathrm{pc})\end{array}$ \\
\hline Lupus 1 & 19 & $6.0 \pm 1.1$ & $167_{-26}^{+37}$ \\
Lupus 2 & 10 & $5.9 \pm 1.1$ & $169_{-26}^{+39}$ \\
Lupus 3 & 42 & $5.8 \pm 1.9$ & $172_{-43}^{+84}$ \\
Lupus 4 & 7 & $5.2 \pm 1.0$ & $192_{-31}^{+46}$ \\
Lupus (off-cloud) & 36 & $7.5 \pm 1.9$ & $133_{-27}^{+45}$ \\
\hline Lupus (full sample) & 114 & $6.4 \pm 1.9$ & $156_{-36}^{+66}$ \\
\hline
\end{tabular}

Full sample: the average parallax and standard deviation is $\bar{\pi}=$ $(6.4 \pm 1.9)$ mas. The average error on parallaxes is $\bar{\sigma}_{\pi}=1.6$ mas and the average precision of the parallaxes derived in this paper is $25 \%$. We note the existence of background and foreground populations (as anticipated in Sect. 2), confirming that the Lupus complex occupies a large volume in space.

Off-cloud population: the WTTSs that form this population are not only scattered in angular extent but also in depth. We derived individual parallaxes for 15 stars in this region. The closest star, RXJ1534.6-4003, is at $87_{-17}^{+27}$ pc while the remotest star, RXJ1525.0-3604, is at $188_{-24}^{+33} \mathrm{pc}$. Different scenarios have been suggested to explain the dispersed population of WTTSs: the stars may have been formed in the vicinity of the clouds and have reached their present location because of the velocity dispersion in the group (Wichmann et al. 1997a); they might have been ejected with high velocities by dynamical interactions in multiple systems (Sterzik \& Durisen 1995); star formation could have occurred in small cloudlets that have now dispersed (Feigelson 1996). One important parameter in this discussion is the stellar age that can now be accurately determined for those stars with individual parallaxes (age determination goes beyond the scope of the present work and will be discussed in a forthcoming paper).

Lupus 1, 2 and 4: altogether they contain 32\% of moving group members. While Lupus 1 and 2 are located approximately at the same average distance and exhibit the same depth, Lupus 4 is more distant and its stars are more dispersed along the line of sight. We derived individual parallaxes for 5 stars in Lupus 1 and one star in Lupus 2. Our results for Lupus 4 are less accurate, because we computed only tentative parallaxes and our sample is biased towards CTTSs as mentioned before. However, Hip 78092 is a HAeBe star projected in the direction of Lupus 4 and HiPPARCos results for this star, $\pi_{H I P 97}=(5.04 \pm 1.18)$ mas and $\pi_{H I P 07}=(4.29 \pm 0.98)$ mas, confirm the approximate distance to the cloud derived in this paper.

Lupus 3: we find significant depth effects (tens of parsec) in Lupus 3 while the stars in the remaining clouds are less dispersed along the line of sight. One possibility to explain this result is the existence of various components along the line of sight that lie at different distances. We computed the average parallax and standard deviation of the CTTSs (16 stars) and WTTSs (24 stars) in Lupus 3 and find

$$
\begin{aligned}
& \bar{\pi}_{\text {CTTS }}=(4.8 \pm 0.8) \mathrm{mas}, \\
& \bar{\pi}_{\text {WTTS }}=(6.5 \pm 2.2) \mathrm{mas} .
\end{aligned}
$$

We note that the standard deviation of the WTTSs is more than twice as large as the one of the CTTSs. The depth of Lupus 3 derived from the the closest and remotest parts of this cloud is in good agreement with Lombardi et al. (2008, see Sect. 2). This preliminary result must be confirmed by further investigations since we derived accurate parallaxes for only a small number of stars. 


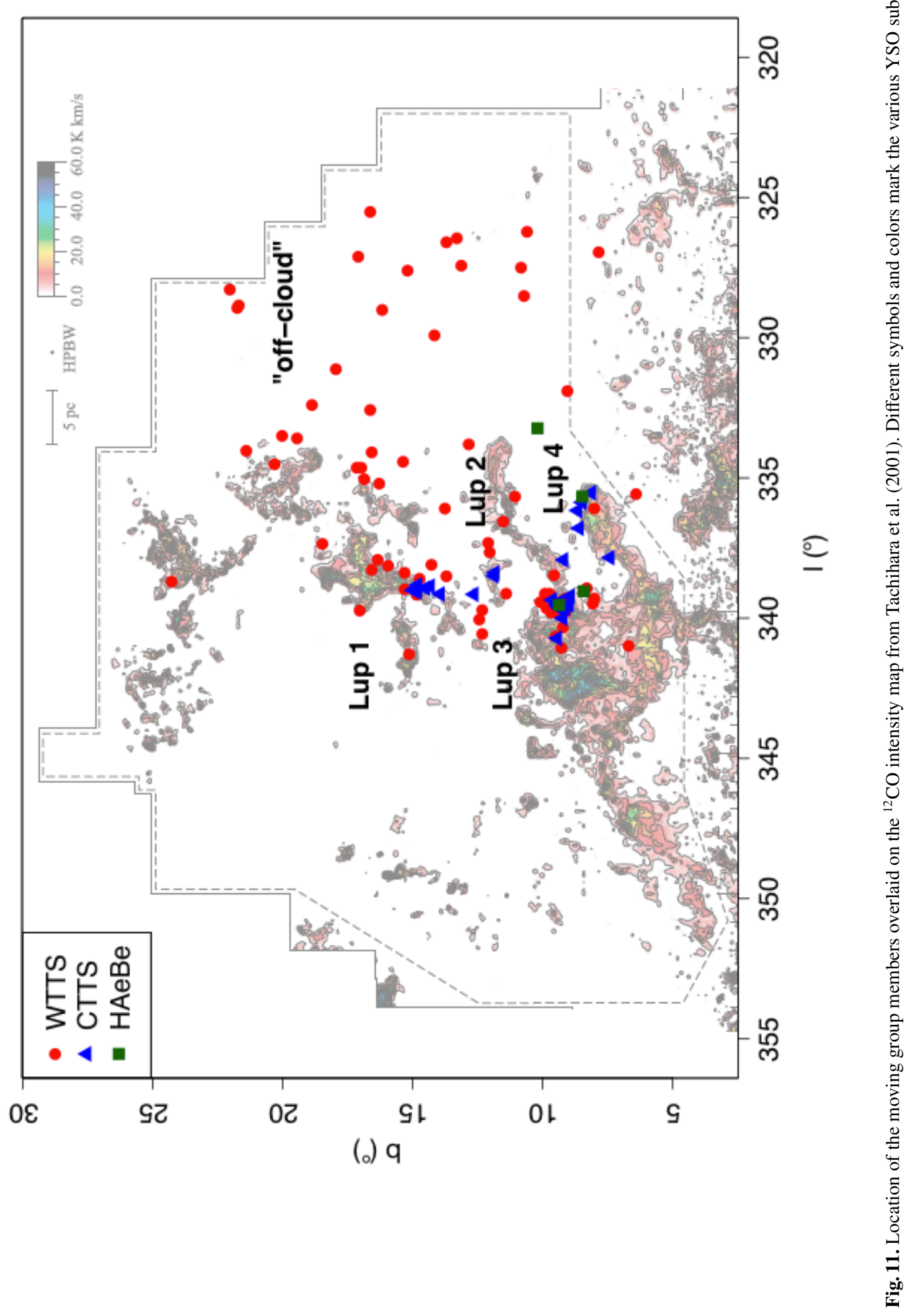




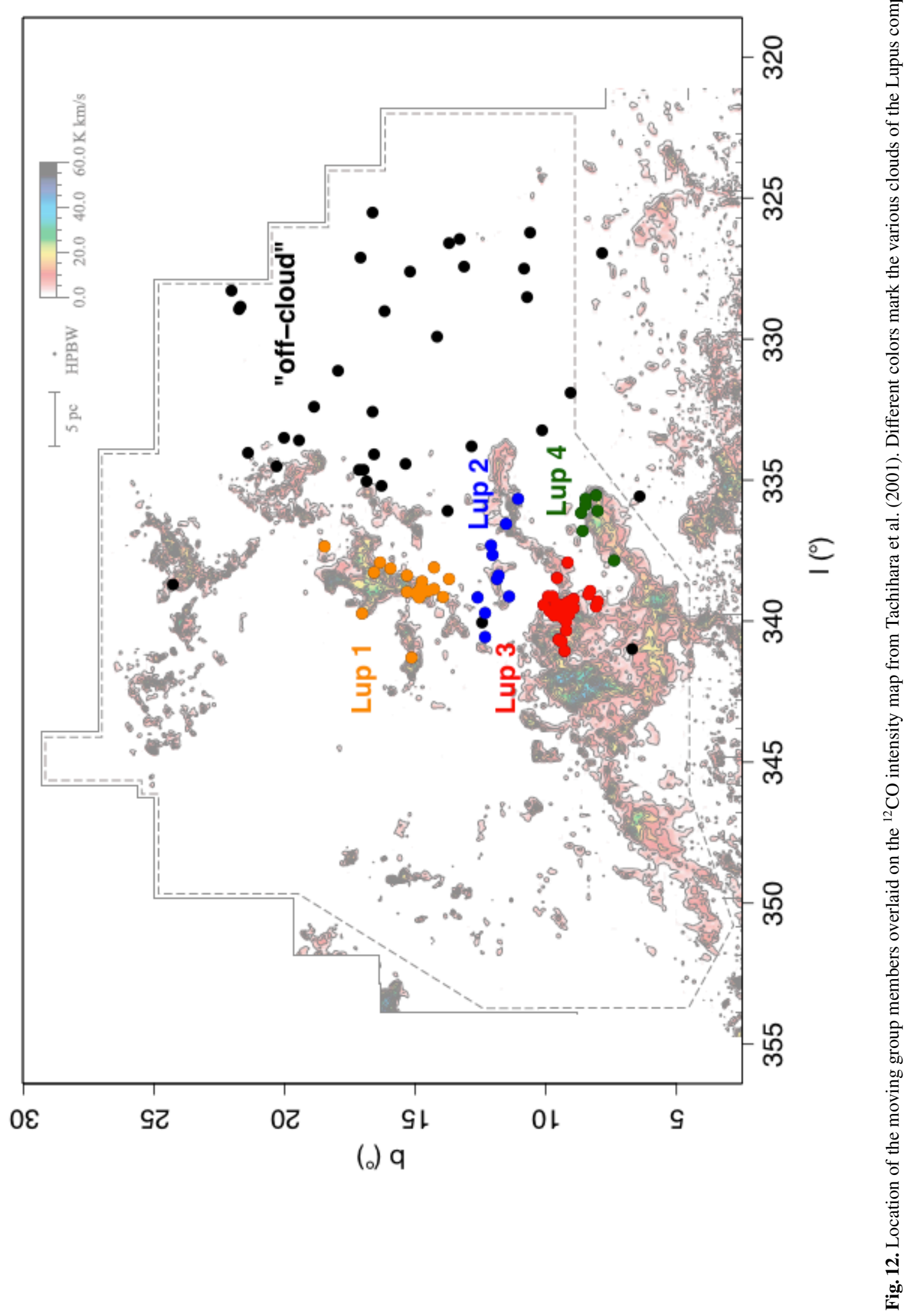


P.A.B. Galli et al.: A kinematic study and membership analysis of the Lupus star-forming region
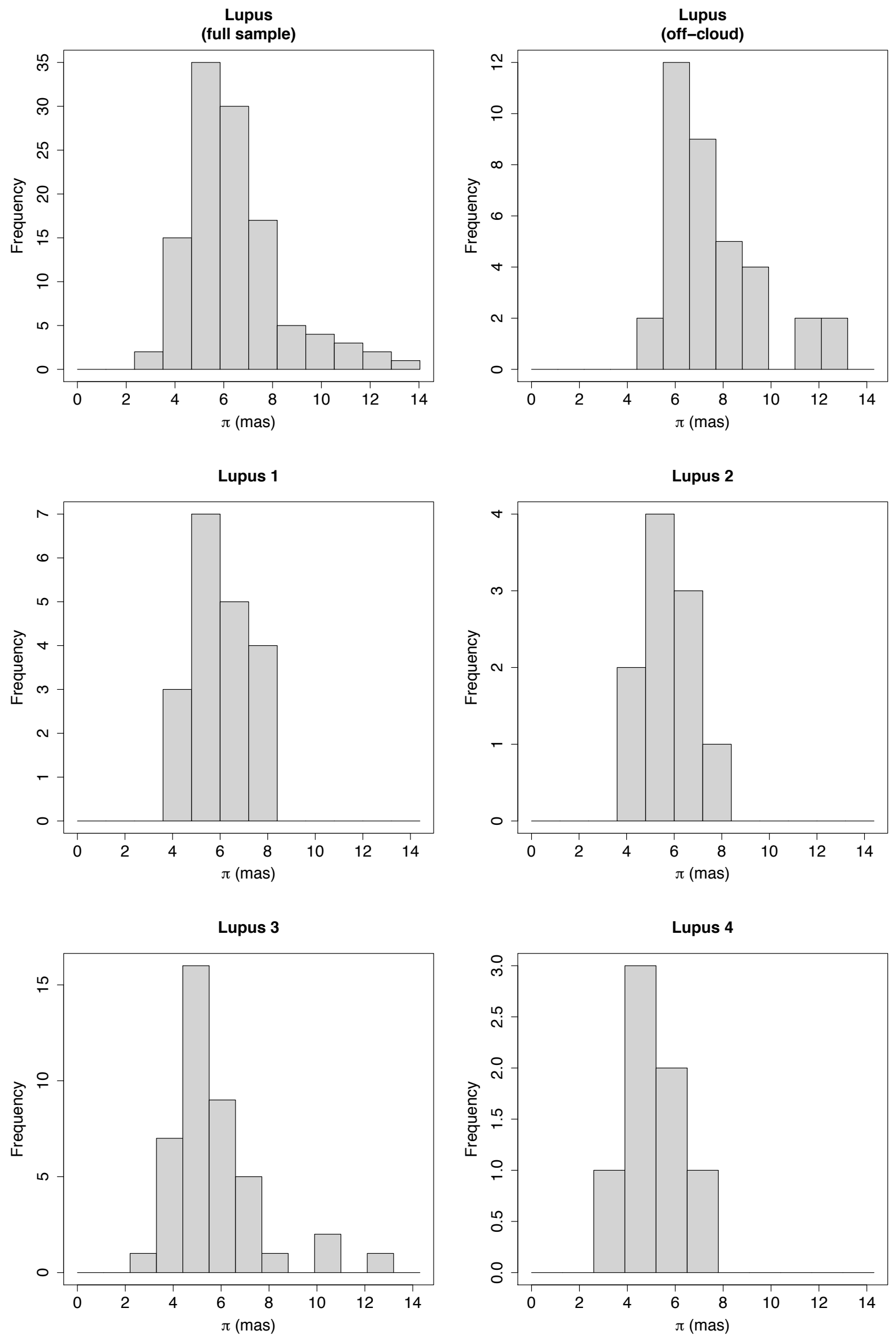

Fig. 13. Histogram of parallaxes for the various subgroups of the Lupus complex. 


\section{Conclusions}

We have identified a moving group of 114 stars in the Lupus SFR by applying our new $\mathrm{CP}$ search method, which made it possible to perform a membership analysis and to distinguish between Lupus and UCL stars. We applied the CP search method to different subsets of association members and confirmed our solution with Monte Carlo simulations. Because of the close proximity to the UCL subgroup and the difficulties encountered in separating these groups, we claim that we detected a minimum moving group in our CP analysis that may not contain all stars kinematically associated to Lupus. We derived accurate parallaxes for members with known RVs that we define as the Lupus core moving group and used the group spatial velocity to tentatively calculate approximate parallaxes for the remaining stars.

The determination of individual parallaxes is restricted to moving group members with known RVs. The RVs of many stars in the group either have never been measured or are of low quality and cannot be used to derive accurate parallaxes. We presented new RV measurements for 52 PMS stars of our starting sample of Lupus candidate stars based on spectroscopic observations performed with FEROS. We encourage observers to employ the available precise spectrographs to perform spectroscopic surveys of this complex SFR. Additional observations will also allow one to detect new binaries and confirm the youth of many PMS candidates.

We show that the CTTSs are located close or within the molecular clouds while the WTTSs are more dispersed in both angular extent and depth. We find evidence of substantial depth effects in Lupus 3 that must be confirmed by further investigations. These results represent a first step to better understand the structure of the Lupus cloud complex. The distances derived in this paper will be used in a forthcoming paper to investigate the physical properties of Lupus stars.

Acknowledgements. It is a pleasure to thank Johny Setiawan and Maren Mohler for their assistance with the FEROS Data Reduction Software and crosscorrelation techniques used for radial velocity computations. This research was funded by a doctoral fellowship from FAPESP and made use of the SIMBAD database operated at the CDS, Strasbourg, France.

\section{References}

Appenzeller, I. \& Mundt, R. 1989, A\&A Rev., 1, 291

Baranne, A., Queloz, D., Mayor, M., et al. 1996, A\&AS, 119, 373

Barbier-Brossat, M. \& Figon, P. 2000, A\&AS, 142, 217

Barbier-Brossat, M., Petit, M., \& Figon, P. 1994, A\&AS, 108, 603

Basri, G., Martin, E. L., \& Bertout, C. 1991, A\&A, 252, 625

Bertout, C. 1987, in IAU Symposium, Vol. 122, Circumstellar Matter, ed. I. Appenzeller \& C. Jordan, 23-38

Bertout, C., Basri, G., \& Bouvier, J. 1988, ApJ, 330, 350

Bertout, C. \& Genova, F. 2006, A\&A, 460, 499

Bertout, C., Robichon, N., \& Arenou, F. 1999, A\&A, 352, 574

Bobylev, V. V. 2006, Astronomy Letters, 32, 816

Comerón, F. 2008, The Lupus Clouds, ed. Reipurth, B., 295

Correia, S., Zinnecker, H., Ratzka, T., \& Sterzik, M. F. 2006, A\&A, 459, 909

de Bruijne, J. H. J. 1999, MNRAS, 310, 585

de Zeeuw, P. T., Hoogerwerf, R., de Bruijne, J. H. J., Brown, A. G. A., \& Blaauw, A. 1999, AJ, 117, 354

Dubath, P., Reipurth, B., \& Mayor, M. 1996, A\&A, 308, 107

Ducourant, C., Teixeira, R., Périé, J. P., et al. 2005, A\&A, 438, 769

Duflot, M., Figon, P., \& Meyssonnier, N. 1995, A\&AS, 114, 269

Dzib, S., Loinard, L., Mioduszewski, A. J., et al. 2010, ApJ, 718, 610

Dzib, S., Loinard, L., Rodríguez, L. F., Mioduszewski, A. J., \& Torres, R. M. 2011, ApJ, 733, 71

ESA. 1997, VizieR Online Data Catalog, 1239, 0

Feigelson, E. D. 1996, ApJ, 468, 306

Galli, P. A. B., Teixeira, R., Ducourant, C., Bertout, C., \& Benevides-Soares, P. 2012, A\&A, 538, A23

Ghez, A. M., McCarthy, D. W., Patience, J. L., \& Beck, T. L. 1997, ApJ, 481, 378
Girard, T. M., van Altena, W. F., Zacharias, N., et al. 2011, AJ, 142, 15

Gontcharov, G. A. 2006, Astronomy Letters, 32, 759

Gregorio-Hetem, J., Lepine, J. R. D., Quast, G. R., Torres, C. A. O., \& de La Reza, R. 1992, AJ, 103, 549

Grenier, S., Burnage, R., Faraggiana, R., et al. 1999, A\&AS, 135, 503

Guenther, E. W., Esposito, M., Mundt, R., et al. 2007, A\&A, 467, 1147

Herbig, G. H. 1962, Advances in Astronomy and Astrophysics, 1, 47

Herbig, G. H. \& Bell, K. R. 1988, Third Catalog of Emission-Line Stars of the

Orion Population : 3 : 1988, ed. Herbig, G. H. \& Bell, K. R.

Høg, E., Fabricius, C., Makarov, V. V., et al. 2000, A\&A, 355, L27

Hughes, J., Hartigan, P., \& Clampitt, L. 1993, AJ, 105, 571

James, D. J., Melo, C., Santos, N. C., \& Bouvier, J. 2006, A\&A, 446, 971

Johnson, D. R. H. \& Soderblom, D. R. 1987, AJ, 93, 864

Jones, B. F. \& Herbig, G. H. 1979, AJ, 84, 1872

Joy, A. H. 1945, ApJ, 102, 168

Kaufer, A., Stahl, O., Tubbesing, S., et al. 1999, The Messenger, 95, 8

Kharchenko, N. V., Scholz, R.-D., Piskunov, A. E., Röser, S., \& Schilbach, E. 2007, Astronomische Nachrichten, 328, 889

Knude, J. \& Hog, E. 1998, A\&A, 338, 897

Knude, J. \& Nielsen, A. S. 2001, A\&A, 373, 714

Krautter, J., Wichmann, R., Schmitt, J. H. M. M., et al. 1997, A\&AS, 123, 329

Loinard, L., Torres, R. M., Mioduszewski, A. J., et al. 2007, ApJ, 671, 546

Lombardi, M., Lada, C. J., \& Alves, J. 2008, A\&A, 480, 785

López Martí, B., Jiménez-Esteban, F., \& Solano, E. 2011, A\&A, 529, A108

Madsen, S., Dravins, D., \& Lindegren, L. 2002, A\&A, 381, 446

Makarov, V. V. 2007, ApJ, 658, 480

Malaroda, S., Levato, H., \& Galliani, S. 2006, VizieR Online Data Catalog, 3249

Mamajek, E. E. 2005, ApJ, 634, 1385

Mamajek, E. E., Meyer, M. R., \& Liebert, J. 2002, AJ, 124, 1670

Mathieu, R. D. 1986, Highlights of Astronomy, 7, 481

Melo, C. H. F. 2003, A\&A, 410, 269

Merín, B., Jørgensen, J., Spezzi, L., et al. 2008, ApJS, 177, 551

Nordström, B., Mayor, M., Andersen, J., et al. 2004, A\&A, 418, 989

Preibisch, T. \& Mamajek, E. 2008, The Nearest OB Association: ScorpiusCentaurus (Sco OB2), ed. Reipurth, B., 235

Racine, R. 1968, AJ, 73, 233

Reipurth, B., Pedrosa, A., \& Lago, M. T. V. T. 1996, A\&AS, 120, 229

Roeser, S., Demleitner, M., \& Schilbach, E. 2010, AJ, 139, 2440

Sartori, M. J., Lépine, J. R. D., \& Dias, W. S. 2003, A\&A, 404, 913

Schwartz, R. D. 1977, ApJS, 35, 161

Setiawan, J., Pasquini, L., da Silva, L., von der Lühe, O., \& Hatzes, A. 2003, A\&A, 397, 1151

Sterzik, M. F. \& Durisen, R. H. 1995, A\&A, 304, L9

Tachihara, K., Toyoda, S., Onishi, T., et al. 2001, PASJ, 53, 1081

Torres, C. A. O., Quast, G. R., da Silva, L., et al. 2006, A\&A, 460, 695

Torres, R. M., Loinard, L., Mioduszewski, A. J., et al. 2012, ApJ, 747, 18

Torres, R. M., Loinard, L., Mioduszewski, A. J., \& Rodríguez, L. F. 2007, ApJ, 671,1813

Torres, R. M., Loinard, L., Mioduszewski, A. J., \& Rodríguez, L. F. 2009, ApJ, 698,242

Udry, S., Mayor, M., \& Queloz, D. 1999, in Astronomical Society of the Pacific Conference Series, Vol. 185, IAU Colloq. 170: Precise Stellar Radial Velocities, ed. J. B. Hearnshaw \& C. D. Scarfe, 367

van Leeuwen, F. 2007, A\&A, 474, 653

Voges, W., Aschenbach, B., Boller, T., et al. 1999, A\&A, 349, 389

Walter, F. M. 1986, ApJ, 306, 573

Weise, P. 2010, PhD thesis, University of Heidelberg

Weise, P., Launhardt, R., Setiawan, J., \& Henning, T. 2010, A\&A, 517, A88

White, R. J., Gabor, J. M., \& Hillenbrand, L. A. 2007, AJ, 133, 2524

Wichmann, R., Covino, E., Alcalá, J. M., et al. 1999, MNRAS, 307, 909

Wichmann, R., Krautter, J., Covino, E., et al. 1997a, A\&A, 320, 185

Wichmann, R., Sterzik, M., Krautter, J., Metanomski, A., \& Voges, W. 1997b, A\&A, 326, 211

Zacharias, N., Finch, C., Girard, T., et al. 2010, AJ, 139, 2184

Zacharias, N., Finch, C. T., Girard, T. M., et al. 2012, VizieR Online Data Catalog, 1322, 0 


\section{Appendix A: Notes on radial velocities}

One important point is that the observed RVs for Lupus stars are expected to be low as compared to other SFRs, such as TaurusAuriga and Chamaeleon (see Bertout \& Genova 2006; James et al. 2006). The average value and standard deviation of the RVs presented in Table 2 for Lupus stars is $\bar{V}_{r}=(2.7 \pm 1.9) \mathrm{km} / \mathrm{s}$ and the average error is $\sigma_{V_{r}}=0.5 \mathrm{~km} / \mathrm{s}$. Here we investigate the errors on parallaxes and space velocities caused by uncertainties in RVs. A small variation $\Delta V_{r}$ in RVs accounts for the variation $\Delta \pi$ in parallaxes that can be approximated by

$\Delta \pi \simeq \frac{A \mu_{\|}}{\tan \lambda}\left(\frac{\Delta V_{r}}{V_{r}^{2}}\right)$.

This shifts the space velocity of the star by $\Delta V_{\text {space }}$ that is given as

$\Delta V_{\text {space }} \simeq \frac{A \mu_{\|}}{\sin \lambda}\left(\frac{\Delta \pi}{\pi^{2}}\right)$.

Using average values of position $(\alpha, \delta)=\left(237^{\circ},-38^{\circ}\right)$, proper motion $\left(\mu_{\alpha}^{*}, \mu_{\delta}\right)=(-15,-23)$ mas $/ \mathrm{yr}, \mathrm{RV}\left(V_{r}=2.7 \mathrm{~km} / \mathrm{s}\right)$, distance $(d=150 \mathrm{pc})$ and the CP solution derived in Sect. 5.2 .4 for the 114 moving group members, we note that a small shift $\Delta V_{r}=0.5 \mathrm{~km} / \mathrm{s}$ accounts for $\Delta \pi \simeq 2$ mas and consequently $\Delta V_{\text {space }} \simeq 5 \mathrm{~km} / \mathrm{s}$. We thus emphasize that high-precision RVs are needed to derive reliable kinematic parallaxes of Lupus stars. 
Apêndice D

\section{Proper motion and densification of the International Celestial Reference Frame in the direction of the Galactic bulge}

Ramachrisna Teixeira, Phillip Andreas Brenner Galli, Paulo Benevides-Soares, Jean François Le Campion, Messias Fidêncio, Alberto Krone-Martins, Christine Ducourant e Dimitris Sinachopoulos.

Publicado no periódico Astronomy \& Astrophyiscs, vol. 534, p. A91 (2011) 
Apêndice D. Proper motion and densification of the International Celestial Reference Frame in the direction of the 


\title{
Proper motion and densification of the International Celestial Reference Frame in the direction of the Galactic bulge ${ }^{\star}, \star \star$
}

\author{
R. Teixeira ${ }^{1,2}$, P. A. B. Galli ${ }^{1}$, P. Benevides-Soares ${ }^{1}$, J. F. Le Campion ${ }^{2}$, M. Fidêncio ${ }^{1}$, \\ A. Krone-Martins ${ }^{1,3}$, C. Ducourant ${ }^{2,1}$, and D. Sinachopoulos ${ }^{1,4}$ \\ ${ }^{1}$ Instituto de Astronomia, Geofísica e Ciências Atmosféricas, Universidade de São Paulo, Rua do Matão, 1226 - Cidade Universitária, \\ 05508-900 São Paulo - SP, Brazil \\ e-mail: teixeira@astro.iag.usp.br \\ 2 Observatoire Aquitaine des Sciences de l'Univers, CNRS-UMR 5804, BP 89, 33270 Floirac, France \\ 3 Faculdade de Ciências, Universidade de Lisboa, Ed. C8, Campo Grande, 1749-016 Lisboa, Portugal \\ ${ }^{4}$ Institute of Astronomy and Astrophysics, National Observatory of Athens, I. Metaxa \& Bas. Pavlou, 15236 Palea Penteli, Greece
}

Received 7 July 2011 / Accepted 30 August 2011

\begin{abstract}
Context. The definition, construction and realization of a reference system is one of the oldest and most fundamental tasks of astronomy. Currently, the International Celestial Reference System (ICRS), realized by the International Celestial Reference Frame (ICRF) or ICRF2, is the reference system adopted by the IAU. It is based on the very precise VLBI positions of a few hundred compact extragalactic radio sources. Despite its excellent precision, the ICRF is far from providing a reference system that is available for the entire sky and accessible to all observers. The ICRF has to be densified and extended to other wavelengths, mainly to the optical domain where the astronomical activities are more intense. For this reason, the IAU has recommended and encouraged works in this direction over the years. Many of them were developed to give sometimes good positions and proper motions, but they are very limited in magnitude, while others are extremely dense and deep in magnitude but have low accuracy, mainly for the proper motions. Nevertheless, all these contributions are very important because they are complementary.

Aims. We present a homogeneous and precise optical astrometric catalog that extends the ICRF in the direction of 12 low-extinction windows of the Galactic bulge and provides at the same time a useful database for kinematic studies.

Methods. The catalog was constructed on a total of 9 observational epochs spread over 15 years of observations with the CCD meridian circle of the Abrahão de Moraes Observatory (Valinhos - Brazil). The Galactic Bulge Valinhos Observatory Catalog (GBVOC) contains only those stars with at least 6 observational epochs and a time base of 10 years or more. We also imposed a minimum of 3 observations per star in each observational epoch and 20 observations in total.

Results. The catalog provides positions and proper motions for about 11600 stars with a mean internal precision of about 22 mas and $4.0 \mathrm{mas} / \mathrm{yr}$, which ensures a high quality and reliable extension of the ICRF in this direction. The GBVOC is extremely dense with more than 3500 stars per square degree. Comparison of the GBVOC with other densification works allows us to estimate the external precision of the GBVOC to be better than 42.0 mas and $5.5 \mathrm{mas} / \mathrm{yr}$ in position and proper motion.
\end{abstract}

Key words. Galaxy: bulge - astrometry - reference systems - dust, extinction - catalogs - proper motions

\section{Introduction}

The definition, construction, and realization of a reference system is the first step to study the positions and motions of any body. As in its beginning astronomy focused on the positions and motions of the celestial bodies, we can say that the realization of a reference system is one of the oldest and most fundamental task in astronomy.

The practical problems in realizing a reference system arise from the need to obtain at any given instant the coordinates of any celestial body as accurately as possible in an inertial reference system. This need requires that the adopted reference system (conventional reference system) be accessible, available, homogeneous, and rigid. In other words, it must be realized by a

* Based on observations collected with the CCD meridian circle of the Abrahão de Moraes Observatory - Valinhos - Brazil.

$\star \star$ Full Table 2 is only available at the CDS via anonymous ftp to cdsarc.u-strasbg.fr $(130.79 .128 .5)$ or via

http://cdsarc.u-strasbg.fr/viz-bin/qcat?]/A+A/534/A91 very homogeneous, accurate, dense, and multiwavelength catalog. This implies having a catalog with very accurate positions of a very large number of extragalactic objects or very accurate positions and proper motions of a large number of stars in the case of a stellar-based reference frame. Over time, in the past two centuries, this issue was somewhat solved by a series of fundamental catalogs (Fricke 1985) where the successor represented an extension and improvement of the predecessor.

In 1991 the IAU adopted a new reference system, the International Celestial Reference System (ICRS; Arias et al. 1995; Feissel \& Mignard 1998) realized by the very accurate VLBI positions of extragalactic radio sources in the International Celestial Reference Frame (ICRF; Ma et al. 1998). They satisfactorily solve some of these aspects such as the inertially for example. On this occasion, the IAU ended the tradition of fundamental catalogs and reference systems based on solar system dynamics and introduced a new strategy that was more coherent 
with the current observational capabilities and needs of astronomical research (see for example, Capitaine et al. 2000; Seidelmann \& Kovalevsky 2002). The extremely low density of ICRF or ICRF2 (Fey et al. 2010) and also the fact that it is based on radio positions while most of the astronomical works are done in the optical domain, implies that this reference system must be extended to this domain and densified by optical stellarbased catalogs. The density of the ICRF2 is less than 1 source (defining source or not) by $10^{\circ} \times 10^{\circ}$.

The primary optical realization of the ICRS is ensured by the Hipparcos catalog (ESA 1997), whose astrometric parameters were determined from spatial observations and, until now, gives the best astrometric parameters of all catalogs. The Hipparcos catalog is very limited for magnitudes, essentially brighter than $V \simeq 10$, and it is not as dense since it contains only 3 stars by $1^{\circ} \times 1^{\circ}$, as would be necessary for astronomical research. Therefore some others optical extensions of the ICRF were built to satisfy the requirements cited before: SPM (Platais et al. 1998), Tycho2 (Hög et al. 2000), UCAC2 (Zacharias et al. 2004), PM2000 (Ducourant et al. 2006), PPMX (Röser et al. 2008), UCAC3 (Zacharias et al. 2010), PPMXL (Röser et al. 2010), and UCAC4 (Zacharias 2011, priv. comm.) and many more. On average we can say that we have a mean precision better than $100 \mathrm{mas}$ in postion and $10 \mathrm{mas} / \mathrm{yr}$ in proper motion for these catalogs.

Of course, all these astrometric catalogs play an important role in the realization of the ICRS in the optical domain, but none fully meets the requirements such as quality, accessibility, availability, rigidity, etc. Moreover, in some cases, even if their overall quality is good, punctual proper motion works may be compromised by inaccuracy or large errors.

In this work we present an accurate and homogeneous catalog that aims to extends and densify the ICRF in the optical domain in a limited but very important region of the sky, the direction of 12 low-extinction windows of the Galactic bulge (Blanco 1988; Blanco \& Terndrup 1989; Dominici et al. 1999). This Galactic Bulge Valinhos Observatory Catalog (GBVOC) is based exclusively on CCD meridian circle observations at the Abrahão de Moraes Observatory, Valinhos - Brazil (Viateau et al. 1999). The GBVOC contains a total of 11619 stars with a very rich observational history, ensuring a very high quality for its positions and proper motions. With this catalog, more than the realization of the ICRS in the optical domain, we also aim at its reliable utilization in works such as determination of memberships and stellar kinematics.

The regions considered here are important because they are the only possible access to the Galactic bulge in the optical domain. The bulge is difficult to access because of the large amount of dust and gas, hence the high extinction, so the lower extinction windows, such as Baade's window (Baade 1951) and others found in Blanco (1988) and Blanco \& Terndrup (1989) offer privileged insight into the Galactic bulge (Dominici et al. 1999).

The observational material used in the construction of this catalog is presented in Sect. 2. In Sect. 3 we present the treatment of the observations. In Sect. 4 we present the catalog. In Sect. 5 we compare our results in position and proper motion with those from some of the most important astrometric catalogs. Finally, Sect. 6 discusses our results and draws a conclusion about our work.

\section{Observations}

All observations used here to compose this catalog were realized over 15 years (1996-2010, mean epoch 2002.2), with a single instrument, the Valinhos CCD meridian circle (Viateau et al. 1999; Dominici et al. 1999; Teixeira et al. 2000). This instrument is installed at the Abrahão de Moraes Observatory in Valinhos Brazil $\left(\phi=-23^{\circ} 00^{\prime} 06^{\prime \prime}, \lambda=+46^{\circ} 58^{\prime} 03^{\prime \prime}\right)$ and was automatized in 1995 with the introduction of a CCD micrometer (Viateau et al. 1999).

The CCD meridian circle works in drift scan mode, observing a field with a defined size in declination (14') and an arbitrary length in right ascension. In general, but not always and not for all low-extinction windows, we observed $20 \mathrm{~min}$ in right ascension for each field ensuring enough reference stars (Tycho-2) for the reduction. During the observation, the telescope was fixed and the electric charges moved along the columns of the CCD with the same velocity as the transit.

The diameter of the objective of the telescope is $0.19 \mathrm{~m}$ and its focal distance is $2.6 \mathrm{~m}$ resulting in a scale of 1 pixel $=1.5^{\prime \prime}$ for squares pixels of $19 \mu$. The Thomson 7895A CCD has a matrix of $512 \times 512$ pixels cooled down to $-40{ }^{\circ} \mathrm{C}$. The observations were carried out with a filter CG495+BG38 defining a bandpass of 5200 to $6800 \AA$ (Dominici et al. 1999; Teixeira et al. 2000). Even if this filter does not exactly match the Johnson $V$ one, we use the letter $V$ because it is also in the visual band, i.e., the letter $V$ does not indicate the filter but only the band. The exposure time is $51^{\mathrm{s}} / \cos (\delta)$.

The observational program was started in 1996 and finished in 2010. Unfortunately, in this period, some technical problems (2000 to 2005) meant we were able to observe only nine years, so they constituted a maximum of nine observational epochs to calculate the proper motions.

This program received an important stimulus in the 24th General Assembly of the IAU Manchester in 2000, in a "Densification of reference frame" meeting proposed by Dafydd Wyn Evans and also by the IAU Working Group, "Astrometry by small ground-based telescopes" led by William Thuillot.

In the total we observed more than one hundred thousand stars. However, the catalog contains only the stars that passed by the filters presented in the Sect. 4: minimum number of observation by star and minimum number of observational epoch.

\section{Data reduction}

For each low-extinction window, data were processed using the Bordeaux reduction pipeline (Ducourant et al. 2006), which takes the following steps. First, each observing strip is measured by fitting a two-dimensional Gaussian to each detected object, which leads to a catalog of ( $x, y$, flux). Then the measurements are preliminarily reduced classically using the Tycho- 2 catalog as reference to produce a catalog of $(\alpha, \delta, \mathrm{mag})$ and associated errors for each detected object of each strip.

The third step corresponds to the global fit of all observations of the considered window, which allows estimating the stellar parameters of all detected sources (Eichhorn 1960; Ducourant \& Rapaport 1991; Benevides-Soares \& Teixeira 1992; Teixeira et al. 1998). This process is iterative and the convergence is reached after two or three iterations. During this process, observations of a star are weighted with the mean residual of the strips where the star has been measured. During the global fit, a constraint is applied to Tycho- 2 stars so that no drift in the origin of the system is allowed. Finally a catalog of positions and proper motions is derived that contains stellar parameters and associated errors for each object. 
Table 1. Coordinates and statistic properties for each of 12 lowextinction windows in the Galactic bulge direction.

\begin{tabular}{|c|c|c|c|c|c|c|c|}
\hline id & $\begin{array}{l}\text { RA } \\
(\mathrm{hm})\end{array}$ & $\begin{array}{l}\text { Dec } \\
\left({ }^{\circ} \prime\right)\end{array}$ & Stars & $\sigma_{\delta}$ & $\sigma_{\alpha}$ & $\begin{array}{l}\sigma_{\mu_{\alpha} \cos \delta}(\mathrm{mas} / \mathrm{yr}\end{array}$ & $\sigma_{\mu_{\delta}}$ \\
\hline $\mathrm{BE}$ & 1810 & -3145 & 1169 & 21.1 & 23.7 & 3.9 & 4.4 \\
\hline BG & 1818 & -3251 & 1259 & 21.4 & 22.7 & 4.0 & 4.2 \\
\hline BJ & 1840 & -3449 & 524 & 16.7 & 18.2 & 3.2 & 3.4 \\
\hline LA & 1656 & -5244 & 1378 & 22.5 & 23.5 & 4.2 & 4.4 \\
\hline LB & 1718 & -4805 & 842 & 20.7 & 22.0 & 3.9 & 4.2 \\
\hline LC & 1729 & -4520 & 1256 & 22.1 & 22.9 & 4.1 & 4.2 \\
\hline LD & 1744 & -4041 & 980 & 18.9 & 21.1 & 3.6 & 4.0 \\
\hline LI & 1804 & -3347 & 987 & 24.2 & 26.4 & 4.6 & 5.1 \\
\hline LR & 1823 & -2610 & 892 & 21.5 & 24.0 & 4.0 & 4.4 \\
\hline LT & 1834 & -2123 & 907 & 24.9 & 26.9 & 4.2 & 4.5 \\
\hline LU & 1851 & -1318 & 542 & 19.9 & 21.4 & 3.7 & 3.9 \\
\hline LV & 1857 & -1023 & 883 & 21.3 & 22.2 & 4.1 & 4.3 \\
\hline
\end{tabular}

Notes. The windows are identified by a local identifiers as in Dominici et al. (1999).

\section{The catalog}

The catalog was constructed only with those stars with 20 observations or more, observed in at least six different years and at least three times each year. Moreover, we selected only the stars with a time base equal to ten years or more. This filtering ensures a good compromise between the number of observations, the observational epochs, and the time base, giving excellent precision on positions and proper motions. Finally, the stars constituting the GBVOC are spread in a region of $5 \mathrm{~min}$ in right ascension by $14^{\prime}$ in declination around each low-extinction window whose sizes are estimated about $20^{\prime} \times 20^{\prime}$. The final resulting catalog contains 11619 stars covering a total zone of more or less $15.0^{\circ} \times 0.2^{\circ}\left(12\right.$ regions of $\left.5 \mathrm{~min} \times 14^{\prime}\right)$ giving a density of more than 3500 stars by $1^{\circ} \times 1^{\circ}$.

The central coordinates of each low-extinction window are given in Table 1, where we also give some statistics such as the number of stars, the mean internal precision on positions, and proper motions in each window direction.

In a general way, this catalog provides precise and homogeneous positions and proper motions in the ICRF as realized by the Tycho- 2 catalog (Hög et al. 2000). In this way, it represents a very good densification in the optical domain of the ICRF reaching a limiting magnitude around 16.0 mag in the $V$ band, as shown in Fig. 1. This limitation in magnitude means that only a few stars in our catalog belong to the bulge, most of them being foreground stars.

As we can see in Fig. 2, where we present the distribution of the number of observations per star, most of the stars of the GBVOC have a very good observational history. Most stars were observed more than 50 times and the average number of observations per star is higher than 80 in both coordinates. The number of observations in right ascension and declination is not exactly the same as a consequence of independent eliminations in the reduction process, but this is only the case for very few stars.

This relatively very large number of observations ensures the high quality of the resulting positions. On average we have a mean internal precision of about 22 mas in both coordinates, as can be seen in the distribution of the internal precision in both coordinates shown in Fig. 3.

In Fig. 4 we show the internal precision on position as a function of magnitude. In this figure, dark is used for RA and red for Dec. We can note that even at the limit of magnitude the mean internal precision of the GBVOC positions remains

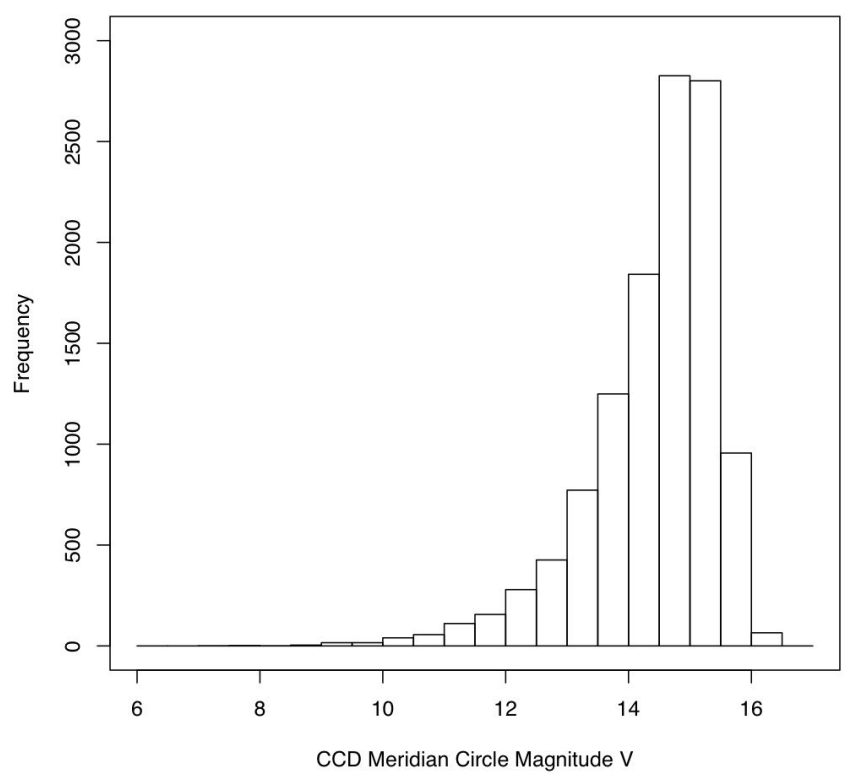

Fig. 1. Distribution of the stars by $V$-band magnitudes in the Galactic Bulge Valinhos Observatory Catalog (GBVOC).
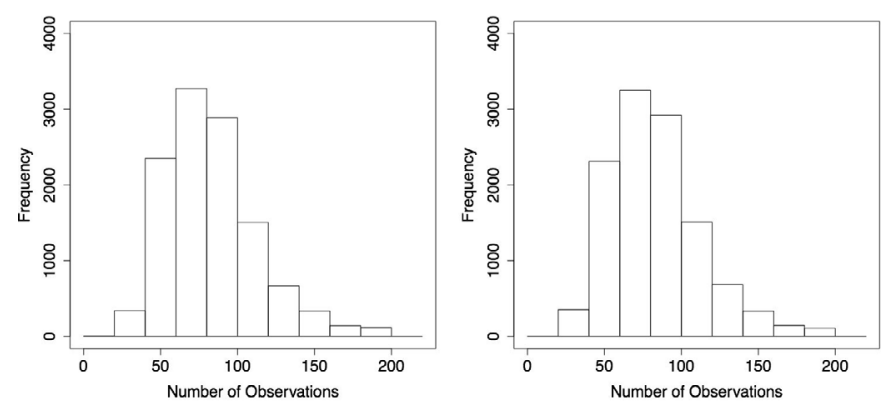

Fig. 2. Distribution of number of observations in right ascension (left panel) and declination (right panel) of the stars composing the GBVOC.
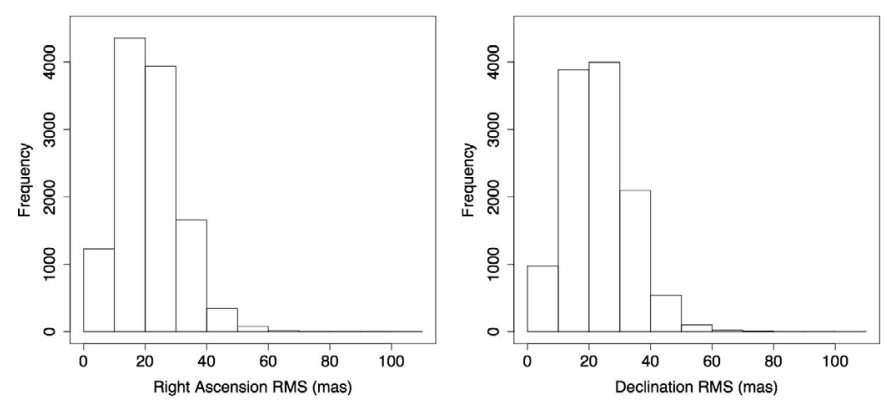

Fig. 3. Distribution of the internal precision achieved (rms) in right ascension (left panel) and declination (right panel).

highly compatible with those given in the best astrometric catalogs. Each point represents the mean of the rms of hundreds of stars. Only the first point is an exception because it results from only a few bright stars. For these stars the images were mostly saturated, which explains their lower quality. The limit of saturation in the Valinhos CCD meridian circle is about 8.5 mag.

The scenario is the same for proper motions. We provide homogeneous and precise proper motions for all stars. Their mean internal precision is about $4.0 \mathrm{mas} / \mathrm{yr}$, which is also compatible with the best astrometric catalogs. Of course, this precision depends on magnitudes and degrades for faint objects. In Fig. 5 we present the distribution of the proper motions of our 


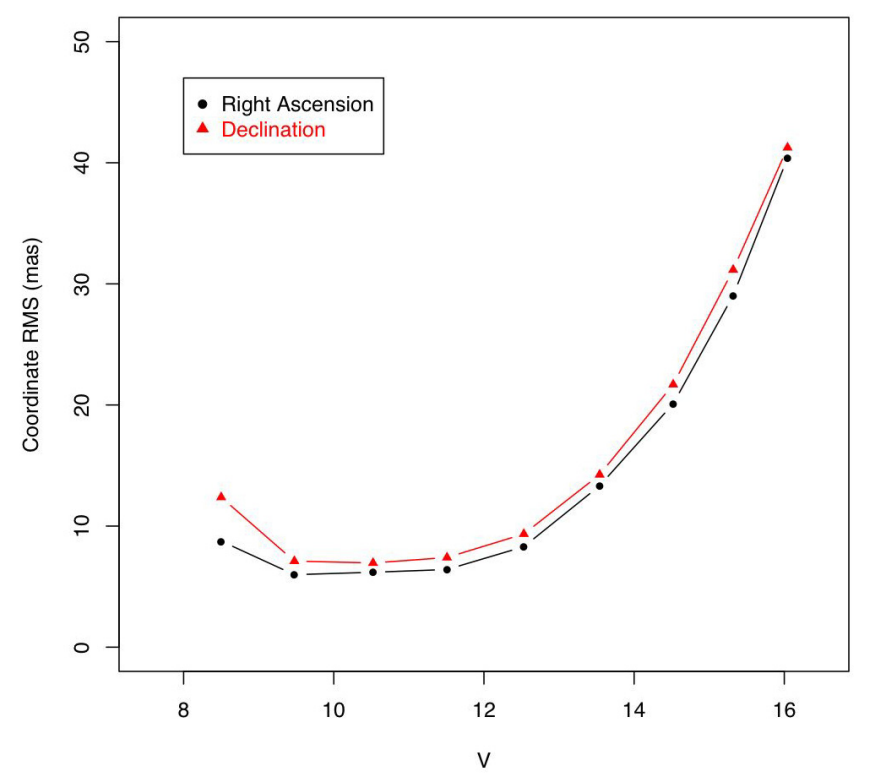

Fig. 4. Mean internal positional precision in right ascension (dark) and declination (red) as function of magnitude.
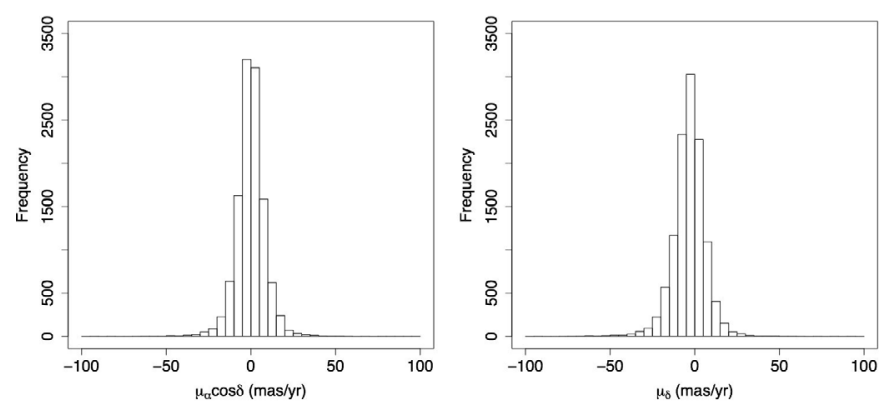

Fig. 5. Distribution of the proper motions in right ascension (left panel) and declination (right panel) in the GBVOC.
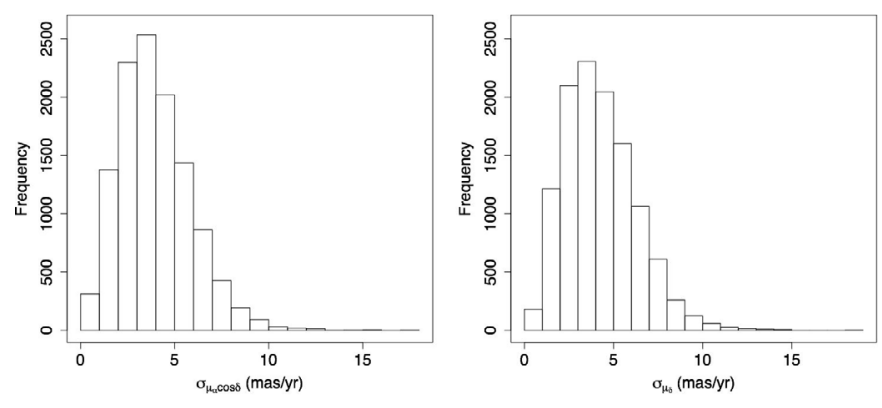

Fig. 6. Distribution of the internal precision achieved (rms) in right ascension (left panel) and declination (right panel) proper motions.

catalog. In Fig. 6 we show the distribution of the internal errors in proper motion. As can be noted here the distribution is very sharp (0-10 mas/yr), reflecting the high homogeneity of our proper motions.

In Fig. 7 we show the variation in the mean internal precision in proper motion as a function of magnitude. In this figure we can see that on average the internal errors in our proper motions in the best interval (until $V \simeq 14.0$ ) are lower than 2 mas/yr. Again, the first point does not follow the general trend because it is the mean of only a few bright stars whose images were frequently saturated. The quality of the positions and proper motions given in the GBVOC associated to the tight control of the

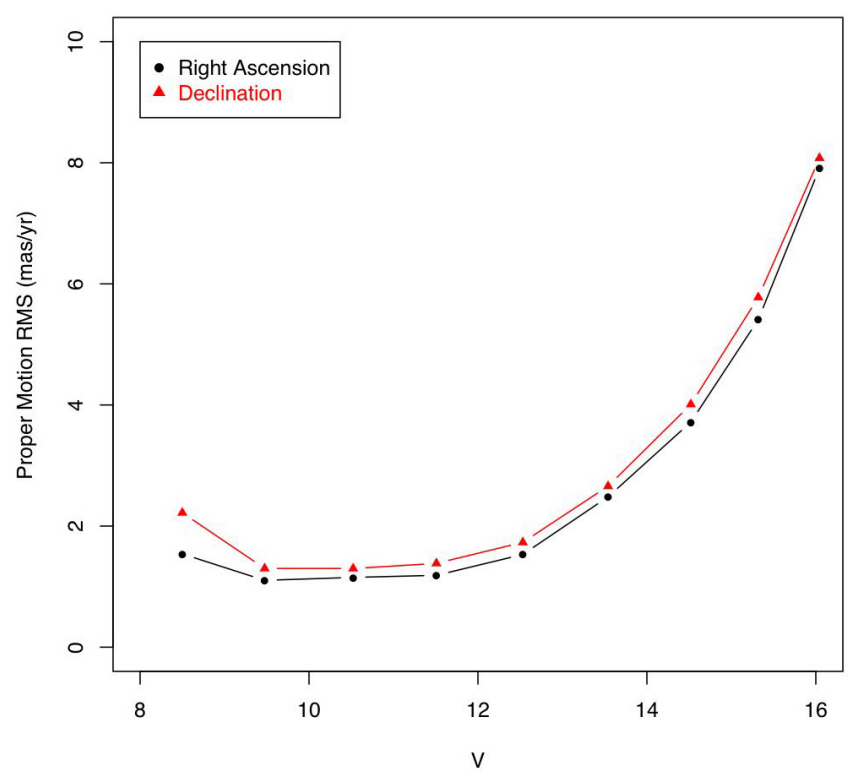

Fig. 7. Mean internal precision in right ascension (dark) and declination (red) proper motion as function of magnitude.

whole process of astrometric reduction transforms this artisanal work into an important extension of the ICRF in a very interesting region of the sky.

Although GBVOC is an astrometric catalog, we used the $J H K$ photometry of the 2MASS for calculating the infrared colors of our stars in order to estimate their effective temperature. We found 2MASS $J H K$ photometry for $98.2 \%$ of the GBVOC stars and we used it to compute their $J-K$ and $H-K$ colors. In their recent publication Worthey \& Lee (2011) present an empirical $U B V R I J H K$ color-temperature calibration for stars. We used this calibration and the $J-K$ and $H-K$ colors to derive the $T_{\text {eff }}$, which corresponds to the stars in our catalog.

We were not able to consider the reddening correction, which corresponds to the stars of GBVOC, because we do not know their distances. But since the stars in our catalog have $V \leq 16 \mathrm{mag}$, they are not so far so that they cannot be members of the Galactic bulge, since they are foreground stars. In any case, GBVOC contains stars that are in fields with low extinction. Nishiyama et al. $(2005,2006,2008)$ determined the ratios of total to selective extinction directly from observations in the optical $V$ band and near-infrared, and obtained $A_{V}: A_{J}: A_{H}: A_{K} \mathrm{~S}=$ 1:0.188:0.108:0.062 for reddened fields near the Galactic center. Considering these extinction ratios, it is not very important to apply a reddening correction to the $J-K$ and $H-K$ colors of the GBVOC stars.

Considering that GBVOC stars are in or close to Galactic disk we restricted our search to part of the Worthey \& Lee (2011) calibration concerning the population I stars with $[\mathrm{Fe} / \mathrm{H}] \geq 0.0$.

To calculate the $T_{\text {eff }}$ of each GBVOC star we calculated first the differences $\Delta(J-K)=(J-K)_{2 \text { MASS }}-(J-K)_{\text {Worthey }}$ and $\Delta(H-K)=(H-K)_{2 \mathrm{MASS}}-(H-K)_{\text {Worthey }}$ for all corresponding colors in the calibration. Then we determined as the effective temperature of a star the $T_{\text {eff }}$ value of the Worthey \& Lee (2011) calibration, which corresponds to the value of $\min \left(\Delta(J-K)^{2}+\right.$ $\left.\Delta(H-K)^{2}\right)$.

We present the distribution of the effective temperatures of the GBVOC stars in our sample in Fig. 8. We note that most of the stars have temperatures lower than $7000 \mathrm{~K}(98.5 \%)$. A large part of them have approximately solar temperatures of 


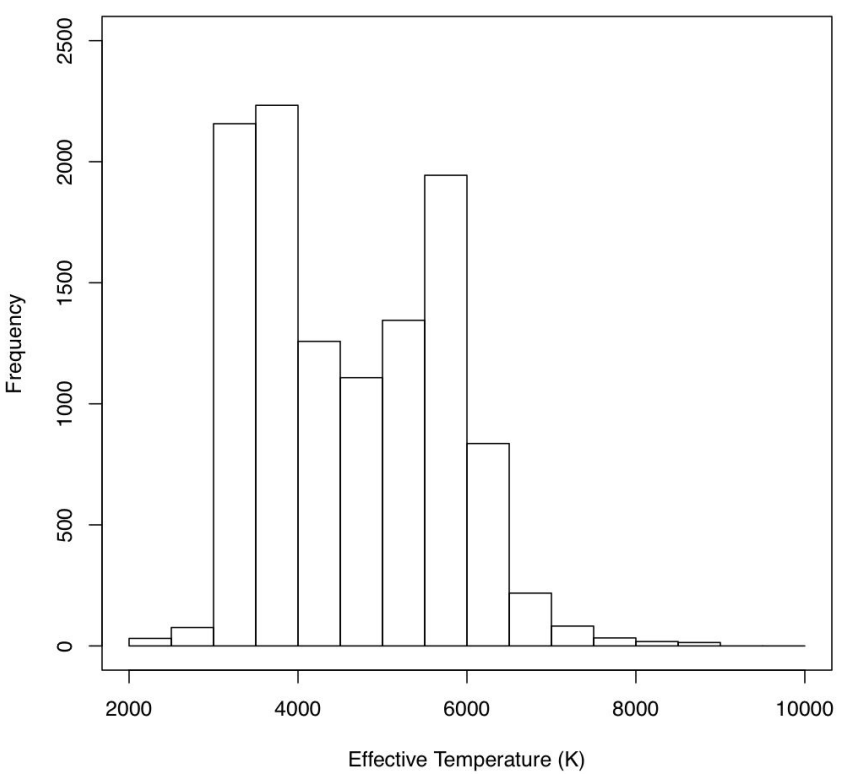

Fig. 8. Distribution of the effective temperature $T_{\text {eff }}$ of the stars of GBVOC.

$T_{\mathrm{eff}} \approx 5800 \pm 500 \mathrm{~K}(28.5 \%)$. Finally, many of the GBVOC stars are cool, with temperatures of $3500 \pm 500 \mathrm{~K}(38.5 \%)$.

The calibration classified $40 \%$ of these cool stars as having at least solar surface gravity $(\log g \geq 4$.0) with a mean magnitude of $11.6 \pm 0.9 \mathrm{mag}$, while an additional $32 \%$ of the stars in the same group of cool stars were classified as having $\log g \leq 1.0$, and their calculated mean magnitude is $10.3 \pm 1.0 \mathrm{mag}$.

Cool stars with $\log g \leq 1.0$ do not only show a tendency to be brighter than cool ones with $\log g \geq 4.0$, but they also have a tendency to be cooler, since their average effective temperature is $3420 \pm 200 \mathrm{~K}$, while stars with $\log g \geq 4.0$ have an average of $3620 \pm 250 \mathrm{~K}$. This means that cool stars classified with a low surface gravity of $\log g \leq 1.0$ are brighter and redder that the corresponding ones with at least solar surface gravity. Although the $\log g$ estimation derived by our method is not reliable, this finding does not contradict to stellar theories.

We compared our $T_{\text {eff }}$ estimation with values taken from the literature for all GBVOC stars brighter than $V=10 \mathrm{mag}$. The precision is about $70 \mathrm{~K}$, but the accuracy is disturbed by reddening, giving about $500 \mathrm{~K}$ systematically lower values.

Finally, in Fig. 9 and Table 2 we conclude this overview of the Galactic Bulge Valinhos Observatory Catalog by showing the distribution of the mean epochs and an extract of the catalog. The mean epoch of our catalog for both coordinates is around 2002. In a few cases, the mean epoch is not exactly the same in both coordinates as a consequence of independent elimination of measurements. Figure 9 illustrates the distribution of the mean epoch in both coordinates.

In Table 2 we present an extract of the GBVOC where we provide all essential information to use it properly. The meaning of each column is in general clear, except the "ident" column which gives a local identifier and the " $J$ ", " $H$ ", and " $K$ " columns that give the 2MASS magnitudes (Cutri et al. 2003). The capital letter " $T$ " after the 2MASS identifier indicates that this star is also presented in the Tycho 2 catalog. The $V$ magnitude given in this table corresponds to the magnitude observed with the Valinhos CCD meridian circle that is very close to the Tycho $V$ magnitude (Dominici et al. 1999). The positions are given to the mean epoch of observations.
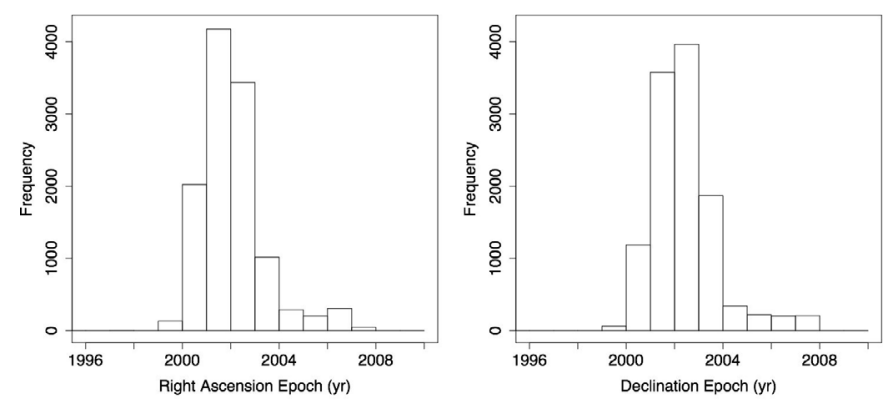

Fig. 9. Mean epoch distribution of the right ascension (left panel) and declination (right panel) in the GBVOC.

The catalog is divided by windows, as in Table 1 in which the stars are listed in order of ascending right ascension.

\section{Analysis and external precision}

Despite the very good internal precision of our astrometric measurements, it is important to discuss and evaluate the quality of our results by comparing them with other astrometric catalogs, as independently as possible. At the present time, there are a few very good astrometric catalogs such as Tycho-2 (Hög et al. 2000), PPMX (Röser et al. 2008) and UCAC3 (Zacharias et al. 2010) for instance. Each of these catalogs attempts to answer some of the basic requirements for the realization of a reference system, but despite their good quality, no one fills it completely. Soon, we will have the UCAC4 (Zacharias 2011, priv. comm.), which has not been published yet, but is used here as requested by the referee of this paper.

For example, Tycho-2 gives very precise positions and proper motions but is very limited in magnitude. The UCAC3 is deep in magnitude, relatively dense but the quality of its positions and proper motions is not as high as one would prefer, and much lower than those of Tycho-2. Moreover, now, this catalog is practically outdated (Zacharias 2011, priv. comm.). The PPMX, on the other hand, is much denser than Tycho- 2 but much less than UCAC3 and UCAC4. Its position and proper motion system is very accurate for its more precise components, but the catalog contains relatively few faint stars.

In fact, such comparisons of catalogs only crudely estimate our uncertainties. The difficulty comes, for instance, from the fact that the internal precisions are not necessarily the best representation of their quality. Even so, these comparisons are important and allow us to obtain, at least, an estimate of the upper limit of the errors in our catalog.

\subsection{Tycho-2 comparison}

The Tycho- 2 catalog (Hög et al. 2000) has about 2.5 million stars that are brighter than $V \simeq 12$ mag, nearly complete until $V \simeq$ $11 \mathrm{mag}$. This catalog results of the combination of various other catalogs and the astrometric observations of the sky mapper of the Hipparcos satellite. Their positions and proper motions are very precise, reaching $\simeq 60$ mas and $\simeq 2.5 \mathrm{mas} / \mathrm{yr}$.

This precision makes it an interesting reference for evaluate the external quality of the Galactic Bulge Valinhos Observatory Catalog. Of course, as we took this catalog as a reference in our reduction, our results depend on the Tycho- 2 positions and proper motions. However, owing to the global strategy of reduction applied here, this dependence is very weak, concerning 


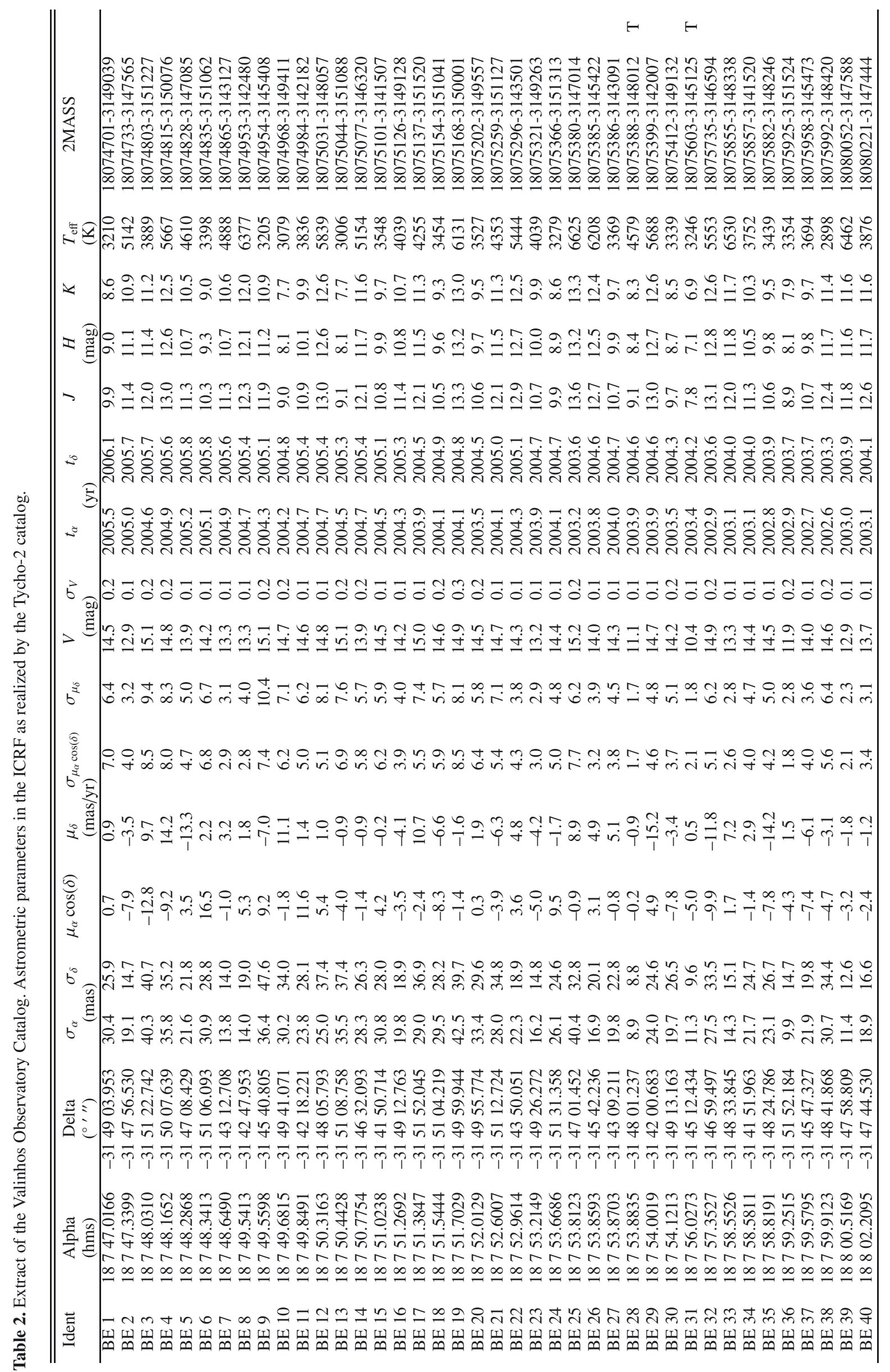



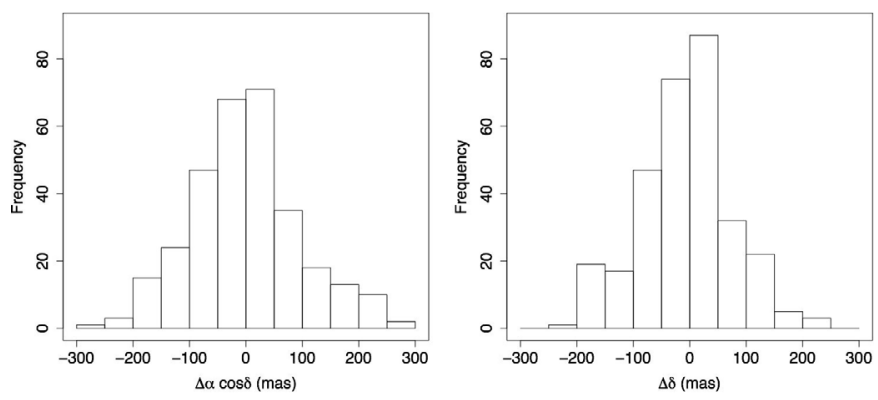

Fig. 10. Distribution of the differences GBVOC - Tycho 2 in right ascension (left panel) and declination (right panel).

only the orientation of the axes (Ducourant \& Rapaport 1991; Benevides-Soares \& Teixeira 1992; Teixeira et al. 1992). Even as the reference of the GBVOC reduction, Tycho- 2 can still give us a reliable estimate of the quality of our position and proper motion measurements.

First of all, we compared the GBVOC positions with those from Tycho-2 at the epoch 2000.0 (published epoch) in Fig. 10. We found 271 Tycho-2 stars in the GBVOC. In fact, this number should be higher, but because our purpose is to evaluate the GBVOC external precision, we selected here stars for which there was no doubt of the cross identification. Of course, some stars may have escaped from our filter, but certainly in an insignificant proportion. This is valid for the other comparisons.

As a result, we obtain a mean difference of -11.8 mas in right ascension and -6.7 mas in declination, with dispersions of 76.0 mas and 72.3 mas, respectively. These values show good agreement between our positions and those from the Tycho-2 catalog. The distribution of the mean differences (GBVOC Tycho-2) shown in Fig. 10 indicate that there are no significant systematic effects in our position system. The agreement, estimated by the dispersion, which can be considered good, could be even better if many stars were not in the magnitude limit of the Tycho- 2 catalog, where its quality is not very good (Hög et al. 2000). Moreover, we have a few bright stars here whose images were saturated in the CCD meridian circle.

If we take the mean errors on the Tycho- 2 right ascension and declination for these common stars into account, 56.7 mas and 69.5 mas, we can estimate the external precision of our positions to 50.6 mas and 19.9 mas in right ascension and declination. This difference between the estimated external precision in right ascension, and declination comes from the reference cata$\log$, Tycho-2, where the mean errors of the two coordinates are significantly different. Even so, we can say from this comparison that the external precision in the GBVOC positions are, on average, better than 50 mas.

In the case of the proper motions, the mean difference are $-1.2 \mathrm{mas} / \mathrm{yr}$ and $0.2 \mathrm{mas} / \mathrm{yr}$ with dispersions of $4.1 \mathrm{mas} / \mathrm{yr}$ and $5.1 \mathrm{mas} / \mathrm{yr}$ in right ascension and declination, respectively. These values suggest very good agreement between the proper motions of the two catalogs. If we also consider the Tycho- 2 proper motion errors, we conclude that the mean external precision of our proper motion is $3.9 \mathrm{mas} / \mathrm{yr}$, hence very near of the internal mean precision.

Figure 11 gives the comparison between our proper motions and those from the Tycho-2 catalog for 271 common stars. This figure leaves no doubt about the good agreement between these proper motions. The good distribution along the diagonal confirms the absence of systematic effects between these two catalogs in the considered region. Unfortunately, the Tycho-2
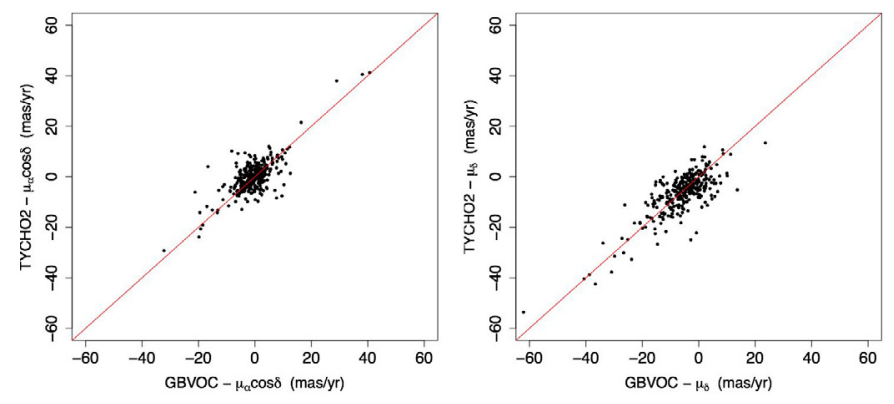

Fig. 11. Proper motion comparison with Tycho 2. Right ascension proper motion (left panel) and declination proper motion (right panel).

limiting magnitude restricts this comparison to the brighter stars where the meridian measurements are better, as in Fig. 7.

To summarize, for the brighter segment of our catalog we have in average, an external precision estimated from comparison with Tycho- 2 catalog better than 50 mas on position and $4 \mathrm{mas} / \mathrm{yr}$ on proper motion.

\subsection{UCAC4 comparison}

To estimate the external precision of our catalog for the fainter stars, initially we used the UCAC3 catalog (Zacharias et al. 2010) and then, following the suggestion of the referee of this paper, we worked with the new version of this series, the UCAC4 (Zacharias 2011, priv. comm.). We therefore took advantage of a beta version of the UCAC4 catalog kindly provided by Dr. N. Zacharias (2011, priv. comm.). In the very near future, the UCAC4 will be one of the most important astrometric catalogs with positions and proper motions better than those from UCAC3 (15-100 mas and 1-10 mas/yr). The UCAC4 like its predecessor will contain more than 100 million objects covering the whole sky. Its astrometric parameters result from the recent astrographic observations of the two hemispheres combined with those from many earlier catalogs.

Certanly, this catalog will be a very important extension to the ICRF mainly thanks to its amount of stars and limiting magnitude $(R \simeq 16)$. Its internal astrometric precision is better than that of UCAC3, and it probably presents a degradation in the fainter segment where, in general, the measured proper motions are only based on a few points, frequently on only two to four points (observational epochs). Nevertheless, we took it as an external reference to evaluate the GBVOC position and proper motion quality mainly for the faint stars, because almost all of our stars are present in the UCAC4 catalog.

In Fig. 12 we can see the distribution of the differences, at the epoch 2000.0 (published epoch), in right ascension (left) and declination (right) for about 8000 common stars. We were very restrictive in the selection of common stars working only with those separated by at most 200 mas. In this case we obtain a mean difference in right ascension and declination of 45.6 mas and -17.6 mas with dispersions of 60.4 mas and 43.2 mas. Taking the mean errors for these UCAC4 positions into account (22.9 mas in right ascension and 22.7 mas in declination), we can estimate the external precision for the GBVOC in 55.9 mas in right ascension and 36.8 mas in declination. The mean difference in right ascension suggests a significant systematic deviation in this coordinate between both catalogs. This deviation can also be seen in Fig. 12. Only based on this comparison we cannot decide which catalog is responsible for this effect. We also 

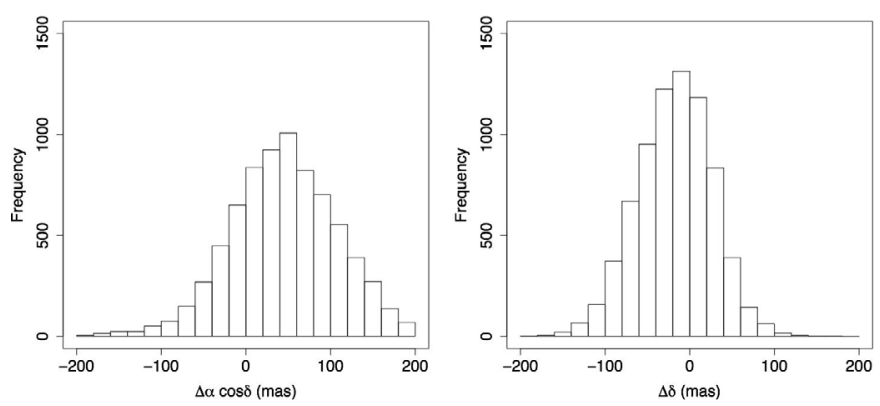

Fig. 12. Distribution of the differences GBVOC - UCAC4 in right ascension (left panel) and declination (right panel).
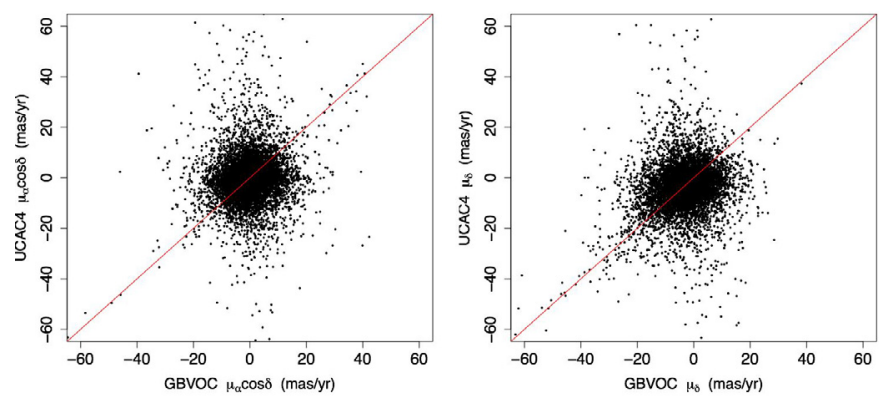

Fig. 13. Proper motion comparison with UCAC4. Right ascension proper motion (left panel) and declination proper motion (right panel).

note that the external errors based on the UCAC4 positions are twice the internal ones.

For the proper motions the mean differences are $0.6 \mathrm{mas} / \mathrm{yr}$ and $0.5 \mathrm{mas} / \mathrm{yr}$ with a dispersion of $10.1 \mathrm{mas} / \mathrm{yr}$ and $10.0 \mathrm{mas} / \mathrm{yr}$ in right ascension and declination respectively. The dispersions are greater than what we observe from the Tycho- 2 comparison, and it is probably a consequence of our working with fainter stars for which the quality of astrometric measurements is lower. When taking the UCAC4 mean internal errors into account for these stars (4.2 mas/yr and $4.1 \mathrm{mas} / \mathrm{yr}$ ), the estimated external mean errors for the GBVOC proper motions are $9.2 \mathrm{mas} / \mathrm{yr}$ and $9.1 \mathrm{mas} / \mathrm{yr}$. These values are very high and far from our internal estimate. The reason for that can be attributed to the relatively large dispersion of the differences in the case of the faint stars as showed in the following figures and discussion.

In Fig. 13 we illustrate the comparison between the UCAC4 and GBVOC proper motions. The behavior seen in these figures is quite unexpected. We expected a much larger dispersion than for Tycho-2 but still with a diagonal trend and without the vertical structure that we can see.

We note in these figures that, while the UCAC4 proper motions spread and reachs high values (we even have some points outside the graphic), in general, GBVOC remains concentrated between -20 mas/yr to $20 \mathrm{mas} / \mathrm{yr}$. We cannot be sure of the origin of this behavior, but it is clear that the UCAC4 proper motions are much more dispersed than ours.

Although our aim in this paper is not to test UCAC4, the unexpected behavior seen in Fig. 13 forces us to conduct a more detailed study to better understand what is happening. First of all, in our comparison with the Tycho- 2 catalog, we are led to believe that this difference comes from the faint stars, since our agreement with Tycho-2 (magnitude limit about $V \simeq 12.0$ ) is very good. To confirm that, we present in Fig. 14 the same comparisons but highlighting in orange the stars brighter than 12.0. As we can see, our proper motions are in good agreement with those from UCAC4 for the bright stars, and as a consequence
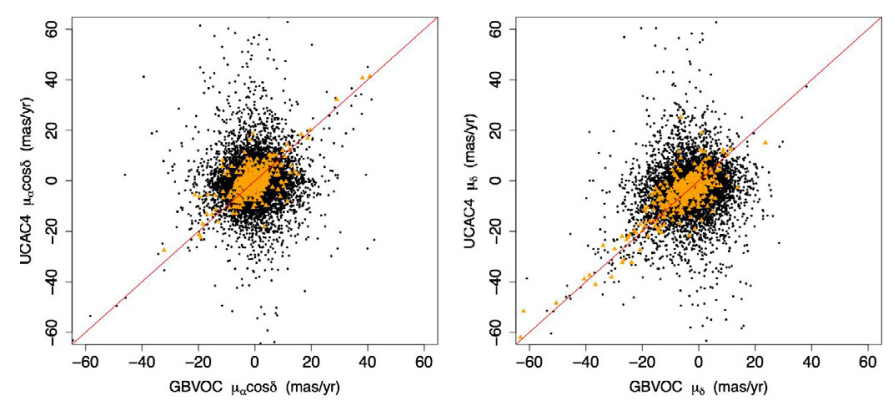

Fig. 14. Proper motion comparison with UCAC4. Right ascension proper motion (left panel) and declination proper motion (right panel) black dots $=$ all stars, red dots $=$ stars brighter than $V \simeq 12$.
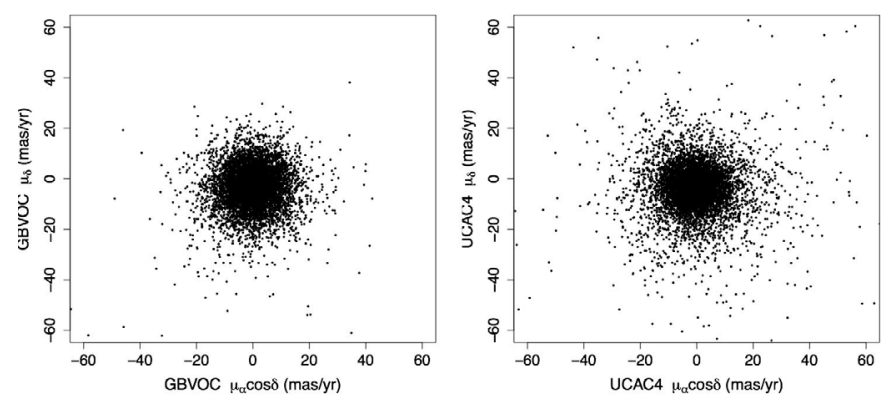

Fig. 15. Vector point diagram for Valinhos proper motions (left panel) and for UCAC4 proper motions (right panel).

the vertical structures seen in Figs. 13 are due to the faint stars. Unfortunately, this figure does not reveal the origin of the problem.

In Fig. 15 we plotted the proper motions in declination as a function of the proper motions in right ascension for both catalogs, GBVOC in the left panel and UCAC4 in the right. As we can see, the results obtained with the GBVOC is noticeably less spread than those with UCAC4. It is very difficult to explain the origin of these differences. We think that, since most stars are faint, the expected behavior is a less dispersed figure, as we see in the case of GBVOC proper motions. The large dispersion in UCAC4 could come from some bad proper motion determinations as a consequence of the automation in dealing with a huge amount of data and also from the higher density of stars in the direction of low-extinction windows.

The following diagrams give the GBVOC (left) and UCAC4 (right) proper motions as a function of the UCAC4 magnitude. In these cases we expected greater dispersion for the fainter stars as consequence of the lower precision. In other words, as the proper motion errors is larger for the fainter stars the dispersion should increase for higher magnitudes. It is easy to see this behavior in both catalogs. However, we also can see an unexpected concentration of larger proper motions from UCAC4 around magnitude 14 . The more likely in this case would be to have this concentration around the brighter stars. So, apparently the structure that we see in the comparison GBVOC $\times$ UCAC4 (Fig. 13) comes from UCAC4.

In either case, we cannot say for sure what the problem with the larger differences is. Since our goal in this paper is not to discuss the beta version of the UCAC4 catalog, we leave a more detailed analysis to more specific work in this direction.

Considering the behavior seen in these figures, only the comparison with UCAC4 is not sufficient for estimating the external qualities of our positions and proper motions. Moreover, 

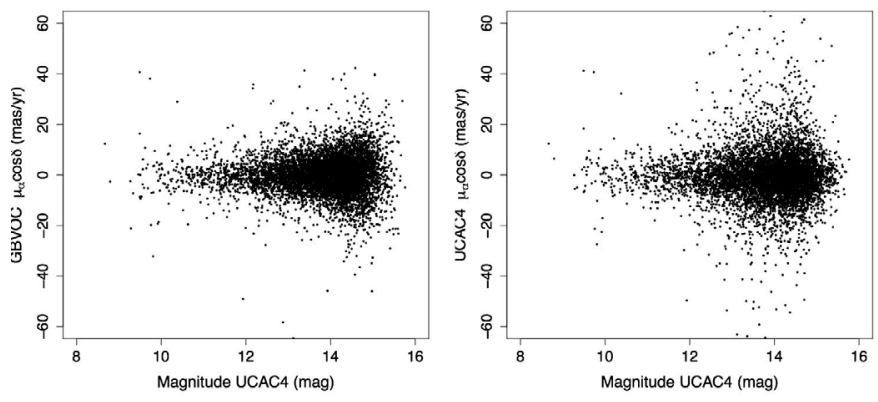

Fig. 16. Right ascension proper motion as a function of the UCAC4 magnitude: GBVOC (left panel) and UCAC4 (right panel).
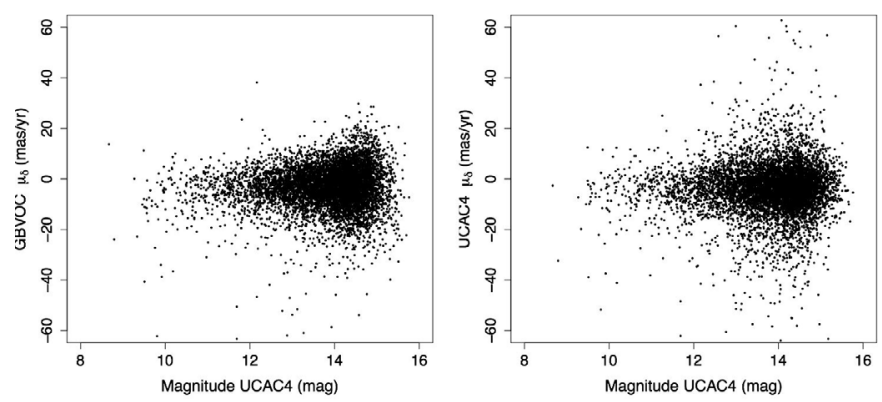

Fig. 17. Declination proper motion as a function of the UCAC4 magnitude: GBVOC (left panel) and UCAC4 (right panel).

the results of this comparison must be seen as encouraging independent measurement of the same astrometric quantities.

\subsection{PPMX comparison}

Owing to the observed and unexpected structure in Fig. 13, we decided to consider another catalog for comparison. An other possibility for estimating the GBVOC external precision is the recent PPMX catalog (Röser et al. 2008). The PPM-Extended catalog, PPMX, is another important extension of the ICRF in the optical domain. It contains about 20 million stars up to $V \simeq 15-16 \mathrm{mag}$, with a typical precision in proper motion of 1-2 mas/yr (Röser et al. 2008). Of course, this catalog is less dense than UCAC4, and the number of common stars is much smaller.

As in Sect. 5.2 we compared our positions and proper motions with those from PPMX catalog for 950 common stars. The obtained mean differences in right ascension and declination at the PPMX published epoch (2000.0) are 30.2 mas and -20.4 mas with dispersions of 58.7 mas and 55.4 mas. Taking the PPMX mean internal errors into account for these stars (34.7 mas and 35.2 mas), we estimated the GBVOC external mean errors in 47.3 mas and 42.8 mas in right ascension and declination. This comparison may be seen in Fig. 18.

The mean differences in proper motions in right ascension and declination are $-1.8 \mathrm{mas} / \mathrm{yr}$ and $2.9 \mathrm{mas} / \mathrm{yr}$ with dispersions of $11.4 \mathrm{mas} / \mathrm{yr}$ and $12.3 \mathrm{mas} / \mathrm{yr}$. Considering that the mean of the internal PPMX errors in proper motion in both coordinates is $9.8 \mathrm{mas} / \mathrm{yr}$ for these common stars, we can estimate the GBVOC external mean proper motion error as about $6.5 \mathrm{mas} / \mathrm{yr}$, not much larger than the internal proper motion error 4.0 mas/yr. The mean differences in position and proper motion are lower than the mean PPMX internal errors, suggesting no significant systematic deviation between both catalogs.
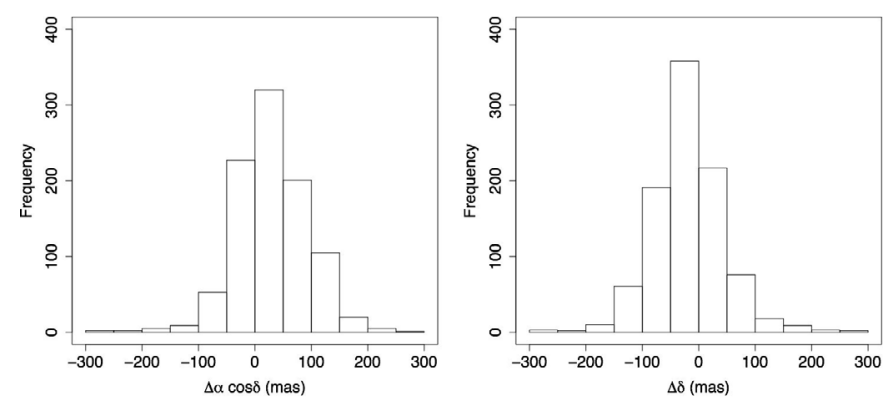

Fig. 18. Distribution of the differences GBVOC - PPMX in right ascension (left panel) and declination (right panel).
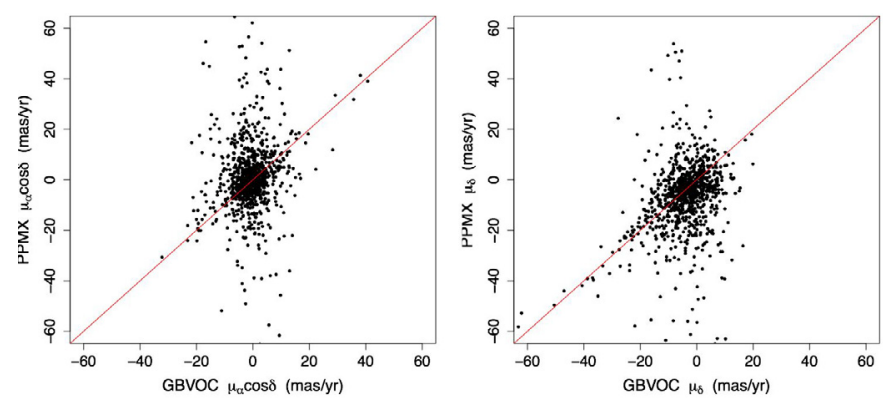

Fig. 19. Proper motion comparison with PPMX. Right ascension proper motion (left panel) and declination proper motion (right panel).
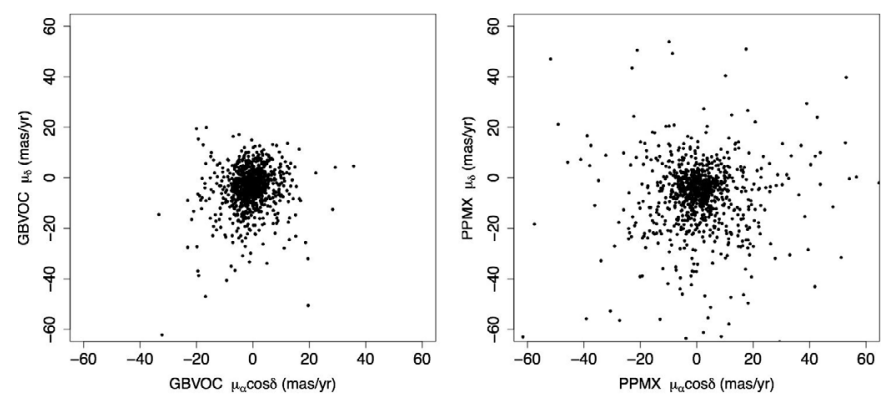

Fig. 20. Vector point diagram for Valinhos proper motions (left panel) and for PPMX proper motions (right panel).

In Fig. 19 we illustrate the results of the comparison between the PPMX and GBVOC proper motions. We do not have the clear diagonal trend one could expect. More or less as in the previous case (UCAC4), we can see a dispersion in the PPMX proper motions when the GBVOC values stay between -20 and $20 \mathrm{mas} / \mathrm{yr}$. The observed proper motion dispersion is lower than that of the UCAC4 comparison probably because we are working here with few common stars.

In Fig. 20 we plot the declination proper motion as a function of the right ascension proper motion for both catalogs. This suggests that the pattern seen in Fig. 19 comes from the differences in the measured proper motions.

Since the PPMX catalog is composed of three components (flags "S", "H", and "O", Röser et al. 2008) to better estimate our external precision, we chose to compare our positions and proper motions only to the best stars from the PPMX. We therefore constructed others graphs (Figs. 21 and 22) but only with those stars whose PPMX internal position and proper motion precision is highest, i.e., for the stars with a flag " $S$ " (survey) and " $H$ " (highest precision). We found 620 common stars that give mean differences in right ascension and declination of 21.4 mas and -18.0 mas with dispersion of 50.6 mas and 47.3 mas, resulting 

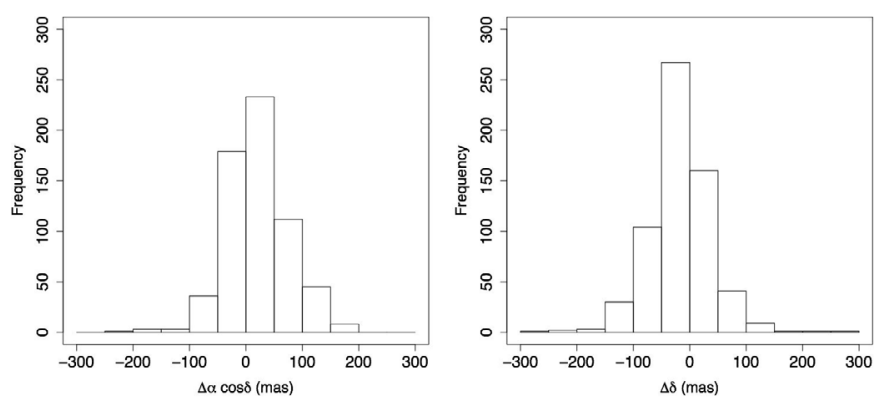

Fig. 21. Distribution of the differences GBVOC - PPMX (flags "S" and "H") in right ascension (left panel) and declination (right panel).
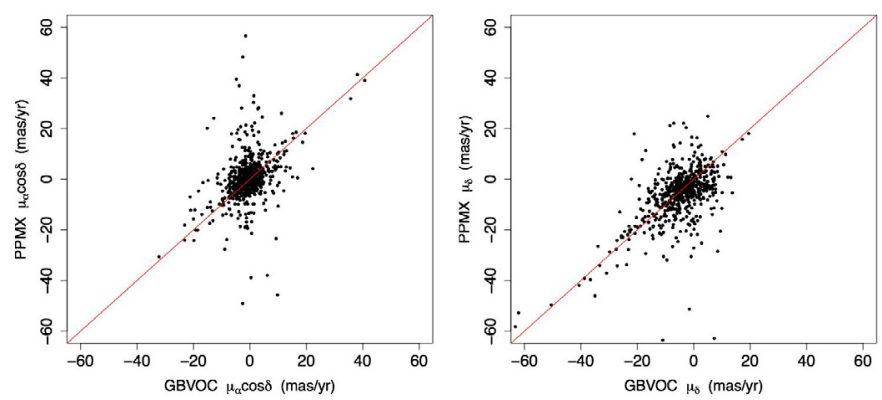

Fig. 22. Proper motion comparison with PPMX (flags "S" and "H"). Right ascension proper motion (left panel) and declination proper motion (right panel).

in an external error estimate for our positions of 40.8 mas in right ascension and 36.5 mas in declination.

For the proper motion, we obtained mean differences of $-0.7 \mathrm{mas} / \mathrm{yr}$ and $0.9 \mathrm{mas} / \mathrm{yr}$ in right ascension and declination proper motions with dispersion, respectively, of $6.7 \mathrm{mas} / \mathrm{yr}$ and $7.5 \mathrm{mas} / \mathrm{yr}$. Here there is no systematic effect. As we can see, the perpendicular structure to the abscissas axis almost disappeared, suggesting that the structure in Fig. 19 comes from the PPMX stars with lower accuracy. Considering that the mean internal errors for this segment of the PPMX catalog are 5.4 mas/yr and $5.5 \mathrm{mas} / \mathrm{yr}$ in proper motion in right ascension and declination, we can estimate the mean external precision of our proper motions as about $4.0 \mathrm{mas} / \mathrm{yr}$ and $5.1 \mathrm{mas} / \mathrm{yr}$, very close to the internal mean precision of GBVOC.

With this selection of PPMX stars ("S" and " $\mathrm{H}$ " stars), we could only compare the stars that are brighter than $V \simeq 14.0$ mag. The results in this case are not very different from those with the complete PPMX. From this part of the PPMX catalog, we can say that our positions are better than 40.8 mas, and the proper motions are better than 5.1 mas/yr.

\section{Conclusions}

We present an homogeneous and high-precision astrometric catalog that realizes the ICRF in the optical domain in the region around the lower extinction windows of the Galactic bulge. This catalog can be especially useful as a secondary reference frame and for kinematic studies in these regions.

For an external evaluation of the quality of our catalog, we considered three of the main astrometric catalogs. First, we compared the GBVOC positions and proper motions with those from the Tycho- 2 catalog. In this case we could estimate the mean external precision of our positions as better than 50 mas. For the proper motions, our estimation is of $3.9 \mathrm{mas} / \mathrm{yr}$, which is fully compatible with our internal estimates.

To test the fainter stars, we compared our results with the positions and proper motions from the UCAC4 and PPMX catalogs. From UCAC4 we could estimate a positional precision of about in 46.0 mas and $9.1 \mathrm{mas} / \mathrm{yr}$ in proper motion. Using the PPMX catalog we obtained 35.0 mas in position and $6.5 \mathrm{mas} / \mathrm{yr}$ in proper motion. On the other hand, taking the best of the PPMX catalog we obtain a mean positional external precision around 39.0 mas and in proper motion about 4.6 mas/yr. Eliminating the extreme cases from these comparisons we can estimate our external precision as better than 42.0 mas for the positions and $5.5 \mathrm{mas} / \mathrm{yr}$ for the proper motions.

Given this, we are sure that we are offering the community a good and homogeneous catalog that can be very useful for works on the Galactic bulge. At the same time, our comparison could reveal inconsistencies in some of the most important astrometric catalogs emphasizing the importance of artisanal astrometric works.

Finally, we again stress that the quality of the positions and proper motions in the Galactic Bulge Valinhos Observatory Catalog associated to the tight control of the whole process of astrometric reduction thanks to using relatively few stars transforms this work into an important extension of the ICRF in a very interesting region of the sky.

Acknowledgements. We would like to thank the Brazilian Organism FAPESP, CAPES, and CNPq and the French organizations COFECUB and CNRS. We are also grateful to the referee N. Zacharias for his comments, corrections, and suggestions and for the beta version of UCAC4.

\section{References}

Arias, E. F., Charlot, P., Feissel, M., \& Lestrade, J. F. 1995, A\&A, 303, 604 Baade, W. 1951, Publ. Obs. Univ. Mich., 10, 7

Benevides-Soares, P., \& Teixeira, R. 1992, A\&A, 253, 307

Blanco, V. M. 1988, AJ, 95, 1400

Blanco, V. M., \& Terndrup, D. M. 1989, AJ, 98, 843

Capitaine, N., Guinot, B., \& McCarthy, D. D. 2000, A\&A, 355, 398

Cutri, R. M., Skrustskie, M. F., Van Dyk, S., et al. 2003, 2MASS all Sky Catalog of Point Sources (Pasadena: IPAC/Caltech)

Dominici, T., Teixeira, R., Horvath, J., Medina Tanco, G., \& Benevides-Soares, P. 1999, A\&A, 136, 261

Ducourant, C., \& Rapaport, M. 1991, A\&A, 241, 303

Ducourant, C., Le Campion, J. F., Rapaport, M., et al. 2006, A\&A, 448, 1235

Eichhorn, H. 1960, Astron. Narchr., 285, 233

ESA 1997, HIPPARCOS and Tycho catalogue, ESA SP, 1200

Feissel, M., \& Mignard, F. 1998, A\&A, 331, L33

Fey, A. L., Gordon, D., \& Jacobs, C. S. 2010, IERS/IVS Working Group, IERS Technical Note, 35

Fricke, W. 1985, CeMec, 36, 207

Hög, E., Fabricius, C., Makarov, V., et al. 2000, A\&A, 355, L27

Ma, C., Arias, F. E., Eubanks, T. M., et al. 1998, AJ, 116, 516

Nishiyama, S., Nagata, T., Baba, D., et al. 2005, ApJ, 621, L105

Nishiyama, S., Nagata, T., Kusakabe, N., et al. 2006, ApJ, 638, 839

Nishiyama, S., Nagata, T., Tamura, M., et al. 2008, ApJ, 680, 1174

Platais, I., Girard, M. T., Kozhurina-Platais, V., et al. 1998, AJ, 116, 2556

Roëser, S., Schilbach, E., Schwan, H., et al. 2008, A\&A, 488, 491

Roëser, S., Demkeitner, M., Schilbach, E., et al. 2010, AJ, 139, 2440

Seidelmann, P. K., \& Kovalesvsky, J. 2002, A\&A, 392, 341

Teixeira, R., Requieme, Y., Benevides-Soares, P., \& Rapaport, M. 1992, A\&A, 264, 307

Teixeira, R., Camargo, J. I. B., Benevides-Soares, P., \& Requieme, Y. 1998, A\&A, 333, 1107

Teixeira, R., Ducourant, C., Sartori, M. J., et al. 2000, A\&A, 361, 1143

Viateau, B., Réquième, Y., Le Campion, J. F., et al. 1999, A\&AS, 134, 173

Worthey, G., \& Lee, H. 2011, ApJS, 193, 1

Zacharias, N., Urban, S., Zacharias, M., et al. 2004, AJ, 127, 3043

Zacharias, N., Finch, C., Girard, T., et al. 2010, AJ, 139, 2184 